Florida International University FIU Digital Commons

9-14-2016

\title{
Multilevel modeling issues and the measurement of stress is multilevel data
}

Tyler Stout

Florida International University, tstou001@fiu.edu

DOI: $10.25148 /$ etd.FIDC001246

Follow this and additional works at: https://digitalcommons.fiu.edu/etd

Part of the Industrial and Organizational Psychology Commons, and the Quantitative Psychology Commons

\section{Recommended Citation}

Stout, Tyler, "Multilevel modeling issues and the measurement of stress is multilevel data" (2016). FIU Electronic Theses and Dissertations. 2979.

https://digitalcommons.fiu.edu/etd/2979

This work is brought to you for free and open access by the University Graduate School at FIU Digital Commons. It has been accepted for inclusion in FIU Electronic Theses and Dissertations by an authorized administrator of FIU Digital Commons. For more information, please contact dcc@fiu.edu. 


\section{FLORIDA INTERNATIONAL UNIVERSITY}

Miami, Florida

MULTILEVEL MODELING ISSUES AND MEASUREMENT OF STRESS IN

\section{MULTILEVEL DATA}

A dissertation submitted in partial fulfillment of the

requirements for the degree of

DOCTOR OF PHILOSOPHY

in

PSYCHOLOGY

by

Tyler James Stout

2016 
To: Dean Michael R. Heithaus

College of Arts, Sciences, and Education

This dissertation, written by Tyler James Stout, and entitled Multilevel Modeling Issues and Measurement of Stress in Multilevel Data, having been approved in respect to style and intellectual content, is referred to you for judgement.

We have read this dissertation and recommend that it be approved.

Chockalingam Viswesvaran

\begin{tabular}{rr}
\hline Stacy Frazier \\
\hline Wensong Wu \\
\hline Stefany Coxe, Major Professor
\end{tabular}

Date of Defense: September 15, 2016

The dissertation of Tyler James Stout is approved.

Dean Michael R. Heithaus

College of Arts, Sciences, and Education

Andrés G. Gil

Vice President for Research and Economic Development and Dean of the University Graduate School

Florida International University, 2016 


\title{
ABSTRACT OF THE DISSERTATION
}

MULTILEVEL MODELING ISSUES AND MEASUREMENT OF STRESS IN

\section{MULTILEVEL DATA}

\author{
by
}

Tyler James Stout

Florida International University, 2016

Miami, Florida

\section{Professor Stefany Coxe, Major Professor}

Multilevel datasets are commonly used and increasingly popular in research in the organizational and other social sciences. These models are complex and have many elements beyond those found in more traditional linear models. However, research on how multilevel models perform is lacking.

The current paper examined the impact of common factors (average cluster size, cluster size distribution, average number of clusters, strength of the intraclass correlation coefficient, and effect sizes of individual and cluster level variables, and their interaction) in multilevel datasets. Monte Carlo data simulation was used across 6,144 factorcombination conditions. The results of study factors on observed intraclass correlation coefficients, calculated design effect, and empirical design effect are discussed.

The results of this study have implications for both researchers in both academic and applied fields. The scale of the simulation variables allow it to be germane to datasets from across the social sciences. However, the nature of data simulation and analysis is such that there are still many elements that can and should be accounted for in future research. 


\section{TABLE OF CONTENTS}

CHAPTER

PAGE

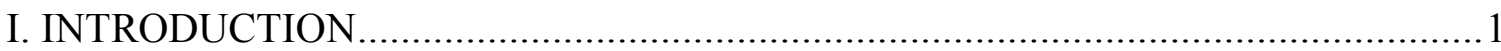

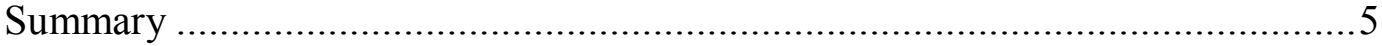

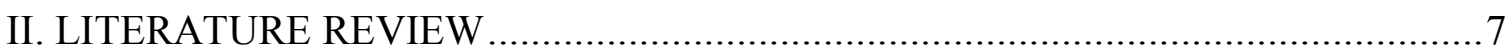

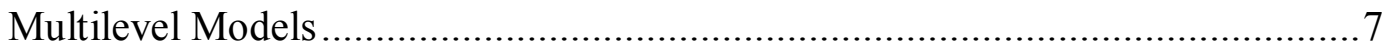

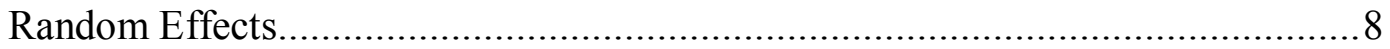

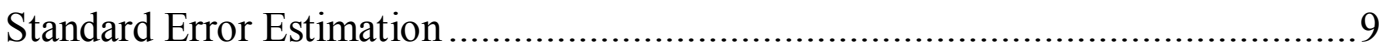

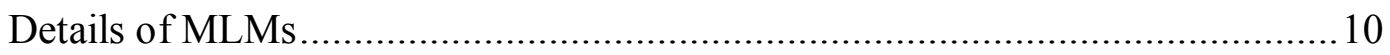

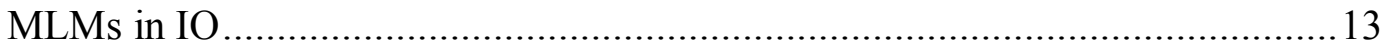

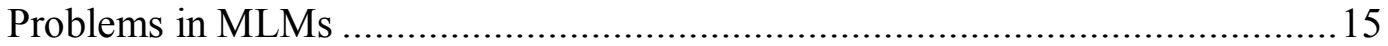

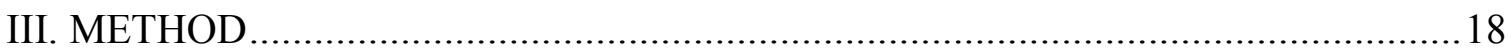

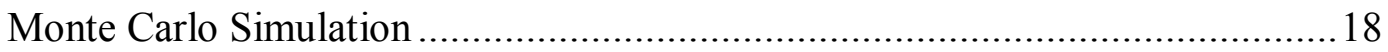

Data Generation and Values in the Current Paper .............................................19

Number of Clusters (k) and Average Sample Size Per Cluster (m) ..........20

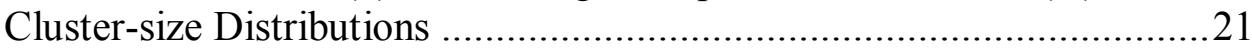

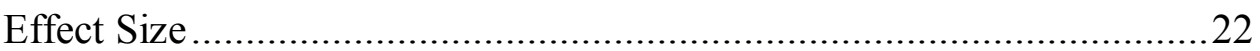

Level 1 and Level 2 Predictor Variables ..............................................22

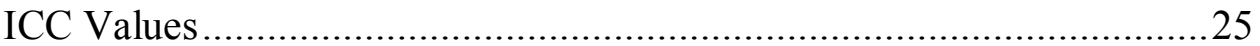

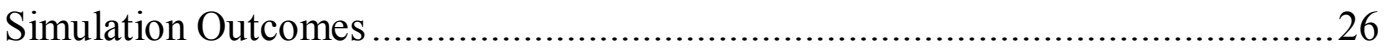

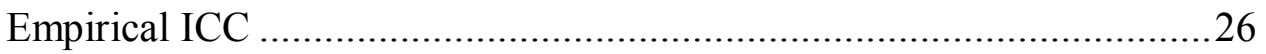

Empirical Model Design Effect ..........................................................26

Empirical Predictor Design Effects .....................................................2

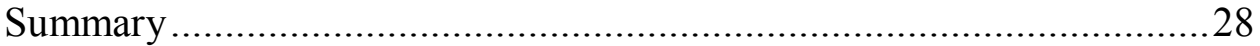

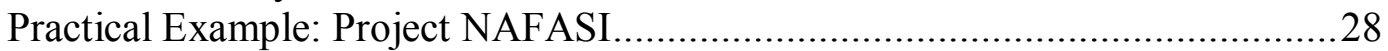

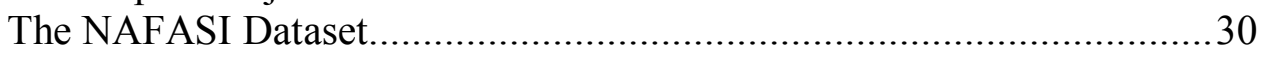

NAFASI Variables and Target Analysis ..................................................31

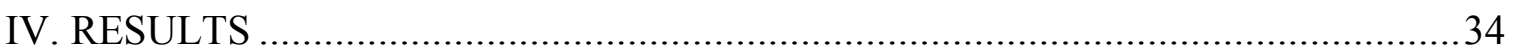

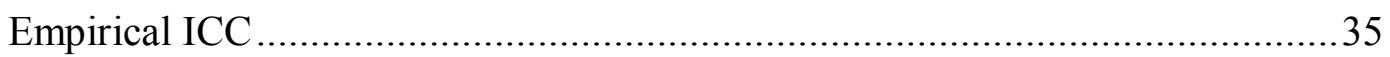

Empirical ICC-impacting factors ......................................................... 35

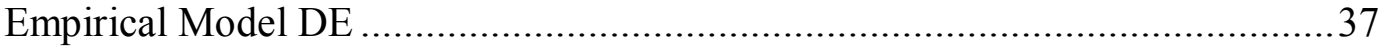

Factors Impacting Empirical Model Design Effect..................................37

Empirical Predictor Design Effects ...............................................................38

Factors Influencing Empirical Predictor Design Effects ..........................39

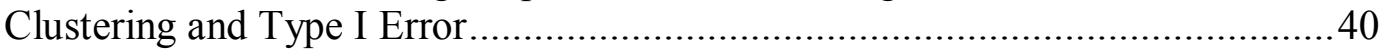

Clustering and Type II Error .......................................................................4 
V. DISCUSSION.

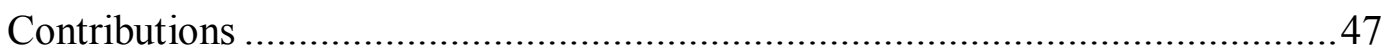

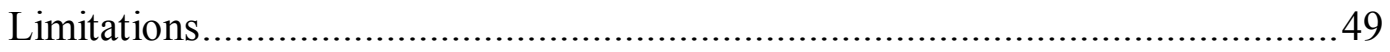

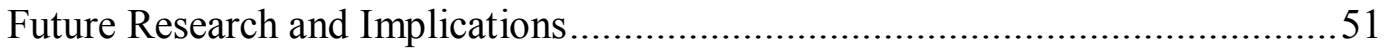

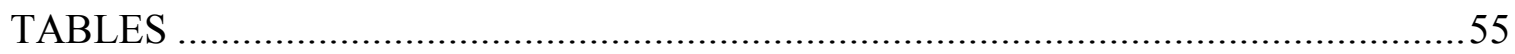

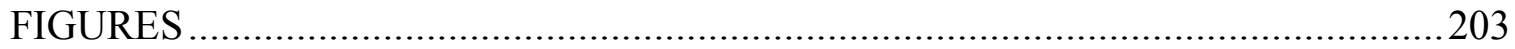

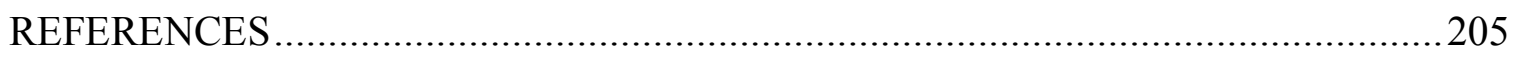

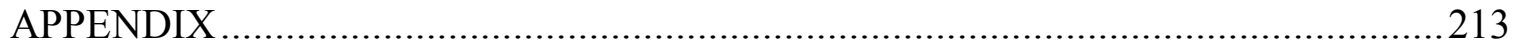

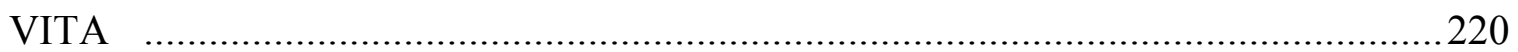




\section{LIST OF TABLES}

TABLE

PAGE

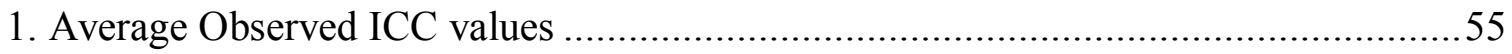

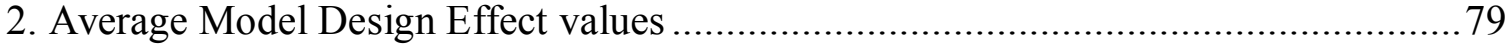

3. Average Design Effect values for the Intercept ............................................... 103

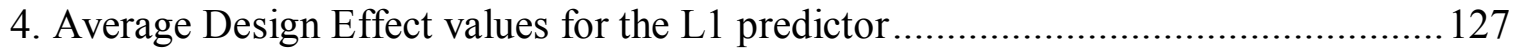

5. Average Design Effect values for the L2 predictor............................................. 151

6. Average Design Effect values for the cross-level interaction ............................. 175

7. Selected Articles from The Journal of Applied Psychology, Academy of

Management Journal, and Personnel Psychology (2012 - Spring 2015)

Featuring Multilevel Models ..... 


\section{Chapter I}

\section{INTRODUCTION}

Behaviors and their consequences do not occur in a vacuum. Accordingly, the goal of statistics and statistical methods in the applied sciences is to establish context. There are several methods of establishing this context. The first that students of statistics and psychological methods usually encounter is the inclusion of covariates, moderators, and mediators in their statistical models. The widespread adoption of SEM software makes this process relatively easy.

Context for behaviors can also be established across time, using longitudinal models. Cross-sectional data provides only a snapshot of behavior. However, crosssectional data cannot typically be used to determine causality. Longitudinal data and models, by examining the development and change of variables temporally, allow for the assessment of growth and establish a frame for behavior over time. However, true longitudinal data (collected from the same individuals over time) is notoriously difficult to collect and manage.

Finally, context can be established environmentally. The individual as a constituent of and within larger social hierarchies is at the core of the study of industrialorganizational (IO) psychology. Individuals work under managers, or in teams, or within departments. Through the use of multilevel models (MLMs), researchers can investigate effects across units such as teams, management units, departments, or organizations. In other words, MLMs allow researchers to evaluate the ecology in which behaviors, actions, and reactions occur for an individual. 
In addition to being useful for evaluating the ecology and context for behavior, MLMs can be used to examine the effects of ecology and context itself. The ecological or aggregation fallacy - the misattribution of individual effects to the group level - reflects the dangers of ignoring the level to which the information belongs (Hox, 2010). Hierarchical or ecological data (also called "clustered" data) refers to data in which observations are to some degree related to other observations. This typically occurs when data are sampled from individuals who are more similar to one another in some ecological way (e.g., employees working for the same manager) than they are to other individuals in the sample. When clustering occurs, traditional statistical methods (such as linear regression) that do not take clustering into account return estimates that exhibit deflated standard errors (SEs), which in turn increase the risk of Type I error. Multilevel modeling eliminates the risk of Type I error due to standard errors that are deflated via non-independence.

There are two main reasons to use MLMs. The first is when clustering/dependence is seen as a nuisance; MLMs are used in order to obtain accurate standard errors and type I error rates. The second is to examine clustering/dependence itself as a phenomenon of interest (Snijders \& Bosker, 2012), such as by examining cross-level effects. In the former case, a researcher might alternatively use an analytic strategy based on Generalized Estimating Equations (GEE) or robust standard error estimation (see McNeish, Stapleton, \& Silverman, 2016). When clustered data are evaluated using GEE, the impact of clustering is removed or partialled out of the analysis. In the latter case, MLMs are the preferred method of analysis. 
MLMs are often used in organizational research because of the specific constructs involved. As described above, any research that includes participants clustered or nested within units - such as groups and teams, manager-subordinate dyads, departments, or physical locations (branches) of an organization - can be evaluated using MLMs. Even within research on the same dependent variable, such as job performance, there are a variety of ways to implement analysis using MLMs. For example, a researcher wishing to measure the impact of a training intervention on job performance might examine how performance changes across teams with different levels of team engagement or cohesion. Alternately, the researcher may choose a different cluster variable (such as the physical region or location in which the team operates or which manager respondents report to) or may examine the interaction between individual differences and variables at the team level (e.g., mean tenure of team members).

MLMs allow researchers to appropriately address research questions when data are clustered, but they are not without their own challenges and assumptions. Of particular interest in this paper is the implicit assumption of a uniform distribution of cluster sizes (e.g., there are 5 participants in each and every cluster). In the real world, clusters only very rarely follow such a distribution, and then usually only in cases where the researcher forces them to be equal a priori as part of the research design. Examples of equal cluster sizes exist, such as in team settings (e.g.., Eddy, Tannenbaum, \& Mathieu, 2013) in which participants are assigned to teams of a specific size (5 in this case). However, unequal cluster sizes are the rule rather than the exception in organizational research. Chun and Choi's (2014) study contains 930 participants (n) across 145 clusters $(k)$, with an average of 7.99 participants per cluster $(m)$ and a standard deviation $\left(S D_{m}\right)$ of 
3.47, for example. Other researchers, such as Probst (2015) reported an even wider degree of variability $\left(m=37.52, S D_{m}=78.17\right)$ in their dataset $(n=1238, k=33)$. In order to ensure relevance and utility for researchers and practitioners in the organizational science field, the current paper explores recent literature across three leading IO journals - The Journal of Applied Psychology, The Academy of Management Journal, and Personnel Psychology - in order to provide direction and plausible parameters for implementing data simulation aimed at recreating "typical" organizational data.

Concern over the distribution and makeup of cluster sizes in MLMs is not simply a piece of statistical minutia. Much like clustering itself, unequal cluster sizes can cause substantial problems in interpretation of results if left unchecked. This stems from the fact that estimates specific to MLMs, such as the design effect - a measure of how much clustering in the dataset is actually impacting SE - is calculated using the average number of observations per cluster. If the distribution of the cluster sizes is skewed - meaning that the mean value $m$ is not representative of the distribution - then researchers could be under or over estimating the full impact of clustering in the dataset.

Thus, a review of current organizational literature is important not only for its own sake, but also to identify and target areas of particular interest and concern in I-O literature. This strategy can create a backdrop to examine potential problems with MLMs themselves. Naturally, published studies have many complexities and vary in several ways. For example, even the small review undertaken for the current paper uncovered a wide variety of both sample sizes, ranging from 82 (Huang, Hsieh, \& He, 2014) to 15,200 (Debus, Probst, Konig, \& Kleimann, 2012), and total number of clusters in the dataset, 
ranging from 11 (Grandey, Chi, \& Diamond, 2013; Hirst, Van Knippenberg, Zhou, Quintane, \& Zhu, 2015) to 741 (Reiche et al., 2014).

As a result of the nuanced nature of MLMs themselves, the wide variety of elements contained therein, and the array of values these elements may take, a strictly mathematical approach toward assessing and addressing this problem is infeasible, and may even be impossible. This dissertation uses a Monte Carlo (MC) simulation method to address the impact of unequal cluster sizes on MLM estimates. MC simulations were originally suggested by Metropolis and Ulam (1949). The simulation method can vary the factors of interest - evaluating additional relevant factors such as effect size and model specification - and evaluate how model estimates change in turn (see Paxton, Curren, Bollen, Kirby, \& Chen, 2001 for an overview). In a MC simulation, population values are specified, allowing researchers to draw conclusions about the statistical method used. In order to practically demonstrate the impact of the MC simulations on realworld data, this dissertation applies the findings provided by the $\mathrm{MC}$ simulations to an actual dataset. For the current paper, the target data is the Nurturing All Families through After-School Improvement (NAFASI) dataset. The NAFASI dataset contains information on 141 employees and 593 parent-child dyads across 32 parks in the Chicago municipal park system. Information in the NAFASI dataset was collected on a variety of variables, but primarily focuses on the impact of climate and culture on employee stress. The data was collected under a National Institute for Health (NIH) RO1 grant (\#RO1MH081049).

\section{Summary}

There is widespread usage of MLMs in the organizational science literature. However, the utility of these models is, as with any statistical procedure, contingent on 
the estimates it derives. In the case of MLMs, some of these estimates are based on the assumption that the number of observations per cluster $m$ is uniform across clusters in the dataset. Real-world non-experimental datasets rarely, if ever, meet this criterion. In order to assess the impact of the divergence of actual datasets from ideal datasets on estimates provided by MLMs, the current paper implements a MC simulation. This simulation presents conclusions derived from datasets that vary across several MLM-relevant factors (described below). In addition, it also extends the findings of the MC simulation to an existing dataset which features a clustered data structure. 


\section{Chapter II}

\section{LITERATURE REVIEW}

\section{Multilevel Models}

Multiple linear regression (also known as the general linear model, GLM) is a powerful and commonly understood analytic tool for modern behavioral research. However, it is not without weaknesses. Among these weaknesses are the assumptions associated with multiple regression, such as a conditionally normal distributed outcome variable and statistically independent observations. While violations of normality can be addressed within multiple regression using techniques such as bootstrapping, datasets with a high degree of non-independence (whether by accident or design) are better handled with different models that are extensions of multiple regression, such as multilevel models (MLMs; Raudenbush \& Bryk, 2002; Hox, 2010; Snijders \& Bosker, 2012).

Multilevel models are commonly used in organizational research; similar approaches with slightly different names are used in other fields. MLMs are also known as hierarchical linear models (HLM, Raudenbush \& Bryk, 2002), mixed models (MM, Littell, Milliken, Stroup, \& Wolfinger, 1999), and random effects models (as well as other designations). In general, these models are used to evaluate relationships between variables when observations are clustered (or "nested") within some higher order classification variable. Statistically speaking, MLMs are extensions of linear regression for use in datasets in which the assumption of independence has been violated. The primary strengths of MLM analysis for non-independent observations fall into two 
domains: identifying and modeling random effects and correctly estimating standard errors.

Random Effects. MLMs have an advantage over standard linear regression in that they are able to model random effects. In linear regression, any relationship between variables is assumed to apply to all subjects: if a regression coefficient $\left(\beta_{\mathrm{x}}\right)$ is positive, for example, an increase in predictor variable $\mathrm{X}$ is expected to be associated with an increase in the outcome variable (Y) for all cases. Suppose that the coefficient value for conscientiousness $(\mathrm{X})$ on performance $(\mathrm{Y})$ is $2.43\left(\beta_{\mathrm{x}}=2.43\right)$. This would mean that for any given case, a one-unit increase in conscientiousness score would be associated with an average 2.43 increase in job performance. The standard error bands around this point indicate a range of values into which the general population effect (i.e., the "true" effect of $\mathrm{X}$ on $\mathrm{Y}$ ) would fall. In the real world, however, the starting value (intercept), slope value (coefficient), and the associated variance around the prediction line (the error terms), may differ depending on membership in a clustering variable. To extend the above example, a manager (A) who espouses high conscientiousness may foster an environment in which high conscientiousness employees thrive, meaning that the conscientiousness-on-performance coefficient in this cluster (i.e. employees under this manager) may be larger - since the relationship for individuals in this cluster is comparatively stronger - than it is for individuals in the rest of the sample. Another manager (B) may not foster such an environment; no matter how disciplined and organized an individual (facets of conscientiousness) is, they are unable to leverage these traits to their advantage. In this case, the relationship would be weaker. It is also possible that the laissez-faire leadership style associated with manager B lowers output of their 
subordinates at mean (i.e., intercept) levels of conscientiousness as compared to manager A and other managers in the sample, even if a subordinate in the "manager A cluster" has the same level of individual conscientiousness as a subordinate in the "manager B cluster". Thus, employees have different values for any conscientiousness-onperformance linear regression depending on their cluster membership.

In statistical terms, multiple linear regression (GLM) posits a single, average effect of the predictor variable $(\mathrm{X})$ on the outcome variable $(\mathrm{Y})$, called the fixed effect, while MLMs make it possible to also model cluster-specific effects of $\mathrm{X}$ on $\mathrm{Y}$ as well as the between-cluster variation in these effects, called random effects. When only cluster differences in intercepts (i.e., outcome means) are modeled, the model is referred to as a "random intercept" model. When only cluster differences in slopes (i.e., relationship between predictor(s) and outcome) are modeled, the model is referred to as a "random slope" model. It is also possible to include both random intercept and the random slope terms in the model, which allows for clusters to differ in both intercept and slope terms. MLMs add to the knowledge gained from GLMs by addressing variability in probabilistic relationships across clusters.

Standard Error Estimation. In the GLM, the standard error (SE) provides an estimate of how variable the observed mean is (due to sampling error) and is used to calculate a confidence interval. A confidence interval is a range that should capture the true value of the estimate (e.g., regression coefficient) with some degree of confidence (usually 95\%). When observations are clustered, the GLM assumption of independence is not met. A sample of size $n$ that includes clustering results in fewer than $n$ independent pieces of information; knowing about one observation provides information about other 
observations in the same cluster. Standard errors and tests of inference based on $n$ independent observations will understate the amount of variance and therefore underestimate standard errors. This can potentially lead to spurious effects being identified as significant ones, as the deflated standard errors lead to narrower confidence intervals that no longer contain 0 . By correctly accounting for clustering in the data using MLM, this source of standard error deflation is eliminated, preventing reductions in statistical power and increases in Type I error rates.

Details of MLMs. As with all statistical models, MLMs are not without their own special considerations and complications. First, any researcher using the analysis should consider that predictor variables can stem from either of two levels of data - data at the level of the individual and data at the level of the cluster - and that considerations such as sample size and effect size may hinge on which level a researcher wishes to target their research questions. In addition, researchers should ascertain not just the presence of but the extent of clustering present in the dataset. While high levels of clustering may bias results, it is possible that clustering in the dataset has an inconsequential impact on the analyses performed on it. Thus, the degree to which any clustering present in the dataset practically influences the estimate of SE also needs to be assessed.

While observations in a dataset may be clustered, the extent of the clustering and the extent to which clustering may influence the results if left unaddressed is also at issue. The mere presence of a cluster variable does not imply that data clustering is influencing results. It is possible that there is little or even no variation between managers on subordinate performance. If there is negligible non-independence occurring in a given dataset then in the interest of simplicity a GLM-based analysis which ignores the effect of 
the cluster variable might be preferable to a MLM-based model. In such a case, a MLM could introduce unnecessary estimation terms without providing either of the advantages described above. On the other hand, if there is a great deal of non-independence in a dataset then ignoring clustering by using a GLM will deflate standard errors and inflate Type I error rates. An MLM-based analysis will be able to account for the betweencluster variance and be more appropriate.

Researchers can use the intraclass correlation coefficient (ICC) to quantitatively evaluate how much variance can be attributed to clustering. The ICC (also notated by the letter $r h o, \rho$ ) is calculated by estimating an unconditional model (also called a random effects ANOVA); a model with no predictors of the outcome variable. The output from this model completely partitions the total variance into a portion attributed to L2 (cluster) and a portion attributed to L1 (individual). The $\mathrm{L} 2$ variance is denoted by $\tau_{00}$ while the $\mathrm{L} 1$ variance is denoted by $\sigma_{\mathrm{e}}^{2}$; the total variance is the sum of the $\mathrm{L} 1$ and $\mathrm{L} 2$ variance values, $\left(\tau_{00}+\sigma^{2}\right)$. The intraclass correlation is the proportion of variance that is due to L2 clustering, or $\rho=\tau_{00} /\left(\tau_{00}+\sigma^{2}\right)$. An ICC of .25, for example, would mean that $25 \%$ of the overall variance in $\mathrm{Y}$ is between clusters. The complement of this value $(1-\rho)$ provides the proportion of overall variance in $\mathrm{Y}$ that is within cluster. MLMs are predicated on the idea that individuals within clusters are more similar to individuals within their own cluster than they are to individuals from other; the ICC allows researchers to quantify the magnitude of this non-independence.

Individual observations and the clusters into which they are nested can come in a variety of forms, depending on the research setting. In educational research, for example, students are clustered within classrooms. Scores for children in the same classroom are 
more like one another than scores from children in different classrooms. A similar example in organizational research is employees who are clustered within district managers, within teams, or within departments. Information can exist at the level of the individual (individual engagement) or at the level of the cluster (team engagement). These variables occur within different domains, even when they roughly refer to the same concept.

Because of the potential confusion of variables and the level in which they occur, variables in MLMs can be referred to as being Level 1 (L1) variables, Level 2 (L2) variables, or cluster variables. An L1 variable refers to variables measured at the lowest (often individual) level of the model. Participant-specific scores on variables such as stress, engagement, intelligence, or other individual differences (e.g., personality) are examples of $L 1$ variables.

An L2 variable refers to variables that are measured or implemented at the level in which individual observations are clustered. The values for L2 variables are usually applied to all individuals within the cluster, giving individual the same value on the L2 variable. L2 variables can be exemplified by leader conscientiousness, the industry in which the organization operates, team engagement, or the geographic/physical location of an organizational branch.

Depending on how the L2 variable is conceptualized it can be termed either an integral (also called structural) or contextual L2 variable (Lazarsfeld \& Menzel, 1971; Susser, 1994). Integral L2 variables are those that are created based on information derived from lower-level units. From the examples above, team engagement (aggregated from individual-level data) would be an integral L2 variable. Contextual L2 variables, on 
the other hand, inform lower-level observations, and are often a single value applied to all units universally within the cluster. From the list above, organizational industry would be an example of a contextual variable. While these variables are functionally identical, they are often interpreted differently when estimating MLMs.

The cluster variable in a dataset denotes the entity that observations are nested within. The selection and designation of a cluster variable is essential to MLMs. In an organizational setting, a cluster could be a department, a manager, or a team. In practice, the cluster variable in a dataset is often given its own code (e.g., Team 7 or Location 59) in order to allow observations within that cluster to be grouped. However, it is often aspects associated with the cluster variable and not the cluster variable itself that a researcher wishes to study, particularly when using integral L2 variables: While individual observations of engagement (from individual employees) may be clustered (or "nested") within teams, it is not the team (the cluster variable) but aggregated team engagement itself that a researcher may wish to examine when gauging performance or turnover intentions as an outcome variable of interest.

\section{$M L M s$ in $I O$}

Research utilizing MLMs are common in IO psychology. A review of recent articles (2012 - late spring of 2015) in The Journal of Applied Psychology, The Academy of Management Journal, and Personnel Psychology (see Table 1) reveals studies across a wide variety of subjects, including creativity (e.g., Chang, Jia, Takeuchi, \& Cai, 2014; Hirst, Van Knippenberg, Zhou, Quintane, \& Zhu, 2015), training reactions (e.g., Harman, Ellington, Surface, \& Thompson, 2014), culture and commitment (e.g., Fisher, 2014), groups and teams (e.g., Chang et al., 2014; Farmer, Van Dyne, \& Kamdar, 2015; 
Gonzalez-Mule, DeGreest, McCormick, Seong, \& Brown, 2014) and leadership (e.g., Chen, Zhu, \& Zhou, 2015; Dong, Liao, Chuang, Zhou \& Campbell, 2015; Farh \& Chen, 2014; Liu, Wang, Chang, Shi, Zhou, \& Shao, 2014).

MLM analysis is appropriate for many of these research questions. Culture (Fisher, 2014) is necessarily a shared or group perception. The impact of leadership (Zhang, LePine, Buckman, \& Wei, 2014) occurs in a multilevel environment, with employees nested within leaders. In addition, the focus on individual traits and differences common in organizational literature and their interaction with contextual variables (e.g., Harman, Ellington, Surface, \& Thompson, 2014; Probst, 2015) often necessitates measurement and analysis of both L1 (individual) and L2 (contextual) variables, as well as their interaction. Organizational literature, which is often focused on interventions aimed at improving group or individual outcomes within the organization, (e.g., Eddy, Tannebaum, \& Matthieu, 2013) is likewise amenable to examination using MLMs.

While many organizational research studies share qualities, they also vary in many respects. Table 1 shows an overview of these aspects that are relevant to the current study. Although the disparities between studies will be described in more detail below, it should be noted that studies vary widely on overall sample size, the number of clusters present in the dataset, the average number of participants in each cluster, the standard deviation of the average number of participants per cluster, as well as the overall range of participants between clusters. This variability is partly due to the wide variety of settings in which organizational science is studied. 
Given the extensive need for use of MLMs in organizational research, there is room in the literature for research examining aspects MLMs and how they are applied. Indeed, existing research in the organizational science field has already tackled topics relevant to multilevel modeling such as the aggregation of individual data to the group level (e.g., LeBreton \& Senter, 2008; Mierlo, Vermunt, \& Rutte, 2009; Wang \& Maxwell, 2015), the effects of sparse clusters (clusters with few participants per cluster) on MLM estimation (e.g., McNeish, 2014), and dealing with cross-level interactions in MLM (Aguinis \& Culpepper, 2015), among others. Nonetheless, many issues remain untouched by the existing literature. Issues intrinsic to multilevel data, such as uneven cluster sizes, have been unexamined by the organizational science literature; this is the focus of this study. Methodological researchers in other areas (Eldridge, Ashby, \& Kerry, 2006; Lauer, Kleinmann, \& Reich, 2015; You et al., 2011) have skirted this issue, but primarily only with a focus on estimating a priori power for cluster randomized trials. As described below, the current study examines the impact of uneven clusters in MLMs on the empirical ICC, model DE, and predictor DE.

\section{Problems in MLMs}

Multilevel models offer data analysts a powerful approach to solving some of the shortcomings associated with the GLM, as well as for assessing the effects of hierarchical context associated with many research questions. The increase in utility and complexity, however, is accompanied by potential problems. These problems stem from the fact that there are now two (or more) levels of information and data to manage. The first level, of course, contains ordinary observations present in the dataset. However, as MLMs could be considered a variance-partitioning technique, the second level also contains explains 
variance of the dependent variable, and is often informed by or created from data from the first level. As previously described, the amount of variance in the dependent variable that is due to clustering is measured by the ICC: the higher the ICC, the greater the degree of clustering present in the dataset. A second value, the design effect, estimates the degree to which clustering actually impacts SEs; the design effect is calculated (in part) using the ICC. As a result, problems inherent in the calculation of ICC are echoed in the calculation of the design effect. The discussion below details the impact of uneven cluster sizes on these two estimates.

The ICC is a single value that applies to the entire sample. In practice, however, a single value may not necessarily capture the clustering present in each cluster. Some clusters may have a very small L1 variance (i.e., all individuals in a cluster have similar scores), while others may have a very large L1 variance (i.e., individuals in a cluster have very different scores). This problem could be exacerbated by uneven cluster sizes. Consider an example in which one manager supervises 40 employees and another manager supervises 3 employees. The larger cluster contributes more to the total variance than the smaller cluster, but there may be more or less variance between individuals in the larger cluster relative to the smaller cluster. When there are several clusters with a small number of cases per cluster, a condition known as cluster sparseness results. This sparseness results in inflated Type I error rates (even in MLMs) because group level (L2) variance terms are overestimated. This effect occurs when cluster sizes are very small $(\sim 2)$, but quickly evaporates with only slightly larger ( $\geq 5)$ cluster sizes (Clarke, 2008). Thus, even though the use of MLMs to address non-independence does not eliminate the 
possibility of inflated Type I error, MLMs can greatly minimize the occurrence of Type I error when designed well.

In addition to carefully choosing the cluster variable itself and examining the extent of clustering present in the dataset via the ICC, researchers should also estimate the degree to which unchecked data clustering would actually inflate SE values. This is done through the estimation of the design effect (DE or $\left.\mathrm{D}_{\text {eff }}\right)$. The DE, introduced by Kish (1965), can be estimated in one of two ways. The first method (demonstrated by Equation 1) is to estimate it directly using the ICC:

$$
\mathrm{D}_{\mathrm{eff}}=1+(m-1) \rho
$$

where $m$ is the number of observations in each cluster and $\rho$ is the ICC. The second is to run a MLM, note the standard error for this model, and then run an identical model without the random effects component(s) (i.e, a standard GLM). The ratio of the MLM variance to the GLM variance is an empirical, or formal, estimate of the design effect (Henry, 1990). The latter, conceptual method of estimation requires access to data. However, in many cases, such as an a priori power analysis (Eldridge, Ashby, \& Kerry, 2006; McNeish, 2014), a researcher may wish to estimate the DE to determine the sample size for the proposed research. The DE formula shown in Equation 1 includes a single value, $m$, for the number of observations in each cluster; this doesn't allow for consideration of unequal cluster sizes. Research that provides guidance regarding the impact of unequal cluster sizes on these calculations is necessary. 


\section{Chapter III}

\section{METHOD}

\section{Monte Carlo Simulation}

The advantages of HLM also bring with them added complexities, as described above. Of particular note for this paper is the impact of uneven cluster sizes on estimation of ICC and, subsequently, DE. There has been substantial research on the effect of cluster sparseness (small samples within clusters) on estimation in MLMs, however, there has been much less research on uneven cluster sizes themselves. In order to assess the impact of uneven clusters on the estimation of the bias produced in model estimates (specifically the empirical ICC, model DE, and predictor DE), the current paper will implement a Monte Carlo (MC) data simulation.

Monte Carlo data simulations (Metropolis \& Ulam, 1949) are the empirical counterpart to mathematical proofs. When attempting to define the behavior of statistical models under certain conditions (e.g., a given sample size) there are two options available to researchers. The first is to derive mathematical proofs based on the mathematical and statistical assumptions of the model. This solution is exact, but is impractical for many complex statistical models. The second option, empirical Monte Carlo data simulation, can be used for complex situations in which mathematical proofs are intractable or impossible. As a simple example, consider the probability of rolling two dice and obtaining a result that adds up to 7 . The traditional, mathematical proof approach would use the probability of various die values to compute the joint probability of a sum of 7 . The empirical simulation approach would roll two actual (or computer generated) dice 1000 times to observe how often a sum of 7 was obtained. 
Although the rolling of a pair of dice 1000 times is certainly feasible and is relatively easy, when dealing with more complicated paradigms, the requisite time and effort to obtain the data associated with these models is often prohibitive, especially when dealing with procedures as complex as MLMs. Instead, the current study, like all modern MC simulations, uses computer software and processing to generate these data. SAS 9.3 was used to generate data according to user-specified sample parameters (e.g., sample size, effect size of independent variables; described below). Since the true (i.e., population) values of these distributions are known, the effects of interest here are how the statistical model performs, rather than statistical inference (as is of interest in traditional methods). One thousand replications of each condition were produced, and each replication was analyzed using MIXED procedure in SAS. The data is aggregated across all datasets within a condition in order to examine the effects of the study predictor variables on the outcomes of interest. For the current paper, there are three outcomes germane to MLMs that are of concern: empirical ICC (EICC), empirical model DE (MDE), and empirical predictor design effect (PDE).

\section{Data Generation and Values in the Current Paper}

Based on the above review of organizational literature, the following section outlines several elements common to MLMs. The literature features datasets with a wide variety of characteristics. Chiefly, they differ in terms of overall sample size, the number of clusters, ICC values, effect size, treatment of the independent variable, and the distribution of cluster sizes within the dataset. The following section will examine plausible values for each element to be included in the data simulation, as well as why 
some values of these elements may influence study outcomes (described in the following section.

Number of Clusters ( $k$ ) and Average Sample Size Per Cluster (m). Sample size is a common consideration in simulation studies because of its key role in statistical power, as well as several related considerations (e.g., type I error, bias in mean estimates, etc.). In MLMs, there are multiple sample sizes to consider: total sample size (n) and number of clusters (k). Extant research that using multilevel models report a wide variety of total sample sizes, from less than $200(n=160$ in Harman et al., 2014) to more than $15,000(n$ $=15,200$ in Debus, 2012). Many samples are in the range of 200-300 participants (e.g, Chen et al., 2015; deJong, Curseu, \& Leenders, 2014; Farmer et al., 2015).

The number of clusters present also varies across studies. Given that the focus of the current paper is the effects of differential cluster sizes, simulation conditions based strictly along the lines of total sample size (e.g., 100, 300, and 500 subjects) would not fullly address the aims of the paper. A sample size of 300 subjects could include 30 clusters of 10 individuals each or 100 clusters of 3 individuals each. For example, Farmer et al. (2015) and Chen et al. (2015) report sample sizes of 236 and 238 respectively; the former study has 52 clusters and the latter 31 .

In order to account for both a range of sample sizes and the varying number of clusters between studies, the current study considers a range of number of clusters $(25,50$, $75,100)$ crossed with a range of participants per cluster $(5,10,20)$. Therefore, the current simulation will include 12 possible total sample sizes (3 numbers of average observations per cluster $(m)$ by 4 number of clusters), allowing for a total sample size ranging from 125 to 2000 . This wide range of sample sizes reflects sample sizes that are common in 
the organizational science literature. Naturally, a combination of low values on $k$ and/or $m$ can impact MLM-centric estimates that rely on them. As a component of the MDE equation (Eq. 1) above, for example, a large $m$ could produce a large DE, even if ICC values are small.

Cluster Size Distributions. The current paper will simulate conditions in which cluster sizes vary and those in which cluster sizes do not vary. Cluster sizes will follow one of two distributions. For constant cluster sizes, a uniform distribution in which all clusters are of equal size will be used. Constant cluster size is commonly assumed in MLMs, especially for power calculations. Though less common in practice than unequal cluster sizes, uniform cluster sizes are plausible; for example, research on dual-earner couples (Hahn \& Doorman, 2013) naturally features two individuals per cluster.

For unequal cluster sizes, a truncated Poisson distribution based on the binomial approximation of the Poisson distribution will be used. Poisson distributions are positively skewed, with a long right tail and are commonly used for positive, discrete outcomes such as count outcomes. However, standard Poisson distributions include values of 0 . As it is impossible to have zero observations in a cluster, a truncated Poisson distribution - which eliminates 0's - was used (see Appendix 1).

Data reported in the literature often feature cluster distributions similar to a truncated Poisson distribution. de Jong, Curseu, and Leenders (2014) report that their cluster sizes ranged from 3 to 13, with an average cluster size of 4.6. Chang et al. (2014) report a cluster size range of 2 to 22 individuals, with an average cluster size of 8.24 individuals. Fisher's (2014) sample $(\mathrm{N}=6,264)$ contained cluster sizes ranging from 4-36 individuals, with an average cluster size of 18. 
The wide variety of research areas and their corresponding cluster structures means that many possible means and shapes of distribution are possible. In an attempt to replicate conditions found in the literature, the cluster sizes for the current paper followed 6 possible distributions. The first three conditions included clusters with uniform distributions - which reflects common assumptions present in discussions related to power in MLMs (vis. Equation 1) - MLMs and have 5, 10, and 20 observations per cluster, respectively. The fourth through sixth conditions included clusters with truncated Poisson distributions, likewise with means of 5, 10, and 20 participants per cluster, respectively.

Effect Size. In order to account for the wide variety of models present in the literature, the current study also examines how various predictor effect sizes (ES) impact the outcomes of interest. In inferential statistics based on the analysis of group-mean differences, Cohen's $d$ is most often used to represent the magnitude of effect size. Cohen (1992) suggested that values of .2, .5 and .8 represent small, medium, and large effect sizes respectively. Extensions of this value can be used to further allow interpretation of results. In order to account for the canonical values established by Cohen and in order to evaluate a null effect, the current paper simulated data across $d$ values of $0, .2, .5$, and .8.

Level 1 and Level 2 predictor variables. In order to obtain unbiased effect estimates for research questions of interest, researchers using MLMs must take care to appropriately center predictors. Fortunately, there are several guides and explanations available (Enders \& Tofighi, 2007; Kreft, de Leeuw, \& Aiken, 1995; Peugh \& Enders, 2005) to assist researchers in selecting the appropriate centering strategy. There are two main types of variable centering in MLMs. The first, centering within cluster (CWC), 
should be used when L1 predictor variable is the focus of the research question. Centering within cluster removes between cluster variation and essentially treats clustering itself as a nuisance. The second strategy, grand mean centering (GMC), should be used when the L2 predictor variable is the focus of the research question. Grand mean centering maintains variation across clusters. Appropriate use of these centering methods is necessary in order to have unbiased estimates of the effects of interest (i.e., the effect of the $\mathrm{L} 1$ predictor on an outcome, unconfounded by variability across clusters).

When clusters have unequal sample sizes, GMC may be minimally impacted because the values used to estimate the grand mean are derived from all participants $(n)$. However, CWC may be more impacted because the mean value around which a cluster is centered is based on varying numbers of individuals. In a dataset within uneven $m$, the mean value around which one cluster is centered may be derived from a cluster with 2 individuals, and another's from a cluster with 16, for example. While it is possible that the first cluster genuinely has only 2 individuals (and thus the values from that cluster would reflect that cluster's "true" values), in many real-world cases it is likely that clusters with a small number of observations are underrepresented. As MLMs estimate effects across clusters, these estimates are likely biased. Thus, depending on the centering strategy used (Enders \& Tofighi, 2007; Kreft et al., 1995; Peugh \& Enders, 2005) both estimates themselves and, as a result, bias could be affected. Each centering strategy, after all, produces different estimates, and any included interaction terms, being based on centered predictors, will likewise be exposed to this same threat to estimation and statistical accuracy. 
In order to address the concerns outlined above, the current study will include three types of predictor: The first is an L1 predictor, with unique values for all cases in all clusters in each dataset. The second is an L2 predictor, which has identical values for cases within each cluster, but different values between each cluster, as would be typically be seen in real-world datasets which feature aggregation of measurements to the cluster level or measurements applicable to every observation within a cluster (e.g., average weekly workload or managerial scores on extroversion). The third is a cross-level interaction term between the first two terms. All three predictors are centered per the recommendations above, with the $\mathrm{L} 1$ variable values being centered within cluster (CWC), L2 variables being centered at the grand mean (GMC) and the interaction term being the product of these two measurements.

As these variables are crossed with ES (see above), any given condition is represented by one of five variable-ES configurations: a null model in which there is no population ES of any of the variables (i.e., the ESs for the L1, L2, and interaction predictor are set to 0$)$, a model in which there is some ES (.2, 5, or .8) of the L1 variable, but not the L2 or interaction terms, a model in which there is some ES of the L2 variable, but not for the L1 or interaction terms, a model in which there is some ES of the interaction term, but not for either the L1 or the L2 terms, and lastly, a model which has some combination of non-null effect sizes for multiple terms. That is, two or more of the terms have an ES of .2 or higher. This means that there is a matrix of $4 * 4 * 4$ effect size combinations, resulting in 64 possible models. Of these 64 , one is a null model, four are "L1 effects only" models, four are "L2 effects only" models, three are "interaction effects 
only" models, and the remaining 51 are models that have some combination of non-null ESs for two or more predictors.

ICC Values. Any evaluation of MLMs must address the magnitude of the ICC. As described above, the ICC is derived by dividing the variance in the outcome variable $d u e$ to the clustering variable by the total variance in the outcome variable. For example, a dependent variable with an ICC of . 2 has $20 \%$ of the variance explained by the clustering variable (i.e., differences between clusters), meaning that the remaining $80 \%$ of the variance would be at L1 (i.e., differences between individuals within a cluster). ICC can also be conceptualized as describing how non-independent observations are across clusters.

The ICC value is a single value that applies to all clusters for a given outcome variable. Uneven cluster sizes may result in ICC values that are more representative of the clusters with a larger number of participants. For the current paper, four levels of ICC will be examined: $0, .1, .3$, and .5 . An ICC of 0 indicates that no clustering is present in the dataset and values of the dependent variable are statistically independent. An ICC of .5 indicates that half of the variance in the dependent variable is due to clustering, i.e., due to differences between clusters. The literature review of recent organizational research outlined in Table 1 contains many examples of studies that report ICC values of approximately .1 (e.g., Chang, 2014; Gong, Kim, Lee, \& Zhu, 2013; Gonzalez-Mule et al., 2014) and .3 (e.g., Chen, Liu, \& Portnoy, 2012; Debus, 2012; Eddy et al., 2013). Studies reporting ICC values of around .5, while certainly less common, are still represented in the literature (Chen, Farh, Campbell-Bush, Wu, \& Wu, 2013; Reiche et al., 2014) Thus, the levels of the ICC factor present in the current simulations encompass a 
wide variety of possible outcomes present in datasets. In order to avoid confusion with the empirical ICC (described below), the simulated population values of the ICC are, in the current paper, referred to as the PICC.

\section{Simulation Outcomes}

Empirical ICC. As stated above, the intraclass-correlation coefficient (denoted $\rho$ ) is a ratio of cluster level variance to total variance in the outcome variable $y$. Thus:

(2) $\rho=\tau_{00} /\left(\tau_{00}+\sigma^{2}\right)$

The denominator term in (2) is calculated by summing the cluster-level (L2) variance $\left(\tau_{00}\right)$ and the individual-level (L1) variance $\left(\sigma^{2}\right)$. It is important to evaluate the empirical ICC because the ICC informs the DE, statistical power calculations, and estimates of standard error.

The current paper evaluates the average empirical ICC (EICC) in each simulation condition. This means that despite the 'true' population values of ICC used in data simulation, the observed values ICC have some deviation (lower or higher) than this value. Thus, in order to be flagged as biased, an observed ICC would need to be outside a typical expected range. Previous research (Donner \& Wells, 1986) has established ICC bands of about .30 (or .15 one-sided) as acceptable. The current study uses this range as a guideline for determining biased ICCs.

Empirical Model Design Effect. As mentioned above the DE is an estimate of the degree of variance deflation due to clustering in a dataset. In a dataset with a DE of 4 , for example, the variance in the actual [clustered, non-independent] dataset is 4 times greater than a hypothetical dataset featuring perfect random sampling, and thus true independence. Per Equation (1), the DE is informed largely by the ICC and $m$, the 
average number of cases per cluster. Thus, as either of these values increases, the DE likewise increases.

Due to sampling error there is always some amount of clustering (and thus some level of SE deflation) in datasets. The problem for researchers is whether or not this deflation is problematic enough to merit consideration by using cluster-robust methods for model estimation such as MLM. While it is unclear where DE values become large enough to always be problematic, previous simulation research (Muthen \& Satorra, 1995) has suggested that even datasets with low levels of DE $(\sim 2)$ - occurring with ICC levels as low as .05 - can exaggerate Type-I error rates. As a result, any condition which reports an average empirical DE for the model (MDE) of larger than 2 was flagged as problematic.

It is important to note that both ICC and MDE are calculated for the entire model. Hence, neither considers the effect of clustering on the individual independent variables. In order to elucidate this, the current paper also examines design effects calculated for each predictor, based on the definition of the design effect.

Empirical Predictor Design Effects. As mentioned above, the design effect calculated in Equation (2) design effect can alternately be conceptualized as:

(3) $\frac{\text { squared standard errors under complex design }}{\text { squared standard errors under standard design }}$ where the 'complex' design is the design under consideration (MLM) and the 'standard' design is analysis done under simple random sampling (SRS). Where these ratios are greater than one, SRS can be said to be preferable to MLMs, and when these ratios are less than one MLMs are preferred. Consequently, evaluating the empirical predictor DEs (PDEs) can be used as a way to determine the efficiency of individual estimators in a 
given model, akin to the variance inflation factor (VIF) statistic common in regression analyses. As mentioned above, simulation studies establish the universe of known or correct values and then simulate data around those values. This means that in the current study, MLMs are always the correct choice for analysis. Examining both the MDEs and the PDEs allows the current study to evaluate how using the inappropriate (i.e., standard) design influences estimates.

Summary. The current paper simulated data based on 6 different factors common to MLMs. These elements are the distribution of cluster size (uniform/Poisson), the $m$ (5, 10 , and 20 individuals per cluster) and $k$-size $(25,50,75$, and 100 clusters $)$, the PICC values $(0, .1, .3$, and .5$)$, and effect size $(d=0, .2, .5$ and .8 magnitude) across the three types of predictor variables (L1 predictor, L2 predictor, the cross-level L1-L2 interaction. All factors are fully crossed, resulting in a 2 × 3 × 4 × 3 × 4 × 4 × 4 design, or 6144 conditions. Each condition was replicated 1,000 times, yielding 6,144,000 total datasets for analysis.

Practical Example: Project NAFASI

The Nurturing All Families through After School Improvement (NAFASI; NIH RO1MH081049) project aims to improve program quality for children who are at-risk both socially and behaviorally. Data for this study were collected from children, parents, and workers at 44 parks in the Chicago municipal park system. By examining the organizational context in which these programs operate, NAFASI aims to assist youth indirectly by evaluating the programs themselves, as well as the work environment of the employees that run them. The work of Glisson and colleagues (Glisson \& James, 2002; Glisson \& Schoenwald, 2005; Hemmelgarn, Glisson, \& James, 2006) demonstrates that 
the social context in the work unit contributes uniquely to providing high-quality services and outcomes for behaviorally at-risk children.

Glisson's (2002) model (Figure 1) suggests that setting-level elements such as culture, structure, procedures, and policies impact employees' individual and group perceptions of climate in the organization. These perceptions, in turn, affect employees' attitudes and behavior. Glisson's model joins several similar models and examinations of culture and performance already present in the organizational literature (e.g., Dension, 1990; Hartnell, Ou, \& Kinicki, 2011; Hofstede, 1980; Schein, 2010). However, it distinguishes itself by suggesting pathways for culture to influence perceptions, and for perceptions to influence attitudes and performance.

Using Glisson's model as a base, a measure known as the Organizational Social Context (OSC) scale was developed. This scale measures three dimensions of culture (proficiency, rigidity, resistance) and three dimensions of climate (engagement, functionality, stress), as well as morale, which assesses job attitudes (commitment and satisfaction). In the NAFASI project, measurement of organizational context via the OSC is used to inform employee outcomes (such as job stress). These outcomes, in turn, are thought to impact the quality of service provided by the after-school park programs. Assessing and managing the quality of after-school programs is especially important for creating a positive impact on the behavior, social skills, and social development of urban and low-income children (Gottfredson et al., 2004; Pierce et al., 1999; Vandell et al., 2005). For this reason, NAFASI data collection targeted both employees - for information regarding climate, culture, and personal perceptions - as well as children and parents, in order to gather information on program quality and behavioral and social outcomes. 
The NAFASI Dataset. Data collection in applied settings is rarely straightforward. For the NAFASI project in particular, which requires responses from both organizational constituents (employees) and stakeholders (children and their parents), data collection and the resulting dataset are quite complicated. The resulting design is inherently multilevel (individuals nested within parks) and features integral L2 variables (aggregated employee perceptions of organizational climate and culture within park, based on the OSC measure). Analysis on the dataset using MLMs is most appropriate, accounting for clustering in the data (across parks) and allowing for examination of L2 variables (aggregated employee perceptions of culture) on a L1 outcome (parent and child perceptions of program quality).

The analysis of the NAFASI dataset is not straightforward. First, each park involved in the NAFASI project is a different size, both in terms of physical space as well as the number of employees and children served. Second, OSC values are aggregated values based on all employees, but only if a sufficient number of employees respond to the measure. As a result, the OSC measure is missing for parks with either low numbers of employees or low response rates. While this problem could potentially be addressed by using standard missing data techniques (e.g., full-information maximum likelihood, multiple imputation, etc.), the multilevel nature of the dataset and the L2 aggregation of the data for this variable complicate the implementation of these strategies. As a result, all parks missing values on all OSC variables were omitted from analysis. This resulted in the usage of only 32 parks and their associated data in the final analyses.

After elimination of parks with inadequate response to allow for aggregation on the OSC variable, the final dataset contains values for park staff $(n=141)$ and values 
from attending children and their parents $(n=593)$. With 32 parks in the final dataset, there were an average of 18.53 matched child/parent respondents per park. The range of respondents per park (between 3 and 55) is similar to some reported samples in the organizational literature (e.g., 14-68 in Hirst, et al., 2015). Similar to other published studies, the NAFASI dataset does not contain a uniform cluster-size distribution assumed by MLM calculations (see Figure 2). The number of clusters, the overall sample size, the range of participants across clusters, and the average number of participants per cluster approximates values found in extant organizational literature. In addition, the frame of the variables involved (the impact of conditions in an organization on stakeholder perceptions) mirrors analytic strategies common in research featuring nested datasets in top IO journals. This similarity makes the NAFASI dataset ideal for demonstrating the effect(s) of uneven cluster sizes in MLMs. As a result, following the simulation study, the current paper proposes to apply the findings of the MC simulation to the NAFASI dataset in order to show the effects, if any, of uneven cluster sizes on both the results of the analysis and its interpretation.

NAFASI Variables and Target Analysis. Among the variables present in the NAFASI dataset are three scales that will serve as the target of supplementary analysis for the current paper. The first is the Organizational Social Context (OSC) scale (Glisson, 2007), which measures three organizational climate factors (stress, engagement, and functionality) as well as three organizational culture factors (rigidity, proficiency, and resistance). The second is the After-school Environment Scale (ASES; Rosenthal \& Vandell, 1996), and measures program quality from the child's perspective. Finally, the third scale is a measurement of program quality from the parent's perpsecive, and is a 
hybrid scale combining six items from the Assessing School-age child care Quality scale (O'Connor, 1991), three items from Kids with My Kid (Vandell, 2005) and 10 items from a parent satisfaction scale (Rosenthal \& Vandell, 1996). As the OSC was developed in clinical settings, one of the primary contributions of the NAFASI project is to move measurement using this scale into more universal settings (i.e., public parks).

Another primary objective of the NAFASI project is to assess the impact of organizational climate and culture on worker perceptions and effectiveness (Figure 1). Thus, analysis undertaken for the project estimates the relationship between the OSC and parent and child perceptions of program effectiveness using parks as a clustering variable and aggregated OSC scores from employees within parks as an independent L2 (structural) variable. The relationships between each of the six dimensions of OSC (a structural L2 variable) and both parent and child perceptions of program quality (L1 variables) were assessed using MLMs.

A single predictor, single outcome model including a random intercept was conducted for each of the 18 analyses described above. Random intercept models were chosen primarily to evaluate the impact of [centered] mean differences between parks. In addition, the small(er) sample size in this dataset made it difficult for random-slope or random-intercept-and-slope models to be estimated effectively, making random-intercept only models the best practical and theoretical choice. In addition to the predictor and outcome variable in each model, each analysis was re-run using the addition of three control variables: a dummy code for region (south and central compared to north Chicago), park enrollment, and averaged proportion of children with a parent-reported 
mental-health need via the Strengths and Difficulties Questionnaire (SDQ; Goodman, 2001) for the park.

In the current study, the impact of clustering and the usage of MLMs on the NAFASI project dataset is examined following the Monte Carlo simulation. Estimates from models in the NAFASI study are compared to similar conditions in the Monte Carlo simulation. This re-evaluation illustrates the impact of elements of MLMs (e.g., varying ICC or effect size magnitudes) on the previously-run analyses in the NAFASI dataset. 


\title{
Chapter IV
}

\begin{abstract}
RESULTS
In line with the simulation factors and conditions described above, a Monte Carlo simulation was performed using randomly generated data. As mentioned before, there are four levels of $k$-size $(25,50,75,100)$, three levels of cluster size $m(5,10,20)$, two levels of cluster size distribution (uniform, Poisson), four levels of effect size $(0, .2, .5, .8)$ across three predictors (L1, L2, and interaction), and four levels of ICC. Across all factors there were thus 6,144 conditions.

Three different models were run on each dataset: the null or unconditional mixed model, which is a model including no predictors and a random intercept; a model including a L1 predictor (centered within cluster), a L2 predictor (centered at the grand mean), an interaction effect between these two variables, and a random intercept term; and a model including the three predictors, but no random component (essentially an ordinary least squares (OLS) regression model). The results from the null models were used to calculate the EICC and thus the MDE for each dataset. In order to obtain the PDEs, the standard errors from the mixed model were compared to the standard errors from the regression model for each predictor. The outcome values (the observed ICC, observed model DE, and the observed DE for each estimator) were then averaged across the 1,000 datasets in each condition. Tables of the empirical ICC (EICC), empirical model DE (MDE), and empirical predictor DE (PDE) were then examined to look for abnormal or extreme values beyond what would ordinarily be expected.
\end{abstract}




\section{Empirical ICC}

The first outcome evaluated by the data simulation was the observed ICC $(\rho)$. As a measure of the amount of clustering in the dataset, ICC can be used to detect the presence of cluster-level variance, if not necessarily the necessity of using MLMs to evaluate hypotheses. Previous authors (Donner \& Wells, 1986; Ip, Wasserman, \& Barkin, 2011) note that the ICC, like any estimate, cannot be estimated from a single dataset, and the true (population) ICC is contained within a range around a given sample estimate.

However, as there are many moving parts in MLMs (e.g., $n, m$, and $k$-size) research establishing calculations for these expected ranges for EICCs has not been conducted. Donner \& Wells (1986) performed a Monte Carlo simulation across ICC levels and estimation methods for samples with $k$ of 25 , and 50 using various estimation methods. Using the method they recommend - Smith's (1956) procedure based on the SE of $\rho$ - ICC bands of around .30 were common across $n$-sizes in datasets exhibiting ICC values present in the current study $(\rho=0, .1, .3, .5)$. Thus, for the current paper, any condition with an observed EICC estimated .15 lower or higher than the simulated PICC was flagged as extreme. Thus, in $(\mathrm{P}) \mathrm{ICC}=.3$ conditions, values below .15 and above .45 were considered discrepant. Naturally, in the conditions in which PICC was simulated at the 0 and the .1 level, the lower bound is truncated at 0 . As expected, all conditions in the current study exhibited positive EICC values.

Empirical ICC-impacting factors. The below tables (Table 1) indicate which simulation conditions contained abnormal observed ICC (OICC) values. Among the simulation factors, effect size (particularly of the L2 variable but also of the cross-level interaction term) seemed to have a strong impact on the EICC, which was particularly 
inflated in the in $d=.5$ and $d=.8$ conditions. This phenomenon was magnified such that in conditions with a large L2 effect and large cross-level interaction effect, the ICC was almost always inflated, unless mollified by the presence of a strong L1 effect (see below).

L1 effect size also impacted observed ICC, but in the opposite direction, deflating estimates in conditions where the L1-variable effect size was strong (i.e., .5 or .8). The cross-level interaction effect magnified this trend, and conditions with stronger interaction effects and L1 effects were very often deflated. In the presence of a stronger L2 effect, however, this trend was often negated.

Simulated PICC values themselves also seemed to play a role on EICC. Again, some small degree of EICC is to be expected even when the true PICC is 0 , in either simulated or practical datasets. In the simulated ICC conditions, those with lower true ICC simulated values (i.e., 0 or .1) were the most susceptible to inflation, while those with higher simulated values (i.e., .3 or .5 ) were more susceptible to deflation.

As ICC is bounded at the lower end by 0 , it is impossible for the lower-ICC conditions to exhibit abnormal deflation. Nonetheless, in $\rho=.1$ conditions, in no case did OICC dip below .03. Interestingly, while the $\rho=.5$ conditions exhibited deflation, in some cases reaching around .23 (in conditions with a strong L1 and interaction effect, but no L2 effect), inflation was limited to (in the most extreme cases) .67. So although lower PICC conditions were firmly bounded by 0 , higher PICC conditions seemed to have a "soft" ceiling.

The impact of the effect sizes of the three predictors and PICC values on EICC are apparent. However, the other simulation factors (distribution type, as well as $m$ and $k$ size) seemed to have little to no impact on the variability of EICC. 


\section{Empirical Model DE}

Per formula (1) above, overall model design effect (MDE) is essentially a function of the average number of cases present in clusters in a dataset $(m)$ and the ICC $(\rho)$. While the design effect has been developed in previous research, primarily by Kish (1965), other authors (Gabler, Haeder, \& Lahiri, 1999; Park \& Lee, 2001; Sarndal, Swenson, \& Wretman, 1992) have examined it as well. However, there is little guidance available to researchers regarding exactly what values of MDEs are problematic in model estimation. Indeed, the only suggestions available come from other empirical (i.e., Monte Carlo) simulations on MLMs (Muthen \& Satorra, 1995), which demonstrate that DEs of 2 and above can distort the results of hypothesis testing and inflate Type I error rates. Thus, the current paper uses a DE of 2 as an indicator of where clustering becomes problematic enough to warrant concern. Table 2 displays the observed DE across all simulation conditions.

Factors Impacting Empirical Model DE. Across conditions, ICC levels (both PICC and EICC) seemed to have a large impact on DE, as would be expected. With a few exceptions (discussed below), DE was always above the target value of 2 when PICC values were .3 or higher. This occurred across cluster distribution types and cluster sizes.

Another factor related to observed DE is the cluster size $(m)$ in the datasets. Specifically, in conditions with $m=10$ and 20, inflated DE values were much more common than in those with five cases per cluster. This is to be expected, however, given that $m$ is one of the two primary values in the equation used in determining DE.

The effect sizes of the model predictors also played a role on the magnitude of the design effect. Specifically, even in datasets with lower levels of ICC, moderate and 
strong L2 effects created large design effects. However, high DEs were also demonstrated in conditions in which the L2 variable had a small or null effect and ICC levels are low, particularly if a moderate or strong effect of the cross-level interaction was also present. Conditions like these are examples of cases in which a high degree of non-independence is present in the dataset, even if this is not accompanied by an actual L2 or cluster effect.

Simulation conditions were also varied in terms of cluster-size distribution (Uniform or Poisson) and number of clusters. However, independent trends in DE across levels of these factors did not emerge.

\section{Empirical Predictor Design Effects}

In addition to evaluating trends in empirical ICC and overall model DE, the current study also examined how the factors present in the simulation impacted the ratio of standard errors of predictors in mixed versus OLS regression analyses. To obtain values of this estimate, all datasets were analyzed both with an MLM (as described above) and with linear regression (i.e., using a standard GLM that does not account for clustering).

Per formula (3) above, the ratio of mixed to SRS-based SEs for each predictor in each model was then calculated. Where this ratio was less than 1 (i.e., mixed model SEs were smaller than SRS model SEs), this metric indicates that the SE estimates produced by SRS-assumed models are too small, resulting in a greater likelihood of making a Type I error.

Tables $3,4,5$, and 6 present the empirical predictor design effects for the intercept term, the L1 predictor, the L2 predictor, and the cross-level interaction, 
respectively. It should be noted, however, that although estimates for the formal design effect are available for the intercept and L2 terms, these estimates are misleading and as such are not appropriate for discussion here. In order to obtain unbiased estimates of the impact of the L1 variable across levels of the L2 variable, the L1 predictor was centered within cluster (CWC) and the L2 predictor was centered using grand-mean centering (GMC). In addition, CWC is also appropriate for examining the influence of a betweenlevel interaction; the third predictor in the models of the current study. These decisions were made following the suggestions of previous research (Enders \& Tofighi, 2007; Kreft, de Leeuw, \& Aiken, 1995). While the combination of these centered variables yields interpretable and unbiased estimates for the $\mathrm{L} 1$ and interaction terms, it results in point estimates and associated SEs based on a mix of between-cluster and betweenperson effects for the L2 term; a bias which feeds into the intercept term. This makes SEs and thus PDEs uninterpretable and biased for both the L2 variable and the intercept term.

Factors Influencing Empirical Predictor Design Effects. As the ICC represents the amount of clustering present in the dataset, it is natural that it should influence the empirical design effects (EDEs). Indeed, this was case for both the L1 predictor and the interaction term. For both terms, higher levels of ICC lead to smaller MLM/SRS SE ratios. Thus, in datasets with high levels of clustering, mixed models are - unsurprisingly - more unbiased than their conventional counterparts.

Compared to their impact on the previous two outcomes, the L1, L2, and interaction term effect sizes have a more moderate impact on EDEs. In general, SRSbased models became more biased as L1, L2, and interaction effect sizes increased. This occurred for both the L1 and the interaction term, although in general the values for the 
EDE were stronger for the L1 variable than they were for the interaction term. EDE values indicated preference for MLMs when L1 and interaction effect sizes were high, across all other factors. L2 effect sizes, meanwhile, had a very small effect on the EDEs of both the L1 variable and the interaction term.

The number of clusters in each dataset $(k)$ also had a noticeable, if slight, impact on the EDE of both the L1 and the interaction terms. This effect was more noticeable in conditions where $m$ was set at 5 or 10 , and was weaker but still noticeable in conditions where $m$ was set at 20 . Both the dataset $m$-distribution type and the $m$-size itself, however, did not seem to impact EDEs on either the L1 or the interaction variables.

\section{Clustering and Type I Error}

The current study included models in which some or all of the predictors were simulated to have a null effect size (i.e, $d=0$ ). As a result, this design allows for examination of Type I error rates across other factors present in the simulation. The impetus for using MLMs instead of SRS-based models in clustered datasets is that ignoring clustering (SRS) should cause the analysis to report Type I error more often than accounting for it (MLM), via deflated SEs. Thus, in datasets with large amounts of clustering (i.e., high ICCs) there should be higher levels of Type I error in SRS/OLS models than there is in MLMs.

Surprisingly, however, this was not borne out in the conditions present in the current study. Across levels of $m, k$, and ICC, both SRS and MLM-based analysis approaches exhibited roughly equal levels of Type I error. Type I error rates were particularly high for the L1 predictor when ICC and the interaction effect size were both high, reaching levels of around $60 \%$ in many of these cases. The interaction effect 
seemed to be much less susceptible to this influence, with error rates capping out at about $6 \%$. As above, there is no distinction between cluster size distribution type and Type I error rates.

\section{Clustering and Type II Error}

Despite mutual poor showing in terms of Type I error, MLMs fared much better than their SRS counterparts when it came to Type II error rates. When ESs were low (for either the L1 or the interaction term) the Type II rate was always larger for SRS models than it was for MLMs, often by $20 \%$ or more, and over $30 \%$ in some cases. MLMs seemed particularly robust to Type II error for the interaction term, only exceeding the target threshold $(\beta \geq 20 \%)$ in the datasets with the very fewest of observations $(m=5, k=$ 25). Error rates in the SRS models fell below the target values for the interaction term beginning in conditions with 250 or more observations. However, even in these conditions, the target threshold was exceeded for the interaction term in instances where its ES was low (i.e., $d=.2$ ), a value commonly seen in organizational research.

Type II error for the L1 predictor effect, on the other hand, exhibited higher magnitudes (reaching nearly $60 \%$ for SRS models with low $N$ and small ESs) while also persisting into larger- $N$ datasets. Datasets with 500 subjects analyzed with SRS methods still exhibited Type II error rates above the 20\% threshold for the L1 term when ES was small (i.e., .2). In short, MLMs fared much better than their SRS counterparts in terms of Type II error, both exhibiting lower rates and diminishing in prevalence much more quickly. 
Applied Example: NAFASI

As a guided example of a practical use of MLMs, consider the Project NAFASI dataset. The dataset for consideration $(k=32, m=18.53$, unbalanced positively-skewed distribution of cluster sizes) has parameters that are similar to levels of factors roughly similar to those present in the data simulation $(k=25, m=20$, truncated Poissondistributed $m$-size). While the absolute $n$ of the NAFASI dataset (593) is slightly larger than the sizes present in the simulation conditions $(\sim 500), n$ itself was not present in the simulation analysis and, per the simulation results above, is largely confounded with the number of clusters and the size of each.

The NAFASI project analyses primarily regress child and parent reports of program quality (a L1 variable) on all six dimensions of the OSC scale. Although the OSC survey solicits responses from individual employees, the nature of the scale scoring is such that these values are aggregated to the park-level, making the OSC a L2 variable. Thus the model is of the configuration L2 -> L1.

The full analyses integrate several other L2 variables and accompanying interaction effects into the model, which the data simulation above was not designed to accommodate. However, given that the main effects of all the OSC factors were - with or without covariates - not significant for child reports of program quality and only significant for one factor (Proficiency) for parent reports of program quality, it is likely that the effect sizes of these variables are either null or small. As a result, conditions similar to the NAFASI models exist in the current dataset (L1 predictor $=0, \mathrm{~L} 2$ predictor $=.0$ or .2 , and interaction $=0$ ). Further, the ICCs of child/parent reports of program 
quality in the NAFASI dataset is about .15 and .25 respectively. Thus, the conditions in the data simulation of:

(a) (Poisson, $m=20, k=25, \mathrm{ICC}=.1, \mathrm{~L} 1=0, \mathrm{~L} 2=0$, Interaction $=0$ )

(b) (Poisson, $m=20, k=25, \mathrm{ICC}=.1, \mathrm{~L} 1=0, \mathrm{~L} 2=.2$, Interaction $=0$ )

(c) (Poisson, $m=20, k=25, \mathrm{ICC}=.3, \mathrm{~L} 1=0, \mathrm{~L} 2=0$, Interaction $=0$ )

(d) (Poisson, $m=20, k=25, \mathrm{ICC}=.3, \mathrm{~L} 1=0, \mathrm{~L} 2=.2$, Interaction $=0$ )

most closely represent those in the NAFASI project dataset, with (a) and (b) approximating the models with parent program-quality as a dependent variable, and (c) and (d) approximating the models with child program-quality as a dependent variable.

These conditions reported, respectively, an EICC of .09 and .14 for (a) and (b) and of .28 and .31 for (c) and (d). A MDE of 2.75 and 3.67 for (a) and (b) respectively and 6.40 and 6.97 for (c) and (d) respectively was also observed. Conditions (a) and (b) reported identical PDEs for both the L1 variable (.91) and the interaction term (.97). PDEs for (c) and (d) were .73 and .72 for the L1 term and .77 and .78 for the interaction term, respectively.

It should be noted that in condition (b) the average EICC was exactly equal to the ICC reported by the NAFASI models. The actual NAFASI models also reported MDEs of around 3.63 (parent) and 5.38 (child). The parent-report MDE is quite similar to the calculated DE of condition (b). Thus, this condition does a good job of representing the NAFASI models featuring parent reports of program quality as a dependent variable. 


\section{Chapter V}

\section{DISCUSSION}

The current study offers several interesting points of discussion regarding mixed models. Taken together, the results suggest that population ICC had strong effects on the EICC, the MDE, and the PDE of the L1 variable and the cross-level interaction term. The population effect sizes of the independent variables (i.e., L1, L2, and the interaction term) influenced the EICC and MDE, with L2 effect size strongly impacting both outcomes. Population effect size also influenced PDEs for the L1 and interaction terms, although not to the extent that population ICC did.

The number of clusters $(k)$, which is often of great concern to researchers working with ecological or nested datasets and constructs, had a profoundly small impact on the study outcomes. Outside of slightly attenuating the PDEs, any conclusions about the outcomes in a dataset with 25 clusters could likewise be made for a dataset with 100 clusters. Even situations in which number of clusters might ordinarily be expected to play a profound role - such as the inability of a model to converge during estimation - were not apparent in this study. Although in some cases random effects were not able to be estimated, the overall model itself was still estimated, even when effect sizes for all predictors were low or null.

The other factor contributing to the number of cases in each dataset, cluster size $(m)$, produced a few findings of note. Average cluster size had a large impact on the MDE. Conditions with $m$-sizes of 20 had MDEs often many times larger than conditions with only 5 subjects per cluster. However, considering that the formula for calculating $\mathrm{DE}$ is essentially the product of $m$ and ICC, this is not surprising. However, $m$ also had a 
slight but noticeable impact on the PDE. In larger $m$ conditions, the PDE for the interaction term was lower. This means that where cross-level interaction effects were concerned, GLM/SRS estimates were more biased that their MLM counterparts in datasets featuring a large number of clusters. This occurred even in datasets featuring a small or null effect size of the L2 variable, but was naturally more pronounced in conditions with high ICC, where the SE-correcting effect of MLMs was much stronger.

However, this trend was reversed for the L1 variable: In conditions with a low number of cases per cluster MLMs exhibited less bias than GLMs than they did in datasets with larger numbers of cases per cluster. Given that one purpose of MLMs is to correct for the effects of clustering/non-independence in the data, this effect is naturally more pronounced in situations in which data was highly clustered (i.e., with a high ICC), but is still strongly evident even in conditions with little to no clustering. Also, it understandably strengthens in situations where there is both a large L2 and a large crosslevel interaction effect size, as these conditions reflect a larger amount of "ambient" clustering due to between-group variance on the independent variable. However, it persists even when the effect sizes of these predictors are null.

Finally, the shape of the $m$-size distributions seemed to be almost completely irrelevant to the outcome variables evaluated, despite being the focal factor of the current study. In fact, the average EICCs, MDEs, and PDEs were identical to the hundredth decimal value across all six distribution $/ m$-size combinations. In addition, the Poissondistribution versus uniform-distribution distinction made no difference, even when combined with any other single factor (e.g., PICC); results from one type of distribution 
were largely redundant with the results of the other. In sum, the use of MLMs is prevalent in organizational research and related fields.

The current paper examined the impact of several factors present in MLMs on the outcomes of EICCs, MDE, and PDE. The results indicate that, compared to datasets with balanced cluster sizes, unbalanced cluster sizes (of the commonly encountered distribution examined here) did not strongly impact these outcomes. While number of clusters $(k)$ also appeared to have small to no effects on the simulation outcomes, average cluster size $(m)$ impacted the model DE as expected by Equation (1) as well as the EDE in a limited fashion. However, the primary drivers of OICC, calculated DE, and EDE seemed to be the interplay of the ICC and the L1 predictor, L2 predictor, and cross-level interaction effect sizes.

In addition, the current paper extends the results of the MC simulation to a practical, real-world applications via the NAFASI dataset. One disadvantage of simulation studies is that their execution can be very sterile; free from practical concerns such as missing data, alternate model specifications (e.g., covariates), as well as psychometric elements such as reliability. Using the NAFASI dataset and other existing publications in the organizational science literature as a lens to focus its results allows a degree of ecological validity for the data simulation presented here. As indicated in the results section above, simulation conditions approximating those found in the NAFASI dataset and models provided roughly similar results. This illustrates the propriety of using MLMs as an analytic approach both in that dataset, and others conducted in similar environments. 


\section{Contributions}

The current study makes several important contributions to the literature on MLMs in the applied sciences. First, it is unique in that it compares ideal datasets featuring a uniform distribution with those that more closely approximate what would be seen in actual research. Despite the fact that this distinction seemed to be unrelated to any of the current outcome variables, it remains an important contribution: All or at least most previous Monte Carlo simulations on mixed models (e.g., Lai \& Kwok, 2015; Muthen \& Satorra, 1995) have simulated data using only uniform distributions, often mentioning this as a weakness when presenting their findings (see also Clarke, 2008). Also, statistics common in MLMs, such as the DE formula in Equation (1) above, rely on the assumption of uniform cluster sizes within a dataset. Thus, empirically examining the differences between uniformly-distributed and more "real world" datasets allows researchers to use these metrics and MLMs in general with more confidence and allows more transportability of research findings.

Second, the current study compares not only the impact of the simulation factors themselves, but also allows for comparison of the outcome variables by including two different conceptualizations of the design effect. The MDE functions as a sort of global DE, providing information on how much clustering in the dataset is deflating SEs for the overall model. The PDEs, meanwhile, show how bias in each individual predictor term contributes to overall bias in the model (i.e., the MDE).

As a result, the current study contributes to the literature by considering not only SE deflation in the overall model, but also which predictors are driving that deflation. 
There were many instances - particularly when ICC was low but the L2 predictor effect size was high - that large DEs were projected for the model using Formula (1) but did not actually manifest for individual predictors. Far more common, however, were instances in which the MDE was below 2, yet a strong PDE emerged on the L1 predictor variable; a phenomenon exacerbated by stronger interaction effect sizes. This phenomenon was present in conditions even with a null PICC, and grew concomitantly stronger with higher levels of PICC. For researchers, this means that the calculation of a large MDE during the research design phase does not necessarily guarantee SE deflation for individual predictors, nor does a small MDE prevent SE deflation.

Nonetheless, in many cases calculated model DE remained an accurate predictor of SE deflation. Not only were MDE and PDE in agreement, but recent research (Lai \& Kwok, 2015) has suggested that DEs as low as 1.1 may be cause for concern in many situations and warrant the use of MLMs or similar methods. Thus, it is less an issue of the calculation of DE itself, and more the reliance upon a common rule of thumb ("Use MLMs when DE is greater than 2") that is at issue.

The third contribution of the current study is its scope. With 6,144 conditions across 6 factors, the study allows for great nuance in examining the several moving parts present in MLMs. Understanding MLMs, their components, and the terminology associated with them goes far in making them accessible to researchers. In addition, the levels of each factor present in the simulation allow it to cover a broad range of possible datasets and make the findings applicable to numerous fields of social, behavioral, and medical research. 
Finally, the current paper allows for examination of the volatility of elements of MLMs themselves. Specifically, EICC merits consideration. The bounds for the point at which observed ICC was considered to be an aberration in the current paper was set at .15 due to previous research (Donner \& Wells, 1986) which suggested that an overall range of about .30 was common for ICC estimates based on datasets with 50 clusters. Restated, observed ICC in any condition could be expected to be between .15 above or below the target ICC value. So in conditions where ICC was simulated at .50, actual ICC values across levels of other conditions should fall between .35 and .65 .

However, ranges of the EICC in the current study varied across levels of PICC, such that EICCs occurred in a band of about .42 in the PICC $=.5$ conditions, about .47 in the $\mathrm{PICC}=.3$ conditions, and about .50 in the $\mathrm{PICC}=.1$ conditions. The actual EICC values were highest when the L1 and interaction effect sizes were null and the L2 effect sizes were strong, and weakest when L1 and interaction effect sizes were high and L2 effect sizes were null. Thus as ICC decreased, the variance of within-condition estimates increased. This means that when only small or even incidental amounts of clustering are present, as is the case in the majority of datasets in the social sciences, ICC has the largest potential range. This means that a large DE may be impacting results even when clustering is expected neither ecologically nor theoretically. That is, when a researcher is either unaware of and/or has no reason to expect clustering in the dataset.

\section{Limitations}

Despite the contributions of the current study to literature on MLMs, it has a number of shortcomings. First, the centering strategy chosen in the current paper obviously impacts the ability of the study to draw conclusions to the widest range of 
research questions possible. However, the decision to center in a way that allowed for valid interpretation of the $\mathrm{L} 1$ and cross-level interaction effects is a defensible one, as most organizational research targets variables of this type. Nonetheless, centering the L1 predicting using GMC instead of CWC would allow for additional (and different) interpretation of study factors.

Second, the current study focused primarily on the estimation and interpretation of fixed effects; the study ignored random slope and random intercept/slope models. Instead, the MLMs used in the current study were exclusively random-intercept only models. While including random slope models or combined models in the analysis would greatly expand the interpretation and application of the simulation factors, it would also greatly increase the complexity of the simulation itself and drastically increase the complexity of the results.

Third, while the current study indeed covers a broad range of dataset characteristics that researchers are likely to encounter, it is heavily targeted at crosssectional rather than longitudinal research. Not only do longitudinal models differ from cross-sectional models in the interpretation of estimates, but they also feature conditions outside those present in the current study. For example, ICCs in longitudinal MLMs are often much higher than those in cross-sectional designs, because repeated observations come from the same person.

Fourth, only datasets with two levels of data were used in the current study. Examples of research questions with three or more levels are becoming increasingly common. However, the majority of multilevel research, particularly in the organizational sciences, still involves two levels. Estimating and evaluating more complicated datasets 
is more complex; given the already complex nature of the current study and the dominance of two-level models in the literature, two-level-only models were simulated and evaluated.

Finally, the actual model evaluated in the current study is a simple one. It features only three independent variables, and only a single interaction/moderator. Due to the accessibility and popularity of structural equation modeling and its accompanying estimation methods, researchers have grown accustomed to evaluating models with several main and interaction effects, complex factor structures, mediation, multiple outcomes, and any combination thereof. Even simple models make for complicated simulations, particularly when several levels of several factors are present. Simulating the variety of complicated models used in modern research is possible, but prohibitive in regards to the time required and the scope of the ensuing analysis.

\section{Future Research and Implications}

In the discussion in this section, several points have been made that point to directions for future research or are relevant to researchers already working in the field. The first possible direction for future research, naturally, is to address the shortcomings of the current study as mentioned above. With slightly different values of some factors (i.e, population ICC), it could be adapted to evaluate longitudinal MLMs. Alternately, additional terms could be simulated that would allow for three-level models, models with multiple L1 and L2 variables or models that include random slopes in addition to random intercepts.

Additionally, the potential values for some factors could be expanded. Extant organizational research reviewed above already provides examples of datasets which 
feature $n$ or $k$ sizes that far exceed those contained in the current study (e.g., Fisher, 2014; Liu et al., 2012). Other practical factors, such as the presence of missing data, could also be considered.

Another potential implication for future research is the impact of $N$-size with differential $k$-size on model estimates. Researchers - especially those interested in L2 or cluster-level variables - are often advised that, given the same number of overall observations, more clusters are preferable to more observations per cluster (Snijders, 2005). For example, if a researcher has a target $\mathrm{N}$ of 500, a dataset with 100 clusters $(k)$ and 5 subjects per cluster $(\mathrm{m})$ is preferable to a dataset with 50 clusters and 10 subjects per cluster, which is itself is preferable to a dataset with 25 clusters and 20 subjects per cluster.

The current study features several $k$ and $m$ combinations which provide identical $\mathrm{N}$-sizes. Specifically, there are two different configurations of $\mathrm{N}=250$ datasets $(\mathrm{m}=5, \mathrm{k}$ $=50 ; m=10, k=25)$, three different configurations of $\mathrm{N}=500$ datasets $(m=5, k=100$; $m=10, k=50 ; m=20, k=25)$, and two different configurations of $\mathrm{N}=1000$ datasets $(m$ $=10, k=100 ; m=20, k=50)$ available in the current study. Thus small, medium, and large datasets (vis. organizational literature) are available for comparison.

When comparing the small datasets, the $m=5$ conditions performed nearly as well as the $m=10$ conditions in terms of OICC and PDEs, with Type II error rates very slightly favoring $m=10$ datasets and Type I error rates slightly favoring $m=5$ conditions. MDE, being derived in part from $m$ size, unsurprisingly favored the smaller $m$ conditions, with these conditions reporting lower MDEs than their larger- $m$ counterparts. This pattern continued for the medium and large datasets. Initially, this seems to challenge the idea 
that more clusters are necessary to accurately obtain estimates. However, as the centering strategy employed in the current paper prevented the inclusion of L2 (cluster) effects, and the "more $k$ is better than fewer $k$ " recommendation is primarily targeted at these effects, these conclusions should be interpreted cautiously.

Despite the plentiful directions for future research, the current study already provides potential applications for both applied and theoretical researchers. Most interesting is the corroboration of existing research (e.g., Lai \& Kwok, 2015; Muthen \& Satorra, 1995); this study also finds that distortion of estimates may still occur even when levels of clustering or resulting DEs are low.

As a result, it may be tempting for researchers to use MLMs or other robust methods in order to estimate model parameters and accompanying significance tests, even when these methods are not theoretically/ecologically warranted or justified. This pressure is compounded by the fact that MLMs, as a newer procedure, may be seen as somehow better than more familiar methods. In addition, usage by prominent or rival researchers may engender a "me too" effect, where the analysis is favored because it is trendy or viewed as necessary and not because it is appropriate. While MLMs are indeed powerful, researchers are advised to carefully consider their modeling options, as alternate and more appropriate methods may exist (see McNeish, Stapleton, and Silverman, 2016).

Despite their potential pitfalls, however, researchers considering analysis using multilevel approaches should be relieved to hear that the distribution of participants per cluster (uniform vs. Poisson) was practically irrelevant for the purposes of model estimates: OICCs, MDEs, and PDEs were functionally identical for both types of 
conditions, as were Type I and II error rates. Multilevel models and their kin have several moving parts and can be very complex to manage. However, in at least the current study, researchers are advised to pay closer attention to the amount of clustering present in the dataset, rather than how the clusters themselves are distributed. 
Table 1 .

Average Observed ICC values: Uniform Distribution conditions $(\mathrm{m}=5, \mathrm{k}=25)$

\begin{tabular}{|c|c|c|c|c|c|c|c|c|c|c|c|c|c|c|c|c|c|}
\hline \multirow{3}{*}{$\begin{array}{c}\text { L1 } \\
\text { Effect }\end{array}$} & \multirow{3}{*}{$\begin{array}{c}\text { Interaction } \\
\text { Effect }\end{array}$} & \multicolumn{12}{|c|}{ ICC Values } & \multicolumn{4}{|c|}{0.5} \\
\hline & & \multicolumn{16}{|c|}{ L2 Effect } \\
\hline & & 0 & 0.2 & 0.5 & 0.8 & 0 & 0.2 & 0.5 & 0.8 & 0 & 0.2 & 0.5 & 0.8 & 0 & 0.2 & 0.5 & 0.8 \\
\hline \multirow{4}{*}{0} & 0 & 0.02 & 0.06 & 0.27 & 0.49 & 0.09 & 0.14 & 0.32 & 0.51 & 0.28 & 0.31 & 0.43 & 0.57 & 0.47 & 0.49 & 0.56 & 0.65 \\
\hline & 0.2 & 0.02 & 0.06 & 0.25 & 0.47 & 0.09 & 0.13 & 0.30 & 0.50 & 0.27 & 0.29 & 0.41 & 0.55 & 0.46 & 0.48 & 0.55 & 0.63 \\
\hline & 0.5 & 0.02 & 0.05 & 0.20 & 0.40 & 0.07 & 0.10 & 0.25 & 0.43 & 0.22 & 0.24 & 0.36 & 0.49 & 0.39 & 0.41 & 0.48 & 0.57 \\
\hline & 0.8 & 0.02 & 0.04 & 0.15 & 0.32 & 0.05 & 0.08 & 0.18 & 0.34 & 0.16 & 0.18 & 0.27 & 0.40 & 0.31 & 0.32 & 0.38 & 0.48 \\
\hline \multirow{4}{*}{0.2} & 0 & 0.02 & 0.06 & 0.26 & 0.47 & 0.09 & 0.13 & 0.31 & 0.50 & 0.27 & 0.29 & 0.42 & 0.56 & 0.46 & 0.47 & 0.55 & 0.63 \\
\hline & 0.2 & 0.02 & 0.06 & 0.25 & 0.45 & 0.08 & 0.12 & 0.29 & 0.48 & 0.25 & 0.29 & 0.40 & 0.53 & 0.45 & 0.46 & 0.53 & 0.62 \\
\hline & 0.5 & 0.02 & 0.05 & 0.20 & 0.39 & 0.07 & 0.10 & 0.24 & 0.42 & 0.21 & 0.24 & 0.34 & 0.48 & 0.38 & 0.40 & 0.46 & 0.56 \\
\hline & 0.8 & 0.03 & 0.04 & 0.15 & 0.31 & 0.06 & 0.08 & 0.19 & 0.33 & 0.16 & 0.17 & 0.26 & 0.39 & 0.31 & 0.31 & 0.38 & 0.47 \\
\hline \multirow{4}{*}{0.5} & 0 & 0.02 & 0.05 & 0.21 & 0.41 & 0.07 & 0.10 & 0.25 & 0.43 & 0.22 & 0.24 & 0.35 & 0.48 & 0.39 & 0.41 & 0.48 & 0.57 \\
\hline & 0.2 & 0.02 & 0.05 & 0.20 & 0.39 & 0.07 & 0.10 & 0.24 & 0.41 & 0.21 & 0.24 & 0.34 & 0.48 & 0.38 & 0.40 & 0.47 & 0.56 \\
\hline & 0.5 & 0.02 & 0.04 & 0.17 & 0.34 & 0.06 & 0.08 & 0.20 & 0.36 & 0.18 & 0.20 & 0.30 & 0.43 & 0.34 & 0.35 & 0.41 & 0.51 \\
\hline & 0.8 & 0.02 & 0.04 & 0.13 & 0.28 & 0.05 & 0.07 & 0.16 & 0.30 & 0.14 & 0.16 & 0.23 & 0.35 & 0.28 & 0.28 & 0.34 & 0.43 \\
\hline \multirow{4}{*}{0.8} & 0 & 0.02 & 0.04 & 0.15 & 0.32 & 0.05 & 0.08 & 0.19 & 0.34 & 0.16 & 0.18 & 0.27 & 0.39 & 0.31 & 0.32 & 0.39 & 0.47 \\
\hline & 0.2 & 0.02 & 0.04 & 0.15 & 0.31 & 0.05 & 0.08 & 0.18 & 0.33 & 0.15 & 0.18 & 0.26 & 0.39 & 0.30 & 0.31 & 0.37 & 0.47 \\
\hline & 0.5 & 0.02 & 0.04 & 0.13 & 0.28 & 0.05 & 0.06 & 0.16 & 0.30 & 0.14 & 0.16 & 0.24 & 0.36 & 0.28 & 0.28 & 0.35 & 0.44 \\
\hline & 0.8 & 0.03 & 0.04 & 0.11 & 0.23 & 0.04 & 0.06 & 0.13 & 0.26 & 0.12 & 0.13 & 0.20 & 0.30 & 0.23 & 0.24 & 0.30 & 0.38 \\
\hline
\end{tabular}

Note: Italicized values indicate conditional deviance of .15 or more above specified value, while bold values indicate conditional deviance of .15 or more below the specified value. ICC = Intraclass Correlation Coefficient. 
Table 1 cont.

Average Observed ICC values: Uniform Distribution conditions $(\mathrm{m}=5, \mathrm{k}=50)$

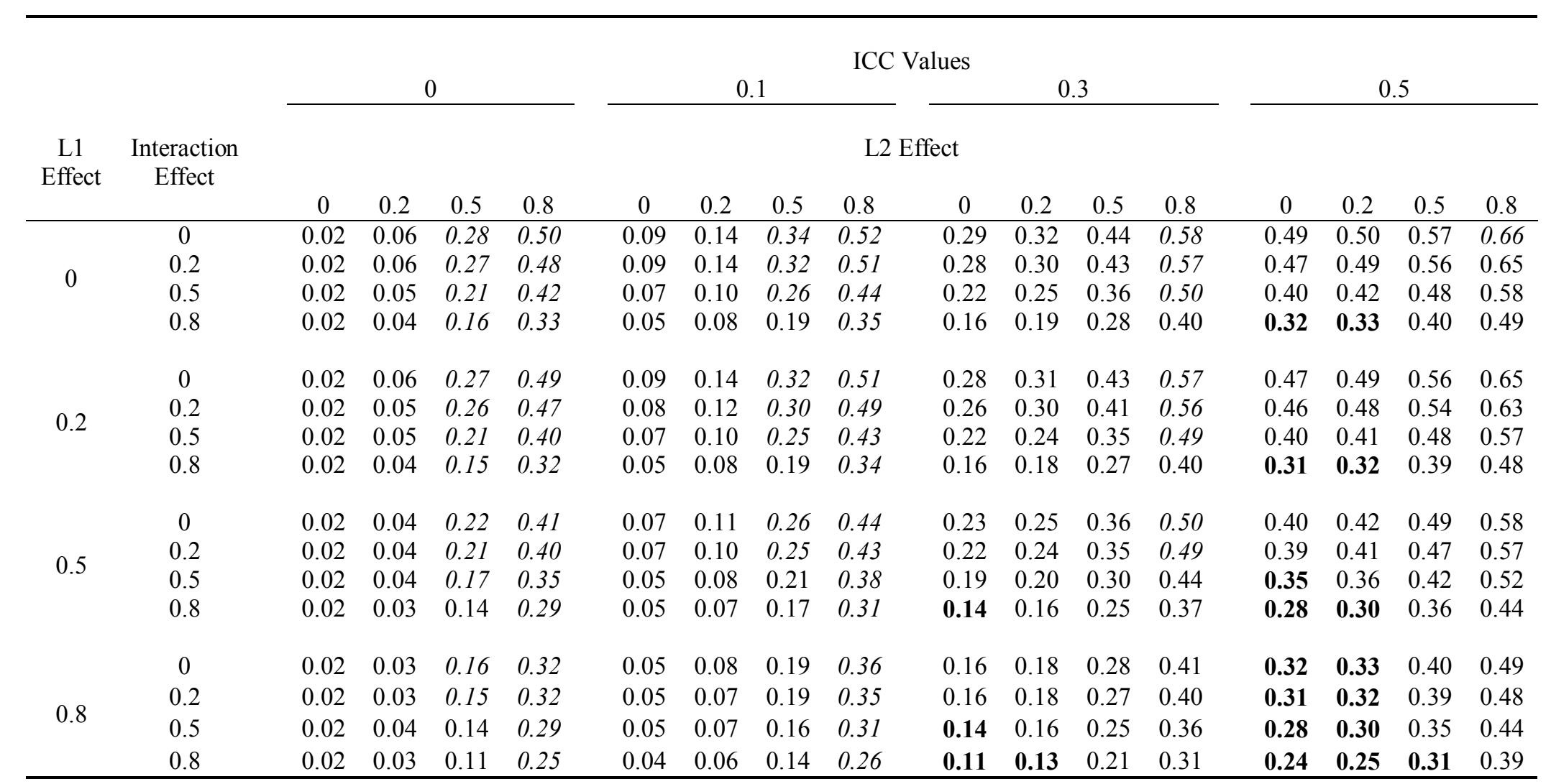

Note: Italicized values indicate conditional deviance of .15 or more above specified value, while bold values indicate conditional deviance of .15 or more below the specified value. ICC = Intraclass Correlation Coefficient. 
Table 1 cont.

Average Observed ICC values: Uniform Distribution conditions $(\mathrm{m}=5, \mathrm{k}=75)$

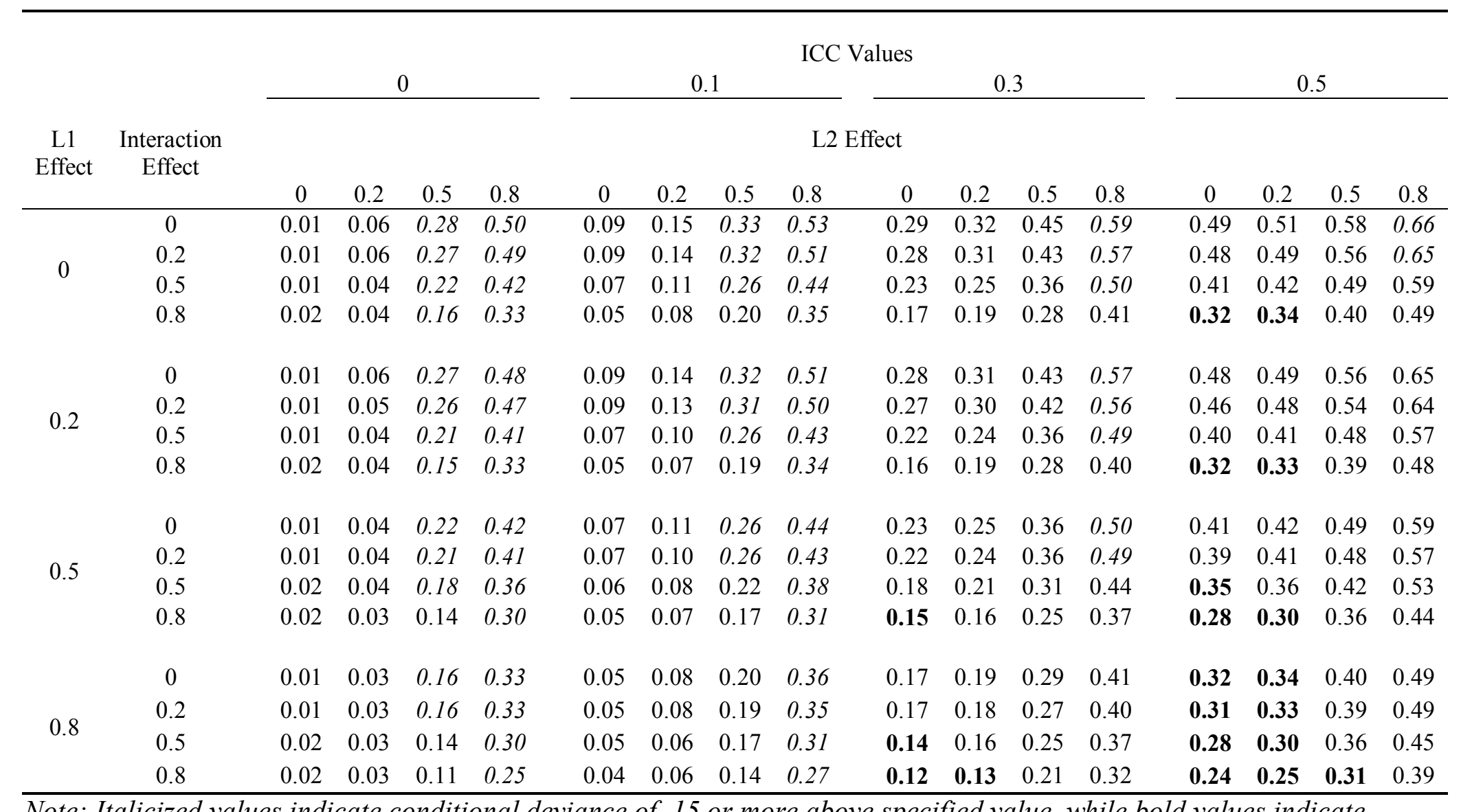

Note: Italicized values indicate conditional deviance of .15 or more above specified value, while bold values indicate

conditional deviance of .15 or more below the specified value. ICC = Intraclass Correlation Coefficient. 
Table 1 cont.

Average Observed ICC values: Uniform Distribution conditions $(\mathrm{m}=5, \mathrm{k}=100)$

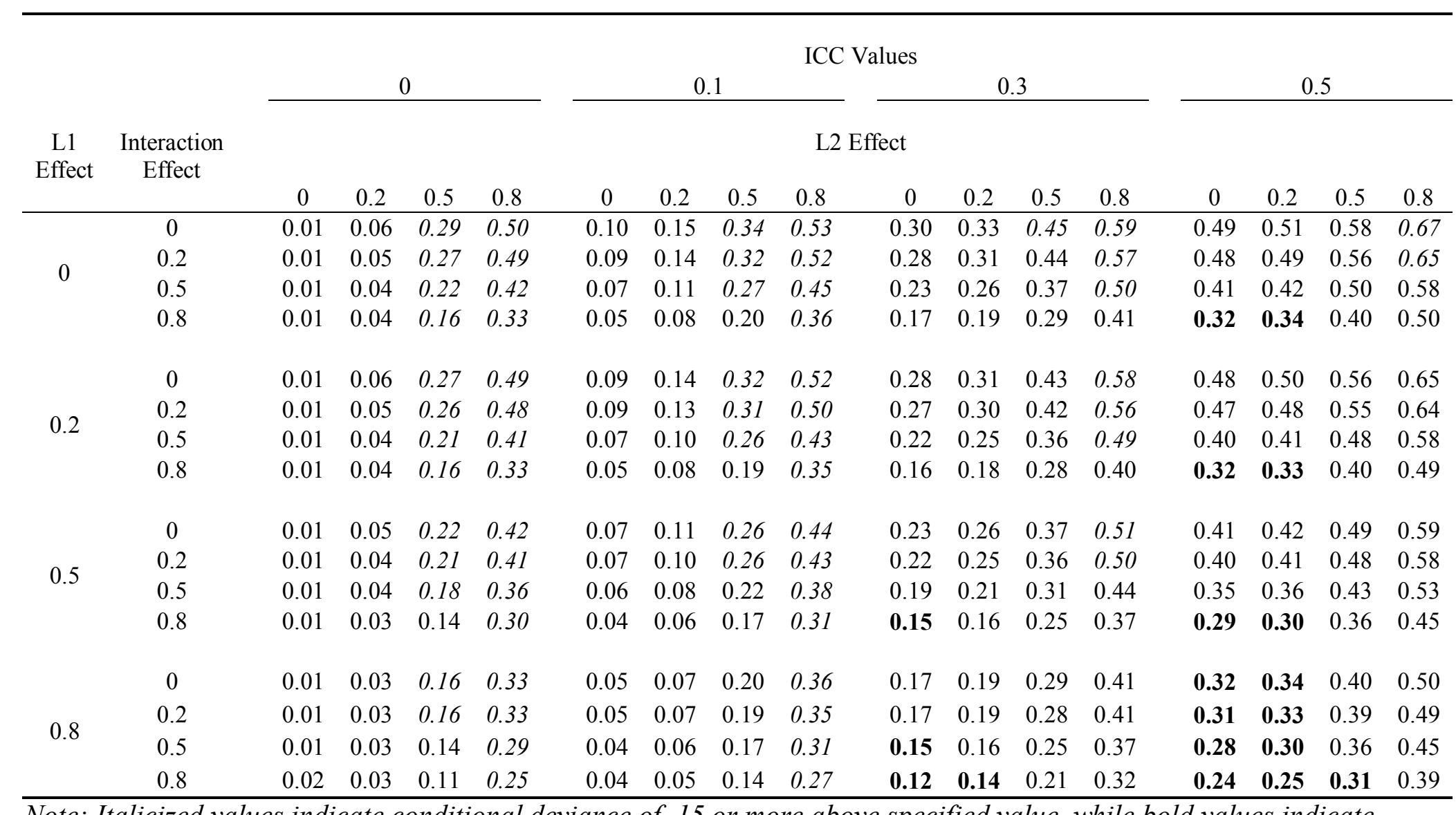

Note: Italicized values indicate conditional deviance of .15 or more above specified value, while bold values indicate

conditional deviance of .15 or more below the specified value. ICC = Intraclass Correlation Coefficient. 
Table 1 cont.

Average Observed ICC values: Uniform Distribution conditions $(\mathrm{m}=10, \mathrm{k}=25)$

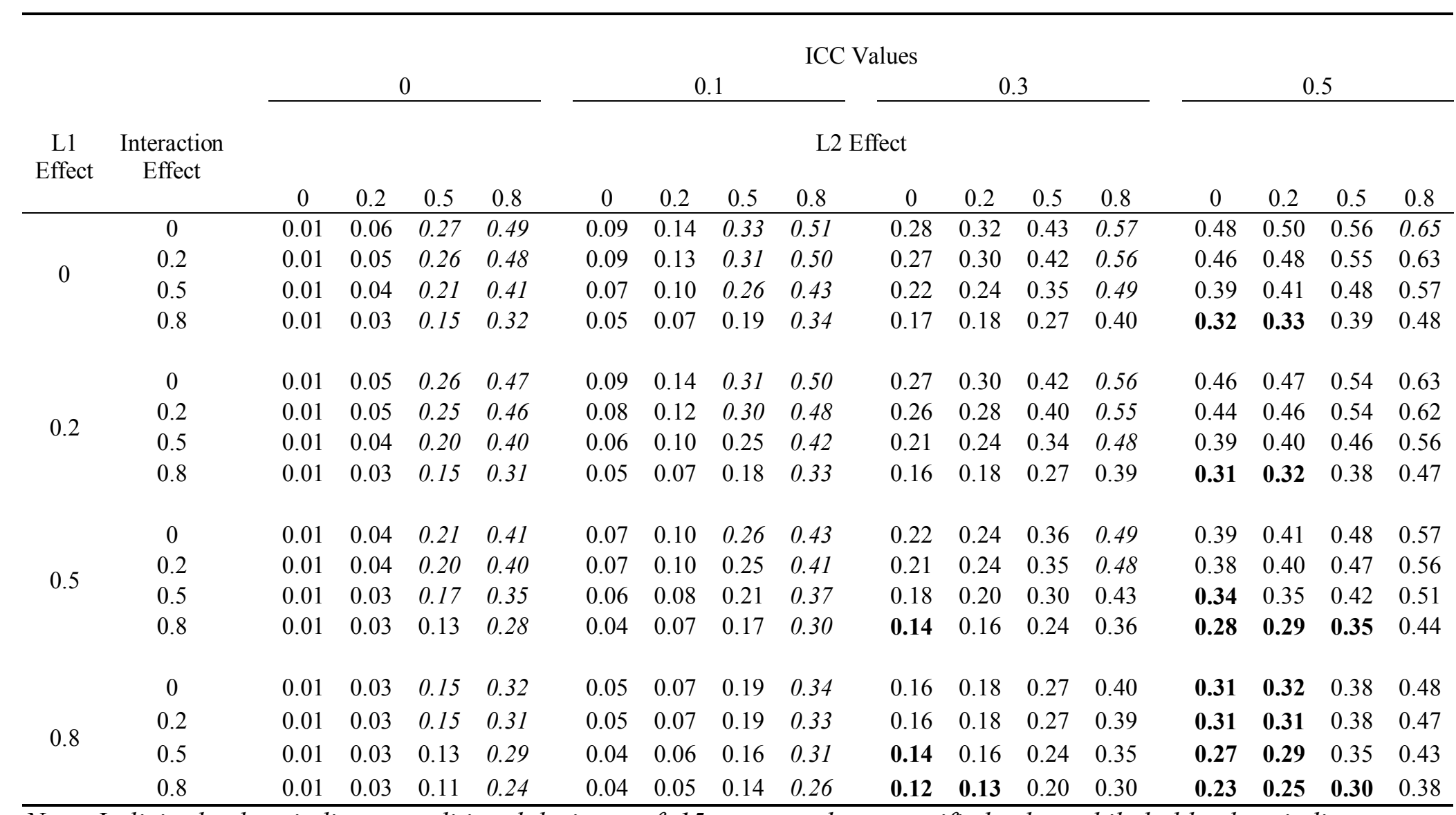

Note: Italicized values indicate conditional deviance of .15 or more above specified value, while bold values indicate conditional deviance of .15 or more below the specified value. ICC = Intraclass Correlation Coefficient. 
Table 1 cont.

Average Observed ICC values: Uniform Distribution conditions $(\mathrm{m}=10, \mathrm{k}=50)$

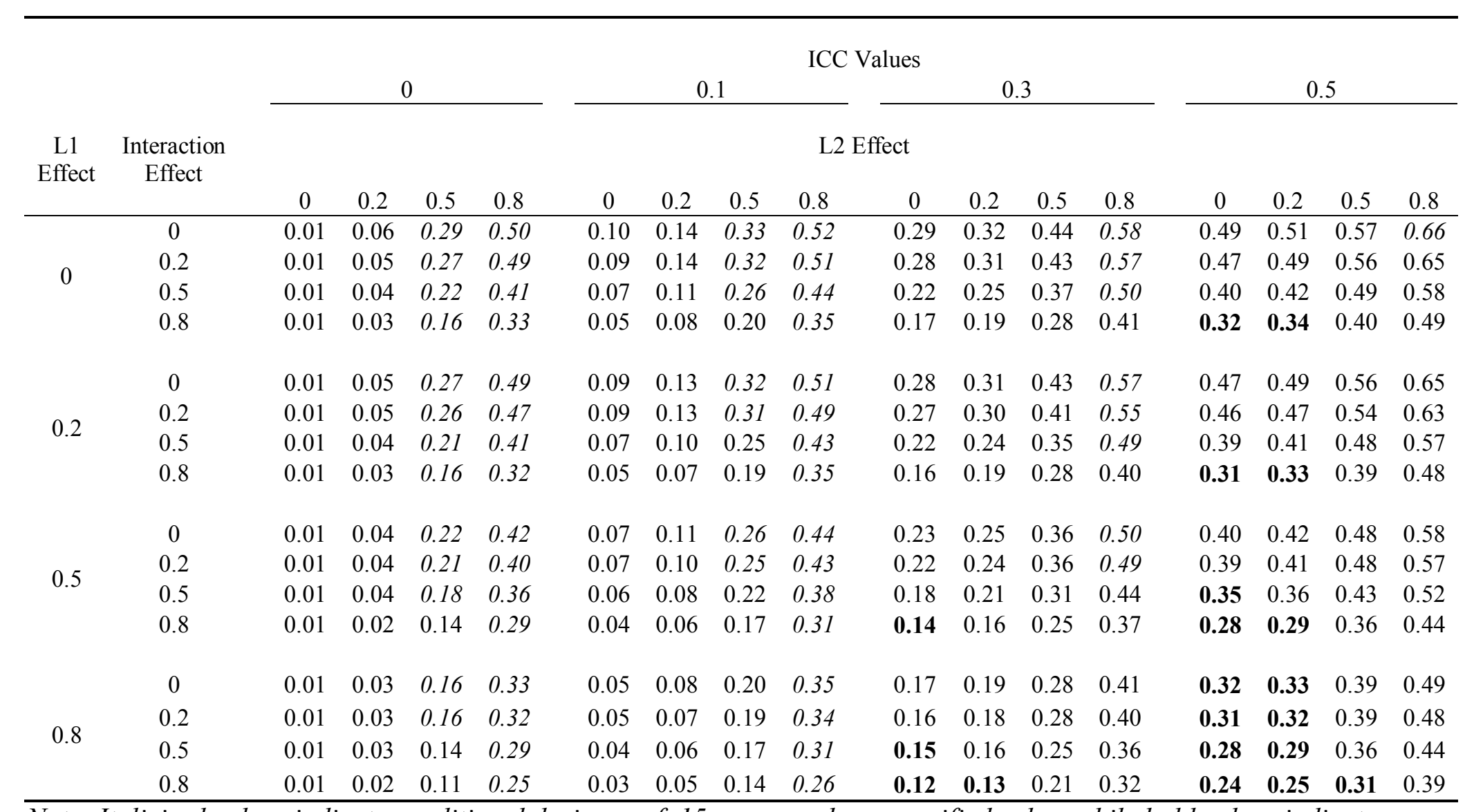

Note: Italicized values indicate conditional deviance of .15 or more above specified value, while bold values indicate

conditional deviance of .15 or more below the specified value. ICC = Intraclass Correlation Coefficient. 
Table 1 cont.

Average Observed ICC values: Uniform Distribution conditions $(\mathrm{m}=10, \mathrm{k}=75)$

\begin{tabular}{|c|c|c|c|c|c|c|c|c|c|c|c|c|c|c|c|c|c|}
\hline \multirow{4}{*}{$\begin{array}{c}\text { L1 } \\
\text { Effect }\end{array}$} & \multirow{4}{*}{$\begin{array}{c}\text { Interaction } \\
\text { Effect }\end{array}$} & \multicolumn{16}{|c|}{ ICC Values } \\
\hline & & \multicolumn{4}{|c|}{0} & \multicolumn{4}{|c|}{0.1} & \multicolumn{4}{|c|}{0.3} & \multicolumn{4}{|c|}{0.5} \\
\hline & & \multicolumn{16}{|c|}{ L2 Effect } \\
\hline & & 0 & 0.2 & 0.5 & 0.8 & 0 & 0.2 & 0.5 & 0.8 & 0 & 0.2 & 0.5 & 0.8 & 0 & 0.2 & 0.5 & 0.8 \\
\hline \multirow{4}{*}{0} & 0 & 0.01 & 0.06 & 0.28 & 0.51 & 0.10 & 0.15 & 0.34 & 0.53 & 0.30 & 0.33 & 0.45 & 0.59 & 0.49 & 0.51 & 0.58 & 0.67 \\
\hline & 0.2 & 0.01 & 0.06 & 0.27 & 0.49 & 0.09 & 0.14 & 0.32 & 0.51 & 0.28 & 0.31 & 0.43 & 0.57 & 0.48 & 0.50 & 0.57 & 0.65 \\
\hline & 0.5 & 0.01 & 0.04 & 0.22 & 0.42 & 0.07 & 0.11 & 0.26 & 0.45 & 0.23 & 0.26 & 0.37 & 0.50 & 0.41 & 0.42 & 0.49 & 0.59 \\
\hline & 0.8 & 0.01 & 0.03 & 0.16 & 0.33 & 0.05 & 0.08 & 0.20 & 0.35 & 0.17 & 0.19 & 0.29 & 0.41 & 0.33 & 0.34 & 0.40 & 0.49 \\
\hline \multirow{4}{*}{0.2} & 0 & 0.01 & 0.06 & 0.27 & 0.49 & 0.09 & 0.14 & 0.32 & 0.51 & 0.28 & 0.31 & 0.43 & 0.57 & 0.47 & 0.49 & 0.56 & 0.65 \\
\hline & 0.2 & 0.01 & 0.05 & 0.26 & 0.47 & 0.09 & 0.13 & 0.31 & 0.50 & 0.27 & 0.30 & 0.42 & 0.56 & 0.46 & 0.48 & 0.55 & 0.64 \\
\hline & 0.5 & 0.01 & 0.04 & 0.21 & 0.41 & 0.07 & 0.10 & 0.26 & 0.43 & 0.22 & 0.25 & 0.35 & 0.49 & 0.40 & 0.41 & 0.48 & 0.57 \\
\hline & 0.8 & 0.01 & 0.03 & 0.16 & 0.32 & 0.05 & 0.07 & 0.19 & 0.34 & 0.17 & 0.19 & 0.28 & 0.41 & 0.32 & 0.33 & 0.39 & 0.48 \\
\hline \multirow{4}{*}{0.5} & 0 & 0.01 & 0.04 & 0.22 & 0.42 & 0.07 & 0.11 & 0.26 & 0.44 & 0.23 & 0.25 & 0.37 & 0.50 & 0.41 & 0.43 & 0.49 & 0.58 \\
\hline & 0.2 & 0.01 & 0.04 & 0.21 & 0.41 & 0.07 & 0.10 & 0.26 & 0.43 & 0.22 & 0.25 & 0.36 & 0.49 & 0.40 & 0.41 & 0.48 & 0.58 \\
\hline & 0.5 & 0.01 & 0.03 & 0.18 & 0.36 & 0.06 & 0.09 & 0.22 & 0.38 & 0.19 & 0.21 & 0.31 & 0.44 & 0.35 & 0.36 & 0.43 & 0.52 \\
\hline & 0.8 & 0.01 & 0.03 & 0.14 & 0.29 & 0.04 & 0.07 & 0.17 & 0.31 & 0.15 & 0.16 & 0.25 & 0.37 & 0.29 & 0.30 & 0.36 & 0.45 \\
\hline \multirow{4}{*}{0.8} & 0 & 0.01 & 0.03 & 0.16 & 0.33 & 0.05 & 0.08 & 0.20 & 0.35 & 0.17 & 0.19 & 0.28 & 0.41 & 0.32 & 0.34 & 0.40 & 0.49 \\
\hline & 0.2 & 0.01 & 0.03 & 0.16 & 0.32 & 0.05 & 0.07 & 0.19 & 0.35 & 0.17 & 0.18 & 0.28 & 0.41 & 0.32 & 0.33 & 0.40 & 0.48 \\
\hline & 0.5 & 0.01 & 0.03 & 0.14 & 0.29 & 0.04 & 0.07 & 0.17 & 0.32 & 0.14 & 0.17 & 0.25 & 0.37 & 0.28 & 0.29 & 0.36 & 0.45 \\
\hline & 0.8 & 0.01 & 0.02 & 0.11 & 0.25 & 0.03 & 0.05 & 0.14 & 0.27 & 0.12 & 0.14 & 0.21 & 0.32 & 0.24 & 0.25 & 0.31 & 0.39 \\
\hline
\end{tabular}

Note: Italicized values indicate conditional deviance of .15 or more above specified value, while bold values indicate

conditional deviance of .15 or more below the specified value. ICC = Intraclass Correlation Coefficient. 
Table 1 cont.

Average Observed ICC values: Uniform Distribution conditions $(\mathrm{m}=10, \mathrm{k}=100)$

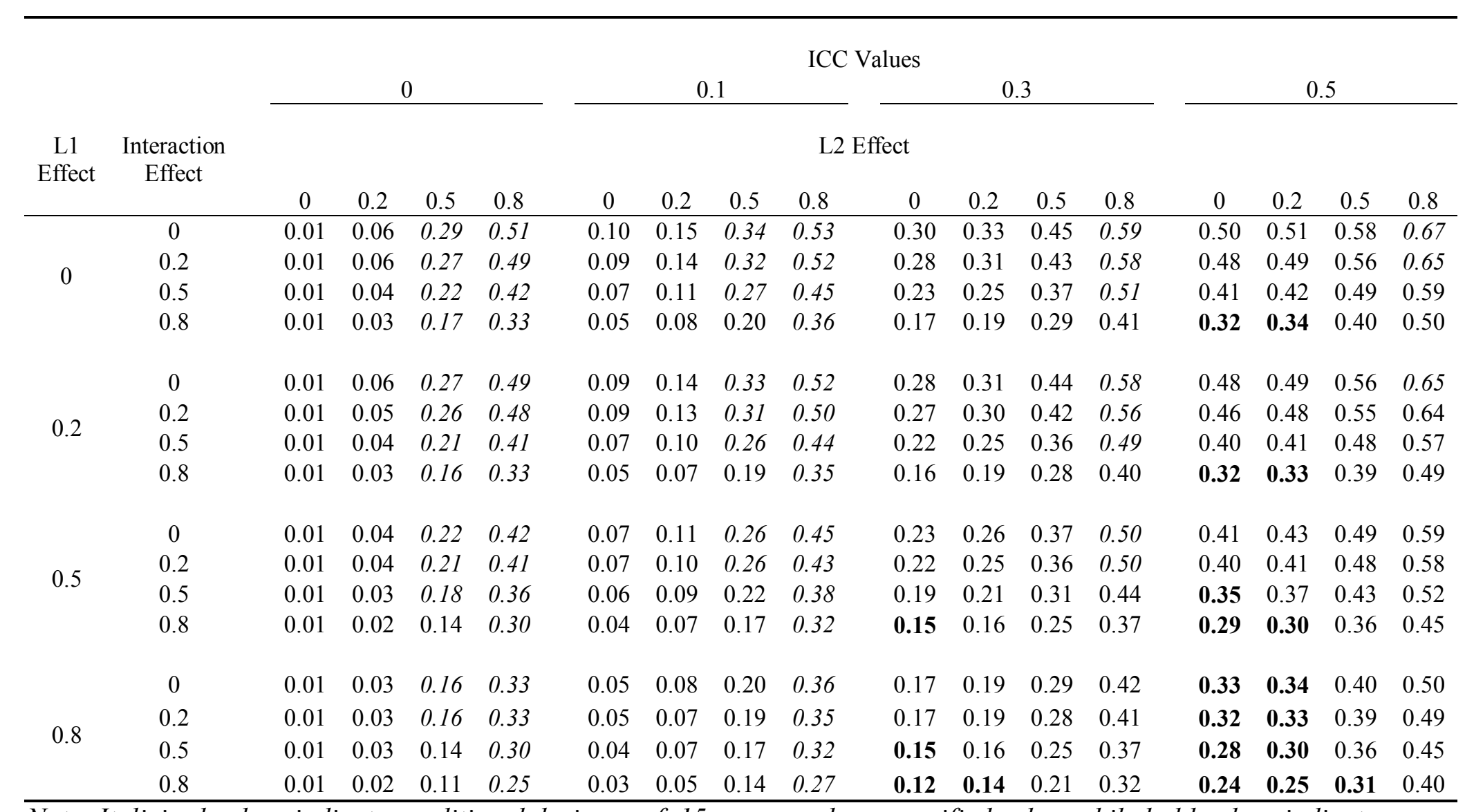

Note: Italicized values indicate conditional deviance of .15 or more above specified value, while bold values indicate

conditional deviance of .15 or more below the specified value. ICC = Intraclass Correlation Coefficient. 
Table 1 cont.

Average Observed ICC values: Uniform Distribution conditions $(\mathrm{m}=20, \mathrm{k}=25)$

\begin{tabular}{|c|c|c|c|c|c|c|c|c|c|c|c|c|c|c|c|c|c|}
\hline \multirow{3}{*}{$\begin{array}{c}\text { L1 } \\
\text { Effect }\end{array}$} & \multirow{3}{*}{$\begin{array}{l}\text { Interaction } \\
\text { Effect }\end{array}$} & \multicolumn{16}{|c|}{ ICC Values } \\
\hline & & \multicolumn{4}{|c|}{0} & \multicolumn{4}{|c|}{0.1} & \multicolumn{4}{|c|}{0.3} & \multicolumn{4}{|c|}{0.5} \\
\hline & & 0 & 0.2 & 0.5 & 0.8 & 0 & 0.2 & 0.5 & 0.8 & 0 & 0.2 & 0.5 & 0.8 & 0 & 0.2 & 0.5 & 0.8 \\
\hline \multirow{4}{*}{0} & 0 & 0.00 & 0.06 & 0.27 & 0.49 & 0.09 & 0.14 & 0.32 & 0.52 & 0.29 & 0.32 & 0.44 & 0.57 & 0.48 & 0.50 & 0.56 & 0.65 \\
\hline & 0.2 & 0.00 & 0.05 & 0.27 & 0.47 & 0.09 & 0.13 & 0.31 & 0.50 & 0.27 & 0.30 & 0.42 & 0.56 & 0.46 & 0.48 & 0.55 & 0.63 \\
\hline & 0.5 & 0.01 & 0.04 & 0.21 & 0.41 & 0.07 & 0.10 & 0.26 & 0.43 & 0.22 & 0.25 & 0.35 & 0.49 & 0.39 & 0.42 & 0.48 & 0.57 \\
\hline & 0.8 & 0.01 & 0.03 & 0.15 & 0.32 & 0.05 & 0.07 & 0.19 & 0.34 & 0.17 & 0.19 & 0.28 & 0.40 & 0.31 & 0.33 & 0.39 & 0.48 \\
\hline \multirow{4}{*}{0.2} & 0 & 0.00 & 0.05 & 0.26 & 0.47 & 0.09 & 0.14 & 0.32 & 0.49 & 0.27 & 0.30 & 0.42 & 0.56 & 0.46 & 0.48 & 0.55 & 0.64 \\
\hline & 0.2 & 0.01 & 0.05 & 0.25 & 0.46 & 0.08 & 0.13 & 0.30 & 0.48 & 0.26 & 0.29 & 0.40 & 0.54 & 0.44 & 0.46 & 0.53 & 0.62 \\
\hline & 0.5 & 0.01 & 0.04 & 0.21 & 0.39 & 0.06 & 0.10 & 0.25 & 0.42 & 0.21 & 0.24 & 0.34 & 0.48 & 0.39 & 0.40 & 0.47 & 0.56 \\
\hline & 0.8 & 0.01 & 0.03 & 0.15 & 0.31 & 0.05 & 0.07 & 0.19 & 0.34 & 0.16 & 0.18 & 0.27 & 0.39 & 0.31 & 0.32 & 0.38 & 0.47 \\
\hline \multirow{4}{*}{0.5} & 0 & 0.00 & 0.04 & 0.22 & 0.40 & 0.07 & 0.11 & 0.26 & 0.43 & 0.22 & 0.25 & 0.35 & 0.49 & 0.39 & 0.41 & 0.48 & 0.57 \\
\hline & 0.2 & 0.01 & 0.04 & 0.21 & 0.39 & 0.06 & 0.10 & 0.25 & 0.42 & 0.21 & 0.23 & 0.34 & 0.48 & 0.39 & 0.40 & 0.47 & 0.56 \\
\hline & 0.5 & 0.01 & 0.03 & 0.17 & 0.35 & 0.06 & 0.08 & 0.21 & 0.37 & 0.18 & 0.20 & 0.30 & 0.43 & 0.34 & 0.36 & 0.42 & 0.51 \\
\hline & 0.8 & 0.01 & 0.02 & 0.13 & 0.29 & 0.04 & 0.06 & 0.17 & 0.30 & 0.14 & 0.16 & 0.24 & 0.36 & 0.28 & 0.29 & 0.35 & 0.43 \\
\hline \multirow{4}{*}{0.8} & 0 & 0.00 & 0.03 & 0.16 & 0.32 & 0.05 & 0.07 & 0.19 & 0.34 & 0.16 & 0.19 & 0.28 & 0.40 & 0.31 & 0.32 & 0.39 & 0.48 \\
\hline & 0.2 & 0.01 & 0.03 & 0.15 & 0.32 & 0.04 & 0.07 & 0.19 & 0.34 & 0.16 & 0.18 & 0.26 & 0.39 & 0.30 & 0.32 & 0.38 & 0.47 \\
\hline & 0.5 & 0.01 & 0.02 & 0.14 & 0.29 & 0.04 & 0.06 & 0.17 & 0.30 & 0.14 & 0.16 & 0.24 & 0.36 & 0.27 & 0.29 & 0.35 & 0.44 \\
\hline & 0.8 & 0.01 & 0.02 & 0.11 & 0.25 & 0.03 & 0.05 & 0.14 & 0.26 & 0.12 & 0.13 & 0.20 & 0.30 & 0.23 & 0.25 & 0.30 & 0.38 \\
\hline
\end{tabular}

Note: Italicized values indicate conditional deviance of .15 or more above specified value, while bold values indicate conditional deviance of .15 or more below the specified value. ICC = Intraclass Correlation Coefficient. 
Table 1 cont.

Average Observed ICC values: Uniform Distribution conditions $(\mathrm{m}=20, \mathrm{k}=50)$

\begin{tabular}{|c|c|c|c|c|c|c|c|c|c|c|c|c|c|c|c|c|c|}
\hline \multirow{3}{*}{$\begin{array}{c}\text { L1 } \\
\text { Effect }\end{array}$} & \multirow{3}{*}{$\begin{array}{l}\text { Interaction } \\
\text { Effect }\end{array}$} & \multicolumn{16}{|c|}{ ICC Values } \\
\hline & & \multicolumn{4}{|c|}{0} & \multicolumn{4}{|c|}{0.1} & \multicolumn{4}{|c|}{0.3} & \multicolumn{4}{|c|}{0.5} \\
\hline & & 0 & 0.2 & 0.5 & 0.8 & 0 & 0.2 & 0.5 & 0.8 & 0 & 0.2 & 0.5 & 0.8 & 0 & 0.2 & 0.5 & 0.8 \\
\hline \multirow{4}{*}{0} & 0 & 0.00 & 0.06 & 0.28 & 0.50 & 0.10 & 0.15 & 0.33 & 0.53 & 0.29 & 0.32 & 0.45 & 0.59 & 0.49 & 0.50 & 0.58 & 0.66 \\
\hline & 0.2 & 0.00 & 0.06 & 0.27 & 0.49 & 0.09 & 0.14 & 0.32 & 0.51 & 0.28 & 0.31 & 0.43 & 0.57 & 0.47 & 0.49 & 0.56 & 0.65 \\
\hline & 0.5 & 0.00 & 0.04 & 0.22 & 0.42 & 0.07 & 0.11 & 0.26 & 0.44 & 0.23 & 0.25 & 0.36 & 0.50 & 0.41 & 0.42 & 0.49 & 0.58 \\
\hline & 0.8 & 0.00 & 0.03 & 0.16 & 0.33 & 0.05 & 0.08 & 0.20 & 0.35 & 0.17 & 0.19 & 0.28 & 0.41 & 0.32 & 0.34 & 0.40 & 0.49 \\
\hline \multirow{4}{*}{0.2} & 0 & 0.00 & 0.06 & 0.27 & 0.49 & 0.09 & 0.14 & 0.32 & 0.51 & 0.28 & 0.31 & 0.43 & 0.57 & 0.48 & 0.49 & 0.56 & 0.65 \\
\hline & 0.2 & 0.00 & 0.05 & 0.26 & 0.47 & 0.09 & 0.13 & 0.31 & 0.50 & 0.27 & 0.30 & 0.42 & 0.56 & 0.46 & 0.48 & 0.54 & 0.63 \\
\hline & 0.5 & 0.00 & 0.04 & 0.21 & 0.41 & 0.07 & 0.10 & 0.25 & 0.43 & 0.22 & 0.24 & 0.35 & 0.49 & 0.39 & 0.41 & 0.48 & 0.57 \\
\hline & 0.8 & 0.00 & 0.03 & 0.16 & 0.32 & 0.05 & 0.07 & 0.19 & 0.35 & 0.16 & 0.18 & 0.28 & 0.40 & 0.31 & 0.33 & 0.39 & 0.48 \\
\hline \multirow{4}{*}{0.5} & 0 & 0.00 & 0.04 & 0.22 & 0.42 & 0.07 & 0.11 & 0.26 & 0.44 & 0.23 & 0.25 & 0.37 & 0.50 & 0.40 & 0.42 & 0.49 & 0.58 \\
\hline & 0.2 & 0.00 & 0.04 & 0.21 & 0.41 & 0.07 & 0.11 & 0.25 & 0.43 & 0.22 & 0.24 & 0.36 & 0.49 & 0.39 & 0.41 & 0.48 & 0.57 \\
\hline & 0.5 & 0.00 & 0.03 & 0.18 & 0.36 & 0.06 & 0.09 & 0.22 & 0.38 & 0.18 & 0.21 & 0.31 & 0.44 & 0.35 & 0.36 & 0.42 & 0.52 \\
\hline & 0.8 & 0.00 & 0.02 & 0.14 & 0.29 & 0.04 & 0.06 & 0.17 & 0.31 & 0.15 & 0.16 & 0.25 & 0.37 & 0.29 & 0.29 & 0.36 & 0.45 \\
\hline \multirow{4}{*}{0.8} & 0 & 0.00 & 0.03 & 0.16 & 0.33 & 0.05 & 0.08 & 0.20 & 0.35 & 0.17 & 0.19 & 0.28 & 0.41 & 0.32 & 0.33 & 0.40 & 0.49 \\
\hline & 0.2 & 0.00 & 0.03 & 0.16 & 0.33 & 0.05 & 0.07 & 0.19 & 0.35 & 0.16 & 0.18 & 0.28 & 0.40 & 0.31 & 0.33 & 0.39 & 0.48 \\
\hline & 0.5 & 0.00 & 0.02 & 0.14 & 0.29 & 0.04 & 0.06 & 0.17 & 0.31 & 0.15 & 0.16 & 0.25 & 0.37 & 0.28 & 0.29 & 0.36 & 0.44 \\
\hline & 0.8 & 0.00 & 0.02 & 0.11 & 0.24 & 0.03 & 0.05 & 0.14 & 0.27 & 0.12 & 0.13 & 0.21 & 0.32 & 0.24 & 0.25 & 0.30 & 0.39 \\
\hline
\end{tabular}

Note: Italicized values indicate conditional deviance of .15 or more above specified value, while bold values indicate conditional deviance of .15 or more below the specified value. ICC = Intraclass Correlation Coefficient. 
Table 1 cont.

Average Observed ICC values: Uniform Distribution conditions $(\mathrm{m}=20, \mathrm{k}=75)$

\begin{tabular}{|c|c|c|c|c|c|c|c|c|c|c|c|c|c|c|c|c|c|}
\hline \multirow{4}{*}{$\begin{array}{c}\text { L1 } \\
\text { Effect }\end{array}$} & \multirow{4}{*}{$\begin{array}{l}\text { Interaction } \\
\text { Effect }\end{array}$} & \multicolumn{16}{|c|}{ ICC Values } \\
\hline & & \multicolumn{4}{|c|}{0} & \multicolumn{4}{|c|}{0.1} & \multicolumn{4}{|c|}{0.3} & \multicolumn{4}{|c|}{0.5} \\
\hline & & \multicolumn{16}{|c|}{ L2 Effect } \\
\hline & & 0 & 0.2 & 0.5 & 0.8 & 0 & 0.2 & 0.5 & 0.8 & 0 & 0.2 & 0.5 & 0.8 & 0 & 0.2 & 0.5 & 0.8 \\
\hline \multirow{4}{*}{0} & 0 & 0.00 & 0.06 & 0.29 & 0.50 & 0.10 & 0.15 & 0.34 & 0.53 & 0.29 & 0.33 & 0.45 & 0.59 & 0.49 & 0.51 & 0.58 & 0.67 \\
\hline & 0.2 & 0.00 & 0.06 & 0.27 & 0.49 & 0.09 & 0.14 & 0.32 & 0.51 & 0.28 & 0.31 & 0.43 & 0.57 & 0.48 & 0.49 & 0.56 & 0.65 \\
\hline & 0.5 & 0.00 & 0.04 & 0.22 & 0.42 & 0.07 & 0.11 & 0.27 & 0.45 & 0.23 & 0.26 & 0.37 & 0.51 & 0.41 & 0.42 & 0.49 & 0.59 \\
\hline & 0.8 & 0.00 & 0.03 & 0.16 & 0.33 & 0.05 & 0.08 & 0.20 & 0.35 & 0.17 & 0.19 & 0.28 & 0.41 & 0.32 & 0.34 & 0.40 & 0.49 \\
\hline \multirow{4}{*}{0.2} & 0 & 0.00 & 0.06 & 0.28 & 0.49 & 0.09 & 0.14 & 0.32 & 0.51 & 0.28 & 0.31 & 0.43 & 0.57 & 0.48 & 0.49 & 0.56 & 0.65 \\
\hline & 0.2 & 0.00 & 0.05 & 0.26 & 0.48 & 0.09 & 0.13 & 0.31 & 0.50 & 0.27 & 0.30 & 0.42 & 0.56 & 0.46 & 0.48 & 0.55 & 0.64 \\
\hline & 0.5 & 0.00 & 0.04 & 0.21 & 0.41 & 0.07 & 0.10 & 0.26 & 0.43 & 0.22 & 0.25 & 0.35 & 0.49 & 0.40 & 0.41 & 0.48 & 0.57 \\
\hline & 0.8 & 0.00 & 0.03 & 0.16 & 0.33 & 0.05 & 0.08 & 0.20 & 0.35 & 0.17 & 0.19 & 0.28 & 0.41 & 0.32 & 0.33 & 0.39 & 0.48 \\
\hline \multirow{4}{*}{0.5} & 0 & 0.00 & 0.04 & 0.22 & 0.42 & 0.07 & 0.11 & 0.26 & 0.44 & 0.23 & 0.25 & 0.37 & 0.50 & 0.41 & 0.42 & 0.49 & 0.59 \\
\hline & 0.2 & 0.00 & 0.04 & 0.21 & 0.41 & 0.07 & 0.11 & 0.26 & 0.43 & 0.22 & 0.25 & 0.36 & 0.49 & 0.40 & 0.42 & 0.48 & 0.57 \\
\hline & 0.5 & 0.00 & 0.03 & 0.18 & 0.36 & 0.06 & 0.09 & 0.22 & 0.38 & 0.19 & 0.21 & 0.31 & 0.44 & 0.35 & 0.37 & 0.43 & 0.52 \\
\hline & 0.8 & 0.00 & 0.02 & 0.14 & 0.29 & 0.04 & 0.07 & 0.17 & 0.32 & 0.15 & 0.17 & 0.25 & 0.37 & 0.29 & 0.30 & 0.36 & 0.45 \\
\hline \multirow{4}{*}{0.8} & 0 & 0.00 & 0.03 & 0.16 & 0.33 & 0.05 & 0.08 & 0.20 & 0.36 & 0.17 & 0.19 & 0.29 & 0.41 & 0.32 & 0.34 & 0.40 & 0.49 \\
\hline & 0.2 & 0.00 & 0.03 & 0.16 & 0.32 & 0.05 & 0.07 & 0.19 & 0.35 & 0.16 & 0.19 & 0.28 & 0.41 & 0.32 & 0.33 & 0.39 & 0.48 \\
\hline & 0.5 & 0.00 & 0.03 & 0.14 & 0.29 & 0.04 & 0.07 & 0.17 & 0.31 & 0.15 & 0.16 & 0.25 & 0.37 & 0.28 & 0.30 & 0.36 & 0.45 \\
\hline & 0.8 & 0.00 & 0.02 & 0.11 & 0.25 & 0.03 & 0.05 & 0.14 & 0.27 & 0.12 & 0.14 & 0.21 & 0.32 & 0.24 & 0.25 & 0.31 & 0.39 \\
\hline
\end{tabular}

Note: Italicized values indicate conditional deviance of .15 or more above specified value, while bold values indicate conditional deviance of .15 or more below the specified value. ICC = Intraclass Correlation Coefficient. 
Table 1 cont.

Average Observed ICC values: Uniform Distribution conditions $(\mathrm{m}=20, \mathrm{k}=100)$

\begin{tabular}{|c|c|c|c|c|c|c|c|c|c|c|c|c|c|c|c|c|c|}
\hline \multirow{4}{*}{$\begin{array}{c}\text { L1 } \\
\text { Effect }\end{array}$} & \multirow{4}{*}{$\begin{array}{l}\text { Interaction } \\
\text { Effect }\end{array}$} & \multicolumn{16}{|c|}{ ICC Values } \\
\hline & & \multicolumn{4}{|c|}{0} & \multicolumn{4}{|c|}{0.1} & \multicolumn{4}{|c|}{0.3} & \multicolumn{4}{|c|}{0.5} \\
\hline & & \multicolumn{16}{|c|}{ L2 Effect } \\
\hline & & 0 & 0.2 & 0.5 & 0.8 & 0 & 0.2 & 0.5 & 0.8 & 0 & 0.2 & 0.5 & 0.8 & 0 & 0.2 & 0.5 & 0.8 \\
\hline \multirow{4}{*}{0} & 0 & 0.00 & 0.06 & 0.29 & 0.51 & 0.10 & 0.15 & 0.34 & 0.53 & 0.30 & 0.33 & 0.45 & 0.59 & 0.49 & 0.51 & 0.58 & 0.67 \\
\hline & 0.2 & 0.00 & 0.06 & 0.27 & 0.49 & 0.09 & 0.14 & 0.32 & 0.52 & 0.28 & 0.31 & 0.44 & 0.58 & 0.48 & 0.50 & 0.56 & 0.65 \\
\hline & 0.5 & 0.00 & 0.04 & 0.22 & 0.42 & 0.07 & 0.11 & 0.26 & 0.45 & 0.23 & 0.26 & 0.37 & 0.51 & 0.41 & 0.43 & 0.50 & 0.59 \\
\hline & 0.8 & 0.00 & 0.03 & 0.16 & 0.33 & 0.05 & 0.08 & 0.20 & 0.36 & 0.17 & 0.19 & 0.29 & 0.42 & 0.33 & 0.34 & 0.40 & 0.50 \\
\hline \multirow{4}{*}{0.2} & 0 & 0.00 & 0.06 & 0.27 & 0.49 & 0.09 & 0.14 & 0.32 & 0.52 & 0.28 & 0.31 & 0.44 & 0.57 & 0.48 & 0.49 & 0.56 & 0.65 \\
\hline & 0.2 & 0.00 & 0.05 & 0.27 & 0.48 & 0.09 & 0.13 & 0.31 & 0.50 & 0.27 & 0.30 & 0.42 & 0.56 & 0.46 & 0.48 & 0.55 & 0.64 \\
\hline & 0.5 & 0.00 & 0.04 & 0.21 & 0.41 & 0.07 & 0.10 & 0.26 & 0.43 & 0.22 & 0.25 & 0.36 & 0.50 & 0.40 & 0.41 & 0.48 & 0.58 \\
\hline & 0.8 & 0.00 & 0.03 & 0.16 & 0.33 & 0.05 & 0.08 & 0.19 & 0.35 & 0.17 & 0.19 & 0.28 & 0.41 & 0.32 & 0.33 & 0.40 & 0.49 \\
\hline \multirow{4}{*}{0.5} & 0 & 0.00 & 0.04 & 0.22 & 0.42 & 0.07 & 0.11 & 0.27 & 0.45 & 0.23 & 0.26 & 0.37 & 0.50 & 0.41 & 0.42 & 0.50 & 0.59 \\
\hline & 0.2 & 0.00 & 0.04 & 0.21 & 0.41 & 0.07 & 0.11 & 0.26 & 0.43 & 0.22 & 0.25 & 0.36 & 0.49 & 0.40 & 0.42 & 0.48 & 0.57 \\
\hline & 0.5 & 0.00 & 0.03 & 0.18 & 0.36 & 0.06 & 0.09 & 0.22 & 0.38 & 0.19 & 0.21 & 0.31 & 0.44 & 0.35 & 0.36 & 0.43 & 0.53 \\
\hline & 0.8 & 0.00 & 0.02 & 0.14 & 0.30 & 0.04 & 0.07 & 0.17 & 0.32 & 0.15 & 0.17 & 0.25 & 0.37 & 0.29 & 0.30 & 0.36 & 0.45 \\
\hline \multirow{4}{*}{0.8} & 0 & 0.00 & 0.03 & 0.16 & 0.33 & 0.05 & 0.08 & 0.20 & 0.36 & 0.17 & 0.19 & 0.29 & 0.41 & 0.32 & 0.34 & 0.40 & 0.49 \\
\hline & 0.2 & 0.00 & 0.03 & 0.16 & 0.33 & 0.05 & 0.08 & 0.19 & 0.35 & 0.17 & 0.19 & 0.28 & 0.41 & 0.32 & 0.33 & 0.40 & 0.49 \\
\hline & 0.5 & 0.00 & 0.03 & 0.14 & 0.30 & 0.04 & 0.07 & 0.17 & 0.32 & 0.15 & 0.17 & 0.25 & 0.37 & 0.29 & 0.30 & 0.36 & 0.45 \\
\hline & 0.8 & 0.00 & 0.02 & 0.12 & 0.25 & 0.03 & 0.05 & 0.14 & 0.27 & 0.12 & 0.14 & 0.21 & 0.32 & 0.24 & 0.25 & 0.31 & 0.39 \\
\hline
\end{tabular}

Note: Italicized values indicate conditional deviance of .15 or more above specified value, while bold values indicate conditional deviance of .15 or more below the specified value. ICC = Intraclass Correlation Coefficient. 
Table 1 cont.

Average Observed ICC values: Poisson Distribution conditions $(\mathrm{m}=5, \mathrm{k}=25)$

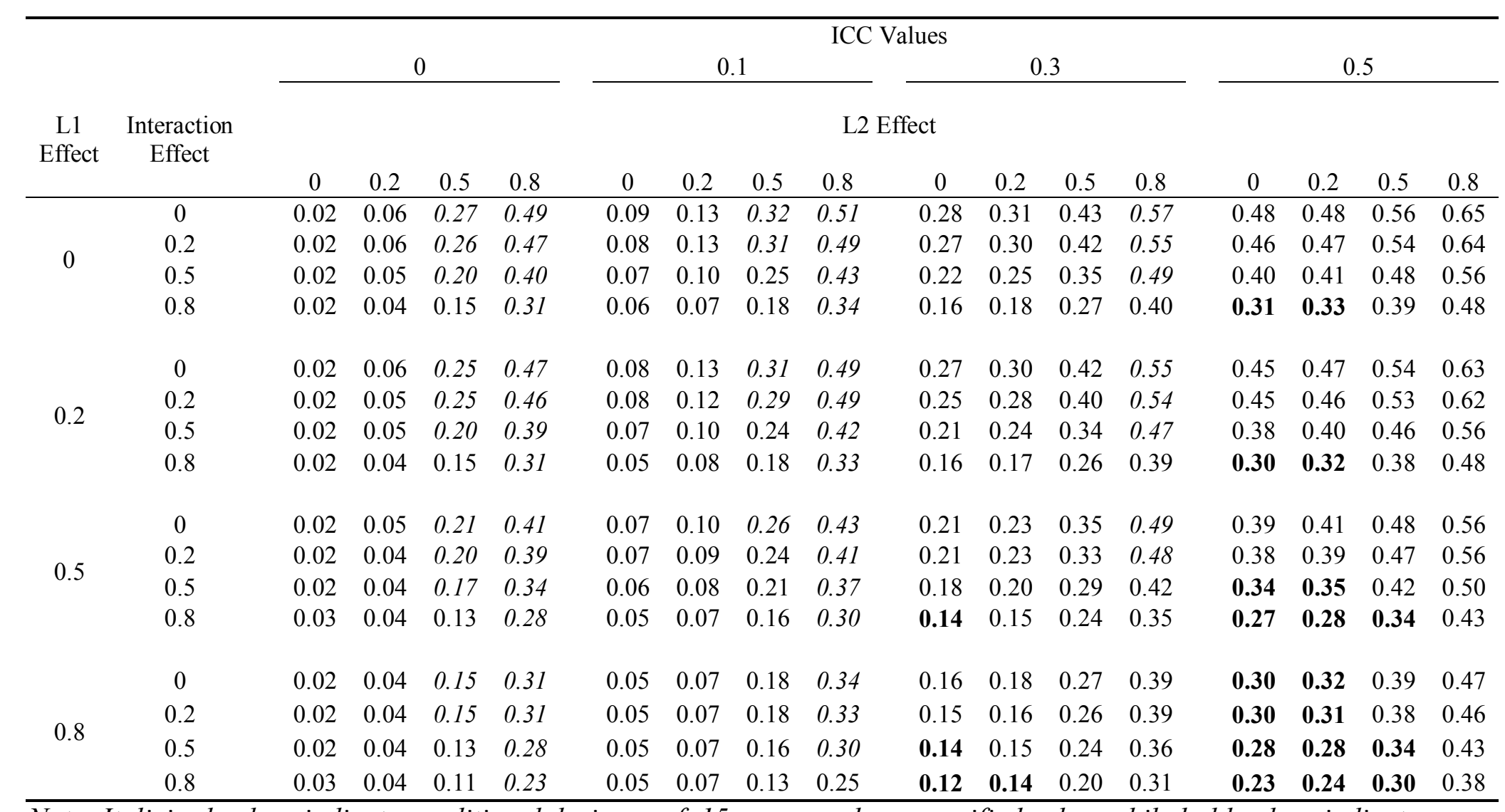

Note: Italicized values indicate conditional deviance of .15 or more above specified value, while bold values indicate conditional deviance of .15 or more below the specified value. ICC = Intraclass Correlation Coefficient. 
Table 1 cont.

Average Observed ICC values: Poisson Distribution conditions $(\mathrm{m}=5, \mathrm{k}=50)$

\begin{tabular}{|c|c|c|c|c|c|c|c|c|c|c|c|c|c|c|c|c|c|}
\hline \multirow{3}{*}{$\begin{array}{c}\text { L1 } \\
\text { Effect }\end{array}$} & \multirow{3}{*}{$\begin{array}{c}\text { Interaction } \\
\text { Effect }\end{array}$} & \multicolumn{12}{|c|}{ ICC Values } & \multicolumn{4}{|c|}{0.5} \\
\hline & & \multicolumn{16}{|c|}{ L2 Effect } \\
\hline & & 0 & 0.2 & 0.5 & 0.8 & 0 & 0.2 & 0.5 & 0.8 & 0 & 0.2 & 0.5 & 0.8 & 0 & 0.2 & 0.5 & 0.8 \\
\hline \multirow{4}{*}{0} & 0 & 0.01 & 0.06 & 0.28 & 0.50 & 0.09 & 0.14 & 0.33 & 0.52 & 0.29 & 0.32 & 0.44 & 0.58 & 0.48 & 0.50 & 0.57 & 0.66 \\
\hline & 0.2 & 0.01 & 0.06 & 0.27 & 0.48 & 0.09 & 0.13 & 0.31 & 0.51 & 0.27 & 0.31 & 0.43 & 0.56 & 0.47 & 0.49 & 0.56 & 0.65 \\
\hline & 0.5 & 0.02 & 0.04 & 0.22 & 0.41 & 0.07 & 0.11 & 0.26 & 0.44 & 0.23 & 0.25 & 0.36 & 0.50 & 0.40 & 0.42 & 0.49 & 0.58 \\
\hline & 0.8 & 0.02 & 0.04 & 0.16 & 0.33 & 0.05 & 0.08 & 0.20 & 0.35 & 0.17 & 0.19 & 0.28 & 0.41 & 0.32 & 0.33 & 0.40 & 0.49 \\
\hline \multirow{4}{*}{0.2} & 0 & 0.02 & 0.06 & 0.27 & 0.48 & 0.09 & 0.13 & 0.32 & 0.50 & 0.28 & 0.31 & 0.43 & 0.57 & 0.47 & 0.49 & 0.55 & 0.65 \\
\hline & 0.2 & 0.02 & 0.05 & 0.26 & 0.47 & 0.08 & 0.13 & 0.30 & 0.50 & 0.27 & 0.29 & 0.42 & 0.55 & 0.46 & 0.47 & 0.54 & 0.63 \\
\hline & 0.5 & 0.02 & 0.04 & 0.21 & 0.41 & 0.07 & 0.10 & 0.25 & 0.43 & 0.22 & 0.24 & 0.35 & 0.49 & 0.39 & 0.41 & 0.48 & 0.56 \\
\hline & 0.8 & 0.02 & 0.04 & 0.16 & 0.31 & 0.05 & 0.07 & 0.18 & 0.34 & 0.17 & 0.18 & 0.27 & 0.40 & 0.31 & 0.32 & 0.39 & 0.48 \\
\hline \multirow{4}{*}{0.5} & 0 & 0.01 & 0.05 & 0.22 & 0.42 & 0.07 & 0.11 & 0.26 & 0.44 & 0.22 & 0.25 & 0.36 & 0.50 & 0.40 & 0.42 & 0.49 & 0.58 \\
\hline & 0.2 & 0.01 & 0.04 & 0.21 & 0.40 & 0.07 & 0.10 & 0.25 & 0.43 & 0.22 & 0.24 & 0.35 & 0.49 & 0.39 & 0.40 & 0.47 & 0.57 \\
\hline & 0.5 & 0.02 & 0.04 & 0.18 & 0.36 & 0.06 & 0.09 & 0.22 & 0.38 & 0.18 & 0.20 & 0.31 & 0.43 & 0.34 & 0.36 & 0.42 & 0.51 \\
\hline & 0.8 & 0.02 & 0.03 & 0.14 & 0.29 & 0.05 & 0.07 & 0.16 & 0.31 & 0.15 & 0.16 & 0.25 & 0.37 & 0.28 & 0.30 & 0.36 & 0.44 \\
\hline \multirow{4}{*}{0.8} & 0 & 0.01 & 0.03 & 0.16 & 0.33 & 0.05 & 0.08 & 0.19 & 0.35 & 0.17 & 0.19 & 0.28 & 0.40 & 0.32 & 0.33 & 0.39 & 0.49 \\
\hline & 0.2 & 0.01 & 0.03 & 0.15 & 0.32 & 0.05 & 0.07 & 0.19 & 0.35 & 0.16 & 0.18 & 0.27 & 0.40 & 0.31 & 0.33 & 0.39 & 0.48 \\
\hline & 0.5 & 0.02 & 0.03 & 0.14 & 0.29 & 0.05 & 0.07 & 0.17 & 0.31 & 0.14 & 0.16 & 0.25 & 0.37 & 0.29 & 0.29 & 0.35 & 0.45 \\
\hline & 0.8 & 0.02 & 0.03 & 0.11 & 0.25 & 0.04 & 0.05 & 0.14 & 0.27 & 0.12 & 0.14 & 0.21 & 0.31 & 0.24 & 0.25 & 0.31 & 0.39 \\
\hline
\end{tabular}

Note: Italicized values indicate conditional deviance of .15 or more above specified value, while bold values indicate

conditional deviance of .15 or more below the specified value. ICC = Intraclass Correlation Coefficient. 
Table 1 cont.

Average Observed ICC values: Poisson Distribution conditions $(\mathrm{m}=5, \mathrm{k}=75)$

\begin{tabular}{|c|c|c|c|c|c|c|c|c|c|c|c|c|c|c|c|c|c|}
\hline \multirow{3}{*}{$\begin{array}{c}\text { L1 } \\
\text { Effect }\end{array}$} & \multirow{3}{*}{$\begin{array}{l}\text { Interaction } \\
\text { Effect }\end{array}$} & \multicolumn{16}{|c|}{ ICC Values } \\
\hline & & \multicolumn{4}{|c|}{0} & \multicolumn{4}{|c|}{0.1} & \multicolumn{4}{|c|}{0.3} & \multicolumn{4}{|c|}{0.5} \\
\hline & & 0 & 0.2 & 0.5 & 0.8 & 0 & 0.2 & 0.5 & 0.8 & 0 & 0.2 & 0.5 & 0.8 & 0 & 0.2 & 0.5 & 0.8 \\
\hline \multirow{4}{*}{0} & 0 & 0.01 & 0.06 & 0.28 & 0.50 & 0.10 & 0.14 & 0.34 & 0.53 & 0.29 & 0.32 & 0.45 & 0.59 & 0.49 & 0.51 & 0.58 & 0.67 \\
\hline & 0.2 & 0.01 & 0.06 & 0.27 & 0.49 & 0.09 & 0.14 & 0.32 & 0.51 & 0.28 & 0.31 & 0.43 & 0.57 & 0.48 & 0.49 & 0.56 & 0.65 \\
\hline & 0.5 & 0.01 & 0.04 & 0.22 & 0.42 & 0.07 & 0.11 & 0.26 & 0.44 & 0.23 & 0.25 & 0.36 & 0.50 & 0.41 & 0.42 & 0.49 & 0.58 \\
\hline & 0.8 & 0.01 & 0.03 & 0.16 & 0.33 & 0.05 & 0.08 & 0.20 & 0.36 & 0.17 & 0.19 & 0.29 & 0.41 & 0.32 & 0.33 & 0.40 & 0.49 \\
\hline \multirow{4}{*}{0.2} & 0 & 0.01 & 0.06 & 0.27 & 0.49 & 0.09 & 0.13 & 0.32 & 0.51 & 0.28 & 0.31 & 0.43 & 0.57 & 0.47 & 0.49 & 0.56 & 0.65 \\
\hline & 0.2 & 0.01 & 0.05 & 0.26 & 0.47 & 0.09 & 0.13 & 0.31 & 0.50 & 0.27 & 0.30 & 0.42 & 0.56 & 0.46 & 0.48 & 0.55 & 0.64 \\
\hline & 0.5 & 0.01 & 0.04 & 0.21 & 0.41 & 0.07 & 0.10 & 0.26 & 0.43 & 0.22 & 0.24 & 0.36 & 0.49 & 0.40 & 0.41 & 0.48 & 0.58 \\
\hline & 0.8 & 0.02 & 0.03 & 0.16 & 0.32 & 0.05 & 0.08 & 0.19 & 0.35 & 0.16 & 0.19 & 0.28 & 0.40 & 0.32 & 0.33 & 0.40 & 0.49 \\
\hline \multirow{4}{*}{0.5} & 0 & 0.01 & 0.04 & 0.22 & 0.42 & 0.07 & 0.11 & 0.26 & 0.44 & 0.23 & 0.25 & 0.37 & 0.51 & 0.41 & 0.42 & 0.49 & 0.58 \\
\hline & 0.2 & 0.01 & 0.04 & 0.21 & 0.41 & 0.07 & 0.10 & 0.25 & 0.43 & 0.22 & 0.24 & 0.36 & 0.49 & 0.39 & 0.41 & 0.48 & 0.57 \\
\hline & 0.5 & 0.01 & 0.04 & 0.18 & 0.36 & 0.06 & 0.09 & 0.22 & 0.38 & 0.18 & 0.21 & 0.31 & 0.44 & 0.35 & 0.36 & 0.43 & 0.52 \\
\hline & 0.8 & 0.02 & 0.03 & 0.14 & 0.29 & 0.04 & 0.06 & 0.17 & 0.31 & 0.15 & 0.16 & 0.25 & 0.37 & 0.28 & 0.29 & 0.36 & 0.45 \\
\hline \multirow{4}{*}{0.8} & 0 & 0.01 & 0.03 & 0.16 & 0.33 & 0.05 & 0.08 & 0.19 & 0.35 & 0.17 & 0.19 & 0.28 & 0.41 & 0.32 & 0.33 & 0.40 & 0.49 \\
\hline & 0.2 & 0.01 & 0.03 & 0.16 & 0.32 & 0.05 & 0.08 & 0.19 & 0.35 & 0.16 & 0.18 & 0.28 & 0.40 & 0.32 & 0.33 & 0.39 & 0.48 \\
\hline & 0.5 & 0.01 & 0.03 & 0.14 & 0.29 & 0.04 & 0.06 & 0.17 & 0.31 & 0.14 & 0.16 & 0.25 & 0.37 & 0.28 & 0.30 & 0.36 & 0.45 \\
\hline & 0.8 & 0.02 & 0.03 & 0.11 & 0.24 & 0.04 & 0.05 & 0.14 & 0.27 & 0.12 & 0.13 & 0.21 & 0.32 & 0.24 & 0.25 & 0.31 & 0.39 \\
\hline
\end{tabular}

Note: Italicized values indicate conditional deviance of .15 or more above specified value, while bold values indicate conditional deviance of .15 or more below the specified value. ICC = Intraclass Correlation Coefficient. 
Table 1 cont.

Average Observed ICC values: Poisson Distribution conditions $(\mathrm{m}=5, \mathrm{k}=100)$

\begin{tabular}{|c|c|c|c|c|c|c|c|c|c|c|c|c|c|c|c|c|c|}
\hline \multirow{3}{*}{$\begin{array}{c}\text { L1 } \\
\text { Effect }\end{array}$} & \multirow{3}{*}{$\begin{array}{l}\text { Interaction } \\
\text { Effect }\end{array}$} & \multicolumn{16}{|c|}{ ICC Values } \\
\hline & & \multicolumn{4}{|c|}{0} & \multicolumn{4}{|c|}{0.1} & \multicolumn{4}{|c|}{0.3} & \multicolumn{4}{|c|}{0.5} \\
\hline & & 0 & 0.2 & 0.5 & 0.8 & 0 & 0.2 & 0.5 & 0.8 & 0 & 0.2 & 0.5 & 0.8 & 0 & 0.2 & 0.5 & 0.8 \\
\hline \multirow{4}{*}{0} & 0 & 0.01 & 0.06 & 0.29 & 0.51 & 0.10 & 0.15 & 0.34 & 0.53 & 0.30 & 0.33 & 0.45 & 0.59 & 0.49 & 0.51 & 0.58 & 0.66 \\
\hline & 0.2 & 0.01 & 0.06 & 0.27 & 0.49 & 0.09 & 0.14 & 0.32 & 0.52 & 0.28 & 0.31 & 0.44 & 0.58 & 0.48 & 0.49 & 0.56 & 0.65 \\
\hline & 0.5 & 0.01 & 0.04 & 0.22 & 0.42 & 0.07 & 0.11 & 0.26 & 0.45 & 0.23 & 0.25 & 0.36 & 0.51 & 0.41 & 0.42 & 0.50 & 0.59 \\
\hline & 0.8 & 0.01 & 0.03 & 0.16 & 0.33 & 0.05 & 0.08 & 0.20 & 0.36 & 0.17 & 0.19 & 0.28 & 0.41 & 0.32 & 0.34 & 0.41 & 0.49 \\
\hline \multirow{4}{*}{0.2} & 0 & 0.01 & 0.06 & 0.27 & 0.49 & 0.09 & 0.14 & 0.32 & 0.52 & 0.28 & 0.31 & 0.44 & 0.57 & 0.48 & 0.49 & 0.56 & 0.65 \\
\hline & 0.2 & 0.01 & 0.05 & 0.26 & 0.47 & 0.09 & 0.13 & 0.31 & 0.50 & 0.27 & 0.30 & 0.42 & 0.56 & 0.46 & 0.48 & 0.55 & 0.64 \\
\hline & 0.5 & 0.01 & 0.04 & 0.21 & 0.41 & 0.07 & 0.10 & 0.26 & 0.43 & 0.22 & 0.24 & 0.36 & 0.49 & 0.40 & 0.41 & 0.48 & 0.58 \\
\hline & 0.8 & 0.01 & 0.03 & 0.16 & 0.33 & 0.05 & 0.08 & 0.19 & 0.35 & 0.17 & 0.19 & 0.28 & 0.41 & 0.32 & 0.33 & 0.39 & 0.48 \\
\hline \multirow{4}{*}{0.5} & 0 & 0.01 & 0.04 & 0.22 & 0.42 & 0.07 & 0.11 & 0.26 & 0.44 & 0.23 & 0.25 & 0.37 & 0.50 & 0.41 & 0.42 & 0.49 & 0.59 \\
\hline & 0.2 & 0.01 & 0.04 & 0.21 & 0.41 & 0.07 & 0.10 & 0.26 & 0.43 & 0.22 & 0.25 & 0.36 & 0.50 & 0.40 & 0.41 & 0.49 & 0.58 \\
\hline & 0.5 & 0.01 & 0.04 & 0.18 & 0.36 & 0.06 & 0.09 & 0.22 & 0.38 & 0.19 & 0.21 & 0.31 & 0.44 & 0.35 & 0.37 & 0.43 & 0.52 \\
\hline & 0.8 & 0.01 & 0.03 & 0.14 & 0.29 & 0.04 & 0.06 & 0.17 & 0.32 & 0.15 & 0.16 & 0.25 & 0.37 & 0.29 & 0.30 & 0.36 & 0.45 \\
\hline \multirow{4}{*}{0.8} & 0 & 0.01 & 0.03 & 0.16 & 0.33 & 0.05 & 0.08 & 0.19 & 0.36 & 0.17 & 0.19 & 0.29 & 0.41 & 0.32 & 0.34 & 0.40 & 0.50 \\
\hline & 0.2 & 0.01 & 0.03 & 0.16 & 0.32 & 0.05 & 0.07 & 0.19 & 0.35 & 0.16 & 0.18 & 0.28 & 0.40 & 0.31 & 0.33 & 0.40 & 0.49 \\
\hline & 0.5 & 0.01 & 0.03 & 0.14 & 0.29 & 0.04 & 0.06 & 0.17 & 0.31 & 0.15 & 0.16 & 0.25 & 0.37 & 0.28 & 0.30 & 0.36 & 0.45 \\
\hline & 0.8 & 0.01 & 0.03 & 0.11 & 0.25 & 0.04 & 0.05 & 0.14 & 0.27 & 0.12 & 0.14 & 0.21 & 0.32 & 0.24 & 0.25 & 0.31 & 0.40 \\
\hline
\end{tabular}

Note: Italicized values indicate conditional deviance of .15 or more above specified value, while bold values indicate conditional deviance of .15 or more below the specified value. ICC = Intraclass Correlation Coefficient. 
Table 1 cont.

Average Observed ICC values: Poisson Distribution conditions $(\mathrm{m}=10, \mathrm{k}=25)$

\begin{tabular}{|c|c|c|c|c|c|c|c|c|c|c|c|c|c|c|c|c|c|}
\hline \multirow{3}{*}{$\begin{array}{c}\text { L1 } \\
\text { Effect }\end{array}$} & \multirow{3}{*}{$\begin{array}{l}\text { Interaction } \\
\text { Effect }\end{array}$} & \multicolumn{16}{|c|}{ ICC Values } \\
\hline & & \multicolumn{4}{|c|}{0} & \multicolumn{4}{|c|}{0.1} & \multicolumn{4}{|c|}{0.3} & \multicolumn{4}{|c|}{0.5} \\
\hline & & 0 & 0.2 & 0.5 & 0.8 & 0 & 0.2 & 0.5 & 0.8 & 0 & 0.2 & 0.5 & 0.8 & 0 & 0.2 & 0.5 & 0.8 \\
\hline \multirow{4}{*}{0} & 0 & 0.01 & 0.06 & 0.27 & 0.48 & 0.09 & 0.14 & 0.32 & 0.52 & 0.29 & 0.32 & 0.43 & 0.57 & 0.48 & 0.49 & 0.56 & 0.65 \\
\hline & 0.2 & 0.01 & 0.05 & 0.26 & 0.47 & 0.09 & 0.13 & 0.31 & 0.49 & 0.27 & 0.30 & 0.42 & 0.56 & 0.46 & 0.48 & 0.55 & 0.63 \\
\hline & 0.5 & 0.01 & 0.04 & 0.21 & 0.41 & 0.07 & 0.10 & 0.25 & 0.43 & 0.22 & 0.25 & 0.35 & 0.48 & 0.39 & 0.41 & 0.47 & 0.57 \\
\hline & 0.8 & 0.01 & 0.03 & 0.16 & 0.32 & 0.05 & 0.08 & 0.19 & 0.34 & 0.17 & 0.18 & 0.28 & 0.40 & 0.31 & 0.33 & 0.39 & 0.48 \\
\hline \multirow{4}{*}{0.2} & 0 & 0.01 & 0.05 & 0.26 & 0.47 & 0.09 & 0.13 & 0.31 & 0.50 & 0.27 & 0.30 & 0.42 & 0.56 & 0.46 & 0.48 & 0.55 & 0.64 \\
\hline & 0.2 & 0.01 & 0.05 & 0.25 & 0.46 & 0.08 & 0.13 & 0.29 & 0.48 & 0.26 & 0.29 & 0.41 & 0.54 & 0.45 & 0.47 & 0.53 & 0.62 \\
\hline & 0.5 & 0.01 & 0.04 & 0.20 & 0.40 & 0.06 & 0.10 & 0.24 & 0.42 & 0.21 & 0.24 & 0.35 & 0.48 & 0.39 & 0.40 & 0.47 & 0.56 \\
\hline & 0.8 & 0.01 & 0.03 & 0.15 & 0.31 & 0.05 & 0.07 & 0.18 & 0.33 & 0.16 & 0.18 & 0.27 & 0.39 & 0.31 & 0.32 & 0.38 & 0.47 \\
\hline \multirow{4}{*}{0.5} & 0 & 0.01 & 0.04 & 0.21 & 0.40 & 0.07 & 0.10 & 0.26 & 0.43 & 0.22 & 0.24 & 0.36 & 0.49 & 0.39 & 0.41 & 0.48 & 0.57 \\
\hline & 0.2 & 0.01 & 0.04 & 0.20 & 0.39 & 0.07 & 0.10 & 0.24 & 0.42 & 0.21 & 0.24 & 0.34 & 0.48 & 0.38 & 0.40 & 0.46 & 0.56 \\
\hline & 0.5 & 0.01 & 0.03 & 0.17 & 0.35 & 0.05 & 0.08 & 0.21 & 0.37 & 0.18 & 0.20 & 0.30 & 0.43 & 0.34 & 0.35 & 0.42 & 0.51 \\
\hline & 0.8 & 0.01 & 0.03 & 0.13 & 0.28 & 0.04 & 0.06 & 0.16 & 0.31 & 0.15 & 0.16 & 0.24 & 0.36 & 0.27 & 0.29 & 0.35 & 0.44 \\
\hline \multirow{4}{*}{0.8} & 0 & 0.01 & 0.03 & 0.15 & 0.32 & 0.05 & 0.07 & 0.19 & 0.34 & 0.16 & 0.18 & 0.27 & 0.40 & 0.31 & 0.32 & 0.39 & 0.48 \\
\hline & 0.2 & 0.01 & 0.03 & 0.15 & 0.31 & 0.04 & 0.07 & 0.18 & 0.34 & 0.16 & 0.18 & 0.26 & 0.39 & 0.31 & 0.32 & 0.38 & 0.47 \\
\hline & 0.5 & 0.01 & 0.03 & 0.13 & 0.28 & 0.04 & 0.06 & 0.16 & 0.30 & 0.14 & 0.16 & 0.24 & 0.36 & 0.27 & 0.29 & 0.34 & 0.43 \\
\hline & 0.8 & 0.01 & 0.02 & 0.11 & 0.24 & 0.03 & 0.05 & 0.14 & 0.26 & 0.11 & 0.13 & 0.20 & 0.30 & 0.23 & 0.25 & 0.30 & 0.38 \\
\hline
\end{tabular}

Note: Italicized values indicate conditional deviance of .15 or more above specified value, while bold values indicate conditional deviance of .15 or more below the specified value. ICC = Intraclass Correlation Coefficient. 
Table 1 cont.

Average Observed ICC values: Poisson Distribution conditions $(\mathrm{m}=10, \mathrm{k}=50)$

\begin{tabular}{|c|c|c|c|c|c|c|c|c|c|c|c|c|c|c|c|c|c|}
\hline \multirow{4}{*}{$\begin{array}{c}\text { L1 } \\
\text { Effect }\end{array}$} & \multirow{4}{*}{$\begin{array}{l}\text { Interaction } \\
\text { Effect }\end{array}$} & \multicolumn{16}{|c|}{ ICC Values } \\
\hline & & \multicolumn{4}{|c|}{0} & \multicolumn{3}{|c|}{0.1} & & \multicolumn{4}{|c|}{0.3} & \multicolumn{4}{|c|}{0.5} \\
\hline & & \multicolumn{16}{|c|}{ L2 Effect } \\
\hline & & 0 & 0.2 & 0.5 & 0.8 & 0 & 0.2 & 0.5 & 0.8 & 0 & 0.2 & 0.5 & 0.8 & 0 & 0.2 & 0.5 & 0.8 \\
\hline \multirow{4}{*}{0} & 0 & 0.01 & 0.06 & 0.28 & 0.50 & 0.10 & 0.15 & 0.33 & 0.53 & 0.29 & 0.32 & 0.45 & 0.58 & 0.49 & 0.50 & 0.57 & 0.66 \\
\hline & 0.2 & 0.01 & 0.05 & 0.27 & 0.49 & 0.09 & 0.14 & 0.32 & 0.51 & 0.28 & 0.30 & 0.43 & 0.57 & 0.47 & 0.49 & 0.56 & 0.65 \\
\hline & 0.5 & 0.01 & 0.04 & 0.22 & 0.41 & 0.07 & 0.11 & 0.26 & 0.44 & 0.23 & 0.25 & 0.36 & 0.50 & 0.40 & 0.42 & 0.49 & 0.58 \\
\hline & 0.8 & 0.01 & 0.03 & 0.16 & 0.33 & 0.05 & 0.08 & 0.20 & 0.36 & 0.17 & 0.19 & 0.28 & 0.41 & 0.32 & 0.34 & 0.40 & 0.49 \\
\hline \multirow{4}{*}{0.2} & 0 & 0.01 & 0.05 & 0.27 & 0.49 & 0.09 & 0.14 & 0.32 & 0.51 & 0.28 & 0.31 & 0.43 & 0.57 & 0.47 & 0.49 & 0.56 & 0.65 \\
\hline & 0.2 & 0.01 & 0.05 & 0.26 & 0.47 & 0.09 & 0.13 & 0.31 & 0.49 & 0.27 & 0.29 & 0.41 & 0.55 & 0.46 & 0.48 & 0.54 & 0.64 \\
\hline & 0.5 & 0.01 & 0.04 & 0.21 & 0.41 & 0.07 & 0.10 & 0.25 & 0.43 & 0.22 & 0.24 & 0.36 & 0.49 & 0.40 & 0.41 & 0.48 & 0.57 \\
\hline & 0.8 & 0.01 & 0.03 & 0.15 & 0.32 & 0.05 & 0.08 & 0.19 & 0.35 & 0.16 & 0.19 & 0.28 & 0.40 & 0.32 & 0.33 & 0.39 & 0.48 \\
\hline \multirow{4}{*}{0.5} & 0 & 0.01 & 0.04 & 0.22 & 0.41 & 0.07 & 0.11 & 0.26 & 0.44 & 0.22 & 0.25 & 0.36 & 0.50 & 0.41 & 0.42 & 0.49 & 0.58 \\
\hline & 0.2 & 0.01 & 0.04 & 0.21 & 0.41 & 0.07 & 0.10 & 0.26 & 0.43 & 0.22 & 0.24 & 0.35 & 0.49 & 0.39 & 0.41 & 0.47 & 0.57 \\
\hline & 0.5 & 0.01 & 0.03 & 0.18 & 0.36 & 0.06 & 0.08 & 0.22 & 0.38 & 0.19 & 0.21 & 0.31 & 0.44 & 0.35 & 0.36 & 0.43 & 0.52 \\
\hline & 0.8 & 0.01 & 0.03 & 0.14 & 0.29 & 0.04 & 0.07 & 0.17 & 0.31 & 0.14 & 0.17 & 0.25 & 0.37 & 0.29 & 0.29 & 0.35 & 0.45 \\
\hline \multirow{4}{*}{0.8} & 0 & 0.01 & 0.03 & 0.16 & 0.33 & 0.05 & 0.08 & 0.20 & 0.35 & 0.17 & 0.19 & 0.28 & 0.41 & 0.32 & 0.33 & 0.40 & 0.48 \\
\hline & 0.2 & 0.01 & 0.03 & 0.16 & 0.32 & 0.05 & 0.07 & 0.19 & 0.34 & 0.16 & 0.18 & 0.28 & 0.40 & 0.31 & 0.33 & 0.39 & 0.48 \\
\hline & 0.5 & 0.01 & 0.03 & 0.14 & 0.29 & 0.04 & 0.06 & 0.17 & 0.31 & 0.14 & 0.16 & 0.25 & 0.37 & 0.28 & 0.29 & 0.36 & 0.44 \\
\hline & 0.8 & 0.01 & 0.02 & 0.11 & 0.25 & 0.04 & 0.05 & 0.14 & 0.26 & 0.12 & 0.14 & 0.21 & 0.32 & 0.24 & 0.25 & 0.31 & 0.39 \\
\hline
\end{tabular}

Note: Italicized values indicate conditional deviance of .15 or more above specified value, while bold values indicate conditional deviance of .15 or more below the specified value. ICC = Intraclass Correlation Coefficient. 
Table 1 cont.

Average Observed ICC values: Poisson Distribution conditions $(\mathrm{m}=10, \mathrm{k}=75)$

\begin{tabular}{|c|c|c|c|c|c|c|c|c|c|c|c|c|c|c|c|c|c|}
\hline \multirow{3}{*}{$\begin{array}{c}\text { L1 } \\
\text { Effect }\end{array}$} & \multirow{3}{*}{$\begin{array}{l}\text { Interaction } \\
\text { Effect }\end{array}$} & \multicolumn{16}{|c|}{ ICC Values } \\
\hline & & \multicolumn{4}{|c|}{0} & \multicolumn{4}{|c|}{0.1} & \multicolumn{4}{|c|}{0.3} & \multicolumn{4}{|c|}{0.5} \\
\hline & & 0 & 0.2 & 0.5 & 0.8 & 0 & 0.2 & 0.5 & 0.8 & 0 & 0.2 & 0.5 & 0.8 & 0 & 0.2 & 0.5 & 0.8 \\
\hline \multirow{4}{*}{0} & 0 & 0.01 & 0.06 & 0.29 & 0.51 & 0.10 & 0.15 & 0.34 & 0.53 & 0.30 & 0.32 & 0.45 & 0.59 & 0.49 & 0.51 & 0.58 & 0.66 \\
\hline & 0.2 & 0.01 & 0.06 & 0.27 & 0.49 & 0.09 & 0.14 & 0.32 & 0.51 & 0.28 & 0.31 & 0.43 & 0.57 & 0.48 & 0.49 & 0.56 & 0.65 \\
\hline & 0.5 & 0.01 & 0.04 & 0.22 & 0.42 & 0.07 & 0.11 & 0.26 & 0.44 & 0.23 & 0.26 & 0.37 & 0.50 & 0.41 & 0.42 & 0.49 & 0.59 \\
\hline & 0.8 & 0.01 & 0.03 & 0.16 & 0.33 & 0.05 & 0.08 & 0.20 & 0.35 & 0.17 & 0.19 & 0.29 & 0.41 & 0.33 & 0.34 & 0.40 & 0.49 \\
\hline \multirow{4}{*}{0.2} & 0 & 0.01 & 0.05 & 0.27 & 0.49 & 0.09 & 0.14 & 0.33 & 0.51 & 0.28 & 0.31 & 0.44 & 0.57 & 0.48 & 0.49 & 0.56 & 0.65 \\
\hline & 0.2 & 0.01 & 0.05 & 0.26 & 0.47 & 0.09 & 0.13 & 0.31 & 0.50 & 0.27 & 0.30 & 0.42 & 0.56 & 0.46 & 0.48 & 0.55 & 0.64 \\
\hline & 0.5 & 0.01 & 0.04 & 0.21 & 0.41 & 0.07 & 0.10 & 0.26 & 0.43 & 0.22 & 0.25 & 0.36 & 0.49 & 0.40 & 0.41 & 0.48 & 0.57 \\
\hline & 0.8 & 0.01 & 0.03 & 0.16 & 0.32 & 0.05 & 0.07 & 0.19 & 0.35 & 0.17 & 0.19 & 0.28 & 0.41 & 0.32 & $\mathbf{0 . 3 3}$ & 0.40 & 0.49 \\
\hline \multirow{4}{*}{0.5} & 0 & 0.01 & 0.04 & 0.22 & 0.42 & 0.07 & 0.11 & 0.26 & 0.44 & 0.23 & 0.25 & 0.37 & 0.50 & 0.41 & 0.43 & 0.49 & 0.58 \\
\hline & 0.2 & 0.01 & 0.04 & 0.21 & 0.41 & 0.07 & 0.10 & 0.25 & 0.43 & 0.22 & 0.24 & 0.36 & 0.49 & 0.40 & 0.41 & 0.48 & 0.57 \\
\hline & 0.5 & 0.01 & 0.03 & 0.18 & 0.36 & 0.06 & 0.09 & 0.22 & 0.38 & 0.19 & 0.21 & 0.31 & 0.44 & 0.35 & 0.36 & 0.43 & 0.52 \\
\hline & 0.8 & 0.01 & 0.03 & 0.14 & 0.29 & 0.04 & 0.06 & 0.17 & 0.32 & 0.15 & 0.16 & 0.25 & 0.37 & 0.28 & 0.30 & 0.36 & 0.45 \\
\hline \multirow{4}{*}{0.8} & 0 & 0.01 & 0.03 & 0.16 & 0.33 & 0.05 & 0.08 & 0.20 & 0.35 & 0.17 & 0.19 & 0.28 & 0.41 & 0.32 & 0.34 & 0.40 & 0.49 \\
\hline & 0.2 & 0.01 & 0.03 & 0.16 & 0.33 & 0.05 & 0.07 & 0.19 & 0.35 & 0.16 & 0.18 & 0.28 & 0.40 & 0.32 & 0.33 & 0.39 & 0.49 \\
\hline & 0.5 & 0.01 & 0.03 & 0.14 & 0.29 & 0.04 & 0.06 & 0.17 & 0.32 & 0.15 & 0.17 & 0.25 & 0.37 & 0.29 & 0.30 & 0.36 & 0.45 \\
\hline & 0.8 & 0.01 & 0.02 & 0.11 & 0.24 & 0.03 & 0.05 & 0.14 & 0.27 & 0.12 & 0.14 & 0.21 & 0.32 & 0.24 & 0.25 & 0.31 & 0.39 \\
\hline
\end{tabular}

Note: Italicized values indicate conditional deviance of .15 or more above specified value, while bold values indicate conditional deviance of .15 or more below the specified value. ICC = Intraclass Correlation Coefficient. 
Table 1 cont.

Average Observed ICC values: Poisson Distribution conditions $(\mathrm{m}=10, \mathrm{k}=100)$

\begin{tabular}{|c|c|c|c|c|c|c|c|c|c|c|c|c|c|c|c|c|c|}
\hline \multirow{3}{*}{$\begin{array}{c}\text { L1 } \\
\text { Effect }\end{array}$} & \multirow{3}{*}{$\begin{array}{l}\text { Interaction } \\
\text { Effect }\end{array}$} & \multicolumn{16}{|c|}{ ICC Values } \\
\hline & & \multicolumn{4}{|c|}{0} & \multicolumn{4}{|c|}{0.1} & \multicolumn{4}{|c|}{0.3} & \multicolumn{4}{|c|}{0.5} \\
\hline & & 0 & 0.2 & 0.5 & 0.8 & 0 & 0.2 & 0.5 & 0.8 & 0 & 0.2 & 0.5 & 0.8 & 0 & 0.2 & 0.5 & 0.8 \\
\hline \multirow{4}{*}{0} & 0 & 0.00 & 0.06 & 0.29 & 0.51 & 0.10 & 0.15 & 0.34 & 0.53 & 0.30 & 0.33 & 0.45 & 0.59 & 0.49 & 0.51 & 0.58 & 0.67 \\
\hline & 0.2 & 0.01 & 0.06 & 0.27 & 0.49 & 0.09 & 0.14 & 0.32 & 0.51 & 0.28 & 0.31 & 0.44 & 0.58 & 0.48 & 0.49 & 0.56 & 0.65 \\
\hline & 0.5 & 0.01 & 0.04 & 0.22 & 0.42 & 0.07 & 0.11 & 0.27 & 0.45 & 0.23 & 0.25 & 0.37 & 0.51 & 0.41 & 0.42 & 0.49 & 0.58 \\
\hline & 0.8 & 0.01 & 0.03 & 0.16 & 0.33 & 0.05 & 0.08 & 0.20 & 0.36 & 0.17 & 0.19 & 0.28 & 0.42 & 0.32 & 0.34 & 0.40 & 0.50 \\
\hline \multirow{4}{*}{0.2} & 0 & 0.01 & 0.06 & 0.27 & 0.49 & 0.09 & 0.14 & 0.32 & 0.51 & 0.28 & 0.31 & 0.44 & 0.58 & 0.48 & 0.49 & 0.56 & 0.65 \\
\hline & 0.2 & 0.01 & 0.05 & 0.26 & 0.47 & 0.09 & 0.13 & 0.31 & 0.50 & 0.27 & 0.30 & 0.42 & 0.56 & 0.46 & 0.48 & 0.55 & 0.64 \\
\hline & 0.5 & 0.01 & 0.04 & 0.21 & 0.41 & 0.07 & 0.11 & 0.26 & 0.44 & 0.22 & 0.25 & 0.36 & 0.50 & 0.40 & 0.41 & 0.48 & 0.58 \\
\hline & 0.8 & 0.01 & 0.03 & 0.16 & 0.33 & 0.05 & 0.08 & 0.20 & 0.35 & 0.17 & 0.19 & 0.28 & 0.41 & 0.32 & 0.33 & 0.39 & 0.49 \\
\hline \multirow{4}{*}{0.5} & 0 & 0.01 & 0.04 & 0.22 & 0.42 & 0.07 & 0.11 & 0.27 & 0.44 & 0.23 & 0.26 & 0.37 & 0.51 & 0.41 & 0.43 & 0.49 & 0.59 \\
\hline & 0.2 & 0.01 & 0.04 & 0.21 & 0.41 & 0.07 & 0.10 & 0.26 & 0.43 & 0.22 & 0.25 & 0.36 & 0.49 & 0.40 & 0.42 & 0.48 & 0.58 \\
\hline & 0.5 & 0.01 & 0.03 & 0.18 & 0.36 & 0.06 & 0.09 & 0.22 & 0.39 & 0.19 & 0.21 & 0.31 & 0.44 & 0.35 & 0.37 & 0.43 & 0.53 \\
\hline & 0.8 & 0.01 & 0.02 & 0.14 & 0.29 & 0.04 & 0.06 & 0.17 & 0.32 & 0.15 & 0.16 & 0.25 & 0.37 & 0.28 & 0.30 & 0.36 & 0.45 \\
\hline \multirow{4}{*}{0.8} & 0 & 0.01 & 0.03 & 0.16 & 0.33 & 0.05 & 0.08 & 0.20 & 0.36 & 0.17 & 0.19 & 0.29 & 0.41 & 0.32 & 0.34 & 0.40 & 0.49 \\
\hline & 0.2 & 0.01 & 0.03 & 0.16 & 0.33 & 0.05 & 0.07 & 0.19 & 0.35 & 0.16 & 0.19 & 0.28 & 0.41 & 0.32 & 0.33 & 0.39 & 0.49 \\
\hline & 0.5 & 0.01 & 0.03 & 0.14 & 0.30 & 0.04 & 0.07 & 0.17 & 0.32 & 0.14 & 0.16 & 0.25 & 0.37 & 0.28 & 0.30 & 0.36 & 0.45 \\
\hline & 0.8 & 0.01 & 0.02 & 0.11 & 0.25 & 0.03 & 0.05 & 0.14 & 0.27 & 0.12 & 0.14 & 0.21 & 0.32 & 0.24 & 0.25 & 0.31 & 0.40 \\
\hline
\end{tabular}

Note: Italicized values indicate conditional deviance of .15 or more above specified value, while bold values indicate conditional deviance of .15 or more below the specified value. ICC = Intraclass Correlation Coefficient. 
Table 1 cont.

Average Observed ICC values: Poisson Distribution conditions $(\mathrm{m}=20, \mathrm{k}=25)$

\begin{tabular}{|c|c|c|c|c|c|c|c|c|c|c|c|c|c|c|c|c|c|}
\hline \multirow{3}{*}{$\begin{array}{c}\text { L1 } \\
\text { Effect }\end{array}$} & \multirow{3}{*}{$\begin{array}{l}\text { Interaction } \\
\text { Effect }\end{array}$} & \multicolumn{16}{|c|}{ ICC Values } \\
\hline & & \multicolumn{4}{|c|}{0} & \multicolumn{4}{|c|}{0.1} & \multicolumn{4}{|c|}{0.3} & \multicolumn{4}{|c|}{0.5} \\
\hline & & 0 & 0.2 & 0.5 & 0.8 & 0 & 0.2 & 0.5 & 0.8 & 0 & 0.2 & 0.5 & 0.8 & 0 & 0.2 & 0.5 & 0.8 \\
\hline \multirow{4}{*}{0} & 0 & 0.00 & 0.06 & 0.27 & 0.49 & 0.09 & 0.14 & 0.33 & 0.52 & 0.28 & 0.31 & 0.44 & 0.57 & 0.48 & 0.49 & 0.56 & 0.65 \\
\hline & 0.2 & 0.00 & 0.05 & 0.27 & 0.47 & 0.09 & 0.13 & 0.31 & 0.50 & 0.27 & 0.30 & 0.42 & 0.56 & 0.47 & 0.48 & 0.55 & 0.64 \\
\hline & 0.5 & 0.01 & 0.04 & 0.21 & 0.41 & 0.07 & 0.10 & 0.26 & 0.43 & 0.22 & 0.25 & 0.36 & 0.50 & 0.40 & 0.41 & 0.48 & 0.57 \\
\hline & 0.8 & 0.01 & 0.03 & 0.16 & 0.32 & 0.05 & 0.08 & 0.19 & 0.34 & 0.17 & 0.18 & 0.27 & 0.40 & 0.31 & 0.33 & 0.39 & 0.48 \\
\hline \multirow{4}{*}{0.2} & 0 & 0.00 & 0.05 & 0.27 & 0.48 & 0.09 & 0.13 & 0.31 & 0.50 & 0.27 & 0.30 & 0.42 & 0.56 & 0.46 & 0.48 & 0.55 & 0.64 \\
\hline & 0.2 & 0.00 & 0.05 & 0.25 & 0.46 & 0.08 & 0.13 & 0.30 & 0.49 & 0.26 & 0.29 & 0.41 & 0.54 & 0.45 & 0.46 & 0.53 & 0.63 \\
\hline & 0.5 & 0.01 & 0.04 & 0.20 & 0.40 & 0.06 & 0.10 & 0.25 & 0.42 & 0.21 & 0.24 & 0.35 & 0.48 & 0.39 & 0.40 & 0.47 & 0.56 \\
\hline & 0.8 & 0.01 & 0.03 & 0.15 & 0.32 & 0.05 & 0.07 & 0.18 & 0.34 & 0.16 & 0.18 & 0.27 & 0.39 & 0.31 & 0.32 & 0.39 & 0.47 \\
\hline \multirow{4}{*}{0.5} & 0 & 0.00 & 0.04 & 0.21 & 0.41 & 0.07 & 0.10 & 0.26 & 0.43 & 0.22 & 0.25 & 0.36 & 0.50 & 0.40 & 0.41 & 0.48 & 0.57 \\
\hline & 0.2 & 0.00 & 0.04 & 0.20 & 0.40 & 0.07 & 0.10 & 0.25 & 0.42 & 0.21 & 0.24 & 0.35 & 0.48 & 0.38 & 0.40 & 0.46 & 0.56 \\
\hline & 0.5 & 0.01 & 0.03 & 0.17 & 0.35 & 0.06 & 0.08 & 0.21 & 0.37 & 0.18 & 0.20 & 0.30 & 0.43 & 0.34 & 0.35 & 0.42 & 0.51 \\
\hline & 0.8 & 0.01 & 0.02 & 0.13 & 0.28 & 0.04 & 0.06 & 0.16 & 0.30 & 0.14 & 0.16 & 0.24 & 0.36 & 0.28 & 0.29 & 0.35 & 0.44 \\
\hline \multirow{4}{*}{0.8} & 0 & 0.00 & 0.03 & 0.16 & 0.32 & 0.05 & 0.07 & 0.19 & 0.35 & 0.16 & 0.19 & 0.28 & 0.40 & 0.31 & 0.32 & 0.39 & 0.48 \\
\hline & 0.2 & 0.01 & 0.03 & 0.15 & 0.31 & 0.04 & 0.07 & 0.19 & 0.34 & 0.16 & 0.18 & 0.27 & 0.39 & 0.30 & 0.32 & 0.38 & 0.47 \\
\hline & 0.5 & 0.01 & 0.02 & 0.13 & 0.28 & 0.04 & 0.06 & 0.17 & 0.31 & 0.14 & 0.16 & 0.24 & 0.36 & 0.28 & 0.29 & 0.35 & 0.43 \\
\hline & 0.8 & 0.01 & 0.02 & 0.11 & 0.24 & 0.03 & 0.05 & 0.13 & 0.26 & 0.12 & 0.13 & 0.20 & 0.31 & 0.24 & 0.25 & 0.30 & 0.38 \\
\hline
\end{tabular}

Note: Italicized values indicate conditional deviance of .15 or more above specified value, while bold values indicate conditional deviance of .15 or more below the specified value. ICC = Intraclass Correlation Coefficient. 
Table 1 cont.

Average Observed ICC values: Poisson Distribution conditions $(\mathrm{m}=20, \mathrm{k}=50)$

\begin{tabular}{|c|c|c|c|c|c|c|c|c|c|c|c|c|c|c|c|c|c|}
\hline \multirow{3}{*}{$\begin{array}{c}\text { L1 } \\
\text { Effect }\end{array}$} & \multirow{3}{*}{$\begin{array}{l}\text { Interaction } \\
\text { Effect }\end{array}$} & \multicolumn{16}{|c|}{ ICC Values } \\
\hline & & \multicolumn{4}{|c|}{0} & \multicolumn{4}{|c|}{0.1} & \multicolumn{4}{|c|}{0.3} & \multicolumn{4}{|c|}{0.5} \\
\hline & & 0 & 0.2 & 0.5 & 0.8 & 0 & 0.2 & 0.5 & 0.8 & 0 & 0.2 & 0.5 & 0.8 & 0 & 0.2 & 0.5 & 0.8 \\
\hline \multirow{4}{*}{0} & 0 & 0.00 & 0.06 & 0.28 & 0.50 & 0.10 & 0.14 & 0.34 & 0.53 & 0.29 & 0.32 & 0.45 & 0.59 & 0.49 & 0.50 & 0.57 & 0.66 \\
\hline & 0.2 & 0.00 & 0.06 & 0.27 & 0.49 & 0.09 & 0.14 & 0.32 & 0.51 & 0.28 & 0.31 & 0.43 & 0.57 & 0.47 & 0.49 & 0.56 & 0.65 \\
\hline & 0.5 & 0.00 & 0.04 & 0.22 & 0.42 & 0.07 & 0.11 & 0.26 & 0.44 & 0.23 & 0.25 & 0.36 & 0.50 & 0.41 & 0.42 & 0.49 & 0.58 \\
\hline & 0.8 & 0.00 & 0.03 & 0.16 & 0.33 & 0.05 & 0.08 & 0.20 & 0.35 & 0.17 & 0.19 & 0.28 & 0.41 & 0.32 & 0.33 & 0.40 & 0.49 \\
\hline \multirow{4}{*}{0.2} & 0 & 0.00 & 0.06 & 0.27 & 0.48 & 0.09 & 0.14 & 0.32 & 0.51 & 0.28 & 0.31 & 0.43 & 0.57 & 0.47 & 0.49 & 0.56 & 0.65 \\
\hline & 0.2 & 0.00 & 0.05 & 0.26 & 0.47 & 0.09 & 0.13 & 0.31 & 0.50 & 0.27 & 0.30 & 0.42 & 0.55 & 0.46 & 0.48 & 0.54 & 0.63 \\
\hline & 0.5 & 0.00 & 0.04 & 0.21 & 0.41 & 0.07 & 0.10 & 0.25 & 0.43 & 0.22 & 0.25 & 0.35 & 0.49 & 0.39 & 0.41 & 0.48 & 0.57 \\
\hline & 0.8 & 0.00 & 0.03 & 0.16 & 0.32 & 0.05 & 0.08 & 0.19 & 0.35 & 0.17 & 0.19 & 0.28 & 0.40 & 0.32 & 0.33 & 0.39 & 0.48 \\
\hline \multirow{4}{*}{0.5} & 0 & 0.00 & 0.04 & 0.22 & 0.42 & 0.07 & 0.11 & 0.26 & 0.44 & 0.23 & 0.25 & 0.37 & 0.50 & 0.41 & 0.42 & 0.49 & 0.58 \\
\hline & 0.2 & 0.00 & 0.04 & 0.21 & 0.41 & 0.07 & 0.10 & 0.26 & 0.43 & 0.22 & 0.24 & 0.35 & 0.49 & 0.40 & 0.41 & 0.48 & 0.57 \\
\hline & 0.5 & 0.00 & 0.03 & 0.18 & 0.36 & 0.05 & 0.08 & 0.22 & 0.38 & 0.18 & 0.21 & 0.31 & 0.44 & 0.35 & 0.36 & 0.43 & 0.52 \\
\hline & 0.8 & 0.00 & 0.02 & 0.14 & 0.29 & 0.04 & 0.06 & 0.17 & 0.31 & 0.15 & 0.16 & 0.25 & 0.37 & 0.29 & 0.30 & 0.36 & 0.44 \\
\hline \multirow{4}{*}{0.8} & 0 & 0.00 & 0.03 & 0.16 & 0.33 & 0.05 & 0.08 & 0.20 & 0.36 & 0.17 & 0.19 & 0.28 & 0.41 & 0.32 & 0.33 & 0.40 & 0.49 \\
\hline & 0.2 & 0.00 & 0.03 & 0.16 & 0.32 & 0.05 & 0.08 & 0.19 & 0.35 & 0.16 & 0.18 & 0.28 & 0.40 & 0.31 & 0.32 & 0.39 & 0.48 \\
\hline & 0.5 & 0.00 & 0.03 & 0.14 & 0.29 & 0.04 & 0.07 & 0.17 & 0.31 & 0.14 & 0.16 & 0.25 & 0.37 & 0.28 & 0.30 & 0.36 & 0.44 \\
\hline & 0.8 & 0.00 & 0.02 & 0.11 & 0.25 & 0.03 & 0.05 & 0.14 & 0.27 & 0.12 & 0.13 & 0.21 & 0.31 & 0.24 & 0.25 & 0.31 & 0.39 \\
\hline
\end{tabular}

Note: Italicized values indicate conditional deviance of .15 or more above specified value, while bold values indicate conditional deviance of .15 or more below the specified value. ICC = Intraclass Correlation Coefficient. 
Table 1 cont.

Average Observed ICC values: Poisson Distribution conditions $(\mathrm{m}=20, \mathrm{k}=75)$

\begin{tabular}{|c|c|c|c|c|c|c|c|c|c|c|c|c|c|c|c|c|c|}
\hline \multirow{3}{*}{$\begin{array}{c}\text { L1 } \\
\text { Effect }\end{array}$} & \multirow{3}{*}{$\begin{array}{c}\text { Interaction } \\
\text { Effect }\end{array}$} & \multicolumn{16}{|c|}{ ICC Values } \\
\hline & & \multicolumn{4}{|c|}{0} & \multicolumn{3}{|c|}{0.1} & & \multicolumn{4}{|c|}{0.3} & \multicolumn{4}{|c|}{0.5} \\
\hline & & 0 & 0.2 & 0.5 & 0.8 & 0 & 0.2 & 0.5 & 0.8 & 0 & 0.2 & 0.5 & 0.8 & 0 & 0.2 & 0.5 & 0.8 \\
\hline \multirow{4}{*}{0} & 0 & 0.00 & 0.06 & 0.29 & 0.50 & 0.10 & 0.15 & 0.33 & 0.53 & 0.29 & 0.33 & 0.45 & 0.59 & 0.49 & 0.51 & 0.58 & 0.66 \\
\hline & 0.2 & 0.00 & 0.06 & 0.27 & 0.49 & 0.09 & 0.14 & 0.32 & 0.52 & 0.28 & 0.31 & 0.43 & 0.57 & 0.48 & 0.49 & 0.56 & 0.65 \\
\hline & 0.5 & 0.00 & 0.04 & 0.22 & 0.42 & 0.07 & 0.11 & 0.26 & 0.45 & 0.23 & 0.26 & 0.37 & 0.50 & 0.41 & 0.42 & 0.49 & 0.59 \\
\hline & 0.8 & 0.00 & 0.03 & 0.16 & 0.33 & 0.05 & 0.08 & 0.20 & 0.36 & 0.17 & 0.19 & 0.29 & 0.41 & 0.32 & 0.34 & 0.40 & 0.49 \\
\hline \multirow{4}{*}{0.2} & 0 & 0.00 & 0.06 & 0.27 & 0.49 & 0.09 & 0.14 & 0.32 & 0.51 & 0.28 & 0.31 & 0.43 & 0.57 & 0.48 & 0.49 & 0.56 & 0.65 \\
\hline & 0.2 & 0.00 & 0.05 & 0.26 & 0.48 & 0.09 & 0.13 & 0.31 & 0.50 & 0.27 & 0.30 & 0.42 & 0.56 & 0.46 & 0.48 & 0.55 & 0.64 \\
\hline & 0.5 & 0.00 & 0.04 & 0.21 & 0.41 & 0.07 & 0.10 & 0.26 & 0.43 & 0.22 & 0.25 & 0.36 & 0.49 & 0.40 & 0.41 & 0.48 & 0.57 \\
\hline & 0.8 & 0.00 & 0.03 & 0.16 & 0.33 & 0.05 & 0.08 & 0.20 & 0.35 & 0.16 & 0.19 & 0.28 & 0.41 & 0.32 & 0.33 & 0.39 & 0.49 \\
\hline \multirow{4}{*}{0.5} & 0 & 0.00 & 0.04 & 0.22 & 0.42 & 0.07 & 0.11 & 0.27 & 0.45 & 0.23 & 0.26 & 0.37 & 0.51 & 0.41 & 0.42 & 0.50 & 0.58 \\
\hline & 0.2 & 0.00 & 0.04 & 0.21 & 0.41 & 0.07 & 0.10 & 0.26 & 0.43 & 0.22 & 0.25 & 0.36 & 0.49 & 0.40 & 0.41 & 0.48 & 0.57 \\
\hline & 0.5 & 0.00 & 0.03 & 0.18 & 0.36 & 0.06 & 0.09 & 0.22 & 0.38 & 0.19 & 0.21 & 0.31 & 0.44 & 0.35 & 0.36 & 0.43 & 0.52 \\
\hline & 0.8 & 0.00 & 0.02 & 0.14 & 0.30 & 0.04 & 0.07 & 0.17 & 0.32 & 0.14 & 0.17 & 0.25 & 0.37 & 0.29 & 0.30 & 0.36 & 0.45 \\
\hline \multirow{4}{*}{0.8} & 0 & 0.00 & 0.03 & 0.16 & 0.33 & 0.05 & 0.08 & 0.20 & 0.36 & 0.17 & 0.19 & 0.28 & 0.41 & 0.32 & 0.34 & 0.40 & 0.49 \\
\hline & 0.2 & 0.00 & 0.03 & 0.16 & 0.33 & 0.05 & 0.07 & 0.19 & 0.35 & 0.16 & 0.19 & 0.28 & 0.41 & 0.32 & 0.33 & 0.40 & 0.49 \\
\hline & 0.5 & 0.00 & 0.03 & 0.14 & 0.29 & 0.04 & 0.07 & 0.17 & 0.32 & 0.14 & 0.16 & 0.25 & 0.37 & 0.28 & 0.30 & 0.36 & 0.45 \\
\hline & 0.8 & 0.00 & 0.02 & 0.11 & 0.25 & 0.03 & 0.05 & 0.14 & 0.27 & 0.12 & 0.13 & 0.21 & 0.32 & 0.24 & 0.25 & 0.31 & 0.39 \\
\hline
\end{tabular}

Note: Italicized values indicate conditional deviance of .15 or more above specified value, while bold values indicate conditional deviance of .15 or more below the specified value. ICC = Intraclass Correlation Coefficient. 
Table 1 cont.

Average Observed ICC values: Poisson Distribution conditions $(\mathrm{m}=20, \mathrm{k}=100)$

\begin{tabular}{|c|c|c|c|c|c|c|c|c|c|c|c|c|c|c|c|c|c|}
\hline \multirow{3}{*}{$\begin{array}{c}\text { L1 } \\
\text { Effect }\end{array}$} & \multirow{3}{*}{$\begin{array}{l}\text { Interaction } \\
\text { Effect }\end{array}$} & \multicolumn{16}{|c|}{ ICC Values } \\
\hline & & \multicolumn{4}{|c|}{0} & \multicolumn{4}{|c|}{0.1} & \multicolumn{4}{|c|}{0.3} & \multicolumn{4}{|c|}{0.5} \\
\hline & & 0 & 0.2 & 0.5 & 0.8 & 0 & 0.2 & 0.5 & 0.8 & 0 & 0.2 & 0.5 & 0.8 & 0 & 0.2 & 0.5 & 0.8 \\
\hline \multirow{4}{*}{0} & 0 & 0.00 & 0.06 & 0.29 & 0.51 & 0.10 & 0.15 & 0.34 & 0.53 & 0.30 & 0.32 & 0.45 & 0.59 & 0.49 & 0.51 & 0.58 & 0.67 \\
\hline & 0.2 & 0.00 & 0.06 & 0.27 & 0.49 & 0.09 & 0.14 & 0.32 & 0.52 & 0.28 & 0.31 & 0.44 & 0.58 & 0.48 & 0.50 & 0.57 & 0.65 \\
\hline & 0.5 & 0.00 & 0.04 & 0.22 & 0.42 & 0.07 & 0.11 & 0.27 & 0.45 & 0.23 & 0.26 & 0.37 & 0.51 & 0.41 & 0.43 & 0.50 & 0.59 \\
\hline & 0.8 & 0.00 & 0.03 & 0.16 & 0.33 & 0.05 & 0.08 & 0.20 & 0.36 & 0.17 & 0.19 & 0.29 & 0.41 & 0.33 & 0.34 & 0.40 & 0.49 \\
\hline \multirow{4}{*}{0.2} & 0 & 0.00 & 0.06 & 0.27 & 0.49 & 0.09 & 0.14 & 0.32 & 0.52 & 0.28 & 0.31 & 0.43 & 0.58 & 0.48 & 0.49 & 0.56 & 0.65 \\
\hline & 0.2 & 0.00 & 0.05 & 0.26 & 0.47 & 0.09 & 0.13 & 0.31 & 0.50 & 0.27 & 0.30 & 0.42 & 0.56 & 0.46 & 0.48 & 0.55 & 0.64 \\
\hline & 0.5 & 0.00 & 0.04 & 0.21 & 0.41 & 0.07 & 0.11 & 0.26 & 0.43 & 0.22 & 0.25 & 0.36 & 0.50 & 0.40 & 0.41 & 0.48 & 0.58 \\
\hline & 0.8 & 0.00 & 0.03 & 0.16 & 0.33 & 0.05 & 0.08 & 0.19 & 0.35 & 0.17 & 0.19 & 0.28 & 0.41 & 0.32 & 0.33 & 0.40 & 0.49 \\
\hline \multirow{4}{*}{0.5} & 0 & 0.00 & 0.04 & 0.22 & 0.42 & 0.07 & 0.11 & 0.26 & 0.45 & 0.23 & 0.26 & 0.37 & 0.51 & 0.41 & 0.43 & 0.49 & 0.59 \\
\hline & 0.2 & 0.00 & 0.04 & 0.22 & 0.41 & 0.07 & 0.10 & 0.26 & 0.43 & 0.22 & 0.25 & 0.36 & 0.49 & 0.40 & 0.41 & 0.49 & 0.58 \\
\hline & 0.5 & 0.00 & 0.03 & 0.18 & 0.36 & 0.06 & 0.09 & 0.22 & 0.39 & 0.19 & 0.21 & 0.31 & 0.44 & 0.35 & 0.36 & 0.43 & 0.53 \\
\hline & 0.8 & 0.00 & 0.02 & 0.14 & 0.29 & 0.04 & 0.07 & 0.17 & 0.32 & 0.15 & 0.16 & 0.25 & 0.37 & 0.29 & 0.30 & 0.36 & 0.45 \\
\hline \multirow{4}{*}{0.8} & 0 & 0.00 & 0.03 & 0.16 & 0.33 & 0.05 & 0.08 & 0.20 & 0.35 & 0.17 & 0.19 & 0.29 & 0.41 & 0.32 & 0.34 & 0.40 & 0.49 \\
\hline & 0.2 & 0.00 & 0.03 & 0.16 & 0.33 & 0.05 & 0.08 & 0.19 & 0.35 & 0.17 & 0.19 & 0.28 & 0.41 & 0.32 & 0.33 & 0.39 & 0.49 \\
\hline & 0.5 & 0.00 & 0.02 & 0.14 & 0.30 & 0.04 & 0.07 & 0.17 & 0.32 & 0.15 & 0.17 & 0.25 & 0.37 & 0.29 & 0.30 & 0.36 & 0.45 \\
\hline & 0.8 & 0.00 & 0.02 & 0.12 & 0.25 & 0.03 & 0.05 & 0.14 & 0.27 & 0.12 & 0.14 & 0.21 & 0.32 & 0.24 & 0.25 & 0.31 & 0.40 \\
\hline
\end{tabular}

Note: Italicized values indicate conditional deviance of .15 or more above specified value, while bold values indicate conditional deviance of .15 or more below the specified value. ICC = Intraclass Correlation Coefficient. 
Table 2.

Average Model Design Effect values: Uniform Distribution conditions $(\mathrm{m}=5, \mathrm{k}=25)$

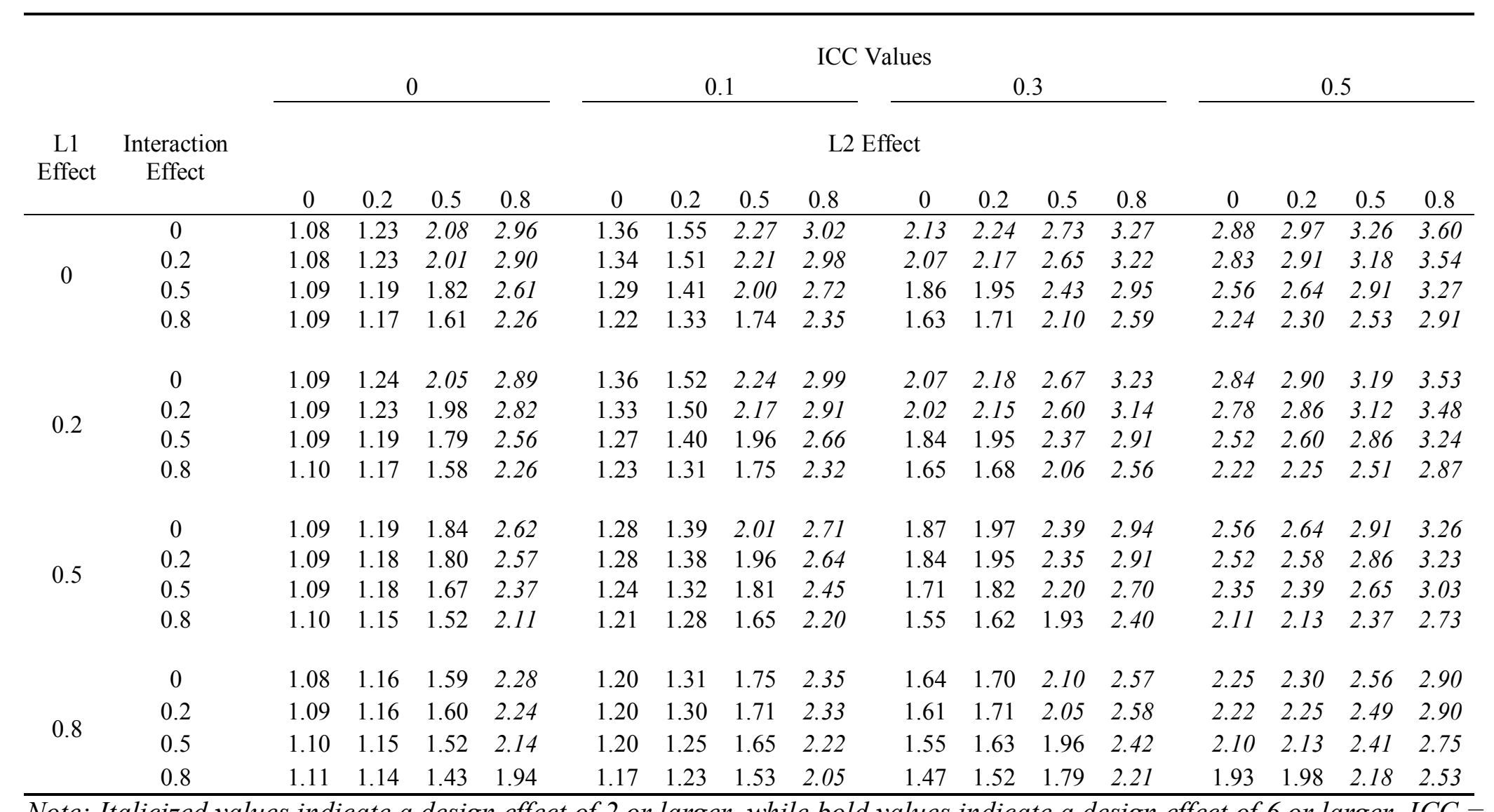

Note: Italicized values indicate a design effect of 2 or larger, while bold values indicate a design effect of 6 or larger. ICC $=$ Intraclass Correlation Coefficient. 
Table 2 cont.

Average Model Design Effect values: Uniform Distribution conditions ( $\mathrm{m}=5, \mathrm{k}=50)$

\begin{tabular}{|c|c|c|c|c|c|c|c|c|c|c|c|c|c|c|c|c|c|}
\hline \multirow{3}{*}{$\begin{array}{c}\text { L1 } \\
\text { Effect }\end{array}$} & \multirow{3}{*}{$\begin{array}{c}\text { Interaction } \\
\text { Effect }\end{array}$} & \multicolumn{16}{|c|}{ ICC Values } \\
\hline & & \multicolumn{4}{|c|}{0} & \multicolumn{4}{|c|}{0.1} & \multicolumn{4}{|c|}{0.3} & \multicolumn{4}{|c|}{0.5} \\
\hline & & 0 & 0.2 & 0.5 & 0.8 & 0 & 0.2 & 0.5 & 0.8 & 0 & 0.2 & 0.5 & 0.8 & 0 & 0.2 & 0.5 & 0.8 \\
\hline \multirow{4}{*}{0} & 0 & 1.06 & 1.24 & 2.13 & 3.00 & 1.37 & 1.58 & 2.34 & 3.09 & 2.17 & 2.28 & 2.77 & 3.33 & 2.97 & 3.02 & 3.29 & 3.64 \\
\hline & 0.2 & 1.06 & 1.22 & 2.07 & 2.93 & 1.36 & 1.55 & 2.27 & 3.05 & 2.11 & 2.22 & 2.72 & 3.28 & 2.87 & 2.94 & 3.23 & 3.58 \\
\hline & 0.5 & 1.07 & 1.18 & 1.85 & 2.66 & 1.29 & 1.42 & 2.04 & 2.75 & 1.89 & 2.00 & 2.43 & 2.99 & 2.61 & 2.67 & 2.94 & 3.33 \\
\hline & 0.8 & 1.07 & 1.15 & 1.64 & 2.30 & 1.22 & 1.30 & 1.77 & 2.42 & 1.65 & 1.77 & 2.12 & 2.62 & 2.27 & 2.34 & 2.60 & 2.95 \\
\hline \multirow{4}{*}{0.2} & 0 & 1.06 & 1.22 & 2.08 & 2.94 & 1.36 & 1.54 & 2.28 & 3.04 & 2.11 & 2.24 & 2.70 & 3.28 & 2.88 & 2.97 & 3.24 & 3.59 \\
\hline & 0.2 & 1.06 & 1.22 & 2.03 & 2.88 & 1.34 & 1.50 & 2.21 & 2.98 & 2.05 & 2.19 & 2.66 & 3.23 & 2.83 & 2.90 & 3.17 & 3.53 \\
\hline & 0.5 & 1.07 & 1.18 & 1.82 & 2.61 & 1.28 & 1.40 & 2.00 & 2.71 & 1.87 & 1.98 & 2.41 & 2.95 & 2.59 & 2.64 & 2.91 & 3.28 \\
\hline & 0.8 & 1.07 & 1.16 & 1.62 & 2.29 & 1.21 & 1.30 & 1.76 & 2.36 & 1.65 & 1.73 & 2.08 & 2.60 & 2.25 & 2.29 & 2.55 & 2.91 \\
\hline \multirow{4}{*}{0.5} & 0 & 1.06 & 1.18 & 1.87 & 2.65 & 1.29 & 1.42 & 2.05 & 2.75 & 1.91 & 2.01 & 2.46 & 3.01 & 2.61 & 2.68 & 2.96 & 3.33 \\
\hline & 0.2 & 1.06 & 1.18 & 1.84 & 2.62 & 1.27 & 1.40 & 2.02 & 2.74 & 1.87 & 1.97 & 2.39 & 2.96 & 2.58 & 2.63 & 2.90 & 3.27 \\
\hline & 0.5 & 1.07 & 1.16 & 1.70 & 2.42 & 1.22 & 1.34 & 1.82 & 2.52 & 1.74 & 1.80 & 2.21 & 2.74 & 2.39 & 2.45 & 2.68 & 3.07 \\
\hline & 0.8 & 1.09 & 1.14 & 1.54 & 2.16 & 1.19 & 1.27 & 1.68 & 2.25 & 1.58 & 1.65 & 1.99 & 2.48 & 2.13 & 2.19 & 2.43 & 2.77 \\
\hline \multirow{4}{*}{0.8} & 0 & 1.06 & 1.14 & 1.63 & 2.29 & 1.20 & 1.31 & 1.77 & 2.42 & 1.66 & 1.74 & 2.13 & 2.64 & 2.29 & 2.32 & 2.59 & 2.94 \\
\hline & 0.2 & 1.07 & 1.14 & 1.62 & 2.28 & 1.20 & 1.30 & 1.75 & 2.39 & 1.65 & 1.73 & 2.09 & 2.62 & 2.25 & 2.30 & 2.57 & 2.92 \\
\hline & 0.5 & 1.07 & 1.15 & 1.55 & 2.16 & 1.19 & 1.27 & 1.66 & 2.26 & 1.57 & 1.64 & 1.98 & 2.46 & 2.13 & 2.19 & 2.40 & 2.77 \\
\hline & 0.8 & 1.08 & 1.13 & 1.46 & 1.99 & 1.15 & 1.23 & 1.56 & 2.06 & 1.46 & 1.52 & 1.83 & 2.26 & 1.96 & 1.99 & 2.22 & 2.55 \\
\hline
\end{tabular}

Note: Italicized values indicate a design effect of 2 or larger, while bold values indicate a design effect of 6 or larger. ICC $=$ Intraclass Correlation Coefficient. 
Table 2 cont.

Average Model Design Effect values: Uniform Distribution conditions ( $\mathrm{m}=5, \mathrm{k}=75)$

\begin{tabular}{|c|c|c|c|c|c|c|c|c|c|c|c|c|c|c|c|c|c|}
\hline \multirow{4}{*}{$\begin{array}{c}\text { L1 } \\
\text { Effect }\end{array}$} & \multirow{4}{*}{$\begin{array}{c}\text { Interaction } \\
\text { Effect }\end{array}$} & \multicolumn{16}{|c|}{ ICC Values } \\
\hline & & \multicolumn{4}{|c|}{0} & \multirow{2}{*}{\multicolumn{3}{|c|}{0.1}} & & \multicolumn{4}{|c|}{0.3} & \multicolumn{4}{|c|}{0.5} \\
\hline & & \multicolumn{9}{|c|}{$\overline{\mathrm{L} 2}$ Effect } & & & & & & & \\
\hline & & 0 & 0.2 & 0.5 & 0.8 & 0 & 0.2 & 0.5 & 0.8 & 0 & 0.2 & 0.5 & 0.8 & 0 & 0.2 & 0.5 & 0.8 \\
\hline \multirow{4}{*}{0} & 0 & 1.05 & 1.24 & 2.14 & 3.02 & 1.37 & 1.59 & 2.34 & 3.11 & 2.18 & 2.29 & 2.79 & 3.35 & 2.96 & 3.03 & 3.31 & 3.66 \\
\hline & 0.2 & 1.05 & 1.22 & 2.09 & 2.94 & 1.36 & 1.55 & 2.29 & 3.06 & 2.12 & 2.24 & 2.73 & 3.29 & 2.91 & 2.98 & 3.24 & 3.61 \\
\hline & 0.5 & 1.05 & 1.18 & 1.87 & 2.67 & 1.29 & 1.42 & 2.04 & 2.77 & 1.92 & 2.01 & 2.45 & 3.01 & 2.63 & 2.68 & 2.97 & 3.35 \\
\hline & 0.8 & 1.07 & 1.15 & 1.64 & 2.32 & 1.20 & 1.31 & 1.80 & 2.41 & 1.68 & 1.76 & 2.13 & 2.63 & 2.30 & 2.34 & 2.60 & 2.97 \\
\hline \multirow{4}{*}{0.2} & 0 & 1.05 & 1.23 & 2.08 & 2.94 & 1.36 & 1.55 & 2.29 & 3.05 & 2.12 & 2.25 & 2.73 & 3.29 & 2.90 & 2.97 & 3.25 & 3.60 \\
\hline & 0.2 & 1.06 & 1.21 & 2.04 & 2.89 & 1.35 & 1.53 & 2.23 & 2.99 & 2.07 & 2.19 & 2.67 & 3.23 & 2.85 & 2.90 & 3.18 & 3.55 \\
\hline & 0.5 & 1.06 & 1.17 & 1.85 & 2.63 & 1.27 & 1.41 & 2.03 & 2.73 & 1.88 & 1.98 & 2.43 & 2.97 & 2.58 & 2.65 & 2.93 & 3.29 \\
\hline & 0.8 & 1.06 & 1.14 & 1.62 & 2.30 & 1.20 & 1.29 & 1.76 & 2.38 & 1.66 & 1.75 & 2.12 & 2.61 & 2.27 & 2.33 & 2.58 & 2.93 \\
\hline \multirow{4}{*}{0.5} & 0 & 1.05 & 1.17 & 1.88 & 2.68 & 1.28 & 1.44 & 2.05 & 2.78 & 1.90 & 2.01 & 2.46 & 3.01 & 2.64 & 2.68 & 2.97 & 3.34 \\
\hline & 0.2 & 1.05 & 1.17 & 1.85 & 2.63 & 1.27 & 1.42 & 2.03 & 2.73 & 1.87 & 1.98 & 2.43 & 2.97 & 2.58 & 2.64 & 2.91 & 3.29 \\
\hline & 0.5 & 1.06 & 1.15 & 1.72 & 2.43 & 1.23 & 1.34 & 1.87 & 2.52 & 1.74 & 1.84 & 2.22 & 2.76 & 2.38 & 2.45 & 2.70 & 3.11 \\
\hline & 0.8 & 1.07 & 1.13 & 1.55 & 2.18 & 1.18 & 1.27 & 1.69 & 2.25 & 1.58 & 1.65 & 2.00 & 2.48 & 2.14 & 2.18 & 2.43 & 2.78 \\
\hline \multirow{4}{*}{0.8} & 0 & 1.06 & 1.13 & 1.65 & 2.32 & 1.20 & 1.30 & 1.80 & 2.42 & 1.66 & 1.76 & 2.15 & 2.64 & 2.29 & 2.34 & 2.61 & 2.98 \\
\hline & 0.2 & 1.06 & 1.14 & 1.63 & 2.32 & 1.19 & 1.31 & 1.77 & 2.39 & 1.66 & 1.73 & 2.10 & 2.62 & 2.26 & 2.30 & 2.57 & 2.94 \\
\hline & 0.5 & 1.06 & 1.12 & 1.55 & 2.18 & 1.18 & 1.26 & 1.68 & 2.25 & 1.58 & 1.65 & 1.98 & 2.48 & 2.13 & 2.18 & 2.44 & 2.79 \\
\hline & 0.8 & 1.07 & 1.12 & 1.45 & 1.99 & 1.16 & 1.22 & 1.55 & 2.07 & 1.47 & 1.54 & 1.83 & 2.27 & 1.96 & 2.00 & 2.22 & 2.56 \\
\hline
\end{tabular}

Note: Italicized values indicate a design effect of 2 or larger, while bold values indicate a design effect of 6 or larger. ICC = Intraclass Correlation Coefficient. 
Table 2 cont.

Average Model Design Effect values: Uniform Distribution conditions ( $\mathrm{m}=5, \mathrm{k}=100)$

\begin{tabular}{|c|c|c|c|c|c|c|c|c|c|c|c|c|c|c|c|c|c|}
\hline \multirow{3}{*}{$\begin{array}{c}\text { L1 } \\
\text { Effect }\end{array}$} & \multirow{3}{*}{$\begin{array}{c}\text { Interaction } \\
\text { Effect }\end{array}$} & \multicolumn{16}{|c|}{ ICC Values } \\
\hline & & \multicolumn{4}{|c|}{0} & \multicolumn{4}{|c|}{0.1} & \multicolumn{4}{|c|}{0.3} & \multicolumn{4}{|c|}{0.5} \\
\hline & & 0 & 0.2 & 0.5 & 0.8 & 0 & 0.2 & 0.5 & 0.8 & 0 & 0.2 & 0.5 & 0.8 & 0 & 0.2 & 0.5 & 0.8 \\
\hline \multirow{4}{*}{0} & 0 & 1.04 & 1.23 & 2.14 & 3.02 & 1.39 & 1.59 & 2.35 & 3.12 & 2.18 & 2.30 & 2.80 & 3.36 & 2.97 & 3.04 & 3.31 & 3.67 \\
\hline & 0.2 & 1.05 & 1.22 & 2.10 & 2.97 & 1.37 & 1.54 & 2.30 & 3.06 & 2.13 & 2.26 & 2.74 & 3.29 & 2.91 & 2.98 & 3.25 & 3.61 \\
\hline & 0.5 & 1.05 & 1.18 & 1.88 & 2.68 & 1.29 & 1.43 & 2.06 & 2.78 & 1.91 & 2.02 & 2.47 & 3.01 & 2.63 & 2.69 & 2.99 & 3.34 \\
\hline & 0.8 & 1.05 & 1.14 & 1.65 & 2.33 & 1.21 & 1.32 & 1.80 & 2.43 & 1.67 & 1.76 & 2.15 & 2.66 & 2.30 & 2.34 & 2.61 & 2.98 \\
\hline \multirow{4}{*}{0.2} & 0 & 1.04 & 1.22 & 2.09 & 2.96 & 1.36 & 1.56 & 2.29 & 3.07 & 2.12 & 2.25 & 2.74 & 3.30 & 2.91 & 2.98 & 3.25 & 3.60 \\
\hline & 0.2 & 1.04 & 1.21 & 2.06 & 2.91 & 1.35 & 1.52 & 2.24 & 2.99 & 2.08 & 2.21 & 2.68 & 3.23 & 2.87 & 2.90 & 3.19 & 3.55 \\
\hline & 0.5 & 1.05 & 1.18 & 1.84 & 2.63 & 1.26 & 1.41 & 2.02 & 2.73 & 1.89 & 1.98 & 2.42 & 2.98 & 2.60 & 2.66 & 2.93 & 3.30 \\
\hline & 0.8 & 1.06 & 1.14 & 1.64 & 2.30 & 1.19 & 1.30 & 1.78 & 2.40 & 1.66 & 1.74 & 2.12 & 2.61 & 2.27 & 2.32 & 2.59 & 2.95 \\
\hline \multirow{4}{*}{0.5} & 0 & 1.05 & 1.18 & 1.89 & 2.69 & 1.28 & 1.43 & 2.05 & 2.77 & 1.91 & 2.03 & 2.46 & 3.02 & 2.63 & 2.69 & 2.97 & 3.34 \\
\hline & 0.2 & 1.04 & 1.18 & 1.85 & 2.64 & 1.26 & 1.42 & 2.03 & 2.73 & 1.89 & 1.98 & 2.44 & 2.99 & 2.59 & 2.65 & 2.93 & 3.30 \\
\hline & 0.5 & 1.06 & 1.14 & 1.71 & 2.44 & 1.23 & 1.34 & 1.87 & 2.53 & 1.75 & 1.83 & 2.24 & 2.76 & 2.41 & 2.46 & 2.73 & 3.10 \\
\hline & 0.8 & 1.06 & 1.13 & 1.55 & 2.18 & 1.17 & 1.26 & 1.68 & 2.26 & 1.59 & 1.66 & 2.00 & 2.48 & 2.15 & 2.20 & 2.43 & 2.78 \\
\hline \multirow{4}{*}{0.8} & 0 & 1.05 & 1.13 & 1.64 & 2.34 & 1.20 & 1.30 & 1.79 & 2.43 & 1.69 & 1.76 & 2.14 & 2.65 & 2.30 & 2.35 & 2.61 & 2.98 \\
\hline & 0.2 & 1.05 & 1.13 & 1.63 & 2.31 & 1.20 & 1.29 & 1.77 & 2.39 & 1.66 & 1.74 & 2.13 & 2.62 & 2.26 & 2.32 & 2.58 & 2.95 \\
\hline & 0.5 & 1.05 & 1.11 & 1.56 & 2.17 & 1.18 & 1.26 & 1.69 & 2.25 & 1.59 & 1.64 & 1.99 & 2.47 & 2.14 & 2.19 & 2.44 & 2.79 \\
\hline & 0.8 & 1.06 & 1.11 & 1.46 & 1.99 & 1.15 & 1.21 & 1.57 & 2.07 & 1.49 & 1.54 & 1.85 & 2.28 & 1.95 & 2.01 & 2.23 & 2.56 \\
\hline
\end{tabular}


Table 2 cont.

Average Model Design Effect values: Uniform Distribution conditions ( $\mathrm{m}=10, \mathrm{k}=25)$

\begin{tabular}{|c|c|c|c|c|c|c|c|c|c|c|c|c|c|c|c|c|c|}
\hline \multirow{2}{*}{$\begin{array}{c}\text { L1 } \\
\text { Effect }\end{array}$} & \multirow{2}{*}{$\begin{array}{l}\text { Interaction } \\
\text { Effect }\end{array}$} & \multicolumn{16}{|c|}{ ICC Values } \\
\hline & & 0 & 0.2 & 0.5 & 0.8 & 0 & 0.2 & 0.5 & 0.8 & 0 & 0.2 & 0.5 & 0.8 & 0 & 0.2 & 0.5 & 0.8 \\
\hline \multirow{4}{*}{0} & 0 & 1.09 & 1.51 & 3.46 & 5.40 & 1.84 & 2.28 & 3.93 & 5.59 & 3.54 & 3.84 & 4.90 & 6.13 & 5.31 & 5.48 & 6.03 & 6.85 \\
\hline & 0.2 & 1.09 & 1.49 & 3.36 & 5.28 & 1.77 & 2.17 & 3.81 & 5.50 & 3.45 & 3.70 & 4.78 & 6.06 & 5.14 & 5.33 & 5.98 & 6.70 \\
\hline & 0.5 & 1.09 & 1.37 & 2.88 & 4.65 & 1.61 & 1.93 & 3.30 & 4.87 & 2.98 & 3.20 & 4.18 & 5.41 & 4.55 & 4.69 & 5.31 & 6.15 \\
\hline & 0.8 & 1.10 & 1.28 & 2.38 & 3.85 & 1.45 & 1.65 & 2.72 & 4.09 & 2.50 & 2.66 & 3.46 & 4.59 & 3.85 & 3.95 & 4.51 & 5.28 \\
\hline \multirow{4}{*}{0.2} & 0 & 1.09 & 1.47 & 3.37 & 5.26 & 1.77 & 2.22 & 3.79 & 5.52 & 3.43 & 3.71 & 4.75 & 6.06 & 5.17 & 5.27 & 5.88 & 6.71 \\
\hline & 0.2 & 1.09 & 1.46 & 3.26 & 5.15 & 1.75 & 2.12 & 3.69 & 5.33 & 3.33 & 3.55 & 4.63 & 5.91 & 4.97 & 5.17 & 5.82 & 6.56 \\
\hline & 0.5 & 1.10 & 1.36 & 2.81 & 4.56 & 1.57 & 1.86 & 3.21 & 4.76 & 2.90 & 3.13 & 4.07 & 5.33 & 4.47 & 4.60 & 5.18 & 6.03 \\
\hline & 0.8 & 1.11 & 1.28 & 2.36 & 3.79 & 1.42 & 1.64 & 2.64 & 4.00 & 2.46 & 2.61 & 3.40 & 4.50 & 3.78 & 3.86 & 4.44 & 5.26 \\
\hline \multirow{4}{*}{0.5} & 0 & 1.09 & 1.37 & 2.90 & 4.66 & 1.60 & 1.91 & 3.32 & 4.86 & 2.95 & 3.20 & 4.21 & 5.40 & 4.55 & 4.66 & 5.30 & 6.15 \\
\hline & 0.2 & 1.10 & 1.36 & 2.82 & 4.58 & 1.59 & 1.87 & 3.21 & 4.73 & 2.86 & 3.15 & 4.11 & 5.29 & 4.41 & 4.58 & 5.19 & 6.04 \\
\hline & 0.5 & 1.10 & 1.30 & 2.57 & 4.11 & 1.50 & 1.75 & 2.87 & 4.33 & 2.65 & 2.82 & 3.71 & 4.89 & 4.10 & 4.18 & 4.77 & 5.56 \\
\hline & 0.8 & 1.12 & 1.25 & 2.19 & 3.53 & 1.38 & 1.59 & 2.49 & 3.74 & 2.28 & 2.42 & 3.17 & 4.21 & 3.52 & 3.59 & 4.11 & 4.92 \\
\hline \multirow{4}{*}{0.8} & 0 & 1.09 & 1.28 & 2.37 & 3.89 & 1.42 & 1.66 & 2.72 & 4.07 & 2.44 & 2.64 & 3.47 & 4.62 & 3.82 & 3.92 & 4.46 & 5.32 \\
\hline & 0.2 & 1.09 & 1.27 & 2.37 & 3.83 & 1.41 & 1.63 & 2.67 & 3.99 & 2.43 & 2.61 & 3.41 & 4.55 & 3.77 & 3.82 & 4.44 & 5.25 \\
\hline & 0.5 & 1.10 & 1.24 & 2.18 & 3.57 & 1.36 & 1.56 & 2.46 & 3.76 & 2.27 & 2.42 & 3.19 & 4.17 & 3.47 & 3.61 & 4.17 & 4.91 \\
\hline & 0.8 & 1.12 & 1.23 & 1.96 & 3.13 & 1.33 & 1.46 & 2.22 & 3.32 & 2.07 & 2.19 & 2.82 & 3.74 & 3.09 & 3.21 & 3.70 & 4.45 \\
\hline
\end{tabular}

Note: Italicized values indicate a design effect of 2 or larger, while bold values indicate a design effect of 6 or larger. ICC $=$ Intraclass Correlation Coefficient. 
Table 2 cont.

Average Model Design Effect values: Uniform Distribution conditions $(\mathrm{m}=10, \mathrm{k}=50)$

\begin{tabular}{|c|c|c|c|c|c|c|c|c|c|c|c|c|c|c|c|c|c|}
\hline \multirow{4}{*}{$\begin{array}{c}\text { L1 } \\
\text { Effect }\end{array}$} & \multirow{4}{*}{$\begin{array}{c}\text { Interaction } \\
\text { Effect }\end{array}$} & \multicolumn{16}{|c|}{ ICC Values } \\
\hline & & \multicolumn{4}{|c|}{0} & \multicolumn{3}{|c|}{0.1} & & \multicolumn{4}{|c|}{0.3} & \multicolumn{4}{|c|}{0.5} \\
\hline & & \multicolumn{16}{|c|}{ L2 Effect } \\
\hline & & 0 & 0.2 & 0.5 & 0.8 & 0 & 0.2 & 0.5 & 0.8 & 0 & 0.2 & 0.5 & 0.8 & 0 & 0.2 & 0.5 & 0.8 \\
\hline \multirow{4}{*}{0} & 0 & 1.06 & 1.52 & 3.57 & 5.52 & 1.86 & 2.29 & 4.00 & 5.71 & 3.61 & 3.92 & 5.00 & 6.25 & 5.41 & 5.56 & 6.16 & 6.96 \\
\hline & 0.2 & 1.06 & 1.49 & 3.45 & 5.37 & 1.81 & 2.23 & 3.87 & 5.60 & 3.51 & 3.79 & 4.85 & 6.14 & 5.22 & 5.41 & 6.02 & 6.83 \\
\hline & 0.5 & 1.07 & 1.37 & 2.94 & 4.72 & 1.64 & 1.97 & 3.35 & 4.96 & 3.02 & 3.23 & 4.29 & 5.49 & 4.61 & 4.76 & 5.42 & 6.25 \\
\hline & 0.8 & 1.08 & 1.27 & 2.43 & 3.95 & 1.45 & 1.68 & 2.76 & 4.18 & 2.52 & 2.69 & 3.55 & 4.69 & 3.91 & 4.02 & 4.59 & 5.44 \\
\hline \multirow{4}{*}{0.2} & 0 & 1.06 & 1.49 & 3.42 & 5.37 & 1.81 & 2.20 & 3.89 & 5.60 & 3.51 & 3.78 & 4.84 & 6.12 & 5.27 & 5.38 & 6.03 & 6.84 \\
\hline & 0.2 & 1.07 & 1.46 & 3.33 & 5.23 & 1.77 & 2.16 & 3.75 & 5.44 & 3.40 & 3.66 & 4.72 & 5.98 & 5.15 & 5.27 & 5.90 & 6.71 \\
\hline & 0.5 & 1.07 & 1.36 & 2.89 & 4.66 & 1.60 & 1.92 & 3.23 & 4.90 & 2.97 & 3.17 & 4.18 & 5.40 & 4.55 & 4.69 & 5.30 & 6.14 \\
\hline & 0.8 & 1.09 & 1.27 & 2.40 & 3.88 & 1.44 & 1.66 & 2.72 & 4.11 & 2.48 & 2.68 & 3.52 & 4.63 & 3.80 & 3.95 & 4.53 & 5.32 \\
\hline \multirow{4}{*}{0.5} & 0 & 1.07 & 1.39 & 2.98 & 4.76 & 1.62 & 1.95 & 3.36 & 4.97 & 3.04 & 3.28 & 4.24 & 5.51 & 4.64 & 4.80 & 5.36 & 6.24 \\
\hline & 0.2 & 1.06 & 1.36 & 2.88 & 4.63 & 1.61 & 1.92 & 3.28 & 4.89 & 2.99 & 3.19 & 4.20 & 5.39 & 4.55 & 4.69 & 5.30 & 6.12 \\
\hline & 0.5 & 1.07 & 1.32 & 2.58 & 4.22 & 1.50 & 1.76 & 2.94 & 4.41 & 2.65 & 2.85 & 3.79 & 4.98 & 4.14 & 4.23 & 4.87 & 5.70 \\
\hline & 0.8 & 1.09 & 1.22 & 2.23 & 3.64 & 1.40 & 1.58 & 2.52 & 3.80 & 2.30 & 2.48 & 3.26 & 4.32 & 3.55 & 3.65 & 4.20 & 4.97 \\
\hline \multirow{4}{*}{0.8} & 0 & 1.06 & 1.27 & 2.44 & 4.00 & 1.44 & 1.68 & 2.76 & 4.17 & 2.51 & 2.67 & 3.54 & 4.71 & 3.89 & 3.99 & 4.54 & 5.38 \\
\hline & 0.2 & 1.08 & 1.25 & 2.40 & 3.90 & 1.43 & 1.67 & 2.69 & 4.09 & 2.47 & 2.66 & 3.48 & 4.60 & 3.82 & 3.92 & 4.53 & 5.34 \\
\hline & 0.5 & 1.07 & 1.23 & 2.26 & 3.62 & 1.38 & 1.56 & 2.52 & 3.82 & 2.32 & 2.48 & 3.23 & 4.27 & 3.52 & 3.64 & 4.21 & 4.98 \\
\hline & 0.8 & 1.07 & 1.19 & 2.02 & 3.23 & 1.30 & 1.46 & 2.26 & 3.37 & 2.06 & 2.20 & 2.89 & 3.84 & 3.17 & 3.29 & 3.76 & 4.52 \\
\hline
\end{tabular}

Note: Italicized values indicate a design effect of 2 or larger, while bold values indicate a design effect of 6 or larger. ICC $=$ Intraclass Correlation Coefficient. 
Table 2 cont.

Average Model Design Effect values: Uniform Distribution conditions ( $\mathrm{m}=10, \mathrm{k}=75)$

\begin{tabular}{|c|c|c|c|c|c|c|c|c|c|c|c|c|c|c|c|c|c|}
\hline \multirow{3}{*}{$\begin{array}{c}\text { L1 } \\
\text { Effect }\end{array}$} & \multirow{3}{*}{$\begin{array}{c}\text { Interaction } \\
\text { Effect }\end{array}$} & \multicolumn{16}{|c|}{ ICC Values } \\
\hline & & \multicolumn{4}{|c|}{0} & \multicolumn{4}{|c|}{0.1} & \multicolumn{4}{|c|}{0.3} & \multicolumn{4}{|c|}{0.5} \\
\hline & & 0 & 0.2 & 0.5 & 0.8 & 0 & 0.2 & 0.5 & 0.8 & 0 & 0.2 & 0.5 & 0.8 & 0 & 0.2 & 0.5 & 0.8 \\
\hline \multirow{4}{*}{0} & 0 & 1.06 & 1.54 & 3.55 & 5.58 & 1.88 & 2.31 & 4.02 & 5.77 & 3.66 & 3.94 & 5.04 & 6.30 & 5.41 & 5.57 & 6.19 & 6.99 \\
\hline & 0.2 & 1.06 & 1.50 & 3.47 & 5.40 & 1.83 & 2.26 & 3.90 & 5.62 & 3.53 & 3.78 & 4.89 & 6.15 & 5.30 & 5.47 & 6.09 & 6.87 \\
\hline & 0.5 & 1.06 & 1.40 & 2.99 & 4.78 & 1.66 & 1.97 & 3.37 & 5.01 & 3.08 & 3.31 & 4.29 & 5.53 & 4.70 & 4.81 & 5.43 & 6.27 \\
\hline & 0.8 & 1.07 & 1.28 & 2.46 & 3.99 & 1.45 & 1.70 & 2.79 & 4.19 & 2.53 & 2.70 & 3.57 & 4.73 & 3.93 & 4.03 & 4.61 & 5.43 \\
\hline \multirow{4}{*}{0.2} & 0 & 1.06 & 1.50 & 3.46 & 5.40 & 1.84 & 2.24 & 3.89 & 5.61 & 3.54 & 3.82 & 4.88 & 6.14 & 5.25 & 5.44 & 6.07 & 6.86 \\
\hline & 0.2 & 1.05 & 1.46 & 3.35 & 5.27 & 1.79 & 2.18 & 3.78 & 5.47 & 3.42 & 3.66 & 4.76 & 6.04 & 5.17 & 5.30 & 5.92 & 6.74 \\
\hline & 0.5 & 1.06 & 1.36 & 2.90 & 4.70 & 1.61 & 1.93 & 3.30 & 4.90 & 3.00 & 3.24 & 4.19 & 5.43 & 4.57 & 4.70 & 5.35 & 6.16 \\
\hline & 0.8 & 1.07 & 1.25 & 2.42 & 3.92 & 1.43 & 1.67 & 2.73 & 4.10 & 2.49 & 2.69 & 3.51 & 4.65 & 3.87 & 3.98 & 4.52 & 5.33 \\
\hline \multirow{4}{*}{0.5} & 0 & 1.06 & 1.38 & 3.00 & 4.77 & 1.63 & 1.97 & 3.38 & 5.00 & 3.06 & 3.29 & 4.30 & 5.53 & 4.68 & 4.84 & 5.44 & 6.25 \\
\hline & 0.2 & 1.06 & 1.37 & 2.91 & 4.67 & 1.60 & 1.94 & 3.32 & 4.91 & 2.99 & 3.22 & 4.22 & 5.45 & 4.58 & 4.72 & 5.35 & 6.18 \\
\hline & 0.5 & 1.06 & 1.30 & 2.59 & 4.21 & 1.50 & 1.77 & 2.97 & 4.43 & 2.68 & 2.87 & 3.79 & 4.97 & 4.14 & 4.27 & 4.86 & 5.71 \\
\hline & 0.8 & 1.07 & 1.23 & 2.28 & 3.65 & 1.37 & 1.59 & 2.52 & 3.83 & 2.32 & 2.48 & 3.23 & 4.34 & 3.58 & 3.68 & 4.25 & 5.02 \\
\hline \multirow{4}{*}{0.8} & 0 & 1.05 & 1.27 & 2.44 & 3.99 & 1.44 & 1.69 & 2.79 & 4.18 & 2.52 & 2.72 & 3.55 & 4.72 & 3.89 & 4.02 & 4.59 & 5.43 \\
\hline & 0.2 & 1.07 & 1.26 & 2.43 & 3.92 & 1.44 & 1.67 & 2.72 & 4.16 & 2.49 & 2.66 & 3.52 & 4.65 & 3.85 & 3.96 & 4.57 & 5.36 \\
\hline & 0.5 & 1.07 & 1.23 & 2.27 & 3.64 & 1.37 & 1.59 & 2.55 & 3.84 & 2.29 & 2.49 & 3.24 & 4.30 & 3.56 & 3.65 & 4.22 & 5.04 \\
\hline & 0.8 & 1.07 & 1.19 & 2.02 & 3.24 & 1.31 & 1.47 & 2.26 & 3.42 & 2.07 & 2.23 & 2.89 & 3.87 & 3.17 & 3.28 & 3.77 & 4.53 \\
\hline
\end{tabular}

Note: Italicized values indicate a design effect of 2 or larger, while bold values indicate a design effect of 6 or larger. ICC $=$ Intraclass Correlation Coefficient. 
Table 2 cont.

Average Model Design Effect values: Uniform Distribution conditions ( $\mathrm{m}=10, \mathrm{k}=100)$

\begin{tabular}{|c|c|c|c|c|c|c|c|c|c|c|c|c|c|c|c|c|c|}
\hline \multirow{3}{*}{$\begin{array}{c}\text { L1 } \\
\text { Effect }\end{array}$} & \multirow{3}{*}{$\begin{array}{c}\text { Interaction } \\
\text { Effect }\end{array}$} & \multicolumn{16}{|c|}{ ICC Values } \\
\hline & & \multicolumn{4}{|c|}{0} & \multicolumn{4}{|c|}{0.1} & \multicolumn{4}{|c|}{0.3} & \multicolumn{4}{|c|}{0.5} \\
\hline & & 0 & 0.2 & 0.5 & 0.8 & 0 & 0.2 & 0.5 & 0.8 & 0 & 0.2 & 0.5 & 0.8 & 0 & 0.2 & 0.5 & 0.8 \\
\hline \multirow{4}{*}{0} & 0 & 1.05 & 1.53 & 3.59 & 5.56 & 1.89 & 2.33 & 4.04 & 5.80 & 3.67 & 3.96 & 5.05 & 6.32 & 5.47 & 5.59 & 6.20 & 7.01 \\
\hline & 0.2 & 1.05 & 1.51 & 3.46 & 5.40 & 1.82 & 2.27 & 3.91 & 5.65 & 3.55 & 3.81 & 4.91 & 6.20 & 5.31 & 5.45 & 6.08 & 6.88 \\
\hline & 0.5 & 1.05 & 1.38 & 3.00 & 4.80 & 1.63 & 1.99 & 3.39 & 5.02 & 3.07 & 3.29 & 4.31 & 5.58 & 4.69 & 4.82 & 5.45 & 6.27 \\
\hline & 0.8 & 1.06 & 1.27 & 2.49 & 4.00 & 1.44 & 1.70 & 2.80 & 4.22 & 2.53 & 2.73 & 3.58 & 4.72 & 3.91 & 4.05 & 4.63 & 5.47 \\
\hline \multirow{4}{*}{0.2} & 0 & 1.05 & 1.51 & 3.45 & 5.40 & 1.83 & 2.26 & 3.94 & 5.64 & 3.55 & 3.82 & 4.92 & 6.18 & 5.31 & 5.44 & 6.07 & 6.87 \\
\hline & 0.2 & 1.05 & 1.46 & 3.34 & 5.29 & 1.79 & 2.19 & 3.80 & 5.51 & 3.43 & 3.69 & 4.79 & 6.07 & 5.16 & 5.32 & 5.95 & 6.75 \\
\hline & 0.5 & 1.05 & 1.36 & 2.93 & 4.69 & 1.61 & 1.93 & 3.32 & 4.92 & 3.01 & 3.23 & 4.24 & 5.45 & 4.57 & 4.72 & 5.36 & 6.17 \\
\hline & 0.8 & 1.06 & 1.28 & 2.42 & 3.94 & 1.43 & 1.67 & 2.75 & 4.15 & 2.48 & 2.68 & 3.52 & 4.64 & 3.85 & 3.99 & 4.55 & 5.37 \\
\hline \multirow{4}{*}{0.5} & 0 & 1.05 & 1.39 & 3.00 & 4.79 & 1.64 & 1.98 & 3.38 & 5.01 & 3.05 & 3.30 & 4.32 & 5.54 & 4.70 & 4.83 & 5.44 & 6.29 \\
\hline & 0.2 & 1.05 & 1.37 & 2.93 & 4.68 & 1.61 & 1.93 & 3.32 & 4.91 & 3.00 & 3.23 & 4.22 & 5.47 & 4.60 & 4.72 & 5.34 & 6.19 \\
\hline & 0.5 & 1.06 & 1.31 & 2.64 & 4.26 & 1.50 & 1.77 & 2.99 & 4.46 & 2.68 & 2.90 & 3.80 & 5.00 & 4.15 & 4.30 & 4.88 & 5.69 \\
\hline & 0.8 & 1.06 & 1.22 & 2.26 & 3.66 & 1.38 & 1.60 & 2.55 & 3.85 & 2.31 & 2.48 & 3.25 & 4.34 & 3.57 & 3.69 & 4.23 & 5.06 \\
\hline \multirow{4}{*}{0.8} & 0 & 1.05 & 1.28 & 2.47 & 4.01 & 1.45 & 1.71 & 2.80 & 4.22 & 2.53 & 2.72 & 3.57 & 4.75 & 3.93 & 4.03 & 4.62 & 5.47 \\
\hline & 0.2 & 1.06 & 1.26 & 2.44 & 3.95 & 1.43 & 1.66 & 2.75 & 4.15 & 2.50 & 2.68 & 3.52 & 4.66 & 3.85 & 3.98 & 4.54 & 5.40 \\
\hline & 0.5 & 1.06 & 1.23 & 2.28 & 3.66 & 1.38 & 1.59 & 2.55 & 3.85 & 2.32 & 2.48 & 3.26 & 4.34 & 3.56 & 3.69 & 4.25 & 5.06 \\
\hline & 0.8 & 1.07 & 1.19 & 2.03 & 3.23 & 1.30 & 1.47 & 2.27 & 3.43 & 2.09 & 2.23 & 2.88 & 3.87 & 3.17 & 3.28 & 3.79 & 4.57 \\
\hline
\end{tabular}

Note: Italicized values indicate a design effect of 2 or larger, while bold values indicate a design effect of 6 or larger. ICC $=$ Intraclass Correlation Coefficient. 
Table 2 cont.

Average Model Design Effect values: Uniform Distribution conditions $(\mathrm{m}=20, \mathrm{k}=25)$

\begin{tabular}{|c|c|c|c|c|c|c|c|c|c|c|c|c|c|c|c|c|c|}
\hline \multirow{4}{*}{$\begin{array}{c}\text { L1 } \\
\text { Effect }\end{array}$} & \multirow{4}{*}{$\begin{array}{c}\text { Interaction } \\
\text { Effect }\end{array}$} & \multicolumn{16}{|c|}{ ICC Values } \\
\hline & & \multicolumn{4}{|c|}{0} & \multicolumn{4}{|c|}{0.1} & \multicolumn{4}{|c|}{0.3} & \multicolumn{4}{|c|}{0.5} \\
\hline & & & & & & & & & $\mathrm{L} 2$ & & & & & & & & \\
\hline & & 0 & 0.2 & 0.5 & 0.8 & 0 & 0.2 & 0.5 & 0.8 & 0 & 0.2 & 0.5 & 0.8 & 0 & 0.2 & 0.5 & 0.8 \\
\hline \multirow{4}{*}{0} & 0 & 1.09 & 2.07 & 6.20 & 10.32 & 2.77 & 3.62 & 7.17 & 10.81 & 6.47 & 7.00 & 9.35 & 11.92 & 10.10 & 10.51 & 11.59 & 13.36 \\
\hline & 0.2 & 1.09 & 2.00 & 6.05 & 10.02 & 2.70 & 3.55 & 6.93 & 10.46 & 6.13 & 6.72 & 8.99 & 11.65 & 9.83 & 10.14 & 11.43 & 13.06 \\
\hline & 0.5 & 1.10 & 1.74 & 5.02 & 8.74 & 2.34 & 2.98 & 5.86 & 9.23 & 5.24 & 5.71 & 7.74 & 10.32 & 8.46 & 8.89 & 10.14 & 11.85 \\
\hline & 0.8 & 1.11 & 1.53 & 3.91 & 7.05 & 1.95 & 2.41 & 4.61 & 7.55 & 4.16 & 4.52 & 6.29 & 8.63 & 6.98 & 7.25 & 8.41 & 10.08 \\
\hline \multirow{4}{*}{0.2} & 0 & 1.09 & 2.04 & 6.00 & 10.02 & 2.70 & 3.59 & 6.99 & 10.40 & 6.18 & 6.65 & 9.00 & 11.60 & 9.71 & 10.11 & 11.43 & 13.10 \\
\hline & 0.2 & 1.10 & 1.96 & 5.80 & 9.77 & 2.57 & 3.45 & 6.64 & 10.13 & 5.89 & 6.52 & 8.68 & 11.35 & 9.45 & 9.79 & 11.12 & 12.87 \\
\hline & 0.5 & 1.10 & 1.74 & 4.90 & 8.48 & 2.22 & 2.94 & 5.68 & 9.02 & 5.08 & 5.58 & 7.52 & 10.12 & 8.37 & 8.59 & 9.94 & 11.66 \\
\hline & 0.8 & 1.11 & 1.56 & 3.88 & 6.96 & 1.88 & 2.38 & 4.54 & 7.39 & 4.04 & 4.48 & 6.17 & 8.42 & 6.87 & 7.07 & 8.26 & 9.94 \\
\hline \multirow{4}{*}{0.5} & 0 & 1.09 & 1.79 & 5.09 & 8.69 & 2.30 & 3.00 & 5.89 & 9.20 & 5.15 & 5.68 & 7.73 & 10.31 & 8.47 & 8.80 & 10.04 & 11.84 \\
\hline & 0.2 & 1.10 & 1.73 & 4.93 & 8.50 & 2.21 & 2.89 & 5.73 & 9.05 & 5.06 & 5.44 & 7.50 & 10.03 & 8.39 & 8.57 & 9.94 & 11.67 \\
\hline & 0.5 & 1.11 & 1.62 & 4.29 & 7.62 & 2.05 & 2.59 & 4.99 & 7.97 & 4.48 & 4.83 & 6.73 & 9.15 & 7.46 & 7.77 & 8.93 & 10.64 \\
\hline & 0.8 & 1.11 & 1.46 & 3.54 & 6.42 & 1.77 & 2.23 & 4.16 & 6.79 & 3.75 & 4.03 & 5.58 & 7.85 & 6.28 & 6.50 & 7.64 & 9.24 \\
\hline \multirow{4}{*}{0.8} & 0 & 1.09 & 1.55 & 4.01 & 7.11 & 1.92 & 2.40 & 4.64 & 7.49 & 4.10 & 4.55 & 6.26 & 8.58 & 6.89 & 7.17 & 8.35 & 10.08 \\
\hline & 0.2 & 1.10 & 1.53 & 3.90 & 7.02 & 1.84 & 2.37 & 4.59 & 7.42 & 4.01 & 4.42 & 6.02 & 8.43 & 6.79 & 7.06 & 8.27 & 9.88 \\
\hline & 0.5 & 1.10 & 1.46 & 3.58 & 6.46 & 1.75 & 2.20 & 4.14 & 6.79 & 3.73 & 3.99 & 5.64 & 7.75 & 6.19 & 6.53 & 7.58 & 9.28 \\
\hline & 0.8 & 1.12 & 1.38 & 3.13 & 5.69 & 1.61 & 1.96 & 3.58 & 5.91 & 3.24 & 3.55 & 4.86 & 6.78 & 5.42 & 5.79 & 6.70 & 8.22 \\
\hline
\end{tabular}

Note: Italicized values indicate a design effect of 2 or larger, while bold values indicate a design effect of 6 or larger. ICC $=$ Intraclass Correlation Coefficient. 
Table 2 cont.

Average Model Design Effect values: Uniform Distribution conditions $(\mathrm{m}=20, \mathrm{k}=50)$

\begin{tabular}{|c|c|c|c|c|c|c|c|c|c|c|c|c|c|c|c|c|c|}
\hline \multirow{4}{*}{$\begin{array}{c}\text { L1 } \\
\text { Effect }\end{array}$} & \multirow{4}{*}{$\begin{array}{c}\text { Interaction } \\
\text { Effect }\end{array}$} & \multicolumn{16}{|c|}{ ICC Values } \\
\hline & & \multicolumn{4}{|c|}{0} & \multicolumn{4}{|c|}{0.1} & \multicolumn{4}{|c|}{0.3} & \multicolumn{4}{|c|}{0.5} \\
\hline & & & & & & & & & $\overline{\mathrm{L} 2 \mathrm{I}}$ & & & & & & & & \\
\hline & & 0 & 0.2 & 0.5 & 0.8 & 0 & 0.2 & 0.5 & 0.8 & 0 & 0.2 & 0.5 & 0.8 & 0 & 0.2 & 0.5 & 0.8 \\
\hline \multirow{4}{*}{0} & 0 & 1.07 & 2.12 & 6.38 & 10.52 & 2.84 & 3.77 & 7.34 & 10.99 & 6.58 & 7.14 & 9.46 & 12.12 & 10.31 & 10.58 & 11.93 & 13.54 \\
\hline & 0.2 & 1.07 & 2.07 & 6.15 & 10.24 & 2.74 & 3.61 & 7.10 & 10.65 & 6.33 & 6.89 & 9.15 & 11.83 & 9.96 & 10.30 & 11.63 & 13.33 \\
\hline & 0.5 & 1.06 & 1.80 & 5.11 & 8.97 & 2.37 & 3.04 & 5.94 & 9.41 & 5.33 & 5.81 & 7.90 & 10.52 & 8.72 & 9.04 & 10.36 & 12.02 \\
\hline & 0.8 & 1.09 & 1.55 & 4.06 & 7.28 & 1.94 & 2.48 & 4.75 & 7.69 & 4.19 & 4.58 & 6.39 & 8.78 & 7.10 & 7.37 & 8.58 & 10.25 \\
\hline \multirow{4}{*}{0.2} & 0 & 1.07 & 2.06 & 6.14 & 10.25 & 2.73 & 3.64 & 7.03 & 10.76 & 6.28 & 6.83 & 9.19 & 11.88 & 10.07 & 10.31 & 11.65 & 13.29 \\
\hline & 0.2 & 1.07 & 2.01 & 5.92 & 9.93 & 2.64 & 3.50 & 6.86 & 10.45 & 6.12 & 6.62 & 8.93 & 11.59 & 9.76 & 10.07 & 11.34 & 13.04 \\
\hline & 0.5 & 1.07 & 1.76 & 5.01 & 8.72 & 2.27 & 2.98 & 5.78 & 9.13 & 5.22 & 5.61 & 7.68 & 10.32 & 8.48 & 8.78 & 10.07 & 11.82 \\
\hline & 0.8 & 1.09 & 1.53 & 4.01 & 7.15 & 1.90 & 2.42 & 4.62 & 7.60 & 4.11 & 4.46 & 6.29 & 8.67 & 6.95 & 7.19 & 8.47 & 10.17 \\
\hline \multirow{4}{*}{0.5} & 0 & 1.06 & 1.81 & 5.13 & 8.93 & 2.34 & 3.08 & 6.02 & 9.41 & 5.34 & 5.82 & 7.95 & 10.52 & 8.69 & 9.02 & 10.32 & 12.04 \\
\hline & 0.2 & 1.07 & 1.79 & 4.99 & 8.73 & 2.28 & 3.01 & 5.84 & 9.15 & 5.15 & 5.63 & 7.78 & 10.28 & 8.48 & 8.80 & 10.07 & 11.87 \\
\hline & 0.5 & 1.07 & 1.64 & 4.38 & 7.82 & 2.06 & 2.62 & 5.13 & 8.25 & 4.51 & 4.96 & 6.83 & 9.32 & 7.59 & 7.88 & 9.06 & 10.90 \\
\hline & 0.8 & 1.09 & 1.47 & 3.65 & 6.57 & 1.79 & 2.23 & 4.21 & 6.94 & 3.80 & 4.11 & 5.74 & 7.94 & 6.43 & 6.60 & 7.75 & 9.49 \\
\hline \multirow{4}{*}{0.8} & 0 & 1.07 & 1.55 & 4.07 & 7.26 & 1.93 & 2.45 & 4.73 & 7.73 & 4.21 & 4.59 & 6.34 & 8.76 & 7.04 & 7.32 & 8.59 & 10.35 \\
\hline & 0.2 & 1.07 & 1.55 & 3.98 & 7.22 & 1.92 & 2.42 & 4.66 & 7.59 & 4.13 & 4.51 & 6.23 & 8.64 & 6.91 & 7.26 & 8.46 & 10.14 \\
\hline & 0.5 & 1.08 & 1.47 & 3.64 & 6.55 & 1.79 & 2.22 & 4.24 & 6.95 & 3.78 & 4.10 & 5.69 & 8.03 & 6.38 & 6.59 & 7.76 & 9.42 \\
\hline & 0.8 & 1.09 & 1.36 & 3.16 & 5.65 & 1.64 & 1.99 & 3.67 & 6.07 & 3.26 & 3.56 & 4.97 & 7.04 & 5.52 & 5.70 & 6.78 & 8.46 \\
\hline
\end{tabular}

Note: Italicized values indicate a design effect of 2 or larger, while bold values indicate a design effect of 6 or larger. ICC $=$

Intraclass Correlation Coefficient. 
Table 2 cont.

Average Model Design Effect values: Uniform Distribution conditions $(\mathrm{m}=20, \mathrm{k}=75)$

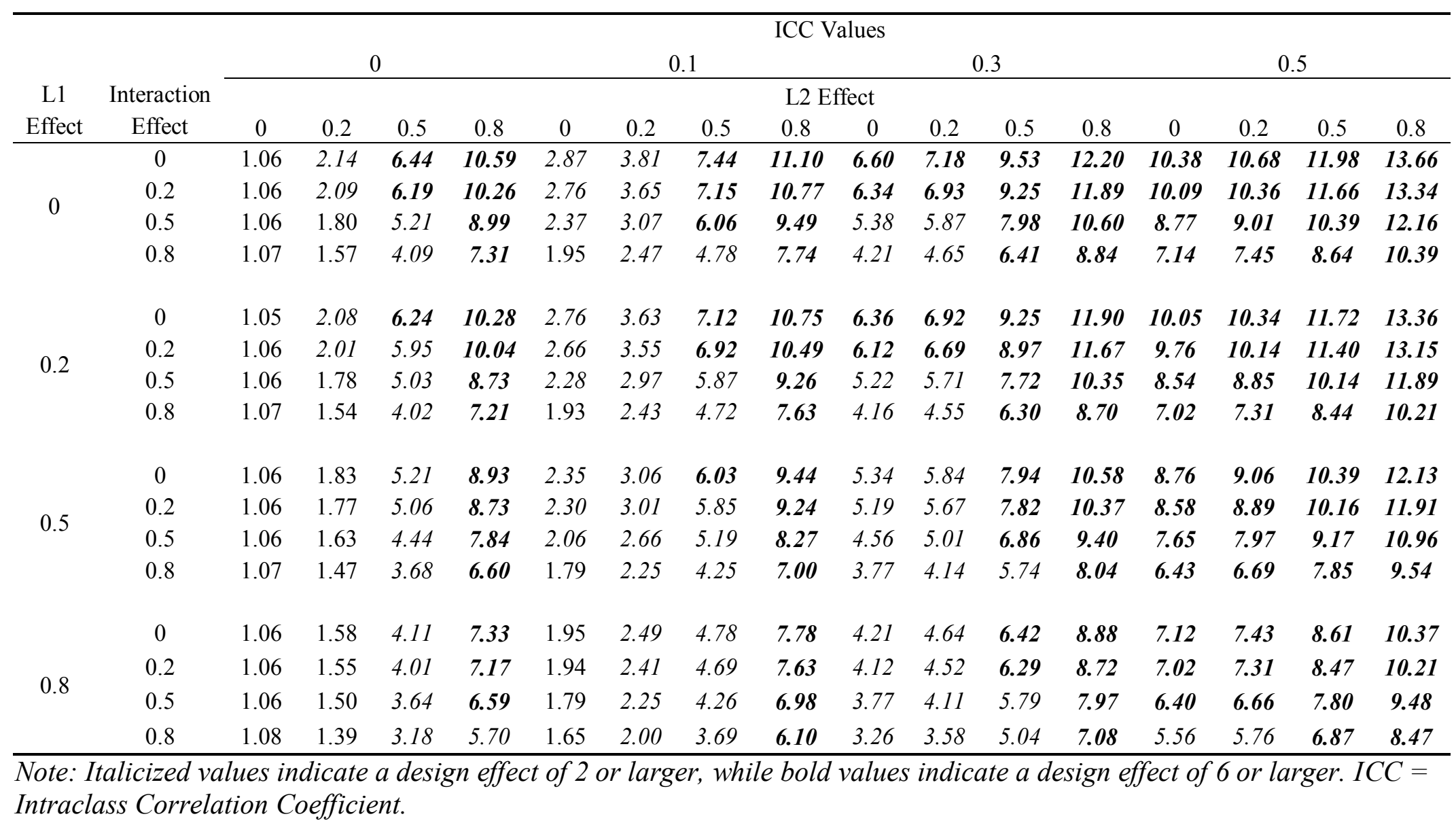


Table 2 cont.

Average Model Design Effect values: Uniform Distribution conditions ( $\mathrm{m}=20, \mathrm{k}=100)$

\begin{tabular}{|c|c|c|c|c|c|c|c|c|c|c|c|c|c|c|c|c|c|}
\hline \multirow{3}{*}{$\begin{array}{c}\text { L1 } \\
\text { Effect }\end{array}$} & \multirow{3}{*}{$\begin{array}{c}\text { Interaction } \\
\text { Effect }\end{array}$} & \multicolumn{16}{|c|}{ ICC Values } \\
\hline & & \multicolumn{5}{|c|}{0} & \multicolumn{2}{|c|}{0.1} & & \multicolumn{4}{|c|}{0.3} & \multicolumn{4}{|c|}{0.5} \\
\hline & & & & & \multicolumn{13}{|c|}{ L2 Effect } \\
\hline & & 0 & 0.2 & 0.5 & 0.8 & 0 & 0.2 & 0.5 & 0.8 & 0 & 0.2 & 0.5 & 0.8 & 0 & 0.2 & 0.5 & 0.8 \\
\hline \multirow{4}{*}{0} & 0 & 1.05 & 2.16 & 6.46 & 10.62 & 2.86 & 3.83 & 7.44 & 11.07 & 6.65 & 7.22 & 9.54 & 12.27 & 10.39 & 10.71 & 12.01 & 13.66 \\
\hline & 0.2 & 1.05 & 2.08 & 6.22 & 10.37 & 2.76 & 3.67 & 7.13 & 10.79 & 6.40 & 6.93 & 9.27 & 11.94 & 10.13 & 10.41 & 11.68 & 13.36 \\
\hline & 0.5 & 1.05 & 1.81 & 5.23 & 9.02 & 2.36 & 3.08 & 6.03 & 9.48 & 5.36 & 5.88 & 8.00 & 10.62 & 8.83 & 9.12 & 10.43 & 12.20 \\
\hline & 0.8 & 1.07 & 1.58 & 4.11 & 7.35 & 1.96 & 2.50 & 4.79 & 7.81 & 4.27 & 4.63 & 6.44 & 8.90 & 7.20 & 7.43 & 8.63 & 10.42 \\
\hline \multirow{4}{*}{0.2} & 0 & 1.05 & 2.07 & 6.21 & 10.34 & 2.76 & 3.66 & 7.13 & 10.83 & 6.40 & 6.94 & 9.29 & 11.91 & 10.15 & 10.39 & 11.72 & 13.40 \\
\hline & 0.2 & 1.05 & 2.03 & 6.04 & 10.07 & 2.68 & 3.52 & 6.93 & 10.51 & 6.14 & 6.70 & 9.02 & 11.69 & 9.78 & 10.08 & 11.44 & 13.16 \\
\hline & 0.5 & 1.05 & 1.79 & 5.04 & 8.76 & 2.32 & 2.98 & 5.89 & 9.25 & 5.21 & 5.72 & 7.81 & 10.42 & 8.63 & 8.84 & 10.20 & 12.01 \\
\hline & 0.8 & 1.06 & 1.56 & 4.03 & 7.19 & 1.93 & 2.45 & 4.68 & 7.68 & 4.18 & 4.55 & 6.34 & 8.76 & 7.07 & 7.24 & 8.54 & 10.25 \\
\hline \multirow{4}{*}{0.5} & 0 & 1.05 & 1.82 & 5.20 & 9.04 & 2.36 & 3.09 & 6.05 & 9.50 & 5.36 & 5.85 & 8.01 & 10.59 & 8.80 & 9.03 & 10.41 & 12.16 \\
\hline & 0.2 & 1.06 & 1.80 & 5.07 & 8.81 & 2.30 & 3.02 & 5.89 & 9.24 & 5.23 & 5.70 & 7.80 & 10.39 & 8.58 & 8.93 & 10.19 & 11.92 \\
\hline & 0.5 & 1.06 & 1.66 & 4.45 & 7.87 & 2.07 & 2.66 & 5.15 & 8.31 & 4.56 & 4.99 & 6.93 & 9.38 & 7.65 & 7.93 & 9.17 & 10.98 \\
\hline & 0.8 & 1.07 & 1.46 & 3.71 & 6.61 & 1.81 & 2.25 & 4.27 & 7.00 & 3.79 & 4.15 & 5.75 & 8.03 & 6.43 & 6.67 & 7.90 & 9.53 \\
\hline \multirow{4}{*}{0.8} & 0 & 1.05 & 1.57 & 4.11 & 7.34 & 1.95 & 2.48 & 4.78 & 7.78 & 4.21 & 4.66 & 6.44 & 8.86 & 7.15 & 7.43 & 8.68 & 10.38 \\
\hline & 0.2 & 1.05 & 1.55 & 4.05 & 7.24 & 1.93 & 2.44 & 4.69 & 7.66 & 4.17 & 4.56 & 6.33 & 8.73 & 7.02 & 7.32 & 8.52 & 10.25 \\
\hline & 0.5 & 1.06 & 1.48 & 3.70 & 6.65 & 1.79 & 2.25 & 4.27 & 7.03 & 3.78 & 4.16 & 5.77 & 8.06 & 6.43 & 6.67 & 7.82 & 9.58 \\
\hline & 0.8 & 1.07 & 1.38 & 3.20 & 5.74 & 1.64 & 2.02 & 3.69 & 6.11 & 3.27 & 3.59 & 5.01 & 7.09 & 5.61 & 5.83 & 6.86 & 8.49 \\
\hline
\end{tabular}

Note: Italicized values indicate a design effect of 2 or larger, while bold values indicate a design effect of 6 or larger. ICC $=$ Intraclass Correlation Coefficient. 
Table 2 cont.

Average Model Design Effect values: Poisson Distribution conditions ( $\mathrm{m}=5, \mathrm{k}=25)$

\begin{tabular}{|c|c|c|c|c|c|c|c|c|c|c|c|c|c|c|c|c|c|}
\hline \multirow{4}{*}{$\begin{array}{c}\text { L1 } \\
\text { Effect }\end{array}$} & \multirow{4}{*}{$\begin{array}{c}\text { Interaction } \\
\text { Effect }\end{array}$} & \multicolumn{16}{|c|}{ ICC Values } \\
\hline & & \multicolumn{5}{|c|}{0} & \multicolumn{2}{|c|}{0.1} & & \multicolumn{4}{|c|}{0.3} & \multicolumn{4}{|c|}{0.5} \\
\hline & & \multicolumn{16}{|c|}{ L2 Effect } \\
\hline & & 0 & 0.2 & 0.5 & 0.8 & 0 & 0.2 & 0.5 & 0.8 & 0 & 0.2 & 0.5 & 0.8 & 0 & 0.2 & 0.5 & 0.8 \\
\hline \multirow{4}{*}{0} & 0 & 1.08 & 1.24 & 2.10 & 2.94 & 1.37 & 1.54 & 2.27 & 3.04 & 2.11 & 2.23 & 2.72 & 3.29 & 2.90 & 2.94 & 3.23 & 3.59 \\
\hline & 0.2 & 1.08 & 1.22 & 2.02 & 2.89 & 1.33 & 1.53 & 2.24 & 2.94 & 2.09 & 2.20 & 2.69 & 3.21 & 2.84 & 2.89 & 3.18 & 3.56 \\
\hline & 0.5 & 1.09 & 1.19 & 1.80 & 2.60 & 1.28 & 1.42 & 1.98 & 2.71 & 1.88 & 1.98 & 2.41 & 2.94 & 2.59 & 2.63 & 2.91 & 3.26 \\
\hline & 0.8 & 1.10 & 1.16 & 1.60 & 2.25 & 1.23 & 1.29 & 1.74 & 2.34 & 1.64 & 1.71 & 2.09 & 2.59 & 2.24 & 2.31 & 2.56 & 2.93 \\
\hline \multirow{4}{*}{0.2} & 0 & 1.08 & 1.23 & 2.02 & 2.89 & 1.34 & 1.53 & 2.23 & 2.98 & 2.08 & 2.18 & 2.67 & 3.21 & 2.82 & 2.88 & 3.16 & 3.52 \\
\hline & 0.2 & 1.08 & 1.21 & 2.00 & 2.82 & 1.33 & 1.49 & 2.17 & 2.94 & 2.02 & 2.11 & 2.59 & 3.16 & 2.79 & 2.83 & 3.11 & 3.48 \\
\hline & 0.5 & 1.08 & 1.19 & 1.79 & 2.55 & 1.28 & 1.40 & 1.97 & 2.67 & 1.83 & 1.95 & 2.35 & 2.88 & 2.52 & 2.61 & 2.86 & 3.22 \\
\hline & 0.8 & 1.09 & 1.17 & 1.59 & 2.24 & 1.21 & 1.32 & 1.74 & 2.33 & 1.64 & 1.69 & 2.06 & 2.56 & 2.20 & 2.28 & 2.53 & 2.90 \\
\hline \multirow{4}{*}{0.5} & 0 & 1.08 & 1.19 & 1.83 & 2.63 & 1.28 & 1.40 & 2.03 & 2.71 & 1.84 & 1.93 & 2.39 & 2.95 & 2.56 & 2.62 & 2.90 & 3.26 \\
\hline & 0.2 & 1.08 & 1.18 & 1.80 & 2.57 & 1.26 & 1.37 & 1.96 & 2.65 & 1.82 & 1.92 & 2.33 & 2.90 & 2.53 & 2.57 & 2.87 & 3.22 \\
\hline & 0.5 & 1.09 & 1.18 & 1.68 & 2.36 & 1.24 & 1.34 & 1.83 & 2.46 & 1.74 & 1.80 & 2.18 & 2.69 & 2.36 & 2.40 & 2.67 & 3.00 \\
\hline & 0.8 & 1.10 & 1.17 & 1.54 & 2.12 & 1.20 & 1.26 & 1.64 & 2.19 & 1.56 & 1.61 & 1.97 & 2.39 & 2.09 & 2.13 & 2.38 & 2.73 \\
\hline \multirow{4}{*}{0.8} & 0 & 1.07 & 1.15 & 1.62 & 2.25 & 1.21 & 1.28 & 1.73 & 2.35 & 1.65 & 1.72 & 2.09 & 2.56 & 2.21 & 2.28 & 2.54 & 2.89 \\
\hline & 0.2 & 1.08 & 1.15 & 1.60 & 2.23 & 1.21 & 1.29 & 1.72 & 2.32 & 1.61 & 1.66 & 2.05 & 2.55 & 2.19 & 2.25 & 2.53 & 2.85 \\
\hline & 0.5 & 1.09 & 1.14 & 1.53 & 2.11 & 1.20 & 1.27 & 1.63 & 2.19 & 1.54 & 1.62 & 1.97 & 2.44 & 2.10 & 2.13 & 2.37 & 2.73 \\
\hline & 0.8 & 1.10 & 1.14 & 1.44 & 1.93 & 1.19 & 1.26 & 1.54 & 2.00 & 1.49 & 1.55 & 1.79 & 2.23 & 1.94 & 1.96 & 2.19 & 2.52 \\
\hline
\end{tabular}

Note: Italicized values indicate a design effect of 2 or larger, while bold values indicate a design effect of 6 or larger. ICC $=$ Intraclass Correlation Coefficient. 
Table 2 cont.

Average Model Design Effect values: Poisson Distribution conditions ( $\mathrm{m}=5, \mathrm{k}=50)$

\begin{tabular}{|c|c|c|c|c|c|c|c|c|c|c|c|c|c|c|c|c|c|}
\hline \multirow{4}{*}{$\begin{array}{c}\text { L1 } \\
\text { Effect }\end{array}$} & \multirow{4}{*}{$\begin{array}{c}\text { Interaction } \\
\text { Effect }\end{array}$} & \multicolumn{16}{|c|}{ ICC Values } \\
\hline & & \multicolumn{4}{|c|}{0} & \multirow{2}{*}{\multicolumn{3}{|c|}{0.1}} & & \multicolumn{4}{|c|}{0.3} & \multicolumn{4}{|c|}{0.5} \\
\hline & & & & & & & & & & & & & & & & & \\
\hline & & 0 & 0.2 & 0.5 & 0.8 & 0 & 0.2 & 0.5 & 0.8 & 0 & 0.2 & 0.5 & 0.8 & 0 & 0.2 & 0.5 & 0.8 \\
\hline \multirow{4}{*}{0} & 0 & 1.06 & 1.24 & 2.12 & 3.01 & 1.38 & 1.58 & 2.32 & 3.08 & 2.14 & 2.28 & 2.77 & 3.34 & 2.94 & 3.01 & 3.29 & 3.64 \\
\hline & 0.2 & 1.05 & 1.23 & 2.06 & 2.94 & 1.36 & 1.54 & 2.26 & 3.03 & 2.10 & 2.22 & 2.71 & 3.26 & 2.89 & 2.94 & 3.24 & 3.59 \\
\hline & 0.5 & 1.07 & 1.18 & 1.87 & 2.66 & 1.28 & 1.43 & 2.02 & 2.76 & 1.91 & 2.00 & 2.44 & 3.00 & 2.62 & 2.69 & 2.95 & 3.33 \\
\hline & 0.8 & 1.06 & 1.14 & 1.62 & 2.30 & 1.21 & 1.30 & 1.79 & 2.39 & 1.68 & 1.75 & 2.14 & 2.63 & 2.26 & 2.33 & 2.59 & 2.96 \\
\hline \multirow{4}{*}{0.2} & 0 & 1.06 & 1.22 & 2.07 & 2.93 & 1.35 & 1.54 & 2.27 & 3.02 & 2.10 & 2.23 & 2.72 & 3.28 & 2.89 & 2.94 & 3.22 & 3.58 \\
\hline & 0.2 & 1.06 & 1.22 & 2.03 & 2.88 & 1.33 & 1.52 & 2.22 & 2.98 & 2.07 & 2.18 & 2.66 & 3.22 & 2.83 & 2.88 & 3.17 & 3.52 \\
\hline & 0.5 & 1.07 & 1.17 & 1.83 & 2.62 & 1.27 & 1.40 & 1.99 & 2.72 & 1.87 & 1.98 & 2.40 & 2.94 & 2.57 & 2.63 & 2.92 & 3.26 \\
\hline & 0.8 & 1.07 & 1.14 & 1.63 & 2.25 & 1.21 & 1.30 & 1.74 & 2.36 & 1.66 & 1.73 & 2.09 & 2.61 & 2.24 & 2.30 & 2.54 & 2.92 \\
\hline \multirow{4}{*}{0.5} & 0 & 1.06 & 1.19 & 1.87 & 2.66 & 1.28 & 1.43 & 2.03 & 2.75 & 1.89 & 1.98 & 2.44 & 2.99 & 2.61 & 2.66 & 2.95 & 3.31 \\
\hline & 0.2 & 1.06 & 1.16 & 1.84 & 2.60 & 1.27 & 1.41 & 2.00 & 2.72 & 1.86 & 1.96 & 2.42 & 2.96 & 2.55 & 2.62 & 2.90 & 3.27 \\
\hline & 0.5 & 1.07 & 1.16 & 1.71 & 2.42 & 1.23 & 1.34 & 1.87 & 2.52 & 1.73 & 1.81 & 2.24 & 2.74 & 2.37 & 2.46 & 2.70 & 3.06 \\
\hline & 0.8 & 1.07 & 1.14 & 1.56 & 2.16 & 1.19 & 1.27 & 1.65 & 2.24 & 1.58 & 1.65 & 2.01 & 2.47 & 2.13 & 2.19 & 2.43 & 2.77 \\
\hline \multirow{4}{*}{0.8} & 0 & 1.05 & 1.13 & 1.63 & 2.30 & 1.21 & 1.30 & 1.78 & 2.40 & 1.66 & 1.74 & 2.11 & 2.61 & 2.27 & 2.34 & 2.57 & 2.94 \\
\hline & 0.2 & 1.06 & 1.13 & 1.61 & 2.26 & 1.20 & 1.29 & 1.76 & 2.38 & 1.64 & 1.72 & 2.10 & 2.60 & 2.24 & 2.31 & 2.55 & 2.92 \\
\hline & 0.5 & 1.07 & 1.12 & 1.54 & 2.17 & 1.19 & 1.27 & 1.69 & 2.23 & 1.57 & 1.65 & 1.99 & 2.46 & 2.14 & 2.17 & 2.41 & 2.79 \\
\hline & 0.8 & 1.07 & 1.13 & 1.44 & 1.99 & 1.16 & 1.21 & 1.56 & 2.07 & 1.47 & 1.55 & 1.82 & 2.25 & 1.97 & 2.00 & 2.23 & 2.54 \\
\hline
\end{tabular}


Table 2 cont.

Average Model Design Effect values: Poisson Distribution conditions ( $\mathrm{m}=5, \mathrm{k}=75)$

\begin{tabular}{|c|c|c|c|c|c|c|c|c|c|c|c|c|c|c|c|c|c|}
\hline \multirow{4}{*}{$\begin{array}{c}\text { L1 } \\
\text { Effect }\end{array}$} & \multirow{4}{*}{$\begin{array}{c}\text { Interaction } \\
\text { Effect }\end{array}$} & \multicolumn{16}{|c|}{ ICC Values } \\
\hline & & \multicolumn{4}{|c|}{0} & \multirow{2}{*}{\multicolumn{3}{|c|}{0.1}} & & \multicolumn{4}{|c|}{0.3} & \multicolumn{4}{|c|}{0.5} \\
\hline & & & & & & & & & & & & & & & & & \\
\hline & & 0 & 0.2 & 0.5 & 0.8 & 0 & 0.2 & 0.5 & 0.8 & 0 & 0.2 & 0.5 & 0.8 & 0 & 0.2 & 0.5 & 0.8 \\
\hline \multirow{4}{*}{0} & 0 & 1.05 & 1.23 & 2.13 & 3.00 & 1.39 & 1.58 & 2.34 & 3.11 & 2.17 & 2.29 & 2.79 & 3.35 & 2.97 & 3.03 & 3.31 & 3.66 \\
\hline & 0.2 & 1.05 & 1.22 & 2.07 & 2.95 & 1.36 & 1.55 & 2.28 & 3.04 & 2.12 & 2.24 & 2.73 & 3.29 & 2.90 & 2.98 & 3.25 & 3.61 \\
\hline & 0.5 & 1.06 & 1.17 & 1.88 & 2.68 & 1.28 & 1.43 & 2.05 & 2.77 & 1.91 & 2.00 & 2.45 & 3.02 & 2.64 & 2.69 & 2.98 & 3.33 \\
\hline & 0.8 & 1.06 & 1.14 & 1.66 & 2.33 & 1.21 & 1.30 & 1.79 & 2.42 & 1.68 & 1.75 & 2.15 & 2.64 & 2.28 & 2.34 & 2.61 & 2.96 \\
\hline \multirow{4}{*}{0.2} & 0 & 1.05 & 1.23 & 2.06 & 2.94 & 1.36 & 1.54 & 2.29 & 3.05 & 2.13 & 2.23 & 2.73 & 3.28 & 2.89 & 2.97 & 3.25 & 3.60 \\
\hline & 0.2 & 1.05 & 1.21 & 2.05 & 2.88 & 1.34 & 1.52 & 2.22 & 3.00 & 2.07 & 2.18 & 2.68 & 3.23 & 2.85 & 2.91 & 3.19 & 3.54 \\
\hline & 0.5 & 1.05 & 1.16 & 1.84 & 2.63 & 1.27 & 1.42 & 2.02 & 2.72 & 1.87 & 1.98 & 2.43 & 2.97 & 2.59 & 2.65 & 2.91 & 3.30 \\
\hline & 0.8 & 1.06 & 1.13 & 1.63 & 2.30 & 1.21 & 1.30 & 1.77 & 2.39 & 1.66 & 1.76 & 2.12 & 2.61 & 2.27 & 2.32 & 2.58 & 2.94 \\
\hline \multirow{4}{*}{0.5} & 0 & 1.05 & 1.18 & 1.88 & 2.68 & 1.27 & 1.42 & 2.05 & 2.77 & 1.91 & 2.01 & 2.47 & 3.03 & 2.64 & 2.69 & 2.96 & 3.33 \\
\hline & 0.2 & 1.05 & 1.17 & 1.85 & 2.62 & 1.26 & 1.41 & 2.01 & 2.73 & 1.87 & 1.98 & 2.43 & 2.97 & 2.58 & 2.65 & 2.91 & 3.28 \\
\hline & 0.5 & 1.06 & 1.15 & 1.71 & 2.43 & 1.23 & 1.34 & 1.87 & 2.52 & 1.73 & 1.83 & 2.24 & 2.76 & 2.39 & 2.44 & 2.72 & 3.09 \\
\hline & 0.8 & 1.06 & 1.13 & 1.57 & 2.17 & 1.18 & 1.26 & 1.69 & 2.25 & 1.59 & 1.65 & 2.00 & 2.47 & 2.13 & 2.18 & 2.44 & 2.79 \\
\hline \multirow{4}{*}{0.8} & 0 & 1.05 & 1.13 & 1.64 & 2.33 & 1.20 & 1.30 & 1.78 & 2.41 & 1.66 & 1.75 & 2.13 & 2.64 & 2.29 & 2.33 & 2.60 & 2.96 \\
\hline & 0.2 & 1.05 & 1.12 & 1.62 & 2.29 & 1.20 & 1.31 & 1.77 & 2.39 & 1.65 & 1.74 & 2.10 & 2.60 & 2.27 & 2.32 & 2.56 & 2.93 \\
\hline & 0.5 & 1.06 & 1.12 & 1.55 & 2.17 & 1.17 & 1.26 & 1.67 & 2.25 & 1.57 & 1.65 & 2.00 & 2.48 & 2.12 & 2.18 & 2.43 & 2.78 \\
\hline & 0.8 & 1.07 & 1.12 & 1.45 & 1.98 & 1.15 & 1.21 & 1.56 & 2.08 & 1.48 & 1.54 & 1.83 & 2.28 & 1.96 & 2.00 & 2.23 & 2.57 \\
\hline
\end{tabular}

Note: Italicized values indicate a design effect of 2 or larger, while bold values indicate a design effect of 6 or larger. ICC = Intraclass Correlation Coefficient. 
Table 2 cont.

Average Model Design Effect values: Poisson Distribution conditions $(\mathrm{m}=5, \mathrm{k}=100)$

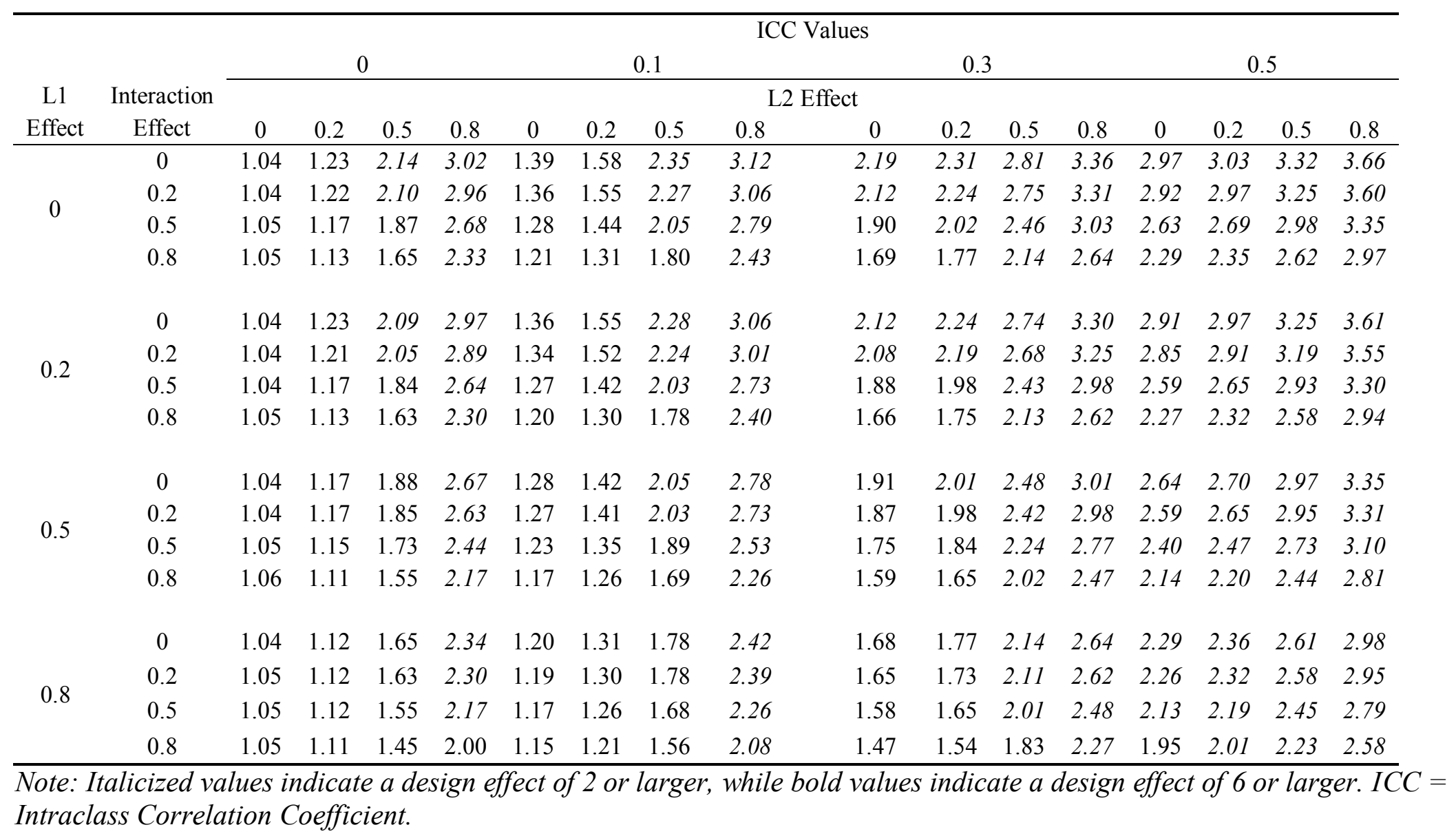


Table 2 cont.

Average Model Design Effect values: Poisson Distribution conditions $(\mathrm{m}=10, \mathrm{k}=25)$

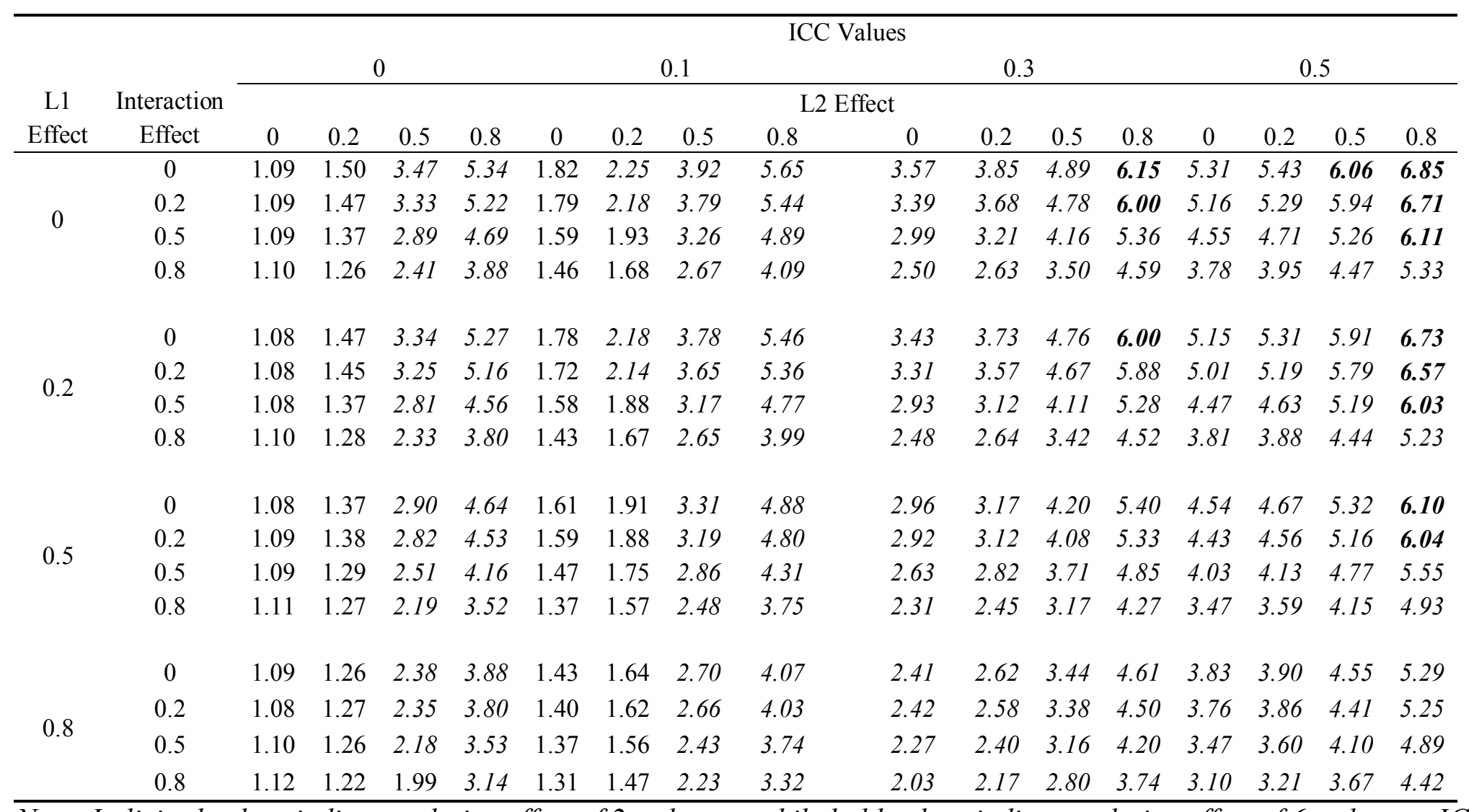

Note: Italicized values indicate a design effect of 2 or larger, while bold values indicate a design effect of 6 or larger. ICC $=$ Intraclass Correlation Coefficient. 
Table 2 cont.

Average Model Design Effect values: Poisson Distribution conditions $(\mathrm{m}=10, \mathrm{k}=50)$

\begin{tabular}{|c|c|c|c|c|c|c|c|c|c|c|c|c|c|c|c|c|c|}
\hline \multirow{4}{*}{$\begin{array}{c}\text { L1 } \\
\text { Effect }\end{array}$} & \multirow{4}{*}{$\begin{array}{l}\text { Interaction } \\
\text { Effect }\end{array}$} & \multicolumn{16}{|c|}{ ICC Values } \\
\hline & & \multicolumn{4}{|c|}{0} & \multirow{2}{*}{\multicolumn{3}{|c|}{0.1}} & & \multicolumn{4}{|c|}{0.3} & \multicolumn{4}{|c|}{0.5} \\
\hline & & \multicolumn{13}{|c|}{ L2 Effect } & & & \\
\hline & & 0 & 0.2 & 0.5 & 0.8 & 0 & 0.2 & 0.5 & 0.8 & 0 & 0.2 & 0.5 & 0.8 & 0 & 0.2 & 0.5 & 0.8 \\
\hline \multirow{4}{*}{0} & 0 & 1.06 & 1.51 & 3.54 & 5.53 & 1.86 & 2.31 & 3.99 & 5.73 & 3.64 & 3.89 & 5.02 & 6.25 & 5.38 & 5.54 & 6.14 & 6.95 \\
\hline & 0.2 & 1.06 & 1.49 & 3.40 & 5.38 & 1.80 & 2.25 & 3.89 & 5.55 & 3.51 & 3.74 & 4.90 & 6.14 & 5.25 & 5.41 & 6.02 & 6.81 \\
\hline & 0.5 & 1.07 & 1.37 & 2.95 & 4.72 & 1.62 & 1.96 & 3.33 & 4.97 & 3.03 & 3.26 & 4.26 & 5.51 & 4.64 & 4.80 & 5.39 & 6.20 \\
\hline & 0.8 & 1.08 & 1.27 & 2.43 & 3.95 & 1.45 & 1.70 & 2.77 & 4.20 & 2.52 & 2.69 & 3.52 & 4.67 & 3.86 & 4.03 & 4.58 & 5.38 \\
\hline \multirow{4}{*}{0.2} & 0 & 1.06 & 1.49 & 3.41 & 5.37 & 1.82 & 2.23 & 3.87 & 5.58 & 3.53 & 3.77 & 4.85 & 6.13 & 5.27 & 5.41 & 6.00 & 6.81 \\
\hline & 0.2 & 1.07 & 1.46 & 3.31 & 5.25 & 1.77 & 2.18 & 3.75 & 5.45 & 3.42 & 3.65 & 4.72 & 5.98 & 5.13 & 5.30 & 5.89 & 6.73 \\
\hline & 0.5 & 1.07 & 1.36 & 2.89 & 4.66 & 1.60 & 1.94 & 3.27 & 4.87 & 2.97 & 3.18 & 4.20 & 5.43 & 4.58 & 4.69 & 5.29 & 6.13 \\
\hline & 0.8 & 1.08 & 1.26 & 2.39 & 3.92 & 1.44 & 1.68 & 2.73 & 4.12 & 2.47 & 2.67 & 3.49 & 4.62 & 3.85 & 3.93 & 4.51 & 5.33 \\
\hline \multirow{4}{*}{0.5} & 0 & 1.06 & 1.38 & 2.97 & 4.73 & 1.61 & 1.96 & 3.36 & 5.00 & 3.01 & 3.24 & 4.28 & 5.50 & 4.66 & 4.79 & 5.37 & 6.22 \\
\hline & 0.2 & 1.07 & 1.36 & 2.88 & 4.65 & 1.59 & 1.92 & 3.30 & 4.84 & 2.97 & 3.16 & 4.16 & 5.43 & 4.52 & 4.67 & 5.27 & 6.15 \\
\hline & 0.5 & 1.07 & 1.31 & 2.58 & 4.21 & 1.51 & 1.75 & 2.95 & 4.43 & 2.69 & 2.87 & 3.77 & 4.93 & 4.14 & 4.28 & 4.86 & 5.64 \\
\hline & 0.8 & 1.08 & 1.24 & 2.25 & 3.59 & 1.38 & 1.59 & 2.52 & 3.79 & 2.30 & 2.49 & 3.22 & 4.31 & 3.59 & 3.62 & 4.18 & 5.01 \\
\hline \multirow{4}{*}{0.8} & 0 & 1.06 & 1.27 & 2.43 & 3.98 & 1.43 & 1.69 & 2.76 & 4.15 & 2.51 & 2.70 & 3.56 & 4.65 & 3.87 & 4.01 & 4.58 & 5.36 \\
\hline & 0.2 & 1.06 & 1.26 & 2.42 & 3.91 & 1.42 & 1.64 & 2.72 & 4.10 & 2.46 & 2.64 & 3.48 & 4.57 & 3.82 & 3.93 & 4.54 & 5.36 \\
\hline & 0.5 & 1.07 & 1.23 & 2.25 & 3.59 & 1.37 & 1.58 & 2.53 & 3.80 & 2.29 & 2.44 & 3.23 & 4.29 & 3.53 & 3.64 & 4.21 & 4.99 \\
\hline & 0.8 & 1.09 & 1.20 & 2.03 & 3.23 & 1.32 & 1.47 & 2.26 & 3.38 & 2.08 & 2.23 & 2.86 & 3.85 & 3.16 & 3.27 & 3.77 & 4.49 \\
\hline
\end{tabular}

Note: Italicized values indicate a design effect of 2 or larger, while bold values indicate a design effect of 6 or larger. ICC = Intraclass Correlation Coefficient. 
Table 2 cont.

Average Model Design Effect values: Poisson Distribution conditions $(\mathrm{m}=10, \mathrm{k}=75)$

\begin{tabular}{|c|c|c|c|c|c|c|c|c|c|c|c|c|c|c|c|c|c|}
\hline \multirow{4}{*}{$\begin{array}{c}\text { L1 } \\
\text { Effect }\end{array}$} & \multirow{4}{*}{$\begin{array}{c}\text { Interaction } \\
\text { Effect }\end{array}$} & \multicolumn{16}{|c|}{ ICC Values } \\
\hline & & \multicolumn{4}{|c|}{0} & \multirow{2}{*}{\multicolumn{3}{|c|}{0.1}} & & \multicolumn{4}{|c|}{0.3} & \multicolumn{4}{|c|}{0.5} \\
\hline & & \multicolumn{13}{|c|}{ L2 Effect } & & & \\
\hline & & 0 & 0.2 & 0.5 & 0.8 & 0 & 0.2 & 0.5 & 0.8 & 0 & 0.2 & 0.5 & 0.8 & 0 & 0.2 & 0.5 & 0.8 \\
\hline \multirow{4}{*}{0} & 0 & 1.05 & 1.53 & 3.59 & 5.55 & 1.89 & 2.32 & 4.02 & 5.78 & 3.67 & 3.92 & 5.03 & 6.30 & 5.41 & 5.56 & 6.19 & 6.96 \\
\hline & 0.2 & 1.05 & 1.50 & 3.44 & 5.40 & 1.83 & 2.25 & 3.88 & 5.62 & 3.52 & 3.75 & 4.90 & 6.17 & 5.29 & 5.45 & 6.05 & 6.83 \\
\hline & 0.5 & 1.05 & 1.38 & 2.98 & 4.77 & 1.64 & 1.98 & 3.38 & 5.00 & 3.03 & 3.30 & 4.32 & 5.53 & 4.68 & 4.80 & 5.45 & 6.27 \\
\hline & 0.8 & 1.07 & 1.27 & 2.46 & 4.00 & 1.45 & 1.69 & 2.78 & 4.19 & 2.52 & 2.72 & 3.58 & 4.72 & 3.93 & 4.02 & 4.61 & 5.43 \\
\hline \multirow{4}{*}{0.2} & 0 & 1.05 & 1.49 & 3.47 & 5.40 & 1.82 & 2.27 & 3.93 & 5.63 & 3.53 & 3.81 & 4.93 & 6.17 & 5.29 & 5.43 & 6.04 & 6.85 \\
\hline & 0.2 & 1.05 & 1.47 & 3.37 & 5.26 & 1.78 & 2.19 & 3.79 & 5.50 & 3.42 & 3.67 & 4.76 & 6.03 & 5.15 & 5.29 & 5.94 & 6.75 \\
\hline & 0.5 & 1.06 & 1.36 & 2.90 & 4.69 & 1.61 & 1.94 & 3.30 & 4.90 & 2.98 & 3.22 & 4.22 & 5.44 & 4.57 & 4.71 & 5.33 & 6.16 \\
\hline & 0.8 & 1.06 & 1.26 & 2.43 & 3.91 & 1.44 & 1.67 & 2.75 & 4.13 & 2.50 & 2.68 & 3.52 & 4.65 & 3.85 & 3.96 & 4.56 & 5.39 \\
\hline \multirow{4}{*}{0.5} & 0 & 1.06 & 1.38 & 2.98 & 4.79 & 1.63 & 1.97 & 3.37 & 4.98 & 3.05 & 3.29 & 4.33 & 5.54 & 4.66 & 4.84 & 5.45 & 6.26 \\
\hline & 0.2 & 1.05 & 1.36 & 2.92 & 4.69 & 1.60 & 1.94 & 3.29 & 4.90 & 2.97 & 3.20 & 4.21 & 5.43 & 4.57 & 4.71 & 5.33 & 6.16 \\
\hline & 0.5 & 1.06 & 1.29 & 2.61 & 4.22 & 1.51 & 1.77 & 2.95 & 4.44 & 2.67 & 2.89 & 3.77 & 4.96 & 4.13 & 4.26 & 4.87 & 5.71 \\
\hline & 0.8 & 1.06 & 1.24 & 2.24 & 3.65 & 1.37 & 1.58 & 2.54 & 3.85 & 2.33 & 2.47 & 3.25 & 4.34 & 3.55 & 3.67 & 4.23 & 5.04 \\
\hline \multirow{4}{*}{0.8} & 0 & 1.05 & 1.27 & 2.47 & 3.97 & 1.44 & 1.69 & 2.77 & 4.19 & 2.51 & 2.71 & 3.56 & 4.71 & 3.91 & 4.03 & 4.61 & 5.43 \\
\hline & 0.2 & 1.06 & 1.26 & 2.42 & 3.95 & 1.43 & 1.66 & 2.73 & 4.14 & 2.48 & 2.65 & 3.50 & 4.63 & 3.85 & 3.94 & 4.55 & 5.38 \\
\hline & 0.5 & 1.06 & 1.23 & 2.25 & 3.65 & 1.37 & 1.58 & 2.56 & 3.85 & 2.31 & 2.50 & 3.27 & 4.29 & 3.57 & 3.68 & 4.23 & 5.03 \\
\hline & 0.8 & 1.07 & 1.20 & 2.01 & 3.20 & 1.31 & 1.46 & 2.28 & 3.42 & 2.08 & 2.22 & 2.88 & 3.86 & 3.17 & 3.28 & 3.77 & 4.53 \\
\hline
\end{tabular}

Note: Italicized values indicate a design effect of 2 or larger, while bold values indicate a design effect of 6 or larger. ICC $=$ Intraclass Correlation Coefficient. 
Table 2 cont.

Average Model Design Effect values: Poisson Distribution conditions ( $\mathrm{m}=10, \mathrm{k}=100)$

\begin{tabular}{|c|c|c|c|c|c|c|c|c|c|c|c|c|c|c|c|c|c|}
\hline \multirow{3}{*}{$\begin{array}{c}\text { L1 } \\
\text { Effect }\end{array}$} & \multirow{4}{*}{$\begin{array}{c}\text { Interaction } \\
\text { Effect }\end{array}$} & \multicolumn{16}{|c|}{ ICC Values } \\
\hline & & \multicolumn{5}{|c|}{0} & \multicolumn{2}{|c|}{0.1} & & \multicolumn{4}{|c|}{0.3} & \multicolumn{4}{|c|}{0.5} \\
\hline & & \multicolumn{16}{|c|}{ L2 Effect } \\
\hline & & 0 & 0.2 & 0.5 & 0.8 & 0 & 0.2 & 0.5 & 0.8 & 0 & 0.2 & 0.5 & 0.8 & 0 & 0.2 & 0.5 & 0.8 \\
\hline \multirow{4}{*}{0} & 0 & 1.04 & 1.54 & 3.59 & 5.59 & 1.88 & 2.34 & 4.05 & 5.80 & 3.67 & 3.94 & 5.06 & 6.32 & 5.44 & 5.59 & 6.21 & 6.99 \\
\hline & 0.2 & 1.05 & 1.51 & 3.45 & 5.43 & 1.82 & 2.27 & 3.92 & 5.62 & 3.54 & 3.81 & 4.93 & 6.18 & 5.31 & 5.45 & 6.07 & 6.86 \\
\hline & 0.5 & 1.05 & 1.38 & 2.99 & 4.80 & 1.64 & 1.99 & 3.41 & 5.02 & 3.06 & 3.29 & 4.31 & 5.57 & 4.68 & 4.82 & 5.45 & 6.26 \\
\hline & 0.8 & 1.06 & 1.27 & 2.45 & 4.00 & 1.46 & 1.69 & 2.78 & 4.22 & 2.55 & 2.73 & 3.56 & 4.74 & 3.92 & 4.03 & 4.62 & 5.47 \\
\hline \multirow{4}{*}{0.2} & 0 & 1.05 & 1.52 & 3.47 & 5.40 & 1.83 & 2.26 & 3.92 & 5.63 & 3.55 & 3.80 & 4.93 & 6.18 & 5.32 & 5.44 & 6.07 & 6.88 \\
\hline & 0.2 & 1.05 & 1.49 & 3.35 & 5.27 & 1.79 & 2.20 & 3.78 & 5.50 & 3.45 & 3.70 & 4.78 & 6.05 & 5.16 & 5.31 & 5.94 & 6.74 \\
\hline & 0.5 & 1.05 & 1.36 & 2.91 & 4.69 & 1.62 & 1.95 & 3.31 & 4.92 & 2.99 & 3.24 & 4.23 & 5.46 & 4.60 & 4.73 & 5.34 & 6.18 \\
\hline & 0.8 & 1.05 & 1.26 & 2.42 & 3.94 & 1.45 & 1.68 & 2.76 & 4.15 & 2.50 & 2.67 & 3.53 & 4.66 & 3.85 & 3.99 & 4.55 & 5.37 \\
\hline \multirow{4}{*}{0.5} & 0 & 1.05 & 1.39 & 3.00 & 4.80 & 1.64 & 1.98 & 3.39 & 4.99 & 3.06 & 3.30 & 4.30 & 5.56 & 4.69 & 4.85 & 5.44 & 6.28 \\
\hline & 0.2 & 1.05 & 1.38 & 2.92 & 4.69 & 1.62 & 1.94 & 3.30 & 4.90 & 2.99 & 3.23 & 4.22 & 5.43 & 4.60 & 4.74 & 5.36 & 6.20 \\
\hline & 0.5 & 1.05 & 1.30 & 2.63 & 4.26 & 1.51 & 1.77 & 2.96 & 4.48 & 2.69 & 2.90 & 3.80 & 4.98 & 4.14 & 4.30 & 4.88 & 5.74 \\
\hline & 0.8 & 1.05 & 1.22 & 2.26 & 3.65 & 1.38 & 1.58 & 2.54 & 3.86 & 2.33 & 2.48 & 3.26 & 4.35 & 3.56 & 3.68 & 4.24 & 5.03 \\
\hline \multirow{4}{*}{0.8} & 0 & 1.05 & 1.27 & 2.48 & 4.01 & 1.45 & 1.70 & 2.77 & 4.22 & 2.51 & 2.73 & 3.57 & 4.70 & 3.91 & 4.03 & 4.61 & 5.44 \\
\hline & 0.2 & 1.05 & 1.26 & 2.45 & 3.94 & 1.43 & 1.67 & 2.75 & 4.14 & 2.48 & 2.70 & 3.52 & 4.65 & 3.85 & 3.96 & 4.54 & 5.37 \\
\hline & 0.5 & 1.06 & 1.23 & 2.26 & 3.66 & 1.38 & 1.59 & 2.54 & 3.85 & 2.30 & 2.48 & 3.27 & 4.33 & 3.56 & 3.67 & 4.25 & 5.03 \\
\hline & 0.8 & 1.06 & 1.19 & 2.02 & 3.24 & 1.31 & 1.48 & 2.28 & 3.42 & 2.09 & 2.24 & 2.90 & 3.85 & 3.18 & 3.26 & 3.76 & 4.56 \\
\hline
\end{tabular}

Note: Italicized values indicate a design effect of 2 or larger, while bold values indicate a design effect of 6 or larger. ICC $=$ Intraclass Correlation Coefficient. 
Table 2 cont.

Average Model Design Effect values: Poisson Distribution conditions $(\mathrm{m}=20, \mathrm{k}=25)$

\begin{tabular}{|c|c|c|c|c|c|c|c|c|c|c|c|c|c|c|c|c|c|}
\hline \multirow{2}{*}{$\begin{array}{c}\text { L1 } \\
\text { Effect }\end{array}$} & \multirow{2}{*}{$\begin{array}{c}\text { Interaction } \\
\text { Effect }\end{array}$} & \multicolumn{16}{|c|}{ ICC Values } \\
\hline & & 0 & 0.2 & 0.5 & 0.8 & 0 & 0.2 & 0.5 & 0.8 & 0 & 0.2 & 0.5 & 0.8 & 0 & 0.2 & 0.5 & 0.8 \\
\hline \multirow{4}{*}{0} & 0 & 1.09 & 2.09 & 6.21 & 10.28 & 2.75 & 3.67 & 7.22 & 10.80 & 6.40 & 6.97 & 9.27 & 11.90 & 10.03 & 10.37 & 11.72 & 13.41 \\
\hline & 0.2 & 1.08 & 2.02 & 6.05 & 9.99 & 2.68 & 3.56 & 6.92 & 10.51 & 6.22 & 6.67 & 8.99 & 11.62 & 9.86 & 10.07 & 11.38 & 13.07 \\
\hline & 0.5 & 1.10 & 1.78 & 5.00 & 8.74 & 2.29 & 2.96 & 5.85 & 9.12 & 5.17 & 5.69 & 7.78 & 10.41 & 8.51 & 8.83 & 10.17 & 11.90 \\
\hline & 0.8 & 1.11 & 1.52 & 3.97 & 7.06 & 1.93 & 2.45 & 4.64 & 7.55 & 4.15 & 4.51 & 6.17 & 8.60 & 6.92 & 7.26 & 8.38 & 10.14 \\
\hline \multirow{4}{*}{0.2} & 0 & 1.09 & 1.99 & 6.06 & 10.07 & 2.64 & 3.55 & 6.89 & 10.47 & 6.16 & 6.77 & 8.93 & 11.64 & 9.82 & 10.08 & 11.39 & 13.10 \\
\hline & 0.2 & 1.09 & 1.95 & 5.74 & 9.71 & 2.60 & 3.46 & 6.71 & 10.25 & 5.98 & 6.46 & 8.75 & 11.27 & 9.51 & 9.78 & 11.11 & 12.88 \\
\hline & 0.5 & 1.10 & 1.72 & 4.87 & 8.56 & 2.21 & 2.90 & 5.67 & 9.00 & 5.07 & 5.52 & 7.60 & 10.16 & 8.33 & 8.61 & 9.96 & 11.64 \\
\hline & 0.8 & 1.11 & 1.51 & 3.87 & 6.99 & 1.89 & 2.36 & 4.49 & 7.40 & 4.09 & 4.49 & 6.13 & 8.50 & 6.85 & 7.07 & 8.38 & 10.01 \\
\hline \multirow{4}{*}{0.5} & 0 & 1.09 & 1.77 & 5.05 & 8.71 & 2.25 & 2.98 & 5.86 & 9.17 & 5.20 & 5.71 & 7.75 & 10.42 & 8.58 & 8.84 & 10.14 & 11.83 \\
\hline & 0.2 & 1.08 & 1.73 & 4.88 & 8.56 & 2.24 & 2.94 & 5.72 & 8.95 & 5.07 & 5.50 & 7.63 & 10.13 & 8.27 & 8.57 & 9.83 & 11.70 \\
\hline & 0.5 & 1.10 & 1.62 & 4.28 & 7.62 & 2.05 & 2.58 & 5.02 & 8.04 & 4.46 & 4.81 & 6.74 & 9.15 & 7.44 & 7.71 & 8.89 & 10.64 \\
\hline & 0.8 & 1.11 & 1.45 & 3.54 & 6.39 & 1.75 & 2.20 & 4.11 & 6.76 & 3.65 & 4.01 & 5.61 & 7.80 & 6.28 & 6.57 & 7.67 & 9.34 \\
\hline \multirow{4}{*}{0.8} & 0 & 1.09 & 1.54 & 4.00 & 7.12 & 1.89 & 2.38 & 4.63 & 7.57 & 4.12 & 4.53 & 6.26 & 8.55 & 6.89 & 7.13 & 8.36 & 10.05 \\
\hline & 0.2 & 1.10 & 1.53 & 3.89 & 6.97 & 1.84 & 2.36 & 4.56 & 7.45 & 3.99 & 4.38 & 6.09 & 8.38 & 6.77 & 7.15 & 8.24 & 9.94 \\
\hline & 0.5 & 1.10 & 1.44 & 3.54 & 6.37 & 1.78 & 2.19 & 4.15 & 6.84 & 3.69 & 3.97 & 5.64 & 7.77 & 6.30 & 6.48 & 7.57 & 9.26 \\
\hline & 0.8 & 1.11 & 1.36 & 3.06 & 5.52 & 1.64 & 1.96 & 3.54 & 5.96 & 3.26 & 3.54 & 4.80 & 6.85 & 5.49 & 5.71 & 6.70 & 8.27 \\
\hline
\end{tabular}

Note: Italicized values indicate a design effect of 2 or larger, while bold values indicate a design effect of 6 or larger. ICC $=$ Intraclass Correlation Coefficient. 
Table 2 cont.

Average Model Design Effect values: Poisson Distribution conditions $(\mathrm{m}=20, \mathrm{k}=50)$

\begin{tabular}{|c|c|c|c|c|c|c|c|c|c|c|c|c|c|c|c|c|c|}
\hline \multirow{2}{*}{$\begin{array}{c}\text { L1 } \\
\text { Effect }\end{array}$} & \multirow{2}{*}{$\begin{array}{c}\text { Interaction } \\
\text { Effect }\end{array}$} & \multicolumn{16}{|c|}{ ICC Values } \\
\hline & & 0 & 0.2 & 0.5 & 0.8 & 0 & 0.2 & 0.5 & 0.8 & 0 & 0.2 & 0.5 & 0.8 & 0 & 0.2 & 0.5 & 0.8 \\
\hline \multirow{4}{*}{0} & 0 & 1.06 & 1.06 & 1.05 & 1.06 & 2.83 & 2.81 & 2.84 & 2.85 & 6.58 & 6.54 & 6.53 & 6.54 & 10.26 & 10.20 & 10.23 & 10.28 \\
\hline & 0.2 & 1.09 & 1.09 & 1.10 & 1.09 & 2.87 & 2.89 & 2.87 & 2.89 & 6.55 & 6.53 & 6.55 & 6.57 & 10.22 & 10.27 & 10.37 & 10.28 \\
\hline & 0.5 & 1.34 & 1.33 & 1.34 & 1.34 & 3.12 & 3.12 & 3.12 & 3.13 & 6.73 & 6.70 & 6.71 & 6.71 & 10.35 & 10.37 & 10.30 & 10.30 \\
\hline & 0.8 & 1.87 & 1.85 & 1.87 & 1.86 & 3.56 & 3.59 & 3.57 & 3.53 & 6.97 & 6.98 & 7.00 & 6.98 & 10.54 & 10.52 & 10.50 & 10.50 \\
\hline \multirow{4}{*}{0.2} & 0 & 1.10 & 1.09 & 1.10 & 1.09 & 2.88 & 2.90 & 2.86 & 2.87 & 6.60 & 6.57 & 6.51 & 6.55 & 10.25 & 10.22 & 10.29 & 10.28 \\
\hline & 0.2 & 1.13 & 1.13 & 1.13 & 1.13 & 2.91 & 2.92 & 2.94 & 2.97 & 6.56 & 6.54 & 6.59 & 6.58 & 10.37 & 10.32 & 10.28 & 10.31 \\
\hline & 0.5 & 1.40 & 1.39 & 1.39 & 1.39 & 3.16 & 3.14 & 3.17 & 3.17 & 6.76 & 6.76 & 6.77 & 6.74 & 10.35 & 10.35 & 10.34 & 10.35 \\
\hline & 0.8 & 1.91 & 1.89 & 1.91 & 1.89 & 3.60 & 3.65 & 3.65 & 3.64 & 7.04 & 7.04 & 7.05 & 7.02 & 10.56 & 10.53 & 10.55 & 10.52 \\
\hline \multirow{4}{*}{0.5} & 0 & 1.35 & 1.35 & 1.35 & 1.35 & 3.15 & 3.14 & 3.13 & 3.13 & 6.70 & 6.72 & 6.73 & 6.74 & 10.43 & 10.39 & 10.35 & 10.32 \\
\hline & 0.2 & 1.39 & 1.40 & 1.40 & 1.39 & 3.17 & 3.17 & 3.18 & 3.18 & 6.79 & 6.69 & 6.75 & 6.73 & 10.40 & 10.38 & 10.37 & 10.44 \\
\hline & 0.5 & 1.69 & 1.70 & 1.68 & 1.71 & 3.42 & 3.41 & 3.41 & 3.41 & 6.86 & 6.95 & 6.83 & 6.95 & 10.43 & 10.47 & 10.53 & 10.41 \\
\hline & 0.8 & 2.22 & 2.22 & 2.27 & 2.19 & 3.85 & 3.84 & 3.86 & 3.86 & 7.15 & 7.13 & 7.17 & 7.16 & 10.63 & 10.58 & 10.61 & 10.61 \\
\hline \multirow{4}{*}{0.8} & 0 & 1.88 & 1.90 & 1.89 & 1.89 & 3.61 & 3.61 & 3.61 & 3.58 & 7.07 & 7.02 & 7.03 & 7.01 & 10.53 & 10.56 & 10.46 & 10.55 \\
\hline & 0.2 & 1.97 & 1.97 & 1.96 & 1.96 & 3.66 & 3.66 & 3.64 & 3.65 & 6.98 & 7.04 & 7.05 & 7.05 & 10.51 & 10.50 & 10.56 & 10.55 \\
\hline & 0.5 & 2.28 & 2.25 & 2.24 & 2.25 & 3.88 & 3.89 & 3.83 & 3.88 & 7.14 & 7.18 & 7.18 & 7.24 & 10.57 & 10.64 & 10.61 & 10.56 \\
\hline & 0.8 & 2.69 & 2.72 & 2.74 & 2.71 & 4.33 & 4.29 & 4.26 & 4.26 & 7.48 & 7.40 & 7.42 & 7.47 & 10.79 & 10.75 & 10.77 & 10.71 \\
\hline
\end{tabular}

Note: Italicized values indicate a design effect of 2 or larger, while bold values indicate a design effect of 6 or larger. ICC $=$ Intraclass Correlation Coefficient. 
Table 2 cont.

Average Model Design Effect values: Poisson Distribution conditions $(\mathrm{m}=20, \mathrm{k}=75)$

\begin{tabular}{|c|c|c|c|c|c|c|c|c|c|c|c|c|c|c|c|c|c|}
\hline \multirow{3}{*}{$\begin{array}{c}\text { L1 } \\
\text { Effect }\end{array}$} & \multirow{3}{*}{$\begin{array}{c}\text { Interaction } \\
\text { Effect }\end{array}$} & \multicolumn{16}{|c|}{ ICC Values } \\
\hline & & \multicolumn{16}{|c|}{ L2 Effect } \\
\hline & & 0 & 0.2 & 0.5 & 0.8 & 0 & 0.2 & 0.5 & 0.8 & 0 & 0.2 & 0.5 & 0.8 & 0 & 0.2 & 0.5 & 0.8 \\
\hline \multirow{4}{*}{0} & 0 & 1.05 & 2.13 & 6.48 & 10.59 & 2.88 & 3.77 & 7.36 & 11.01 & 6.57 & 7.20 & 9.54 & 12.17 & 10.32 & 10.65 & 11.95 & 13.63 \\
\hline & 0.2 & 1.06 & 2.08 & 6.22 & 10.22 & 2.76 & 3.65 & 7.09 & 10.80 & 6.35 & 6.87 & 9.23 & 11.89 & 10.05 & 10.35 & 11.66 & 13.37 \\
\hline & 0.5 & 1.06 & 1.82 & 5.24 & 8.97 & 2.38 & 3.08 & 6.02 & 9.47 & 5.39 & 5.88 & 7.94 & 10.57 & 8.77 & 9.05 & 10.37 & 12.17 \\
\hline & 0.8 & 1.07 & 1.55 & 4.09 & 7.31 & 1.97 & 2.46 & 4.75 & 7.78 & 4.24 & 4.62 & 6.43 & 8.83 & 7.15 & 7.45 & 8.67 & 10.37 \\
\hline \multirow{4}{*}{0.2} & 0 & 1.06 & 2.06 & 6.18 & 10.27 & 2.76 & 3.64 & 7.16 & 10.76 & 6.33 & 6.93 & 9.22 & 11.88 & 10.05 & 10.40 & 11.69 & 13.35 \\
\hline & 0.2 & 1.05 & 2.03 & 5.95 & 10.04 & 2.65 & 3.50 & 6.90 & 10.49 & 6.13 & 6.70 & 8.97 & 11.57 & 9.80 & 10.10 & 11.42 & 13.09 \\
\hline & 0.5 & 1.06 & 1.79 & 5.00 & 8.77 & 2.30 & 2.98 & 5.88 & 9.22 & 5.19 & 5.70 & 7.81 & 10.38 & 8.58 & 8.84 & 10.17 & 11.92 \\
\hline & 0.8 & 1.07 & 1.56 & 4.07 & 7.19 & 1.93 & 2.44 & 4.71 & 7.63 & 4.13 & 4.56 & 6.29 & 8.71 & 7.05 & 7.23 & 8.46 & 10.23 \\
\hline \multirow{4}{*}{0.5} & 0 & 1.06 & 1.82 & 5.18 & 8.96 & 2.38 & 3.09 & 6.05 & 9.48 & 5.39 & 5.86 & 7.96 & 10.62 & 8.79 & 9.06 & 10.44 & 12.08 \\
\hline & 0.2 & 1.05 & 1.80 & 5.01 & 8.76 & 2.32 & 2.99 & 5.87 & 9.25 & 5.21 & 5.69 & 7.76 & 10.36 & 8.57 & 8.83 & 10.12 & 11.88 \\
\hline & 0.5 & 1.07 & 1.63 & 4.41 & 7.80 & 2.07 & 2.65 & 5.17 & 8.31 & 4.52 & 4.98 & 6.85 & 9.42 & 7.65 & 7.89 & 9.20 & 10.95 \\
\hline & 0.8 & 1.07 & 1.47 & 3.64 & 6.64 & 1.83 & 2.24 & 4.27 & 7.02 & 3.75 & 4.14 & 5.76 & 8.03 & 6.45 & 6.66 & 7.82 & 9.51 \\
\hline \multirow{4}{*}{0.8} & 0 & 1.05 & 1.58 & 4.09 & 7.31 & 1.94 & 2.46 & 4.79 & 7.78 & 4.20 & 4.63 & 6.41 & 8.85 & 7.10 & 7.40 & 8.67 & 10.36 \\
\hline & 0.2 & 1.06 & 1.55 & 4.03 & 7.18 & 1.92 & 2.41 & 4.69 & 7.62 & 4.12 & 4.53 & 6.33 & 8.75 & 7.00 & 7.27 & 8.51 & 10.23 \\
\hline & 0.5 & 1.06 & 1.49 & 3.64 & 6.60 & 1.81 & 2.25 & 4.23 & 7.00 & 3.75 & 4.13 & 5.78 & 8.07 & 6.41 & 6.64 & 7.89 & 9.52 \\
\hline & 0.8 & 1.07 & 1.38 & 3.17 & 5.73 & 1.65 & 1.99 & 3.69 & 6.06 & 3.25 & 3.56 & 4.94 & 7.08 & 5.58 & 5.81 & 6.84 & 8.41 \\
\hline
\end{tabular}

Note: Italicized values indicate a design effect of 2 or larger, while bold values indicate a design effect of 6 or larger. ICC $=$ Intraclass Correlation Coefficient. 
Table 2 cont.

Average Model Design Effect values: Poisson Distribution conditions $(\mathrm{m}=20, \mathrm{k}=100)$

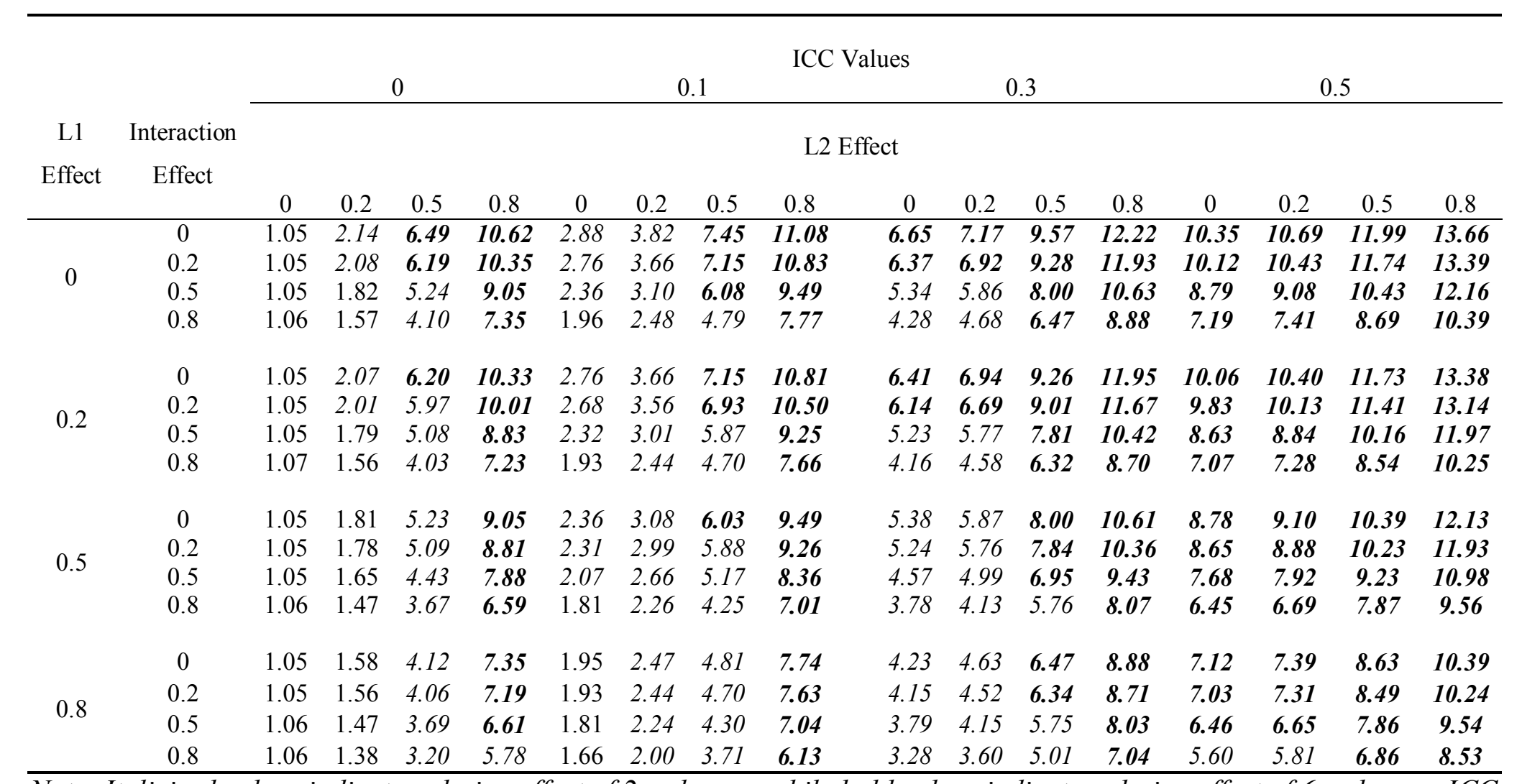

Note: Italicized values indicate a design effect of 2 or larger, while bold values indicate a design effect of 6 or larger. ICC $=$ Intraclass Correlation Coefficient. 
Table 3.

Average Design Effect values for the Intercept: Uniform Distribution conditions $(\mathrm{m}=5, \mathrm{k}=25)$

\begin{tabular}{|c|c|c|c|c|c|c|c|c|c|c|c|c|c|c|c|c|c|}
\hline \multirow{4}{*}{$\begin{array}{c}\text { L1 } \\
\text { Effect }\end{array}$} & \multirow{4}{*}{$\begin{array}{c}\text { Interaction } \\
\text { Effect }\end{array}$} & \multicolumn{16}{|c|}{ ICC Values } \\
\hline & & \multicolumn{4}{|c|}{0} & \multicolumn{4}{|c|}{0.1} & \multicolumn{4}{|c|}{0.3} & \multicolumn{4}{|c|}{0.5} \\
\hline & & & & & & & & & & & & & & & & & \\
\hline & & 0 & 0.2 & 0.5 & 0.8 & 0 & 0.2 & 0.5 & 0.8 & 0 & 0.2 & 0.5 & 0.8 & 0 & 0.2 & 0.5 & 0.8 \\
\hline \multirow{4}{*}{0} & 0 & 1.08 & 1.07 & 1.06 & 1.08 & 1.34 & 1.35 & 1.35 & 1.35 & 2.10 & 2.09 & 2.09 & 2.09 & 2.85 & 2.88 & 2.88 & 2.88 \\
\hline & 0.2 & 1.10 & 1.10 & 1.10 & 1.11 & 1.38 & 1.38 & 1.36 & 1.38 & 2.11 & 2.10 & 2.11 & 2.11 & 2.89 & 2.88 & 2.89 & 2.91 \\
\hline & 0.5 & 1.25 & 1.25 & 1.25 & 1.26 & 1.56 & 1.55 & 1.55 & 1.58 & 2.22 & 2.21 & 2.24 & 2.23 & 2.94 & 2.94 & 2.96 & 2.96 \\
\hline & 0.8 & 1.56 & 1.56 & 1.56 & 1.57 & 1.83 & 1.85 & 1.81 & 1.85 & 2.39 & 2.39 & 2.42 & 2.41 & 3.04 & 3.03 & 3.01 & 3.05 \\
\hline \multirow{4}{*}{0.2} & 0 & 1.10 & 1.11 & 1.10 & 1.11 & 1.40 & 1.39 & 1.38 & 1.39 & 2.12 & 2.10 & 2.12 & 2.12 & 2.88 & 2.88 & 2.90 & 2.89 \\
\hline & 0.2 & 1.14 & 1.13 & 1.13 & 1.13 & 1.42 & 1.43 & 1.41 & 1.41 & 2.14 & 2.15 & 2.13 & 2.11 & 2.90 & 2.91 & 2.89 & 2.90 \\
\hline & 0.5 & 1.30 & 1.30 & 1.29 & 1.28 & 1.57 & 1.59 & 1.58 & 1.59 & 2.25 & 2.26 & 2.26 & 2.26 & 2.94 & 2.97 & 2.95 & 2.97 \\
\hline & 0.8 & 1.59 & 1.58 & 1.59 & 1.61 & 1.84 & 1.86 & 1.86 & 1.84 & 2.45 & 2.41 & 2.44 & 2.44 & 3.06 & 3.06 & 3.05 & 3.05 \\
\hline \multirow{3}{*}{0.5} & 0 & 1.27 & 1.27 & 1.27 & 1.28 & 1.57 & 1.55 & 1.58 & 1.56 & 2.24 & 2.24 & 2.25 & 2.23 & 2.94 & 2.96 & 2.96 & 2.95 \\
\hline & 0.2 & 1.31 & 1.30 & 1.30 & 1.30 & 1.62 & 1.59 & 1.59 & 1.58 & 2.26 & 2.27 & 2.25 & 2.25 & 2.96 & 2.95 & 2.97 & 2.95 \\
\hline & 0.5 & 1.47 & 1.47 & 1.45 & 1.47 & 1.74 & 1.73 & 1.73 & 1.73 & 2.37 & 2.39 & 2.37 & 2.34 & 3.03 & 3.00 & 3.01 & 3.02 \\
\hline \multirow{4}{*}{0.8} & 0 & 1.61 & 1.62 & 1.59 & 1.62 & 1.86 & 1.89 & 1.88 & 1.87 & 2.45 & 2.43 & 2.46 & 2.43 & 3.09 & 3.07 & 3.09 & 3.07 \\
\hline & 0.2 & 1.63 & 1.65 & 1.65 & 1.65 & 1.88 & 1.91 & 1.88 & 1.91 & 2.46 & 2.47 & 2.44 & 2.46 & 3.08 & 3.08 & 3.05 & 3.09 \\
\hline & 0.5 & 1.80 & 1.76 & 1.79 & 1.78 & 2.02 & 2.00 & 2.03 & 2.00 & 2.53 & 2.55 & 2.54 & 2.53 & 3.14 & 3.11 & 3.15 & 3.13 \\
\hline & 0.8 & 2.00 & 2.00 & 2.02 & 2.01 & 2.20 & 2.20 & 2.21 & 2.24 & 2.69 & 2.71 & 2.69 & 2.68 & 3.21 & 3.21 & 3.19 & 3.20 \\
\hline
\end{tabular}

Note: ICC = Intraclass Correlation Coefficient. 
Table 3 cont.

Average Design Effect values for the Intercept: Uniform Distribution conditions $(\mathrm{m}=5, \mathrm{k}=50)$

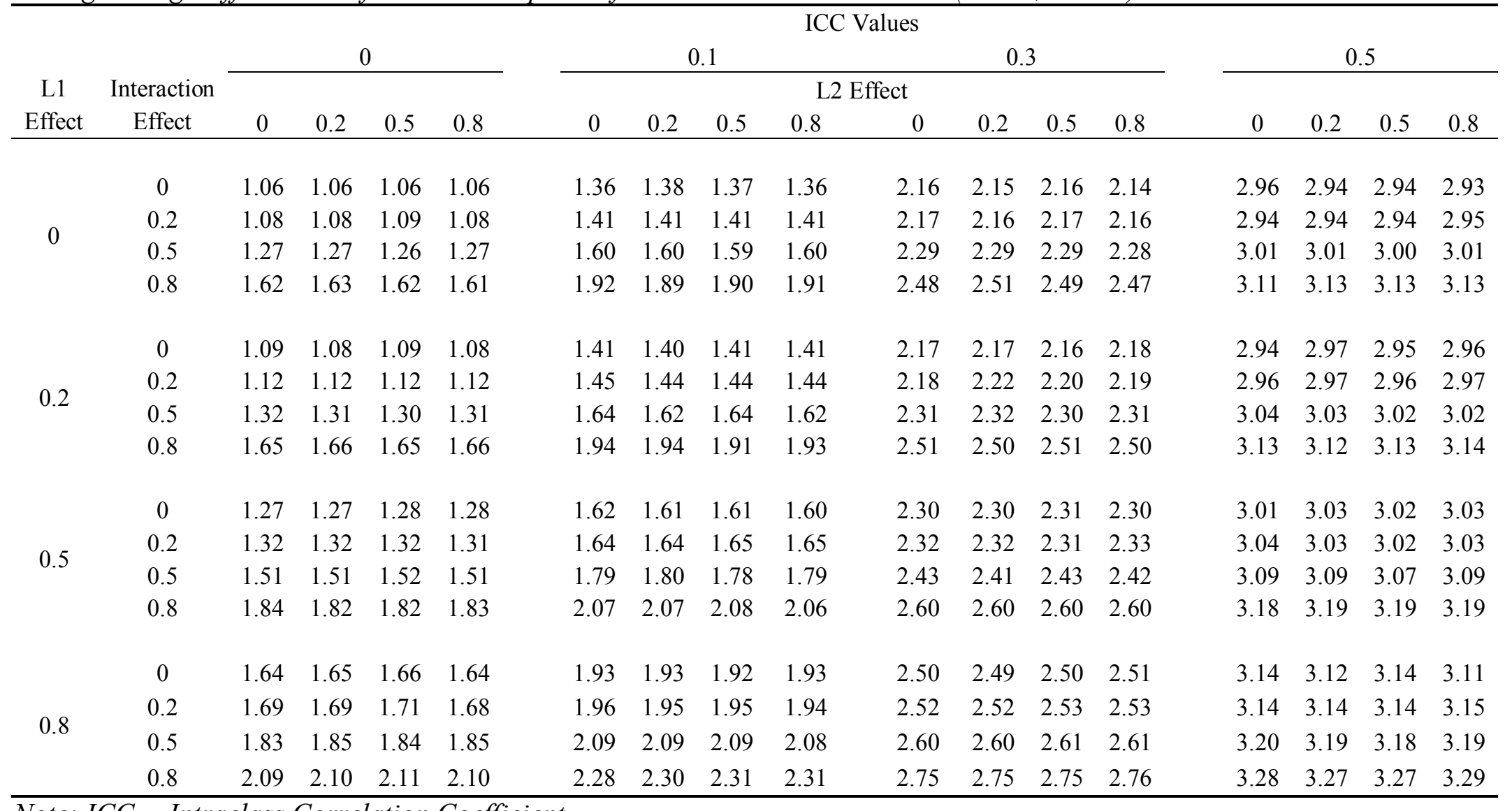

Note: ICC $=$ Intraclass Correlation Coefficient . 
Table 3 cont.

Average Design Effect values for the Intercept: Uniform Distribution conditions $(\mathrm{m}=5, \mathrm{k}=75)$

\begin{tabular}{|c|c|c|c|c|c|c|c|c|c|c|c|c|c|c|c|c|c|}
\hline \multirow{4}{*}{$\begin{array}{c}\text { L1 } \\
\text { Effect }\end{array}$} & \multirow{4}{*}{$\begin{array}{c}\text { Interaction } \\
\text { Effect }\end{array}$} & \multicolumn{16}{|c|}{ ICC Values } \\
\hline & & \multicolumn{4}{|c|}{0} & \multirow{2}{*}{\multicolumn{3}{|c|}{0.1}} & & \multicolumn{4}{|c|}{0.3} & \multicolumn{4}{|c|}{0.5} \\
\hline & & & & & & & & & \multicolumn{2}{|c|}{ L2 Effect } & & & & & & & \\
\hline & & 0 & 0.2 & 0.5 & 0.8 & 0 & 0.2 & 0.5 & 0.8 & 0 & 0.2 & 0.5 & 0.8 & 0 & 0.2 & 0.5 & 0.8 \\
\hline \multirow{4}{*}{0} & 0 & 1.05 & 1.05 & 1.05 & 1.05 & 1.36 & 1.38 & 1.37 & 1.37 & 2.17 & 2.16 & 2.17 & 2.16 & 2.96 & 2.96 & 2.95 & 2.95 \\
\hline & 0.2 & 1.08 & 1.07 & 1.08 & 1.07 & 1.41 & 1.42 & 1.42 & 1.41 & 2.18 & 2.19 & 2.19 & 2.19 & 2.98 & 2.98 & 2.97 & 2.98 \\
\hline & 0.5 & 1.27 & 1.27 & 1.28 & 1.28 & 1.62 & 1.61 & 1.60 & 1.61 & 2.32 & 2.31 & 2.31 & 2.31 & 3.03 & 3.02 & 3.04 & 3.05 \\
\hline & 0.8 & 1.66 & 1.65 & 1.65 & 1.64 & 1.91 & 1.92 & 1.92 & 1.92 & 2.51 & 2.50 & 2.50 & 2.50 & 3.16 & 3.14 & 3.15 & 3.13 \\
\hline \multirow{4}{*}{0.2} & 0 & 1.08 & 1.08 & 1.08 & 1.07 & 1.42 & 1.41 & 1.43 & 1.42 & 2.19 & 2.19 & 2.19 & 2.20 & 2.97 & 2.97 & 2.98 & 2.96 \\
\hline & 0.2 & 1.12 & 1.12 & 1.11 & 1.11 & 1.46 & 1.47 & 1.45 & 1.45 & 2.21 & 2.21 & 2.21 & 2.20 & 2.99 & 2.98 & 2.98 & 2.99 \\
\hline & 0.5 & 1.32 & 1.31 & 1.32 & 1.33 & 1.65 & 1.65 & 1.66 & 1.65 & 2.34 & 2.33 & 2.34 & 2.33 & 3.05 & 3.04 & 3.05 & 3.05 \\
\hline & 0.8 & 1.68 & 1.68 & 1.67 & 1.68 & 1.96 & 1.95 & 1.97 & 1.93 & 2.52 & 2.54 & 2.53 & 2.52 & 3.16 & 3.17 & 3.16 & 3.15 \\
\hline \multirow{4}{*}{0.5} & 0 & 1.29 & 1.28 & 1.28 & 1.28 & 1.63 & 1.63 & 1.61 & 1.62 & 2.31 & 2.31 & 2.31 & 2.32 & 3.05 & 3.03 & 3.04 & 3.03 \\
\hline & 0.2 & 1.33 & 1.33 & 1.33 & 1.32 & 1.66 & 1.65 & 1.66 & 1.64 & 2.34 & 2.34 & 2.35 & 2.34 & 3.04 & 3.04 & 3.05 & 3.05 \\
\hline & 0.5 & 1.53 & 1.53 & 1.53 & 1.53 & 1.82 & 1.82 & 1.82 & 1.83 & 2.44 & 2.46 & 2.44 & 2.45 & 3.10 & 3.12 & 3.10 & 3.12 \\
\hline & 0.8 & 1.85 & 1.85 & 1.84 & 1.86 & 2.09 & 2.11 & 2.10 & 2.08 & 2.62 & 2.62 & 2.62 & 2.62 & 3.21 & 3.21 & 3.21 & 3.21 \\
\hline \multirow{4}{*}{0.8} & 0 & 1.67 & 1.67 & 1.66 & 1.67 & 1.94 & 1.94 & 1.94 & 1.94 & 2.51 & 2.52 & 2.52 & 2.52 & 3.16 & 3.14 & 3.16 & 3.16 \\
\hline & 0.2 & 1.70 & 1.70 & 1.70 & 1.70 & 1.97 & 1.97 & 1.97 & 1.97 & 2.54 & 2.54 & 2.54 & 2.53 & 3.16 & 3.15 & 3.17 & 3.16 \\
\hline & 0.5 & 1.87 & 1.87 & 1.86 & 1.87 & 2.11 & 2.10 & 2.12 & 2.10 & 2.64 & 2.63 & 2.63 & 2.64 & 3.21 & 3.21 & 3.22 & 3.21 \\
\hline & 0.8 & 2.13 & 2.13 & 2.14 & 2.12 & 2.34 & 2.35 & 2.33 & 2.34 & 2.77 & 2.78 & 2.78 & 2.78 & 3.30 & 3.30 & 3.29 & 3.29 \\
\hline
\end{tabular}

Note: ICC = Intraclass Correlation Coefficient. 
Table 3 cont.

Average Design Effect values for the Intercept: Uniform Distribution conditions $(\mathrm{m}=5, \mathrm{k}=100)$

\begin{tabular}{|c|c|c|c|c|c|c|c|c|c|c|c|c|c|c|c|c|c|}
\hline \multirow{3}{*}{$\begin{array}{c}\text { L1 } \\
\text { Effect }\end{array}$} & \multirow{3}{*}{$\begin{array}{c}\text { Interaction } \\
\text { Effect }\end{array}$} & \multicolumn{12}{|c|}{ ICC Values } & \multicolumn{4}{|c|}{0.5} \\
\hline & & \multicolumn{16}{|c|}{ L2 Effect } \\
\hline & & 0 & 0.2 & 0.5 & 0.8 & 0 & 0.2 & 0.5 & 0.8 & 0 & 0.2 & 0.5 & 0.8 & 0 & 0.2 & 0.5 & 0.8 \\
\hline \multirow{4}{*}{0} & 0 & 1.04 & 1.04 & 1.04 & 1.04 & 1.38 & 1.39 & 1.39 & 1.38 & 2.18 & 2.17 & 2.18 & 2.17 & 2.97 & 2.96 & 2.97 & 2.97 \\
\hline & 0.2 & 1.07 & 1.07 & 1.08 & 1.07 & 1.43 & 1.42 & 1.42 & 1.42 & 2.20 & 2.21 & 2.21 & 2.20 & 2.98 & 2.98 & 2.98 & 2.99 \\
\hline & 0.5 & 1.29 & 1.28 & 1.29 & 1.28 & 1.62 & 1.62 & 1.62 & 1.61 & 2.31 & 2.33 & 2.33 & 2.32 & 3.05 & 3.04 & 3.05 & 3.05 \\
\hline & 0.8 & 1.65 & 1.66 & 1.65 & 1.65 & 1.93 & 1.95 & 1.93 & 1.94 & 2.51 & 2.53 & 2.53 & 2.53 & 3.16 & 3.15 & 3.15 & 3.16 \\
\hline \multirow{4}{*}{0.2} & 0 & 1.07 & 1.07 & 1.07 & 1.07 & 1.41 & 1.43 & 1.43 & 1.42 & 2.20 & 2.20 & 2.21 & 2.19 & 2.98 & 2.99 & 2.98 & 2.97 \\
\hline & 0.2 & 1.10 & 1.11 & 1.11 & 1.10 & 1.46 & 1.46 & 1.46 & 1.47 & 2.22 & 2.23 & 2.22 & 2.21 & 3.01 & 2.98 & 2.99 & 2.99 \\
\hline & 0.5 & 1.32 & 1.33 & 1.32 & 1.31 & 1.65 & 1.65 & 1.66 & 1.66 & 2.35 & 2.34 & 2.34 & 2.35 & 3.06 & 3.06 & 3.06 & 3.06 \\
\hline & 0.8 & 1.68 & 1.70 & 1.70 & 1.69 & 1.96 & 1.97 & 1.97 & 1.97 & 2.54 & 2.54 & 2.55 & 2.54 & 3.17 & 3.16 & 3.17 & 3.17 \\
\hline \multirow{4}{*}{0.5} & 0 & 1.29 & 1.29 & 1.29 & 1.29 & 1.63 & 1.62 & 1.62 & 1.62 & 2.31 & 2.33 & 2.31 & 2.33 & 3.05 & 3.04 & 3.05 & 3.05 \\
\hline & 0.2 & 1.33 & 1.34 & 1.33 & 1.33 & 1.65 & 1.66 & 1.66 & 1.66 & 2.36 & 2.34 & 2.35 & 2.35 & 3.06 & 3.06 & 3.06 & 3.06 \\
\hline & 0.5 & 1.55 & 1.54 & 1.53 & 1.53 & 1.84 & 1.83 & 1.83 & 1.83 & 2.46 & 2.44 & 2.46 & 2.44 & 3.13 & 3.12 & 3.11 & 3.12 \\
\hline & 0.8 & 1.85 & 1.87 & 1.88 & 1.86 & 2.10 & 2.11 & 2.10 & 2.11 & 2.63 & 2.63 & 2.63 & 2.64 & 3.22 & 3.22 & 3.22 & 3.20 \\
\hline \multirow{4}{*}{0.8} & 0 & 1.68 & 1.67 & 1.67 & 1.68 & 1.95 & 1.95 & 1.94 & 1.94 & 2.54 & 2.54 & 2.53 & 2.53 & 3.17 & 3.16 & 3.16 & 3.17 \\
\hline & 0.2 & 1.70 & 1.72 & 1.70 & 1.71 & 1.98 & 1.97 & 1.97 & 1.97 & 2.55 & 2.55 & 2.56 & 2.56 & 3.17 & 3.17 & 3.17 & 3.18 \\
\hline & 0.5 & 1.87 & 1.86 & 1.88 & 1.89 & 2.12 & 2.11 & 2.12 & 2.12 & 2.64 & 2.64 & 2.63 & 2.64 & 3.22 & 3.22 & 3.23 & 3.21 \\
\hline & 0.8 & 2.14 & 2.14 & 2.14 & 2.14 & 2.34 & 2.33 & 2.34 & 2.35 & 2.81 & 2.80 & 2.80 & 2.80 & 3.31 & 3.32 & 3.31 & 3.31 \\
\hline
\end{tabular}

Note: ICC = Intraclass Correlation Coefficient. 
Table 3 cont.

Average Design Effect values for the Intercept: Uniform Distribution conditions ( $\mathrm{m}=10, \mathrm{k}=25)$

\begin{tabular}{|c|c|c|c|c|c|c|c|c|c|c|c|c|c|c|c|c|c|}
\hline \multirow{3}{*}{$\begin{array}{c}\text { L1 } \\
\text { Effect }\end{array}$} & \multirow{3}{*}{$\begin{array}{c}\text { Interaction } \\
\text { Effect }\end{array}$} & \multicolumn{16}{|c|}{ ICC Values } \\
\hline & & \multicolumn{4}{|c|}{0} & \multicolumn{3}{|c|}{0.1} & & \multicolumn{4}{|c|}{0.3} & \multicolumn{4}{|c|}{0.5} \\
\hline & & 0 & 0.2 & 0.5 & 0.8 & 0 & 0.2 & 0.5 & 0.8 & 0 & 0.2 & 0.5 & 0.8 & 0 & 0.2 & 0.5 & 0.8 \\
\hline \multirow{4}{*}{0} & 0 & 1.08 & 1.08 & 1.07 & 1.08 & 1.79 & 1.80 & 1.78 & 1.75 & 3.47 & 3.49 & 3.47 & 3.44 & 5.22 & 5.25 & 5.18 & 5.19 \\
\hline & 0.2 & 1.10 & 1.11 & 1.11 & 1.11 & 1.81 & 1.80 & 1.81 & 1.82 & 3.50 & 3.50 & 3.48 & 3.50 & 5.20 & 5.24 & 5.28 & 5.22 \\
\hline & 0.5 & 1.29 & 1.28 & 1.28 & 1.29 & 2.04 & 2.03 & 2.05 & 2.04 & 3.63 & 3.65 & 3.64 & 3.62 & 5.28 & 5.29 & 5.28 & 5.35 \\
\hline & 0.8 & 1.68 & 1.69 & 1.68 & 1.67 & 2.38 & 2.37 & 2.39 & 2.37 & 3.87 & 3.85 & 3.81 & 3.88 & 5.45 & 5.42 & 5.42 & 5.37 \\
\hline \multirow{4}{*}{0.2} & 0 & 1.10 & 1.11 & 1.10 & 1.11 & 1.81 & 1.85 & 1.83 & 1.83 & 3.48 & 3.51 & 3.49 & 3.55 & 5.24 & 5.21 & 5.20 & 5.18 \\
\hline & 0.2 & 1.13 & 1.14 & 1.12 & 1.14 & 1.87 & 1.85 & 1.87 & 1.85 & 3.53 & 3.50 & 3.52 & 3.51 & 5.19 & 5.25 & 5.26 & 5.23 \\
\hline & 0.5 & 1.32 & 1.34 & 1.34 & 1.33 & 2.05 & 2.04 & 2.08 & 2.07 & 3.63 & 3.66 & 3.64 & 3.69 & 5.31 & 5.30 & 5.31 & 5.33 \\
\hline & 0.8 & 1.73 & 1.74 & 1.75 & 1.72 & 2.39 & 2.42 & 2.42 & 2.38 & 3.90 & 3.91 & 3.88 & 3.85 & 5.44 & 5.39 & 5.46 & 5.43 \\
\hline \multirow{4}{*}{0.5} & 0 & 1.31 & 1.31 & 1.29 & 1.30 & 2.04 & 2.03 & 2.04 & 2.07 & 3.62 & 3.65 & 3.63 & 3.62 & 5.30 & 5.28 & 5.30 & 5.35 \\
\hline & 0.2 & 1.35 & 1.34 & 1.35 & 1.36 & 2.10 & 2.06 & 2.06 & 2.07 & 3.61 & 3.67 & 3.66 & 3.67 & 5.27 & 5.30 & 5.30 & 5.35 \\
\hline & 0.5 & 1.54 & 1.55 & 1.57 & 1.56 & 2.30 & 2.31 & 2.27 & 2.30 & 3.82 & 3.81 & 3.77 & 3.79 & 5.44 & 5.37 & 5.40 & 5.40 \\
\hline & 0.8 & 1.94 & 1.92 & 1.91 & 1.88 & 2.59 & 2.63 & 2.59 & 2.61 & 4.01 & 3.99 & 3.98 & 3.98 & 5.55 & 5.48 & 5.51 & 5.51 \\
\hline \multirow{4}{*}{0.8} & 0 & 1.75 & 1.74 & 1.73 & 1.76 & 2.43 & 2.45 & 2.41 & 2.43 & 3.88 & 3.89 & 3.88 & 3.87 & 5.45 & 5.45 & 5.43 & 5.42 \\
\hline & 0.2 & 1.78 & 1.77 & 1.76 & 1.80 & 2.46 & 2.46 & 2.48 & 2.45 & 3.92 & 3.90 & 3.91 & 3.91 & 5.48 & 5.40 & 5.46 & 5.45 \\
\hline & 0.5 & 1.98 & 1.99 & 1.97 & 2.00 & 2.62 & 2.63 & 2.58 & 2.66 & 4.03 & 4.04 & 4.04 & 4.02 & 5.49 & 5.53 & 5.51 & 5.50 \\
\hline & 0.8 & 2.34 & 2.33 & 2.27 & 2.32 & 2.97 & 2.97 & 2.94 & 2.89 & 4.25 & 4.19 & 4.18 & 4.17 & 5.62 & 5.64 & 5.61 & 5.65 \\
\hline
\end{tabular}

Note: ICC = Intraclass Correlation Coefficient. 
Table 3 cont.

Average Design Effect values for the Intercept: Uniform Distribution conditions $(\mathrm{m}=10, \mathrm{k}=50)$

\begin{tabular}{|c|c|c|c|c|c|c|c|c|c|c|c|c|c|c|c|c|c|}
\hline \multirow{3}{*}{$\begin{array}{c}\text { L1 } \\
\text { Effect }\end{array}$} & \multirow{3}{*}{$\begin{array}{c}\text { Interaction } \\
\text { Effect }\end{array}$} & \multicolumn{16}{|c|}{ ICC Values } \\
\hline & & \multicolumn{4}{|c|}{0} & \multicolumn{3}{|c|}{0.1} & & \multicolumn{4}{|c|}{0.3} & \multicolumn{4}{|c|}{0.5} \\
\hline & & 0 & 0.2 & 0.5 & 0.8 & 0 & 0.2 & 0.5 & 0.8 & 0 & 0.2 & 0.5 & 0.8 & 0 & 0.2 & 0.5 & 0.8 \\
\hline \multirow{4}{*}{0} & 0 & 1.06 & 1.06 & 1.05 & 1.06 & 1.84 & 1.83 & 1.84 & 1.82 & 3.57 & 3.61 & 3.59 & 3.57 & 5.37 & 5.37 & 5.35 & 5.37 \\
\hline & 0.2 & 1.08 & 1.08 & 1.09 & 1.09 & 1.88 & 1.88 & 1.90 & 1.89 & 3.61 & 3.61 & 3.61 & 3.60 & 5.34 & 5.37 & 5.36 & 5.38 \\
\hline & 0.5 & 1.31 & 1.30 & 1.29 & 1.31 & 2.11 & 2.11 & 2.11 & 2.09 & 3.74 & 3.71 & 3.75 & 3.76 & 5.40 & 5.42 & 5.45 & 5.47 \\
\hline & 0.8 & 1.77 & 1.76 & 1.77 & 1.73 & 2.48 & 2.49 & 2.49 & 2.49 & 4.00 & 3.98 & 4.00 & 3.99 & 5.57 & 5.57 & 5.59 & 5.59 \\
\hline \multirow{4}{*}{0.2} & 0 & 1.09 & 1.09 & 1.08 & 1.10 & 1.88 & 1.85 & 1.89 & 1.87 & 3.61 & 3.61 & 3.61 & 3.59 & 5.39 & 5.36 & 5.37 & 5.38 \\
\hline & 0.2 & 1.12 & 1.13 & 1.13 & 1.13 & 1.94 & 1.92 & 1.92 & 1.95 & 3.64 & 3.64 & 3.63 & 3.64 & 5.40 & 5.39 & 5.39 & 5.36 \\
\hline & 0.5 & 1.36 & 1.35 & 1.38 & 1.37 & 2.15 & 2.14 & 2.13 & 2.16 & 3.78 & 3.75 & 3.78 & 3.78 & 5.46 & 5.46 & 5.47 & 5.48 \\
\hline & 0.8 & 1.81 & 1.81 & 1.82 & 1.80 & 2.53 & 2.52 & 2.53 & 2.51 & 4.00 & 4.02 & 4.04 & 4.02 & 5.58 & 5.59 & 5.60 & 5.58 \\
\hline \multirow{4}{*}{0.5} & 0 & 1.32 & 1.32 & 1.33 & 1.31 & 2.12 & 2.11 & 2.11 & 2.12 & 3.76 & 3.76 & 3.74 & 3.74 & 5.45 & 5.46 & 5.41 & 5.47 \\
\hline & 0.2 & 1.36 & 1.36 & 1.37 & 1.37 & 2.17 & 2.14 & 2.15 & 2.16 & 3.81 & 3.77 & 3.78 & 3.77 & 5.47 & 5.47 & 5.46 & 5.48 \\
\hline & 0.5 & 1.59 & 1.62 & 1.62 & 1.61 & 2.37 & 2.34 & 2.36 & 2.37 & 3.89 & 3.90 & 3.92 & 3.96 & 5.54 & 5.51 & 5.55 & 5.54 \\
\hline & 0.8 & 2.03 & 2.03 & 2.01 & 2.02 & 2.74 & 2.71 & 2.70 & 2.74 & 4.12 & 4.16 & 4.17 & 4.12 & 5.66 & 5.64 & 5.65 & 5.61 \\
\hline \multirow{4}{*}{0.8} & 0 & 1.77 & 1.81 & 1.79 & 1.81 & 2.52 & 2.50 & 2.52 & 2.55 & 4.01 & 4.00 & 4.03 & 4.00 & 5.60 & 5.58 & 5.57 & 5.56 \\
\hline & 0.2 & 1.85 & 1.84 & 1.83 & 1.84 & 2.56 & 2.56 & 2.55 & 2.55 & 4.03 & 4.03 & 4.05 & 4.03 & 5.61 & 5.57 & 5.60 & 5.60 \\
\hline & 0.5 & 2.04 & 2.04 & 2.05 & 2.04 & 2.75 & 2.72 & 2.72 & 2.73 & 4.16 & 4.15 & 4.13 & 4.17 & 5.64 & 5.65 & 5.66 & 5.66 \\
\hline & 0.8 & 2.42 & 2.41 & 2.45 & 2.44 & 3.03 & 3.04 & 3.03 & 3.05 & 4.34 & 4.35 & 4.37 & 4.39 & 5.80 & 5.79 & 5.78 & 5.76 \\
\hline
\end{tabular}

Note: ICC = Intraclass Correlation Coefficient. 
Table 3 cont.

Average Design Effect values for the Intercept: Uniform Distribution conditions $(\mathrm{m}=10, \mathrm{k}=75)$

\begin{tabular}{|c|c|c|c|c|c|c|c|c|c|c|c|c|c|c|c|c|c|}
\hline \multirow{3}{*}{$\begin{array}{c}\text { L1 } \\
\text { Effect }\end{array}$} & \multirow{3}{*}{$\begin{array}{c}\text { Interaction } \\
\text { Effect }\end{array}$} & \multicolumn{16}{|c|}{ ICC Values } \\
\hline & & \multicolumn{16}{|c|}{ L2 Effect } \\
\hline & & 0 & 0.2 & 0.5 & 0.8 & 0 & 0.2 & 0.5 & 0.8 & 0 & 0.2 & 0.5 & 0.8 & 0 & 0.2 & 0.5 & 0.8 \\
\hline \multirow{4}{*}{0} & 0 & 1.05 & 1.05 & 1.05 & 1.05 & 1.86 & 1.85 & 1.86 & 1.85 & 3.64 & 3.63 & 3.63 & 3.61 & 5.38 & 5.40 & 5.40 & 5.39 \\
\hline & 0.2 & 1.08 & 1.09 & 1.09 & 1.08 & 1.91 & 1.91 & 1.91 & 1.91 & 3.65 & 3.64 & 3.64 & 3.64 & 5.43 & 5.46 & 5.44 & 5.44 \\
\hline & 0.5 & 1.32 & 1.33 & 1.32 & 1.31 & 2.15 & 2.12 & 2.14 & 2.13 & 3.81 & 3.81 & 3.78 & 3.80 & 5.50 & 5.49 & 5.49 & 5.50 \\
\hline & 0.8 & 1.80 & 1.79 & 1.80 & 1.79 & 2.52 & 2.54 & 2.52 & 2.52 & 4.04 & 4.03 & 4.04 & 4.03 & 5.64 & 5.61 & 5.61 & 5.59 \\
\hline \multirow{4}{*}{0.2} & 0 & 1.09 & 1.08 & 1.09 & 1.08 & 1.92 & 1.89 & 1.93 & 1.89 & 3.65 & 3.67 & 3.66 & 3.66 & 5.38 & 5.43 & 5.44 & 5.42 \\
\hline & 0.2 & 1.12 & 1.12 & 1.13 & 1.12 & 1.96 & 1.95 & 1.95 & 1.95 & 3.67 & 3.66 & 3.66 & 3.70 & 5.44 & 5.43 & 5.42 & 5.44 \\
\hline & 0.5 & 1.38 & 1.36 & 1.38 & 1.38 & 2.17 & 2.17 & 2.18 & 2.17 & 3.84 & 3.84 & 3.81 & 3.83 & 5.52 & 5.50 & 5.52 & 5.49 \\
\hline & 0.8 & 1.83 & 1.83 & 1.83 & 1.83 & 2.56 & 2.58 & 2.55 & 2.54 & 4.07 & 4.06 & 4.07 & 4.06 & 5.66 & 5.64 & 5.62 & 5.62 \\
\hline \multirow{4}{*}{0.5} & 0 & 1.33 & 1.32 & 1.33 & 1.33 & 2.14 & 2.14 & 2.14 & 2.13 & 3.80 & 3.80 & 3.79 & 3.79 & 5.51 & 5.51 & 5.48 & 5.48 \\
\hline & 0.2 & 1.38 & 1.39 & 1.39 & 1.38 & 2.17 & 2.20 & 2.19 & 2.20 & 3.83 & 3.83 & 3.84 & 3.83 & 5.52 & 5.51 & 5.52 & 5.51 \\
\hline & 0.5 & 1.63 & 1.64 & 1.63 & 1.64 & 2.39 & 2.40 & 2.39 & 2.40 & 3.95 & 3.93 & 3.96 & 3.95 & 5.58 & 5.59 & 5.56 & 5.58 \\
\hline & 0.8 & 2.09 & 2.08 & 2.12 & 2.07 & 2.74 & 2.79 & 2.75 & 2.76 & 4.17 & 4.20 & 4.16 & 4.18 & 5.72 & 5.72 & 5.72 & 5.68 \\
\hline \multirow{4}{*}{0.8} & 0 & 1.82 & 1.80 & 1.82 & 1.82 & 2.54 & 2.55 & 2.53 & 2.54 & 4.04 & 4.07 & 4.05 & 4.07 & 5.62 & 5.62 & 5.61 & 5.61 \\
\hline & 0.2 & 1.88 & 1.86 & 1.87 & 1.86 & 2.59 & 2.57 & 2.58 & 2.59 & 4.08 & 4.08 & 4.10 & 4.08 & 5.66 & 5.64 & 5.65 & 5.65 \\
\hline & 0.5 & 2.09 & 2.09 & 2.09 & 2.08 & 2.76 & 2.78 & 2.79 & 2.79 & 4.17 & 4.21 & 4.19 & 4.20 & 5.71 & 5.68 & 5.70 & 5.71 \\
\hline & 0.8 & 2.48 & 2.46 & 2.48 & 2.48 & 3.10 & 3.10 & 3.08 & 3.11 & 4.41 & 4.42 & 4.42 & 4.42 & 5.83 & 5.83 & 5.81 & 5.83 \\
\hline
\end{tabular}

Note: ICC $=$ Intraclass Correlation Coefficient. 
Table 3 cont.

Average Design Effect values for the Intercept: Uniform Distribution conditions $(\mathrm{m}=10, \mathrm{k}=100)$

\begin{tabular}{|c|c|c|c|c|c|c|c|c|c|c|c|c|c|c|c|c|c|}
\hline \multirow{3}{*}{$\begin{array}{c}\text { L1 } \\
\text { Effect }\end{array}$} & \multirow{3}{*}{$\begin{array}{c}\text { Interaction } \\
\text { Effect }\end{array}$} & \multicolumn{12}{|c|}{ ICC Values } & \multicolumn{4}{|c|}{0.5} \\
\hline & & \multicolumn{16}{|c|}{2 Effect } \\
\hline & & 0 & 0.2 & 0.5 & 0.8 & 0 & 0.2 & 0.5 & 0.8 & 0 & 0.2 & 0.5 & 0.8 & 0 & 0.2 & 0.5 & 0.8 \\
\hline \multirow{4}{*}{0} & 0 & 1.05 & 1.05 & 1.05 & 1.05 & 1.88 & 1.87 & 1.87 & 1.87 & 3.66 & 3.66 & 3.63 & 3.66 & 5.45 & 5.43 & 5.42 & 5.44 \\
\hline & 0.2 & 1.08 & 1.08 & 1.08 & 1.08 & 1.90 & 1.92 & 1.91 & 1.92 & 3.67 & 3.66 & 3.67 & 3.70 & 5.45 & 5.44 & 5.44 & 5.45 \\
\hline & 0.5 & 1.33 & 1.33 & 1.33 & 1.33 & 2.13 & 2.16 & 2.15 & 2.16 & 3.81 & 3.80 & 3.82 & 3.83 & 5.51 & 5.52 & 5.51 & 5.53 \\
\hline & 0.8 & 1.82 & 1.80 & 1.81 & 1.80 & 2.54 & 2.54 & 2.55 & 2.54 & 4.06 & 4.07 & 4.07 & 4.07 & 5.63 & 5.66 & 5.66 & 5.64 \\
\hline \multirow{4}{*}{0.2} & 0 & 1.08 & 1.08 & 1.08 & 1.08 & 1.91 & 1.92 & 1.92 & 1.91 & 3.68 & 3.68 & 3.67 & 3.69 & 5.45 & 5.44 & 5.43 & 5.46 \\
\hline & 0.2 & 1.12 & 1.11 & 1.12 & 1.12 & 1.96 & 1.96 & 1.96 & 1.96 & 3.69 & 3.70 & 3.70 & 3.71 & 5.44 & 5.46 & 5.45 & 5.45 \\
\hline & 0.5 & 1.38 & 1.37 & 1.38 & 1.37 & 2.18 & 2.18 & 2.20 & 2.21 & 3.86 & 3.85 & 3.85 & 3.83 & 5.51 & 5.53 & 5.54 & 5.53 \\
\hline & 0.8 & 1.85 & 1.88 & 1.85 & 1.85 & 2.58 & 2.57 & 2.57 & 2.59 & 4.07 & 4.08 & 4.08 & 4.08 & 5.66 & 5.68 & 5.66 & 5.64 \\
\hline \multirow{4}{*}{0.5} & 0 & 1.33 & 1.33 & 1.33 & 1.33 & 2.15 & 2.15 & 2.14 & 2.14 & 3.80 & 3.81 & 3.82 & 3.80 & 5.53 & 5.52 & 5.51 & 5.55 \\
\hline & 0.2 & 1.39 & 1.38 & 1.38 & 1.38 & 2.19 & 2.19 & 2.19 & 2.19 & 3.85 & 3.84 & 3.84 & 3.85 & 5.54 & 5.54 & 5.54 & 5.55 \\
\hline & 0.5 & 1.65 & 1.66 & 1.65 & 1.63 & 2.40 & 2.40 & 2.41 & 2.43 & 3.96 & 3.98 & 4.00 & 3.98 & 5.60 & 5.62 & 5.58 & 5.62 \\
\hline & 0.8 & 2.09 & 2.08 & 2.09 & 2.11 & 2.78 & 2.80 & 2.80 & 2.79 & 4.22 & 4.20 & 4.21 & 4.23 & 5.73 & 5.73 & 5.72 & 5.75 \\
\hline \multirow{4}{*}{0.8} & 0 & 1.83 & 1.85 & 1.83 & 1.82 & 2.56 & 2.57 & 2.57 & 2.56 & 4.08 & 4.06 & 4.05 & 4.08 & 5.67 & 5.65 & 5.66 & 5.65 \\
\hline & 0.2 & 1.88 & 1.87 & 1.86 & 1.87 & 2.59 & 2.58 & 2.59 & 2.59 & 4.11 & 4.10 & 4.06 & 4.09 & 5.66 & 5.67 & 5.66 & 5.67 \\
\hline & 0.5 & 2.10 & 2.10 & 2.10 & 2.11 & 2.78 & 2.79 & 2.79 & 2.79 & 4.23 & 4.20 & 4.22 & 4.23 & 5.73 & 5.74 & 5.72 & 5.74 \\
\hline & 0.8 & 2.50 & 2.50 & 2.49 & 2.49 & 3.12 & 3.11 & 3.12 & 3.11 & 4.44 & 4.44 & 4.43 & 4.42 & 5.85 & 5.85 & 5.85 & 5.86 \\
\hline
\end{tabular}

Note: ICC = Intraclass Correlation Coefficient. 
Table 3 cont.

Average Design Effect values for the Intercept: Uniform Distribution conditions ( $\mathrm{m}=20, \mathrm{k}=25)$

\begin{tabular}{|c|c|c|c|c|c|c|c|c|c|c|c|c|c|c|c|c|c|}
\hline \multirow{3}{*}{$\begin{array}{c}\text { L1 } \\
\text { Effect }\end{array}$} & \multirow{3}{*}{$\begin{array}{c}\text { Interaction } \\
\text { Effect }\end{array}$} & \multicolumn{16}{|c|}{ ICC Values } \\
\hline & & \multicolumn{4}{|c|}{0} & \multicolumn{3}{|c|}{0.1} & & \multicolumn{4}{|c|}{0.3} & \multicolumn{4}{|c|}{0.5} \\
\hline & & 0 & 0.2 & 0.5 & 0.8 & 0 & 0.2 & 0.5 & 0.8 & 0 & 0.2 & 0.5 & 0.8 & 0 & 0.2 & 0.5 & 0.8 \\
\hline \multirow{4}{*}{0} & 0 & 1.07 & 1.08 & 1.08 & 1.07 & 2.68 & 2.66 & 2.68 & 2.68 & 6.32 & 6.28 & 6.29 & 6.22 & 9.93 & 9.97 & 9.84 & 9.89 \\
\hline & 0.2 & 1.10 & 1.09 & 1.10 & 1.11 & 2.75 & 2.76 & 2.74 & 2.76 & 6.25 & 6.27 & 6.32 & 6.29 & 9.94 & 9.95 & 9.88 & 9.89 \\
\hline & 0.5 & 1.30 & 1.30 & 1.31 & 1.30 & 3.01 & 2.97 & 2.89 & 2.94 & 6.44 & 6.42 & 6.37 & 6.38 & 9.91 & 10.03 & 10.01 & 10.02 \\
\hline & 0.8 & 1.73 & 1.75 & 1.73 & 1.74 & 3.39 & 3.37 & 3.39 & 3.35 & 6.70 & 6.64 & 6.67 & 6.72 & 10.16 & 10.14 & 10.05 & 10.09 \\
\hline \multirow{4}{*}{0.2} & 0 & 1.10 & 1.11 & 1.10 & 1.10 & 2.75 & 2.74 & 2.73 & 2.68 & 6.28 & 6.23 & 6.33 & 6.34 & 9.81 & 9.91 & 9.93 & 9.90 \\
\hline & 0.2 & 1.15 & 1.14 & 1.14 & 1.14 & 2.76 & 2.78 & 2.75 & 2.77 & 6.23 & 6.28 & 6.21 & 6.22 & 9.85 & 9.89 & 9.95 & 9.95 \\
\hline & 0.5 & 1.35 & 1.35 & 1.36 & 1.35 & 2.95 & 2.99 & 2.98 & 3.03 & 6.40 & 6.51 & 6.45 & 6.47 & 10.03 & 10.01 & 10.05 & 10.01 \\
\hline & 0.8 & 1.78 & 1.81 & 1.83 & 1.77 & 3.36 & 3.42 & 3.41 & 3.41 & 6.67 & 6.72 & 6.74 & 6.63 & 10.18 & 10.08 & 10.10 & 10.09 \\
\hline \multirow{4}{*}{0.5} & 0 & 1.32 & 1.34 & 1.32 & 1.31 & 3.00 & 3.00 & 3.00 & 3.01 & 6.40 & 6.42 & 6.45 & 6.39 & 9.95 & 9.99 & 9.92 & 9.96 \\
\hline & 0.2 & 1.37 & 1.35 & 1.36 & 1.37 & 2.97 & 3.01 & 3.01 & 3.05 & 6.45 & 6.40 & 6.44 & 6.38 & 10.09 & 9.98 & 10.02 & 10.05 \\
\hline & 0.5 & 1.62 & 1.63 & 1.61 & 1.59 & 3.27 & 3.25 & 3.24 & 3.18 & 6.61 & 6.55 & 6.63 & 6.57 & 10.11 & 10.11 & 10.08 & 9.99 \\
\hline & 0.8 & 2.03 & 2.06 & 2.05 & 2.04 & 3.65 & 3.63 & 3.62 & 3.62 & 6.90 & 6.77 & 6.83 & 6.80 & 10.19 & 10.15 & 10.23 & 10.20 \\
\hline \multirow{4}{*}{0.8} & 0 & 1.80 & 1.84 & 1.82 & 1.79 & 3.43 & 3.39 & 3.44 & 3.45 & 6.71 & 6.72 & 6.72 & 6.75 & 10.10 & 10.13 & 10.16 & 10.13 \\
\hline & 0.2 & 1.88 & 1.86 & 1.89 & 1.87 & 3.41 & 3.47 & 3.42 & 3.43 & 6.69 & 6.74 & 6.63 & 6.66 & 10.12 & 10.13 & 10.16 & 10.17 \\
\hline & 0.5 & 2.07 & 2.09 & 2.08 & 2.11 & 3.63 & 3.65 & 3.65 & 3.59 & 6.88 & 6.82 & 6.84 & 6.77 & 10.14 & 10.25 & 10.20 & 10.21 \\
\hline & 0.8 & 2.51 & 2.50 & 2.48 & 2.52 & 3.98 & 3.97 & 4.01 & 4.01 & 7.07 & 7.12 & 7.10 & 7.02 & 10.29 & 10.40 & 10.33 & 10.31 \\
\hline
\end{tabular}

Note: ICC = Intraclass Correlation Coefficient. 
Table 3 cont.

Average Design Effect values for the Intercept: Uniform Distribution conditions $(\mathrm{m}=20, \mathrm{k}=50)$

\begin{tabular}{|c|c|c|c|c|c|c|c|c|c|c|c|c|c|c|c|c|c|}
\hline \multirow{4}{*}{$\begin{array}{c}\text { L1 } \\
\text { Effect }\end{array}$} & \multirow{4}{*}{$\begin{array}{l}\text { Interaction } \\
\text { Effect }\end{array}$} & \multicolumn{16}{|c|}{ ICC Values } \\
\hline & & \multicolumn{4}{|c|}{0} & \multirow{2}{*}{\multicolumn{3}{|c|}{0.1}} & & \multicolumn{4}{|c|}{0.3} & \multicolumn{4}{|c|}{0.5} \\
\hline & & & & & & & & & \multicolumn{2}{|c|}{ L2 Effect } & & & & & & & \\
\hline & & 0 & 0.2 & 0.5 & 0.8 & 0 & 0.2 & 0.5 & 0.8 & 0 & 0.2 & 0.5 & 0.8 & 0 & 0.2 & 0.5 & 0.8 \\
\hline \multirow{4}{*}{0} & 0 & 1.06 & 1.06 & 1.06 & 1.06 & 2.80 & 2.80 & 2.80 & 2.81 & 6.49 & 6.50 & 6.47 & 6.50 & 10.21 & 10.19 & 10.21 & 10.22 \\
\hline & 0.2 & 1.09 & 1.10 & 1.09 & 1.10 & 2.85 & 2.85 & 2.86 & 2.84 & 6.52 & 6.52 & 6.51 & 6.54 & 10.17 & 10.22 & 10.23 & 10.21 \\
\hline & 0.5 & 1.32 & 1.32 & 1.33 & 1.33 & 3.12 & 3.06 & 3.09 & 3.08 & 6.65 & 6.65 & 6.65 & 6.65 & 10.30 & 10.33 & 10.34 & 10.35 \\
\hline & 0.8 & 1.85 & 1.86 & 1.83 & 1.84 & 3.52 & 3.53 & 3.53 & 3.49 & 6.87 & 6.86 & 6.93 & 6.87 & 10.44 & 10.42 & 10.41 & 10.40 \\
\hline \multirow{4}{*}{0.2} & 0 & 1.09 & 1.09 & 1.10 & 1.09 & 2.83 & 2.85 & 2.83 & 2.84 & 6.47 & 6.50 & 6.51 & 6.52 & 10.29 & 10.24 & 10.24 & 10.21 \\
\hline & 0.2 & 1.13 & 1.13 & 1.13 & 1.14 & 2.89 & 2.90 & 2.90 & 2.90 & 6.56 & 6.53 & 6.57 & 6.53 & 10.28 & 10.28 & 10.27 & 10.22 \\
\hline & 0.5 & 1.38 & 1.39 & 1.38 & 1.39 & 3.10 & 3.14 & 3.12 & 3.15 & 6.72 & 6.66 & 6.68 & 6.66 & 10.29 & 10.29 & 10.30 & 10.32 \\
\hline & 0.8 & 1.90 & 1.88 & 1.90 & 1.90 & 3.53 & 3.57 & 3.53 & 3.54 & 6.92 & 6.87 & 6.96 & 6.97 & 10.43 & 10.43 & 10.47 & 10.44 \\
\hline \multirow{4}{*}{0.5} & 0 & 1.34 & 1.34 & 1.33 & 1.33 & 3.09 & 3.12 & 3.10 & 3.10 & 6.69 & 6.67 & 6.67 & 6.64 & 10.30 & 10.34 & 10.32 & 10.36 \\
\hline & 0.2 & 1.39 & 1.40 & 1.39 & 1.40 & 3.13 & 3.16 & 3.15 & 3.15 & 6.66 & 6.69 & 6.71 & 6.64 & 10.29 & 10.31 & 10.27 & 10.36 \\
\hline & 0.5 & 1.66 & 1.67 & 1.68 & 1.69 & 3.38 & 3.34 & 3.36 & 3.38 & 6.80 & 6.82 & 6.80 & 6.82 & 10.39 & 10.40 & 10.38 & 10.41 \\
\hline & 0.8 & 2.16 & 2.16 & 2.16 & 2.19 & 3.78 & 3.79 & 3.75 & 3.79 & 7.09 & 7.06 & 7.10 & 7.09 & 10.58 & 10.50 & 10.52 & 10.53 \\
\hline \multirow{4}{*}{0.8} & 0 & 1.86 & 1.86 & 1.87 & 1.88 & 3.53 & 3.52 & 3.52 & 3.51 & 6.96 & 6.94 & 6.92 & 6.90 & 10.42 & 10.44 & 10.46 & 10.46 \\
\hline & 0.2 & 1.92 & 1.94 & 1.94 & 1.92 & 3.59 & 3.59 & 3.59 & 3.57 & 6.99 & 6.97 & 6.94 & 6.96 & 10.43 & 10.52 & 10.51 & 10.44 \\
\hline & 0.5 & 2.17 & 2.20 & 2.21 & 2.19 & 3.77 & 3.79 & 3.80 & 3.78 & 7.14 & 7.11 & 7.12 & 7.14 & 10.53 & 10.52 & 10.48 & 10.51 \\
\hline & 0.8 & 2.69 & 2.63 & 2.66 & 2.63 & 4.19 & 4.17 & 4.23 & 4.21 & 7.33 & 7.37 & 7.38 & 7.35 & 10.65 & 10.59 & 10.60 & 10.70 \\
\hline
\end{tabular}

Note: ICC = Intraclass Correlation Coefficient. 
Table 3 cont.

Average Design Effect values for the Intercept: Uniform Distribution conditions $(\mathrm{m}=20, \mathrm{k}=75)$

\begin{tabular}{|c|c|c|c|c|c|c|c|c|c|c|c|c|c|c|c|c|c|}
\hline \multirow{3}{*}{$\begin{array}{c}\text { L1 } \\
\text { Effect }\end{array}$} & \multirow{3}{*}{$\begin{array}{c}\text { Interaction } \\
\text { Effect }\end{array}$} & \multicolumn{12}{|c|}{ ICC Values } & \multicolumn{4}{|c|}{0.5} \\
\hline & & \multicolumn{16}{|c|}{ L2 Effect } \\
\hline & & 0 & 0.2 & 0.5 & 0.8 & 0 & 0.2 & 0.5 & 0.8 & 0 & 0.2 & 0.5 & 0.8 & 0 & 0.2 & 0.5 & 0.8 \\
\hline \multirow{4}{*}{0} & 0 & 1.05 & 1.05 & 1.05 & 1.05 & 2.85 & 2.84 & 2.84 & 2.83 & 6.55 & 6.53 & 6.55 & 6.53 & 10.32 & 10.31 & 10.28 & 10.29 \\
\hline & 0.2 & 1.08 & 1.08 & 1.09 & 1.08 & 2.88 & 2.89 & 2.90 & 2.89 & 6.56 & 6.61 & 6.60 & 6.58 & 10.35 & 10.30 & 10.30 & 10.32 \\
\hline & 0.5 & 1.33 & 1.34 & 1.34 & 1.35 & 3.14 & 3.12 & 3.14 & 3.12 & 6.76 & 6.73 & 6.74 & 6.68 & 10.40 & 10.36 & 10.40 & 10.42 \\
\hline & 0.8 & 1.88 & 1.89 & 1.85 & 1.87 & 3.54 & 3.56 & 3.57 & 3.58 & 6.97 & 7.01 & 6.95 & 7.02 & 10.52 & 10.53 & 10.53 & 10.53 \\
\hline \multirow{4}{*}{0.2} & 0 & 1.08 & 1.08 & 1.09 & 1.08 & 2.88 & 2.87 & 2.88 & 2.86 & 6.59 & 6.59 & 6.58 & 6.58 & 10.30 & 10.28 & 10.33 & 10.31 \\
\hline & 0.2 & 1.13 & 1.12 & 1.12 & 1.13 & 2.93 & 2.95 & 2.96 & 2.93 & 6.60 & 6.63 & 6.61 & 6.63 & 10.31 & 10.37 & 10.34 & 10.36 \\
\hline & 0.5 & 1.39 & 1.41 & 1.39 & 1.40 & 3.13 & 3.15 & 3.17 & 3.19 & 6.76 & 6.79 & 6.74 & 6.71 & 10.39 & 10.41 & 10.40 & 10.41 \\
\hline & 0.8 & 1.93 & 1.93 & 1.92 & 1.93 & 3.60 & 3.60 & 3.59 & 3.61 & 7.01 & 7.02 & 7.03 & 6.99 & 10.53 & 10.61 & 10.55 & 10.55 \\
\hline \multirow{4}{*}{0.5} & 0 & 1.36 & 1.36 & 1.35 & 1.36 & 3.13 & 3.12 & 3.13 & 3.15 & 6.73 & 6.74 & 6.73 & 6.72 & 10.39 & 10.43 & 10.41 & 10.42 \\
\hline & 0.2 & 1.41 & 1.40 & 1.40 & 1.40 & 3.17 & 3.20 & 3.18 & 3.17 & 6.76 & 6.75 & 6.81 & 6.76 & 10.44 & 10.47 & 10.43 & 10.43 \\
\hline & 0.5 & 1.68 & 1.69 & 1.71 & 1.69 & 3.41 & 3.42 & 3.41 & 3.44 & 6.89 & 6.92 & 6.90 & 6.93 & 10.49 & 10.52 & 10.49 & 10.50 \\
\hline & 0.8 & 2.22 & 2.21 & 2.23 & 2.20 & 3.81 & 3.85 & 3.83 & 3.84 & 7.17 & 7.15 & 7.17 & 7.21 & 10.61 & 10.63 & 10.66 & 10.66 \\
\hline \multirow{4}{*}{0.8} & 0 & 1.89 & 1.90 & 1.90 & 1.90 & 3.59 & 3.60 & 3.60 & 3.61 & 6.99 & 7.03 & 7.05 & 7.09 & 10.54 & 10.58 & 10.56 & 10.55 \\
\hline & 0.2 & 1.95 & 1.95 & 1.97 & 1.95 & 3.65 & 3.59 & 3.62 & 3.61 & 7.01 & 7.04 & 7.05 & 7.03 & 10.58 & 10.58 & 10.56 & 10.56 \\
\hline & 0.5 & 2.22 & 2.25 & 2.23 & 2.24 & 3.83 & 3.87 & 3.86 & 3.86 & 7.18 & 7.12 & 7.19 & 7.21 & 10.62 & 10.64 & 10.61 & 10.62 \\
\hline & 0.8 & 2.74 & 2.72 & 2.72 & 2.72 & 4.26 & 4.25 & 4.24 & 4.28 & 7.40 & 7.46 & 7.47 & 7.43 & 10.76 & 10.73 & 10.78 & 10.80 \\
\hline
\end{tabular}

Note: ICC = Intraclass Correlation Coefficient. 
Table 3 cont.

Average Design Effect values for the Intercept: Uniform Distribution conditions $(\mathrm{m}=20, \mathrm{k}=100)$

\begin{tabular}{|c|c|c|c|c|c|c|c|c|c|c|c|c|c|c|c|c|c|}
\hline \multirow{3}{*}{$\begin{array}{c}\text { L1 } \\
\text { Effect }\end{array}$} & \multirow{3}{*}{$\begin{array}{c}\text { Interaction } \\
\text { Effect }\end{array}$} & \multicolumn{16}{|c|}{ ICC Values } \\
\hline & & \multicolumn{4}{|c|}{0} & \multicolumn{3}{|c|}{0.1} & & \multicolumn{4}{|c|}{0.3} & \multicolumn{4}{|c|}{0.5} \\
\hline & & 0 & 0.2 & 0.5 & 0.8 & 0 & 0.2 & 0.5 & 0.8 & 0 & 0.2 & 0.5 & 0.8 & 0 & 0.2 & 0.5 & 0.8 \\
\hline \multirow{4}{*}{0} & 0 & 1.05 & 1.05 & 1.05 & 1.05 & 2.84 & 2.87 & 2.84 & 2.84 & 6.61 & 6.61 & 6.55 & 6.57 & 10.35 & 10.37 & 10.37 & 10.34 \\
\hline & 0.2 & 1.08 & 1.08 & 1.08 & 1.09 & 2.88 & 2.91 & 2.89 & 2.90 & 6.64 & 6.61 & 6.63 & 6.64 & 10.40 & 10.38 & 10.33 & 10.36 \\
\hline & 0.5 & 1.35 & 1.35 & 1.35 & 1.34 & 3.14 & 3.14 & 3.14 & 3.15 & 6.75 & 6.78 & 6.74 & 6.74 & 10.49 & 10.46 & 10.44 & 10.46 \\
\hline & 0.8 & 1.90 & 1.89 & 1.89 & 1.89 & 3.59 & 3.61 & 3.58 & 3.59 & 7.06 & 7.04 & 7.03 & 7.06 & 10.61 & 10.61 & 10.53 & 10.62 \\
\hline \multirow{4}{*}{0.2} & 0 & 1.08 & 1.08 & 1.08 & 1.08 & 2.89 & 2.90 & 2.90 & 2.89 & 6.64 & 6.61 & 6.62 & 6.61 & 10.41 & 10.36 & 10.38 & 10.36 \\
\hline & 0.2 & 1.12 & 1.12 & 1.13 & 1.12 & 2.95 & 2.93 & 2.95 & 2.94 & 6.63 & 6.65 & 6.67 & 6.66 & 10.35 & 10.36 & 10.39 & 10.41 \\
\hline & 0.5 & 1.41 & 1.42 & 1.41 & 1.40 & 3.20 & 3.18 & 3.20 & 3.20 & 6.78 & 6.82 & 6.79 & 6.82 & 10.49 & 10.45 & 10.46 & 10.49 \\
\hline & 0.8 & 1.94 & 1.95 & 1.95 & 1.95 & 3.64 & 3.65 & 3.61 & 3.65 & 7.09 & 7.07 & 7.10 & 7.09 & 10.63 & 10.56 & 10.62 & 10.57 \\
\hline \multirow{4}{*}{0.5} & 0 & 1.36 & 1.36 & 1.36 & 1.36 & 3.15 & 3.16 & 3.13 & 3.15 & 6.76 & 6.77 & 6.77 & 6.81 & 10.47 & 10.39 & 10.47 & 10.48 \\
\hline & 0.2 & 1.43 & 1.42 & 1.41 & 1.40 & 3.18 & 3.21 & 3.21 & 3.22 & 6.82 & 6.79 & 6.77 & 6.80 & 10.46 & 10.51 & 10.48 & 10.44 \\
\hline & 0.5 & 1.71 & 1.72 & 1.72 & 1.71 & 3.44 & 3.45 & 3.42 & 3.43 & 6.93 & 6.93 & 6.93 & 6.92 & 10.53 & 10.54 & 10.51 & 10.57 \\
\hline & 0.8 & 2.24 & 2.22 & 2.25 & 2.22 & 3.87 & 3.86 & 3.87 & 3.85 & 7.21 & 7.21 & 7.19 & 7.20 & 10.68 & 10.67 & 10.75 & 10.70 \\
\hline \multirow{4}{*}{0.8} & 0 & 1.92 & 1.91 & 1.91 & 1.92 & 3.59 & 3.59 & 3.59 & 3.63 & 7.02 & 7.07 & 7.02 & 7.02 & 10.59 & 10.61 & 10.62 & 10.58 \\
\hline & 0.2 & 1.97 & 1.95 & 1.97 & 1.97 & 3.66 & 3.64 & 3.65 & 3.67 & 7.11 & 7.10 & 7.07 & 7.09 & 10.61 & 10.64 & 10.64 & 10.63 \\
\hline & 0.5 & 2.27 & 2.23 & 2.25 & 2.23 & 3.85 & 3.87 & 3.89 & 3.86 & 7.20 & 7.24 & 7.21 & 7.24 & 10.70 & 10.67 & 10.66 & 10.70 \\
\hline & 0.8 & 2.74 & 2.72 & 2.75 & 2.72 & 4.29 & 4.29 & 4.30 & 4.31 & 7.45 & 7.50 & 7.46 & 7.50 & 10.84 & 10.84 & 10.82 & 10.82 \\
\hline
\end{tabular}

Note: ICC = Intraclass Correlation Coefficient. 
Table 3 cont.

Average Design Effect values for the Intercept: Poisson Distribution conditions $(\mathrm{m}=5, \mathrm{k}=25)$

\begin{tabular}{|c|c|c|c|c|c|c|c|c|c|c|c|c|c|c|c|c|c|}
\hline \multirow{4}{*}{$\begin{array}{c}\text { L1 } \\
\text { Effect }\end{array}$} & \multirow{4}{*}{$\begin{array}{c}\text { Interaction } \\
\text { Effect }\end{array}$} & \multicolumn{16}{|c|}{ ICC Values } \\
\hline & & \multicolumn{4}{|c|}{0} & \multicolumn{4}{|c|}{0.1} & \multicolumn{4}{|c|}{0.3} & \multicolumn{4}{|c|}{0.5} \\
\hline & & & & & & \multicolumn{8}{|c|}{ L2 Effect } & \multirow[b]{2}{*}{0} & \multirow[b]{2}{*}{0.2} & \multirow[b]{2}{*}{0.5} & \multirow[b]{2}{*}{0.8} \\
\hline & & 0 & 0.2 & 0.5 & 0.8 & 0 & 0.2 & 0.5 & 0.8 & 0 & 0.2 & 0.5 & 0.8 & & & & \\
\hline \multirow{4}{*}{0} & 0 & 1.08 & 1.08 & 1.08 & 1.08 & 1.40 & 1.39 & 1.40 & 1.39 & 2.21 & 2.21 & 2.18 & 2.19 & 3.03 & 2.99 & 3.01 & 3.02 \\
\hline & 0.2 & 1.11 & 1.10 & 1.11 & 1.10 & 1.42 & 1.43 & 1.45 & 1.42 & 2.24 & 2.24 & 2.24 & 2.21 & 3.04 & 3.01 & 3.02 & 3.04 \\
\hline & 0.5 & 1.28 & 1.28 & 1.26 & 1.26 & 1.61 & 1.63 & 1.59 & 1.61 & 2.38 & 2.38 & 2.38 & 2.34 & 3.12 & 3.09 & 3.11 & 3.08 \\
\hline & 0.8 & 1.65 & 1.62 & 1.60 & 1.64 & 1.97 & 1.93 & 1.93 & 1.93 & 2.61 & 2.56 & 2.61 & 2.59 & 3.26 & 3.28 & 3.29 & 3.28 \\
\hline \multirow{4}{*}{0.2} & 0 & 1.11 & 1.11 & 1.11 & 1.10 & 1.43 & 1.44 & 1.43 & 1.44 & 2.25 & 2.24 & 2.23 & 2.22 & 3.01 & 3.01 & 3.03 & 3.02 \\
\hline & 0.2 & 1.14 & 1.12 & 1.14 & 1.14 & 1.46 & 1.46 & 1.47 & 1.47 & 2.25 & 2.24 & 2.24 & 2.25 & 3.07 & 3.05 & 3.06 & 3.05 \\
\hline & 0.5 & 1.31 & 1.30 & 1.32 & 1.31 & 1.66 & 1.67 & 1.65 & 1.66 & 2.37 & 2.41 & 2.39 & 2.39 & 3.14 & 3.16 & 3.13 & 3.11 \\
\hline & 0.8 & 1.63 & 1.66 & 1.65 & 1.68 & 1.98 & 1.98 & 1.99 & 1.97 & 2.61 & 2.63 & 2.60 & 2.63 & 3.26 & 3.29 & 3.29 & 3.27 \\
\hline \multirow{4}{*}{0.5} & 0 & 1.29 & 1.30 & 1.28 & 1.27 & 1.64 & 1.64 & 1.64 & 1.64 & 2.35 & 2.33 & 2.37 & 2.37 & 3.14 & 3.13 & 3.12 & 3.14 \\
\hline & 0.2 & 1.32 & 1.32 & 1.31 & 1.32 & 1.67 & 1.62 & 1.66 & 1.66 & 2.39 & 2.40 & 2.38 & 2.39 & 3.15 & 3.14 & 3.16 & 3.14 \\
\hline & 0.5 & 1.51 & 1.53 & 1.51 & 1.50 & 1.86 & 1.86 & 1.83 & 1.85 & 2.57 & 2.55 & 2.50 & 2.50 & 3.22 & 3.21 & 3.23 & 3.19 \\
\hline & 0.8 & 1.86 & 1.88 & 1.87 & 1.85 & 2.14 & 2.14 & 2.15 & 2.14 & 2.74 & 2.71 & 2.74 & 2.70 & 3.36 & 3.33 & 3.36 & 3.33 \\
\hline \multirow{4}{*}{0.8} & 0 & 1.66 & 1.66 & 1.70 & 1.65 & 1.99 & 1.97 & 1.98 & 1.99 & 2.65 & 2.65 & 2.64 & 2.59 & 3.29 & 3.28 & 3.25 & 3.29 \\
\hline & 0.2 & 1.72 & 1.73 & 1.71 & 1.71 & 2.04 & 2.04 & 2.02 & 1.99 & 2.64 & 2.61 & 2.63 & 2.66 & 3.28 & 3.32 & 3.30 & 3.27 \\
\hline & 0.5 & 1.94 & 1.88 & 1.89 & 1.89 & 2.20 & 2.20 & 2.18 & 2.17 & 2.73 & 2.72 & 2.77 & 2.75 & 3.36 & 3.37 & 3.34 & 3.35 \\
\hline & 0.8 & 2.16 & 2.21 & 2.19 & 2.20 & 2.48 & 2.45 & 2.42 & 2.41 & 2.95 & 2.96 & 2.96 & 2.96 & 3.51 & 3.47 & 3.50 & 3.50 \\
\hline
\end{tabular}

Note: ICC $=$ Intraclass Correlation Coefficient. 
Table 3 cont.

Average Design Effect values for the Intercept: Poisson Distribution conditions $(\mathrm{m}=5, \mathrm{k}=50)$

\begin{tabular}{|c|c|c|c|c|c|c|c|c|c|c|c|c|c|c|c|c|c|}
\hline \multirow{4}{*}{$\begin{array}{c}\text { L1 } \\
\text { Effect }\end{array}$} & \multirow{4}{*}{$\begin{array}{c}\text { Interaction } \\
\text { Effect }\end{array}$} & \multicolumn{16}{|c|}{ ICC Values } \\
\hline & & \multicolumn{4}{|c|}{0} & \multirow{2}{*}{\multicolumn{3}{|c|}{0.1}} & & \multicolumn{4}{|c|}{0.3} & \multicolumn{4}{|c|}{0.5} \\
\hline & & & & & & & & & \multicolumn{2}{|c|}{ L2 Effect } & & & & & & & \\
\hline & & 0 & 0.2 & 0.5 & 0.8 & 0 & 0.2 & 0.5 & 0.8 & 0 & 0.2 & 0.5 & 0.8 & 0 & 0.2 & 0.5 & 0.8 \\
\hline \multirow{4}{*}{0} & 0 & 1.07 & 1.07 & 1.07 & 1.07 & 1.42 & 1.43 & 1.42 & 1.43 & 2.25 & 2.26 & 2.26 & 2.26 & 3.06 & 3.07 & 3.07 & 3.05 \\
\hline & 0.2 & 1.09 & 1.10 & 1.09 & 1.09 & 1.46 & 1.47 & 1.46 & 1.46 & 2.28 & 2.28 & 2.29 & 2.27 & 3.09 & 3.09 & 3.10 & 3.09 \\
\hline & 0.5 & 1.29 & 1.29 & 1.28 & 1.30 & 1.67 & 1.67 & 1.64 & 1.67 & 2.43 & 2.42 & 2.43 & 2.43 & 3.17 & 3.19 & 3.18 & 3.17 \\
\hline & 0.8 & 1.66 & 1.67 & 1.69 & 1.70 & 2.01 & 2.00 & 2.02 & 2.00 & 2.68 & 2.65 & 2.66 & 2.66 & 3.32 & 3.33 & 3.32 & 3.33 \\
\hline \multirow{4}{*}{0.2} & 0 & 1.10 & 1.09 & 1.08 & 1.10 & 1.45 & 1.46 & 1.48 & 1.46 & 2.28 & 2.30 & 2.29 & 2.27 & 3.09 & 3.09 & 3.07 & 3.08 \\
\hline & 0.2 & 1.13 & 1.12 & 1.12 & 1.13 & 1.50 & 1.51 & 1.50 & 1.51 & 2.33 & 2.32 & 2.31 & 2.31 & 3.11 & 3.10 & 3.11 & 3.11 \\
\hline & 0.5 & 1.33 & 1.31 & 1.32 & 1.33 & 1.71 & 1.70 & 1.71 & 1.71 & 2.46 & 2.45 & 2.45 & 2.44 & 3.19 & 3.18 & 3.21 & 3.18 \\
\hline & 0.8 & 1.76 & 1.73 & 1.71 & 1.73 & 2.06 & 2.05 & 2.05 & 2.04 & 2.70 & 2.70 & 2.68 & 2.70 & 3.34 & 3.33 & 3.33 & 3.34 \\
\hline \multirow{4}{*}{0.5} & 0 & 1.28 & 1.30 & 1.30 & 1.30 & 1.69 & 1.67 & 1.67 & 1.68 & 2.42 & 2.43 & 2.43 & 2.44 & 3.17 & 3.17 & 3.17 & 3.15 \\
\hline & 0.2 & 1.33 & 1.33 & 1.34 & 1.33 & 1.72 & 1.73 & 1.72 & 1.73 & 2.46 & 2.46 & 2.48 & 2.46 & 3.19 & 3.17 & 3.19 & 3.20 \\
\hline & 0.5 & 1.55 & 1.56 & 1.55 & 1.57 & 1.91 & 1.90 & 1.90 & 1.90 & 2.59 & 2.58 & 2.60 & 2.59 & 3.25 & 3.29 & 3.26 & 3.26 \\
\hline & 0.8 & 1.93 & 1.94 & 1.93 & 1.93 & 2.24 & 2.23 & 2.22 & 2.24 & 2.82 & 2.82 & 2.83 & 2.83 & 3.44 & 3.43 & 3.42 & 3.40 \\
\hline \multirow{4}{*}{0.8} & 0 & 1.72 & 1.71 & 1.73 & 1.72 & 2.06 & 2.05 & 2.06 & 2.02 & 2.69 & 2.68 & 2.68 & 2.67 & 3.33 & 3.36 & 3.30 & 3.33 \\
\hline & 0.2 & 1.75 & 1.76 & 1.76 & 1.75 & 2.09 & 2.07 & 2.08 & 2.08 & 2.70 & 2.70 & 2.69 & 2.70 & 3.33 & 3.37 & 3.35 & 3.34 \\
\hline & 0.5 & 1.95 & 1.95 & 1.96 & 1.96 & 2.26 & 2.24 & 2.25 & 2.25 & 2.84 & 2.82 & 2.82 & 2.81 & 3.46 & 3.43 & 3.42 & 3.43 \\
\hline & 0.8 & 2.26 & 2.28 & 2.27 & 2.27 & 2.53 & 2.52 & 2.52 & 2.54 & 3.02 & 3.04 & 3.01 & 3.02 & 3.58 & 3.57 & 3.57 & 3.54 \\
\hline
\end{tabular}

Note: ICC $=$ Intraclass Correlation Coefficient. 
Table 3 cont.

Average Design Effect values for the Intercept: Poisson Distribution conditions $(\mathrm{m}=5, \mathrm{k}=75)$

\begin{tabular}{|c|c|c|c|c|c|c|c|c|c|c|c|c|c|c|c|c|c|}
\hline \multirow{4}{*}{$\begin{array}{c}\text { L1 } \\
\text { Effect }\end{array}$} & \multirow{4}{*}{$\begin{array}{l}\text { Interaction } \\
\text { Effect }\end{array}$} & \multicolumn{16}{|c|}{ ICC Values } \\
\hline & & \multicolumn{4}{|c|}{0} & \multirow{2}{*}{\multicolumn{3}{|c|}{0.1}} & & \multicolumn{4}{|c|}{0.3} & \multicolumn{4}{|c|}{0.5} \\
\hline & & & & & & & & & \multicolumn{2}{|c|}{ L2 Effect } & & & & & & & \\
\hline & & 0 & 0.2 & 0.5 & 0.8 & 0 & 0.2 & 0.5 & 0.8 & 0 & 0.2 & 0.5 & 0.8 & 0 & 0.2 & 0.5 & 0.8 \\
\hline \multirow{4}{*}{0} & 0 & 1.06 & 1.05 & 1.05 & 1.05 & 1.44 & 1.44 & 1.43 & 1.42 & 2.27 & 2.28 & 2.28 & 2.27 & 3.10 & 3.09 & 3.08 & 3.10 \\
\hline & 0.2 & 1.08 & 1.08 & 1.08 & 1.08 & 1.47 & 1.48 & 1.48 & 1.47 & 2.31 & 2.31 & 2.31 & 2.31 & 3.10 & 3.12 & 3.12 & 3.11 \\
\hline & 0.5 & 1.30 & 1.29 & 1.29 & 1.29 & 1.68 & 1.69 & 1.68 & 1.68 & 2.45 & 2.45 & 2.44 & 2.45 & 3.21 & 3.19 & 3.20 & 3.18 \\
\hline & 0.8 & 1.71 & 1.72 & 1.72 & 1.71 & 2.06 & 2.04 & 2.04 & 2.03 & 2.69 & 2.70 & 2.70 & 2.67 & 3.33 & 3.35 & 3.33 & 3.33 \\
\hline \multirow{4}{*}{0.2} & 0 & 1.08 & 1.08 & 1.08 & 1.08 & 1.48 & 1.47 & 1.47 & 1.49 & 2.32 & 2.30 & 2.31 & 2.31 & 3.09 & 3.11 & 3.10 & 3.10 \\
\hline & 0.2 & 1.12 & 1.11 & 1.12 & 1.11 & 1.52 & 1.52 & 1.52 & 1.52 & 2.33 & 2.34 & 2.34 & 2.33 & 3.13 & 3.13 & 3.12 & 3.13 \\
\hline & 0.5 & 1.34 & 1.33 & 1.33 & 1.34 & 1.72 & 1.74 & 1.75 & 1.73 & 2.47 & 2.48 & 2.48 & 2.49 & 3.21 & 3.21 & 3.21 & 3.22 \\
\hline & 0.8 & 1.76 & 1.74 & 1.76 & 1.77 & 2.09 & 2.09 & 2.08 & 2.08 & 2.71 & 2.73 & 2.72 & 2.72 & 3.37 & 3.38 & 3.35 & 3.36 \\
\hline \multirow{4}{*}{0.5} & 0 & 1.30 & 1.30 & 1.29 & 1.31 & 1.68 & 1.69 & 1.70 & 1.68 & 2.46 & 2.46 & 2.46 & 2.46 & 3.21 & 3.19 & 3.19 & 3.19 \\
\hline & 0.2 & 1.35 & 1.34 & 1.34 & 1.35 & 1.73 & 1.74 & 1.74 & 1.72 & 2.47 & 2.47 & 2.48 & 2.47 & 3.21 & 3.22 & 3.20 & 3.21 \\
\hline & 0.5 & 1.59 & 1.56 & 1.58 & 1.57 & 1.94 & 1.93 & 1.94 & 1.92 & 2.58 & 2.60 & 2.61 & 2.62 & 3.30 & 3.28 & 3.30 & 3.28 \\
\hline & 0.8 & 1.97 & 1.96 & 1.98 & 1.98 & 2.24 & 2.25 & 2.26 & 2.24 & 2.86 & 2.83 & 2.83 & 2.83 & 3.44 & 3.42 & 3.44 & 3.43 \\
\hline \multirow{4}{*}{0.8} & 0 & 1.74 & 1.74 & 1.74 & 1.74 & 2.06 & 2.08 & 2.06 & 2.07 & 2.70 & 2.71 & 2.70 & 2.70 & 3.35 & 3.35 & 3.36 & 3.35 \\
\hline & 0.2 & 1.78 & 1.77 & 1.78 & 1.77 & 2.10 & 2.11 & 2.11 & 2.11 & 2.73 & 2.72 & 2.73 & 2.71 & 3.38 & 3.37 & 3.37 & 3.35 \\
\hline & 0.5 & 1.99 & 1.98 & 1.99 & 1.98 & 2.27 & 2.26 & 2.28 & 2.28 & 2.84 & 2.85 & 2.85 & 2.84 & 3.42 & 3.44 & 3.44 & 3.45 \\
\hline & 0.8 & 2.32 & 2.32 & 2.30 & 2.30 & 2.55 & 2.53 & 2.56 & 2.56 & 3.04 & 3.04 & 3.06 & 3.05 & 3.57 & 3.57 & 3.56 & 3.56 \\
\hline
\end{tabular}

Note: ICC $=$ Intraclass Correlation Coefficient. 
Table 3 cont.

Average Design Effect values for the Intercept: Poisson Distribution conditions $(\mathrm{m}=5, \mathrm{k}=100)$

\begin{tabular}{|c|c|c|c|c|c|c|c|c|c|c|c|c|c|c|c|c|c|}
\hline \multirow{4}{*}{$\begin{array}{c}\text { L1 } \\
\text { Effect }\end{array}$} & \multirow{4}{*}{$\begin{array}{c}\text { Interaction } \\
\text { Effect }\end{array}$} & \multicolumn{16}{|c|}{ ICC Values } \\
\hline & & \multicolumn{4}{|c|}{0} & \multirow{2}{*}{\multicolumn{3}{|c|}{0.1}} & & \multicolumn{4}{|c|}{0.3} & \multicolumn{4}{|c|}{0.5} \\
\hline & & \multirow[b]{2}{*}{0} & \multirow[b]{2}{*}{0.2} & \multirow[b]{2}{*}{0.5} & \multirow[b]{2}{*}{0.8} & & & & \multicolumn{2}{|c|}{ L2 Effect } & \multirow[b]{2}{*}{0.2} & \multirow[b]{2}{*}{0.5} & \multirow[b]{2}{*}{0.8} & \multirow[b]{2}{*}{0} & \multirow[b]{2}{*}{0.2} & \multirow[b]{2}{*}{0.5} & \multirow[b]{2}{*}{0.8} \\
\hline & & & & & & 0 & 0.2 & 0.5 & 0.8 & 0 & & & & & & & \\
\hline \multirow{4}{*}{0} & 0 & 1.05 & 1.04 & 1.05 & 1.05 & 1.45 & 1.44 & 1.44 & 1.45 & 2.30 & 2.29 & 2.30 & 2.30 & 3.09 & 3.09 & 3.10 & 3.08 \\
\hline & 0.2 & 1.08 & 1.07 & 1.08 & 1.07 & 1.48 & 1.48 & 1.48 & 1.49 & 2.31 & 2.31 & 2.32 & 2.32 & 3.12 & 3.11 & 3.11 & 3.12 \\
\hline & 0.5 & 1.31 & 1.29 & 1.29 & 1.30 & 1.70 & 1.71 & 1.69 & 1.70 & 2.45 & 2.46 & 2.45 & 2.46 & 3.20 & 3.18 & 3.20 & 3.20 \\
\hline & 0.8 & 1.72 & 1.71 & 1.73 & 1.72 & 2.06 & 2.06 & 2.06 & 2.06 & 2.72 & 2.71 & 2.70 & 2.71 & 3.35 & 3.34 & 3.37 & 3.35 \\
\hline \multirow{4}{*}{0.2} & 0 & 1.07 & 1.08 & 1.08 & 1.07 & 1.48 & 1.48 & 1.47 & 1.49 & 2.31 & 2.31 & 2.32 & 2.31 & 3.11 & 3.12 & 3.11 & 3.12 \\
\hline & 0.2 & 1.11 & 1.11 & 1.11 & 1.11 & 1.53 & 1.52 & 1.53 & 1.52 & 2.34 & 2.34 & 2.35 & 2.35 & 3.13 & 3.12 & 3.12 & 3.13 \\
\hline & 0.5 & 1.33 & 1.34 & 1.33 & 1.34 & 1.74 & 1.74 & 1.75 & 1.74 & 2.48 & 2.48 & 2.49 & 2.49 & 3.21 & 3.22 & 3.21 & 3.21 \\
\hline & 0.8 & 1.75 & 1.76 & 1.77 & 1.76 & 2.09 & 2.09 & 2.10 & 2.10 & 2.73 & 2.72 & 2.74 & 2.73 & 3.37 & 3.35 & 3.37 & 3.38 \\
\hline \multirow{4}{*}{0.5} & 0 & 1.30 & 1.30 & 1.30 & 1.30 & 1.69 & 1.70 & 1.70 & 1.71 & 2.47 & 2.46 & 2.47 & 2.44 & 3.20 & 3.21 & 3.20 & 3.22 \\
\hline & 0.2 & 1.35 & 1.36 & 1.35 & 1.36 & 1.74 & 1.73 & 1.75 & 1.75 & 2.47 & 2.48 & 2.49 & 2.50 & 3.22 & 3.21 & 3.23 & 3.22 \\
\hline & 0.5 & 1.58 & 1.59 & 1.58 & 1.58 & 1.95 & 1.94 & 1.95 & 1.95 & 2.63 & 2.62 & 2.62 & 2.63 & 3.30 & 3.30 & 3.32 & 3.30 \\
\hline & 0.8 & 1.99 & 1.98 & 1.98 & 1.97 & 2.26 & 2.26 & 2.27 & 2.27 & 2.86 & 2.85 & 2.86 & 2.84 & 3.44 & 3.45 & 3.44 & 3.44 \\
\hline \multirow{4}{*}{0.8} & 0 & 1.74 & 1.74 & 1.76 & 1.75 & 2.07 & 2.09 & 2.06 & 2.07 & 2.72 & 2.72 & 2.71 & 2.72 & 3.36 & 3.36 & 3.37 & 3.37 \\
\hline & 0.2 & 1.80 & 1.78 & 1.78 & 1.78 & 2.10 & 2.11 & 2.12 & 2.11 & 2.73 & 2.73 & 2.74 & 2.73 & 3.38 & 3.37 & 3.37 & 3.38 \\
\hline & 0.5 & 1.99 & 1.99 & 1.98 & 1.98 & 2.29 & 2.26 & 2.28 & 2.28 & 2.85 & 2.84 & 2.85 & 2.86 & 3.43 & 3.44 & 3.45 & 3.45 \\
\hline & 0.8 & 2.30 & 2.32 & 2.33 & 2.33 & 2.57 & 2.55 & 2.57 & 2.57 & 3.06 & 3.06 & 3.05 & 3.07 & 3.57 & 3.58 & 3.58 & 3.59 \\
\hline
\end{tabular}

Note: ICC $=$ Intraclass Correlation Coefficient. 
Table 3 cont.

Average Design Effect values for the Intercept: Poisson Distribution conditions $(\mathrm{m}=10, \mathrm{k}=25)$

\begin{tabular}{|c|c|c|c|c|c|c|c|c|c|c|c|c|c|c|c|c|c|}
\hline \multirow{4}{*}{$\begin{array}{c}\text { L1 } \\
\text { Effect }\end{array}$} & \multirow{4}{*}{$\begin{array}{l}\text { Interaction } \\
\text { Effect }\end{array}$} & \multicolumn{16}{|c|}{ ICC Values } \\
\hline & & \multicolumn{4}{|c|}{0} & \multirow{2}{*}{\multicolumn{3}{|c|}{0.1}} & & \multicolumn{4}{|c|}{0.3} & \multicolumn{4}{|c|}{0.5} \\
\hline & & \multirow[b]{2}{*}{0} & \multirow[b]{2}{*}{0.2} & \multirow[b]{2}{*}{0.5} & \multirow[b]{2}{*}{0.8} & & & & \multicolumn{2}{|c|}{ L2 Effect } & \multirow[b]{2}{*}{0.2} & \multirow[b]{2}{*}{0.5} & \multirow[b]{2}{*}{0.8} & \multirow[b]{2}{*}{0} & \multirow[b]{2}{*}{0.2} & \multirow[b]{2}{*}{0.5} & \multirow[b]{2}{*}{0.8} \\
\hline & & & & & & 0 & 0.2 & 0.5 & 0.8 & 0 & & & & & & & \\
\hline \multirow{4}{*}{0} & 0 & 1.08 & 1.07 & 1.07 & 1.07 & 1.82 & 1.82 & 1.82 & 1.81 & 3.57 & 3.58 & 3.56 & 3.55 & 5.32 & 5.32 & 5.34 & 5.31 \\
\hline & 0.2 & 1.11 & 1.11 & 1.10 & 1.11 & 1.87 & 1.85 & 1.88 & 1.85 & 3.53 & 3.60 & 3.58 & 3.54 & 5.34 & 5.35 & 5.35 & 5.34 \\
\hline & 0.5 & 1.28 & 1.32 & 1.29 & 1.30 & 2.07 & 2.09 & 2.08 & 2.08 & 3.74 & 3.73 & 3.75 & 3.68 & 5.39 & 5.41 & 5.38 & 5.39 \\
\hline & 0.8 & 1.71 & 1.69 & 1.74 & 1.69 & 2.49 & 2.49 & 2.45 & 2.50 & 4.02 & 3.98 & 4.00 & 3.95 & 5.53 & 5.58 & 5.53 & 5.59 \\
\hline \multirow{4}{*}{0.2} & 0 & 1.11 & 1.11 & 1.11 & 1.11 & 1.87 & 1.86 & 1.87 & 1.86 & 3.58 & 3.60 & 3.59 & 3.60 & 5.31 & 5.35 & 5.32 & 5.40 \\
\hline & 0.2 & 1.13 & 1.14 & 1.15 & 1.15 & 1.89 & 1.91 & 1.90 & 1.90 & 3.58 & 3.58 & 3.64 & 3.63 & 5.31 & 5.36 & 5.34 & 5.31 \\
\hline & 0.5 & 1.34 & 1.34 & 1.35 & 1.33 & 2.11 & 2.14 & 2.12 & 2.14 & 3.77 & 3.75 & 3.74 & 3.78 & 5.45 & 5.45 & 5.41 & 5.42 \\
\hline & 0.8 & 1.74 & 1.76 & 1.77 & 1.77 & 2.52 & 2.54 & 2.49 & 2.50 & 4.06 & 4.01 & 4.04 & 4.02 & 5.59 & 5.55 & 5.58 & 5.59 \\
\hline \multirow{4}{*}{0.5} & 0 & 1.31 & 1.33 & 1.29 & 1.32 & 2.12 & 2.10 & 2.11 & 2.13 & 3.74 & 3.72 & 3.74 & 3.70 & 5.43 & 5.43 & 5.45 & 5.43 \\
\hline & 0.2 & 1.35 & 1.37 & 1.37 & 1.35 & 2.15 & 2.16 & 2.13 & 2.14 & 3.79 & 3.78 & 3.77 & 3.77 & 5.43 & 5.41 & 5.46 & 5.50 \\
\hline & 0.5 & 1.58 & 1.59 & 1.57 & 1.59 & 2.35 & 2.39 & 2.37 & 2.34 & 3.92 & 3.90 & 3.95 & 3.87 & 5.52 & 5.46 & 5.55 & 5.51 \\
\hline & 0.8 & 2.00 & 2.03 & 2.00 & 1.98 & 2.72 & 2.68 & 2.74 & 2.73 & 4.18 & 4.15 & 4.17 & 4.16 & 5.65 & 5.66 & 5.66 & 5.69 \\
\hline \multirow{4}{*}{0.8} & 0 & 1.78 & 1.76 & 1.78 & 1.78 & 2.54 & 2.52 & 2.50 & 2.52 & 3.99 & 4.02 & 4.02 & 4.02 & 5.61 & 5.60 & 5.69 & 5.63 \\
\hline & 0.2 & 1.82 & 1.82 & 1.83 & 1.81 & 2.56 & 2.54 & 2.56 & 2.56 & 4.07 & 4.02 & 4.02 & 4.06 & 5.62 & 5.57 & 5.60 & 5.59 \\
\hline & 0.5 & 2.02 & 2.08 & 2.04 & 2.03 & 2.74 & 2.75 & 2.73 & 2.73 & 4.16 & 4.17 & 4.17 & 4.20 & 5.66 & 5.67 & 5.63 & 5.67 \\
\hline & 0.8 & 2.46 & 2.41 & 2.46 & 2.46 & 3.09 & 3.11 & 3.07 & 3.10 & 4.40 & 4.41 & 4.39 & 4.39 & 5.81 & 5.80 & 5.78 & 5.81 \\
\hline
\end{tabular}

Note: ICC $=$ Intraclass Correlation Coefficient. 
Table 3 cont.

Average Design Effect values for the Intercept: Poisson Distribution conditions $(\mathrm{m}=10, \mathrm{k}=50)$

\begin{tabular}{|c|c|c|c|c|c|c|c|c|c|c|c|c|c|c|c|c|c|}
\hline \multirow{4}{*}{$\begin{array}{c}\text { L1 } \\
\text { Effect }\end{array}$} & \multirow{4}{*}{$\begin{array}{l}\text { Interaction } \\
\text { Effect }\end{array}$} & \multicolumn{16}{|c|}{ ICC Values } \\
\hline & & \multicolumn{4}{|c|}{0} & \multirow{2}{*}{\multicolumn{3}{|c|}{0.1}} & & \multicolumn{4}{|c|}{0.3} & \multicolumn{4}{|c|}{0.5} \\
\hline & & & & & & & & & \multicolumn{2}{|c|}{ L2 Effect } & & & & & & & \\
\hline & & 0 & 0.2 & 0.5 & 0.8 & 0 & 0.2 & 0.5 & 0.8 & 0 & 0.2 & 0.5 & 0.8 & 0 & 0.2 & 0.5 & 0.8 \\
\hline \multirow{4}{*}{0} & 0 & 1.06 & 1.06 & 1.06 & 1.06 & 1.88 & 1.89 & 1.87 & 1.88 & 3.68 & 3.67 & 3.64 & 3.65 & 5.42 & 5.44 & 5.43 & 5.47 \\
\hline & 0.2 & 1.09 & 1.09 & 1.09 & 1.09 & 1.92 & 1.93 & 1.92 & 1.90 & 3.70 & 3.67 & 3.71 & 3.71 & 5.46 & 5.48 & 5.47 & 5.45 \\
\hline & 0.5 & 1.31 & 1.32 & 1.31 & 1.31 & 2.16 & 2.15 & 2.16 & 2.17 & 3.84 & 3.84 & 3.84 & 3.83 & 5.54 & 5.53 & 5.54 & 5.53 \\
\hline & 0.8 & 1.82 & 1.78 & 1.82 & 1.81 & 2.59 & 2.59 & 2.61 & 2.60 & 4.12 & 4.13 & 4.10 & 4.09 & 5.70 & 5.75 & 5.72 & 5.70 \\
\hline \multirow{4}{*}{0.2} & 0 & 1.09 & 1.10 & 1.09 & 1.09 & 1.94 & 1.92 & 1.92 & 1.94 & 3.72 & 3.69 & 3.67 & 3.69 & 5.49 & 5.47 & 5.45 & 5.45 \\
\hline & 0.2 & 1.13 & 1.12 & 1.13 & 1.14 & 1.98 & 1.98 & 1.97 & 1.96 & 3.75 & 3.72 & 3.74 & 3.74 & 5.48 & 5.51 & 5.48 & 5.53 \\
\hline & 0.5 & 1.37 & 1.38 & 1.39 & 1.36 & 2.21 & 2.23 & 2.21 & 2.22 & 3.89 & 3.86 & 3.90 & 3.89 & 5.58 & 5.56 & 5.57 & 5.57 \\
\hline & 0.8 & 1.85 & 1.84 & 1.84 & 1.86 & 2.63 & 2.63 & 2.61 & 2.62 & 4.16 & 4.16 & 4.14 & 4.16 & 5.75 & 5.71 & 5.72 & 5.72 \\
\hline \multirow{4}{*}{0.5} & 0 & 1.32 & 1.33 & 1.33 & 1.33 & 2.17 & 2.20 & 2.18 & 2.16 & 3.84 & 3.83 & 3.87 & 3.84 & 5.57 & 5.58 & 5.53 & 5.53 \\
\hline & 0.2 & 1.38 & 1.39 & 1.38 & 1.39 & 2.21 & 2.23 & 2.23 & 2.22 & 3.90 & 3.87 & 3.89 & 3.90 & 5.56 & 5.58 & 5.59 & 5.56 \\
\hline & 0.5 & 1.63 & 1.66 & 1.65 & 1.65 & 2.46 & 2.42 & 2.46 & 2.46 & 4.06 & 4.03 & 4.04 & 4.03 & 5.64 & 5.69 & 5.70 & 5.65 \\
\hline & 0.8 & 2.11 & 2.12 & 2.07 & 2.12 & 2.85 & 2.86 & 2.86 & 2.83 & 4.29 & 4.33 & 4.29 & 4.30 & 5.88 & 5.78 & 5.81 & 5.79 \\
\hline \multirow{4}{*}{0.8} & 0 & 1.84 & 1.85 & 1.85 & 1.84 & 2.59 & 2.62 & 2.60 & 2.60 & 4.15 & 4.15 & 4.15 & 4.13 & 5.71 & 5.74 & 5.72 & 5.67 \\
\hline & 0.2 & 1.88 & 1.88 & 1.90 & 1.87 & 2.63 & 2.64 & 2.65 & 2.65 & 4.15 & 4.15 & 4.18 & 4.13 & 5.73 & 5.73 & 5.75 & 5.77 \\
\hline & 0.5 & 2.14 & 2.13 & 2.14 & 2.15 & 2.86 & 2.85 & 2.87 & 2.85 & 4.29 & 4.29 & 4.30 & 4.31 & 5.82 & 5.84 & 5.82 & 5.80 \\
\hline & 0.8 & 2.55 & 2.54 & 2.56 & 2.51 & 3.25 & 3.22 & 3.20 & 3.21 & 4.54 & 4.59 & 4.52 & 4.56 & 5.97 & 5.99 & 5.94 & 5.96 \\
\hline
\end{tabular}

Note: ICC $=$ Intraclass Correlation Coefficient. 
Table 3 cont.

Average Design Effect values for the Intercept: Poisson Distribution conditions $(\mathrm{m}=10, \mathrm{k}=75)$

\begin{tabular}{|c|c|c|c|c|c|c|c|c|c|c|c|c|c|c|c|c|c|}
\hline \multirow{4}{*}{$\begin{array}{c}\text { L1 } \\
\text { Effect }\end{array}$} & \multirow{4}{*}{$\begin{array}{l}\text { Interaction } \\
\text { Effect }\end{array}$} & \multicolumn{16}{|c|}{ ICC Values } \\
\hline & & \multicolumn{4}{|c|}{0} & \multirow{2}{*}{\multicolumn{3}{|c|}{0.1}} & & \multicolumn{4}{|c|}{0.3} & \multicolumn{4}{|c|}{0.5} \\
\hline & & & & & & & & & \multicolumn{2}{|c|}{ L2 Effect } & & & & & & & \\
\hline & & 0 & 0.2 & 0.5 & 0.8 & 0 & 0.2 & 0.5 & 0.8 & 0 & 0.2 & 0.5 & 0.8 & 0 & 0.2 & 0.5 & 0.8 \\
\hline \multirow{4}{*}{0} & 0 & 1.05 & 1.05 & 1.05 & 1.06 & 1.92 & 1.90 & 1.92 & 1.91 & 3.71 & 3.70 & 3.70 & 3.70 & 5.47 & 5.48 & 5.49 & 5.47 \\
\hline & 0.2 & 1.09 & 1.09 & 1.09 & 1.09 & 1.96 & 1.96 & 1.96 & 1.96 & 3.72 & 3.69 & 3.73 & 3.74 & 5.52 & 5.53 & 5.52 & 5.49 \\
\hline & 0.5 & 1.32 & 1.33 & 1.33 & 1.32 & 2.20 & 2.21 & 2.19 & 2.20 & 3.86 & 3.90 & 3.92 & 3.89 & 5.59 & 5.57 & 5.61 & 5.57 \\
\hline & 0.8 & 1.83 & 1.84 & 1.83 & 1.83 & 2.62 & 2.61 & 2.62 & 2.60 & 4.16 & 4.16 & 4.16 & 4.20 & 5.79 & 5.73 & 5.74 & 5.72 \\
\hline \multirow{4}{*}{0.2} & 0 & 1.09 & 1.08 & 1.08 & 1.09 & 1.95 & 1.97 & 1.97 & 1.95 & 3.74 & 3.75 & 3.77 & 3.73 & 5.50 & 5.51 & 5.48 & 5.48 \\
\hline & 0.2 & 1.12 & 1.12 & 1.13 & 1.12 & 2.00 & 1.99 & 2.01 & 2.01 & 3.76 & 3.76 & 3.76 & 3.75 & 5.51 & 5.51 & 5.54 & 5.53 \\
\hline & 0.5 & 1.38 & 1.38 & 1.38 & 1.38 & 2.25 & 2.25 & 2.24 & 2.23 & 3.91 & 3.92 & 3.91 & 3.91 & 5.59 & 5.60 & 5.62 & 5.61 \\
\hline & 0.8 & 1.89 & 1.89 & 1.89 & 1.87 & 2.67 & 2.65 & 2.66 & 2.66 & 4.22 & 4.19 & 4.20 & 4.19 & 5.78 & 5.77 & 5.78 & 5.81 \\
\hline \multirow{4}{*}{0.5} & 0 & 1.34 & 1.34 & 1.33 & 1.34 & 2.20 & 2.21 & 2.22 & 2.21 & 3.89 & 3.90 & 3.91 & 3.91 & 5.58 & 5.62 & 5.62 & 5.61 \\
\hline & 0.2 & 1.39 & 1.39 & 1.40 & 1.39 & 2.24 & 2.25 & 2.23 & 2.25 & 3.92 & 3.91 & 3.92 & 3.90 & 5.61 & 5.62 & 5.62 & 5.62 \\
\hline & 0.5 & 1.68 & 1.65 & 1.66 & 1.65 & 2.48 & 2.48 & 2.48 & 2.45 & 4.06 & 4.08 & 4.06 & 4.07 & 5.69 & 5.71 & 5.69 & 5.72 \\
\hline & 0.8 & 2.13 & 2.15 & 2.16 & 2.14 & 2.87 & 2.88 & 2.89 & 2.89 & 4.34 & 4.34 & 4.37 & 4.36 & 5.87 & 5.84 & 5.84 & 5.85 \\
\hline \multirow{4}{*}{0.8} & 0 & 1.86 & 1.85 & 1.87 & 1.84 & 2.63 & 2.63 & 2.63 & 2.64 & 4.17 & 4.18 & 4.17 & 4.21 & 5.77 & 5.79 & 5.76 & 5.75 \\
\hline & 0.2 & 1.91 & 1.91 & 1.90 & 1.90 & 2.68 & 2.67 & 2.68 & 2.69 & 4.21 & 4.19 & 4.20 & 4.19 & 5.79 & 5.75 & 5.79 & 5.77 \\
\hline & 0.5 & 2.15 & 2.16 & 2.15 & 2.18 & 2.90 & 2.87 & 2.91 & 2.90 & 4.36 & 4.36 & 4.36 & 4.34 & 5.86 & 5.86 & 5.86 & 5.87 \\
\hline & 0.8 & 2.59 & 2.63 & 2.58 & 2.58 & 3.26 & 3.25 & 3.28 & 3.26 & 4.60 & 4.58 & 4.60 & 4.58 & 6.01 & 6.01 & 6.03 & 5.99 \\
\hline
\end{tabular}

Note: ICC $=$ Intraclass Correlation Coefficient. 
Table 3 cont.

Average Design Effect values for the Intercept: Poisson Distribution conditions $(\mathrm{m}=10, \mathrm{k}=100)$

\begin{tabular}{|c|c|c|c|c|c|c|c|c|c|c|c|c|c|c|c|c|c|}
\hline \multirow{4}{*}{$\begin{array}{c}\text { L1 } \\
\text { Effect }\end{array}$} & \multirow{4}{*}{$\begin{array}{c}\text { Interaction } \\
\text { Effect }\end{array}$} & \multicolumn{16}{|c|}{ ICC Values } \\
\hline & & \multicolumn{4}{|c|}{0} & \multicolumn{4}{|c|}{0.1} & \multicolumn{4}{|c|}{0.3} & \multicolumn{4}{|c|}{0.5} \\
\hline & & \multicolumn{12}{|c|}{ L2 Effect } & & & & \\
\hline & & 0 & 0.2 & 0.5 & 0.8 & 0 & 0.2 & 0.5 & 0.8 & 0 & 0.2 & 0.5 & 0.8 & 0 & 0.2 & 0.5 & 0.8 \\
\hline \multirow{4}{*}{0} & 0 & 1.05 & 1.05 & 1.05 & 1.05 & 1.92 & 1.92 & 1.93 & 1.92 & 3.73 & 3.73 & 3.71 & 3.72 & 5.51 & 5.51 & 5.52 & 5.50 \\
\hline & 0.2 & 1.08 & 1.08 & 1.08 & 1.08 & 1.95 & 1.97 & 1.98 & 1.97 & 3.75 & 3.76 & 3.77 & 3.74 & 5.53 & 5.53 & 5.53 & 5.51 \\
\hline & 0.5 & 1.34 & 1.34 & 1.34 & 1.35 & 2.20 & 2.21 & 2.22 & 2.22 & 3.90 & 3.89 & 3.91 & 3.91 & 5.60 & 5.60 & 5.62 & 5.59 \\
\hline & 0.8 & 1.84 & 1.85 & 1.84 & 1.84 & 2.65 & 2.63 & 2.63 & 2.65 & 4.21 & 4.20 & 4.18 & 4.20 & 5.79 & 5.77 & 5.77 & 5.79 \\
\hline \multirow{4}{*}{0.2} & 0 & 1.08 & 1.08 & 1.09 & 1.08 & 1.97 & 1.97 & 1.97 & 1.97 & 3.77 & 3.74 & 3.75 & 3.76 & 5.55 & 5.53 & 5.51 & 5.56 \\
\hline & 0.2 & 1.12 & 1.13 & 1.12 & 1.12 & 2.02 & 2.02 & 2.01 & 2.02 & 3.79 & 3.78 & 3.79 & 3.79 & 5.54 & 5.54 & 5.56 & 5.54 \\
\hline & 0.5 & 1.40 & 1.39 & 1.38 & 1.40 & 2.25 & 2.26 & 2.26 & 2.25 & 3.93 & 3.95 & 3.94 & 3.96 & 5.62 & 5.65 & 5.65 & 5.65 \\
\hline & 0.8 & 1.89 & 1.88 & 1.89 & 1.89 & 2.68 & 2.68 & 2.68 & 2.71 & 4.22 & 4.21 & 4.24 & 4.22 & 5.78 & 5.81 & 5.78 & 5.75 \\
\hline \multirow{4}{*}{0.5} & 0 & 1.34 & 1.35 & 1.34 & 1.33 & 2.21 & 2.22 & 2.22 & 2.20 & 3.92 & 3.92 & 3.90 & 3.90 & 5.62 & 5.64 & 5.60 & 5.61 \\
\hline & 0.2 & 1.39 & 1.40 & 1.39 & 1.39 & 2.28 & 2.26 & 2.25 & 2.26 & 3.94 & 3.95 & 3.94 & 3.92 & 5.66 & 5.65 & 5.63 & 5.65 \\
\hline & 0.5 & 1.67 & 1.68 & 1.67 & 1.68 & 2.50 & 2.48 & 2.50 & 2.52 & 4.10 & 4.11 & 4.08 & 4.10 & 5.71 & 5.76 & 5.72 & 5.71 \\
\hline & 0.8 & 2.17 & 2.17 & 2.15 & 2.16 & 2.89 & 2.90 & 2.89 & 2.90 & 4.36 & 4.36 & 4.35 & 4.37 & 5.88 & 5.86 & 5.87 & 5.86 \\
\hline \multirow{4}{*}{0.8} & 0 & 1.87 & 1.86 & 1.88 & 1.88 & 2.65 & 2.65 & 2.65 & 2.65 & 4.17 & 4.21 & 4.19 & 4.20 & 5.78 & 5.78 & 5.76 & 5.76 \\
\hline & 0.2 & 1.93 & 1.92 & 1.93 & 1.91 & 2.69 & 2.69 & 2.69 & 2.69 & 4.22 & 4.26 & 4.22 & 4.22 & 5.81 & 5.81 & 5.80 & 5.82 \\
\hline & 0.5 & 2.18 & 2.18 & 2.19 & 2.18 & 2.90 & 2.91 & 2.89 & 2.91 & 4.34 & 4.35 & 4.37 & 4.37 & 5.87 & 5.86 & 5.91 & 5.88 \\
\hline & 0.8 & 2.63 & 2.60 & 2.61 & 2.62 & 3.29 & 3.28 & 3.27 & 3.28 & 4.63 & 4.63 & 4.62 & 4.63 & 6.03 & 6.02 & 6.03 & 6.03 \\
\hline
\end{tabular}

Note: ICC $=$ Intraclass Correlation Coefficient. 
Table 3 cont.

Average Design Effect values for the Intercept: Poisson Distribution conditions $(\mathrm{m}=20, \mathrm{k}=25)$

\begin{tabular}{|c|c|c|c|c|c|c|c|c|c|c|c|c|c|c|c|c|c|}
\hline \multirow{4}{*}{$\begin{array}{c}\text { L1 } \\
\text { Effect }\end{array}$} & \multirow{4}{*}{$\begin{array}{c}\text { Interaction } \\
\text { Effect }\end{array}$} & \multicolumn{16}{|c|}{ ICC Values } \\
\hline & & \multicolumn{4}{|c|}{0} & \multirow{2}{*}{\multicolumn{3}{|c|}{0.1}} & & \multicolumn{4}{|c|}{0.3} & \multicolumn{4}{|c|}{0.5} \\
\hline & & \multicolumn{9}{|c|}{ L2 Effect } & & & & \multirow[b]{2}{*}{0} & \multirow[b]{2}{*}{0.2} & \multirow[b]{2}{*}{0.5} & \multirow[b]{2}{*}{0.8} \\
\hline & & 0 & 0.2 & 0.5 & 0.8 & 0 & 0.2 & 0.5 & 0.8 & 0 & 0.2 & 0.5 & 0.8 & & & & \\
\hline \multirow{4}{*}{0} & 0 & 1.07 & 1.08 & 1.08 & 1.08 & 2.70 & 2.71 & 2.74 & 2.74 & 6.29 & 6.29 & 6.30 & 6.28 & 9.96 & 9.97 & 10.04 & 10.06 \\
\hline & 0.2 & 1.10 & 1.10 & 1.11 & 1.12 & 2.76 & 2.79 & 2.78 & 2.78 & 6.38 & 6.29 & 6.30 & 6.33 & 10.07 & 9.94 & 10.01 & 9.98 \\
\hline & 0.5 & 1.32 & 1.31 & 1.31 & 1.31 & 2.98 & 2.99 & 3.02 & 3.07 & 6.44 & 6.50 & 6.50 & 6.52 & 10.06 & 10.06 & 10.08 & 10.10 \\
\hline & 0.8 & 1.78 & 1.79 & 1.80 & 1.72 & 3.37 & 3.43 & 3.42 & 3.41 & 6.75 & 6.75 & 6.68 & 6.78 & 10.18 & 10.26 & 10.19 & 10.17 \\
\hline \multirow{4}{*}{0.2} & 0 & 1.10 & 1.10 & 1.11 & 1.11 & 2.72 & 2.78 & 2.76 & 2.79 & 6.30 & 6.39 & 6.26 & 6.32 & 10.00 & 9.95 & 9.96 & 10.00 \\
\hline & 0.2 & 1.14 & 1.13 & 1.15 & 1.15 & 2.83 & 2.86 & 2.81 & 2.83 & 6.36 & 6.33 & 6.39 & 6.35 & 9.98 & 10.00 & 9.96 & 10.00 \\
\hline & 0.5 & 1.36 & 1.35 & 1.34 & 1.36 & 2.98 & 3.03 & 3.04 & 3.03 & 6.54 & 6.46 & 6.53 & 6.54 & 10.02 & 10.08 & 10.12 & 10.08 \\
\hline & 0.8 & 1.84 & 1.83 & 1.81 & 1.81 & 3.45 & 3.44 & 3.42 & 3.44 & 6.79 & 6.80 & 6.79 & 6.78 & 10.13 & 10.18 & 10.35 & 10.24 \\
\hline \multirow{4}{*}{0.5} & 0 & 1.32 & 1.30 & 1.30 & 1.32 & 2.98 & 3.03 & 3.02 & 3.01 & 6.51 & 6.54 & 6.50 & 6.55 & 10.19 & 10.12 & 10.09 & 10.07 \\
\hline & 0.2 & 1.37 & 1.36 & 1.37 & 1.38 & 3.06 & 3.10 & 3.11 & 3.01 & 6.55 & 6.50 & 6.50 & 6.56 & 10.04 & 10.04 & 10.07 & 10.14 \\
\hline & 0.5 & 1.62 & 1.63 & 1.61 & 1.62 & 3.33 & 3.28 & 3.29 & 3.26 & 6.68 & 6.58 & 6.63 & 6.62 & 10.12 & 10.19 & 10.07 & 10.17 \\
\hline & 0.8 & 2.09 & 2.07 & 2.13 & 2.09 & 3.62 & 3.70 & 3.65 & 3.67 & 6.79 & 6.89 & 6.90 & 6.87 & 10.30 & 10.34 & 10.33 & 10.38 \\
\hline \multirow{4}{*}{0.8} & 0 & 1.83 & 1.84 & 1.85 & 1.82 & 3.44 & 3.42 & 3.44 & 3.53 & 6.79 & 6.84 & 6.76 & 6.70 & 10.16 & 10.24 & 10.26 & 10.28 \\
\hline & 0.2 & 1.89 & 1.87 & 1.91 & 1.92 & 3.48 & 3.55 & 3.50 & 3.52 & 6.75 & 6.78 & 6.74 & 6.82 & 10.21 & 10.35 & 10.29 & 10.31 \\
\hline & 0.5 & 2.13 & 2.12 & 2.14 & 2.10 & 3.74 & 3.68 & 3.72 & 3.70 & 6.92 & 6.87 & 6.97 & 6.90 & 10.41 & 10.29 & 10.32 & 10.30 \\
\hline & 0.8 & 2.57 & 2.58 & 2.62 & 2.65 & 4.08 & 4.16 & 4.05 & 4.14 & 7.22 & 7.23 & 7.13 & 7.20 & 10.45 & 10.42 & 10.45 & 10.41 \\
\hline
\end{tabular}

Note: ICC $=$ Intraclass Correlation Coefficient. 
Table 3 cont.

Average Design Effect values for the Intercept: Poisson Distribution conditions $(\mathrm{m}=20, \mathrm{k}=50)$

\begin{tabular}{|c|c|c|c|c|c|c|c|c|c|c|c|c|c|c|c|c|c|}
\hline \multirow{4}{*}{$\begin{array}{c}\text { L1 } \\
\text { Effect }\end{array}$} & \multirow{4}{*}{$\begin{array}{c}\text { Interaction } \\
\text { Effect }\end{array}$} & \multicolumn{16}{|c|}{ ICC Values } \\
\hline & & \multicolumn{4}{|c|}{0} & \multirow{2}{*}{\multicolumn{3}{|c|}{0.1}} & & \multicolumn{4}{|c|}{0.3} & \multicolumn{4}{|c|}{0.5} \\
\hline & & \multicolumn{9}{|c|}{ L2 Effect } & & & & \multirow[b]{2}{*}{0} & \multirow[b]{2}{*}{0.2} & \multirow[b]{2}{*}{0.5} & \multirow[b]{2}{*}{0.8} \\
\hline & & 0 & 0.2 & 0.5 & 0.8 & 0 & 0.2 & 0.5 & 0.8 & 0 & 0.2 & 0.5 & 0.8 & & & & \\
\hline \multirow{4}{*}{0} & 0 & 1.06 & 1.06 & 1.05 & 1.06 & 2.83 & 2.81 & 2.84 & 2.85 & 6.58 & 6.54 & 6.53 & 6.54 & 10.26 & 10.20 & 10.23 & 10.28 \\
\hline & 0.2 & 1.09 & 1.09 & 1.10 & 1.09 & 2.87 & 2.89 & 2.87 & 2.89 & 6.55 & 6.53 & 6.55 & 6.57 & 10.22 & 10.27 & 10.37 & 10.28 \\
\hline & 0.5 & 1.34 & 1.33 & 1.34 & 1.34 & 3.12 & 3.12 & 3.12 & 3.13 & 6.73 & 6.70 & 6.71 & 6.71 & 10.35 & 10.37 & 10.30 & 10.30 \\
\hline & 0.8 & 1.87 & 1.85 & 1.87 & 1.86 & 3.56 & 3.59 & 3.57 & 3.53 & 6.97 & 6.98 & 7.00 & 6.98 & 10.54 & 10.52 & 10.50 & 10.50 \\
\hline \multirow{4}{*}{0.2} & 0 & 1.10 & 1.09 & 1.10 & 1.09 & 2.88 & 2.90 & 2.86 & 2.87 & 6.60 & 6.57 & 6.51 & 6.55 & 10.25 & 10.22 & 10.29 & 10.28 \\
\hline & 0.2 & 1.13 & 1.13 & 1.13 & 1.13 & 2.91 & 2.92 & 2.94 & 2.97 & 6.56 & 6.54 & 6.59 & 6.58 & 10.37 & 10.32 & 10.28 & 10.31 \\
\hline & 0.5 & 1.40 & 1.39 & 1.39 & 1.39 & 3.16 & 3.14 & 3.17 & 3.17 & 6.76 & 6.76 & 6.77 & 6.74 & 10.35 & 10.35 & 10.34 & 10.35 \\
\hline & 0.8 & 1.91 & 1.89 & 1.91 & 1.89 & 3.60 & 3.65 & 3.65 & 3.64 & 7.04 & 7.04 & 7.05 & 7.02 & 10.56 & 10.53 & 10.55 & 10.52 \\
\hline \multirow{4}{*}{0.5} & 0 & 1.35 & 1.35 & 1.35 & 1.35 & 3.15 & 3.14 & 3.13 & 3.13 & 6.70 & 6.72 & 6.73 & 6.74 & 10.43 & 10.39 & 10.35 & 10.32 \\
\hline & 0.2 & 1.39 & 1.40 & 1.40 & 1.39 & 3.17 & 3.17 & 3.18 & 3.18 & 6.79 & 6.69 & 6.75 & 6.73 & 10.40 & 10.38 & 10.37 & 10.44 \\
\hline & 0.5 & 1.69 & 1.70 & 1.68 & 1.71 & 3.42 & 3.41 & 3.41 & 3.41 & 6.86 & 6.95 & 6.83 & 6.95 & 10.43 & 10.47 & 10.53 & 10.41 \\
\hline & 0.8 & 2.22 & 2.22 & 2.27 & 2.19 & 3.85 & 3.84 & 3.86 & 3.86 & 7.15 & 7.13 & 7.17 & 7.16 & 10.63 & 10.58 & 10.61 & 10.61 \\
\hline \multirow{4}{*}{0.8} & 0 & 1.88 & 1.90 & 1.89 & 1.89 & 3.61 & 3.61 & 3.61 & 3.58 & 7.07 & 7.02 & 7.03 & 7.01 & 10.53 & 10.56 & 10.46 & 10.55 \\
\hline & 0.2 & 1.97 & 1.97 & 1.96 & 1.96 & 3.66 & 3.66 & 3.64 & 3.65 & 6.98 & 7.04 & 7.05 & 7.05 & 10.51 & 10.50 & 10.56 & 10.55 \\
\hline & 0.5 & 2.28 & 2.25 & 2.24 & 2.25 & 3.88 & 3.89 & 3.83 & 3.88 & 7.14 & 7.18 & 7.18 & 7.24 & 10.57 & 10.64 & 10.61 & 10.56 \\
\hline & 0.8 & 2.69 & 2.72 & 2.74 & 2.71 & 4.33 & 4.29 & 4.26 & 4.26 & 7.48 & 7.40 & 7.42 & 7.47 & 10.79 & 10.75 & 10.77 & 10.71 \\
\hline
\end{tabular}

Note: ICC $=$ Intraclass Correlation Coefficient. 
Table 3 cont.

Average Design Effect values for the Intercept: Poisson Distribution conditions $(\mathrm{m}=20, \mathrm{k}=75)$

\begin{tabular}{|c|c|c|c|c|c|c|c|c|c|c|c|c|c|c|c|c|c|}
\hline \multirow{4}{*}{$\begin{array}{c}\text { L1 } \\
\text { Effect }\end{array}$} & \multirow{4}{*}{$\begin{array}{c}\text { Interaction } \\
\text { Effect }\end{array}$} & \multicolumn{16}{|c|}{ ICC Values } \\
\hline & & \multicolumn{4}{|c|}{0} & \multirow{2}{*}{\multicolumn{3}{|c|}{0.1}} & & \multicolumn{4}{|c|}{0.3} & \multicolumn{4}{|c|}{0.5} \\
\hline & & \multicolumn{9}{|c|}{ L2 Effect } & & & & \multirow[b]{2}{*}{0} & \multirow[b]{2}{*}{0.2} & \multirow[b]{2}{*}{0.5} & \multirow[b]{2}{*}{0.8} \\
\hline & & 0 & 0.2 & 0.5 & 0.8 & 0 & 0.2 & 0.5 & 0.8 & 0 & 0.2 & 0.5 & 0.8 & & & & \\
\hline \multirow{4}{*}{0} & 0 & 1.05 & 1.05 & 1.06 & 1.06 & 2.88 & 2.85 & 2.87 & 2.86 & 6.54 & 6.58 & 6.60 & 6.59 & 10.29 & 10.32 & 10.38 & 10.34 \\
\hline & 0.2 & 1.09 & 1.08 & 1.09 & 1.08 & 2.91 & 2.94 & 2.89 & 2.91 & 6.62 & 6.58 & 6.57 & 6.59 & 10.33 & 10.34 & 10.34 & 10.39 \\
\hline & 0.5 & 1.35 & 1.34 & 1.36 & 1.34 & 3.19 & 3.18 & 3.16 & 3.20 & 6.83 & 6.81 & 6.75 & 6.79 & 10.44 & 10.44 & 10.44 & 10.50 \\
\hline & 0.8 & 1.91 & 1.87 & 1.89 & 1.87 & 3.63 & 3.62 & 3.60 & 3.61 & 7.08 & 7.04 & 7.07 & 7.10 & 10.61 & 10.63 & 10.65 & 10.57 \\
\hline \multirow{4}{*}{0.2} & 0 & 1.09 & 1.08 & 1.09 & 1.08 & 2.92 & 2.90 & 2.91 & 2.90 & 6.59 & 6.64 & 6.61 & 6.59 & 10.35 & 10.40 & 10.39 & 10.34 \\
\hline & 0.2 & 1.12 & 1.13 & 1.13 & 1.13 & 2.95 & 2.95 & 2.96 & 2.95 & 6.64 & 6.65 & 6.68 & 6.67 & 10.41 & 10.41 & 10.38 & 10.37 \\
\hline & 0.5 & 1.40 & 1.40 & 1.39 & 1.41 & 3.23 & 3.21 & 3.22 & 3.22 & 6.79 & 6.83 & 6.83 & 6.80 & 10.46 & 10.44 & 10.47 & 10.49 \\
\hline & 0.8 & 1.94 & 1.97 & 1.98 & 1.95 & 3.68 & 3.70 & 3.69 & 3.69 & 7.08 & 7.13 & 7.10 & 7.08 & 10.65 & 10.55 & 10.59 & 10.65 \\
\hline \multirow{4}{*}{0.5} & 0 & 1.37 & 1.36 & 1.35 & 1.35 & 3.21 & 3.18 & 3.18 & 3.18 & 6.83 & 6.82 & 6.80 & 6.78 & 10.49 & 10.45 & 10.50 & 10.39 \\
\hline & 0.2 & 1.40 & 1.42 & 1.41 & 1.42 & 3.25 & 3.21 & 3.23 & 3.19 & 6.84 & 6.83 & 6.81 & 6.81 & 10.48 & 10.47 & 10.42 & 10.48 \\
\hline & 0.5 & 1.72 & 1.71 & 1.69 & 1.72 & 3.45 & 3.48 & 3.46 & 3.48 & 6.93 & 6.98 & 6.91 & 6.97 & 10.54 & 10.52 & 10.57 & 10.57 \\
\hline & 0.8 & 2.25 & 2.25 & 2.27 & 2.23 & 3.96 & 3.88 & 3.90 & 3.93 & 7.21 & 7.29 & 7.25 & 7.29 & 10.75 & 10.69 & 10.71 & 10.72 \\
\hline \multirow{4}{*}{0.8} & 0 & 1.92 & 1.93 & 1.93 & 1.92 & 3.64 & 3.64 & 3.68 & 3.64 & 7.05 & 7.09 & 7.10 & 7.08 & 10.57 & 10.62 & 10.65 & 10.61 \\
\hline & 0.2 & 1.99 & 1.99 & 1.98 & 1.97 & 3.69 & 3.67 & 3.70 & 3.67 & 7.09 & 7.11 & 7.15 & 7.15 & 10.63 & 10.66 & 10.66 & 10.62 \\
\hline & 0.5 & 2.25 & 2.29 & 2.27 & 2.27 & 3.94 & 3.93 & 3.91 & 3.91 & 7.24 & 7.29 & 7.31 & 7.26 & 10.73 & 10.68 & 10.77 & 10.69 \\
\hline & 0.8 & 2.76 & 2.74 & 2.76 & 2.78 & 4.32 & 4.32 & 4.36 & 4.31 & 7.51 & 7.53 & 7.53 & 7.60 & 10.87 & 10.89 & 10.82 & 10.80 \\
\hline
\end{tabular}

Note: ICC $=$ Intraclass Correlation Coefficient. 
Table 3 cont.

Average Design Effect values for the Intercept: Poisson Distribution conditions $(\mathrm{m}=20, \mathrm{k}=100)$

\begin{tabular}{|c|c|c|c|c|c|c|c|c|c|c|c|c|c|c|c|c|c|}
\hline \multirow{4}{*}{$\begin{array}{c}\text { L1 } \\
\text { Effect }\end{array}$} & \multirow{4}{*}{$\begin{array}{c}\text { Interaction } \\
\text { Effect }\end{array}$} & \multicolumn{16}{|c|}{ ICC Values } \\
\hline & & \multicolumn{4}{|c|}{0} & \multirow{2}{*}{\multicolumn{3}{|c|}{0.1}} & & \multicolumn{4}{|c|}{0.3} & \multicolumn{4}{|c|}{0.5} \\
\hline & & \multicolumn{9}{|c|}{ L2 Effect } & & & & \multirow[b]{2}{*}{0} & \multirow[b]{2}{*}{0.2} & \multirow[b]{2}{*}{0.5} & \multirow[b]{2}{*}{0.8} \\
\hline & & 0 & 0.2 & 0.5 & 0.8 & 0 & 0.2 & 0.5 & 0.8 & 0 & 0.2 & 0.5 & 0.8 & & & & \\
\hline \multirow{4}{*}{0} & 0 & 1.04 & 1.04 & 1.04 & 1.05 & 2.89 & 2.90 & 2.89 & 2.87 & 6.65 & 6.58 & 6.60 & 6.65 & 10.35 & 10.37 & 10.39 & 10.38 \\
\hline & 0.2 & 1.09 & 1.08 & 1.08 & 1.08 & 2.92 & 2.93 & 2.92 & 2.94 & 6.65 & 6.65 & 6.68 & 6.64 & 10.41 & 10.45 & 10.42 & 10.44 \\
\hline & 0.5 & 1.35 & 1.35 & 1.35 & 1.35 & 3.18 & 3.18 & 3.20 & 3.17 & 6.79 & 6.81 & 6.82 & 6.83 & 10.48 & 10.50 & 10.56 & 10.46 \\
\hline & 0.8 & 1.93 & 1.93 & 1.91 & 1.94 & 3.63 & 3.65 & 3.65 & 3.65 & 7.15 & 7.12 & 7.15 & 7.16 & 10.69 & 10.64 & 10.67 & 10.65 \\
\hline \multirow{4}{*}{0.2} & 0 & 1.09 & 1.08 & 1.08 & 1.08 & 2.93 & 2.94 & 2.94 & 2.94 & 6.69 & 6.66 & 6.65 & 6.66 & 10.38 & 10.39 & 10.43 & 10.37 \\
\hline & 0.2 & 1.12 & 1.12 & 1.11 & 1.13 & 2.99 & 3.00 & 3.00 & 2.98 & 6.67 & 6.70 & 6.72 & 6.71 & 10.44 & 10.44 & 10.42 & 10.42 \\
\hline & 0.5 & 1.42 & 1.42 & 1.41 & 1.41 & 3.24 & 3.25 & 3.24 & 3.21 & 6.86 & 6.92 & 6.84 & 6.85 & 10.54 & 10.49 & 10.49 & 10.53 \\
\hline & 0.8 & 2.00 & 1.97 & 1.97 & 1.98 & 3.69 & 3.70 & 3.72 & 3.68 & 7.13 & 7.16 & 7.11 & 7.16 & 10.70 & 10.66 & 10.69 & 10.69 \\
\hline \multirow{4}{*}{0.5} & 0 & 1.36 & 1.35 & 1.37 & 1.37 & 3.19 & 3.19 & 3.19 & 3.21 & 6.84 & 6.80 & 6.82 & 6.84 & 10.48 & 10.51 & 10.53 & 10.48 \\
\hline & 0.2 & 1.41 & 1.42 & 1.42 & 1.42 & 3.25 & 3.24 & 3.23 & 3.23 & 6.88 & 6.91 & 6.85 & 6.83 & 10.56 & 10.52 & 10.56 & 10.51 \\
\hline & 0.5 & 1.72 & 1.73 & 1.74 & 1.71 & 3.48 & 3.49 & 3.50 & 3.49 & 6.99 & 7.00 & 7.01 & 7.02 & 10.62 & 10.58 & 10.63 & 10.57 \\
\hline & 0.8 & 2.26 & 2.26 & 2.28 & 2.28 & 3.95 & 3.97 & 3.91 & 3.93 & 7.26 & 7.26 & 7.30 & 7.29 & 10.76 & 10.72 & 10.73 & 10.74 \\
\hline \multirow{4}{*}{0.8} & 0 & 1.94 & 1.94 & 1.93 & 1.92 & 3.66 & 3.66 & 3.66 & 3.67 & 7.12 & 7.11 & 7.13 & 7.16 & 10.60 & 10.64 & 10.63 & 10.66 \\
\hline & 0.2 & 2.00 & 2.00 & 1.98 & 1.98 & 3.70 & 3.70 & 3.69 & 3.74 & 7.16 & 7.10 & 7.18 & 7.14 & 10.68 & 10.69 & 10.65 & 10.65 \\
\hline & 0.5 & 2.29 & 2.27 & 2.29 & 2.28 & 3.95 & 3.97 & 3.95 & 3.96 & 7.32 & 7.31 & 7.29 & 7.29 & 10.77 & 10.74 & 10.78 & 10.73 \\
\hline & 0.8 & 2.77 & 2.81 & 2.76 & 2.79 & 4.39 & 4.36 & 4.37 & 4.35 & 7.56 & 7.61 & 7.60 & 7.58 & 10.91 & 10.89 & 10.87 & 10.90 \\
\hline
\end{tabular}

Note: ICC $=$ Intraclass Correlation Coefficient. 
Table 4.

Average Design Effect values for the L1 predictor: Uniform Distribution conditions $(\mathrm{m}=5, \mathrm{k}=25)$

\begin{tabular}{|c|c|c|c|c|c|c|c|c|c|c|c|c|c|c|c|c|c|}
\hline \multirow{4}{*}{$\begin{array}{c}\text { L1 } \\
\text { Effect }\end{array}$} & \multirow{4}{*}{$\begin{array}{c}\text { Interaction } \\
\text { Effect }\end{array}$} & \multicolumn{16}{|c|}{ ICC Values } \\
\hline & & \multicolumn{4}{|c|}{0} & \multicolumn{4}{|c|}{0.1} & \multicolumn{4}{|c|}{0.3} & \multicolumn{4}{|c|}{0.5} \\
\hline & & \multicolumn{12}{|c|}{ L2 Effect } & & & & \\
\hline & & 0 & 0.2 & 0.5 & 0.8 & 0 & 0.2 & 0.5 & 0.8 & 0 & 0.2 & 0.5 & 0.8 & 0 & 0.2 & 0.5 & 0.8 \\
\hline \multirow{4}{*}{0} & 0 & 0.98 & 0.98 & 0.98 & 0.98 & 0.91 & 0.91 & 0.91 & 0.91 & 0.72 & 0.73 & 0.73 & 0.73 & 0.54 & 0.53 & 0.53 & 0.53 \\
\hline & 0.2 & 0.98 & 0.97 & 0.98 & 0.97 & 0.91 & 0.90 & 0.91 & 0.91 & 0.72 & 0.73 & 0.72 & 0.72 & 0.53 & 0.53 & 0.53 & 0.52 \\
\hline & 0.5 & 0.94 & 0.94 & 0.94 & 0.94 & 0.86 & 0.86 & 0.86 & 0.86 & 0.69 & 0.70 & 0.69 & 0.69 & 0.51 & 0.51 & 0.51 & 0.51 \\
\hline & 0.8 & 0.86 & 0.86 & 0.86 & 0.86 & 0.79 & 0.79 & 0.80 & 0.79 & 0.65 & 0.65 & 0.64 & 0.65 & 0.49 & 0.49 & 0.50 & 0.49 \\
\hline \multirow{4}{*}{0.2} & 0 & 0.97 & 0.97 & 0.97 & 0.97 & 0.90 & 0.90 & 0.90 & 0.90 & 0.72 & 0.72 & 0.72 & 0.72 & 0.53 & 0.53 & 0.53 & 0.53 \\
\hline & 0.2 & 0.97 & 0.97 & 0.97 & 0.97 & 0.90 & 0.89 & 0.90 & 0.90 & 0.72 & 0.71 & 0.72 & 0.72 & 0.53 & 0.52 & 0.53 & 0.53 \\
\hline & 0.5 & 0.92 & 0.92 & 0.93 & 0.93 & 0.86 & 0.85 & 0.85 & 0.85 & 0.69 & 0.68 & 0.69 & 0.69 & 0.51 & 0.51 & 0.51 & 0.51 \\
\hline & 0.8 & 0.85 & 0.86 & 0.85 & 0.85 & 0.79 & 0.79 & 0.78 & 0.79 & 0.64 & 0.65 & 0.64 & 0.64 & 0.49 & 0.49 & 0.49 & 0.49 \\
\hline \multirow{4}{*}{0.5} & 0 & 0.93 & 0.93 & 0.93 & 0.93 & 0.86 & 0.86 & 0.86 & 0.86 & 0.69 & 0.69 & 0.69 & 0.69 & 0.51 & 0.51 & 0.51 & 0.51 \\
\hline & 0.2 & 0.92 & 0.92 & 0.92 & 0.93 & 0.84 & 0.85 & 0.85 & 0.86 & 0.68 & 0.68 & 0.69 & 0.69 & 0.51 & 0.51 & 0.51 & 0.51 \\
\hline & 0.5 & 0.88 & 0.88 & 0.89 & 0.88 & 0.81 & 0.82 & 0.82 & 0.82 & 0.66 & 0.65 & 0.66 & 0.67 & 0.49 & 0.50 & 0.50 & 0.49 \\
\hline & 0.8 & 0.81 & 0.81 & 0.82 & 0.82 & 0.75 & 0.75 & 0.75 & 0.75 & 0.62 & 0.62 & 0.62 & 0.62 & 0.47 & 0.48 & 0.48 & 0.47 \\
\hline \multirow{4}{*}{0.8} & 0 & 0.85 & 0.84 & 0.85 & 0.85 & 0.79 & 0.78 & 0.78 & 0.78 & 0.64 & 0.64 & 0.64 & 0.64 & 0.48 & 0.48 & 0.48 & 0.48 \\
\hline & 0.2 & 0.84 & 0.84 & 0.84 & 0.84 & 0.78 & 0.77 & 0.78 & 0.77 & 0.64 & 0.63 & 0.64 & 0.63 & 0.48 & 0.48 & 0.49 & 0.48 \\
\hline & 0.5 & 0.80 & 0.81 & 0.80 & 0.80 & 0.74 & 0.75 & 0.74 & 0.75 & 0.62 & 0.61 & 0.62 & 0.62 & 0.47 & 0.47 & 0.46 & 0.47 \\
\hline & 0.8 & 0.75 & 0.75 & 0.75 & 0.75 & 0.70 & 0.70 & 0.70 & 0.69 & 0.58 & 0.57 & 0.58 & 0.58 & 0.45 & 0.45 & 0.45 & 0.45 \\
\hline
\end{tabular}

Note: ICC $=$ Intraclass Correlation Coefficient. 
Table 4 cont.

Average Design Effect values for the $L 1$ predictor: Uniform Distribution conditions $(\mathrm{m}=5, \mathrm{k}=50)$

\begin{tabular}{|c|c|c|c|c|c|c|c|c|c|c|c|c|c|c|c|c|c|}
\hline \multirow{4}{*}{$\begin{array}{c}\text { L1 } \\
\text { Effect }\end{array}$} & \multirow{4}{*}{$\begin{array}{c}\text { Interaction } \\
\text { Effect }\end{array}$} & \multicolumn{16}{|c|}{ ICC Values } \\
\hline & & \multicolumn{4}{|c|}{0} & \multicolumn{4}{|c|}{0.1} & \multicolumn{4}{|c|}{0.3} & \multicolumn{4}{|c|}{0.5} \\
\hline & & \multirow[b]{2}{*}{0} & \multirow[b]{2}{*}{0.2} & \multirow[b]{2}{*}{0.5} & \multirow[b]{2}{*}{0.8} & \multirow[b]{2}{*}{0} & \multirow[b]{2}{*}{0.2} & \multirow[b]{2}{*}{0.5} & \multicolumn{2}{|c|}{ L2 Effect } & \multirow[b]{2}{*}{0.2} & \multirow[b]{2}{*}{0.5} & \multirow[b]{2}{*}{0.8} & \multirow[b]{2}{*}{0} & \multirow[b]{2}{*}{0.2} & \multirow[b]{2}{*}{0.5} & \multirow[b]{2}{*}{0.8} \\
\hline & & & & & & & & & 0.8 & 0 & & & & & & & \\
\hline \multirow{4}{*}{0} & 0 & 0.98 & 0.98 & 0.98 & 0.98 & 0.91 & 0.91 & 0.91 & 0.91 & 0.71 & 0.71 & 0.71 & 0.72 & 0.51 & 0.51 & 0.51 & 0.52 \\
\hline & 0.2 & 0.98 & 0.98 & 0.98 & 0.98 & 0.90 & 0.90 & 0.90 & 0.90 & 0.71 & 0.71 & 0.71 & 0.71 & 0.52 & 0.51 & 0.51 & 0.51 \\
\hline & 0.5 & 0.93 & 0.93 & 0.93 & 0.93 & 0.85 & 0.85 & 0.85 & 0.85 & 0.68 & 0.68 & 0.68 & 0.68 & 0.50 & 0.50 & 0.50 & 0.50 \\
\hline & 0.8 & 0.85 & 0.84 & 0.84 & 0.85 & 0.77 & 0.78 & 0.77 & 0.77 & 0.63 & 0.62 & 0.63 & 0.63 & 0.47 & 0.47 & 0.47 & 0.47 \\
\hline \multirow{4}{*}{0.2} & 0 & 0.98 & 0.98 & 0.98 & 0.98 & 0.90 & 0.90 & 0.90 & 0.90 & 0.71 & 0.71 & 0.71 & 0.71 & 0.52 & 0.51 & 0.51 & 0.51 \\
\hline & 0.2 & 0.97 & 0.97 & 0.97 & 0.97 & 0.89 & 0.89 & 0.89 & 0.89 & 0.70 & 0.70 & 0.70 & 0.70 & 0.51 & 0.51 & 0.51 & 0.51 \\
\hline & 0.5 & 0.92 & 0.92 & 0.92 & 0.92 & 0.84 & 0.84 & 0.84 & 0.84 & 0.67 & 0.67 & 0.67 & 0.67 & 0.49 & 0.49 & 0.49 & 0.50 \\
\hline & 0.8 & 0.84 & 0.83 & 0.84 & 0.84 & 0.76 & 0.77 & 0.77 & 0.77 & 0.62 & 0.62 & 0.62 & 0.62 & 0.47 & 0.47 & 0.47 & 0.46 \\
\hline \multirow{4}{*}{0.5} & 0 & 0.93 & 0.93 & 0.93 & 0.93 & 0.85 & 0.85 & 0.85 & 0.85 & 0.67 & 0.67 & 0.67 & 0.68 & 0.50 & 0.49 & 0.50 & 0.49 \\
\hline & 0.2 & 0.92 & 0.92 & 0.92 & 0.92 & 0.84 & 0.84 & 0.84 & 0.84 & 0.67 & 0.67 & 0.67 & 0.67 & 0.49 & 0.49 & 0.50 & 0.49 \\
\hline & 0.5 & 0.87 & 0.87 & 0.87 & 0.87 & 0.80 & 0.80 & 0.81 & 0.80 & 0.64 & 0.65 & 0.64 & 0.64 & 0.48 & 0.48 & 0.48 & 0.48 \\
\hline & 0.8 & 0.79 & 0.80 & 0.80 & 0.79 & 0.73 & 0.73 & 0.73 & 0.73 & 0.60 & 0.60 & 0.60 & 0.60 & 0.45 & 0.45 & 0.45 & 0.45 \\
\hline \multirow{4}{*}{0.8} & 0 & 0.84 & 0.84 & 0.84 & 0.84 & 0.77 & 0.77 & 0.77 & 0.77 & 0.63 & 0.63 & 0.62 & 0.62 & 0.46 & 0.47 & 0.47 & 0.47 \\
\hline & 0.2 & 0.83 & 0.83 & 0.82 & 0.83 & 0.76 & 0.76 & 0.76 & 0.76 & 0.62 & 0.62 & 0.62 & 0.62 & 0.46 & 0.46 & 0.46 & 0.46 \\
\hline & 0.5 & 0.79 & 0.79 & 0.79 & 0.79 & 0.73 & 0.73 & 0.73 & 0.73 & 0.60 & 0.60 & 0.60 & 0.60 & 0.45 & 0.45 & 0.46 & 0.45 \\
\hline & 0.8 & 0.73 & 0.72 & 0.72 & 0.72 & 0.68 & 0.68 & 0.67 & 0.67 & 0.56 & 0.56 & 0.56 & 0.56 & 0.43 & 0.43 & 0.43 & 0.43 \\
\hline
\end{tabular}

Note: ICC = Intraclass Correlation Coefficient. 
Table 4 cont.

Average Design Effect values for the L1 predictor: Uniform Distribution conditions $(\mathrm{m}=5, \mathrm{k}=75)$

\begin{tabular}{|c|c|c|c|c|c|c|c|c|c|c|c|c|c|c|c|c|c|}
\hline \multirow{4}{*}{$\begin{array}{c}\text { L1 } \\
\text { Effect }\end{array}$} & \multirow{4}{*}{$\begin{array}{c}\text { Interaction } \\
\text { Effect }\end{array}$} & \multicolumn{16}{|c|}{ ICC Values } \\
\hline & & \multicolumn{4}{|c|}{0} & \multirow{2}{*}{\multicolumn{3}{|c|}{0.1}} & & \multicolumn{4}{|c|}{0.3} & \multicolumn{4}{|c|}{0.5} \\
\hline & & & & & & & & & \multicolumn{2}{|c|}{ L2 Effect } & & & & & & & \\
\hline & & 0 & 0.2 & 0.5 & 0.8 & 0 & 0.2 & 0.5 & 0.8 & 0 & 0.2 & 0.5 & 0.8 & 0 & 0.2 & 0.5 & 0.8 \\
\hline \multirow{4}{*}{0} & 0 & 0.99 & 0.99 & 0.99 & 0.99 & 0.91 & 0.90 & 0.91 & 0.91 & 0.71 & 0.71 & 0.71 & 0.71 & 0.51 & 0.51 & 0.51 & 0.51 \\
\hline & 0.2 & 0.98 & 0.98 & 0.98 & 0.98 & 0.90 & 0.90 & 0.90 & 0.90 & 0.70 & 0.70 & 0.70 & 0.70 & 0.51 & 0.51 & 0.51 & 0.51 \\
\hline & 0.5 & 0.93 & 0.93 & 0.93 & 0.93 & 0.85 & 0.85 & 0.85 & 0.85 & 0.67 & 0.67 & 0.67 & 0.67 & 0.49 & 0.49 & 0.49 & 0.49 \\
\hline & 0.8 & 0.84 & 0.84 & 0.84 & 0.84 & 0.77 & 0.77 & 0.77 & 0.77 & 0.62 & 0.63 & 0.62 & 0.63 & 0.46 & 0.46 & 0.46 & 0.47 \\
\hline \multirow{4}{*}{0.2} & 0 & 0.98 & 0.98 & 0.98 & 0.98 & 0.90 & 0.90 & 0.89 & 0.90 & 0.70 & 0.70 & 0.70 & 0.70 & 0.51 & 0.51 & 0.50 & 0.51 \\
\hline & 0.2 & 0.97 & 0.97 & 0.97 & 0.97 & 0.89 & 0.88 & 0.89 & 0.89 & 0.70 & 0.70 & 0.70 & 0.70 & 0.50 & 0.51 & 0.50 & 0.50 \\
\hline & 0.5 & 0.92 & 0.92 & 0.92 & 0.92 & 0.84 & 0.84 & 0.84 & 0.84 & 0.67 & 0.67 & 0.67 & 0.67 & 0.49 & 0.49 & 0.49 & 0.49 \\
\hline & 0.8 & 0.83 & 0.83 & 0.83 & 0.83 & 0.76 & 0.76 & 0.76 & 0.77 & 0.62 & 0.62 & 0.62 & 0.62 & 0.46 & 0.46 & 0.46 & 0.46 \\
\hline \multirow{4}{*}{0.5} & 0 & 0.93 & 0.93 & 0.93 & 0.93 & 0.84 & 0.84 & 0.85 & 0.85 & 0.67 & 0.67 & 0.67 & 0.67 & 0.49 & 0.49 & 0.49 & 0.49 \\
\hline & 0.2 & 0.92 & 0.92 & 0.92 & 0.92 & 0.83 & 0.84 & 0.83 & 0.84 & 0.67 & 0.67 & 0.66 & 0.66 & 0.49 & 0.49 & 0.49 & 0.49 \\
\hline & 0.5 & 0.87 & 0.87 & 0.87 & 0.87 & 0.79 & 0.79 & 0.79 & 0.79 & 0.64 & 0.64 & 0.64 & 0.64 & 0.47 & 0.47 & 0.48 & 0.47 \\
\hline & 0.8 & 0.79 & 0.79 & 0.79 & 0.78 & 0.73 & 0.72 & 0.72 & 0.73 & 0.59 & 0.60 & 0.60 & 0.60 & 0.45 & 0.45 & 0.45 & 0.45 \\
\hline \multirow{4}{*}{0.8} & 0 & 0.83 & 0.83 & 0.83 & 0.83 & 0.76 & 0.77 & 0.77 & 0.77 & 0.62 & 0.62 & 0.62 & 0.62 & 0.46 & 0.46 & 0.46 & 0.46 \\
\hline & 0.2 & 0.82 & 0.82 & 0.83 & 0.83 & 0.76 & 0.76 & 0.76 & 0.76 & 0.61 & 0.61 & 0.62 & 0.62 & 0.46 & 0.46 & 0.46 & 0.46 \\
\hline & 0.5 & 0.78 & 0.78 & 0.79 & 0.78 & 0.72 & 0.72 & 0.72 & 0.72 & 0.59 & 0.59 & 0.59 & 0.59 & 0.45 & 0.45 & 0.44 & 0.45 \\
\hline & 0.8 & 0.72 & 0.72 & 0.72 & 0.72 & 0.67 & 0.66 & 0.67 & 0.67 & 0.56 & 0.55 & 0.56 & 0.56 & 0.42 & 0.42 & 0.43 & 0.43 \\
\hline
\end{tabular}

Note: ICC $=$ Intraclass Correlation Coefficient. 
Table 4 cont.

Average Design Effect values for the L1 predictor: Uniform Distribution conditions $(\mathrm{m}=5, \mathrm{k}=100)$

\begin{tabular}{|c|c|c|c|c|c|c|c|c|c|c|c|c|c|c|c|c|c|}
\hline \multirow{4}{*}{$\begin{array}{c}\text { L1 } \\
\text { Effect }\end{array}$} & \multirow{4}{*}{$\begin{array}{c}\text { Interaction } \\
\text { Effect }\end{array}$} & \multicolumn{16}{|c|}{ ICC Values } \\
\hline & & \multicolumn{4}{|c|}{0} & \multicolumn{4}{|c|}{0.1} & \multicolumn{4}{|c|}{0.3} & \multicolumn{4}{|c|}{0.5} \\
\hline & & \multirow[b]{2}{*}{0} & \multirow[b]{2}{*}{0.2} & \multirow[b]{2}{*}{0.5} & \multirow[b]{2}{*}{0.8} & \multirow[b]{2}{*}{0} & \multirow[b]{2}{*}{0.2} & \multirow[b]{2}{*}{0.5} & \multicolumn{2}{|c|}{ L2 Effect } & \multirow[b]{2}{*}{0.2} & \multirow[b]{2}{*}{0.5} & \multirow[b]{2}{*}{0.8} & \multirow[b]{2}{*}{0} & \multirow[b]{2}{*}{0.2} & \multirow[b]{2}{*}{0.5} & \multirow[b]{2}{*}{0.8} \\
\hline & & & & & & & & & 0.8 & 0 & & & & & & & \\
\hline \multirow{4}{*}{0} & 0 & 0.99 & 0.99 & 0.99 & 0.99 & 0.90 & 0.90 & 0.90 & 0.90 & 0.71 & 0.71 & 0.71 & 0.71 & 0.51 & 0.51 & 0.51 & 0.51 \\
\hline & 0.2 & 0.98 & 0.98 & 0.98 & 0.98 & 0.89 & 0.90 & 0.89 & 0.89 & 0.70 & 0.70 & 0.70 & 0.70 & 0.51 & 0.50 & 0.51 & 0.50 \\
\hline & 0.5 & 0.93 & 0.93 & 0.93 & 0.93 & 0.84 & 0.85 & 0.85 & 0.85 & 0.67 & 0.67 & 0.67 & 0.67 & 0.49 & 0.49 & 0.49 & 0.49 \\
\hline & 0.8 & 0.84 & 0.83 & 0.84 & 0.84 & 0.77 & 0.76 & 0.77 & 0.76 & 0.62 & 0.62 & 0.62 & 0.62 & 0.46 & 0.46 & 0.46 & 0.46 \\
\hline \multirow{4}{*}{0.2} & 0 & 0.98 & 0.98 & 0.98 & 0.98 & 0.90 & 0.89 & 0.89 & 0.89 & 0.70 & 0.70 & 0.70 & 0.70 & 0.51 & 0.50 & 0.51 & 0.51 \\
\hline & 0.2 & 0.97 & 0.97 & 0.97 & 0.97 & 0.88 & 0.89 & 0.88 & 0.88 & 0.69 & 0.69 & 0.70 & 0.70 & 0.50 & 0.51 & 0.50 & 0.50 \\
\hline & 0.5 & 0.92 & 0.92 & 0.92 & 0.92 & 0.84 & 0.84 & 0.84 & 0.84 & 0.66 & 0.66 & 0.66 & 0.66 & 0.49 & 0.48 & 0.48 & 0.49 \\
\hline & 0.8 & 0.83 & 0.82 & 0.82 & 0.83 & 0.76 & 0.76 & 0.76 & 0.76 & 0.62 & 0.62 & 0.61 & 0.62 & 0.46 & 0.46 & 0.46 & 0.46 \\
\hline \multirow{4}{*}{0.5} & 0 & 0.93 & 0.93 & 0.93 & 0.93 & 0.84 & 0.84 & 0.85 & 0.85 & 0.67 & 0.67 & 0.67 & 0.67 & 0.49 & 0.49 & 0.49 & 0.49 \\
\hline & 0.2 & 0.92 & 0.92 & 0.92 & 0.92 & 0.84 & 0.83 & 0.83 & 0.83 & 0.66 & 0.66 & 0.66 & 0.66 & 0.49 & 0.49 & 0.48 & 0.49 \\
\hline & 0.5 & 0.86 & 0.87 & 0.87 & 0.87 & 0.79 & 0.79 & 0.79 & 0.79 & 0.63 & 0.64 & 0.64 & 0.64 & 0.47 & 0.47 & 0.47 & 0.47 \\
\hline & 0.8 & 0.79 & 0.78 & 0.78 & 0.78 & 0.72 & 0.72 & 0.72 & 0.72 & 0.59 & 0.59 & 0.59 & 0.59 & 0.44 & 0.44 & 0.45 & 0.45 \\
\hline \multirow{4}{*}{0.8} & 0 & 0.83 & 0.83 & 0.83 & 0.83 & 0.76 & 0.76 & 0.76 & 0.76 & 0.62 & 0.62 & 0.62 & 0.62 & 0.46 & 0.46 & 0.46 & 0.46 \\
\hline & 0.2 & 0.83 & 0.82 & 0.82 & 0.82 & 0.75 & 0.76 & 0.76 & 0.76 & 0.61 & 0.61 & 0.61 & 0.61 & 0.46 & 0.46 & 0.46 & 0.46 \\
\hline & 0.5 & 0.78 & 0.79 & 0.78 & 0.78 & 0.72 & 0.72 & 0.72 & 0.72 & 0.59 & 0.59 & 0.59 & 0.59 & 0.44 & 0.44 & 0.44 & 0.45 \\
\hline & 0.8 & 0.72 & 0.72 & 0.72 & 0.72 & 0.67 & 0.67 & 0.67 & 0.66 & 0.55 & 0.55 & 0.55 & 0.55 & 0.42 & 0.42 & 0.42 & 0.42 \\
\hline
\end{tabular}

Note: ICC $=$ Intraclass Correlation Coefficient. 
Table 4 cont.

Average Design Effect values for the L1 predictor: Uniform Distribution conditions $(\mathrm{m}=10, \mathrm{k}=25)$

\begin{tabular}{|c|c|c|c|c|c|c|c|c|c|c|c|c|c|c|c|c|c|}
\hline \multirow{4}{*}{$\begin{array}{c}\text { L1 } \\
\text { Effect }\end{array}$} & \multirow{4}{*}{$\begin{array}{c}\text { Interaction } \\
\text { Effect }\end{array}$} & \multicolumn{16}{|c|}{ ICC Values } \\
\hline & & \multicolumn{4}{|c|}{0} & \multicolumn{4}{|c|}{0.1} & \multicolumn{4}{|c|}{0.3} & \multicolumn{4}{|c|}{0.5} \\
\hline & & \multirow[b]{2}{*}{0} & \multirow[b]{2}{*}{0.2} & \multirow[b]{2}{*}{0.5} & \multirow[b]{2}{*}{0.8} & \multirow[b]{2}{*}{0} & \multirow[b]{2}{*}{0.2} & \multirow[b]{2}{*}{0.5} & \multicolumn{2}{|c|}{ L2 Effect } & \multirow[b]{2}{*}{0.2} & \multirow[b]{2}{*}{0.5} & \multirow[b]{2}{*}{0.8} & \multirow[b]{2}{*}{0} & \multirow[b]{2}{*}{0.2} & \multirow[b]{2}{*}{0.5} & \multirow[b]{2}{*}{0.8} \\
\hline & & & & & & & & & 0.8 & 0 & & & & & & & \\
\hline \multirow{4}{*}{0} & 0 & 0.99 & 0.99 & 0.99 & 0.99 & 0.91 & 0.91 & 0.91 & 0.92 & 0.73 & 0.72 & 0.73 & 0.73 & 0.53 & 0.53 & 0.54 & 0.53 \\
\hline & 0.2 & 0.99 & 0.99 & 0.99 & 0.99 & 0.91 & 0.91 & 0.91 & 0.91 & 0.72 & 0.72 & 0.72 & 0.72 & 0.53 & 0.53 & 0.52 & 0.53 \\
\hline & 0.5 & 0.97 & 0.97 & 0.97 & 0.97 & 0.88 & 0.89 & 0.88 & 0.88 & 0.71 & 0.71 & 0.71 & 0.71 & 0.52 & 0.52 & 0.52 & 0.52 \\
\hline & 0.8 & 0.92 & 0.92 & 0.92 & 0.93 & 0.85 & 0.85 & 0.85 & 0.85 & 0.68 & 0.68 & 0.69 & 0.68 & 0.51 & 0.51 & 0.51 & 0.51 \\
\hline \multirow{4}{*}{0.2} & 0 & 0.99 & 0.99 & 0.99 & 0.99 & 0.91 & 0.91 & 0.91 & 0.91 & 0.72 & 0.72 & 0.72 & 0.72 & 0.53 & 0.53 & 0.53 & 0.54 \\
\hline & 0.2 & 0.99 & 0.98 & 0.99 & 0.98 & 0.90 & 0.91 & 0.90 & 0.91 & 0.72 & 0.72 & 0.72 & 0.72 & 0.53 & 0.53 & 0.53 & 0.53 \\
\hline & 0.5 & 0.96 & 0.96 & 0.96 & 0.96 & 0.88 & 0.88 & 0.88 & 0.88 & 0.71 & 0.70 & 0.71 & 0.70 & 0.52 & 0.52 & 0.52 & 0.52 \\
\hline & 0.8 & 0.92 & 0.92 & 0.92 & 0.92 & 0.85 & 0.84 & 0.84 & 0.85 & 0.68 & 0.68 & 0.68 & 0.68 & 0.51 & 0.51 & 0.50 & 0.51 \\
\hline \multirow{4}{*}{0.5} & 0 & 0.97 & 0.97 & 0.97 & 0.97 & 0.88 & 0.89 & 0.88 & 0.88 & 0.71 & 0.71 & 0.71 & 0.71 & 0.52 & 0.52 & 0.52 & 0.52 \\
\hline & 0.2 & 0.96 & 0.96 & 0.96 & 0.96 & 0.88 & 0.88 & 0.88 & 0.88 & 0.71 & 0.70 & 0.70 & 0.70 & 0.53 & 0.52 & 0.52 & 0.52 \\
\hline & 0.5 & 0.94 & 0.94 & 0.94 & 0.94 & 0.86 & 0.85 & 0.86 & 0.86 & 0.69 & 0.69 & 0.69 & 0.69 & 0.51 & 0.51 & 0.51 & 0.51 \\
\hline & 0.8 & 0.90 & 0.90 & 0.90 & 0.90 & 0.82 & 0.82 & 0.82 & 0.82 & 0.67 & 0.67 & 0.67 & 0.67 & 0.49 & 0.50 & 0.50 & 0.50 \\
\hline \multirow{4}{*}{0.8} & 0 & 0.92 & 0.92 & 0.92 & 0.92 & 0.84 & 0.84 & 0.84 & 0.84 & 0.68 & 0.68 & 0.68 & 0.68 & 0.51 & 0.51 & 0.51 & 0.51 \\
\hline & 0.2 & 0.91 & 0.91 & 0.92 & 0.91 & 0.84 & 0.84 & 0.84 & 0.84 & 0.68 & 0.68 & 0.68 & 0.68 & 0.50 & 0.51 & 0.50 & 0.51 \\
\hline & 0.5 & 0.89 & 0.89 & 0.89 & 0.89 & 0.82 & 0.82 & 0.82 & 0.82 & 0.66 & 0.66 & 0.66 & 0.66 & 0.50 & 0.50 & 0.50 & 0.50 \\
\hline & 0.8 & 0.85 & 0.85 & 0.86 & 0.85 & 0.78 & 0.78 & 0.78 & 0.79 & 0.64 & 0.65 & 0.65 & 0.65 & 0.49 & 0.48 & 0.49 & 0.48 \\
\hline
\end{tabular}

Note: ICC $=$ Intraclass Correlation Coefficient. 
Table 4 cont.

Average Design Effect values for the L1 predictor: Uniform Distribution conditions $(\mathrm{m}=10, \mathrm{k}=50)$

\begin{tabular}{|c|c|c|c|c|c|c|c|c|c|c|c|c|c|c|c|c|c|}
\hline \multirow{4}{*}{$\begin{array}{c}\text { L1 } \\
\text { Effect }\end{array}$} & \multirow{4}{*}{$\begin{array}{c}\text { Interaction } \\
\text { Effect }\end{array}$} & \multicolumn{16}{|c|}{ ICC Values } \\
\hline & & \multicolumn{4}{|c|}{0} & \multicolumn{4}{|c|}{0.1} & \multicolumn{4}{|c|}{0.3} & \multicolumn{4}{|c|}{0.5} \\
\hline & & \multirow[b]{2}{*}{0} & \multirow[b]{2}{*}{0.2} & \multirow[b]{2}{*}{0.5} & \multirow[b]{2}{*}{0.8} & \multirow[b]{2}{*}{0} & \multirow[b]{2}{*}{0.2} & \multirow[b]{2}{*}{0.5} & \multicolumn{2}{|c|}{ L2 Effect } & \multirow[b]{2}{*}{0.2} & \multirow[b]{2}{*}{0.5} & \multirow[b]{2}{*}{0.8} & \multirow[b]{2}{*}{0} & \multirow[b]{2}{*}{0.2} & \multirow[b]{2}{*}{0.5} & \multirow[b]{2}{*}{0.8} \\
\hline & & & & & & & & & 0.8 & 0 & & & & & & & \\
\hline \multirow{4}{*}{0} & 0 & 0.99 & 0.99 & 0.99 & 0.99 & 0.91 & 0.91 & 0.91 & 0.91 & 0.71 & 0.71 & 0.71 & 0.71 & 0.51 & 0.51 & 0.52 & 0.51 \\
\hline & 0.2 & 0.99 & 0.99 & 0.99 & 0.99 & 0.90 & 0.90 & 0.90 & 0.90 & 0.71 & 0.71 & 0.71 & 0.71 & 0.52 & 0.51 & 0.52 & 0.51 \\
\hline & 0.5 & 0.97 & 0.97 & 0.97 & 0.97 & 0.88 & 0.88 & 0.88 & 0.88 & 0.70 & 0.70 & 0.69 & 0.69 & 0.51 & 0.51 & 0.51 & 0.50 \\
\hline & 0.8 & 0.91 & 0.92 & 0.91 & 0.92 & 0.84 & 0.83 & 0.83 & 0.83 & 0.67 & 0.67 & 0.67 & 0.67 & 0.49 & 0.49 & 0.49 & 0.49 \\
\hline \multirow{4}{*}{0.2} & 0 & 0.99 & 0.99 & 0.99 & 0.99 & 0.90 & 0.91 & 0.90 & 0.90 & 0.71 & 0.71 & 0.71 & 0.71 & 0.51 & 0.52 & 0.51 & 0.51 \\
\hline & 0.2 & 0.99 & 0.99 & 0.99 & 0.99 & 0.90 & 0.90 & 0.90 & 0.89 & 0.71 & 0.71 & 0.71 & 0.71 & 0.51 & 0.51 & 0.51 & 0.52 \\
\hline & 0.5 & 0.96 & 0.96 & 0.96 & 0.96 & 0.87 & 0.87 & 0.87 & 0.87 & 0.69 & 0.69 & 0.69 & 0.69 & 0.50 & 0.50 & 0.50 & 0.50 \\
\hline & 0.8 & 0.91 & 0.91 & 0.91 & 0.91 & 0.83 & 0.83 & 0.83 & 0.83 & 0.67 & 0.66 & 0.66 & 0.66 & 0.49 & 0.49 & 0.49 & 0.49 \\
\hline \multirow{4}{*}{0.5} & 0 & 0.96 & 0.96 & 0.96 & 0.97 & 0.88 & 0.88 & 0.88 & 0.88 & 0.69 & 0.69 & 0.70 & 0.70 & 0.51 & 0.50 & 0.51 & 0.50 \\
\hline & 0.2 & 0.96 & 0.96 & 0.96 & 0.96 & 0.87 & 0.87 & 0.87 & 0.87 & 0.69 & 0.69 & 0.69 & 0.69 & 0.50 & 0.50 & 0.50 & 0.50 \\
\hline & 0.5 & 0.93 & 0.93 & 0.93 & 0.93 & 0.85 & 0.85 & 0.85 & 0.85 & 0.68 & 0.68 & 0.68 & 0.67 & 0.50 & 0.50 & 0.49 & 0.50 \\
\hline & 0.8 & 0.89 & 0.89 & 0.89 & 0.89 & 0.81 & 0.81 & 0.81 & 0.81 & 0.65 & 0.65 & 0.65 & 0.65 & 0.48 & 0.48 & 0.48 & 0.49 \\
\hline \multirow{4}{*}{0.8} & 0 & 0.91 & 0.91 & 0.91 & 0.91 & 0.83 & 0.83 & 0.83 & 0.83 & 0.67 & 0.67 & 0.66 & 0.67 & 0.49 & 0.49 & 0.49 & 0.49 \\
\hline & 0.2 & 0.91 & 0.91 & 0.91 & 0.91 & 0.83 & 0.83 & 0.83 & 0.83 & 0.66 & 0.66 & 0.66 & 0.66 & 0.49 & 0.49 & 0.49 & 0.49 \\
\hline & 0.5 & 0.88 & 0.88 & 0.88 & 0.88 & 0.81 & 0.81 & 0.81 & 0.81 & 0.65 & 0.65 & 0.65 & 0.65 & 0.48 & 0.48 & 0.48 & 0.48 \\
\hline & 0.8 & 0.84 & 0.84 & 0.84 & 0.84 & 0.77 & 0.77 & 0.77 & 0.77 & 0.63 & 0.63 & 0.63 & 0.62 & 0.47 & 0.47 & 0.47 & 0.47 \\
\hline
\end{tabular}

Note: ICC $=$ Intraclass Correlation Coefficient. 
Table 4 cont.

Average Design Effect values for the L1 predictor: Uniform Distribution conditions $(\mathrm{m}=10, \mathrm{k}=75)$

\begin{tabular}{|c|c|c|c|c|c|c|c|c|c|c|c|c|c|c|c|c|c|}
\hline \multirow{4}{*}{$\begin{array}{c}\text { L1 } \\
\text { Effect }\end{array}$} & \multirow{4}{*}{$\begin{array}{c}\text { Interaction } \\
\text { Effect }\end{array}$} & \multicolumn{16}{|c|}{ ICC Values } \\
\hline & & \multicolumn{4}{|c|}{0} & \multicolumn{4}{|c|}{0.1} & \multicolumn{4}{|c|}{0.3} & \multicolumn{4}{|c|}{0.5} \\
\hline & & \multirow[b]{2}{*}{0} & \multirow[b]{2}{*}{0.2} & \multirow[b]{2}{*}{0.5} & \multirow[b]{2}{*}{0.8} & \multirow[b]{2}{*}{0} & \multirow[b]{2}{*}{0.2} & \multirow[b]{2}{*}{0.5} & \multicolumn{2}{|c|}{ L2 Effect } & \multirow[b]{2}{*}{0.2} & \multirow[b]{2}{*}{0.5} & \multirow[b]{2}{*}{0.8} & \multirow[b]{2}{*}{0} & \multirow[b]{2}{*}{0.2} & \multirow[b]{2}{*}{0.5} & \multirow[b]{2}{*}{0.8} \\
\hline & & & & & & & & & 0.8 & 0 & & & & & & & \\
\hline \multirow{4}{*}{0} & 0 & 0.99 & 0.99 & 0.99 & 0.99 & 0.90 & 0.91 & 0.90 & 0.91 & 0.71 & 0.71 & 0.71 & 0.71 & 0.51 & 0.51 & 0.51 & 0.51 \\
\hline & 0.2 & 0.99 & 0.99 & 0.99 & 0.99 & 0.90 & 0.90 & 0.90 & 0.90 & 0.71 & 0.71 & 0.71 & 0.71 & 0.51 & 0.50 & 0.51 & 0.51 \\
\hline & 0.5 & 0.96 & 0.96 & 0.96 & 0.97 & 0.87 & 0.88 & 0.87 & 0.87 & 0.69 & 0.69 & 0.69 & 0.69 & 0.50 & 0.50 & 0.50 & 0.50 \\
\hline & 0.8 & 0.91 & 0.91 & 0.91 & 0.91 & 0.83 & 0.83 & 0.83 & 0.83 & 0.66 & 0.66 & 0.66 & 0.66 & 0.48 & 0.49 & 0.49 & 0.49 \\
\hline \multirow{4}{*}{0.2} & 0 & 0.99 & 0.99 & 0.99 & 0.99 & 0.90 & 0.90 & 0.90 & 0.90 & 0.71 & 0.70 & 0.70 & 0.70 & 0.51 & 0.51 & 0.51 & 0.51 \\
\hline & 0.2 & 0.99 & 0.99 & 0.99 & 0.99 & 0.89 & 0.89 & 0.89 & 0.89 & 0.70 & 0.70 & 0.70 & 0.70 & 0.51 & 0.51 & 0.51 & 0.51 \\
\hline & 0.5 & 0.96 & 0.96 & 0.96 & 0.96 & 0.87 & 0.87 & 0.87 & 0.87 & 0.68 & 0.68 & 0.69 & 0.69 & 0.50 & 0.50 & 0.50 & 0.50 \\
\hline & 0.8 & 0.91 & 0.91 & 0.91 & 0.91 & 0.83 & 0.82 & 0.83 & 0.83 & 0.66 & 0.66 & 0.66 & 0.66 & 0.48 & 0.48 & 0.49 & 0.49 \\
\hline \multirow{4}{*}{0.5} & 0 & 0.96 & 0.96 & 0.96 & 0.96 & 0.87 & 0.87 & 0.87 & 0.87 & 0.69 & 0.69 & 0.69 & 0.69 & 0.50 & 0.50 & 0.50 & 0.50 \\
\hline & 0.2 & 0.96 & 0.96 & 0.96 & 0.96 & 0.87 & 0.87 & 0.87 & 0.87 & 0.69 & 0.69 & 0.68 & 0.69 & 0.50 & 0.50 & 0.50 & 0.50 \\
\hline & 0.5 & 0.93 & 0.93 & 0.93 & 0.93 & 0.85 & 0.84 & 0.85 & 0.84 & 0.67 & 0.67 & 0.67 & 0.67 & 0.49 & 0.49 & 0.49 & 0.49 \\
\hline & 0.8 & 0.88 & 0.88 & 0.88 & 0.88 & 0.81 & 0.80 & 0.81 & 0.80 & 0.65 & 0.64 & 0.65 & 0.65 & 0.48 & 0.48 & 0.48 & 0.48 \\
\hline \multirow{4}{*}{0.8} & 0 & 0.91 & 0.91 & 0.91 & 0.91 & 0.83 & 0.83 & 0.83 & 0.83 & 0.66 & 0.66 & 0.66 & 0.66 & 0.49 & 0.49 & 0.49 & 0.49 \\
\hline & 0.2 & 0.90 & 0.90 & 0.90 & 0.90 & 0.82 & 0.83 & 0.82 & 0.82 & 0.66 & 0.66 & 0.66 & 0.66 & 0.48 & 0.48 & 0.48 & 0.48 \\
\hline & 0.5 & 0.88 & 0.88 & 0.88 & 0.88 & 0.80 & 0.80 & 0.80 & 0.80 & 0.65 & 0.64 & 0.65 & 0.64 & 0.48 & 0.48 & 0.48 & 0.48 \\
\hline & 0.8 & 0.84 & 0.84 & 0.84 & 0.84 & 0.77 & 0.77 & 0.77 & 0.77 & 0.62 & 0.62 & 0.62 & 0.62 & 0.46 & 0.46 & 0.47 & 0.46 \\
\hline
\end{tabular}

Note: ICC $=$ Intraclass Correlation Coefficient. 
Table 4 cont.

Average Design Effect values for the L1 predictor: Uniform Distribution conditions ( $\mathrm{m}=10, \mathrm{k}=100)$

\begin{tabular}{|c|c|c|c|c|c|c|c|c|c|c|c|c|c|c|c|c|c|}
\hline \multirow{4}{*}{$\begin{array}{c}\text { L1 } \\
\text { Effect }\end{array}$} & \multirow{4}{*}{$\begin{array}{c}\text { Interaction } \\
\text { Effect }\end{array}$} & \multicolumn{16}{|c|}{ ICC Values } \\
\hline & & \multicolumn{4}{|c|}{0} & \multicolumn{4}{|c|}{0.1} & \multicolumn{4}{|c|}{0.3} & \multicolumn{4}{|c|}{0.5} \\
\hline & & \multicolumn{12}{|c|}{ L2 Effect } & & & & \\
\hline & & 0 & 0.2 & 0.5 & 0.8 & 0 & 0.2 & 0.5 & 0.8 & 0 & 0.2 & 0.5 & 0.8 & 0 & 0.2 & 0.5 & 0.8 \\
\hline \multirow{4}{*}{0} & 0 & 0.99 & 0.99 & 0.99 & 0.99 & 0.90 & 0.90 & 0.90 & 0.90 & 0.70 & 0.70 & 0.71 & 0.70 & 0.51 & 0.51 & 0.51 & 0.51 \\
\hline & 0.2 & 0.99 & 0.99 & 0.99 & 0.99 & 0.90 & 0.90 & 0.90 & 0.90 & 0.70 & 0.70 & 0.70 & 0.70 & 0.51 & 0.51 & 0.51 & 0.51 \\
\hline & 0.5 & 0.96 & 0.96 & 0.96 & 0.96 & 0.87 & 0.87 & 0.87 & 0.87 & 0.69 & 0.69 & 0.69 & 0.69 & 0.50 & 0.50 & 0.50 & 0.50 \\
\hline & 0.8 & 0.91 & 0.91 & 0.91 & 0.91 & 0.83 & 0.83 & 0.83 & 0.83 & 0.66 & 0.66 & 0.66 & 0.66 & 0.49 & 0.48 & 0.48 & 0.48 \\
\hline \multirow{4}{*}{0.2} & 0 & 0.99 & 0.99 & 0.99 & 0.99 & 0.90 & 0.90 & 0.90 & 0.90 & 0.70 & 0.70 & 0.70 & 0.70 & 0.51 & 0.51 & 0.51 & 0.50 \\
\hline & 0.2 & 0.99 & 0.99 & 0.99 & 0.99 & 0.89 & 0.89 & 0.89 & 0.89 & 0.70 & 0.70 & 0.70 & 0.70 & 0.51 & 0.50 & 0.51 & 0.51 \\
\hline & 0.5 & 0.96 & 0.96 & 0.96 & 0.96 & 0.87 & 0.87 & 0.87 & 0.87 & 0.68 & 0.68 & 0.68 & 0.69 & 0.50 & 0.50 & 0.50 & 0.50 \\
\hline & 0.8 & 0.91 & 0.90 & 0.91 & 0.91 & 0.82 & 0.83 & 0.83 & 0.82 & 0.66 & 0.66 & 0.66 & 0.66 & 0.48 & 0.48 & 0.48 & 0.48 \\
\hline \multirow{4}{*}{0.5} & 0 & 0.96 & 0.96 & 0.96 & 0.96 & 0.87 & 0.87 & 0.87 & 0.87 & 0.69 & 0.69 & 0.69 & 0.69 & 0.50 & 0.50 & 0.50 & 0.49 \\
\hline & 0.2 & 0.96 & 0.96 & 0.96 & 0.96 & 0.87 & 0.87 & 0.87 & 0.87 & 0.68 & 0.68 & 0.68 & 0.68 & 0.50 & 0.50 & 0.50 & 0.49 \\
\hline & 0.5 & 0.93 & 0.93 & 0.93 & 0.93 & 0.84 & 0.84 & 0.84 & 0.84 & 0.67 & 0.67 & 0.67 & 0.67 & 0.49 & 0.49 & 0.49 & 0.49 \\
\hline & 0.8 & 0.88 & 0.88 & 0.88 & 0.88 & 0.80 & 0.80 & 0.80 & 0.80 & 0.64 & 0.64 & 0.64 & 0.64 & 0.47 & 0.47 & 0.48 & 0.47 \\
\hline \multirow{4}{*}{0.8} & 0 & 0.91 & 0.91 & 0.91 & 0.91 & 0.83 & 0.83 & 0.83 & 0.83 & 0.66 & 0.66 & 0.66 & 0.66 & 0.48 & 0.48 & 0.48 & 0.48 \\
\hline & 0.2 & 0.90 & 0.90 & 0.90 & 0.90 & 0.82 & 0.82 & 0.82 & 0.82 & 0.65 & 0.66 & 0.66 & 0.66 & 0.48 & 0.48 & 0.48 & 0.48 \\
\hline & 0.5 & 0.88 & 0.88 & 0.88 & 0.88 & 0.80 & 0.80 & 0.80 & 0.80 & 0.64 & 0.64 & 0.64 & 0.64 & 0.47 & 0.47 & 0.48 & 0.47 \\
\hline & 0.8 & 0.83 & 0.83 & 0.83 & 0.83 & 0.76 & 0.77 & 0.76 & 0.77 & 0.62 & 0.62 & 0.62 & 0.62 & 0.46 & 0.46 & 0.46 & 0.46 \\
\hline
\end{tabular}

Note: ICC $=$ Intraclass Correlation Coefficient. 
Table 4 cont.

Average Design Effect values for the L1 predictor: Uniform Distribution conditions $(\mathrm{m}=20, \mathrm{k}=25)$

\begin{tabular}{|c|c|c|c|c|c|c|c|c|c|c|c|c|c|c|c|c|c|}
\hline \multirow{4}{*}{$\begin{array}{c}\text { L1 } \\
\text { Effect }\end{array}$} & \multirow{4}{*}{$\begin{array}{c}\text { Interaction } \\
\text { Effect }\end{array}$} & \multicolumn{16}{|c|}{ ICC Values } \\
\hline & & \multicolumn{4}{|c|}{0} & \multicolumn{4}{|c|}{0.1} & \multicolumn{4}{|c|}{0.3} & \multicolumn{4}{|c|}{0.5} \\
\hline & & \multirow[b]{2}{*}{0} & \multirow[b]{2}{*}{0.2} & \multirow[b]{2}{*}{0.5} & \multirow[b]{2}{*}{0.8} & \multirow[b]{2}{*}{0} & \multirow[b]{2}{*}{0.2} & \multirow[b]{2}{*}{0.5} & \multicolumn{2}{|c|}{ L2 Effect } & \multirow[b]{2}{*}{0.2} & \multirow[b]{2}{*}{0.5} & \multirow[b]{2}{*}{0.8} & \multirow[b]{2}{*}{0} & \multirow[b]{2}{*}{0.2} & \multirow[b]{2}{*}{0.5} & \multirow[b]{2}{*}{0.8} \\
\hline & & & & & & & & & 0.8 & 0 & & & & & & & \\
\hline \multirow{4}{*}{0} & 0 & 1.00 & 1.00 & 1.00 & 1.00 & 0.91 & 0.91 & 0.91 & 0.91 & 0.72 & 0.72 & 0.72 & 0.73 & 0.53 & 0.53 & 0.53 & 0.53 \\
\hline & 0.2 & 0.99 & 1.00 & 0.99 & 0.99 & 0.91 & 0.91 & 0.91 & 0.91 & 0.72 & 0.72 & 0.72 & 0.72 & 0.53 & 0.53 & 0.53 & 0.53 \\
\hline & 0.5 & 0.98 & 0.98 & 0.98 & 0.98 & 0.89 & 0.90 & 0.90 & 0.90 & 0.71 & 0.71 & 0.72 & 0.72 & 0.53 & 0.52 & 0.53 & 0.53 \\
\hline & 0.8 & 0.96 & 0.96 & 0.96 & 0.96 & 0.87 & 0.88 & 0.87 & 0.88 & 0.70 & 0.70 & 0.70 & 0.70 & 0.52 & 0.52 & 0.52 & 0.52 \\
\hline \multirow{4}{*}{0.2} & 0 & 0.99 & 0.99 & 0.99 & 0.99 & 0.91 & 0.91 & 0.91 & 0.91 & 0.72 & 0.72 & 0.72 & 0.72 & 0.54 & 0.53 & 0.53 & 0.53 \\
\hline & 0.2 & 0.99 & 0.99 & 0.99 & 0.99 & 0.91 & 0.91 & 0.91 & 0.91 & 0.72 & 0.72 & 0.73 & 0.73 & 0.53 & 0.53 & 0.53 & 0.53 \\
\hline & 0.5 & 0.98 & 0.98 & 0.98 & 0.98 & 0.90 & 0.90 & 0.90 & 0.89 & 0.72 & 0.71 & 0.71 & 0.71 & 0.52 & 0.53 & 0.52 & 0.53 \\
\hline & 0.8 & 0.96 & 0.96 & 0.96 & 0.96 & 0.88 & 0.87 & 0.87 & 0.87 & 0.70 & 0.70 & 0.70 & 0.70 & 0.52 & 0.52 & 0.52 & 0.52 \\
\hline \multirow{4}{*}{0.5} & 0 & 0.98 & 0.98 & 0.98 & 0.98 & 0.89 & 0.89 & 0.89 & 0.89 & 0.72 & 0.71 & 0.71 & 0.72 & 0.53 & 0.53 & 0.53 & 0.53 \\
\hline & 0.2 & 0.98 & 0.98 & 0.98 & 0.98 & 0.90 & 0.89 & 0.89 & 0.89 & 0.71 & 0.72 & 0.71 & 0.72 & 0.52 & 0.53 & 0.53 & 0.52 \\
\hline & 0.5 & 0.97 & 0.97 & 0.97 & 0.97 & 0.88 & 0.88 & 0.88 & 0.89 & 0.70 & 0.71 & 0.70 & 0.71 & 0.52 & 0.52 & 0.52 & 0.53 \\
\hline & 0.8 & 0.95 & 0.94 & 0.94 & 0.95 & 0.86 & 0.86 & 0.86 & 0.86 & 0.69 & 0.70 & 0.69 & 0.69 & 0.52 & 0.52 & 0.51 & 0.52 \\
\hline \multirow{4}{*}{0.8} & 0 & 0.96 & 0.96 & 0.96 & 0.96 & 0.87 & 0.87 & 0.87 & 0.87 & 0.70 & 0.70 & 0.70 & 0.70 & 0.52 & 0.52 & 0.52 & 0.52 \\
\hline & 0.2 & 0.95 & 0.95 & 0.95 & 0.95 & 0.87 & 0.87 & 0.87 & 0.87 & 0.70 & 0.70 & 0.70 & 0.70 & 0.52 & 0.52 & 0.52 & 0.52 \\
\hline & 0.5 & 0.94 & 0.94 & 0.94 & 0.94 & 0.86 & 0.86 & 0.86 & 0.86 & 0.69 & 0.69 & 0.69 & 0.70 & 0.52 & 0.51 & 0.52 & 0.52 \\
\hline & 0.8 & 0.92 & 0.92 & 0.92 & 0.92 & 0.84 & 0.84 & 0.84 & 0.84 & 0.68 & 0.68 & 0.68 & 0.68 & 0.51 & 0.51 & 0.51 & 0.51 \\
\hline
\end{tabular}

Note: ICC = Intraclass Correlation Coefficient. 
Table 4 cont.

Average Design Effect values for the L1 predictor: Uniform Distribution conditions $(\mathrm{m}=20, \mathrm{k}=50)$

\begin{tabular}{|c|c|c|c|c|c|c|c|c|c|c|c|c|c|c|c|c|c|}
\hline \multirow{4}{*}{$\begin{array}{c}\text { L1 } \\
\text { Effect }\end{array}$} & \multirow{4}{*}{$\begin{array}{c}\text { Interaction } \\
\text { Effect }\end{array}$} & \multicolumn{16}{|c|}{ ICC Values } \\
\hline & & \multicolumn{4}{|c|}{0} & \multicolumn{4}{|c|}{0.1} & \multicolumn{4}{|c|}{0.3} & \multicolumn{4}{|c|}{0.5} \\
\hline & & \multirow[b]{2}{*}{0} & \multirow[b]{2}{*}{0.2} & \multirow[b]{2}{*}{0.5} & \multirow[b]{2}{*}{0.8} & \multirow[b]{2}{*}{0} & \multirow[b]{2}{*}{0.2} & \multirow[b]{2}{*}{0.5} & \multicolumn{2}{|c|}{ L2 Effect } & \multirow[b]{2}{*}{0.2} & \multirow[b]{2}{*}{0.5} & \multirow[b]{2}{*}{0.8} & \multirow[b]{2}{*}{0} & \multirow[b]{2}{*}{0.2} & \multirow[b]{2}{*}{0.5} & \multirow[b]{2}{*}{0.8} \\
\hline & & & & & & & & & 0.8 & 0 & & & & & & & \\
\hline \multirow{4}{*}{0} & 0 & 1.00 & 1.00 & 1.00 & 1.00 & 0.91 & 0.91 & 0.91 & 0.90 & 0.71 & 0.71 & 0.71 & 0.71 & 0.52 & 0.52 & 0.52 & 0.51 \\
\hline & 0.2 & 1.00 & 0.99 & 1.00 & 0.99 & 0.90 & 0.90 & 0.90 & 0.90 & 0.71 & 0.71 & 0.71 & 0.71 & 0.52 & 0.51 & 0.51 & 0.52 \\
\hline & 0.5 & 0.98 & 0.98 & 0.98 & 0.98 & 0.89 & 0.89 & 0.89 & 0.89 & 0.70 & 0.70 & 0.70 & 0.70 & 0.51 & 0.51 & 0.51 & 0.51 \\
\hline & 0.8 & 0.96 & 0.95 & 0.96 & 0.96 & 0.87 & 0.87 & 0.87 & 0.87 & 0.69 & 0.69 & 0.69 & 0.69 & 0.50 & 0.50 & 0.50 & 0.51 \\
\hline \multirow{4}{*}{0.2} & 0 & 1.00 & 1.00 & 0.99 & 1.00 & 0.90 & 0.90 & 0.90 & 0.90 & 0.71 & 0.71 & 0.71 & 0.71 & 0.51 & 0.51 & 0.51 & 0.52 \\
\hline & 0.2 & 0.99 & 0.99 & 0.99 & 0.99 & 0.90 & 0.90 & 0.90 & 0.90 & 0.71 & 0.71 & 0.71 & 0.71 & 0.51 & 0.51 & 0.51 & 0.51 \\
\hline & 0.5 & 0.98 & 0.98 & 0.98 & 0.98 & 0.89 & 0.89 & 0.89 & 0.89 & 0.70 & 0.70 & 0.70 & 0.70 & 0.51 & 0.51 & 0.51 & 0.51 \\
\hline & 0.8 & 0.95 & 0.95 & 0.95 & 0.95 & 0.87 & 0.86 & 0.87 & 0.87 & 0.69 & 0.69 & 0.69 & 0.69 & 0.50 & 0.50 & 0.50 & 0.50 \\
\hline \multirow{4}{*}{0.5} & 0 & 0.98 & 0.98 & 0.98 & 0.98 & 0.89 & 0.89 & 0.89 & 0.89 & 0.70 & 0.70 & 0.70 & 0.70 & 0.51 & 0.51 & 0.51 & 0.51 \\
\hline & 0.2 & 0.98 & 0.98 & 0.98 & 0.98 & 0.89 & 0.89 & 0.89 & 0.89 & 0.70 & 0.70 & 0.70 & 0.70 & 0.51 & 0.51 & 0.51 & 0.51 \\
\hline & 0.5 & 0.97 & 0.96 & 0.96 & 0.96 & 0.87 & 0.88 & 0.88 & 0.87 & 0.69 & 0.69 & 0.69 & 0.69 & 0.51 & 0.51 & 0.51 & 0.50 \\
\hline & 0.8 & 0.94 & 0.94 & 0.94 & 0.94 & 0.85 & 0.85 & 0.86 & 0.85 & 0.68 & 0.68 & 0.68 & 0.68 & 0.50 & 0.50 & 0.50 & 0.50 \\
\hline \multirow{4}{*}{0.8} & 0 & 0.95 & 0.95 & 0.95 & 0.95 & 0.87 & 0.87 & 0.87 & 0.87 & 0.69 & 0.69 & 0.69 & 0.69 & 0.50 & 0.50 & 0.50 & 0.50 \\
\hline & 0.2 & 0.95 & 0.95 & 0.95 & 0.95 & 0.86 & 0.86 & 0.86 & 0.86 & 0.68 & 0.69 & 0.69 & 0.69 & 0.50 & 0.50 & 0.50 & 0.50 \\
\hline & 0.5 & 0.94 & 0.94 & 0.94 & 0.94 & 0.85 & 0.85 & 0.85 & 0.85 & 0.68 & 0.68 & 0.68 & 0.68 & 0.50 & 0.50 & 0.50 & 0.50 \\
\hline & 0.8 & 0.91 & 0.91 & 0.91 & 0.91 & 0.83 & 0.83 & 0.83 & 0.83 & 0.67 & 0.66 & 0.66 & 0.67 & 0.49 & 0.50 & 0.49 & 0.49 \\
\hline
\end{tabular}

Note: ICC $=$ Intraclass Correlation Coefficient. 
Table 4 cont.

Average Design Effect values for the L1 predictor: Uniform Distribution conditions $(\mathrm{m}=20, \mathrm{k}=75)$

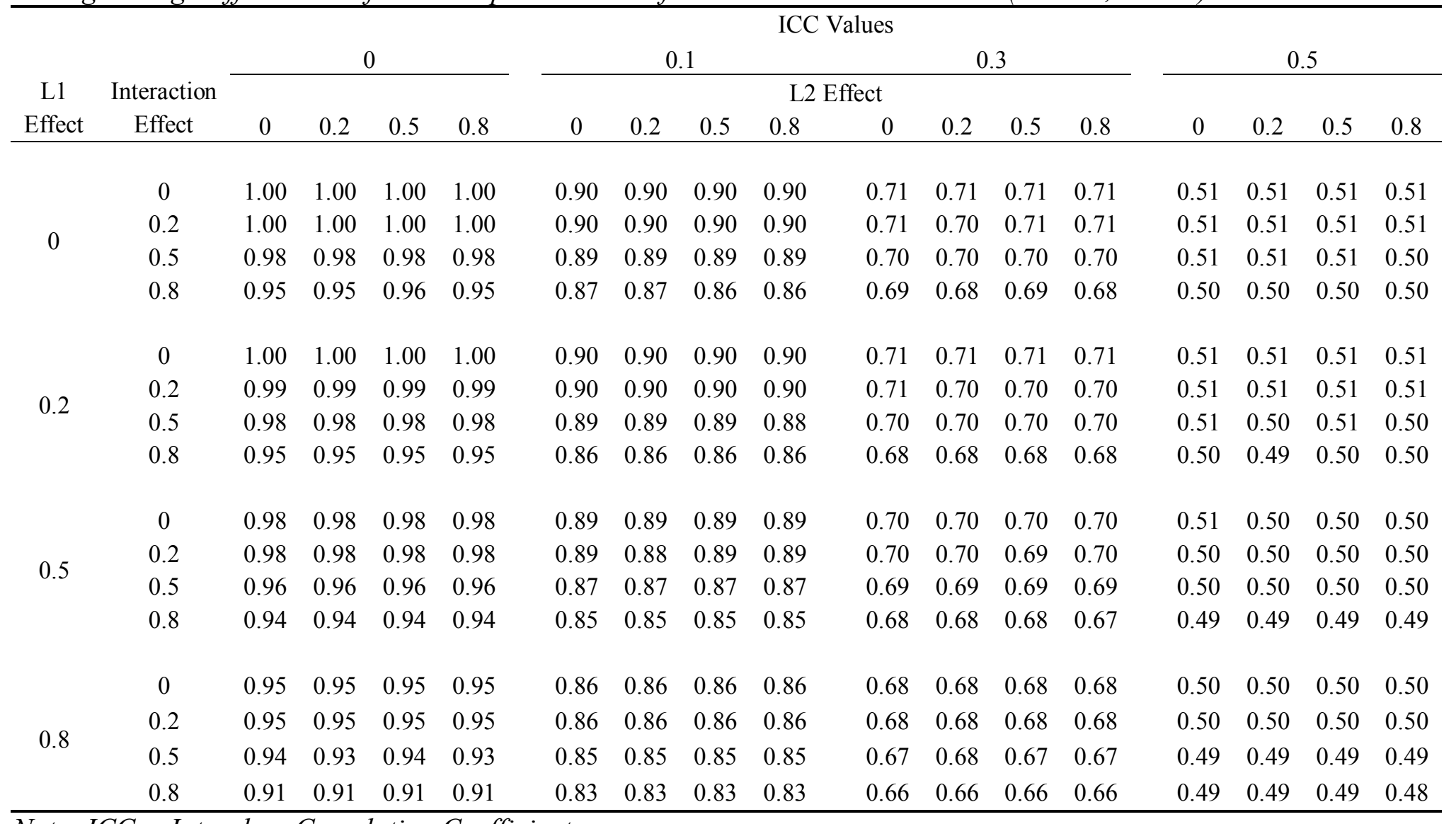

Note: ICC $=$ Intraclass Correlation Coefficient. 
Table 4 cont.

Average Design Effect values for the L1 predictor: Uniform Distribution conditions ( $\mathrm{m}=20, \mathrm{k}=100)$

\begin{tabular}{|c|c|c|c|c|c|c|c|c|c|c|c|c|c|c|c|c|c|}
\hline \multirow{4}{*}{$\begin{array}{c}\text { L1 } \\
\text { Effect }\end{array}$} & \multirow{4}{*}{$\begin{array}{c}\text { Interaction } \\
\text { Effect }\end{array}$} & \multicolumn{16}{|c|}{ ICC Values } \\
\hline & & \multicolumn{4}{|c|}{0} & \multicolumn{4}{|c|}{0.1} & \multicolumn{4}{|c|}{0.3} & \multicolumn{4}{|c|}{0.5} \\
\hline & & \multicolumn{12}{|c|}{ L2 Effect } & & & & \\
\hline & & 0 & 0.2 & 0.5 & 0.8 & 0 & 0.2 & 0.5 & 0.8 & 0 & 0.2 & 0.5 & 0.8 & 0 & 0.2 & 0.5 & 0.8 \\
\hline \multirow{4}{*}{0} & 0 & 1.00 & 1.00 & 1.00 & 1.00 & 0.90 & 0.90 & 0.90 & 0.90 & 0.70 & 0.70 & 0.71 & 0.71 & 0.51 & 0.51 & 0.51 & 0.51 \\
\hline & 0.2 & 1.00 & 1.00 & 1.00 & 1.00 & 0.90 & 0.90 & 0.90 & 0.90 & 0.70 & 0.70 & 0.70 & 0.70 & 0.51 & 0.51 & 0.51 & 0.51 \\
\hline & 0.5 & 0.98 & 0.98 & 0.98 & 0.98 & 0.89 & 0.89 & 0.89 & 0.89 & 0.70 & 0.70 & 0.70 & 0.70 & 0.50 & 0.50 & 0.50 & 0.50 \\
\hline & 0.8 & 0.95 & 0.95 & 0.95 & 0.95 & 0.86 & 0.86 & 0.86 & 0.86 & 0.68 & 0.68 & 0.68 & 0.68 & 0.49 & 0.49 & 0.50 & 0.49 \\
\hline \multirow{4}{*}{0.2} & 0 & 1.00 & 1.00 & 1.00 & 1.00 & 0.90 & 0.90 & 0.90 & 0.90 & 0.70 & 0.70 & 0.70 & 0.70 & 0.50 & 0.51 & 0.51 & 0.51 \\
\hline & 0.2 & 0.99 & 0.99 & 0.99 & 0.99 & 0.90 & 0.90 & 0.90 & 0.90 & 0.70 & 0.70 & 0.70 & 0.70 & 0.51 & 0.51 & 0.51 & 0.50 \\
\hline & 0.5 & 0.98 & 0.98 & 0.98 & 0.98 & 0.88 & 0.89 & 0.88 & 0.88 & 0.70 & 0.69 & 0.70 & 0.69 & 0.50 & 0.50 & 0.50 & 0.50 \\
\hline & 0.8 & 0.95 & 0.95 & 0.95 & 0.95 & 0.86 & 0.86 & 0.86 & 0.86 & 0.68 & 0.68 & 0.68 & 0.68 & 0.49 & 0.50 & 0.49 & 0.50 \\
\hline \multirow{4}{*}{0.5} & 0 & 0.98 & 0.98 & 0.98 & 0.98 & 0.89 & 0.89 & 0.89 & 0.89 & 0.70 & 0.70 & 0.70 & 0.69 & 0.50 & 0.51 & 0.50 & 0.50 \\
\hline & 0.2 & 0.98 & 0.98 & 0.98 & 0.98 & 0.89 & 0.88 & 0.88 & 0.88 & 0.69 & 0.70 & 0.70 & 0.69 & 0.50 & 0.50 & 0.50 & 0.50 \\
\hline & 0.5 & 0.96 & 0.96 & 0.96 & 0.96 & 0.87 & 0.87 & 0.87 & 0.87 & 0.69 & 0.69 & 0.69 & 0.69 & 0.50 & 0.50 & 0.50 & 0.50 \\
\hline & 0.8 & 0.93 & 0.94 & 0.93 & 0.94 & 0.85 & 0.85 & 0.85 & 0.85 & 0.67 & 0.67 & 0.67 & 0.67 & 0.49 & 0.49 & 0.49 & 0.49 \\
\hline \multirow{4}{*}{0.8} & 0 & 0.95 & 0.95 & 0.95 & 0.95 & 0.86 & 0.86 & 0.86 & 0.86 & 0.68 & 0.68 & 0.68 & 0.68 & 0.50 & 0.49 & 0.49 & 0.50 \\
\hline & 0.2 & 0.95 & 0.95 & 0.95 & 0.95 & 0.86 & 0.86 & 0.86 & 0.86 & 0.68 & 0.68 & 0.68 & 0.68 & 0.49 & 0.49 & 0.49 & 0.49 \\
\hline & 0.5 & 0.93 & 0.94 & 0.93 & 0.94 & 0.85 & 0.85 & 0.85 & 0.85 & 0.67 & 0.67 & 0.67 & 0.67 & 0.49 & 0.49 & 0.49 & 0.49 \\
\hline & 0.8 & 0.91 & 0.91 & 0.91 & 0.91 & 0.83 & 0.83 & 0.83 & 0.83 & 0.66 & 0.66 & 0.66 & 0.66 & 0.48 & 0.48 & 0.48 & 0.48 \\
\hline
\end{tabular}

Note: ICC $=$ Intraclass Correlation Coefficient. 
Table 4 cont.

Average Design Effect values for the L1 predictor: Poisson Distribution conditions $(\mathrm{m}=5, \mathrm{k}=25)$

\begin{tabular}{|c|c|c|c|c|c|c|c|c|c|c|c|c|c|c|c|c|c|}
\hline \multirow{4}{*}{$\begin{array}{c}\text { L1 } \\
\text { Effect }\end{array}$} & \multirow{4}{*}{$\begin{array}{c}\text { Interaction } \\
\text { Effect }\end{array}$} & \multicolumn{16}{|c|}{ ICC Values } \\
\hline & & \multicolumn{4}{|c|}{0} & \multicolumn{4}{|c|}{0.1} & \multicolumn{4}{|c|}{0.3} & \multicolumn{4}{|c|}{0.5} \\
\hline & & \multirow[b]{2}{*}{0} & \multirow[b]{2}{*}{0.2} & \multirow[b]{2}{*}{0.5} & \multirow[b]{2}{*}{0.8} & \multirow[b]{2}{*}{0} & \multirow[b]{2}{*}{0.2} & \multirow[b]{2}{*}{0.5} & \multicolumn{2}{|c|}{ L2 Effect } & \multirow[b]{2}{*}{0.2} & \multirow[b]{2}{*}{0.5} & \multirow[b]{2}{*}{0.8} & \multirow[b]{2}{*}{0} & \multirow[b]{2}{*}{0.2} & \multirow[b]{2}{*}{0.5} & \multirow[b]{2}{*}{0.8} \\
\hline & & & & & & & & & 0.8 & 0 & & & & & & & \\
\hline \multirow{4}{*}{0} & 0 & 0.98 & 0.98 & 0.98 & 0.98 & 0.91 & 0.92 & 0.91 & 0.92 & 0.73 & 0.73 & 0.74 & 0.73 & 0.53 & 0.54 & 0.54 & 0.54 \\
\hline & 0.2 & 0.98 & 0.98 & 0.98 & 0.98 & 0.91 & 0.91 & 0.90 & 0.91 & 0.72 & 0.72 & 0.72 & 0.73 & 0.53 & 0.54 & 0.54 & 0.53 \\
\hline & 0.5 & 0.94 & 0.94 & 0.94 & 0.95 & 0.87 & 0.87 & 0.87 & 0.87 & 0.70 & 0.69 & 0.69 & 0.70 & 0.52 & 0.52 & 0.52 & 0.52 \\
\hline & 0.8 & 0.87 & 0.87 & 0.88 & 0.87 & 0.79 & 0.80 & 0.80 & 0.80 & 0.65 & 0.66 & 0.65 & 0.65 & 0.50 & 0.49 & 0.49 & 0.49 \\
\hline \multirow{4}{*}{0.2} & 0 & 0.98 & 0.98 & 0.98 & 0.98 & 0.91 & 0.90 & 0.91 & 0.90 & 0.72 & 0.72 & 0.73 & 0.73 & 0.54 & 0.54 & 0.54 & 0.54 \\
\hline & 0.2 & 0.97 & 0.97 & 0.97 & 0.97 & 0.90 & 0.90 & 0.90 & 0.90 & 0.72 & 0.72 & 0.72 & 0.72 & 0.53 & 0.53 & 0.53 & 0.53 \\
\hline & 0.5 & 0.93 & 0.94 & 0.93 & 0.93 & 0.86 & 0.86 & 0.86 & 0.86 & 0.70 & 0.69 & 0.69 & 0.69 & 0.52 & 0.51 & 0.51 & 0.52 \\
\hline & 0.8 & 0.87 & 0.86 & 0.87 & 0.86 & 0.80 & 0.79 & 0.79 & 0.80 & 0.65 & 0.65 & 0.65 & 0.65 & 0.49 & 0.49 & 0.49 & 0.49 \\
\hline \multirow{4}{*}{0.5} & 0 & 0.94 & 0.94 & 0.94 & 0.94 & 0.86 & 0.86 & 0.86 & 0.86 & 0.70 & 0.70 & 0.70 & 0.70 & 0.52 & 0.52 & 0.52 & 0.52 \\
\hline & 0.2 & 0.93 & 0.93 & 0.93 & 0.93 & 0.86 & 0.87 & 0.86 & 0.86 & 0.69 & 0.69 & 0.69 & 0.69 & 0.51 & 0.52 & 0.51 & 0.51 \\
\hline & 0.5 & 0.89 & 0.89 & 0.89 & 0.89 & 0.82 & 0.82 & 0.82 & 0.82 & 0.66 & 0.66 & 0.67 & 0.67 & 0.50 & 0.50 & 0.50 & 0.51 \\
\hline & 0.8 & 0.82 & 0.82 & 0.82 & 0.83 & 0.76 & 0.76 & 0.76 & 0.76 & 0.63 & 0.63 & 0.62 & 0.63 & 0.48 & 0.48 & 0.48 & 0.48 \\
\hline \multirow{4}{*}{0.8} & 0 & 0.86 & 0.86 & 0.85 & 0.86 & 0.79 & 0.79 & 0.79 & 0.79 & 0.64 & 0.64 & 0.64 & 0.65 & 0.49 & 0.49 & 0.49 & 0.49 \\
\hline & 0.2 & 0.85 & 0.85 & 0.85 & 0.85 & 0.78 & 0.78 & 0.79 & 0.79 & 0.64 & 0.65 & 0.65 & 0.64 & 0.49 & 0.49 & 0.49 & 0.49 \\
\hline & 0.5 & 0.81 & 0.82 & 0.82 & 0.82 & 0.75 & 0.75 & 0.75 & 0.75 & 0.63 & 0.63 & 0.62 & 0.62 & 0.47 & 0.48 & 0.48 & 0.48 \\
\hline & 0.8 & 0.76 & 0.76 & 0.76 & 0.76 & 0.70 & 0.70 & 0.71 & 0.71 & 0.58 & 0.58 & 0.58 & 0.58 & 0.45 & 0.46 & 0.45 & 0.45 \\
\hline
\end{tabular}

Note: ICC $=$ Intraclass Correlation Coefficient. 
Table 4 cont.

Average Design Effect values for the $L 1$ predictor: Poisson Distribution conditions $(\mathrm{m}=5, \mathrm{k}=50)$

\begin{tabular}{|c|c|c|c|c|c|c|c|c|c|c|c|c|c|c|c|c|c|}
\hline \multirow{4}{*}{$\begin{array}{c}\text { L1 } \\
\text { Effect }\end{array}$} & \multirow{4}{*}{$\begin{array}{c}\text { Interaction } \\
\text { Effect }\end{array}$} & \multicolumn{16}{|c|}{ ICC Values } \\
\hline & & \multicolumn{4}{|c|}{0} & \multicolumn{3}{|c|}{0.1} & & \multicolumn{4}{|c|}{0.3} & \multicolumn{4}{|c|}{0.5} \\
\hline & & \multirow[b]{2}{*}{0} & \multirow[b]{2}{*}{0.2} & \multirow[b]{2}{*}{0.5} & \multirow[b]{2}{*}{0.8} & \multirow[b]{2}{*}{0} & \multirow[b]{2}{*}{0.2} & \multirow[b]{2}{*}{0.5} & \multicolumn{2}{|c|}{ L2 Effect } & \multirow[b]{2}{*}{0.2} & \multirow[b]{2}{*}{0.5} & \multirow[b]{2}{*}{0.8} & \multirow[b]{2}{*}{0} & \multirow[b]{2}{*}{0.2} & \multirow[b]{2}{*}{0.5} & \multirow[b]{2}{*}{0.8} \\
\hline & & & & & & & & & 0.8 & 0 & & & & & & & \\
\hline \multirow{4}{*}{0} & 0 & 0.99 & 0.99 & 0.99 & 0.99 & 0.91 & 0.91 & 0.91 & 0.91 & 0.72 & 0.72 & 0.72 & 0.72 & 0.52 & 0.52 & 0.52 & 0.52 \\
\hline & 0.2 & 0.98 & 0.98 & 0.98 & 0.98 & 0.90 & 0.90 & 0.90 & 0.90 & 0.71 & 0.71 & 0.71 & 0.71 & 0.51 & 0.52 & 0.51 & 0.51 \\
\hline & 0.5 & 0.94 & 0.94 & 0.94 & 0.94 & 0.86 & 0.86 & 0.86 & 0.86 & 0.68 & 0.68 & 0.68 & 0.68 & 0.50 & 0.50 & 0.50 & 0.50 \\
\hline & 0.8 & 0.86 & 0.86 & 0.86 & 0.86 & 0.79 & 0.79 & 0.78 & 0.79 & 0.63 & 0.64 & 0.63 & 0.64 & 0.47 & 0.47 & 0.48 & 0.47 \\
\hline \multirow{4}{*}{0.2} & 0 & 0.98 & 0.98 & 0.98 & 0.98 & 0.90 & 0.90 & 0.90 & 0.90 & 0.71 & 0.71 & 0.71 & 0.71 & 0.51 & 0.52 & 0.52 & 0.51 \\
\hline & 0.2 & 0.97 & 0.97 & 0.97 & 0.97 & 0.89 & 0.89 & 0.89 & 0.89 & 0.70 & 0.70 & 0.70 & 0.70 & 0.51 & 0.51 & 0.51 & 0.52 \\
\hline & 0.5 & 0.93 & 0.93 & 0.93 & 0.93 & 0.85 & 0.85 & 0.85 & 0.85 & 0.67 & 0.68 & 0.68 & 0.68 & 0.50 & 0.50 & 0.49 & 0.50 \\
\hline & 0.8 & 0.84 & 0.85 & 0.85 & 0.85 & 0.78 & 0.78 & 0.78 & 0.78 & 0.63 & 0.63 & 0.63 & 0.63 & 0.47 & 0.47 & 0.47 & 0.47 \\
\hline \multirow{4}{*}{0.5} & 0 & 0.94 & 0.94 & 0.94 & 0.94 & 0.85 & 0.86 & 0.86 & 0.85 & 0.68 & 0.68 & 0.68 & 0.68 & 0.50 & 0.50 & 0.50 & 0.50 \\
\hline & 0.2 & 0.93 & 0.93 & 0.93 & 0.93 & 0.85 & 0.84 & 0.85 & 0.84 & 0.67 & 0.68 & 0.67 & 0.67 & 0.50 & 0.50 & 0.50 & 0.50 \\
\hline & 0.5 & 0.89 & 0.88 & 0.89 & 0.88 & 0.80 & 0.81 & 0.81 & 0.81 & 0.65 & 0.65 & 0.65 & 0.65 & 0.49 & 0.48 & 0.48 & 0.49 \\
\hline & 0.8 & 0.81 & 0.81 & 0.81 & 0.81 & 0.74 & 0.74 & 0.74 & 0.74 & 0.60 & 0.61 & 0.61 & 0.60 & 0.46 & 0.46 & 0.46 & 0.46 \\
\hline \multirow{4}{*}{0.8} & 0 & 0.85 & 0.85 & 0.85 & 0.85 & 0.77 & 0.78 & 0.78 & 0.78 & 0.63 & 0.63 & 0.63 & 0.63 & 0.47 & 0.47 & 0.48 & 0.47 \\
\hline & 0.2 & 0.84 & 0.84 & 0.84 & 0.84 & 0.77 & 0.77 & 0.77 & 0.77 & 0.63 & 0.63 & 0.63 & 0.62 & 0.47 & 0.47 & 0.47 & 0.47 \\
\hline & 0.5 & 0.80 & 0.80 & 0.80 & 0.80 & 0.73 & 0.74 & 0.74 & 0.74 & 0.60 & 0.60 & 0.60 & 0.61 & 0.45 & 0.46 & 0.46 & 0.46 \\
\hline & 0.8 & 0.74 & 0.74 & 0.74 & 0.74 & 0.68 & 0.68 & 0.68 & 0.68 & 0.57 & 0.56 & 0.57 & 0.57 & 0.43 & 0.43 & 0.43 & 0.43 \\
\hline
\end{tabular}

Note: ICC = Intraclass Correlation Coefficient. 
Table 4 cont.

Average Design Effect values for the $L 1$ predictor: Poisson Distribution conditions $(\mathrm{m}=5, \mathrm{k}=75)$

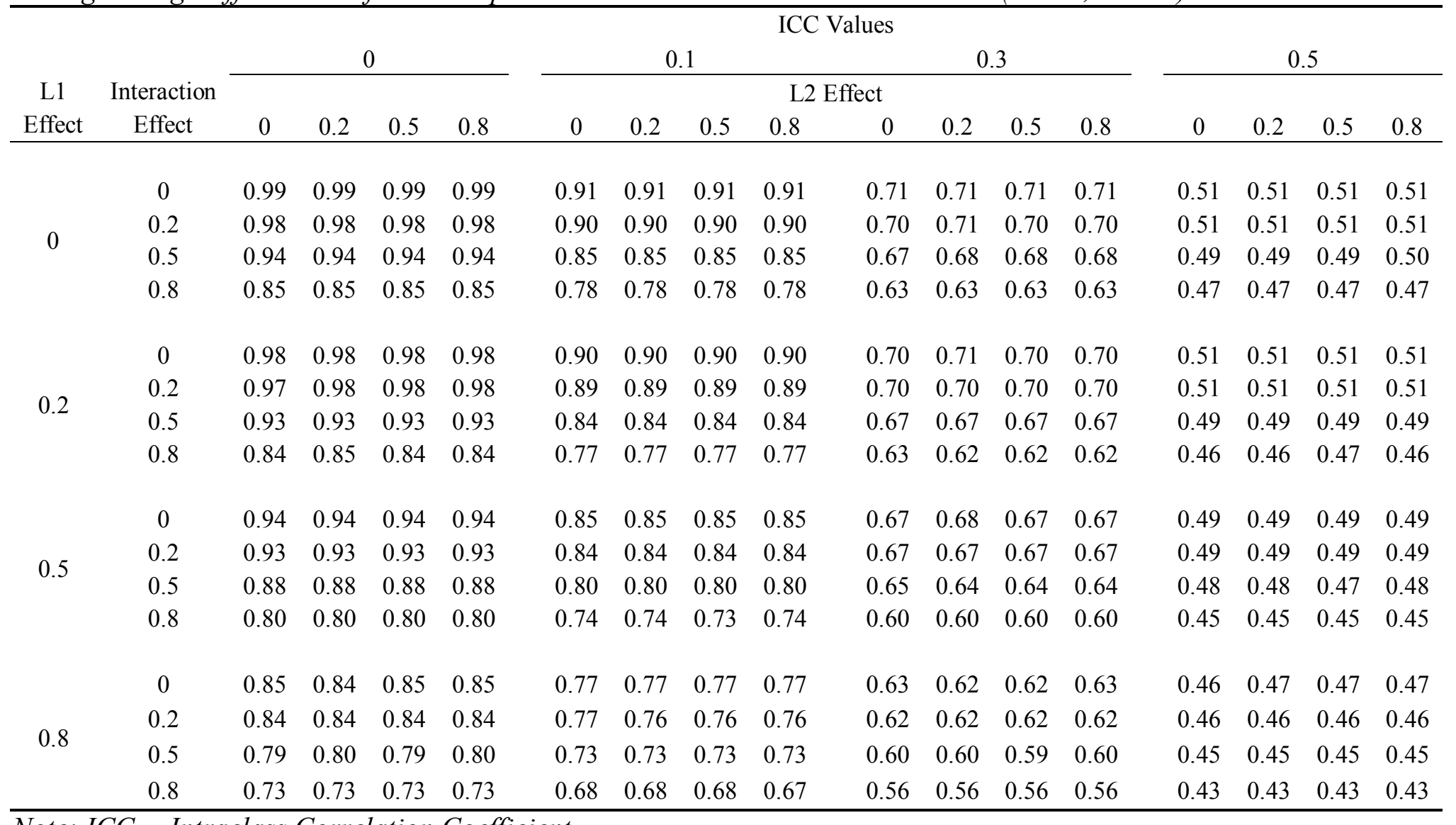

Note: ICC = Intraclass Correlation Coefficient. 
Table 4 cont.

Average Design Effect values for the $L 1$ predictor: Poisson Distribution conditions $(\mathrm{m}=5, \mathrm{k}=100)$

\begin{tabular}{|c|c|c|c|c|c|c|c|c|c|c|c|c|c|c|c|c|c|}
\hline \multirow{4}{*}{$\begin{array}{c}\text { L1 } \\
\text { Effect }\end{array}$} & \multirow{4}{*}{$\begin{array}{c}\text { Interaction } \\
\text { Effect }\end{array}$} & \multicolumn{16}{|c|}{ ICC Values } \\
\hline & & \multicolumn{4}{|c|}{0} & \multicolumn{4}{|c|}{0.1} & \multicolumn{4}{|c|}{0.3} & \multicolumn{4}{|c|}{0.5} \\
\hline & & \multirow[b]{2}{*}{0} & \multirow[b]{2}{*}{0.2} & \multirow[b]{2}{*}{0.5} & \multirow[b]{2}{*}{0.8} & \multirow[b]{2}{*}{0} & \multirow[b]{2}{*}{0.2} & \multirow[b]{2}{*}{0.5} & \multicolumn{2}{|c|}{ L2 Effect } & \multirow[b]{2}{*}{0.2} & \multirow[b]{2}{*}{0.5} & \multirow[b]{2}{*}{0.8} & \multirow[b]{2}{*}{0} & \multirow[b]{2}{*}{0.2} & \multirow[b]{2}{*}{0.5} & \multirow[b]{2}{*}{0.8} \\
\hline & & & & & & & & & 0.8 & 0 & & & & & & & \\
\hline \multirow{4}{*}{0} & 0 & 0.99 & 0.99 & 0.99 & 0.99 & 0.90 & 0.91 & 0.91 & 0.90 & 0.71 & 0.71 & 0.71 & 0.71 & 0.51 & 0.51 & 0.51 & 0.51 \\
\hline & 0.2 & 0.98 & 0.98 & 0.98 & 0.99 & 0.90 & 0.90 & 0.90 & 0.90 & 0.70 & 0.70 & 0.70 & 0.70 & 0.50 & 0.51 & 0.50 & 0.51 \\
\hline & 0.5 & 0.94 & 0.94 & 0.94 & 0.94 & 0.85 & 0.85 & 0.85 & 0.85 & 0.68 & 0.67 & 0.68 & 0.67 & 0.49 & 0.49 & 0.49 & 0.49 \\
\hline & 0.8 & 0.85 & 0.85 & 0.85 & 0.85 & 0.77 & 0.77 & 0.77 & 0.78 & 0.62 & 0.62 & 0.63 & 0.62 & 0.46 & 0.47 & 0.46 & 0.47 \\
\hline \multirow{4}{*}{0.2} & 0 & 0.98 & 0.98 & 0.98 & 0.98 & 0.90 & 0.90 & 0.90 & 0.89 & 0.70 & 0.70 & 0.70 & 0.70 & 0.51 & 0.51 & 0.51 & 0.50 \\
\hline & 0.2 & 0.98 & 0.98 & 0.98 & 0.98 & 0.89 & 0.89 & 0.89 & 0.89 & 0.70 & 0.70 & 0.69 & 0.69 & 0.50 & 0.50 & 0.50 & 0.50 \\
\hline & 0.5 & 0.93 & 0.93 & 0.93 & 0.93 & 0.84 & 0.84 & 0.84 & 0.84 & 0.67 & 0.67 & 0.67 & 0.67 & 0.49 & 0.49 & 0.49 & 0.49 \\
\hline & 0.8 & 0.84 & 0.84 & 0.84 & 0.84 & 0.77 & 0.77 & 0.77 & 0.77 & 0.62 & 0.62 & 0.62 & 0.62 & 0.46 & 0.46 & 0.46 & 0.46 \\
\hline \multirow{4}{*}{0.5} & 0 & 0.94 & 0.94 & 0.94 & 0.94 & 0.85 & 0.85 & 0.85 & 0.85 & 0.67 & 0.67 & 0.67 & 0.68 & 0.49 & 0.49 & 0.49 & 0.49 \\
\hline & 0.2 & 0.93 & 0.92 & 0.93 & 0.93 & 0.84 & 0.84 & 0.84 & 0.84 & 0.67 & 0.67 & 0.67 & 0.67 & 0.49 & 0.49 & 0.48 & 0.49 \\
\hline & 0.5 & 0.88 & 0.88 & 0.88 & 0.88 & 0.80 & 0.80 & 0.80 & 0.80 & 0.64 & 0.64 & 0.64 & 0.64 & 0.47 & 0.47 & 0.47 & 0.47 \\
\hline & 0.8 & 0.80 & 0.80 & 0.80 & 0.80 & 0.73 & 0.73 & 0.73 & 0.73 & 0.59 & 0.60 & 0.59 & 0.60 & 0.45 & 0.45 & 0.45 & 0.45 \\
\hline \multirow{4}{*}{0.8} & 0 & 0.85 & 0.85 & 0.84 & 0.84 & 0.77 & 0.77 & 0.77 & 0.77 & 0.62 & 0.62 & 0.62 & 0.62 & 0.46 & 0.46 & 0.46 & 0.46 \\
\hline & 0.2 & 0.83 & 0.84 & 0.84 & 0.84 & 0.77 & 0.76 & 0.76 & 0.76 & 0.62 & 0.62 & 0.62 & 0.62 & 0.46 & 0.46 & 0.46 & 0.46 \\
\hline & 0.5 & 0.79 & 0.80 & 0.80 & 0.80 & 0.73 & 0.73 & 0.73 & 0.73 & 0.59 & 0.60 & 0.59 & 0.59 & 0.45 & 0.45 & 0.44 & 0.45 \\
\hline & 0.8 & 0.73 & 0.73 & 0.73 & 0.73 & 0.67 & 0.68 & 0.67 & 0.67 & 0.56 & 0.55 & 0.56 & 0.56 & 0.43 & 0.43 & 0.43 & 0.42 \\
\hline
\end{tabular}

Note: ICC = Intraclass Correlation Coefficient. 
Table 4 cont.

Average Design Effect values for the L1 predictor: Poisson Distribution conditions $(\mathrm{m}=10, \mathrm{k}=25)$

\begin{tabular}{|c|c|c|c|c|c|c|c|c|c|c|c|c|c|c|c|c|c|}
\hline \multirow{4}{*}{$\begin{array}{c}\text { L1 } \\
\text { Effect }\end{array}$} & \multirow{4}{*}{$\begin{array}{c}\text { Interaction } \\
\text { Effect }\end{array}$} & \multicolumn{16}{|c|}{ ICC Values } \\
\hline & & \multicolumn{4}{|c|}{0} & \multicolumn{4}{|c|}{0.1} & \multicolumn{4}{|c|}{0.3} & \multicolumn{4}{|c|}{0.5} \\
\hline & & \multirow[b]{2}{*}{0} & \multirow[b]{2}{*}{0.2} & \multirow[b]{2}{*}{0.5} & \multirow[b]{2}{*}{0.8} & \multirow[b]{2}{*}{0} & \multirow[b]{2}{*}{0.2} & \multirow[b]{2}{*}{0.5} & \multicolumn{2}{|c|}{ L2 Effect } & \multirow[b]{2}{*}{0.2} & \multirow[b]{2}{*}{0.5} & \multirow[b]{2}{*}{0.8} & \multirow[b]{2}{*}{0} & \multirow[b]{2}{*}{0.2} & \multirow[b]{2}{*}{0.5} & \multirow[b]{2}{*}{0.8} \\
\hline & & & & & & & & & 0.8 & 0 & & & & & & & \\
\hline \multirow{4}{*}{0} & 0 & 0.99 & 0.99 & 0.99 & 0.99 & 0.91 & 0.92 & 0.91 & 0.92 & 0.72 & 0.73 & 0.73 & 0.73 & 0.53 & 0.54 & 0.53 & 0.54 \\
\hline & 0.2 & 0.99 & 0.99 & 0.99 & 0.99 & 0.91 & 0.91 & 0.91 & 0.91 & 0.73 & 0.72 & 0.72 & 0.73 & 0.53 & 0.53 & 0.53 & 0.53 \\
\hline & 0.5 & 0.97 & 0.97 & 0.97 & 0.97 & 0.89 & 0.89 & 0.89 & 0.89 & 0.71 & 0.71 & 0.71 & 0.72 & 0.53 & 0.53 & 0.53 & 0.53 \\
\hline & 0.8 & 0.93 & 0.93 & 0.92 & 0.93 & 0.84 & 0.85 & 0.85 & 0.84 & 0.68 & 0.69 & 0.68 & 0.69 & 0.52 & 0.51 & 0.52 & 0.51 \\
\hline \multirow{4}{*}{0.2} & 0 & 0.99 & 0.99 & 0.99 & 0.99 & 0.91 & 0.91 & 0.91 & 0.91 & 0.72 & 0.72 & 0.73 & 0.72 & 0.53 & 0.53 & 0.53 & 0.53 \\
\hline & 0.2 & 0.99 & 0.99 & 0.99 & 0.99 & 0.91 & 0.91 & 0.91 & 0.91 & 0.72 & 0.73 & 0.72 & 0.72 & 0.53 & 0.53 & 0.53 & 0.54 \\
\hline & 0.5 & 0.97 & 0.96 & 0.96 & 0.97 & 0.88 & 0.88 & 0.88 & 0.88 & 0.70 & 0.71 & 0.71 & 0.70 & 0.52 & 0.52 & 0.52 & 0.53 \\
\hline & 0.8 & 0.92 & 0.92 & 0.92 & 0.92 & 0.84 & 0.84 & 0.85 & 0.85 & 0.68 & 0.68 & 0.68 & 0.68 & 0.51 & 0.51 & 0.51 & 0.51 \\
\hline \multirow{4}{*}{0.5} & 0 & 0.97 & 0.97 & 0.97 & 0.97 & 0.88 & 0.89 & 0.88 & 0.88 & 0.71 & 0.71 & 0.71 & 0.71 & 0.53 & 0.52 & 0.52 & 0.52 \\
\hline & 0.2 & 0.96 & 0.96 & 0.96 & 0.96 & 0.88 & 0.88 & 0.88 & 0.88 & 0.70 & 0.70 & 0.71 & 0.71 & 0.53 & 0.53 & 0.52 & 0.52 \\
\hline & 0.5 & 0.94 & 0.94 & 0.94 & 0.94 & 0.86 & 0.86 & 0.86 & 0.86 & 0.69 & 0.69 & 0.69 & 0.70 & 0.52 & 0.52 & 0.51 & 0.52 \\
\hline & 0.8 & 0.90 & 0.89 & 0.90 & 0.90 & 0.82 & 0.83 & 0.82 & 0.82 & 0.67 & 0.67 & 0.67 & 0.67 & 0.51 & 0.50 & 0.50 & 0.50 \\
\hline \multirow{4}{*}{0.8} & 0 & 0.92 & 0.92 & 0.92 & 0.92 & 0.84 & 0.84 & 0.85 & 0.84 & 0.69 & 0.68 & 0.68 & 0.68 & 0.51 & 0.51 & 0.50 & 0.51 \\
\hline & 0.2 & 0.92 & 0.92 & 0.92 & 0.92 & 0.84 & 0.84 & 0.84 & 0.84 & 0.68 & 0.68 & 0.68 & 0.68 & 0.51 & 0.51 & 0.51 & 0.51 \\
\hline & 0.5 & 0.90 & 0.89 & 0.89 & 0.90 & 0.82 & 0.82 & 0.82 & 0.82 & 0.67 & 0.67 & 0.67 & 0.66 & 0.50 & 0.50 & 0.50 & 0.50 \\
\hline & 0.8 & 0.85 & 0.86 & 0.85 & 0.85 & 0.79 & 0.79 & 0.79 & 0.79 & 0.65 & 0.64 & 0.65 & 0.65 & 0.49 & 0.49 & 0.49 & 0.49 \\
\hline
\end{tabular}

Note: ICC $=$ Intraclass Correlation Coefficient. 
Table 4 cont.

Average Design Effect values for the L1 predictor: Poisson Distribution conditions $(\mathrm{m}=10, \mathrm{k}=50)$

\begin{tabular}{|c|c|c|c|c|c|c|c|c|c|c|c|c|c|c|c|c|c|}
\hline \multirow{4}{*}{$\begin{array}{c}\text { L1 } \\
\text { Effect }\end{array}$} & \multirow{4}{*}{$\begin{array}{l}\text { Interaction } \\
\text { Effect }\end{array}$} & \multicolumn{16}{|c|}{ ICC Values } \\
\hline & & \multicolumn{4}{|c|}{0} & \multirow{2}{*}{\multicolumn{3}{|c|}{0.1}} & & \multicolumn{4}{|c|}{0.3} & \multicolumn{4}{|c|}{0.5} \\
\hline & & \multirow[b]{2}{*}{0} & \multirow[b]{2}{*}{0.2} & \multirow[b]{2}{*}{0.5} & \multirow[b]{2}{*}{0.8} & & & & \multicolumn{2}{|c|}{ L2 Effect } & \multirow[b]{2}{*}{0.2} & \multirow[b]{2}{*}{0.5} & \multirow[b]{2}{*}{0.8} & \multirow[b]{2}{*}{0} & \multirow[b]{2}{*}{0.2} & \multirow[b]{2}{*}{0.5} & \multirow[b]{2}{*}{0.8} \\
\hline & & & & & & 0 & 0.2 & 0.5 & 0.8 & 0 & & & & & & & \\
\hline \multirow{4}{*}{0} & 0 & 0.99 & 0.99 & 0.99 & 0.99 & 0.91 & 0.91 & 0.91 & 0.91 & 0.71 & 0.71 & 0.72 & 0.72 & 0.52 & 0.52 & 0.52 & 0.51 \\
\hline & 0.2 & 0.99 & 0.99 & 0.99 & 0.99 & 0.90 & 0.90 & 0.90 & 0.91 & 0.71 & 0.71 & 0.71 & 0.71 & 0.52 & 0.52 & 0.52 & 0.52 \\
\hline & 0.5 & 0.97 & 0.97 & 0.97 & 0.97 & 0.88 & 0.88 & 0.88 & 0.88 & 0.70 & 0.70 & 0.70 & 0.70 & 0.51 & 0.51 & 0.51 & 0.51 \\
\hline & 0.8 & 0.92 & 0.92 & 0.92 & 0.92 & 0.84 & 0.84 & 0.83 & 0.83 & 0.67 & 0.67 & 0.67 & 0.67 & 0.50 & 0.49 & 0.49 & 0.49 \\
\hline \multirow{4}{*}{0.2} & 0 & 0.99 & 0.99 & 0.99 & 0.99 & 0.90 & 0.90 & 0.90 & 0.90 & 0.71 & 0.71 & 0.71 & 0.71 & 0.51 & 0.51 & 0.52 & 0.52 \\
\hline & 0.2 & 0.99 & 0.99 & 0.99 & 0.99 & 0.90 & 0.90 & 0.90 & 0.90 & 0.70 & 0.71 & 0.71 & 0.71 & 0.51 & 0.51 & 0.51 & 0.51 \\
\hline & 0.5 & 0.96 & 0.96 & 0.96 & 0.96 & 0.87 & 0.87 & 0.87 & 0.87 & 0.69 & 0.69 & 0.69 & 0.69 & 0.50 & 0.51 & 0.50 & 0.50 \\
\hline & 0.8 & 0.91 & 0.91 & 0.91 & 0.91 & 0.83 & 0.83 & 0.83 & 0.83 & 0.67 & 0.66 & 0.67 & 0.66 & 0.49 & 0.49 & 0.49 & 0.49 \\
\hline \multirow{4}{*}{0.5} & 0 & 0.97 & 0.97 & 0.97 & 0.97 & 0.88 & 0.88 & 0.88 & 0.88 & 0.70 & 0.70 & 0.69 & 0.70 & 0.50 & 0.51 & 0.51 & 0.51 \\
\hline & 0.2 & 0.96 & 0.96 & 0.96 & 0.96 & 0.87 & 0.87 & 0.87 & 0.87 & 0.69 & 0.70 & 0.69 & 0.69 & 0.51 & 0.51 & 0.51 & 0.51 \\
\hline & 0.5 & 0.94 & 0.93 & 0.93 & 0.93 & 0.85 & 0.85 & 0.85 & 0.85 & 0.67 & 0.68 & 0.68 & 0.68 & 0.50 & 0.50 & 0.49 & 0.50 \\
\hline & 0.8 & 0.89 & 0.89 & 0.89 & 0.89 & 0.81 & 0.81 & 0.81 & 0.81 & 0.65 & 0.65 & 0.65 & 0.65 & 0.48 & 0.49 & 0.48 & 0.48 \\
\hline \multirow{4}{*}{0.8} & 0 & 0.91 & 0.91 & 0.91 & 0.91 & 0.83 & 0.83 & 0.83 & 0.83 & 0.67 & 0.67 & 0.67 & 0.67 & 0.49 & 0.49 & 0.49 & 0.50 \\
\hline & 0.2 & 0.91 & 0.91 & 0.91 & 0.91 & 0.83 & 0.83 & 0.83 & 0.83 & 0.67 & 0.67 & 0.66 & 0.67 & 0.49 & 0.49 & 0.49 & 0.49 \\
\hline & 0.5 & 0.88 & 0.89 & 0.89 & 0.88 & 0.81 & 0.81 & 0.81 & 0.81 & 0.65 & 0.65 & 0.65 & 0.65 & 0.48 & 0.48 & 0.48 & 0.48 \\
\hline & 0.8 & 0.84 & 0.84 & 0.84 & 0.85 & 0.77 & 0.77 & 0.77 & 0.77 & 0.63 & 0.63 & 0.63 & 0.63 & 0.47 & 0.47 & 0.47 & 0.47 \\
\hline
\end{tabular}

Note: ICC = Intraclass Correlation Coefficient. 
Table 4 cont.

Average Design Effect values for the L1 predictor: Poisson Distribution conditions $(\mathrm{m}=10, \mathrm{k}=75)$

\begin{tabular}{|c|c|c|c|c|c|c|c|c|c|c|c|c|c|c|c|c|c|}
\hline \multirow{4}{*}{$\begin{array}{c}\text { L1 } \\
\text { Effect }\end{array}$} & \multirow{4}{*}{$\begin{array}{l}\text { Interaction } \\
\text { Effect }\end{array}$} & \multicolumn{16}{|c|}{ ICC Values } \\
\hline & & \multicolumn{4}{|c|}{0} & \multirow{2}{*}{\multicolumn{3}{|c|}{0.1}} & & \multicolumn{4}{|c|}{0.3} & \multicolumn{4}{|c|}{0.5} \\
\hline & & & & & & & & & \multicolumn{2}{|c|}{ L2 Effect } & & & & & & & \\
\hline & & 0 & 0.2 & 0.5 & 0.8 & 0 & 0.2 & 0.5 & 0.8 & 0 & 0.2 & 0.5 & 0.8 & 0 & 0.2 & 0.5 & 0.8 \\
\hline \multirow{4}{*}{0} & 0 & 0.99 & 1.00 & 0.99 & 0.99 & 0.90 & 0.91 & 0.90 & 0.90 & 0.71 & 0.71 & 0.71 & 0.71 & 0.51 & 0.51 & 0.51 & 0.51 \\
\hline & 0.2 & 0.99 & 0.99 & 0.99 & 0.99 & 0.90 & 0.90 & 0.90 & 0.90 & 0.71 & 0.71 & 0.71 & 0.71 & 0.51 & 0.51 & 0.51 & 0.51 \\
\hline & 0.5 & 0.97 & 0.97 & 0.97 & 0.97 & 0.87 & 0.87 & 0.88 & 0.87 & 0.69 & 0.69 & 0.69 & 0.69 & 0.50 & 0.50 & 0.50 & 0.50 \\
\hline & 0.8 & 0.92 & 0.91 & 0.92 & 0.92 & 0.83 & 0.83 & 0.83 & 0.83 & 0.66 & 0.66 & 0.66 & 0.66 & 0.48 & 0.49 & 0.49 & 0.49 \\
\hline \multirow{4}{*}{0.2} & 0 & 0.99 & 0.99 & 0.99 & 0.99 & 0.90 & 0.90 & 0.90 & 0.90 & 0.71 & 0.70 & 0.70 & 0.70 & 0.51 & 0.51 & 0.51 & 0.51 \\
\hline & 0.2 & 0.99 & 0.99 & 0.99 & 0.99 & 0.90 & 0.90 & 0.89 & 0.89 & 0.70 & 0.70 & 0.70 & 0.70 & 0.51 & 0.51 & 0.51 & 0.51 \\
\hline & 0.5 & 0.96 & 0.96 & 0.96 & 0.96 & 0.87 & 0.87 & 0.87 & 0.87 & 0.69 & 0.69 & 0.69 & 0.69 & 0.50 & 0.50 & 0.50 & 0.50 \\
\hline & 0.8 & 0.91 & 0.91 & 0.91 & 0.91 & 0.83 & 0.83 & 0.83 & 0.83 & 0.66 & 0.66 & 0.66 & 0.66 & 0.49 & 0.49 & 0.49 & 0.48 \\
\hline \multirow{4}{*}{0.5} & 0 & 0.96 & 0.97 & 0.97 & 0.97 & 0.87 & 0.87 & 0.87 & 0.87 & 0.69 & 0.69 & 0.69 & 0.69 & 0.50 & 0.50 & 0.50 & 0.50 \\
\hline & 0.2 & 0.96 & 0.96 & 0.96 & 0.96 & 0.87 & 0.87 & 0.87 & 0.87 & 0.69 & 0.69 & 0.69 & 0.69 & 0.50 & 0.50 & 0.50 & 0.50 \\
\hline & 0.5 & 0.93 & 0.93 & 0.93 & 0.93 & 0.85 & 0.85 & 0.85 & 0.85 & 0.67 & 0.67 & 0.67 & 0.67 & 0.49 & 0.49 & 0.49 & 0.49 \\
\hline & 0.8 & 0.89 & 0.88 & 0.88 & 0.88 & 0.81 & 0.81 & 0.81 & 0.81 & 0.65 & 0.65 & 0.64 & 0.64 & 0.48 & 0.48 & 0.48 & 0.48 \\
\hline \multirow{4}{*}{0.8} & 0 & 0.91 & 0.91 & 0.91 & 0.91 & 0.83 & 0.83 & 0.83 & 0.83 & 0.66 & 0.66 & 0.66 & 0.66 & 0.48 & 0.49 & 0.49 & 0.49 \\
\hline & 0.2 & 0.91 & 0.91 & 0.91 & 0.91 & 0.83 & 0.83 & 0.83 & 0.83 & 0.66 & 0.66 & 0.66 & 0.66 & 0.48 & 0.49 & 0.48 & 0.49 \\
\hline & 0.5 & 0.88 & 0.88 & 0.88 & 0.88 & 0.80 & 0.81 & 0.80 & 0.80 & 0.65 & 0.64 & 0.64 & 0.65 & 0.48 & 0.48 & 0.48 & 0.48 \\
\hline & 0.8 & 0.84 & 0.84 & 0.84 & 0.84 & 0.77 & 0.77 & 0.77 & 0.77 & 0.62 & 0.62 & 0.62 & 0.63 & 0.46 & 0.46 & 0.46 & 0.47 \\
\hline
\end{tabular}

Note: ICC = Intraclass Correlation Coefficient. 
Table 4 cont.

Average Design Effect values for the $L 1$ predictor: Poisson Distribution conditions $(\mathrm{m}=10, \mathrm{k}=100)$

\begin{tabular}{|c|c|c|c|c|c|c|c|c|c|c|c|c|c|c|c|c|c|}
\hline \multirow{4}{*}{$\begin{array}{c}\text { L1 } \\
\text { Effect }\end{array}$} & \multirow{4}{*}{$\begin{array}{c}\text { Interaction } \\
\text { Effect }\end{array}$} & \multicolumn{16}{|c|}{ ICC Values } \\
\hline & & \multicolumn{4}{|c|}{0} & \multicolumn{4}{|c|}{0.1} & \multicolumn{4}{|c|}{0.3} & \multicolumn{4}{|c|}{0.5} \\
\hline & & & & & & \multicolumn{8}{|c|}{ L2 Effect } & & & & \\
\hline & & 0 & 0.2 & 0.5 & 0.8 & 0 & 0.2 & 0.5 & 0.8 & 0 & 0.2 & 0.5 & 0.8 & 0 & 0.2 & 0.5 & 0.8 \\
\hline \multirow{4}{*}{0} & 0 & 1.00 & 1.00 & 1.00 & 1.00 & 0.90 & 0.90 & 0.90 & 0.90 & 0.71 & 0.71 & 0.71 & 0.71 & 0.51 & 0.51 & 0.51 & 0.51 \\
\hline & 0.2 & 0.99 & 0.99 & 0.99 & 0.99 & 0.90 & 0.90 & 0.90 & 0.90 & 0.70 & 0.70 & 0.70 & 0.71 & 0.51 & 0.51 & 0.51 & 0.51 \\
\hline & 0.5 & 0.97 & 0.97 & 0.97 & 0.96 & 0.87 & 0.87 & 0.87 & 0.87 & 0.69 & 0.69 & 0.69 & 0.69 & 0.50 & 0.50 & 0.50 & 0.50 \\
\hline & 0.8 & 0.91 & 0.91 & 0.91 & 0.91 & 0.83 & 0.83 & 0.83 & 0.83 & 0.66 & 0.66 & 0.66 & 0.66 & 0.48 & 0.48 & 0.49 & 0.48 \\
\hline \multirow{4}{*}{0.2} & 0 & 0.99 & 0.99 & 0.99 & 0.99 & 0.90 & 0.90 & 0.90 & 0.90 & 0.70 & 0.71 & 0.70 & 0.70 & 0.51 & 0.51 & 0.51 & 0.51 \\
\hline & 0.2 & 0.99 & 0.99 & 0.99 & 0.99 & 0.89 & 0.89 & 0.89 & 0.89 & 0.70 & 0.70 & 0.70 & 0.70 & 0.51 & 0.51 & 0.50 & 0.51 \\
\hline & 0.5 & 0.96 & 0.96 & 0.96 & 0.96 & 0.87 & 0.87 & 0.87 & 0.87 & 0.69 & 0.68 & 0.69 & 0.68 & 0.50 & 0.50 & 0.50 & 0.50 \\
\hline & 0.8 & 0.91 & 0.91 & 0.91 & 0.91 & 0.83 & 0.83 & 0.83 & 0.82 & 0.66 & 0.66 & 0.66 & 0.66 & 0.48 & 0.48 & 0.48 & 0.49 \\
\hline \multirow{4}{*}{0.5} & 0 & 0.96 & 0.96 & 0.97 & 0.97 & 0.87 & 0.87 & 0.87 & 0.88 & 0.69 & 0.69 & 0.69 & 0.69 & 0.50 & 0.50 & 0.50 & 0.50 \\
\hline & 0.2 & 0.96 & 0.96 & 0.96 & 0.96 & 0.87 & 0.87 & 0.87 & 0.87 & 0.69 & 0.68 & 0.69 & 0.69 & 0.50 & 0.50 & 0.50 & 0.50 \\
\hline & 0.5 & 0.93 & 0.93 & 0.93 & 0.93 & 0.84 & 0.85 & 0.84 & 0.84 & 0.67 & 0.67 & 0.67 & 0.67 & 0.49 & 0.49 & 0.49 & 0.49 \\
\hline & 0.8 & 0.88 & 0.88 & 0.88 & 0.88 & 0.80 & 0.80 & 0.80 & 0.80 & 0.64 & 0.64 & 0.64 & 0.64 & 0.48 & 0.48 & 0.47 & 0.48 \\
\hline \multirow{4}{*}{0.8} & 0 & 0.91 & 0.91 & 0.91 & 0.91 & 0.83 & 0.83 & 0.83 & 0.83 & 0.66 & 0.66 & 0.66 & 0.66 & 0.48 & 0.48 & 0.49 & 0.49 \\
\hline & 0.2 & 0.91 & 0.91 & 0.91 & 0.91 & 0.82 & 0.82 & 0.82 & 0.82 & 0.66 & 0.65 & 0.66 & 0.66 & 0.48 & 0.48 & 0.48 & 0.48 \\
\hline & 0.5 & 0.88 & 0.88 & 0.88 & 0.88 & 0.80 & 0.80 & 0.80 & 0.80 & 0.65 & 0.65 & 0.64 & 0.64 & 0.48 & 0.48 & 0.47 & 0.48 \\
\hline & 0.8 & 0.84 & 0.84 & 0.84 & 0.84 & 0.76 & 0.77 & 0.77 & 0.77 & 0.62 & 0.62 & 0.62 & 0.62 & 0.46 & 0.46 & 0.46 & 0.46 \\
\hline
\end{tabular}

Note: ICC $=$ Intraclass Correlation Coefficient. 
Table 4 cont.

Average Design Effect values for the L2 predictor: Poisson Distribution conditions $(\mathrm{m}=20, \mathrm{k}=25)$

\begin{tabular}{|c|c|c|c|c|c|c|c|c|c|c|c|c|c|c|c|c|c|}
\hline \multirow{4}{*}{$\begin{array}{c}\text { L1 } \\
\text { Effect }\end{array}$} & \multirow{4}{*}{$\begin{array}{c}\text { Interaction } \\
\text { Effect }\end{array}$} & \multicolumn{16}{|c|}{ ICC Values } \\
\hline & & \multicolumn{4}{|c|}{0} & \multicolumn{4}{|c|}{0.1} & \multicolumn{4}{|c|}{0.3} & \multicolumn{4}{|c|}{0.5} \\
\hline & & \multirow[b]{2}{*}{0} & \multirow[b]{2}{*}{0.2} & \multirow[b]{2}{*}{0.5} & \multirow[b]{2}{*}{0.8} & \multirow[b]{2}{*}{0} & \multirow[b]{2}{*}{0.2} & \multirow[b]{2}{*}{0.5} & \multicolumn{2}{|c|}{ L2 Effect } & \multirow[b]{2}{*}{0.2} & \multirow[b]{2}{*}{0.5} & \multirow[b]{2}{*}{0.8} & \multirow[b]{2}{*}{0} & \multirow[b]{2}{*}{0.2} & \multirow[b]{2}{*}{0.5} & \multirow[b]{2}{*}{0.8} \\
\hline & & & & & & & & & 0.8 & 0 & & & & & & & \\
\hline \multirow{4}{*}{0} & 0 & 1.00 & 1.00 & 1.00 & 1.00 & 0.91 & 0.91 & 0.91 & 0.91 & 0.73 & 0.72 & 0.72 & 0.73 & 0.54 & 0.53 & 0.53 & 0.53 \\
\hline & 0.2 & 0.99 & 0.99 & 0.99 & 0.99 & 0.91 & 0.91 & 0.91 & 0.91 & 0.72 & 0.73 & 0.72 & 0.72 & 0.53 & 0.53 & 0.53 & 0.53 \\
\hline & 0.5 & 0.98 & 0.98 & 0.98 & 0.98 & 0.90 & 0.90 & 0.90 & 0.89 & 0.72 & 0.72 & 0.71 & 0.71 & 0.53 & 0.53 & 0.53 & 0.53 \\
\hline & 0.8 & 0.96 & 0.96 & 0.96 & 0.96 & 0.88 & 0.88 & 0.88 & 0.88 & 0.70 & 0.70 & 0.71 & 0.70 & 0.53 & 0.52 & 0.52 & 0.52 \\
\hline \multirow{4}{*}{0.2} & 0 & 0.99 & 0.99 & 0.99 & 0.99 & 0.91 & 0.91 & 0.91 & 0.91 & 0.72 & 0.72 & 0.73 & 0.72 & 0.53 & 0.53 & 0.53 & 0.53 \\
\hline & 0.2 & 0.99 & 0.99 & 0.99 & 0.99 & 0.91 & 0.90 & 0.91 & 0.91 & 0.72 & 0.72 & 0.72 & 0.72 & 0.53 & 0.53 & 0.53 & 0.53 \\
\hline & 0.5 & 0.98 & 0.98 & 0.98 & 0.98 & 0.90 & 0.90 & 0.90 & 0.90 & 0.71 & 0.72 & 0.71 & 0.71 & 0.53 & 0.53 & 0.53 & 0.53 \\
\hline & 0.8 & 0.96 & 0.96 & 0.96 & 0.96 & 0.87 & 0.88 & 0.88 & 0.88 & 0.70 & 0.70 & 0.70 & 0.70 & 0.52 & 0.52 & 0.51 & 0.52 \\
\hline \multirow{4}{*}{0.5} & 0 & 0.98 & 0.98 & 0.98 & 0.98 & 0.90 & 0.90 & 0.90 & 0.90 & 0.72 & 0.71 & 0.71 & 0.71 & 0.52 & 0.53 & 0.53 & 0.53 \\
\hline & 0.2 & 0.98 & 0.98 & 0.98 & 0.98 & 0.89 & 0.89 & 0.89 & 0.90 & 0.71 & 0.71 & 0.71 & 0.71 & 0.53 & 0.53 & 0.53 & 0.52 \\
\hline & 0.5 & 0.97 & 0.97 & 0.97 & 0.97 & 0.88 & 0.88 & 0.88 & 0.88 & 0.71 & 0.71 & 0.71 & 0.71 & 0.53 & 0.52 & 0.53 & 0.52 \\
\hline & 0.8 & 0.95 & 0.95 & 0.94 & 0.95 & 0.87 & 0.86 & 0.86 & 0.86 & 0.70 & 0.70 & 0.70 & 0.70 & 0.52 & 0.51 & 0.52 & 0.51 \\
\hline \multirow{4}{*}{0.8} & 0 & 0.96 & 0.96 & 0.96 & 0.96 & 0.87 & 0.88 & 0.87 & 0.87 & 0.70 & 0.70 & 0.70 & 0.70 & 0.52 & 0.52 & 0.52 & 0.52 \\
\hline & 0.2 & 0.96 & 0.96 & 0.95 & 0.95 & 0.87 & 0.87 & 0.87 & 0.87 & 0.70 & 0.70 & 0.70 & 0.70 & 0.52 & 0.52 & 0.52 & 0.52 \\
\hline & 0.5 & 0.94 & 0.94 & 0.94 & 0.94 & 0.86 & 0.86 & 0.86 & 0.86 & 0.69 & 0.70 & 0.69 & 0.70 & 0.51 & 0.52 & 0.52 & 0.52 \\
\hline & 0.8 & 0.92 & 0.92 & 0.92 & 0.92 & 0.84 & 0.84 & 0.84 & 0.84 & 0.68 & 0.68 & 0.68 & 0.68 & 0.51 & 0.51 & 0.51 & 0.51 \\
\hline
\end{tabular}

Note: ICC = Intraclass Correlation Coefficient. 
Table 4 cont.

Average Design Effect values for the L1 predictor: Poisson Distribution conditions $(\mathrm{m}=20, \mathrm{k}=50)$

\begin{tabular}{|c|c|c|c|c|c|c|c|c|c|c|c|c|c|c|c|c|c|}
\hline \multirow{4}{*}{$\begin{array}{c}\text { L1 } \\
\text { Effect }\end{array}$} & \multirow{4}{*}{$\begin{array}{l}\text { Interaction } \\
\text { Effect }\end{array}$} & \multicolumn{16}{|c|}{ ICC Values } \\
\hline & & \multicolumn{4}{|c|}{0} & \multirow{2}{*}{\multicolumn{3}{|c|}{0.1}} & & \multicolumn{4}{|c|}{0.3} & \multicolumn{4}{|c|}{0.5} \\
\hline & & & & & & & & & \multicolumn{2}{|c|}{ L2 Effect } & & & & & & & \\
\hline & & 0 & 0.2 & 0.5 & 0.8 & 0 & 0.2 & 0.5 & 0.8 & 0 & 0.2 & 0.5 & 0.8 & 0 & 0.2 & 0.5 & 0.8 \\
\hline \multirow{4}{*}{0} & 0 & 1.00 & 1.00 & 1.00 & 1.00 & 0.91 & 0.91 & 0.91 & 0.90 & 0.71 & 0.71 & 0.71 & 0.71 & 0.52 & 0.52 & 0.52 & 0.51 \\
\hline & 0.2 & 1.00 & 1.00 & 0.99 & 1.00 & 0.90 & 0.90 & 0.90 & 0.90 & 0.71 & 0.71 & 0.71 & 0.71 & 0.52 & 0.52 & 0.51 & 0.51 \\
\hline & 0.5 & 0.98 & 0.98 & 0.98 & 0.98 & 0.89 & 0.89 & 0.89 & 0.89 & 0.70 & 0.71 & 0.70 & 0.70 & 0.51 & 0.51 & 0.51 & 0.51 \\
\hline & 0.8 & 0.96 & 0.96 & 0.96 & 0.96 & 0.87 & 0.87 & 0.87 & 0.87 & 0.69 & 0.69 & 0.69 & 0.69 & 0.50 & 0.50 & 0.50 & 0.50 \\
\hline \multirow{4}{*}{0.2} & 0 & 1.00 & 1.00 & 1.00 & 1.00 & 0.90 & 0.90 & 0.90 & 0.90 & 0.71 & 0.71 & 0.71 & 0.71 & 0.52 & 0.52 & 0.51 & 0.52 \\
\hline & 0.2 & 0.99 & 0.99 & 0.99 & 0.99 & 0.90 & 0.90 & 0.90 & 0.90 & 0.71 & 0.71 & 0.71 & 0.71 & 0.51 & 0.51 & 0.52 & 0.51 \\
\hline & 0.5 & 0.98 & 0.98 & 0.98 & 0.98 & 0.89 & 0.89 & 0.89 & 0.89 & 0.70 & 0.70 & 0.70 & 0.70 & 0.51 & 0.51 & 0.51 & 0.51 \\
\hline & 0.8 & 0.95 & 0.96 & 0.95 & 0.95 & 0.87 & 0.86 & 0.86 & 0.86 & 0.69 & 0.69 & 0.69 & 0.69 & 0.50 & 0.50 & 0.50 & 0.50 \\
\hline \multirow{4}{*}{0.5} & 0 & 0.98 & 0.98 & 0.98 & 0.98 & 0.89 & 0.89 & 0.89 & 0.89 & 0.70 & 0.70 & 0.70 & 0.70 & 0.51 & 0.51 & 0.51 & 0.51 \\
\hline & 0.2 & 0.98 & 0.98 & 0.98 & 0.98 & 0.89 & 0.89 & 0.89 & 0.89 & 0.70 & 0.70 & 0.70 & 0.70 & 0.51 & 0.51 & 0.51 & 0.51 \\
\hline & 0.5 & 0.97 & 0.96 & 0.97 & 0.96 & 0.88 & 0.88 & 0.88 & 0.88 & 0.70 & 0.69 & 0.70 & 0.69 & 0.51 & 0.51 & 0.50 & 0.51 \\
\hline & 0.8 & 0.94 & 0.94 & 0.94 & 0.94 & 0.85 & 0.85 & 0.85 & 0.85 & 0.68 & 0.68 & 0.68 & 0.68 & 0.50 & 0.50 & 0.50 & 0.50 \\
\hline \multirow{4}{*}{0.8} & 0 & 0.96 & 0.95 & 0.96 & 0.96 & 0.87 & 0.87 & 0.87 & 0.87 & 0.68 & 0.69 & 0.69 & 0.69 & 0.50 & 0.50 & 0.51 & 0.50 \\
\hline & 0.2 & 0.95 & 0.95 & 0.95 & 0.95 & 0.86 & 0.86 & 0.86 & 0.86 & 0.69 & 0.69 & 0.69 & 0.69 & 0.50 & 0.51 & 0.50 & 0.50 \\
\hline & 0.5 & 0.94 & 0.94 & 0.94 & 0.94 & 0.85 & 0.85 & 0.85 & 0.85 & 0.68 & 0.68 & 0.68 & 0.68 & 0.50 & 0.50 & 0.50 & 0.50 \\
\hline & 0.8 & 0.92 & 0.91 & 0.91 & 0.91 & 0.83 & 0.83 & 0.83 & 0.83 & 0.67 & 0.67 & 0.67 & 0.67 & 0.49 & 0.49 & 0.49 & 0.49 \\
\hline
\end{tabular}

Note: ICC = Intraclass Correlation Coefficient. 
Table 4 cont.

Average Design Effect values for the L1 predictor: Poisson Distribution conditions $(\mathrm{m}=20, \mathrm{k}=75)$

\begin{tabular}{|c|c|c|c|c|c|c|c|c|c|c|c|c|c|c|c|c|c|}
\hline \multirow{4}{*}{$\begin{array}{c}\text { L1 } \\
\text { Effect }\end{array}$} & \multirow{4}{*}{$\begin{array}{l}\text { Interaction } \\
\text { Effect }\end{array}$} & \multicolumn{16}{|c|}{ ICC Values } \\
\hline & & \multicolumn{4}{|c|}{0} & \multirow{2}{*}{\multicolumn{3}{|c|}{0.1}} & & \multicolumn{4}{|c|}{0.3} & \multicolumn{4}{|c|}{0.5} \\
\hline & & & & & & & & & \multicolumn{2}{|c|}{ L2 Effect } & & & & & & & \\
\hline & & 0 & 0.2 & 0.5 & 0.8 & 0 & 0.2 & 0.5 & 0.8 & 0 & 0.2 & 0.5 & 0.8 & 0 & 0.2 & 0.5 & 0.8 \\
\hline \multirow{4}{*}{0} & 0 & 1.00 & 1.00 & 1.00 & 1.00 & 0.90 & 0.90 & 0.90 & 0.90 & 0.71 & 0.71 & 0.71 & 0.71 & 0.51 & 0.51 & 0.51 & 0.51 \\
\hline & 0.2 & 1.00 & 1.00 & 1.00 & 1.00 & 0.90 & 0.90 & 0.90 & 0.90 & 0.71 & 0.71 & 0.71 & 0.71 & 0.51 & 0.51 & 0.51 & 0.51 \\
\hline & 0.5 & 0.98 & 0.98 & 0.98 & 0.98 & 0.89 & 0.89 & 0.89 & 0.89 & 0.70 & 0.70 & 0.70 & 0.70 & 0.51 & 0.51 & 0.51 & 0.50 \\
\hline & 0.8 & 0.95 & 0.96 & 0.95 & 0.96 & 0.87 & 0.87 & 0.87 & 0.87 & 0.68 & 0.69 & 0.68 & 0.68 & 0.50 & 0.50 & 0.50 & 0.50 \\
\hline \multirow{4}{*}{0.2} & 0 & 1.00 & 1.00 & 1.00 & 1.00 & 0.90 & 0.90 & 0.90 & 0.90 & 0.71 & 0.71 & 0.71 & 0.71 & 0.51 & 0.51 & 0.51 & 0.51 \\
\hline & 0.2 & 0.99 & 0.99 & 0.99 & 0.99 & 0.90 & 0.90 & 0.90 & 0.90 & 0.71 & 0.71 & 0.70 & 0.70 & 0.51 & 0.51 & 0.51 & 0.51 \\
\hline & 0.5 & 0.98 & 0.98 & 0.98 & 0.98 & 0.89 & 0.89 & 0.89 & 0.89 & 0.70 & 0.70 & 0.70 & 0.70 & 0.50 & 0.51 & 0.51 & 0.51 \\
\hline & 0.8 & 0.95 & 0.95 & 0.95 & 0.95 & 0.86 & 0.86 & 0.86 & 0.86 & 0.68 & 0.68 & 0.68 & 0.68 & 0.50 & 0.50 & 0.50 & 0.50 \\
\hline \multirow{4}{*}{0.5} & 0 & 0.98 & 0.98 & 0.98 & 0.98 & 0.89 & 0.89 & 0.89 & 0.89 & 0.70 & 0.70 & 0.70 & 0.70 & 0.50 & 0.50 & 0.50 & 0.51 \\
\hline & 0.2 & 0.98 & 0.98 & 0.98 & 0.98 & 0.88 & 0.89 & 0.89 & 0.89 & 0.70 & 0.70 & 0.70 & 0.70 & 0.50 & 0.50 & 0.51 & 0.50 \\
\hline & 0.5 & 0.96 & 0.96 & 0.96 & 0.96 & 0.87 & 0.87 & 0.87 & 0.87 & 0.69 & 0.69 & 0.69 & 0.69 & 0.50 & 0.50 & 0.50 & 0.50 \\
\hline & 0.8 & 0.94 & 0.94 & 0.94 & 0.94 & 0.85 & 0.85 & 0.85 & 0.85 & 0.68 & 0.67 & 0.67 & 0.67 & 0.49 & 0.50 & 0.49 & 0.49 \\
\hline \multirow{4}{*}{0.8} & 0 & 0.95 & 0.95 & 0.95 & 0.95 & 0.86 & 0.86 & 0.86 & 0.86 & 0.69 & 0.68 & 0.68 & 0.68 & 0.50 & 0.50 & 0.50 & 0.50 \\
\hline & 0.2 & 0.95 & 0.95 & 0.95 & 0.95 & 0.86 & 0.86 & 0.86 & 0.86 & 0.68 & 0.68 & 0.68 & 0.68 & 0.50 & 0.50 & 0.49 & 0.50 \\
\hline & 0.5 & 0.94 & 0.94 & 0.94 & 0.94 & 0.85 & 0.85 & 0.85 & 0.85 & 0.68 & 0.67 & 0.67 & 0.67 & 0.49 & 0.49 & 0.49 & 0.49 \\
\hline & 0.8 & 0.91 & 0.91 & 0.91 & 0.91 & 0.83 & 0.83 & 0.83 & 0.83 & 0.66 & 0.66 & 0.66 & 0.66 & 0.49 & 0.48 & 0.49 & 0.49 \\
\hline
\end{tabular}

Note: ICC $=$ Intraclass Correlation Coefficient. 
Table 4 cont.

Average Design Effect values for the $L 1$ predictor: Poisson Distribution conditions $(\mathrm{m}=20, \mathrm{k}=100)$

\begin{tabular}{|c|c|c|c|c|c|c|c|c|c|c|c|c|c|c|c|c|c|}
\hline \multirow{4}{*}{$\begin{array}{c}\text { L1 } \\
\text { Effect }\end{array}$} & \multirow{4}{*}{$\begin{array}{c}\text { Interaction } \\
\text { Effect }\end{array}$} & \multicolumn{16}{|c|}{ ICC Values } \\
\hline & & \multicolumn{4}{|c|}{0} & \multicolumn{4}{|c|}{0.1} & \multicolumn{4}{|c|}{0.3} & \multicolumn{4}{|c|}{0.5} \\
\hline & & \multicolumn{12}{|c|}{ L2 Effect } & & & & \\
\hline & & 0 & 0.2 & 0.5 & 0.8 & 0 & 0.2 & 0.5 & 0.8 & 0 & 0.2 & 0.5 & 0.8 & 0 & 0.2 & 0.5 & 0.8 \\
\hline \multirow{4}{*}{0} & 0 & 1.00 & 1.00 & 1.00 & 1.00 & 0.90 & 0.90 & 0.90 & 0.90 & 0.71 & 0.71 & 0.71 & 0.70 & 0.51 & 0.51 & 0.51 & 0.51 \\
\hline & 0.2 & 1.00 & 1.00 & 1.00 & 1.00 & 0.90 & 0.90 & 0.90 & 0.90 & 0.70 & 0.70 & 0.70 & 0.71 & 0.51 & 0.51 & 0.51 & 0.51 \\
\hline & 0.5 & 0.98 & 0.98 & 0.98 & 0.98 & 0.89 & 0.89 & 0.89 & 0.89 & 0.70 & 0.70 & 0.70 & 0.70 & 0.50 & 0.50 & 0.50 & 0.50 \\
\hline & 0.8 & 0.95 & 0.95 & 0.95 & 0.95 & 0.86 & 0.86 & 0.86 & 0.86 & 0.68 & 0.68 & 0.68 & 0.68 & 0.49 & 0.50 & 0.49 & 0.50 \\
\hline \multirow{4}{*}{0.2} & 0 & 1.00 & 1.00 & 1.00 & 1.00 & 0.90 & 0.90 & 0.90 & 0.90 & 0.70 & 0.70 & 0.70 & 0.71 & 0.51 & 0.51 & 0.51 & 0.51 \\
\hline & 0.2 & 0.99 & 0.99 & 0.99 & 0.99 & 0.90 & 0.90 & 0.90 & 0.90 & 0.70 & 0.70 & 0.70 & 0.70 & 0.51 & 0.51 & 0.51 & 0.51 \\
\hline & 0.5 & 0.98 & 0.98 & 0.98 & 0.98 & 0.88 & 0.88 & 0.88 & 0.89 & 0.70 & 0.69 & 0.70 & 0.70 & 0.50 & 0.50 & 0.50 & 0.50 \\
\hline & 0.8 & 0.95 & 0.95 & 0.95 & 0.95 & 0.86 & 0.86 & 0.86 & 0.86 & 0.68 & 0.68 & 0.68 & 0.68 & 0.49 & 0.50 & 0.49 & 0.49 \\
\hline \multirow{4}{*}{0.5} & 0 & 0.98 & 0.98 & 0.98 & 0.98 & 0.89 & 0.89 & 0.89 & 0.89 & 0.70 & 0.70 & 0.70 & 0.70 & 0.50 & 0.50 & 0.50 & 0.50 \\
\hline & 0.2 & 0.98 & 0.98 & 0.98 & 0.98 & 0.88 & 0.88 & 0.89 & 0.89 & 0.69 & 0.69 & 0.70 & 0.70 & 0.50 & 0.50 & 0.50 & 0.50 \\
\hline & 0.5 & 0.96 & 0.96 & 0.96 & 0.96 & 0.87 & 0.87 & 0.87 & 0.87 & 0.69 & 0.69 & 0.69 & 0.69 & 0.50 & 0.50 & 0.50 & 0.50 \\
\hline & 0.8 & 0.94 & 0.94 & 0.94 & 0.94 & 0.85 & 0.85 & 0.85 & 0.85 & 0.68 & 0.67 & 0.67 & 0.67 & 0.49 & 0.49 & 0.49 & 0.49 \\
\hline \multirow{4}{*}{0.8} & 0 & 0.95 & 0.95 & 0.95 & 0.95 & 0.86 & 0.86 & 0.86 & 0.86 & 0.68 & 0.68 & 0.68 & 0.68 & 0.50 & 0.50 & 0.50 & 0.50 \\
\hline & 0.2 & 0.95 & 0.95 & 0.95 & 0.95 & 0.86 & 0.86 & 0.86 & 0.86 & 0.68 & 0.68 & 0.68 & 0.68 & 0.49 & 0.49 & 0.50 & 0.50 \\
\hline & 0.5 & 0.93 & 0.94 & 0.93 & 0.94 & 0.85 & 0.85 & 0.85 & 0.85 & 0.67 & 0.67 & 0.67 & 0.67 & 0.49 & 0.49 & 0.49 & 0.49 \\
\hline & 0.8 & 0.91 & 0.91 & 0.91 & 0.91 & 0.83 & 0.83 & 0.83 & 0.83 & 0.66 & 0.66 & 0.66 & 0.66 & 0.48 & 0.48 & 0.49 & 0.48 \\
\hline
\end{tabular}

Note: ICC $=$ Intraclass Correlation Coefficient. 


\section{Table 5}

Average Design Effect values for the L2 predictor: Uniform Distribution conditions $(\mathrm{m}=5, \mathrm{k}=25)$

\begin{tabular}{|c|c|c|c|c|c|c|c|c|c|c|c|c|c|c|c|c|c|}
\hline \multirow{4}{*}{$\begin{array}{c}\text { L1 } \\
\text { Effect }\end{array}$} & \multirow{4}{*}{$\begin{array}{c}\text { Interaction } \\
\text { Effect }\end{array}$} & \multicolumn{16}{|c|}{ ICC Values } \\
\hline & & \multicolumn{4}{|c|}{0} & \multicolumn{4}{|c|}{0.1} & \multicolumn{4}{|c|}{0.3} & \multicolumn{4}{|c|}{0.5} \\
\hline & & \multicolumn{12}{|c|}{ L2 Effect } & & & & \\
\hline & & 0 & 0.2 & 0.5 & 0.8 & 0 & 0.2 & 0.5 & 0.8 & 0 & 0.2 & 0.5 & 0.8 & 0 & 0.2 & 0.5 & 0.8 \\
\hline \multirow{4}{*}{0} & 0 & 1.08 & 1.07 & 1.06 & 1.08 & 1.34 & 1.35 & 1.35 & 1.35 & 2.10 & 2.09 & 2.09 & 2.09 & 2.85 & 2.88 & 2.88 & 2.88 \\
\hline & 0.2 & 1.10 & 1.10 & 1.10 & 1.11 & 1.38 & 1.38 & 1.36 & 1.38 & 2.11 & 2.10 & 2.11 & 2.11 & 2.89 & 2.88 & 2.89 & 2.91 \\
\hline & 0.5 & 1.25 & 1.25 & 1.25 & 1.26 & 1.56 & 1.55 & 1.55 & 1.58 & 2.22 & 2.21 & 2.24 & 2.23 & 2.94 & 2.94 & 2.96 & 2.96 \\
\hline & 0.8 & 1.56 & 1.56 & 1.56 & 1.57 & 1.83 & 1.85 & 1.81 & 1.85 & 2.39 & 2.39 & 2.42 & 2.41 & 3.04 & 3.03 & 3.01 & 3.05 \\
\hline \multirow{4}{*}{0.2} & 0 & 1.10 & 1.11 & 1.10 & 1.11 & 1.40 & 1.39 & 1.38 & 1.39 & 2.12 & 2.10 & 2.12 & 2.12 & 2.88 & 2.88 & 2.90 & 2.89 \\
\hline & 0.2 & 1.14 & 1.13 & 1.13 & 1.13 & 1.42 & 1.43 & 1.41 & 1.41 & 2.14 & 2.15 & 2.13 & 2.11 & 2.90 & 2.91 & 2.89 & 2.90 \\
\hline & 0.5 & 1.30 & 1.30 & 1.29 & 1.28 & 1.57 & 1.59 & 1.58 & 1.59 & 2.25 & 2.26 & 2.26 & 2.26 & 2.94 & 2.97 & 2.95 & 2.97 \\
\hline & 0.8 & 1.59 & 1.58 & 1.59 & 1.61 & 1.84 & 1.86 & 1.86 & 1.84 & 2.45 & 2.41 & 2.44 & 2.44 & 3.06 & 3.06 & 3.05 & 3.05 \\
\hline \multirow{4}{*}{0.5} & 0 & 1.27 & 1.27 & 1.27 & 1.28 & 1.57 & 1.55 & 1.58 & 1.56 & 2.24 & 2.24 & 2.25 & 2.23 & 2.94 & 2.96 & 2.96 & 2.95 \\
\hline & 0.2 & 1.31 & 1.30 & 1.30 & 1.30 & 1.62 & 1.59 & 1.59 & 1.58 & 2.26 & 2.27 & 2.25 & 2.25 & 2.96 & 2.95 & 2.97 & 2.95 \\
\hline & 0.5 & 1.47 & 1.47 & 1.45 & 1.47 & 1.74 & 1.73 & 1.73 & 1.73 & 2.37 & 2.39 & 2.37 & 2.34 & 3.03 & 3.00 & 3.01 & 3.02 \\
\hline & 0.8 & 1.76 & 1.74 & 1.72 & 1.73 & 1.99 & 2.01 & 2.00 & 1.99 & 2.52 & 2.51 & 2.51 & 2.52 & 3.11 & 3.09 & 3.09 & 3.12 \\
\hline \multirow{4}{*}{0.8} & 0 & 1.61 & 1.62 & 1.59 & 1.62 & 1.86 & 1.89 & 1.88 & 1.87 & 2.45 & 2.43 & 2.46 & 2.43 & 3.09 & 3.07 & 3.09 & 3.07 \\
\hline & 0.2 & 1.63 & 1.65 & 1.65 & 1.65 & 1.88 & 1.91 & 1.88 & 1.91 & 2.46 & 2.47 & 2.44 & 2.46 & 3.08 & 3.08 & 3.05 & 3.09 \\
\hline & 0.5 & 1.80 & 1.76 & 1.79 & 1.78 & 2.02 & 2.00 & 2.03 & 2.00 & 2.53 & 2.55 & 2.54 & 2.53 & 3.14 & 3.11 & 3.15 & 3.13 \\
\hline & 0.8 & 2.00 & 2.00 & 2.02 & 2.01 & 2.20 & 2.20 & 2.21 & 2.24 & 2.69 & 2.71 & 2.69 & 2.68 & 3.21 & 3.21 & 3.19 & 3.20 \\
\hline
\end{tabular}

Note: ICC $=$ Intraclass Correlation Coefficient. 
Table 5 cont.

Average Design Effect values for the L2 predictor: Uniform Distribution conditions $(\mathrm{m}=5, \mathrm{k}=50)$

\begin{tabular}{|c|c|c|c|c|c|c|c|c|c|c|c|c|c|c|c|c|c|}
\hline \multirow{4}{*}{$\begin{array}{c}\text { L1 } \\
\text { Effect }\end{array}$} & \multirow{4}{*}{$\begin{array}{c}\text { Interaction } \\
\text { Effect }\end{array}$} & \multicolumn{16}{|c|}{ ICC Values } \\
\hline & & \multicolumn{4}{|c|}{0} & \multicolumn{4}{|c|}{0.1} & \multicolumn{4}{|c|}{0.3} & \multicolumn{4}{|c|}{0.5} \\
\hline & & & & & & \multicolumn{8}{|c|}{ L2 Effect } & \multirow[b]{2}{*}{0} & \multirow[b]{2}{*}{0.2} & \multirow[b]{2}{*}{0.5} & \multirow[b]{2}{*}{0.8} \\
\hline & & 0 & 0.2 & 0.5 & 0.8 & 0 & 0.2 & 0.5 & 0.8 & 0 & 0.2 & 0.5 & 0.8 & & & & \\
\hline \multirow{4}{*}{0} & 0 & 1.06 & 1.06 & 1.06 & 1.06 & 1.36 & 1.38 & 1.37 & 1.36 & 2.16 & 2.15 & 2.16 & 2.14 & 2.96 & 2.94 & 2.94 & 2.93 \\
\hline & 0.2 & 1.08 & 1.08 & 1.09 & 1.08 & 1.41 & 1.41 & 1.41 & 1.41 & 2.17 & 2.16 & 2.17 & 2.16 & 2.94 & 2.94 & 2.94 & 2.95 \\
\hline & 0.5 & 1.27 & 1.27 & 1.26 & 1.27 & 1.60 & 1.60 & 1.59 & 1.60 & 2.29 & 2.29 & 2.29 & 2.28 & 3.01 & 3.01 & 3.00 & 3.01 \\
\hline & 0.8 & 1.62 & 1.63 & 1.62 & 1.61 & 1.92 & 1.89 & 1.90 & 1.91 & 2.48 & 2.51 & 2.49 & 2.47 & 3.11 & 3.13 & 3.13 & 3.13 \\
\hline \multirow{4}{*}{0.2} & 0 & 1.09 & 1.08 & 1.09 & 1.08 & 1.41 & 1.40 & 1.41 & 1.41 & 2.17 & 2.17 & 2.16 & 2.18 & 2.94 & 2.97 & 2.95 & 2.96 \\
\hline & 0.2 & 1.12 & 1.12 & 1.12 & 1.12 & 1.45 & 1.44 & 1.44 & 1.44 & 2.18 & 2.22 & 2.20 & 2.19 & 2.96 & 2.97 & 2.96 & 2.97 \\
\hline & 0.5 & 1.32 & 1.31 & 1.30 & 1.31 & 1.64 & 1.62 & 1.64 & 1.62 & 2.31 & 2.32 & 2.30 & 2.31 & 3.04 & 3.03 & 3.02 & 3.02 \\
\hline & 0.8 & 1.65 & 1.66 & 1.65 & 1.66 & 1.94 & 1.94 & 1.91 & 1.93 & 2.51 & 2.50 & 2.51 & 2.50 & 3.13 & 3.12 & 3.13 & 3.14 \\
\hline \multirow{4}{*}{0.5} & 0 & 1.27 & 1.27 & 1.28 & 1.28 & 1.62 & 1.61 & 1.61 & 1.60 & 2.30 & 2.30 & 2.31 & 2.30 & 3.01 & 3.03 & 3.02 & 3.03 \\
\hline & 0.2 & 1.32 & 1.32 & 1.32 & 1.31 & 1.64 & 1.64 & 1.65 & 1.65 & 2.32 & 2.32 & 2.31 & 2.33 & 3.04 & 3.03 & 3.02 & 3.03 \\
\hline & 0.5 & 1.51 & 1.51 & 1.52 & 1.51 & 1.79 & 1.80 & 1.78 & 1.79 & 2.43 & 2.41 & 2.43 & 2.42 & 3.09 & 3.09 & 3.07 & 3.09 \\
\hline & 0.8 & 1.84 & 1.82 & 1.82 & 1.83 & 2.07 & 2.07 & 2.08 & 2.06 & 2.60 & 2.60 & 2.60 & 2.60 & 3.18 & 3.19 & 3.19 & 3.19 \\
\hline \multirow{4}{*}{0.8} & 0 & 1.64 & 1.65 & 1.66 & 1.64 & 1.93 & 1.93 & 1.92 & 1.93 & 2.50 & 2.49 & 2.50 & 2.51 & 3.14 & 3.12 & 3.14 & 3.11 \\
\hline & 0.2 & 1.69 & 1.69 & 1.71 & 1.68 & 1.96 & 1.95 & 1.95 & 1.94 & 2.52 & 2.52 & 2.53 & 2.53 & 3.14 & 3.14 & 3.14 & 3.15 \\
\hline & 0.5 & 1.83 & 1.85 & 1.84 & 1.85 & 2.09 & 2.09 & 2.09 & 2.08 & 2.60 & 2.60 & 2.61 & 2.61 & 3.20 & 3.19 & 3.18 & 3.19 \\
\hline & 0.8 & 2.09 & 2.10 & 2.11 & 2.10 & 2.28 & 2.30 & 2.31 & 2.31 & 2.75 & 2.75 & 2.75 & 2.76 & 3.28 & 3.27 & 3.27 & 3.29 \\
\hline
\end{tabular}

Note: ICC = Intraclass Correlation Coefficient. 


\section{Table 5 cont}

Average Design Effect values for the L2 predictor: Uniform Distribution conditions $(\mathrm{m}=5, \mathrm{k}=75)$

\begin{tabular}{|c|c|c|c|c|c|c|c|c|c|c|c|c|c|c|c|c|c|}
\hline \multirow{4}{*}{$\begin{array}{c}\text { L1 } \\
\text { Effect }\end{array}$} & \multirow{4}{*}{$\begin{array}{c}\text { Interaction } \\
\text { Effect }\end{array}$} & \multicolumn{16}{|c|}{ ICC Values } \\
\hline & & \multicolumn{4}{|c|}{0} & \multirow{2}{*}{\multicolumn{3}{|c|}{0.1}} & & \multicolumn{4}{|c|}{0.3} & \multicolumn{4}{|c|}{0.5} \\
\hline & & & & & & & & & \multicolumn{2}{|c|}{ L2 Effect } & & & & & & & \\
\hline & & 0 & 0.2 & 0.5 & 0.8 & 0 & 0.2 & 0.5 & 0.8 & 0 & 0.2 & 0.5 & 0.8 & 0 & 0.2 & 0.5 & 0.8 \\
\hline \multirow{4}{*}{0} & 0 & 1.05 & 1.05 & 1.05 & 1.05 & 1.36 & 1.38 & 1.37 & 1.37 & 2.17 & 2.16 & 2.17 & 2.16 & 2.96 & 2.96 & 2.95 & 2.95 \\
\hline & 0.2 & 1.08 & 1.07 & 1.08 & 1.07 & 1.41 & 1.42 & 1.42 & 1.41 & 2.18 & 2.19 & 2.19 & 2.19 & 2.98 & 2.98 & 2.97 & 2.98 \\
\hline & 0.5 & 1.27 & 1.27 & 1.28 & 1.28 & 1.62 & 1.61 & 1.60 & 1.61 & 2.32 & 2.31 & 2.31 & 2.31 & 3.03 & 3.02 & 3.04 & 3.05 \\
\hline & 0.8 & 1.66 & 1.65 & 1.65 & 1.64 & 1.91 & 1.92 & 1.92 & 1.92 & 2.51 & 2.50 & 2.50 & 2.50 & 3.16 & 3.14 & 3.15 & 3.13 \\
\hline \multirow{4}{*}{0.2} & 0 & 1.08 & 1.08 & 1.08 & 1.07 & 1.42 & 1.41 & 1.43 & 1.42 & 2.19 & 2.19 & 2.19 & 2.20 & 2.97 & 2.97 & 2.98 & 2.96 \\
\hline & 0.2 & 1.12 & 1.12 & 1.11 & 1.11 & 1.46 & 1.47 & 1.45 & 1.45 & 2.21 & 2.21 & 2.21 & 2.20 & 2.99 & 2.98 & 2.98 & 2.99 \\
\hline & 0.5 & 1.32 & 1.31 & 1.32 & 1.33 & 1.65 & 1.65 & 1.66 & 1.65 & 2.34 & 2.33 & 2.34 & 2.33 & 3.05 & 3.04 & 3.05 & 3.05 \\
\hline & 0.8 & 1.68 & 1.68 & 1.67 & 1.68 & 1.96 & 1.95 & 1.97 & 1.93 & 2.52 & 2.54 & 2.53 & 2.52 & 3.16 & 3.17 & 3.16 & 3.15 \\
\hline \multirow{4}{*}{0.5} & 0 & 1.29 & 1.28 & 1.28 & 1.28 & 1.63 & 1.63 & 1.61 & 1.62 & 2.31 & 2.31 & 2.31 & 2.32 & 3.05 & 3.03 & 3.04 & 3.03 \\
\hline & 0.2 & 1.33 & 1.33 & 1.33 & 1.32 & 1.66 & 1.65 & 1.66 & 1.64 & 2.34 & 2.34 & 2.35 & 2.34 & 3.04 & 3.04 & 3.05 & 3.05 \\
\hline & 0.5 & 1.53 & 1.53 & 1.53 & 1.53 & 1.82 & 1.82 & 1.82 & 1.83 & 2.44 & 2.46 & 2.44 & 2.45 & 3.10 & 3.12 & 3.10 & 3.12 \\
\hline & 0.8 & 1.85 & 1.85 & 1.84 & 1.86 & 2.09 & 2.11 & 2.10 & 2.08 & 2.62 & 2.62 & 2.62 & 2.62 & 3.21 & 3.21 & 3.21 & 3.21 \\
\hline \multirow{4}{*}{0.8} & 0 & 1.67 & 1.67 & 1.66 & 1.67 & 1.94 & 1.94 & 1.94 & 1.94 & 2.51 & 2.52 & 2.52 & 2.52 & 3.16 & 3.14 & 3.16 & 3.16 \\
\hline & 0.2 & 1.70 & 1.70 & 1.70 & 1.70 & 1.97 & 1.97 & 1.97 & 1.97 & 2.54 & 2.54 & 2.54 & 2.53 & 3.16 & 3.15 & 3.17 & 3.16 \\
\hline & 0.5 & 1.87 & 1.87 & 1.86 & 1.87 & 2.11 & 2.10 & 2.12 & 2.10 & 2.64 & 2.63 & 2.63 & 2.64 & 3.21 & 3.21 & 3.22 & 3.21 \\
\hline & 0.8 & 2.13 & 2.13 & 2.14 & 2.12 & 2.34 & 2.35 & 2.33 & 2.34 & 2.77 & 2.78 & 2.78 & 2.78 & 3.30 & 3.30 & 3.29 & 3.29 \\
\hline
\end{tabular}

Note: ICC = Intraclass Correlation Coefficient. 
Table 5 cont.

Average Design Effect values for the L2 predictor: Uniform Distribution conditions $(\mathrm{m}=5, \mathrm{k}=100)$

\begin{tabular}{|c|c|c|c|c|c|c|c|c|c|c|c|c|c|c|c|c|c|}
\hline \multirow{4}{*}{$\begin{array}{c}\text { L1 } \\
\text { Effect }\end{array}$} & \multirow{4}{*}{$\begin{array}{c}\text { Interaction } \\
\text { Effect }\end{array}$} & \multicolumn{16}{|c|}{ ICC Values } \\
\hline & & \multicolumn{4}{|c|}{0} & \multirow{2}{*}{\multicolumn{3}{|c|}{0.1}} & & \multicolumn{4}{|c|}{0.3} & \multicolumn{4}{|c|}{0.5} \\
\hline & & \multirow[b]{2}{*}{0} & \multirow[b]{2}{*}{0.2} & \multirow[b]{2}{*}{0.5} & \multirow[b]{2}{*}{0.8} & & & & \multicolumn{2}{|c|}{ L2 Effect } & \multirow[b]{2}{*}{0.2} & \multirow[b]{2}{*}{0.5} & \multirow[b]{2}{*}{0.8} & \multirow[b]{2}{*}{0} & \multirow[b]{2}{*}{0.2} & \multirow[b]{2}{*}{0.5} & \multirow[b]{2}{*}{0.8} \\
\hline & & & & & & 0 & 0.2 & 0.5 & 0.8 & 0 & & & & & & & \\
\hline \multirow{4}{*}{0} & 0 & 1.04 & 1.04 & 1.04 & 1.04 & 1.38 & 1.39 & 1.39 & 1.38 & 2.18 & 2.17 & 2.18 & 2.17 & 2.97 & 2.96 & 2.97 & 2.97 \\
\hline & 0.2 & 1.07 & 1.07 & 1.08 & 1.07 & 1.43 & 1.42 & 1.42 & 1.42 & 2.20 & 2.21 & 2.21 & 2.20 & 2.98 & 2.98 & 2.98 & 2.99 \\
\hline & 0.5 & 1.29 & 1.28 & 1.29 & 1.28 & 1.62 & 1.62 & 1.62 & 1.61 & 2.31 & 2.33 & 2.33 & 2.32 & 3.05 & 3.04 & 3.05 & 3.05 \\
\hline & 0.8 & 1.65 & 1.66 & 1.65 & 1.65 & 1.93 & 1.95 & 1.93 & 1.94 & 2.51 & 2.53 & 2.53 & 2.53 & 3.16 & 3.15 & 3.15 & 3.16 \\
\hline \multirow{4}{*}{0.2} & 0 & 1.07 & 1.07 & 1.07 & 1.07 & 1.41 & 1.43 & 1.43 & 1.42 & 2.20 & 2.20 & 2.21 & 2.19 & 2.98 & 2.99 & 2.98 & 2.97 \\
\hline & 0.2 & 1.10 & 1.11 & 1.11 & 1.10 & 1.46 & 1.46 & 1.46 & 1.47 & 2.22 & 2.23 & 2.22 & 2.21 & 3.01 & 2.98 & 2.99 & 2.99 \\
\hline & 0.5 & 1.32 & 1.33 & 1.32 & 1.31 & 1.65 & 1.65 & 1.66 & 1.66 & 2.35 & 2.34 & 2.34 & 2.35 & 3.06 & 3.06 & 3.06 & 3.06 \\
\hline & 0.8 & 1.68 & 1.70 & 1.70 & 1.69 & 1.96 & 1.97 & 1.97 & 1.97 & 2.54 & 2.54 & 2.55 & 2.54 & 3.17 & 3.16 & 3.17 & 3.17 \\
\hline \multirow{4}{*}{0.5} & 0 & 1.29 & 1.29 & 1.29 & 1.29 & 1.63 & 1.62 & 1.62 & 1.62 & 2.31 & 2.33 & 2.31 & 2.33 & 3.05 & 3.04 & 3.05 & 3.05 \\
\hline & 0.2 & 1.33 & 1.34 & 1.33 & 1.33 & 1.65 & 1.66 & 1.66 & 1.66 & 2.36 & 2.34 & 2.35 & 2.35 & 3.06 & 3.06 & 3.06 & 3.06 \\
\hline & 0.5 & 1.55 & 1.54 & 1.53 & 1.53 & 1.84 & 1.83 & 1.83 & 1.83 & 2.46 & 2.44 & 2.46 & 2.44 & 3.13 & 3.12 & 3.11 & 3.12 \\
\hline & 0.8 & 1.85 & 1.87 & 1.88 & 1.86 & 2.10 & 2.11 & 2.10 & 2.11 & 2.63 & 2.63 & 2.63 & 2.64 & 3.22 & 3.22 & 3.22 & 3.20 \\
\hline \multirow{4}{*}{0.8} & 0 & 1.68 & 1.67 & 1.67 & 1.68 & 1.95 & 1.95 & 1.94 & 1.94 & 2.54 & 2.54 & 2.53 & 2.53 & 3.17 & 3.16 & 3.16 & 3.17 \\
\hline & 0.2 & 1.70 & 1.72 & 1.70 & 1.71 & 1.98 & 1.97 & 1.97 & 1.97 & 2.55 & 2.55 & 2.56 & 2.56 & 3.17 & 3.17 & 3.17 & 3.18 \\
\hline & 0.5 & 1.87 & 1.86 & 1.88 & 1.89 & 2.12 & 2.11 & 2.12 & 2.12 & 2.64 & 2.64 & 2.63 & 2.64 & 3.22 & 3.22 & 3.23 & 3.21 \\
\hline & 0.8 & 2.14 & 2.14 & 2.14 & 2.14 & 2.34 & 2.33 & 2.34 & 2.35 & 2.81 & 2.80 & 2.80 & 2.80 & 3.31 & 3.32 & 3.31 & 3.31 \\
\hline
\end{tabular}

Note: ICC $=$ Intraclass Correlation Coefficient. 
Table 5 cont.

Average Design Effect values for the L2 predictor: Uniform Distribution conditions $(\mathrm{m}=10, \mathrm{k}=25)$

\begin{tabular}{|c|c|c|c|c|c|c|c|c|c|c|c|c|c|c|c|c|c|}
\hline \multirow{4}{*}{$\begin{array}{c}\text { L1 } \\
\text { Effect }\end{array}$} & \multirow{4}{*}{$\begin{array}{c}\text { Interaction } \\
\text { Effect }\end{array}$} & \multicolumn{16}{|c|}{ ICC Values } \\
\hline & & \multicolumn{4}{|c|}{0} & \multirow{2}{*}{\multicolumn{3}{|c|}{0.1}} & & \multicolumn{4}{|c|}{0.3} & \multicolumn{4}{|c|}{0.5} \\
\hline & & \multirow[b]{2}{*}{0} & \multirow[b]{2}{*}{0.2} & \multirow[b]{2}{*}{0.5} & \multirow[b]{2}{*}{0.8} & & & & \multicolumn{2}{|c|}{ L2 Effect } & \multirow[b]{2}{*}{0.2} & \multirow[b]{2}{*}{0.5} & \multirow[b]{2}{*}{0.8} & \multirow[b]{2}{*}{0} & \multirow[b]{2}{*}{0.2} & \multirow[b]{2}{*}{0.5} & \multirow[b]{2}{*}{0.8} \\
\hline & & & & & & 0 & 0.2 & 0.5 & 0.8 & 0 & & & & & & & \\
\hline \multirow{4}{*}{0} & 0 & 1.08 & 1.08 & 1.07 & 1.08 & 1.79 & 1.80 & 1.78 & 1.75 & 3.47 & 3.49 & 3.47 & 3.44 & 5.22 & 5.25 & 5.18 & 5.19 \\
\hline & 0.2 & 1.10 & 1.11 & 1.11 & 1.11 & 1.81 & 1.80 & 1.81 & 1.82 & 3.50 & 3.50 & 3.48 & 3.50 & 5.20 & 5.24 & 5.28 & 5.22 \\
\hline & 0.5 & 1.29 & 1.28 & 1.28 & 1.29 & 2.04 & 2.03 & 2.05 & 2.04 & 3.63 & 3.65 & 3.64 & 3.62 & 5.28 & 5.29 & 5.28 & 5.35 \\
\hline & 0.8 & 1.68 & 1.69 & 1.68 & 1.67 & 2.38 & 2.37 & 2.39 & 2.37 & 3.87 & 3.85 & 3.81 & 3.88 & 5.45 & 5.42 & 5.42 & 5.37 \\
\hline \multirow{4}{*}{0.2} & 0 & 1.10 & 1.11 & 1.10 & 1.11 & 1.81 & 1.85 & 1.83 & 1.83 & 3.48 & 3.51 & 3.49 & 3.55 & 5.24 & 5.21 & 5.20 & 5.18 \\
\hline & 0.2 & 1.13 & 1.14 & 1.12 & 1.14 & 1.87 & 1.85 & 1.87 & 1.85 & 3.53 & 3.50 & 3.52 & 3.51 & 5.19 & 5.25 & 5.26 & 5.23 \\
\hline & 0.5 & 1.32 & 1.34 & 1.34 & 1.33 & 2.05 & 2.04 & 2.08 & 2.07 & 3.63 & 3.66 & 3.64 & 3.69 & 5.31 & 5.30 & 5.31 & 5.33 \\
\hline & 0.8 & 1.73 & 1.74 & 1.75 & 1.72 & 2.39 & 2.42 & 2.42 & 2.38 & 3.90 & 3.91 & 3.88 & 3.85 & 5.44 & 5.39 & 5.46 & 5.43 \\
\hline \multirow{4}{*}{0.5} & 0 & 1.31 & 1.31 & 1.29 & 1.30 & 2.04 & 2.03 & 2.04 & 2.07 & 3.62 & 3.65 & 3.63 & 3.62 & 5.30 & 5.28 & 5.30 & 5.35 \\
\hline & 0.2 & 1.35 & 1.34 & 1.35 & 1.36 & 2.10 & 2.06 & 2.06 & 2.07 & 3.61 & 3.67 & 3.66 & 3.67 & 5.27 & 5.30 & 5.30 & 5.35 \\
\hline & 0.5 & 1.54 & 1.55 & 1.57 & 1.56 & 2.30 & 2.31 & 2.27 & 2.30 & 3.82 & 3.81 & 3.77 & 3.79 & 5.44 & 5.37 & 5.40 & 5.40 \\
\hline & 0.8 & 1.94 & 1.92 & 1.91 & 1.88 & 2.59 & 2.63 & 2.59 & 2.61 & 4.01 & 3.99 & 3.98 & 3.98 & 5.55 & 5.48 & 5.51 & 5.51 \\
\hline \multirow{4}{*}{0.8} & 0 & 1.75 & 1.74 & 1.73 & 1.76 & 2.43 & 2.45 & 2.41 & 2.43 & 3.88 & 3.89 & 3.88 & 3.87 & 5.45 & 5.45 & 5.43 & 5.42 \\
\hline & 0.2 & 1.78 & 1.77 & 1.76 & 1.80 & 2.46 & 2.46 & 2.48 & 2.45 & 3.92 & 3.90 & 3.91 & 3.91 & 5.48 & 5.40 & 5.46 & 5.45 \\
\hline & 0.5 & 1.98 & 1.99 & 1.97 & 2.00 & 2.62 & 2.63 & 2.58 & 2.66 & 4.03 & 4.04 & 4.04 & 4.02 & 5.49 & 5.53 & 5.51 & 5.50 \\
\hline & 0.8 & 2.34 & 2.33 & 2.27 & 2.32 & 2.97 & 2.97 & 2.94 & 2.89 & 4.25 & 4.19 & 4.18 & 4.17 & 5.62 & 5.64 & 5.61 & 5.65 \\
\hline
\end{tabular}

Note: ICC $=$ Intraclass Correlation Coefficient. 
Table 5 cont.

Average Design Effect values for the L2 predictor: Uniform Distribution conditions $(\mathrm{m}=10, \mathrm{k}=50)$

\begin{tabular}{|c|c|c|c|c|c|c|c|c|c|c|c|c|c|c|c|c|c|}
\hline \multirow{4}{*}{$\begin{array}{c}\text { L1 } \\
\text { Effect }\end{array}$} & \multirow{4}{*}{$\begin{array}{c}\text { Interaction } \\
\text { Effect }\end{array}$} & \multicolumn{16}{|c|}{ ICC Values } \\
\hline & & \multicolumn{4}{|c|}{0} & \multicolumn{4}{|c|}{0.1} & \multicolumn{4}{|c|}{0.3} & \multicolumn{4}{|c|}{0.5} \\
\hline & & \multirow[b]{2}{*}{0} & \multirow[b]{2}{*}{0.2} & \multirow[b]{2}{*}{0.5} & \multirow[b]{2}{*}{0.8} & \multirow[b]{2}{*}{0} & \multirow[b]{2}{*}{0.2} & \multirow[b]{2}{*}{0.5} & \multicolumn{2}{|c|}{ L2 Effect } & \multirow[b]{2}{*}{0.2} & \multirow[b]{2}{*}{0.5} & \multirow[b]{2}{*}{0.8} & \multirow[b]{2}{*}{0} & \multirow[b]{2}{*}{0.2} & \multirow[b]{2}{*}{0.5} & \multirow[b]{2}{*}{0.8} \\
\hline & & & & & & & & & 0.8 & 0 & & & & & & & \\
\hline \multirow{4}{*}{0} & 0 & 1.06 & 1.06 & 1.05 & 1.06 & 1.84 & 1.83 & 1.84 & 1.82 & 3.57 & 3.61 & 3.59 & 3.57 & 5.37 & 5.37 & 5.35 & 5.37 \\
\hline & 0.2 & 1.08 & 1.08 & 1.09 & 1.09 & 1.88 & 1.88 & 1.90 & 1.89 & 3.61 & 3.61 & 3.61 & 3.60 & 5.34 & 5.37 & 5.36 & 5.38 \\
\hline & 0.5 & 1.31 & 1.30 & 1.29 & 1.31 & 2.11 & 2.11 & 2.11 & 2.09 & 3.74 & 3.71 & 3.75 & 3.76 & 5.40 & 5.42 & 5.45 & 5.47 \\
\hline & 0.8 & 1.77 & 1.76 & 1.77 & 1.73 & 2.48 & 2.49 & 2.49 & 2.49 & 4.00 & 3.98 & 4.00 & 3.99 & 5.57 & 5.57 & 5.59 & 5.59 \\
\hline \multirow{4}{*}{0.2} & 0 & 1.09 & 1.09 & 1.08 & 1.10 & 1.88 & 1.85 & 1.89 & 1.87 & 3.61 & 3.61 & 3.61 & 3.59 & 5.39 & 5.36 & 5.37 & 5.38 \\
\hline & 0.2 & 1.12 & 1.13 & 1.13 & 1.13 & 1.94 & 1.92 & 1.92 & 1.95 & 3.64 & 3.64 & 3.63 & 3.64 & 5.40 & 5.39 & 5.39 & 5.36 \\
\hline & 0.5 & 1.36 & 1.35 & 1.38 & 1.37 & 2.15 & 2.14 & 2.13 & 2.16 & 3.78 & 3.75 & 3.78 & 3.78 & 5.46 & 5.46 & 5.47 & 5.48 \\
\hline & 0.8 & 1.81 & 1.81 & 1.82 & 1.80 & 2.53 & 2.52 & 2.53 & 2.51 & 4.00 & 4.02 & 4.04 & 4.02 & 5.58 & 5.59 & 5.60 & 5.58 \\
\hline \multirow{4}{*}{0.5} & 0 & 1.32 & 1.32 & 1.33 & 1.31 & 2.12 & 2.11 & 2.11 & 2.12 & 3.76 & 3.76 & 3.74 & 3.74 & 5.45 & 5.46 & 5.41 & 5.47 \\
\hline & 0.2 & 1.36 & 1.36 & 1.37 & 1.37 & 2.17 & 2.14 & 2.15 & 2.16 & 3.81 & 3.77 & 3.78 & 3.77 & 5.47 & 5.47 & 5.46 & 5.48 \\
\hline & 0.5 & 1.59 & 1.62 & 1.62 & 1.61 & 2.37 & 2.34 & 2.36 & 2.37 & 3.89 & 3.90 & 3.92 & 3.96 & 5.54 & 5.51 & 5.55 & 5.54 \\
\hline & 0.8 & 2.03 & 2.03 & 2.01 & 2.02 & 2.74 & 2.71 & 2.70 & 2.74 & 4.12 & 4.16 & 4.17 & 4.12 & 5.66 & 5.64 & 5.65 & 5.61 \\
\hline \multirow{4}{*}{0.8} & 0 & 1.77 & 1.81 & 1.79 & 1.81 & 2.52 & 2.50 & 2.52 & 2.55 & 4.01 & 4.00 & 4.03 & 4.00 & 5.60 & 5.58 & 5.57 & 5.56 \\
\hline & 0.2 & 1.85 & 1.84 & 1.83 & 1.84 & 2.56 & 2.56 & 2.55 & 2.55 & 4.03 & 4.03 & 4.05 & 4.03 & 5.61 & 5.57 & 5.60 & 5.60 \\
\hline & 0.5 & 2.04 & 2.04 & 2.05 & 2.04 & 2.75 & 2.72 & 2.72 & 2.73 & 4.16 & 4.15 & 4.13 & 4.17 & 5.64 & 5.65 & 5.66 & 5.66 \\
\hline & 0.8 & 2.42 & 2.41 & 2.45 & 2.44 & 3.03 & 3.04 & 3.03 & 3.05 & 4.34 & 4.35 & 4.37 & 4.39 & 5.80 & 5.79 & 5.78 & 5.76 \\
\hline
\end{tabular}

Note: ICC $=$ Intraclass Correlation Coefficient. 
Table 5 cont.

Average Design Effect values for the L2 predictor: Uniform Distribution conditions $(\mathrm{m}=10, \mathrm{k}=75)$

\begin{tabular}{|c|c|c|c|c|c|c|c|c|c|c|c|c|c|c|c|c|c|}
\hline \multirow{4}{*}{$\begin{array}{c}\text { L1 } \\
\text { Effect }\end{array}$} & \multirow{4}{*}{$\begin{array}{c}\text { Interaction } \\
\text { Effect }\end{array}$} & \multicolumn{16}{|c|}{ ICC Values } \\
\hline & & \multicolumn{4}{|c|}{0} & \multirow{2}{*}{\multicolumn{3}{|c|}{0.1}} & & \multicolumn{4}{|c|}{0.3} & \multicolumn{4}{|c|}{0.5} \\
\hline & & \multirow[b]{2}{*}{0} & \multirow[b]{2}{*}{0.2} & \multirow[b]{2}{*}{0.5} & \multirow[b]{2}{*}{0.8} & & & & \multicolumn{2}{|c|}{ L2 Effect } & \multirow[b]{2}{*}{0.2} & \multirow[b]{2}{*}{0.5} & \multirow[b]{2}{*}{0.8} & \multirow[b]{2}{*}{0} & \multirow[b]{2}{*}{0.2} & \multirow[b]{2}{*}{0.5} & \multirow[b]{2}{*}{0.8} \\
\hline & & & & & & 0 & 0.2 & 0.5 & 0.8 & 0 & & & & & & & \\
\hline \multirow{4}{*}{0} & 0 & 1.05 & 1.05 & 1.05 & 1.05 & 1.86 & 1.85 & 1.86 & 1.85 & 3.64 & 3.63 & 3.63 & 3.61 & 5.38 & 5.40 & 5.40 & 5.39 \\
\hline & 0.2 & 1.08 & 1.09 & 1.09 & 1.08 & 1.91 & 1.91 & 1.91 & 1.91 & 3.65 & 3.64 & 3.64 & 3.64 & 5.43 & 5.46 & 5.44 & 5.44 \\
\hline & 0.5 & 1.32 & 1.33 & 1.32 & 1.31 & 2.15 & 2.12 & 2.14 & 2.13 & 3.81 & 3.81 & 3.78 & 3.80 & 5.50 & 5.49 & 5.49 & 5.50 \\
\hline & 0.8 & 1.80 & 1.79 & 1.80 & 1.79 & 2.52 & 2.54 & 2.52 & 2.52 & 4.04 & 4.03 & 4.04 & 4.03 & 5.64 & 5.61 & 5.61 & 5.59 \\
\hline \multirow{4}{*}{0.2} & 0 & 1.09 & 1.08 & 1.09 & 1.08 & 1.92 & 1.89 & 1.93 & 1.89 & 3.65 & 3.67 & 3.66 & 3.66 & 5.38 & 5.43 & 5.44 & 5.42 \\
\hline & 0.2 & 1.12 & 1.12 & 1.13 & 1.12 & 1.96 & 1.95 & 1.95 & 1.95 & 3.67 & 3.66 & 3.66 & 3.70 & 5.44 & 5.43 & 5.42 & 5.44 \\
\hline & 0.5 & 1.38 & 1.36 & 1.38 & 1.38 & 2.17 & 2.17 & 2.18 & 2.17 & 3.84 & 3.84 & 3.81 & 3.83 & 5.52 & 5.50 & 5.52 & 5.49 \\
\hline & 0.8 & 1.83 & 1.83 & 1.83 & 1.83 & 2.56 & 2.58 & 2.55 & 2.54 & 4.07 & 4.06 & 4.07 & 4.06 & 5.66 & 5.64 & 5.62 & 5.62 \\
\hline \multirow{4}{*}{0.5} & 0 & 1.33 & 1.32 & 1.33 & 1.33 & 2.14 & 2.14 & 2.14 & 2.13 & 3.80 & 3.80 & 3.79 & 3.79 & 5.51 & 5.51 & 5.48 & 5.48 \\
\hline & 0.2 & 1.38 & 1.39 & 1.39 & 1.38 & 2.17 & 2.20 & 2.19 & 2.20 & 3.83 & 3.83 & 3.84 & 3.83 & 5.52 & 5.51 & 5.52 & 5.51 \\
\hline & 0.5 & 1.63 & 1.64 & 1.63 & 1.64 & 2.39 & 2.40 & 2.39 & 2.40 & 3.95 & 3.93 & 3.96 & 3.95 & 5.58 & 5.59 & 5.56 & 5.58 \\
\hline & 0.8 & 2.09 & 2.08 & 2.12 & 2.07 & 2.74 & 2.79 & 2.75 & 2.76 & 4.17 & 4.20 & 4.16 & 4.18 & 5.72 & 5.72 & 5.72 & 5.68 \\
\hline \multirow{4}{*}{0.8} & 0 & 1.82 & 1.80 & 1.82 & 1.82 & 2.54 & 2.55 & 2.53 & 2.54 & 4.04 & 4.07 & 4.05 & 4.07 & 5.62 & 5.62 & 5.61 & 5.61 \\
\hline & 0.2 & 1.88 & 1.86 & 1.87 & 1.86 & 2.59 & 2.57 & 2.58 & 2.59 & 4.08 & 4.08 & 4.10 & 4.08 & 5.66 & 5.64 & 5.65 & 5.65 \\
\hline & 0.5 & 2.09 & 2.09 & 2.09 & 2.08 & 2.76 & 2.78 & 2.79 & 2.79 & 4.17 & 4.21 & 4.19 & 4.20 & 5.71 & 5.68 & 5.70 & 5.71 \\
\hline & 0.8 & 2.48 & 2.46 & 2.48 & 2.48 & 3.10 & 3.10 & 3.08 & 3.11 & 4.41 & 4.42 & 4.42 & 4.42 & 5.83 & 5.83 & 5.81 & 5.83 \\
\hline
\end{tabular}

Note: ICC $=$ Intraclass Correlation Coefficient. 
Table 5 cont.

Average Design Effect values for the L2 predictor: Uniform Distribution conditions ( $\mathrm{m}=10, \mathrm{k}=100)$

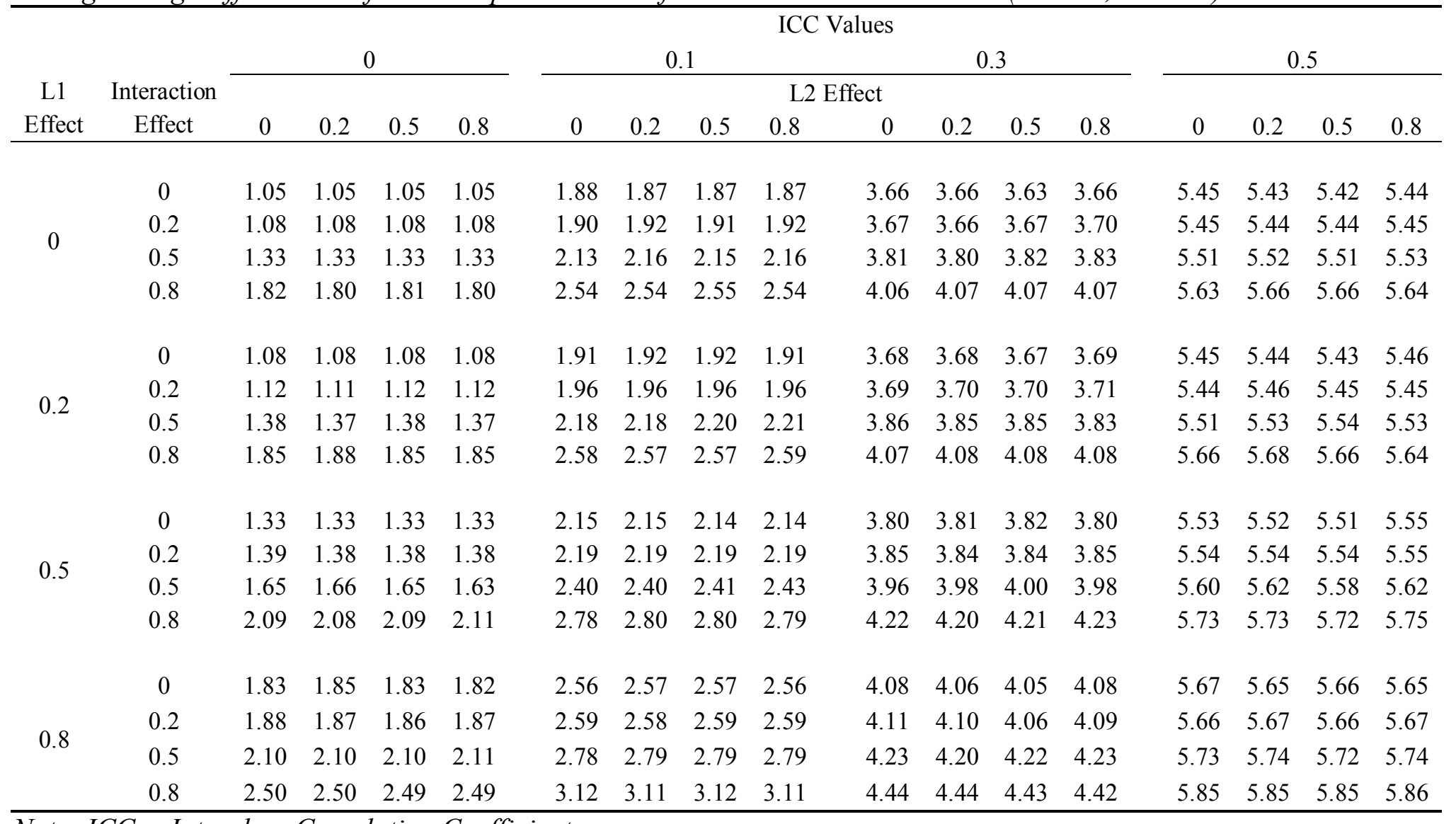

Note: ICC $=$ Intraclass Correlation Coefficient. 
Table 5 cont.

Average Design Effect values for the L2 predictor: Uniform Distribution conditions $(\mathrm{m}=20, \mathrm{k}=25)$

\begin{tabular}{|c|c|c|c|c|c|c|c|c|c|c|c|c|c|c|c|c|c|}
\hline \multirow{4}{*}{$\begin{array}{c}\text { L1 } \\
\text { Effect }\end{array}$} & \multirow{4}{*}{$\begin{array}{c}\text { Interaction } \\
\text { Effect }\end{array}$} & \multicolumn{16}{|c|}{ ICC Values } \\
\hline & & \multicolumn{4}{|c|}{0} & \multirow{2}{*}{\multicolumn{3}{|c|}{0.1}} & & \multicolumn{4}{|c|}{0.3} & \multicolumn{4}{|c|}{0.5} \\
\hline & & \multicolumn{9}{|c|}{ L2 Effect } & & & & \multirow[b]{2}{*}{0} & \multirow[b]{2}{*}{0.2} & \multirow[b]{2}{*}{0.5} & \multirow[b]{2}{*}{0.8} \\
\hline & & 0 & 0.2 & 0.5 & 0.8 & 0 & 0.2 & 0.5 & 0.8 & 0 & 0.2 & 0.5 & 0.8 & & & & \\
\hline \multirow{4}{*}{0} & 0 & 1.07 & 1.08 & 1.08 & 1.07 & 2.68 & 2.66 & 2.68 & 2.68 & 6.32 & 6.28 & 6.29 & 6.22 & 9.93 & 9.97 & 9.84 & 9.89 \\
\hline & 0.2 & 1.10 & 1.09 & 1.10 & 1.11 & 2.75 & 2.76 & 2.74 & 2.76 & 6.25 & 6.27 & 6.32 & 6.29 & 9.94 & 9.95 & 9.88 & 9.89 \\
\hline & 0.5 & 1.30 & 1.30 & 1.31 & 1.30 & 3.01 & 2.97 & 2.89 & 2.94 & 6.44 & 6.42 & 6.37 & 6.38 & 9.91 & 10.03 & 10.01 & 10.02 \\
\hline & 0.8 & 1.73 & 1.75 & 1.73 & 1.74 & 3.39 & 3.37 & 3.39 & 3.35 & 6.70 & 6.64 & 6.67 & 6.72 & 10.16 & 10.14 & 10.05 & 10.09 \\
\hline \multirow{4}{*}{0.2} & 0 & 1.10 & 1.11 & 1.10 & 1.10 & 2.75 & 2.74 & 2.73 & 2.68 & 6.28 & 6.23 & 6.33 & 6.34 & 9.81 & 9.91 & 9.93 & 9.90 \\
\hline & 0.2 & 1.15 & 1.14 & 1.14 & 1.14 & 2.76 & 2.78 & 2.75 & 2.77 & 6.23 & 6.28 & 6.21 & 6.22 & 9.85 & 9.89 & 9.95 & 9.95 \\
\hline & 0.5 & 1.35 & 1.35 & 1.36 & 1.35 & 2.95 & 2.99 & 2.98 & 3.03 & 6.40 & 6.51 & 6.45 & 6.47 & 10.03 & 10.01 & 10.05 & 10.01 \\
\hline & 0.8 & 1.78 & 1.81 & 1.83 & 1.77 & 3.36 & 3.42 & 3.41 & 3.41 & 6.67 & 6.72 & 6.74 & 6.63 & 10.18 & 10.08 & 10.10 & 10.09 \\
\hline \multirow{4}{*}{0.5} & 0 & 1.32 & 1.34 & 1.32 & 1.31 & 3.00 & 3.00 & 3.00 & 3.01 & 6.40 & 6.42 & 6.45 & 6.39 & 9.95 & 9.99 & 9.92 & 9.96 \\
\hline & 0.2 & 1.37 & 1.35 & 1.36 & 1.37 & 2.97 & 3.01 & 3.01 & 3.05 & 6.45 & 6.40 & 6.44 & 6.38 & 10.09 & 9.98 & 10.02 & 10.05 \\
\hline & 0.5 & 1.62 & 1.63 & 1.61 & 1.59 & 3.27 & 3.25 & 3.24 & 3.18 & 6.61 & 6.55 & 6.63 & 6.57 & 10.11 & 10.11 & 10.08 & 9.99 \\
\hline & 0.8 & 2.03 & 2.06 & 2.05 & 2.04 & 3.65 & 3.63 & 3.62 & 3.62 & 6.90 & 6.77 & 6.83 & 6.80 & 10.19 & 10.15 & 10.23 & 10.20 \\
\hline \multirow{4}{*}{0.8} & 0 & 1.80 & 1.84 & 1.82 & 1.79 & 3.43 & 3.39 & 3.44 & 3.45 & 6.71 & 6.72 & 6.72 & 6.75 & 10.10 & 10.13 & 10.16 & 10.13 \\
\hline & 0.2 & 1.88 & 1.86 & 1.89 & 1.87 & 3.41 & 3.47 & 3.42 & 3.43 & 6.69 & 6.74 & 6.63 & 6.66 & 10.12 & 10.13 & 10.16 & 10.17 \\
\hline & 0.5 & 2.07 & 2.09 & 2.08 & 2.11 & 3.63 & 3.65 & 3.65 & 3.59 & 6.88 & 6.82 & 6.84 & 6.77 & 10.14 & 10.25 & 10.20 & 10.21 \\
\hline & 0.8 & 2.51 & 2.50 & 2.48 & 2.52 & 3.98 & 3.97 & 4.01 & 4.01 & 7.07 & 7.12 & 7.10 & 7.02 & 10.29 & 10.40 & 10.33 & 10.31 \\
\hline
\end{tabular}

Note: ICC $=$ Intraclass Correlation Coefficient. 
Table 5 cont.

Average Design Effect values for the L2 predictor: Uniform Distribution conditions $(\mathrm{m}=20, \mathrm{k}=50)$

\begin{tabular}{|c|c|c|c|c|c|c|c|c|c|c|c|c|c|c|c|c|c|}
\hline \multirow{4}{*}{$\begin{array}{c}\text { L1 } \\
\text { Effect }\end{array}$} & \multirow{4}{*}{$\begin{array}{c}\text { Interaction } \\
\text { Effect }\end{array}$} & \multicolumn{16}{|c|}{ ICC Values } \\
\hline & & \multicolumn{4}{|c|}{0} & \multirow{2}{*}{\multicolumn{3}{|c|}{0.1}} & & \multicolumn{4}{|c|}{0.3} & \multicolumn{4}{|c|}{0.5} \\
\hline & & \multicolumn{9}{|c|}{ L2 Effect } & & & & \multirow[b]{2}{*}{0} & \multirow[b]{2}{*}{0.2} & \multirow[b]{2}{*}{0.5} & \multirow[b]{2}{*}{0.8} \\
\hline & & 0 & 0.2 & 0.5 & 0.8 & 0 & 0.2 & 0.5 & 0.8 & 0 & 0.2 & 0.5 & 0.8 & & & & \\
\hline \multirow{4}{*}{0} & 0 & 1.06 & 1.06 & 1.06 & 1.06 & 2.80 & 2.80 & 2.80 & 2.81 & 6.49 & 6.50 & 6.47 & 6.50 & 10.21 & 10.19 & 10.21 & 10.22 \\
\hline & 0.2 & 1.09 & 1.10 & 1.09 & 1.10 & 2.85 & 2.85 & 2.86 & 2.84 & 6.52 & 6.52 & 6.51 & 6.54 & 10.17 & 10.22 & 10.23 & 10.21 \\
\hline & 0.5 & 1.32 & 1.32 & 1.33 & 1.33 & 3.12 & 3.06 & 3.09 & 3.08 & 6.65 & 6.65 & 6.65 & 6.65 & 10.30 & 10.33 & 10.34 & 10.35 \\
\hline & 0.8 & 1.85 & 1.86 & 1.83 & 1.84 & 3.52 & 3.53 & 3.53 & 3.49 & 6.87 & 6.86 & 6.93 & 6.87 & 10.44 & 10.42 & 10.41 & 10.40 \\
\hline \multirow{4}{*}{0.2} & 0 & 1.09 & 1.09 & 1.10 & 1.09 & 2.83 & 2.85 & 2.83 & 2.84 & 6.47 & 6.50 & 6.51 & 6.52 & 10.29 & 10.24 & 10.24 & 10.21 \\
\hline & 0.2 & 1.13 & 1.13 & 1.13 & 1.14 & 2.89 & 2.90 & 2.90 & 2.90 & 6.56 & 6.53 & 6.57 & 6.53 & 10.28 & 10.28 & 10.27 & 10.22 \\
\hline & 0.5 & 1.38 & 1.39 & 1.38 & 1.39 & 3.10 & 3.14 & 3.12 & 3.15 & 6.72 & 6.66 & 6.68 & 6.66 & 10.29 & 10.29 & 10.30 & 10.32 \\
\hline & 0.8 & 1.90 & 1.88 & 1.90 & 1.90 & 3.53 & 3.57 & 3.53 & 3.54 & 6.92 & 6.87 & 6.96 & 6.97 & 10.43 & 10.43 & 10.47 & 10.44 \\
\hline \multirow{4}{*}{0.5} & 0 & 1.34 & 1.34 & 1.33 & 1.33 & 3.09 & 3.12 & 3.10 & 3.10 & 6.69 & 6.67 & 6.67 & 6.64 & 10.30 & 10.34 & 10.32 & 10.36 \\
\hline & 0.2 & 1.39 & 1.40 & 1.39 & 1.40 & 3.13 & 3.16 & 3.15 & 3.15 & 6.66 & 6.69 & 6.71 & 6.64 & 10.29 & 10.31 & 10.27 & 10.36 \\
\hline & 0.5 & 1.66 & 1.67 & 1.68 & 1.69 & 3.38 & 3.34 & 3.36 & 3.38 & 6.80 & 6.82 & 6.80 & 6.82 & 10.39 & 10.40 & 10.38 & 10.41 \\
\hline & 0.8 & 2.16 & 2.16 & 2.16 & 2.19 & 3.78 & 3.79 & 3.75 & 3.79 & 7.09 & 7.06 & 7.10 & 7.09 & 10.58 & 10.50 & 10.52 & 10.53 \\
\hline \multirow{4}{*}{0.8} & 0 & 1.86 & 1.86 & 1.87 & 1.88 & 3.53 & 3.52 & 3.52 & 3.51 & 6.96 & 6.94 & 6.92 & 6.90 & 10.42 & 10.44 & 10.46 & 10.46 \\
\hline & 0.2 & 1.92 & 1.94 & 1.94 & 1.92 & 3.59 & 3.59 & 3.59 & 3.57 & 6.99 & 6.97 & 6.94 & 6.96 & 10.43 & 10.52 & 10.51 & 10.44 \\
\hline & 0.5 & 2.17 & 2.20 & 2.21 & 2.19 & 3.77 & 3.79 & 3.80 & 3.78 & 7.14 & 7.11 & 7.12 & 7.14 & 10.53 & 10.52 & 10.48 & 10.51 \\
\hline & 0.8 & 2.69 & 2.63 & 2.66 & 2.63 & 4.19 & 4.17 & 4.23 & 4.21 & 7.33 & 7.37 & 7.38 & 7.35 & 10.65 & 10.59 & 10.60 & 10.70 \\
\hline
\end{tabular}

Note: ICC = Intraclass Correlation Coefficient. 
Table 5 cont.

Average Design Effect values for the L2 predictor: Uniform Distribution conditions $(\mathrm{m}=20, \mathrm{k}=50)$

\begin{tabular}{|c|c|c|c|c|c|c|c|c|c|c|c|c|c|c|c|c|c|}
\hline \multirow{4}{*}{$\begin{array}{c}\text { L1 } \\
\text { Effect }\end{array}$} & \multirow{4}{*}{$\begin{array}{c}\text { Interaction } \\
\text { Effect }\end{array}$} & \multicolumn{16}{|c|}{ ICC Values } \\
\hline & & \multicolumn{4}{|c|}{0} & \multirow{2}{*}{\multicolumn{3}{|c|}{0.1}} & & \multicolumn{4}{|c|}{0.3} & \multicolumn{4}{|c|}{0.5} \\
\hline & & \multicolumn{9}{|c|}{ L2 Effect } & & & & \multirow[b]{2}{*}{0} & \multirow[b]{2}{*}{0.2} & \multirow[b]{2}{*}{0.5} & \multirow[b]{2}{*}{0.8} \\
\hline & & 0 & 0.2 & 0.5 & 0.8 & 0 & 0.2 & 0.5 & 0.8 & 0 & 0.2 & 0.5 & 0.8 & & & & \\
\hline \multirow{4}{*}{0} & 0 & 1.05 & 1.05 & 1.05 & 1.05 & 2.85 & 2.84 & 2.84 & 2.83 & 6.55 & 6.53 & 6.55 & 6.53 & 10.32 & 10.31 & 10.28 & 10.29 \\
\hline & 0.2 & 1.08 & 1.08 & 1.09 & 1.08 & 2.88 & 2.89 & 2.90 & 2.89 & 6.56 & 6.61 & 6.60 & 6.58 & 10.35 & 10.30 & 10.30 & 10.32 \\
\hline & 0.5 & 1.33 & 1.34 & 1.34 & 1.35 & 3.14 & 3.12 & 3.14 & 3.12 & 6.76 & 6.73 & 6.74 & 6.68 & 10.40 & 10.36 & 10.40 & 10.42 \\
\hline & 0.8 & 1.88 & 1.89 & 1.85 & 1.87 & 3.54 & 3.56 & 3.57 & 3.58 & 6.97 & 7.01 & 6.95 & 7.02 & 10.52 & 10.53 & 10.53 & 10.53 \\
\hline \multirow{4}{*}{0.2} & 0 & 1.08 & 1.08 & 1.09 & 1.08 & 2.88 & 2.87 & 2.88 & 2.86 & 6.59 & 6.59 & 6.58 & 6.58 & 10.30 & 10.28 & 10.33 & 10.31 \\
\hline & 0.2 & 1.13 & 1.12 & 1.12 & 1.13 & 2.93 & 2.95 & 2.96 & 2.93 & 6.60 & 6.63 & 6.61 & 6.63 & 10.31 & 10.37 & 10.34 & 10.36 \\
\hline & 0.5 & 1.39 & 1.41 & 1.39 & 1.40 & 3.13 & 3.15 & 3.17 & 3.19 & 6.76 & 6.79 & 6.74 & 6.71 & 10.39 & 10.41 & 10.40 & 10.41 \\
\hline & 0.8 & 1.93 & 1.93 & 1.92 & 1.93 & 3.60 & 3.60 & 3.59 & 3.61 & 7.01 & 7.02 & 7.03 & 6.99 & 10.53 & 10.61 & 10.55 & 10.55 \\
\hline \multirow{4}{*}{0.5} & 0 & 1.36 & 1.36 & 1.35 & 1.36 & 3.13 & 3.12 & 3.13 & 3.15 & 6.73 & 6.74 & 6.73 & 6.72 & 10.39 & 10.43 & 10.41 & 10.42 \\
\hline & 0.2 & 1.41 & 1.40 & 1.40 & 1.40 & 3.17 & 3.20 & 3.18 & 3.17 & 6.76 & 6.75 & 6.81 & 6.76 & 10.44 & 10.47 & 10.43 & 10.43 \\
\hline & 0.5 & 1.68 & 1.69 & 1.71 & 1.69 & 3.41 & 3.42 & 3.41 & 3.44 & 6.89 & 6.92 & 6.90 & 6.93 & 10.49 & 10.52 & 10.49 & 10.50 \\
\hline & 0.8 & 2.22 & 2.21 & 2.23 & 2.20 & 3.81 & 3.85 & 3.83 & 3.84 & 7.17 & 7.15 & 7.17 & 7.21 & 10.61 & 10.63 & 10.66 & 10.66 \\
\hline \multirow{4}{*}{0.8} & 0 & 1.89 & 1.90 & 1.90 & 1.90 & 3.59 & 3.60 & 3.60 & 3.61 & 6.99 & 7.03 & 7.05 & 7.09 & 10.54 & 10.58 & 10.56 & 10.55 \\
\hline & 0.2 & 1.95 & 1.95 & 1.97 & 1.95 & 3.65 & 3.59 & 3.62 & 3.61 & 7.01 & 7.04 & 7.05 & 7.03 & 10.58 & 10.58 & 10.56 & 10.56 \\
\hline & 0.5 & 2.22 & 2.25 & 2.23 & 2.24 & 3.83 & 3.87 & 3.86 & 3.86 & 7.18 & 7.12 & 7.19 & 7.21 & 10.62 & 10.64 & 10.61 & 10.62 \\
\hline & 0.8 & 2.74 & 2.72 & 2.72 & 2.72 & 4.26 & 4.25 & 4.24 & 4.28 & 7.40 & 7.46 & 7.47 & 7.43 & 10.76 & 10.73 & 10.78 & 10.80 \\
\hline
\end{tabular}

Note: ICC $=$ Intraclass Correlation Coefficient. 
Table 5 cont.

Average Design Effect values for the L2 predictor: Uniform Distribution conditions ( $\mathrm{m}=20, \mathrm{k}=100)$

\begin{tabular}{|c|c|c|c|c|c|c|c|c|c|c|c|c|c|c|c|c|c|}
\hline \multirow{4}{*}{$\begin{array}{c}\text { L1 } \\
\text { Effect }\end{array}$} & \multirow{4}{*}{$\begin{array}{c}\text { Interaction } \\
\text { Effect }\end{array}$} & \multicolumn{16}{|c|}{ ICC Values } \\
\hline & & \multicolumn{4}{|c|}{0} & \multirow{2}{*}{\multicolumn{3}{|c|}{0.1}} & & \multicolumn{4}{|c|}{0.3} & \multicolumn{4}{|c|}{0.5} \\
\hline & & \multicolumn{9}{|c|}{ L2 Effect } & & & & \multirow[b]{2}{*}{0} & \multirow[b]{2}{*}{0.2} & \multirow[b]{2}{*}{0.5} & \multirow[b]{2}{*}{0.8} \\
\hline & & 0 & 0.2 & 0.5 & 0.8 & 0 & 0.2 & 0.5 & 0.8 & 0 & 0.2 & 0.5 & 0.8 & & & & \\
\hline \multirow{4}{*}{0} & 0 & 1.05 & 1.05 & 1.05 & 1.05 & 2.84 & 2.87 & 2.84 & 2.84 & 6.61 & 6.61 & 6.55 & 6.57 & 10.35 & 10.37 & 10.37 & 10.34 \\
\hline & 0.2 & 1.08 & 1.08 & 1.08 & 1.09 & 2.88 & 2.91 & 2.89 & 2.90 & 6.64 & 6.61 & 6.63 & 6.64 & 10.40 & 10.38 & 10.33 & 10.36 \\
\hline & 0.5 & 1.35 & 1.35 & 1.35 & 1.34 & 3.14 & 3.14 & 3.14 & 3.15 & 6.75 & 6.78 & 6.74 & 6.74 & 10.49 & 10.46 & 10.44 & 10.46 \\
\hline & 0.8 & 1.90 & 1.89 & 1.89 & 1.89 & 3.59 & 3.61 & 3.58 & 3.59 & 7.06 & 7.04 & 7.03 & 7.06 & 10.61 & 10.61 & 10.53 & 10.62 \\
\hline \multirow{4}{*}{0.2} & 0 & 1.08 & 1.08 & 1.08 & 1.08 & 2.89 & 2.90 & 2.90 & 2.89 & 6.64 & 6.61 & 6.62 & 6.61 & 10.41 & 10.36 & 10.38 & 10.36 \\
\hline & 0.2 & 1.12 & 1.12 & 1.13 & 1.12 & 2.95 & 2.93 & 2.95 & 2.94 & 6.63 & 6.65 & 6.67 & 6.66 & 10.35 & 10.36 & 10.39 & 10.41 \\
\hline & 0.5 & 1.41 & 1.42 & 1.41 & 1.40 & 3.20 & 3.18 & 3.20 & 3.20 & 6.78 & 6.82 & 6.79 & 6.82 & 10.49 & 10.45 & 10.46 & 10.49 \\
\hline & 0.8 & 1.94 & 1.95 & 1.95 & 1.95 & 3.64 & 3.65 & 3.61 & 3.65 & 7.09 & 7.07 & 7.10 & 7.09 & 10.63 & 10.56 & 10.62 & 10.57 \\
\hline \multirow{4}{*}{0.5} & 0 & 1.36 & 1.36 & 1.36 & 1.36 & 3.15 & 3.16 & 3.13 & 3.15 & 6.76 & 6.77 & 6.77 & 6.81 & 10.47 & 10.39 & 10.47 & 10.48 \\
\hline & 0.2 & 1.43 & 1.42 & 1.41 & 1.40 & 3.18 & 3.21 & 3.21 & 3.22 & 6.82 & 6.79 & 6.77 & 6.80 & 10.46 & 10.51 & 10.48 & 10.44 \\
\hline & 0.5 & 1.71 & 1.72 & 1.72 & 1.71 & 3.44 & 3.45 & 3.42 & 3.43 & 6.93 & 6.93 & 6.93 & 6.92 & 10.53 & 10.54 & 10.51 & 10.57 \\
\hline & 0.8 & 2.24 & 2.22 & 2.25 & 2.22 & 3.87 & 3.86 & 3.87 & 3.85 & 7.21 & 7.21 & 7.19 & 7.20 & 10.68 & 10.67 & 10.75 & 10.70 \\
\hline \multirow{4}{*}{0.8} & 0 & 1.92 & 1.91 & 1.91 & 1.92 & 3.59 & 3.59 & 3.59 & 3.63 & 7.02 & 7.07 & 7.02 & 7.02 & 10.59 & 10.61 & 10.62 & 10.58 \\
\hline & 0.2 & 1.97 & 1.95 & 1.97 & 1.97 & 3.66 & 3.64 & 3.65 & 3.67 & 7.11 & 7.10 & 7.07 & 7.09 & 10.61 & 10.64 & 10.64 & 10.63 \\
\hline & 0.5 & 2.27 & 2.23 & 2.25 & 2.23 & 3.85 & 3.87 & 3.89 & 3.86 & 7.20 & 7.24 & 7.21 & 7.24 & 10.70 & 10.67 & 10.66 & 10.70 \\
\hline & 0.8 & 2.74 & 2.72 & 2.75 & 2.72 & 4.29 & 4.29 & 4.30 & 4.31 & 7.45 & 7.50 & 7.46 & 7.50 & 10.84 & 10.84 & 10.82 & 10.82 \\
\hline
\end{tabular}

Note: ICC $=$ Intraclass Correlation Coefficient. 
Table 5 cont.

Average Design Effect values for the L2 predictor: Poisson Distribution conditions $(\mathrm{m}=5, \mathrm{k}=25)$

\begin{tabular}{|c|c|c|c|c|c|c|c|c|c|c|c|c|c|c|c|c|c|}
\hline \multirow{4}{*}{$\begin{array}{c}\text { L1 } \\
\text { Effect }\end{array}$} & \multirow{4}{*}{$\begin{array}{l}\text { Interaction } \\
\text { Effect }\end{array}$} & \multicolumn{16}{|c|}{ ICC Values } \\
\hline & & \multicolumn{4}{|c|}{0} & \multirow{2}{*}{\multicolumn{3}{|c|}{0.1}} & & \multicolumn{4}{|c|}{0.3} & \multicolumn{4}{|c|}{0.5} \\
\hline & & & & & & & & & \multicolumn{2}{|c|}{ L2 Effect } & & & & & & & \\
\hline & & 0 & 0.2 & 0.5 & 0.8 & 0 & 0.2 & 0.5 & 0.8 & 0 & 0.2 & 0.5 & 0.8 & 0 & 0.2 & 0.5 & 0.8 \\
\hline \multirow{4}{*}{0} & 0 & 1.08 & 1.08 & 1.08 & 1.08 & 1.40 & 1.38 & 1.39 & 1.38 & 2.19 & 2.18 & 2.16 & 2.17 & 2.97 & 2.95 & 2.97 & 2.98 \\
\hline & 0.2 & 1.11 & 1.10 & 1.11 & 1.10 & 1.40 & 1.42 & 1.43 & 1.41 & 2.22 & 2.22 & 2.21 & 2.19 & 3.00 & 2.97 & 2.98 & 3.01 \\
\hline & 0.5 & 1.27 & 1.27 & 1.26 & 1.25 & 1.60 & 1.61 & 1.58 & 1.61 & 2.34 & 2.36 & 2.33 & 2.30 & 3.08 & 3.05 & 3.08 & 3.02 \\
\hline & 0.8 & 1.63 & 1.60 & 1.59 & 1.62 & 1.95 & 1.91 & 1.91 & 1.90 & 2.56 & 2.54 & 2.56 & 2.57 & 3.21 & 3.24 & 3.23 & 3.22 \\
\hline \multirow{4}{*}{0.2} & 0 & 1.11 & 1.11 & 1.10 & 1.10 & 1.42 & 1.43 & 1.42 & 1.42 & 2.21 & 2.22 & 2.20 & 2.19 & 2.95 & 2.97 & 2.98 & 2.97 \\
\hline & 0.2 & 1.14 & 1.12 & 1.13 & 1.13 & 1.45 & 1.45 & 1.46 & 1.45 & 2.23 & 2.22 & 2.21 & 2.22 & 3.01 & 3.00 & 3.03 & 3.00 \\
\hline & 0.5 & 1.30 & 1.29 & 1.31 & 1.30 & 1.65 & 1.66 & 1.64 & 1.65 & 2.34 & 2.37 & 2.36 & 2.36 & 3.10 & 3.12 & 3.10 & 3.07 \\
\hline & 0.8 & 1.62 & 1.64 & 1.63 & 1.66 & 1.95 & 1.96 & 1.96 & 1.94 & 2.56 & 2.59 & 2.55 & 2.59 & 3.21 & 3.22 & 3.24 & 3.19 \\
\hline \multirow{4}{*}{0.5} & 0 & 1.28 & 1.29 & 1.28 & 1.26 & 1.62 & 1.62 & 1.62 & 1.63 & 2.32 & 2.31 & 2.34 & 2.35 & 3.11 & 3.09 & 3.07 & 3.12 \\
\hline & 0.2 & 1.31 & 1.32 & 1.30 & 1.31 & 1.65 & 1.61 & 1.64 & 1.65 & 2.34 & 2.36 & 2.36 & 2.36 & 3.12 & 3.10 & 3.11 & 3.09 \\
\hline & 0.5 & 1.50 & 1.52 & 1.50 & 1.49 & 1.83 & 1.84 & 1.82 & 1.84 & 2.54 & 2.51 & 2.46 & 2.47 & 3.17 & 3.17 & 3.17 & 3.13 \\
\hline & 0.8 & 1.83 & 1.86 & 1.85 & 1.83 & 2.10 & 2.11 & 2.12 & 2.10 & 2.70 & 2.68 & 2.71 & 2.66 & 3.31 & 3.29 & 3.31 & 3.30 \\
\hline \multirow{4}{*}{0.8} & 0 & 1.64 & 1.64 & 1.69 & 1.63 & 1.95 & 1.94 & 1.96 & 1.97 & 2.62 & 2.61 & 2.61 & 2.56 & 3.24 & 3.21 & 3.20 & 3.24 \\
\hline & 0.2 & 1.70 & 1.71 & 1.69 & 1.70 & 2.02 & 2.02 & 2.00 & 1.98 & 2.59 & 2.58 & 2.60 & 2.62 & 3.21 & 3.29 & 3.25 & 3.22 \\
\hline & 0.5 & 1.91 & 1.86 & 1.87 & 1.87 & 2.16 & 2.17 & 2.15 & 2.14 & 2.70 & 2.68 & 2.74 & 2.71 & 3.30 & 3.33 & 3.28 & 3.31 \\
\hline & 0.8 & 2.13 & 2.18 & 2.17 & 2.17 & 2.44 & 2.41 & 2.39 & 2.38 & 2.92 & 2.90 & 2.91 & 2.92 & 3.45 & 3.41 & 3.46 & 3.45 \\
\hline
\end{tabular}

Note: ICC = Intraclass Correlation Coefficient. 
Table 5 cont.

Average Design Effect values for the L2 predictor: Poisson Distribution conditions $(\mathrm{m}=5, \mathrm{k}=50)$

\begin{tabular}{|c|c|c|c|c|c|c|c|c|c|c|c|c|c|c|c|c|c|}
\hline \multirow{4}{*}{$\begin{array}{c}\text { L1 } \\
\text { Effect }\end{array}$} & \multirow{4}{*}{$\begin{array}{c}\text { Interaction } \\
\text { Effect }\end{array}$} & \multicolumn{16}{|c|}{ ICC Values } \\
\hline & & \multicolumn{4}{|c|}{0} & \multirow{2}{*}{\multicolumn{3}{|c|}{0.1}} & & \multicolumn{4}{|c|}{0.3} & \multicolumn{4}{|c|}{0.5} \\
\hline & & & & & & & & & \multicolumn{2}{|c|}{ L2 Effect } & & & & & & & \\
\hline & & 0 & 0.2 & 0.5 & 0.8 & 0 & 0.2 & 0.5 & 0.8 & 0 & 0.2 & 0.5 & 0.8 & 0 & 0.2 & 0.5 & 0.8 \\
\hline \multirow{4}{*}{0} & 0 & 1.07 & 1.06 & 1.07 & 1.07 & 1.42 & 1.42 & 1.42 & 1.42 & 2.23 & 2.25 & 2.25 & 2.24 & 3.03 & 3.05 & 3.04 & 3.03 \\
\hline & 0.2 & 1.09 & 1.09 & 1.09 & 1.09 & 1.46 & 1.46 & 1.45 & 1.45 & 2.26 & 2.27 & 2.28 & 2.25 & 3.07 & 3.07 & 3.07 & 3.07 \\
\hline & 0.5 & 1.29 & 1.29 & 1.27 & 1.29 & 1.66 & 1.66 & 1.63 & 1.66 & 2.41 & 2.40 & 2.41 & 2.42 & 3.14 & 3.16 & 3.15 & 3.15 \\
\hline & 0.8 & 1.65 & 1.66 & 1.68 & 1.69 & 2.00 & 1.98 & 2.00 & 1.99 & 2.67 & 2.63 & 2.64 & 2.64 & 3.29 & 3.31 & 3.29 & 3.31 \\
\hline \multirow{4}{*}{0.2} & 0 & 1.10 & 1.09 & 1.08 & 1.10 & 1.45 & 1.45 & 1.47 & 1.46 & 2.28 & 2.28 & 2.28 & 2.26 & 3.07 & 3.06 & 3.05 & 3.07 \\
\hline & 0.2 & 1.12 & 1.12 & 1.12 & 1.12 & 1.49 & 1.50 & 1.50 & 1.51 & 2.31 & 2.30 & 2.29 & 2.30 & 3.09 & 3.08 & 3.09 & 3.07 \\
\hline & 0.5 & 1.32 & 1.31 & 1.32 & 1.33 & 1.70 & 1.69 & 1.70 & 1.69 & 2.45 & 2.43 & 2.44 & 2.42 & 3.16 & 3.16 & 3.19 & 3.15 \\
\hline & 0.8 & 1.74 & 1.72 & 1.70 & 1.72 & 2.05 & 2.04 & 2.04 & 2.03 & 2.69 & 2.68 & 2.67 & 2.68 & 3.32 & 3.30 & 3.30 & 3.31 \\
\hline \multirow{4}{*}{0.5} & 0 & 1.28 & 1.29 & 1.30 & 1.29 & 1.68 & 1.66 & 1.66 & 1.68 & 2.41 & 2.42 & 2.42 & 2.43 & 3.14 & 3.15 & 3.14 & 3.14 \\
\hline & 0.2 & 1.33 & 1.32 & 1.33 & 1.33 & 1.71 & 1.72 & 1.71 & 1.72 & 2.44 & 2.44 & 2.46 & 2.44 & 3.19 & 3.13 & 3.17 & 3.16 \\
\hline & 0.5 & 1.55 & 1.56 & 1.54 & 1.56 & 1.90 & 1.89 & 1.89 & 1.89 & 2.57 & 2.55 & 2.59 & 2.56 & 3.23 & 3.27 & 3.23 & 3.23 \\
\hline & 0.8 & 1.92 & 1.94 & 1.92 & 1.92 & 2.22 & 2.21 & 2.21 & 2.23 & 2.81 & 2.80 & 2.82 & 2.81 & 3.41 & 3.40 & 3.40 & 3.36 \\
\hline \multirow{4}{*}{0.8} & 0 & 1.70 & 1.70 & 1.72 & 1.72 & 2.05 & 2.03 & 2.04 & 2.01 & 2.67 & 2.66 & 2.67 & 2.66 & 3.30 & 3.34 & 3.27 & 3.31 \\
\hline & 0.2 & 1.74 & 1.75 & 1.75 & 1.74 & 2.08 & 2.06 & 2.06 & 2.07 & 2.69 & 2.67 & 2.67 & 2.69 & 3.30 & 3.34 & 3.33 & 3.31 \\
\hline & 0.5 & 1.95 & 1.94 & 1.95 & 1.95 & 2.25 & 2.23 & 2.23 & 2.22 & 2.82 & 2.81 & 2.79 & 2.78 & 3.42 & 3.41 & 3.39 & 3.41 \\
\hline & 0.8 & 2.24 & 2.27 & 2.25 & 2.28 & 2.51 & 2.50 & 2.51 & 2.53 & 2.99 & 3.01 & 2.97 & 3.00 & 3.53 & 3.53 & 3.52 & 3.50 \\
\hline
\end{tabular}

Note: ICC $=$ Intraclass Correlation Coefficient. 
Table 5 cont.

Average Design Effect values for the L2 predictor: Poisson Distribution conditions $(\mathrm{m}=5, \mathrm{k}=75)$

\begin{tabular}{|c|c|c|c|c|c|c|c|c|c|c|c|c|c|c|c|c|c|}
\hline \multirow{4}{*}{$\begin{array}{c}\text { L1 } \\
\text { Effect }\end{array}$} & \multirow{4}{*}{$\begin{array}{c}\text { Interaction } \\
\text { Effect }\end{array}$} & \multicolumn{16}{|c|}{ ICC Values } \\
\hline & & \multicolumn{4}{|c|}{0} & \multirow{2}{*}{\multicolumn{3}{|c|}{0.1}} & & \multicolumn{4}{|c|}{0.3} & \multicolumn{4}{|c|}{0.5} \\
\hline & & & & & & & & & \multicolumn{2}{|c|}{ L2 Effect } & & & & & & & \\
\hline & & 0 & 0.2 & 0.5 & 0.8 & 0 & 0.2 & 0.5 & 0.8 & 0 & 0.2 & 0.5 & 0.8 & 0 & 0.2 & 0.5 & 0.8 \\
\hline \multirow{4}{*}{0} & 0 & 1.06 & 1.05 & 1.05 & 1.05 & 1.44 & 1.43 & 1.43 & 1.42 & 2.26 & 2.27 & 2.27 & 2.26 & 3.09 & 3.07 & 3.07 & 3.09 \\
\hline & 0.2 & 1.08 & 1.08 & 1.08 & 1.08 & 1.47 & 1.48 & 1.47 & 1.46 & 2.30 & 2.29 & 2.30 & 2.30 & 3.07 & 3.11 & 3.10 & 3.08 \\
\hline & 0.5 & 1.30 & 1.28 & 1.29 & 1.28 & 1.67 & 1.69 & 1.68 & 1.67 & 2.45 & 2.43 & 2.43 & 2.44 & 3.21 & 3.18 & 3.18 & 3.17 \\
\hline & 0.8 & 1.71 & 1.71 & 1.71 & 1.70 & 2.05 & 2.03 & 2.03 & 2.02 & 2.69 & 2.68 & 2.67 & 2.66 & 3.32 & 3.33 & 3.32 & 3.31 \\
\hline \multirow{4}{*}{0.2} & 0 & 1.08 & 1.08 & 1.08 & 1.08 & 1.47 & 1.47 & 1.47 & 1.49 & 2.31 & 2.30 & 2.30 & 2.31 & 3.09 & 3.10 & 3.08 & 3.09 \\
\hline & 0.2 & 1.12 & 1.11 & 1.12 & 1.11 & 1.52 & 1.52 & 1.51 & 1.52 & 2.32 & 2.33 & 2.32 & 2.31 & 3.11 & 3.12 & 3.10 & 3.11 \\
\hline & 0.5 & 1.34 & 1.33 & 1.32 & 1.33 & 1.72 & 1.73 & 1.74 & 1.72 & 2.46 & 2.47 & 2.47 & 2.47 & 3.19 & 3.20 & 3.19 & 3.20 \\
\hline & 0.8 & 1.76 & 1.73 & 1.76 & 1.76 & 2.08 & 2.08 & 2.07 & 2.07 & 2.69 & 2.71 & 2.71 & 2.71 & 3.34 & 3.36 & 3.33 & 3.34 \\
\hline \multirow{4}{*}{0.5} & 0 & 1.30 & 1.30 & 1.29 & 1.30 & 1.67 & 1.69 & 1.70 & 1.68 & 2.45 & 2.45 & 2.45 & 2.45 & 3.19 & 3.17 & 3.17 & 3.17 \\
\hline & 0.2 & 1.35 & 1.34 & 1.34 & 1.35 & 1.72 & 1.73 & 1.73 & 1.72 & 2.46 & 2.46 & 2.47 & 2.47 & 3.18 & 3.22 & 3.18 & 3.20 \\
\hline & 0.5 & 1.58 & 1.56 & 1.58 & 1.57 & 1.93 & 1.93 & 1.93 & 1.91 & 2.58 & 2.59 & 2.60 & 2.61 & 3.29 & 3.26 & 3.29 & 3.26 \\
\hline & 0.8 & 1.96 & 1.95 & 1.97 & 1.97 & 2.23 & 2.23 & 2.26 & 2.23 & 2.85 & 2.81 & 2.82 & 2.82 & 3.42 & 3.40 & 3.42 & 3.40 \\
\hline \multirow{4}{*}{0.8} & 0 & 1.73 & 1.74 & 1.73 & 1.73 & 2.05 & 2.07 & 2.05 & 2.06 & 2.68 & 2.70 & 2.70 & 2.68 & 3.34 & 3.33 & 3.35 & 3.33 \\
\hline & 0.2 & 1.78 & 1.76 & 1.77 & 1.77 & 2.09 & 2.10 & 2.11 & 2.10 & 2.72 & 2.70 & 2.70 & 2.70 & 3.36 & 3.35 & 3.35 & 3.34 \\
\hline & 0.5 & 1.98 & 1.98 & 1.98 & 1.97 & 2.26 & 2.25 & 2.27 & 2.27 & 2.83 & 2.83 & 2.83 & 2.83 & 3.41 & 3.42 & 3.43 & 3.43 \\
\hline & 0.8 & 2.31 & 2.31 & 2.29 & 2.29 & 2.54 & 2.52 & 2.55 & 2.55 & 3.03 & 3.03 & 3.04 & 3.03 & 3.54 & 3.56 & 3.54 & 3.55 \\
\hline
\end{tabular}

Note: ICC $=$ Intraclass Correlation Coefficient. 
Table 5 cont.

Average Design Effect values for the L2 predictor: Poisson Distribution conditions $(\mathrm{m}=5, \mathrm{k}=100)$

\begin{tabular}{|c|c|c|c|c|c|c|c|c|c|c|c|c|c|c|c|c|c|}
\hline \multirow{4}{*}{$\begin{array}{c}\text { L1 } \\
\text { Effect }\end{array}$} & \multirow{4}{*}{$\begin{array}{c}\text { Interaction } \\
\text { Effect }\end{array}$} & \multicolumn{16}{|c|}{ ICC Values } \\
\hline & & \multicolumn{4}{|c|}{0} & \multirow{2}{*}{\multicolumn{3}{|c|}{0.1}} & & \multicolumn{4}{|c|}{0.3} & \multicolumn{4}{|c|}{0.5} \\
\hline & & & & & & & & & \multicolumn{2}{|c|}{ L2 Effect } & & & & & & & \\
\hline & & 0 & 0.2 & 0.5 & 0.8 & 0 & 0.2 & 0.5 & 0.8 & 0 & 0.2 & 0.5 & 0.8 & 0 & 0.2 & 0.5 & 0.8 \\
\hline \multirow{4}{*}{0} & 0 & 1.05 & 1.04 & 1.05 & 1.05 & 1.44 & 1.44 & 1.44 & 1.45 & 2.29 & 2.28 & 2.29 & 2.29 & 3.08 & 3.08 & 3.09 & 3.07 \\
\hline & 0.2 & 1.08 & 1.07 & 1.08 & 1.07 & 1.48 & 1.48 & 1.48 & 1.48 & 2.30 & 2.31 & 2.31 & 2.31 & 3.11 & 3.10 & 3.10 & 3.11 \\
\hline & 0.5 & 1.31 & 1.29 & 1.29 & 1.30 & 1.69 & 1.70 & 1.69 & 1.70 & 2.44 & 2.45 & 2.43 & 2.45 & 3.19 & 3.17 & 3.19 & 3.18 \\
\hline & 0.8 & 1.72 & 1.71 & 1.73 & 1.71 & 2.06 & 2.05 & 2.06 & 2.05 & 2.70 & 2.70 & 2.69 & 2.71 & 3.34 & 3.32 & 3.35 & 3.33 \\
\hline \multirow{4}{*}{0.2} & 0 & 1.07 & 1.08 & 1.08 & 1.07 & 1.48 & 1.48 & 1.47 & 1.49 & 2.30 & 2.30 & 2.31 & 2.30 & 3.10 & 3.10 & 3.10 & 3.11 \\
\hline & 0.2 & 1.11 & 1.11 & 1.11 & 1.11 & 1.52 & 1.52 & 1.53 & 1.52 & 2.34 & 2.33 & 2.34 & 2.34 & 3.11 & 3.11 & 3.11 & 3.12 \\
\hline & 0.5 & 1.33 & 1.33 & 1.33 & 1.34 & 1.73 & 1.74 & 1.75 & 1.74 & 2.47 & 2.48 & 2.48 & 2.48 & 3.20 & 3.21 & 3.20 & 3.20 \\
\hline & 0.8 & 1.75 & 1.76 & 1.76 & 1.76 & 2.08 & 2.08 & 2.09 & 2.10 & 2.72 & 2.70 & 2.72 & 2.72 & 3.35 & 3.34 & 3.36 & 3.37 \\
\hline \multirow{4}{*}{0.5} & 0 & 1.30 & 1.29 & 1.30 & 1.30 & 1.69 & 1.69 & 1.70 & 1.71 & 2.46 & 2.45 & 2.46 & 2.43 & 3.20 & 3.19 & 3.18 & 3.21 \\
\hline & 0.2 & 1.34 & 1.36 & 1.35 & 1.35 & 1.73 & 1.73 & 1.74 & 1.74 & 2.46 & 2.47 & 2.48 & 2.49 & 3.21 & 3.20 & 3.21 & 3.21 \\
\hline & 0.5 & 1.58 & 1.59 & 1.58 & 1.58 & 1.94 & 1.93 & 1.95 & 1.95 & 2.63 & 2.62 & 2.61 & 2.62 & 3.29 & 3.29 & 3.30 & 3.29 \\
\hline & 0.8 & 1.98 & 1.97 & 1.97 & 1.97 & 2.25 & 2.26 & 2.27 & 2.26 & 2.85 & 2.83 & 2.85 & 2.83 & 3.42 & 3.45 & 3.43 & 3.43 \\
\hline \multirow{4}{*}{0.8} & 0 & 1.73 & 1.74 & 1.75 & 1.74 & 2.07 & 2.08 & 2.05 & 2.07 & 2.72 & 2.72 & 2.71 & 2.71 & 3.35 & 3.36 & 3.36 & 3.36 \\
\hline & 0.2 & 1.79 & 1.78 & 1.77 & 1.77 & 2.10 & 2.10 & 2.11 & 2.10 & 2.72 & 2.72 & 2.73 & 2.71 & 3.37 & 3.35 & 3.36 & 3.38 \\
\hline & 0.5 & 1.99 & 1.98 & 1.97 & 1.97 & 2.28 & 2.25 & 2.27 & 2.27 & 2.84 & 2.84 & 2.84 & 2.86 & 3.42 & 3.43 & 3.44 & 3.43 \\
\hline & 0.8 & 2.30 & 2.31 & 2.32 & 2.32 & 2.57 & 2.54 & 2.56 & 2.56 & 3.04 & 3.04 & 3.04 & 3.06 & 3.55 & 3.57 & 3.57 & 3.58 \\
\hline
\end{tabular}

Note: ICC = Intraclass Correlation Coefficient. 
Table 5 cont.

Average Design Effect values for the L2 predictor: Poisson Distribution conditions $(\mathrm{m}=10, \mathrm{k}=25)$

\begin{tabular}{|c|c|c|c|c|c|c|c|c|c|c|c|c|c|c|c|c|c|}
\hline \multirow{4}{*}{$\begin{array}{c}\text { L1 } \\
\text { Effect }\end{array}$} & \multirow{4}{*}{$\begin{array}{l}\text { Interaction } \\
\text { Effect }\end{array}$} & \multicolumn{16}{|c|}{ ICC Values } \\
\hline & & \multicolumn{4}{|c|}{0} & \multirow{2}{*}{\multicolumn{3}{|c|}{0.1}} & & \multicolumn{4}{|c|}{0.3} & \multicolumn{4}{|c|}{0.5} \\
\hline & & & & & & & & & \multicolumn{2}{|c|}{ L2 Effect } & & & & & & & \\
\hline & & 0 & 0.2 & 0.5 & 0.8 & 0 & 0.2 & 0.5 & 0.8 & 0 & 0.2 & 0.5 & 0.8 & 0 & 0.2 & 0.5 & 0.8 \\
\hline \multirow{4}{*}{0} & 0 & 1.08 & 1.07 & 1.07 & 1.07 & 1.81 & 1.81 & 1.81 & 1.80 & 3.56 & 3.55 & 3.53 & 3.52 & 5.27 & 5.27 & 5.31 & 5.27 \\
\hline & 0.2 & 1.11 & 1.11 & 1.10 & 1.11 & 1.86 & 1.84 & 1.87 & 1.84 & 3.49 & 3.58 & 3.56 & 3.52 & 5.31 & 5.33 & 5.29 & 5.30 \\
\hline & 0.5 & 1.28 & 1.31 & 1.29 & 1.30 & 2.05 & 2.08 & 2.07 & 2.07 & 3.72 & 3.70 & 3.71 & 3.65 & 5.34 & 5.35 & 5.35 & 5.36 \\
\hline & 0.8 & 1.70 & 1.68 & 1.74 & 1.68 & 2.47 & 2.47 & 2.43 & 2.49 & 4.00 & 3.95 & 3.97 & 3.92 & 5.49 & 5.54 & 5.49 & 5.56 \\
\hline \multirow{4}{*}{0.2} & 0 & 1.11 & 1.11 & 1.10 & 1.12 & 1.86 & 1.86 & 1.86 & 1.85 & 3.55 & 3.58 & 3.57 & 3.57 & 5.27 & 5.27 & 5.26 & 5.35 \\
\hline & 0.2 & 1.13 & 1.14 & 1.14 & 1.14 & 1.88 & 1.90 & 1.89 & 1.89 & 3.56 & 3.56 & 3.61 & 3.60 & 5.27 & 5.33 & 5.30 & 5.28 \\
\hline & 0.5 & 1.33 & 1.34 & 1.35 & 1.33 & 2.10 & 2.12 & 2.10 & 2.13 & 3.73 & 3.72 & 3.71 & 3.74 & 5.42 & 5.40 & 5.35 & 5.36 \\
\hline & 0.8 & 1.73 & 1.75 & 1.75 & 1.76 & 2.50 & 2.52 & 2.47 & 2.48 & 4.03 & 3.95 & 4.01 & 3.98 & 5.53 & 5.51 & 5.55 & 5.54 \\
\hline \multirow{4}{*}{0.5} & 0 & 1.30 & 1.32 & 1.28 & 1.31 & 2.11 & 2.10 & 2.10 & 2.11 & 3.72 & 3.69 & 3.72 & 3.68 & 5.40 & 5.38 & 5.40 & 5.39 \\
\hline & 0.2 & 1.35 & 1.36 & 1.36 & 1.34 & 2.13 & 2.14 & 2.11 & 2.13 & 3.75 & 3.76 & 3.75 & 3.74 & 5.39 & 5.36 & 5.41 & 5.47 \\
\hline & 0.5 & 1.57 & 1.59 & 1.56 & 1.58 & 2.33 & 2.37 & 2.35 & 2.32 & 3.89 & 3.86 & 3.93 & 3.86 & 5.47 & 5.43 & 5.52 & 5.44 \\
\hline & 0.8 & 1.98 & 2.02 & 1.99 & 1.96 & 2.69 & 2.66 & 2.72 & 2.70 & 4.14 & 4.11 & 4.13 & 4.13 & 5.61 & 5.64 & 5.62 & 5.64 \\
\hline \multirow{4}{*}{0.8} & 0 & 1.77 & 1.75 & 1.77 & 1.77 & 2.52 & 2.51 & 2.49 & 2.50 & 3.95 & 3.99 & 3.99 & 3.98 & 5.55 & 5.59 & 5.66 & 5.58 \\
\hline & 0.2 & 1.81 & 1.81 & 1.82 & 1.80 & 2.54 & 2.52 & 2.54 & 2.54 & 4.04 & 3.99 & 3.99 & 4.01 & 5.57 & 5.53 & 5.57 & 5.54 \\
\hline & 0.5 & 2.01 & 2.07 & 2.03 & 2.02 & 2.72 & 2.74 & 2.71 & 2.72 & 4.13 & 4.13 & 4.14 & 4.17 & 5.60 & 5.62 & 5.57 & 5.64 \\
\hline & 0.8 & 2.45 & 2.40 & 2.44 & 2.46 & 3.06 & 3.08 & 3.05 & 3.07 & 4.38 & 4.39 & 4.39 & 4.35 & 5.74 & 5.76 & 5.74 & 5.79 \\
\hline
\end{tabular}

Note: ICC $=$ Intraclass Correlation Coefficient. 
Table 5 cont.

Average Design Effect values for the L2 predictor: Poisson Distribution conditions $(\mathrm{m}=10, \mathrm{k}=50)$

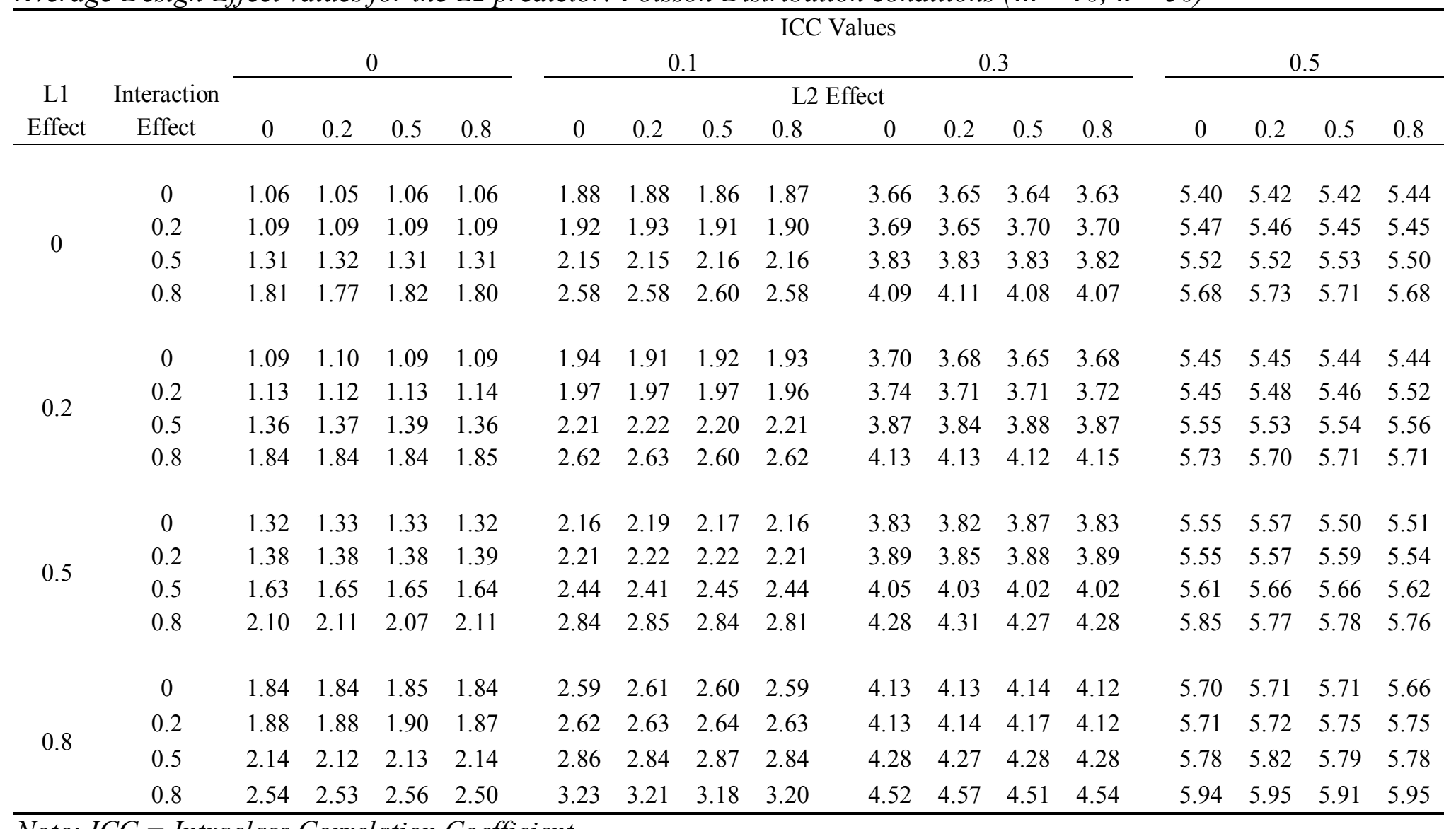

Note: ICC = Intraclass Correlation Coefficient. 
Table 5 cont.

Average Design Effect values for the L2 predictor: Poisson Distribution conditions $(\mathrm{m}=10, \mathrm{k}=75)$

\begin{tabular}{|c|c|c|c|c|c|c|c|c|c|c|c|c|c|c|c|c|c|}
\hline \multirow{4}{*}{$\begin{array}{c}\text { L1 } \\
\text { Effect }\end{array}$} & \multirow{4}{*}{$\begin{array}{l}\text { Interaction } \\
\text { Effect }\end{array}$} & \multicolumn{16}{|c|}{ ICC Values } \\
\hline & & \multicolumn{4}{|c|}{0} & \multirow{2}{*}{\multicolumn{3}{|c|}{0.1}} & & \multicolumn{4}{|c|}{0.3} & \multicolumn{4}{|c|}{0.5} \\
\hline & & & & & & & & & \multicolumn{2}{|c|}{ L2 Effect } & & & & & & & \\
\hline & & 0 & 0.2 & 0.5 & 0.8 & 0 & 0.2 & 0.5 & 0.8 & 0 & 0.2 & 0.5 & 0.8 & 0 & 0.2 & 0.5 & 0.8 \\
\hline \multirow{4}{*}{0} & 0 & 1.05 & 1.05 & 1.05 & 1.06 & 1.92 & 1.90 & 1.92 & 1.91 & 3.71 & 3.68 & 3.69 & 3.68 & 5.46 & 5.46 & 5.48 & 5.46 \\
\hline & 0.2 & 1.09 & 1.09 & 1.09 & 1.08 & 1.96 & 1.95 & 1.95 & 1.96 & 3.71 & 3.68 & 3.72 & 3.73 & 5.50 & 5.52 & 5.50 & 5.48 \\
\hline & 0.5 & 1.32 & 1.32 & 1.33 & 1.32 & 2.20 & 2.20 & 2.19 & 2.20 & 3.85 & 3.90 & 3.92 & 3.88 & 5.58 & 5.56 & 5.59 & 5.56 \\
\hline & 0.8 & 1.82 & 1.84 & 1.83 & 1.83 & 2.61 & 2.61 & 2.61 & 2.60 & 4.14 & 4.15 & 4.14 & 4.18 & 5.77 & 5.70 & 5.71 & 5.71 \\
\hline \multirow{4}{*}{0.2} & 0 & 1.09 & 1.08 & 1.08 & 1.09 & 1.95 & 1.97 & 1.96 & 1.95 & 3.72 & 3.74 & 3.76 & 3.72 & 5.49 & 5.49 & 5.46 & 5.47 \\
\hline & 0.2 & 1.12 & 1.12 & 1.13 & 1.12 & 1.99 & 1.99 & 2.01 & 2.01 & 3.75 & 3.75 & 3.74 & 3.74 & 5.51 & 5.48 & 5.52 & 5.51 \\
\hline & 0.5 & 1.38 & 1.38 & 1.38 & 1.38 & 2.25 & 2.24 & 2.24 & 2.23 & 3.90 & 3.91 & 3.91 & 3.91 & 5.58 & 5.57 & 5.59 & 5.61 \\
\hline & 0.8 & 1.88 & 1.89 & 1.89 & 1.87 & 2.66 & 2.65 & 2.66 & 2.66 & 4.21 & 4.17 & 4.18 & 4.18 & 5.76 & 5.76 & 5.76 & 5.79 \\
\hline \multirow{4}{*}{0.5} & 0 & 1.34 & 1.33 & 1.33 & 1.34 & 2.20 & 2.20 & 2.22 & 2.21 & 3.88 & 3.89 & 3.90 & 3.90 & 5.56 & 5.61 & 5.61 & 5.59 \\
\hline & 0.2 & 1.39 & 1.39 & 1.39 & 1.39 & 2.24 & 2.25 & 2.23 & 2.24 & 3.92 & 3.91 & 3.91 & 3.87 & 5.59 & 5.60 & 5.62 & 5.60 \\
\hline & 0.5 & 1.68 & 1.65 & 1.66 & 1.65 & 2.48 & 2.48 & 2.47 & 2.45 & 4.06 & 4.08 & 4.05 & 4.06 & 5.69 & 5.68 & 5.67 & 5.70 \\
\hline & 0.8 & 2.12 & 2.15 & 2.15 & 2.13 & 2.86 & 2.88 & 2.88 & 2.88 & 4.33 & 4.33 & 4.35 & 4.34 & 5.85 & 5.83 & 5.85 & 5.84 \\
\hline \multirow{4}{*}{0.8} & 0 & 1.85 & 1.85 & 1.86 & 1.84 & 2.63 & 2.62 & 2.63 & 2.64 & 4.16 & 4.16 & 4.15 & 4.19 & 5.75 & 5.78 & 5.74 & 5.75 \\
\hline & 0.2 & 1.91 & 1.91 & 1.89 & 1.90 & 2.67 & 2.66 & 2.68 & 2.68 & 4.20 & 4.18 & 4.20 & 4.17 & 5.77 & 5.74 & 5.77 & 5.75 \\
\hline & 0.5 & 2.15 & 2.15 & 2.15 & 2.17 & 2.89 & 2.86 & 2.90 & 2.90 & 4.34 & 4.35 & 4.36 & 4.32 & 5.85 & 5.84 & 5.84 & 5.86 \\
\hline & 0.8 & 2.58 & 2.62 & 2.57 & 2.58 & 3.26 & 3.24 & 3.27 & 3.25 & 4.59 & 4.56 & 4.58 & 4.56 & 5.99 & 6.00 & 6.00 & 5.98 \\
\hline
\end{tabular}

Note: ICC $=$ Intraclass Correlation Coefficient. 
Table 5 cont.

Average Design Effect values for the L2 predictor: Poisson Distribution conditions $(\mathrm{m}=10, \mathrm{k}=100)$

\begin{tabular}{|c|c|c|c|c|c|c|c|c|c|c|c|c|c|c|c|c|c|}
\hline \multirow{4}{*}{$\begin{array}{c}\text { L1 } \\
\text { Effect }\end{array}$} & \multirow{4}{*}{$\begin{array}{c}\text { Interaction } \\
\text { Effect }\end{array}$} & \multicolumn{16}{|c|}{ ICC Values } \\
\hline & & \multicolumn{4}{|c|}{0} & \multicolumn{4}{|c|}{0.1} & \multicolumn{4}{|c|}{0.3} & \multicolumn{4}{|c|}{0.5} \\
\hline & & \multirow[b]{2}{*}{0} & \multirow[b]{2}{*}{0.2} & \multirow[b]{2}{*}{0.5} & \multirow[b]{2}{*}{0.8} & \multirow[b]{2}{*}{0} & \multirow[b]{2}{*}{0.2} & \multirow[b]{2}{*}{0.5} & \multicolumn{2}{|c|}{ L2 Effect } & \multirow[b]{2}{*}{0.2} & \multirow[b]{2}{*}{0.5} & \multirow[b]{2}{*}{0.8} & \multirow[b]{2}{*}{0} & \multirow[b]{2}{*}{0.2} & \multirow[b]{2}{*}{0.5} & \multirow[b]{2}{*}{0.8} \\
\hline & & & & & & & & & 0.8 & 0 & & & & & & & \\
\hline \multirow{4}{*}{0} & 0 & 1.05 & 1.05 & 1.05 & 1.05 & 1.92 & 1.92 & 1.93 & 1.92 & 3.72 & 3.72 & 3.70 & 3.72 & 5.49 & 5.49 & 5.50 & 5.50 \\
\hline & 0.2 & 1.08 & 1.08 & 1.08 & 1.08 & 1.95 & 1.97 & 1.97 & 1.97 & 3.75 & 3.76 & 3.77 & 3.73 & 5.50 & 5.51 & 5.52 & 5.50 \\
\hline & 0.5 & 1.34 & 1.34 & 1.34 & 1.35 & 2.19 & 2.21 & 2.22 & 2.21 & 3.88 & 3.88 & 3.90 & 3.90 & 5.61 & 5.58 & 5.62 & 5.58 \\
\hline & 0.8 & 1.84 & 1.84 & 1.84 & 1.84 & 2.64 & 2.62 & 2.63 & 2.65 & 4.21 & 4.19 & 4.18 & 4.19 & 5.77 & 5.77 & 5.75 & 5.80 \\
\hline \multirow{4}{*}{0.2} & 0 & 1.08 & 1.08 & 1.09 & 1.08 & 1.97 & 1.97 & 1.96 & 1.96 & 3.76 & 3.73 & 3.75 & 3.75 & 5.53 & 5.52 & 5.50 & 5.55 \\
\hline & 0.2 & 1.12 & 1.13 & 1.12 & 1.12 & 2.01 & 2.02 & 2.01 & 2.01 & 3.79 & 3.77 & 3.77 & 3.77 & 5.52 & 5.53 & 5.56 & 5.52 \\
\hline & 0.5 & 1.40 & 1.39 & 1.37 & 1.40 & 2.25 & 2.26 & 2.26 & 2.24 & 3.92 & 3.95 & 3.94 & 3.94 & 5.60 & 5.64 & 5.64 & 5.64 \\
\hline & 0.8 & 1.88 & 1.88 & 1.89 & 1.89 & 2.67 & 2.68 & 2.67 & 2.70 & 4.21 & 4.21 & 4.23 & 4.21 & 5.77 & 5.79 & 5.77 & 5.74 \\
\hline \multirow{4}{*}{0.5} & 0 & 1.34 & 1.35 & 1.34 & 1.33 & 2.21 & 2.22 & 2.22 & 2.19 & 3.91 & 3.91 & 3.89 & 3.90 & 5.62 & 5.63 & 5.60 & 5.60 \\
\hline & 0.2 & 1.38 & 1.40 & 1.39 & 1.39 & 2.27 & 2.26 & 2.25 & 2.26 & 3.93 & 3.96 & 3.92 & 3.91 & 5.64 & 5.64 & 5.64 & 5.64 \\
\hline & 0.5 & 1.67 & 1.68 & 1.67 & 1.68 & 2.50 & 2.47 & 2.49 & 2.51 & 4.08 & 4.11 & 4.08 & 4.09 & 5.69 & 5.73 & 5.72 & 5.70 \\
\hline & 0.8 & 2.17 & 2.17 & 2.14 & 2.16 & 2.89 & 2.90 & 2.88 & 2.89 & 4.35 & 4.35 & 4.34 & 4.36 & 5.86 & 5.85 & 5.86 & 5.86 \\
\hline \multirow{4}{*}{0.8} & 0 & 1.87 & 1.86 & 1.88 & 1.87 & 2.65 & 2.65 & 2.65 & 2.64 & 4.16 & 4.19 & 4.18 & 4.19 & 5.77 & 5.77 & 5.75 & 5.74 \\
\hline & 0.2 & 1.93 & 1.92 & 1.92 & 1.91 & 2.69 & 2.68 & 2.69 & 2.69 & 4.21 & 4.25 & 4.21 & 4.21 & 5.79 & 5.80 & 5.78 & 5.80 \\
\hline & 0.5 & 2.17 & 2.17 & 2.18 & 2.18 & 2.90 & 2.91 & 2.88 & 2.90 & 4.33 & 4.35 & 4.37 & 4.36 & 5.86 & 5.86 & 5.90 & 5.86 \\
\hline & 0.8 & 2.62 & 2.60 & 2.60 & 2.62 & 3.28 & 3.28 & 3.26 & 3.27 & 4.63 & 4.63 & 4.61 & 4.63 & 6.01 & 6.02 & 6.01 & 6.02 \\
\hline
\end{tabular}

Note: ICC $=$ Intraclass Correlation Coefficient. 
Table 5 cont.

Average Design Effect values for the L2 predictor: Poisson Distribution conditions $(\mathrm{m}=20, \mathrm{k}=25)$

\begin{tabular}{|c|c|c|c|c|c|c|c|c|c|c|c|c|c|c|c|c|c|}
\hline \multirow{4}{*}{$\begin{array}{c}\text { L1 } \\
\text { Effect }\end{array}$} & \multirow{4}{*}{$\begin{array}{c}\text { Interaction } \\
\text { Effect }\end{array}$} & \multicolumn{16}{|c|}{ ICC Values } \\
\hline & & \multicolumn{4}{|c|}{0} & \multirow{2}{*}{\multicolumn{3}{|c|}{0.1}} & & \multicolumn{4}{|c|}{0.3} & \multicolumn{4}{|c|}{0.5} \\
\hline & & \multicolumn{9}{|c|}{ L2 Effect } & & & & \multirow[b]{2}{*}{0} & \multirow[b]{2}{*}{0.2} & \multirow[b]{2}{*}{0.5} & \multirow[b]{2}{*}{0.8} \\
\hline & & 0 & 0.2 & 0.5 & 0.8 & 0 & 0.2 & 0.5 & 0.8 & 0 & 0.2 & 0.5 & 0.8 & & & & \\
\hline \multirow{4}{*}{0} & 0 & 1.07 & 1.08 & 1.08 & 1.08 & 2.69 & 2.71 & 2.74 & 2.73 & 6.26 & 6.26 & 6.28 & 6.26 & 9.92 & 9.94 & 10.00 & 10.01 \\
\hline & 0.2 & 1.10 & 1.10 & 1.11 & 1.12 & 2.75 & 2.78 & 2.78 & 2.77 & 6.35 & 6.27 & 6.27 & 6.33 & 10.04 & 9.92 & 9.97 & 9.95 \\
\hline & 0.5 & 1.32 & 1.31 & 1.30 & 1.31 & 2.96 & 2.97 & 3.01 & 3.06 & 6.41 & 6.47 & 6.48 & 6.50 & 10.01 & 9.99 & 10.04 & 10.04 \\
\hline & 0.8 & 1.78 & 1.79 & 1.79 & 1.72 & 3.36 & 3.41 & 3.41 & 3.40 & 6.71 & 6.74 & 6.67 & 6.75 & 10.12 & 10.25 & 10.13 & 10.13 \\
\hline \multirow{4}{*}{0.2} & 0 & 1.10 & 1.10 & 1.11 & 1.11 & 2.71 & 2.76 & 2.75 & 2.78 & 6.29 & 6.37 & 6.24 & 6.28 & 9.96 & 9.90 & 9.92 & 9.96 \\
\hline & 0.2 & 1.14 & 1.13 & 1.15 & 1.15 & 2.82 & 2.85 & 2.80 & 2.82 & 6.34 & 6.29 & 6.35 & 6.34 & 9.98 & 9.94 & 9.93 & 9.96 \\
\hline & 0.5 & 1.36 & 1.35 & 1.34 & 1.36 & 2.97 & 3.03 & 3.03 & 3.02 & 6.52 & 6.42 & 6.51 & 6.52 & 9.98 & 10.03 & 10.08 & 9.99 \\
\hline & 0.8 & 1.84 & 1.82 & 1.81 & 1.81 & 3.43 & 3.42 & 3.41 & 3.42 & 6.76 & 6.77 & 6.78 & 6.76 & 10.08 & 10.13 & 10.32 & 10.20 \\
\hline \multirow{4}{*}{0.5} & 0 & 1.32 & 1.30 & 1.30 & 1.32 & 2.98 & 3.02 & 3.01 & 3.00 & 6.48 & 6.51 & 6.47 & 6.55 & 10.16 & 10.09 & 10.06 & 10.05 \\
\hline & 0.2 & 1.36 & 1.36 & 1.37 & 1.38 & 3.05 & 3.09 & 3.10 & 3.00 & 6.53 & 6.47 & 6.48 & 6.54 & 10.00 & 10.02 & 10.05 & 10.06 \\
\hline & 0.5 & 1.62 & 1.63 & 1.61 & 1.62 & 3.32 & 3.26 & 3.28 & 3.25 & 6.64 & 6.57 & 6.60 & 6.59 & 10.07 & 10.16 & 10.02 & 10.14 \\
\hline & 0.8 & 2.08 & 2.06 & 2.12 & 2.09 & 3.61 & 3.67 & 3.64 & 3.65 & 6.76 & 6.86 & 6.85 & 6.85 & 10.29 & 10.30 & 10.26 & 10.35 \\
\hline \multirow{4}{*}{0.8} & 0 & 1.83 & 1.83 & 1.84 & 1.81 & 3.43 & 3.41 & 3.43 & 3.50 & 6.76 & 6.78 & 6.73 & 6.68 & 10.10 & 10.20 & 10.24 & 10.27 \\
\hline & 0.2 & 1.88 & 1.87 & 1.90 & 1.92 & 3.47 & 3.54 & 3.48 & 3.49 & 6.74 & 6.76 & 6.71 & 6.80 & 10.18 & 10.32 & 10.29 & 10.26 \\
\hline & 0.5 & 2.13 & 2.11 & 2.14 & 2.09 & 3.72 & 3.66 & 3.71 & 3.68 & 6.89 & 6.85 & 6.94 & 6.88 & 10.34 & 10.25 & 10.24 & 10.27 \\
\hline & 0.8 & 2.56 & 2.56 & 2.61 & 2.64 & 4.07 & 4.13 & 4.03 & 4.13 & 7.20 & 7.18 & 7.09 & 7.17 & 10.42 & 10.39 & 10.43 & 10.37 \\
\hline
\end{tabular}

Note: ICC $=$ Intraclass Correlation Coefficient. 
Table 5 cont.

Average Design Effect values for the L2 predictor: Poisson Distribution conditions $(\mathrm{m}=20, \mathrm{k}=50)$

\begin{tabular}{|c|c|c|c|c|c|c|c|c|c|c|c|c|c|c|c|c|c|}
\hline \multirow{4}{*}{$\begin{array}{c}\text { L1 } \\
\text { Effect }\end{array}$} & \multirow{4}{*}{$\begin{array}{c}\text { Interaction } \\
\text { Effect }\end{array}$} & \multicolumn{16}{|c|}{ ICC Values } \\
\hline & & \multicolumn{4}{|c|}{0} & \multirow{2}{*}{\multicolumn{3}{|c|}{0.1}} & & \multicolumn{4}{|c|}{0.3} & \multicolumn{4}{|c|}{0.5} \\
\hline & & \multicolumn{9}{|c|}{ L2 Effect } & & & & \multirow[b]{2}{*}{0} & \multirow[b]{2}{*}{0.2} & \multirow[b]{2}{*}{0.5} & \multirow[b]{2}{*}{0.8} \\
\hline & & 0 & 0.2 & 0.5 & 0.8 & 0 & 0.2 & 0.5 & 0.8 & 0 & 0.2 & 0.5 & 0.8 & & & & \\
\hline \multirow{4}{*}{0} & 0 & 1.06 & 1.06 & 1.05 & 1.06 & 2.83 & 2.81 & 2.84 & 2.85 & 6.56 & 6.53 & 6.52 & 6.54 & 10.25 & 10.19 & 10.21 & 10.26 \\
\hline & 0.2 & 1.09 & 1.09 & 1.10 & 1.09 & 2.87 & 2.88 & 2.86 & 2.89 & 6.55 & 6.51 & 6.55 & 6.57 & 10.19 & 10.27 & 10.34 & 10.26 \\
\hline & 0.5 & 1.34 & 1.33 & 1.34 & 1.33 & 3.11 & 3.12 & 3.12 & 3.12 & 6.72 & 6.67 & 6.69 & 6.70 & 10.32 & 10.34 & 10.26 & 10.28 \\
\hline & 0.8 & 1.86 & 1.85 & 1.88 & 1.86 & 3.55 & 3.58 & 3.57 & 3.53 & 6.96 & 6.97 & 6.99 & 6.98 & 10.50 & 10.51 & 10.47 & 10.48 \\
\hline \multirow{4}{*}{0.2} & 0 & 1.10 & 1.09 & 1.10 & 1.09 & 2.87 & 2.89 & 2.86 & 2.87 & 6.57 & 6.55 & 6.51 & 6.54 & 10.23 & 10.21 & 10.28 & 10.28 \\
\hline & 0.2 & 1.13 & 1.13 & 1.13 & 1.13 & 2.90 & 2.92 & 2.94 & 2.96 & 6.54 & 6.51 & 6.58 & 6.55 & 10.36 & 10.28 & 10.26 & 10.27 \\
\hline & 0.5 & 1.40 & 1.39 & 1.39 & 1.39 & 3.16 & 3.14 & 3.16 & 3.16 & 6.74 & 6.76 & 6.75 & 6.72 & 10.32 & 10.32 & 10.31 & 10.35 \\
\hline & 0.8 & 1.91 & 1.89 & 1.91 & 1.89 & 3.59 & 3.64 & 3.64 & 3.63 & 7.02 & 7.03 & 7.03 & 7.00 & 10.53 & 10.52 & 10.52 & 10.50 \\
\hline \multirow{4}{*}{0.5} & 0 & 1.34 & 1.35 & 1.35 & 1.35 & 3.14 & 3.14 & 3.12 & 3.13 & 6.67 & 6.69 & 6.70 & 6.73 & 10.39 & 10.40 & 10.34 & 10.30 \\
\hline & 0.2 & 1.39 & 1.40 & 1.40 & 1.39 & 3.17 & 3.16 & 3.18 & 3.19 & 6.77 & 6.67 & 6.74 & 6.70 & 10.37 & 10.36 & 10.35 & 10.42 \\
\hline & 0.5 & 1.69 & 1.70 & 1.68 & 1.71 & 3.41 & 3.42 & 3.42 & 3.41 & 6.85 & 6.94 & 6.81 & 6.93 & 10.40 & 10.45 & 10.51 & 10.38 \\
\hline & 0.8 & 2.22 & 2.22 & 2.26 & 2.19 & 3.85 & 3.84 & 3.85 & 3.85 & 7.15 & 7.13 & 7.16 & 7.14 & 10.62 & 10.56 & 10.61 & 10.58 \\
\hline \multirow{4}{*}{0.8} & 0 & 1.88 & 1.90 & 1.89 & 1.89 & 3.61 & 3.61 & 3.60 & 3.58 & 7.06 & 7.02 & 7.03 & 7.01 & 10.51 & 10.54 & 10.45 & 10.54 \\
\hline & 0.2 & 1.96 & 1.97 & 1.96 & 1.95 & 3.65 & 3.65 & 3.63 & 3.64 & 6.97 & 7.02 & 7.03 & 7.03 & 10.50 & 10.47 & 10.52 & 10.52 \\
\hline & 0.5 & 2.28 & 2.24 & 2.23 & 2.24 & 3.87 & 3.89 & 3.83 & 3.87 & 7.12 & 7.18 & 7.17 & 7.22 & 10.53 & 10.61 & 10.59 & 10.54 \\
\hline & 0.8 & 2.68 & 2.71 & 2.74 & 2.71 & 4.32 & 4.28 & 4.26 & 4.26 & 7.46 & 7.40 & 7.40 & 7.44 & 10.76 & 10.71 & 10.71 & 10.68 \\
\hline
\end{tabular}

Note: ICC $=$ Intraclass Correlation Coefficient. 
Table 5 cont.

Average Design Effect values for the L2 predictor: Poisson Distribution conditions $(\mathrm{m}=20, \mathrm{k}=75)$

\begin{tabular}{|c|c|c|c|c|c|c|c|c|c|c|c|c|c|c|c|c|c|}
\hline \multirow{4}{*}{$\begin{array}{c}\text { L1 } \\
\text { Effect }\end{array}$} & \multirow{4}{*}{$\begin{array}{l}\text { Interaction } \\
\text { Effect }\end{array}$} & \multicolumn{16}{|c|}{ ICC Values } \\
\hline & & \multicolumn{4}{|c|}{0} & \multirow{2}{*}{\multicolumn{3}{|c|}{0.1}} & & \multicolumn{4}{|c|}{0.3} & \multicolumn{4}{|c|}{0.5} \\
\hline & & & & & & & & & \multicolumn{2}{|c|}{ L2 Effect } & & & & & & & \\
\hline & & 0 & 0.2 & 0.5 & 0.8 & 0 & 0.2 & 0.5 & 0.8 & 0 & 0.2 & 0.5 & 0.8 & 0 & 0.2 & 0.5 & 0.8 \\
\hline \multirow{4}{*}{0} & 0 & 1.05 & 1.05 & 1.06 & 1.06 & 2.88 & 2.85 & 2.86 & 2.85 & 6.53 & 6.56 & 6.59 & 6.58 & 10.28 & 10.30 & 10.36 & 10.35 \\
\hline & 0.2 & 1.09 & 1.08 & 1.09 & 1.08 & 2.90 & 2.93 & 2.89 & 2.91 & 6.61 & 6.57 & 6.56 & 6.58 & 10.32 & 10.34 & 10.31 & 10.37 \\
\hline & 0.5 & 1.35 & 1.34 & 1.36 & 1.34 & 3.18 & 3.17 & 3.16 & 3.19 & 6.82 & 6.80 & 6.74 & 6.78 & 10.44 & 10.43 & 10.42 & 10.48 \\
\hline & 0.8 & 1.91 & 1.87 & 1.89 & 1.87 & 3.62 & 3.61 & 3.60 & 3.60 & 7.08 & 7.04 & 7.06 & 7.08 & 10.60 & 10.60 & 10.63 & 10.56 \\
\hline \multirow{4}{*}{0.2} & 0 & 1.09 & 1.08 & 1.09 & 1.08 & 2.93 & 2.90 & 2.90 & 2.89 & 6.59 & 6.63 & 6.61 & 6.58 & 10.33 & 10.37 & 10.37 & 10.31 \\
\hline & 0.2 & 1.12 & 1.13 & 1.13 & 1.12 & 2.94 & 2.95 & 2.96 & 2.95 & 6.63 & 6.64 & 6.66 & 6.67 & 10.39 & 10.40 & 10.37 & 10.35 \\
\hline & 0.5 & 1.39 & 1.40 & 1.39 & 1.41 & 3.22 & 3.21 & 3.22 & 3.21 & 6.78 & 6.82 & 6.81 & 6.79 & 10.43 & 10.41 & 10.44 & 10.47 \\
\hline & 0.8 & 1.94 & 1.97 & 1.98 & 1.95 & 3.68 & 3.69 & 3.69 & 3.68 & 7.07 & 7.11 & 7.10 & 7.07 & 10.63 & 10.52 & 10.58 & 10.67 \\
\hline \multirow{4}{*}{0.5} & 0 & 1.37 & 1.36 & 1.35 & 1.35 & 3.20 & 3.17 & 3.18 & 3.18 & 6.82 & 6.81 & 6.78 & 6.78 & 10.46 & 10.44 & 10.47 & 10.37 \\
\hline & 0.2 & 1.40 & 1.42 & 1.41 & 1.42 & 3.24 & 3.21 & 3.22 & 3.19 & 6.83 & 6.82 & 6.78 & 6.80 & 10.47 & 10.45 & 10.41 & 10.48 \\
\hline & 0.5 & 1.72 & 1.70 & 1.69 & 1.72 & 3.45 & 3.47 & 3.46 & 3.48 & 6.92 & 6.99 & 6.89 & 6.96 & 10.52 & 10.52 & 10.54 & 10.57 \\
\hline & 0.8 & 2.24 & 2.25 & 2.27 & 2.22 & 3.95 & 3.87 & 3.89 & 3.92 & 7.19 & 7.29 & 7.25 & 7.28 & 10.76 & 10.67 & 10.69 & 10.73 \\
\hline \multirow{4}{*}{0.8} & 0 & 1.92 & 1.93 & 1.93 & 1.92 & 3.63 & 3.64 & 3.67 & 3.63 & 7.03 & 7.09 & 7.10 & 7.08 & 10.57 & 10.62 & 10.62 & 10.60 \\
\hline & 0.2 & 1.99 & 1.99 & 1.98 & 1.97 & 3.68 & 3.67 & 3.69 & 3.66 & 7.09 & 7.11 & 7.13 & 7.13 & 10.63 & 10.63 & 10.67 & 10.58 \\
\hline & 0.5 & 2.25 & 2.28 & 2.26 & 2.26 & 3.94 & 3.92 & 3.91 & 3.90 & 7.23 & 7.28 & 7.31 & 7.25 & 10.70 & 10.66 & 10.78 & 10.67 \\
\hline & 0.8 & 2.76 & 2.74 & 2.75 & 2.78 & 4.32 & 4.31 & 4.35 & 4.31 & 7.49 & 7.52 & 7.52 & 7.58 & 10.85 & 10.86 & 10.81 & 10.79 \\
\hline
\end{tabular}

Note: ICC = Intraclass Correlation Coefficient. 
Table 5 cont.

Average Design Effect values for the L2 predictor: Poisson Distribution conditions $(\mathrm{m}=20, \mathrm{k}=100)$

\begin{tabular}{|c|c|c|c|c|c|c|c|c|c|c|c|c|c|c|c|c|c|}
\hline \multirow{4}{*}{$\begin{array}{c}\text { L1 } \\
\text { Effect }\end{array}$} & \multirow{4}{*}{$\begin{array}{c}\text { Interaction } \\
\text { Effect }\end{array}$} & \multicolumn{16}{|c|}{ ICC Values } \\
\hline & & \multicolumn{4}{|c|}{0} & \multirow{2}{*}{\multicolumn{3}{|c|}{0.1}} & & \multicolumn{4}{|c|}{0.3} & \multicolumn{4}{|c|}{0.5} \\
\hline & & \multicolumn{9}{|c|}{ L2 Effect } & & & & \multirow[b]{2}{*}{0} & \multirow[b]{2}{*}{0.2} & \multirow[b]{2}{*}{0.5} & \multirow[b]{2}{*}{0.8} \\
\hline & & 0 & 0.2 & 0.5 & 0.8 & 0 & 0.2 & 0.5 & 0.8 & 0 & 0.2 & 0.5 & 0.8 & & & & \\
\hline \multirow{4}{*}{0} & 0 & 1.04 & 1.04 & 1.04 & 1.05 & 2.89 & 2.90 & 2.88 & 2.86 & 6.64 & 6.57 & 6.60 & 6.65 & 10.34 & 10.37 & 10.38 & 10.36 \\
\hline & 0.2 & 1.09 & 1.08 & 1.08 & 1.08 & 2.92 & 2.93 & 2.92 & 2.94 & 6.64 & 6.64 & 6.67 & 6.63 & 10.41 & 10.45 & 10.42 & 10.43 \\
\hline & 0.5 & 1.35 & 1.35 & 1.35 & 1.35 & 3.17 & 3.18 & 3.20 & 3.17 & 6.78 & 6.81 & 6.81 & 6.83 & 10.47 & 10.50 & 10.55 & 10.45 \\
\hline & 0.8 & 1.93 & 1.92 & 1.91 & 1.94 & 3.63 & 3.65 & 3.65 & 3.65 & 7.14 & 7.11 & 7.14 & 7.15 & 10.69 & 10.64 & 10.66 & 10.63 \\
\hline \multirow{4}{*}{0.2} & 0 & 1.09 & 1.08 & 1.08 & 1.08 & 2.92 & 2.94 & 2.94 & 2.94 & 6.68 & 6.65 & 6.64 & 6.65 & 10.36 & 10.39 & 10.42 & 10.34 \\
\hline & 0.2 & 1.12 & 1.12 & 1.11 & 1.13 & 2.99 & 3.00 & 2.99 & 2.98 & 6.67 & 6.70 & 6.70 & 6.70 & 10.42 & 10.42 & 10.40 & 10.41 \\
\hline & 0.5 & 1.42 & 1.42 & 1.41 & 1.41 & 3.24 & 3.24 & 3.24 & 3.21 & 6.84 & 6.91 & 6.83 & 6.84 & 10.51 & 10.47 & 10.46 & 10.52 \\
\hline & 0.8 & 2.00 & 1.97 & 1.97 & 1.98 & 3.68 & 3.69 & 3.72 & 3.67 & 7.12 & 7.15 & 7.12 & 7.16 & 10.68 & 10.64 & 10.68 & 10.68 \\
\hline \multirow{4}{*}{0.5} & 0 & 1.36 & 1.35 & 1.37 & 1.37 & 3.19 & 3.19 & 3.18 & 3.20 & 6.83 & 6.79 & 6.83 & 6.83 & 10.46 & 10.50 & 10.51 & 10.47 \\
\hline & 0.2 & 1.41 & 1.42 & 1.42 & 1.42 & 3.24 & 3.24 & 3.24 & 3.23 & 6.88 & 6.90 & 6.84 & 6.83 & 10.55 & 10.51 & 10.56 & 10.50 \\
\hline & 0.5 & 1.72 & 1.73 & 1.74 & 1.71 & 3.48 & 3.49 & 3.50 & 3.49 & 6.98 & 6.99 & 6.99 & 7.02 & 10.62 & 10.58 & 10.62 & 10.56 \\
\hline & 0.8 & 2.26 & 2.25 & 2.28 & 2.28 & 3.94 & 3.96 & 3.91 & 3.93 & 7.25 & 7.26 & 7.30 & 7.26 & 10.75 & 10.71 & 10.72 & 10.72 \\
\hline \multirow{4}{*}{0.8} & 0 & 1.94 & 1.94 & 1.93 & 1.92 & 3.66 & 3.66 & 3.66 & 3.67 & 7.13 & 7.10 & 7.13 & 7.15 & 10.59 & 10.64 & 10.61 & 10.64 \\
\hline & 0.2 & 2.00 & 1.99 & 1.98 & 1.98 & 3.70 & 3.70 & 3.70 & 3.74 & 7.15 & 7.09 & 7.18 & 7.14 & 10.66 & 10.70 & 10.63 & 10.63 \\
\hline & 0.5 & 2.29 & 2.27 & 2.29 & 2.28 & 3.95 & 3.97 & 3.94 & 3.96 & 7.31 & 7.30 & 7.28 & 7.28 & 10.76 & 10.71 & 10.77 & 10.73 \\
\hline & 0.8 & 2.77 & 2.80 & 2.76 & 2.78 & 4.38 & 4.36 & 4.37 & 4.34 & 7.56 & 7.60 & 7.59 & 7.58 & 10.91 & 10.88 & 10.87 & 10.89 \\
\hline
\end{tabular}

Note: ICC $=$ Intraclass Correlation Coefficient. 
Table 6.

Average Design Effect values for the cross-level interaction: Uniform Distribution conditions $(\mathrm{m}=5, \mathrm{k}=25)$

\begin{tabular}{|c|c|c|c|c|c|c|c|c|c|c|c|c|c|c|c|c|c|}
\hline \multirow{4}{*}{$\begin{array}{c}\text { L1 } \\
\text { Effect }\end{array}$} & \multirow{4}{*}{$\begin{array}{c}\text { Interaction } \\
\text { Effect }\end{array}$} & \multicolumn{16}{|c|}{ ICC Values } \\
\hline & & \multicolumn{4}{|c|}{0} & \multicolumn{4}{|c|}{0.1} & \multicolumn{4}{|c|}{0.3} & \multicolumn{4}{|c|}{0.5} \\
\hline & & & & & & \multicolumn{8}{|c|}{ L2 Effect } & & & & \\
\hline & & 0 & 0.2 & 0.5 & 0.8 & 0 & 0.2 & 0.5 & 0.8 & 0 & 0.2 & 0.5 & 0.8 & 0 & 0.2 & 0.5 & 0.8 \\
\hline \multirow{4}{*}{0} & 0 & 1.33 & 1.31 & 1.31 & 1.30 & 1.22 & 1.23 & 1.24 & 1.25 & 0.97 & 0.97 & 0.97 & 0.97 & 0.72 & 0.71 & 0.71 & 0.71 \\
\hline & 0.2 & 1.32 & 1.32 & 1.30 & 1.32 & 1.21 & 1.21 & 1.22 & 1.22 & 0.99 & 0.97 & 0.96 & 0.97 & 0.71 & 0.72 & 0.71 & 0.70 \\
\hline & 0.5 & 1.27 & 1.26 & 1.26 & 1.26 & 1.15 & 1.17 & 1.17 & 1.17 & 0.93 & 0.95 & 0.92 & 0.94 & 0.70 & 0.70 & 0.69 & 0.68 \\
\hline & 0.8 & 1.16 & 1.18 & 1.15 & 1.16 & 1.06 & 1.06 & 1.06 & 1.06 & 0.87 & 0.88 & 0.88 & 0.86 & 0.65 & 0.66 & 0.67 & 0.64 \\
\hline \multirow{4}{*}{0.2} & 0 & 1.31 & 1.31 & 1.32 & 1.31 & 1.21 & 1.21 & 1.20 & 1.21 & 0.97 & 0.98 & 0.97 & 0.96 & 0.71 & 0.70 & 0.70 & 0.71 \\
\hline & 0.2 & 1.29 & 1.30 & 1.29 & 1.29 & 1.19 & 1.20 & 1.20 & 1.21 & 0.97 & 0.95 & 0.96 & 0.95 & 0.71 & 0.71 & 0.71 & 0.70 \\
\hline & 0.5 & 1.23 & 1.25 & 1.24 & 1.26 & 1.16 & 1.15 & 1.15 & 1.15 & 0.92 & 0.93 & 0.92 & 0.92 & 0.68 & 0.69 & 0.70 & 0.69 \\
\hline & 0.8 & 1.15 & 1.16 & 1.14 & 1.15 & 1.07 & 1.06 & 1.05 & 1.06 & 0.86 & 0.88 & 0.86 & 0.85 & 0.65 & 0.65 & 0.65 & 0.65 \\
\hline \multirow{4}{*}{0.5} & 0 & 1.25 & 1.26 & 1.26 & 1.28 & 1.16 & 1.16 & 1.17 & 1.17 & 0.93 & 0.92 & 0.93 & 0.93 & 0.70 & 0.69 & 0.69 & 0.68 \\
\hline & 0.2 & 1.25 & 1.24 & 1.27 & 1.25 & 1.13 & 1.16 & 1.15 & 1.14 & 0.92 & 0.92 & 0.94 & 0.93 & 0.69 & 0.69 & 0.68 & 0.69 \\
\hline & 0.5 & 1.18 & 1.19 & 1.20 & 1.19 & 1.10 & 1.10 & 1.11 & 1.09 & 0.88 & 0.87 & 0.87 & 0.90 & 0.67 & 0.67 & 0.67 & 0.67 \\
\hline & 0.8 & 1.08 & 1.09 & 1.11 & 1.10 & 1.02 & 1.01 & 0.99 & 1.01 & 0.83 & 0.84 & 0.83 & 0.83 & 0.64 & 0.64 & 0.64 & 0.64 \\
\hline \multirow{4}{*}{0.8} & 0 & 1.14 & 1.14 & 1.14 & 1.15 & 1.06 & 1.05 & 1.04 & 1.06 & 0.86 & 0.86 & 0.85 & 0.87 & 0.64 & 0.65 & 0.64 & 0.65 \\
\hline & 0.2 & 1.14 & 1.12 & 1.13 & 1.13 & 1.04 & 1.05 & 1.05 & 1.04 & 0.86 & 0.87 & 0.87 & 0.85 & 0.64 & 0.64 & 0.66 & 0.64 \\
\hline & 0.5 & 1.07 & 1.10 & 1.08 & 1.08 & 0.99 & 1.00 & 1.01 & 1.00 & 0.84 & 0.82 & 0.83 & 0.83 & 0.63 & 0.63 & 0.63 & 0.63 \\
\hline & 0.8 & 1.01 & 1.00 & 1.01 & 1.01 & 0.94 & 0.95 & 0.94 & 0.94 & 0.78 & 0.77 & 0.78 & 0.77 & 0.60 & 0.60 & 0.60 & 0.61 \\
\hline
\end{tabular}

Note: ICC $=$ Intraclass Correlation Coefficient. 
Table 6 cont.

Average Design Effect values for the cross-level interaction: Uniform Distribution conditions $(\mathrm{m}=5, \mathrm{k}=50)$

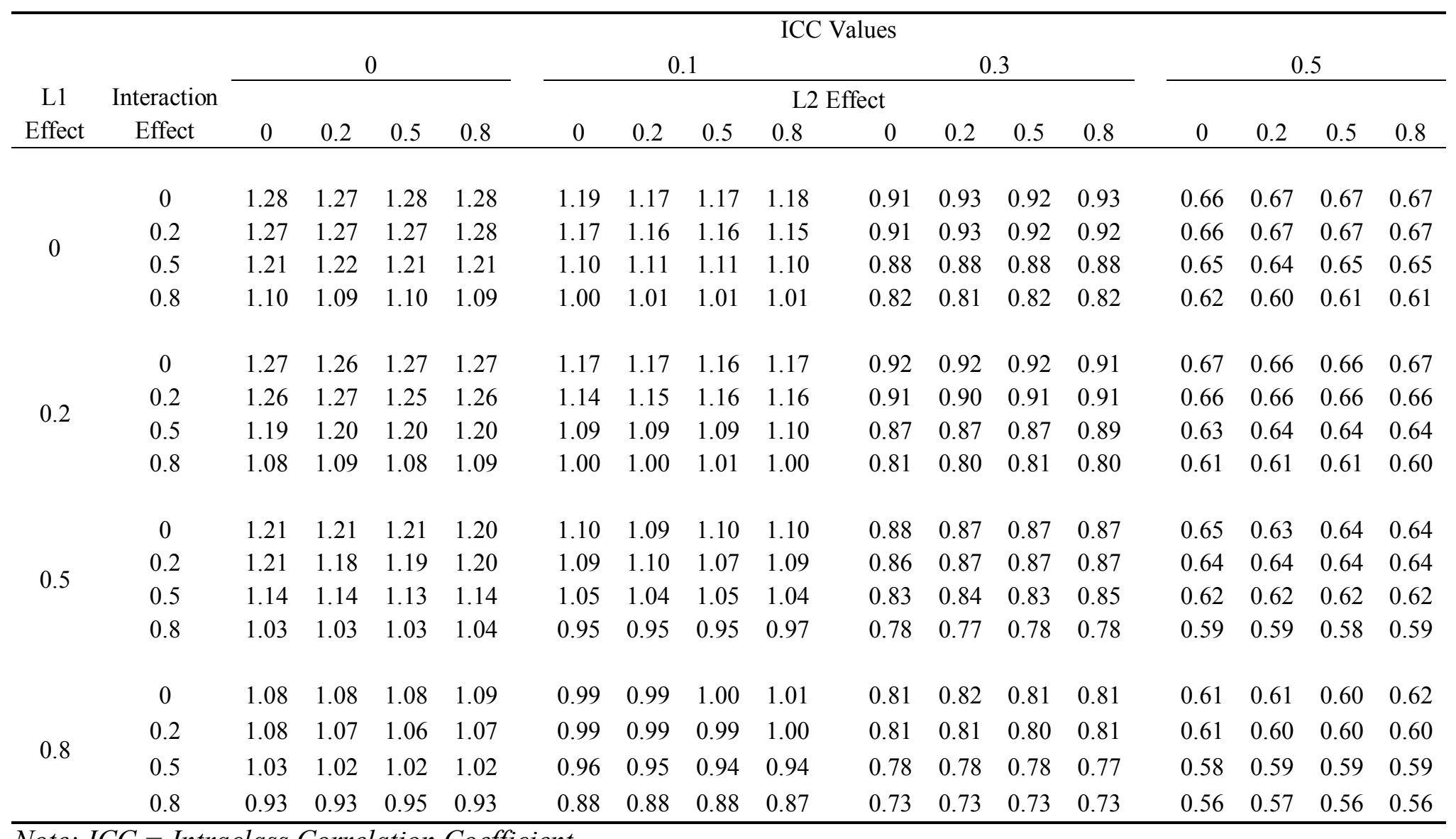

Note: ICC $=$ Intraclass Correlation Coefficient. 


\section{Table 6 cont}

Average Design Effect values for the cross-level interaction: Uniform Distribution conditions $(\mathrm{m}=5, \mathrm{k}=75)$

\begin{tabular}{|c|c|c|c|c|c|c|c|c|c|c|c|c|c|c|c|c|c|}
\hline \multirow{4}{*}{$\begin{array}{c}\text { L1 } \\
\text { Effect }\end{array}$} & \multirow{4}{*}{$\begin{array}{c}\text { Interaction } \\
\text { Effect }\end{array}$} & \multicolumn{16}{|c|}{ ICC Values } \\
\hline & & \multicolumn{4}{|c|}{0} & \multicolumn{4}{|c|}{0.1} & \multicolumn{4}{|c|}{0.3} & \multicolumn{4}{|c|}{0.5} \\
\hline & & \multicolumn{12}{|c|}{ L2 Effect } & & & & \\
\hline & & 0 & 0.2 & 0.5 & 0.8 & 0 & 0.2 & 0.5 & 0.8 & 0 & 0.2 & 0.5 & 0.8 & 0 & 0.2 & 0.5 & 0.8 \\
\hline \multirow{4}{*}{0} & 0 & 1.27 & 1.27 & 1.27 & 1.27 & 1.17 & 1.16 & 1.16 & 1.16 & 0.91 & 0.91 & 0.91 & 0.91 & 0.66 & 0.66 & 0.66 & 0.66 \\
\hline & 0.2 & 1.25 & 1.26 & 1.26 & 1.27 & 1.15 & 1.15 & 1.14 & 1.15 & 0.90 & 0.90 & 0.91 & 0.90 & 0.65 & 0.65 & 0.65 & 0.65 \\
\hline & 0.5 & 1.20 & 1.20 & 1.20 & 1.19 & 1.09 & 1.08 & 1.09 & 1.08 & 0.86 & 0.86 & 0.86 & 0.86 & 0.63 & 0.64 & 0.63 & 0.63 \\
\hline & 0.8 & 1.07 & 1.08 & 1.08 & 1.08 & 0.99 & 0.99 & 0.99 & 0.99 & 0.80 & 0.81 & 0.80 & 0.80 & 0.59 & 0.60 & 0.59 & 0.60 \\
\hline \multirow{4}{*}{0.2} & 0 & 1.26 & 1.24 & 1.26 & 1.26 & 1.15 & 1.15 & 1.15 & 1.15 & 0.90 & 0.90 & 0.90 & 0.90 & 0.64 & 0.65 & 0.65 & 0.65 \\
\hline & 0.2 & 1.25 & 1.24 & 1.24 & 1.25 & 1.13 & 1.13 & 1.14 & 1.14 & 0.89 & 0.90 & 0.89 & 0.90 & 0.64 & 0.65 & 0.65 & 0.65 \\
\hline & 0.5 & 1.18 & 1.19 & 1.17 & 1.18 & 1.07 & 1.07 & 1.06 & 1.07 & 0.85 & 0.86 & 0.86 & 0.86 & 0.62 & 0.62 & 0.62 & 0.62 \\
\hline & 0.8 & 1.06 & 1.06 & 1.07 & 1.07 & 0.98 & 0.97 & 0.97 & 0.98 & 0.79 & 0.79 & 0.79 & 0.80 & 0.59 & 0.59 & 0.59 & 0.59 \\
\hline \multirow{4}{*}{0.5} & 0 & 1.20 & 1.19 & 1.19 & 1.20 & 1.08 & 1.08 & 1.09 & 1.08 & 0.87 & 0.86 & 0.87 & 0.87 & 0.63 & 0.63 & 0.63 & 0.63 \\
\hline & 0.2 & 1.18 & 1.18 & 1.18 & 1.18 & 1.07 & 1.07 & 1.07 & 1.08 & 0.85 & 0.86 & 0.85 & 0.85 & 0.63 & 0.63 & 0.62 & 0.63 \\
\hline & 0.5 & 1.12 & 1.11 & 1.12 & 1.12 & 1.02 & 1.02 & 1.02 & 1.02 & 0.82 & 0.82 & 0.82 & 0.82 & 0.61 & 0.60 & 0.61 & 0.61 \\
\hline & 0.8 & 1.01 & 1.01 & 1.01 & 1.01 & 0.94 & 0.93 & 0.92 & 0.94 & 0.76 & 0.77 & 0.76 & 0.77 & 0.57 & 0.57 & 0.57 & 0.58 \\
\hline \multirow{4}{*}{0.8} & 0 & 1.07 & 1.07 & 1.08 & 1.07 & 0.98 & 0.97 & 0.98 & 0.99 & 0.80 & 0.79 & 0.80 & 0.79 & 0.59 & 0.60 & 0.59 & 0.59 \\
\hline & 0.2 & 1.05 & 1.05 & 1.06 & 1.05 & 0.98 & 0.97 & 0.97 & 0.97 & 0.79 & 0.79 & 0.79 & 0.80 & 0.59 & 0.59 & 0.59 & 0.60 \\
\hline & 0.5 & 1.00 & 1.01 & 1.00 & 1.00 & 0.93 & 0.92 & 0.93 & 0.94 & 0.76 & 0.76 & 0.76 & 0.76 & 0.57 & 0.57 & 0.57 & 0.57 \\
\hline & 0.8 & 0.92 & 0.92 & 0.92 & 0.92 & 0.86 & 0.85 & 0.85 & 0.85 & 0.72 & 0.71 & 0.71 & 0.71 & 0.54 & 0.54 & 0.55 & 0.55 \\
\hline
\end{tabular}

Note: ICC = Intraclass Correlation Coefficient. 


\section{Table 6 cont}

Average Design Effect values for the cross-level interaction: Uniform Distribution conditions $(\mathrm{m}=5, \mathrm{k}=100)$

\begin{tabular}{|c|c|c|c|c|c|c|c|c|c|c|c|c|c|c|c|c|c|}
\hline \multirow{4}{*}{$\begin{array}{c}\text { L1 } \\
\text { Effect }\end{array}$} & \multirow{4}{*}{$\begin{array}{c}\text { Interaction } \\
\text { Effect }\end{array}$} & \multicolumn{16}{|c|}{ ICC Values } \\
\hline & & \multicolumn{4}{|c|}{0} & \multicolumn{4}{|c|}{0.1} & \multicolumn{4}{|c|}{0.3} & \multicolumn{4}{|c|}{0.5} \\
\hline & & \multicolumn{12}{|c|}{ L2 Effect } & & & & \\
\hline & & 0 & 0.2 & 0.5 & 0.8 & 0 & 0.2 & 0.5 & 0.8 & 0 & 0.2 & 0.5 & 0.8 & 0 & 0.2 & 0.5 & 0.8 \\
\hline \multirow{4}{*}{0} & 0 & 1.26 & 1.26 & 1.25 & 1.26 & 1.15 & 1.15 & 1.15 & 1.15 & 0.91 & 0.90 & 0.90 & 0.90 & 0.64 & 0.65 & 0.64 & 0.65 \\
\hline & 0.2 & 1.25 & 1.25 & 1.25 & 1.25 & 1.13 & 1.14 & 1.14 & 1.14 & 0.89 & 0.89 & 0.89 & 0.90 & 0.65 & 0.65 & 0.64 & 0.64 \\
\hline & 0.5 & 1.18 & 1.18 & 1.18 & 1.19 & 1.08 & 1.08 & 1.09 & 1.08 & 0.86 & 0.85 & 0.85 & 0.85 & 0.62 & 0.63 & 0.62 & 0.62 \\
\hline & 0.8 & 1.07 & 1.06 & 1.06 & 1.07 & 0.98 & 0.98 & 0.98 & 0.97 & 0.79 & 0.78 & 0.78 & 0.79 & 0.59 & 0.59 & 0.59 & 0.59 \\
\hline \multirow{4}{*}{0.2} & 0 & 1.25 & 1.24 & 1.25 & 1.25 & 1.13 & 1.14 & 1.14 & 1.14 & 0.89 & 0.89 & 0.89 & 0.89 & 0.65 & 0.64 & 0.64 & 0.64 \\
\hline & 0.2 & 1.24 & 1.24 & 1.23 & 1.25 & 1.13 & 1.14 & 1.12 & 1.13 & 0.89 & 0.89 & 0.89 & 0.88 & 0.64 & 0.64 & 0.64 & 0.64 \\
\hline & 0.5 & 1.17 & 1.18 & 1.17 & 1.18 & 1.07 & 1.07 & 1.07 & 1.08 & 0.84 & 0.84 & 0.85 & 0.84 & 0.62 & 0.61 & 0.61 & 0.62 \\
\hline & 0.8 & 1.06 & 1.06 & 1.05 & 1.06 & 0.97 & 0.97 & 0.97 & 0.97 & 0.79 & 0.78 & 0.78 & 0.78 & 0.59 & 0.59 & 0.58 & 0.59 \\
\hline \multirow{4}{*}{0.5} & 0 & 1.18 & 1.18 & 1.18 & 1.17 & 1.07 & 1.08 & 1.07 & 1.08 & 0.86 & 0.85 & 0.86 & 0.84 & 0.62 & 0.62 & 0.62 & 0.62 \\
\hline & 0.2 & 1.16 & 1.17 & 1.16 & 1.17 & 1.07 & 1.07 & 1.06 & 1.07 & 0.83 & 0.85 & 0.84 & 0.84 & 0.61 & 0.62 & 0.62 & 0.62 \\
\hline & 0.5 & 1.10 & 1.11 & 1.11 & 1.11 & 1.01 & 1.01 & 1.01 & 1.01 & 0.81 & 0.82 & 0.81 & 0.82 & 0.60 & 0.60 & 0.60 & 0.60 \\
\hline & 0.8 & 1.01 & 1.00 & 1.00 & 1.00 & 0.92 & 0.92 & 0.92 & 0.92 & 0.76 & 0.76 & 0.75 & 0.75 & 0.56 & 0.57 & 0.57 & 0.57 \\
\hline \multirow{4}{*}{0.8} & 0 & 1.06 & 1.06 & 1.06 & 1.06 & 0.97 & 0.97 & 0.97 & 0.97 & 0.79 & 0.78 & 0.79 & 0.79 & 0.58 & 0.59 & 0.59 & 0.59 \\
\hline & 0.2 & 1.06 & 1.05 & 1.05 & 1.05 & 0.96 & 0.97 & 0.96 & 0.97 & 0.79 & 0.78 & 0.78 & 0.78 & 0.59 & 0.58 & 0.58 & 0.58 \\
\hline & 0.5 & 1.00 & 0.99 & 1.00 & 0.99 & 0.92 & 0.92 & 0.92 & 0.92 & 0.75 & 0.75 & 0.76 & 0.75 & 0.57 & 0.57 & 0.57 & 0.56 \\
\hline & 0.8 & 0.91 & 0.92 & 0.92 & 0.91 & 0.85 & 0.85 & 0.85 & 0.84 & 0.70 & 0.70 & 0.70 & 0.70 & 0.54 & 0.54 & 0.54 & 0.54 \\
\hline
\end{tabular}

Note: ICC = Intraclass Correlation Coefficient. 


\section{Table 6 cont}

Average Design Effect values for the cross-level interaction: Uniform Distribution conditions $(\mathrm{m}=10, \mathrm{k}=25)$

\begin{tabular}{|c|c|c|c|c|c|c|c|c|c|c|c|c|c|c|c|c|c|}
\hline \multirow{4}{*}{$\begin{array}{c}\text { L1 } \\
\text { Effect }\end{array}$} & \multirow{4}{*}{$\begin{array}{c}\text { Interaction } \\
\text { Effect }\end{array}$} & \multicolumn{16}{|c|}{ ICC Values } \\
\hline & & \multicolumn{4}{|c|}{0} & \multicolumn{4}{|c|}{0.1} & \multicolumn{4}{|c|}{0.3} & \multicolumn{4}{|c|}{0.5} \\
\hline & & \multicolumn{12}{|c|}{ L2 Effect } & & & & \\
\hline & & 0 & 0.2 & 0.5 & 0.8 & 0 & 0.2 & 0.5 & 0.8 & 0 & 0.2 & 0.5 & 0.8 & 0 & 0.2 & 0.5 & 0.8 \\
\hline \multirow{4}{*}{0} & 0 & 1.26 & 1.26 & 1.25 & 1.26 & 1.15 & 1.15 & 1.15 & 1.15 & 0.91 & 0.90 & 0.90 & 0.90 & 0.64 & 0.65 & 0.64 & 0.65 \\
\hline & 0.2 & 1.25 & 1.25 & 1.25 & 1.25 & 1.13 & 1.14 & 1.14 & 1.14 & 0.89 & 0.89 & 0.89 & 0.90 & 0.65 & 0.65 & 0.64 & 0.64 \\
\hline & 0.5 & 1.18 & 1.18 & 1.18 & 1.19 & 1.08 & 1.08 & 1.09 & 1.08 & 0.86 & 0.85 & 0.85 & 0.85 & 0.62 & 0.63 & 0.62 & 0.62 \\
\hline & 0.8 & 1.07 & 1.06 & 1.06 & 1.07 & 0.98 & 0.98 & 0.98 & 0.97 & 0.79 & 0.78 & 0.78 & 0.79 & 0.59 & 0.59 & 0.59 & 0.59 \\
\hline \multirow{4}{*}{0.2} & 0 & 1.25 & 1.24 & 1.25 & 1.25 & 1.13 & 1.14 & 1.14 & 1.14 & 0.89 & 0.89 & 0.89 & 0.89 & 0.65 & 0.64 & 0.64 & 0.64 \\
\hline & 0.2 & 1.24 & 1.24 & 1.23 & 1.25 & 1.13 & 1.14 & 1.12 & 1.13 & 0.89 & 0.89 & 0.89 & 0.88 & 0.64 & 0.64 & 0.64 & 0.64 \\
\hline & 0.5 & 1.17 & 1.18 & 1.17 & 1.18 & 1.07 & 1.07 & 1.07 & 1.08 & 0.84 & 0.84 & 0.85 & 0.84 & 0.62 & 0.61 & 0.61 & 0.62 \\
\hline & 0.8 & 1.06 & 1.06 & 1.05 & 1.06 & 0.97 & 0.97 & 0.97 & 0.97 & 0.79 & 0.78 & 0.78 & 0.78 & 0.59 & 0.59 & 0.58 & 0.59 \\
\hline \multirow{4}{*}{0.5} & 0 & 1.18 & 1.18 & 1.18 & 1.17 & 1.07 & 1.08 & 1.07 & 1.08 & 0.86 & 0.85 & 0.86 & 0.84 & 0.62 & 0.62 & 0.62 & 0.62 \\
\hline & 0.2 & 1.16 & 1.17 & 1.16 & 1.17 & 1.07 & 1.07 & 1.06 & 1.07 & 0.83 & 0.85 & 0.84 & 0.84 & 0.61 & 0.62 & 0.62 & 0.62 \\
\hline & 0.5 & 1.10 & 1.11 & 1.11 & 1.11 & 1.01 & 1.01 & 1.01 & 1.01 & 0.81 & 0.82 & 0.81 & 0.82 & 0.60 & 0.60 & 0.60 & 0.60 \\
\hline & 0.8 & 1.01 & 1.00 & 1.00 & 1.00 & 0.92 & 0.92 & 0.92 & 0.92 & 0.76 & 0.76 & 0.75 & 0.75 & 0.56 & 0.57 & 0.57 & 0.57 \\
\hline \multirow{4}{*}{0.8} & 0 & 1.06 & 1.06 & 1.06 & 1.06 & 0.97 & 0.97 & 0.97 & 0.97 & 0.79 & 0.78 & 0.79 & 0.79 & 0.58 & 0.59 & 0.59 & 0.59 \\
\hline & 0.2 & 1.06 & 1.05 & 1.05 & 1.05 & 0.96 & 0.97 & 0.96 & 0.97 & 0.79 & 0.78 & 0.78 & 0.78 & 0.59 & 0.58 & 0.58 & 0.58 \\
\hline & 0.5 & 1.00 & 0.99 & 1.00 & 0.99 & 0.92 & 0.92 & 0.92 & 0.92 & 0.75 & 0.75 & 0.76 & 0.75 & 0.57 & 0.57 & 0.57 & 0.56 \\
\hline & 0.8 & 0.91 & 0.92 & 0.92 & 0.91 & 0.85 & 0.85 & 0.85 & 0.84 & 0.70 & 0.70 & 0.70 & 0.70 & 0.54 & 0.54 & 0.54 & 0.54 \\
\hline
\end{tabular}

Note: ICC = Intraclass Correlation Coefficient. 


\section{Table 6 cont}

Average Design Effect values for the cross-level interaction: Uniform Distribution conditions $(\mathrm{m}=10, \mathrm{k}=50)$

\begin{tabular}{|c|c|c|c|c|c|c|c|c|c|c|c|c|c|c|c|c|c|}
\hline \multirow{4}{*}{$\begin{array}{c}\text { L1 } \\
\text { Effect }\end{array}$} & \multirow{4}{*}{$\begin{array}{c}\text { Interaction } \\
\text { Effect }\end{array}$} & \multicolumn{16}{|c|}{ ICC Values } \\
\hline & & \multicolumn{4}{|c|}{0} & \multicolumn{4}{|c|}{0.1} & \multicolumn{4}{|c|}{0.3} & \multicolumn{4}{|c|}{0.5} \\
\hline & & \multicolumn{12}{|c|}{ L2 Effect } & & & & \\
\hline & & 0 & 0.2 & 0.5 & 0.8 & 0 & 0.2 & 0.5 & 0.8 & 0 & 0.2 & 0.5 & 0.8 & 0 & 0.2 & 0.5 & 0.8 \\
\hline \multirow{4}{*}{0} & 0 & 1.26 & 1.26 & 1.25 & 1.26 & 1.15 & 1.15 & 1.15 & 1.15 & 0.91 & 0.90 & 0.90 & 0.90 & 0.64 & 0.65 & 0.64 & 0.65 \\
\hline & 0.2 & 1.25 & 1.25 & 1.25 & 1.25 & 1.13 & 1.14 & 1.14 & 1.14 & 0.89 & 0.89 & 0.89 & 0.90 & 0.65 & 0.65 & 0.64 & 0.64 \\
\hline & 0.5 & 1.18 & 1.18 & 1.18 & 1.19 & 1.08 & 1.08 & 1.09 & 1.08 & 0.86 & 0.85 & 0.85 & 0.85 & 0.62 & 0.63 & 0.62 & 0.62 \\
\hline & 0.8 & 1.07 & 1.06 & 1.06 & 1.07 & 0.98 & 0.98 & 0.98 & 0.97 & 0.79 & 0.78 & 0.78 & 0.79 & 0.59 & 0.59 & 0.59 & 0.59 \\
\hline \multirow{4}{*}{0.2} & 0 & 1.25 & 1.24 & 1.25 & 1.25 & 1.13 & 1.14 & 1.14 & 1.14 & 0.89 & 0.89 & 0.89 & 0.89 & 0.65 & 0.64 & 0.64 & 0.64 \\
\hline & 0.2 & 1.24 & 1.24 & 1.23 & 1.25 & 1.13 & 1.14 & 1.12 & 1.13 & 0.89 & 0.89 & 0.89 & 0.88 & 0.64 & 0.64 & 0.64 & 0.64 \\
\hline & 0.5 & 1.17 & 1.18 & 1.17 & 1.18 & 1.07 & 1.07 & 1.07 & 1.08 & 0.84 & 0.84 & 0.85 & 0.84 & 0.62 & 0.61 & 0.61 & 0.62 \\
\hline & 0.8 & 1.06 & 1.06 & 1.05 & 1.06 & 0.97 & 0.97 & 0.97 & 0.97 & 0.79 & 0.78 & 0.78 & 0.78 & 0.59 & 0.59 & 0.58 & 0.59 \\
\hline \multirow{4}{*}{0.5} & 0 & 1.18 & 1.18 & 1.18 & 1.17 & 1.07 & 1.08 & 1.07 & 1.08 & 0.86 & 0.85 & 0.86 & 0.84 & 0.62 & 0.62 & 0.62 & 0.62 \\
\hline & 0.2 & 1.16 & 1.17 & 1.16 & 1.17 & 1.07 & 1.07 & 1.06 & 1.07 & 0.83 & 0.85 & 0.84 & 0.84 & 0.61 & 0.62 & 0.62 & 0.62 \\
\hline & 0.5 & 1.10 & 1.11 & 1.11 & 1.11 & 1.01 & 1.01 & 1.01 & 1.01 & 0.81 & 0.82 & 0.81 & 0.82 & 0.60 & 0.60 & 0.60 & 0.60 \\
\hline & 0.8 & 1.01 & 1.00 & 1.00 & 1.00 & 0.92 & 0.92 & 0.92 & 0.92 & 0.76 & 0.76 & 0.75 & 0.75 & 0.56 & 0.57 & 0.57 & 0.57 \\
\hline \multirow{4}{*}{0.8} & 0 & 1.06 & 1.06 & 1.06 & 1.06 & 0.97 & 0.97 & 0.97 & 0.97 & 0.79 & 0.78 & 0.79 & 0.79 & 0.58 & 0.59 & 0.59 & 0.59 \\
\hline & 0.2 & 1.06 & 1.05 & 1.05 & 1.05 & 0.96 & 0.97 & 0.96 & 0.97 & 0.79 & 0.78 & 0.78 & 0.78 & 0.59 & 0.58 & 0.58 & 0.58 \\
\hline & 0.5 & 1.00 & 0.99 & 1.00 & 0.99 & 0.92 & 0.92 & 0.92 & 0.92 & 0.75 & 0.75 & 0.76 & 0.75 & 0.57 & 0.57 & 0.57 & 0.56 \\
\hline & 0.8 & 0.91 & 0.92 & 0.92 & 0.91 & 0.85 & 0.85 & 0.85 & 0.84 & 0.70 & 0.70 & 0.70 & 0.70 & 0.54 & 0.54 & 0.54 & 0.54 \\
\hline
\end{tabular}

Note: ICC = Intraclass Correlation Coefficient. 


\section{Table 6 cont}

Average Design Effect values for the cross-level interaction: Uniform Distribution conditions $(\mathrm{m}=10, \mathrm{k}=75)$

\begin{tabular}{|c|c|c|c|c|c|c|c|c|c|c|c|c|c|c|c|c|c|}
\hline \multirow{4}{*}{$\begin{array}{c}\text { L1 } \\
\text { Effect }\end{array}$} & \multirow{4}{*}{$\begin{array}{c}\text { Interaction } \\
\text { Effect }\end{array}$} & \multicolumn{16}{|c|}{ ICC Values } \\
\hline & & \multicolumn{4}{|c|}{0} & \multicolumn{4}{|c|}{0.1} & \multicolumn{4}{|c|}{0.3} & \multicolumn{4}{|c|}{0.5} \\
\hline & & \multicolumn{12}{|c|}{ L2 Effect } & & & & \\
\hline & & 0 & 0.2 & 0.5 & 0.8 & 0 & 0.2 & 0.5 & 0.8 & 0 & 0.2 & 0.5 & 0.8 & 0 & 0.2 & 0.5 & 0.8 \\
\hline \multirow{4}{*}{0} & 0 & 1.26 & 1.26 & 1.25 & 1.26 & 1.15 & 1.15 & 1.15 & 1.15 & 0.91 & 0.90 & 0.90 & 0.90 & 0.64 & 0.65 & 0.64 & 0.65 \\
\hline & 0.2 & 1.25 & 1.25 & 1.25 & 1.25 & 1.13 & 1.14 & 1.14 & 1.14 & 0.89 & 0.89 & 0.89 & 0.90 & 0.65 & 0.65 & 0.64 & 0.64 \\
\hline & 0.5 & 1.18 & 1.18 & 1.18 & 1.19 & 1.08 & 1.08 & 1.09 & 1.08 & 0.86 & 0.85 & 0.85 & 0.85 & 0.62 & 0.63 & 0.62 & 0.62 \\
\hline & 0.8 & 1.07 & 1.06 & 1.06 & 1.07 & 0.98 & 0.98 & 0.98 & 0.97 & 0.79 & 0.78 & 0.78 & 0.79 & 0.59 & 0.59 & 0.59 & 0.59 \\
\hline \multirow{4}{*}{0.2} & 0 & 1.25 & 1.24 & 1.25 & 1.25 & 1.13 & 1.14 & 1.14 & 1.14 & 0.89 & 0.89 & 0.89 & 0.89 & 0.65 & 0.64 & 0.64 & 0.64 \\
\hline & 0.2 & 1.24 & 1.24 & 1.23 & 1.25 & 1.13 & 1.14 & 1.12 & 1.13 & 0.89 & 0.89 & 0.89 & 0.88 & 0.64 & 0.64 & 0.64 & 0.64 \\
\hline & 0.5 & 1.17 & 1.18 & 1.17 & 1.18 & 1.07 & 1.07 & 1.07 & 1.08 & 0.84 & 0.84 & 0.85 & 0.84 & 0.62 & 0.61 & 0.61 & 0.62 \\
\hline & 0.8 & 1.06 & 1.06 & 1.05 & 1.06 & 0.97 & 0.97 & 0.97 & 0.97 & 0.79 & 0.78 & 0.78 & 0.78 & 0.59 & 0.59 & 0.58 & 0.59 \\
\hline \multirow{4}{*}{0.5} & 0 & 1.18 & 1.18 & 1.18 & 1.17 & 1.07 & 1.08 & 1.07 & 1.08 & 0.86 & 0.85 & 0.86 & 0.84 & 0.62 & 0.62 & 0.62 & 0.62 \\
\hline & 0.2 & 1.16 & 1.17 & 1.16 & 1.17 & 1.07 & 1.07 & 1.06 & 1.07 & 0.83 & 0.85 & 0.84 & 0.84 & 0.61 & 0.62 & 0.62 & 0.62 \\
\hline & 0.5 & 1.10 & 1.11 & 1.11 & 1.11 & 1.01 & 1.01 & 1.01 & 1.01 & 0.81 & 0.82 & 0.81 & 0.82 & 0.60 & 0.60 & 0.60 & 0.60 \\
\hline & 0.8 & 1.01 & 1.00 & 1.00 & 1.00 & 0.92 & 0.92 & 0.92 & 0.92 & 0.76 & 0.76 & 0.75 & 0.75 & 0.56 & 0.57 & 0.57 & 0.57 \\
\hline \multirow{4}{*}{0.8} & 0 & 1.06 & 1.06 & 1.06 & 1.06 & 0.97 & 0.97 & 0.97 & 0.97 & 0.79 & 0.78 & 0.79 & 0.79 & 0.58 & 0.59 & 0.59 & 0.59 \\
\hline & 0.2 & 1.06 & 1.05 & 1.05 & 1.05 & 0.96 & 0.97 & 0.96 & 0.97 & 0.79 & 0.78 & 0.78 & 0.78 & 0.59 & 0.58 & 0.58 & 0.58 \\
\hline & 0.5 & 1.00 & 0.99 & 1.00 & 0.99 & 0.92 & 0.92 & 0.92 & 0.92 & 0.75 & 0.75 & 0.76 & 0.75 & 0.57 & 0.57 & 0.57 & 0.56 \\
\hline & 0.8 & 0.91 & 0.92 & 0.92 & 0.91 & 0.85 & 0.85 & 0.85 & 0.84 & 0.70 & 0.70 & 0.70 & 0.70 & 0.54 & 0.54 & 0.54 & 0.54 \\
\hline
\end{tabular}

Note: ICC = Intraclass Correlation Coefficient. 


\section{Table 6 cont}

Average Design Effect values for the cross-level interaction: Uniform Distribution conditions $(\mathrm{m}=10, \mathrm{k}=100)$

\begin{tabular}{|c|c|c|c|c|c|c|c|c|c|c|c|c|c|c|c|c|c|}
\hline \multirow{4}{*}{$\begin{array}{c}\text { L1 } \\
\text { Effect }\end{array}$} & \multirow{4}{*}{$\begin{array}{c}\text { Interaction } \\
\text { Effect }\end{array}$} & \multicolumn{16}{|c|}{ ICC Values } \\
\hline & & \multicolumn{4}{|c|}{0} & \multicolumn{4}{|c|}{0.1} & \multicolumn{4}{|c|}{0.3} & \multicolumn{4}{|c|}{0.5} \\
\hline & & \multicolumn{12}{|c|}{ L2 Effect } & & & & \\
\hline & & 0 & 0.2 & 0.5 & 0.8 & 0 & 0.2 & 0.5 & 0.8 & 0 & 0.2 & 0.5 & 0.8 & 0 & 0.2 & 0.5 & 0.8 \\
\hline \multirow{4}{*}{0} & 0 & 1.26 & 1.26 & 1.25 & 1.26 & 1.15 & 1.15 & 1.15 & 1.15 & 0.91 & 0.90 & 0.90 & 0.90 & 0.64 & 0.65 & 0.64 & 0.65 \\
\hline & 0.2 & 1.25 & 1.25 & 1.25 & 1.25 & 1.13 & 1.14 & 1.14 & 1.14 & 0.89 & 0.89 & 0.89 & 0.90 & 0.65 & 0.65 & 0.64 & 0.64 \\
\hline & 0.5 & 1.18 & 1.18 & 1.18 & 1.19 & 1.08 & 1.08 & 1.09 & 1.08 & 0.86 & 0.85 & 0.85 & 0.85 & 0.62 & 0.63 & 0.62 & 0.62 \\
\hline & 0.8 & 1.07 & 1.06 & 1.06 & 1.07 & 0.98 & 0.98 & 0.98 & 0.97 & 0.79 & 0.78 & 0.78 & 0.79 & 0.59 & 0.59 & 0.59 & 0.59 \\
\hline \multirow{4}{*}{0.2} & 0 & 1.25 & 1.24 & 1.25 & 1.25 & 1.13 & 1.14 & 1.14 & 1.14 & 0.89 & 0.89 & 0.89 & 0.89 & 0.65 & 0.64 & 0.64 & 0.64 \\
\hline & 0.2 & 1.24 & 1.24 & 1.23 & 1.25 & 1.13 & 1.14 & 1.12 & 1.13 & 0.89 & 0.89 & 0.89 & 0.88 & 0.64 & 0.64 & 0.64 & 0.64 \\
\hline & 0.5 & 1.17 & 1.18 & 1.17 & 1.18 & 1.07 & 1.07 & 1.07 & 1.08 & 0.84 & 0.84 & 0.85 & 0.84 & 0.62 & 0.61 & 0.61 & 0.62 \\
\hline & 0.8 & 1.06 & 1.06 & 1.05 & 1.06 & 0.97 & 0.97 & 0.97 & 0.97 & 0.79 & 0.78 & 0.78 & 0.78 & 0.59 & 0.59 & 0.58 & 0.59 \\
\hline \multirow{4}{*}{0.5} & 0 & 1.18 & 1.18 & 1.18 & 1.17 & 1.07 & 1.08 & 1.07 & 1.08 & 0.86 & 0.85 & 0.86 & 0.84 & 0.62 & 0.62 & 0.62 & 0.62 \\
\hline & 0.2 & 1.16 & 1.17 & 1.16 & 1.17 & 1.07 & 1.07 & 1.06 & 1.07 & 0.83 & 0.85 & 0.84 & 0.84 & 0.61 & 0.62 & 0.62 & 0.62 \\
\hline & 0.5 & 1.10 & 1.11 & 1.11 & 1.11 & 1.01 & 1.01 & 1.01 & 1.01 & 0.81 & 0.82 & 0.81 & 0.82 & 0.60 & 0.60 & 0.60 & 0.60 \\
\hline & 0.8 & 1.01 & 1.00 & 1.00 & 1.00 & 0.92 & 0.92 & 0.92 & 0.92 & 0.76 & 0.76 & 0.75 & 0.75 & 0.56 & 0.57 & 0.57 & 0.57 \\
\hline \multirow{4}{*}{0.8} & 0 & 1.06 & 1.06 & 1.06 & 1.06 & 0.97 & 0.97 & 0.97 & 0.97 & 0.79 & 0.78 & 0.79 & 0.79 & 0.58 & 0.59 & 0.59 & 0.59 \\
\hline & 0.2 & 1.06 & 1.05 & 1.05 & 1.05 & 0.96 & 0.97 & 0.96 & 0.97 & 0.79 & 0.78 & 0.78 & 0.78 & 0.59 & 0.58 & 0.58 & 0.58 \\
\hline & 0.5 & 1.00 & 0.99 & 1.00 & 0.99 & 0.92 & 0.92 & 0.92 & 0.92 & 0.75 & 0.75 & 0.76 & 0.75 & 0.57 & 0.57 & 0.57 & 0.56 \\
\hline & 0.8 & 0.91 & 0.92 & 0.92 & 0.91 & 0.85 & 0.85 & 0.85 & 0.84 & 0.70 & 0.70 & 0.70 & 0.70 & 0.54 & 0.54 & 0.54 & 0.54 \\
\hline
\end{tabular}

Note: ICC = Intraclass Correlation Coefficient. 


\section{Table 6 cont}

Average Design Effect values for the cross-level interaction: Uniform Distribution conditions $(\mathrm{m}=20, \mathrm{k}=25)$

\begin{tabular}{|c|c|c|c|c|c|c|c|c|c|c|c|c|c|c|c|c|c|}
\hline \multirow{4}{*}{$\begin{array}{c}\text { L1 } \\
\text { Effect }\end{array}$} & \multirow{4}{*}{$\begin{array}{c}\text { Interaction } \\
\text { Effect }\end{array}$} & \multicolumn{16}{|c|}{ ICC Values } \\
\hline & & \multicolumn{4}{|c|}{0} & \multicolumn{4}{|c|}{0.1} & \multicolumn{4}{|c|}{0.3} & \multicolumn{4}{|c|}{0.5} \\
\hline & & \multicolumn{12}{|c|}{ L2 Effect } & & & & \\
\hline & & 0 & 0.2 & 0.5 & 0.8 & 0 & 0.2 & 0.5 & 0.8 & 0 & 0.2 & 0.5 & 0.8 & 0 & 0.2 & 0.5 & 0.8 \\
\hline \multirow{4}{*}{0} & 0 & 1.07 & 1.07 & 1.07 & 1.07 & 0.98 & 0.97 & 0.98 & 0.97 & 0.77 & 0.78 & 0.77 & 0.78 & 0.57 & 0.56 & 0.57 & 0.57 \\
\hline & 0.2 & 1.06 & 1.07 & 1.06 & 1.06 & 0.98 & 0.97 & 0.97 & 0.97 & 0.77 & 0.77 & 0.77 & 0.77 & 0.57 & 0.57 & 0.57 & 0.57 \\
\hline & 0.5 & 1.05 & 1.05 & 1.05 & 1.05 & 0.96 & 0.96 & 0.97 & 0.96 & 0.77 & 0.76 & 0.77 & 0.77 & 0.57 & 0.56 & 0.56 & 0.56 \\
\hline & 0.8 & 1.02 & 1.03 & 1.03 & 1.02 & 0.94 & 0.93 & 0.94 & 0.94 & 0.75 & 0.76 & 0.74 & 0.75 & 0.55 & 0.56 & 0.56 & 0.56 \\
\hline \multirow{4}{*}{0.2} & 0 & 1.06 & 1.07 & 1.07 & 1.07 & 0.97 & 0.97 & 0.96 & 0.98 & 0.77 & 0.78 & 0.76 & 0.77 & 0.57 & 0.57 & 0.57 & 0.57 \\
\hline & 0.2 & 1.06 & 1.07 & 1.07 & 1.06 & 0.97 & 0.97 & 0.97 & 0.97 & 0.78 & 0.77 & 0.78 & 0.78 & 0.57 & 0.57 & 0.56 & 0.56 \\
\hline & 0.5 & 1.04 & 1.05 & 1.05 & 1.05 & 0.97 & 0.97 & 0.96 & 0.96 & 0.77 & 0.76 & 0.76 & 0.76 & 0.56 & 0.56 & 0.56 & 0.56 \\
\hline & 0.8 & 1.03 & 1.02 & 1.02 & 1.03 & 0.93 & 0.94 & 0.93 & 0.93 & 0.75 & 0.75 & 0.75 & 0.75 & 0.55 & 0.56 & 0.55 & 0.55 \\
\hline \multirow{4}{*}{0.5} & 0 & 1.05 & 1.05 & 1.05 & 1.05 & 0.96 & 0.96 & 0.96 & 0.96 & 0.76 & 0.77 & 0.76 & 0.76 & 0.56 & 0.56 & 0.57 & 0.57 \\
\hline & 0.2 & 1.05 & 1.05 & 1.05 & 1.04 & 0.96 & 0.96 & 0.96 & 0.96 & 0.76 & 0.77 & 0.77 & 0.76 & 0.56 & 0.56 & 0.56 & 0.56 \\
\hline & 0.5 & 1.03 & 1.03 & 1.04 & 1.03 & 0.94 & 0.94 & 0.94 & 0.95 & 0.75 & 0.76 & 0.75 & 0.76 & 0.55 & 0.56 & 0.56 & 0.56 \\
\hline & 0.8 & 1.02 & 1.01 & 1.01 & 1.01 & 0.92 & 0.92 & 0.92 & 0.92 & 0.74 & 0.74 & 0.74 & 0.74 & 0.55 & 0.56 & 0.55 & 0.55 \\
\hline \multirow{4}{*}{0.8} & 0 & 1.02 & 1.02 & 1.03 & 1.03 & 0.93 & 0.94 & 0.93 & 0.93 & 0.75 & 0.75 & 0.75 & 0.75 & 0.56 & 0.56 & 0.55 & 0.56 \\
\hline & 0.2 & 1.02 & 1.02 & 1.03 & 1.02 & 0.94 & 0.92 & 0.93 & 0.93 & 0.75 & 0.75 & 0.75 & 0.75 & 0.55 & 0.55 & 0.55 & 0.55 \\
\hline & 0.5 & 1.01 & 1.00 & 1.02 & 1.01 & 0.92 & 0.92 & 0.92 & 0.92 & 0.73 & 0.74 & 0.74 & 0.74 & 0.56 & 0.55 & 0.55 & 0.55 \\
\hline & 0.8 & 0.99 & 0.98 & 0.98 & 0.99 & 0.90 & 0.90 & 0.90 & 0.90 & 0.73 & 0.73 & 0.73 & 0.73 & 0.55 & 0.54 & 0.54 & 0.54 \\
\hline
\end{tabular}

Note: ICC = Intraclass Correlation Coefficient. 
Table 6 cont.

Average Design Effect values for the cross-level interaction: Uniform Distribution conditions $(\mathrm{m}=20, \mathrm{k}=50)$

\begin{tabular}{|c|c|c|c|c|c|c|c|c|c|c|c|c|c|c|c|c|c|}
\hline \multirow{4}{*}{$\begin{array}{c}\text { L1 } \\
\text { Effect }\end{array}$} & \multirow{4}{*}{$\begin{array}{c}\text { Interaction } \\
\text { Effect }\end{array}$} & \multicolumn{16}{|c|}{ ICC Values } \\
\hline & & \multicolumn{4}{|c|}{0} & \multicolumn{4}{|c|}{0.1} & \multicolumn{4}{|c|}{0.3} & \multicolumn{4}{|c|}{0.5} \\
\hline & & \multicolumn{12}{|c|}{ L2 Effect } & & & & \\
\hline & & 0 & 0.2 & 0.5 & 0.8 & 0 & 0.2 & 0.5 & 0.8 & 0 & 0.2 & 0.5 & 0.8 & 0 & 0.2 & 0.5 & 0.8 \\
\hline \multirow{4}{*}{0} & 0 & 1.06 & 1.06 & 1.05 & 1.06 & 0.96 & 0.96 & 0.96 & 0.96 & 0.75 & 0.76 & 0.76 & 0.75 & 0.55 & 0.55 & 0.55 & 0.55 \\
\hline & 0.2 & 1.06 & 1.06 & 1.06 & 1.06 & 0.96 & 0.96 & 0.96 & 0.96 & 0.75 & 0.75 & 0.75 & 0.75 & 0.55 & 0.55 & 0.55 & 0.55 \\
\hline & 0.5 & 1.04 & 1.04 & 1.04 & 1.05 & 0.95 & 0.95 & 0.94 & 0.94 & 0.75 & 0.74 & 0.74 & 0.75 & 0.54 & 0.54 & 0.54 & 0.54 \\
\hline & 0.8 & 1.01 & 1.02 & 1.02 & 1.02 & 0.92 & 0.92 & 0.92 & 0.92 & 0.73 & 0.73 & 0.73 & 0.73 & 0.53 & 0.54 & 0.53 & 0.54 \\
\hline \multirow{4}{*}{0.2} & 0 & 1.06 & 1.05 & 1.05 & 1.06 & 0.96 & 0.95 & 0.96 & 0.96 & 0.75 & 0.75 & 0.75 & 0.75 & 0.54 & 0.54 & 0.54 & 0.55 \\
\hline & 0.2 & 1.06 & 1.06 & 1.05 & 1.06 & 0.96 & 0.96 & 0.95 & 0.96 & 0.75 & 0.75 & 0.75 & 0.75 & 0.54 & 0.54 & 0.54 & 0.55 \\
\hline & 0.5 & 1.04 & 1.04 & 1.04 & 1.04 & 0.95 & 0.94 & 0.94 & 0.94 & 0.74 & 0.75 & 0.74 & 0.74 & 0.54 & 0.54 & 0.54 & 0.54 \\
\hline & 0.8 & 1.01 & 1.01 & 1.01 & 1.01 & 0.92 & 0.92 & 0.92 & 0.92 & 0.73 & 0.73 & 0.73 & 0.73 & 0.53 & 0.53 & 0.53 & 0.53 \\
\hline \multirow{4}{*}{0.5} & 0 & 1.04 & 1.04 & 1.04 & 1.04 & 0.95 & 0.95 & 0.95 & 0.95 & 0.74 & 0.74 & 0.75 & 0.75 & 0.54 & 0.54 & 0.54 & 0.54 \\
\hline & 0.2 & 1.04 & 1.04 & 1.04 & 1.04 & 0.94 & 0.94 & 0.94 & 0.94 & 0.75 & 0.74 & 0.74 & 0.75 & 0.54 & 0.54 & 0.54 & 0.54 \\
\hline & 0.5 & 1.02 & 1.02 & 1.03 & 1.03 & 0.94 & 0.93 & 0.93 & 0.93 & 0.74 & 0.74 & 0.74 & 0.74 & 0.54 & 0.54 & 0.54 & 0.54 \\
\hline & 0.8 & 1.00 & 1.00 & 1.00 & 1.00 & 0.90 & 0.90 & 0.90 & 0.91 & 0.72 & 0.72 & 0.72 & 0.72 & 0.52 & 0.53 & 0.53 & 0.53 \\
\hline \multirow{4}{*}{0.8} & 0 & 1.01 & 1.01 & 1.02 & 1.01 & 0.92 & 0.92 & 0.92 & 0.92 & 0.73 & 0.73 & 0.73 & 0.73 & 0.53 & 0.53 & 0.53 & 0.53 \\
\hline & 0.2 & 1.01 & 1.00 & 1.01 & 1.01 & 0.91 & 0.92 & 0.92 & 0.92 & 0.73 & 0.73 & 0.73 & 0.73 & 0.53 & 0.53 & 0.53 & 0.54 \\
\hline & 0.5 & 1.00 & 1.00 & 0.99 & 0.99 & 0.91 & 0.91 & 0.90 & 0.91 & 0.72 & 0.72 & 0.72 & 0.72 & 0.53 & 0.53 & 0.53 & 0.53 \\
\hline & 0.8 & 0.97 & 0.97 & 0.97 & 0.97 & 0.89 & 0.88 & 0.88 & 0.88 & 0.71 & 0.70 & 0.71 & 0.71 & 0.52 & 0.53 & 0.52 & 0.52 \\
\hline
\end{tabular}

Note: ICC = Intraclass Correlation Coefficient. 
Table 6 cont.

Average Design Effect values for the cross-level interaction: Uniform Distribution conditions $(\mathrm{m}=20, \mathrm{k}=75)$

\begin{tabular}{|c|c|c|c|c|c|c|c|c|c|c|c|c|c|c|c|c|c|}
\hline \multirow{4}{*}{$\begin{array}{c}\text { L1 } \\
\text { Effect }\end{array}$} & \multirow{4}{*}{$\begin{array}{c}\text { Interaction } \\
\text { Effect }\end{array}$} & \multicolumn{16}{|c|}{ ICC Values } \\
\hline & & \multicolumn{4}{|c|}{0} & \multicolumn{4}{|c|}{0.1} & \multicolumn{4}{|c|}{0.3} & \multicolumn{4}{|c|}{0.5} \\
\hline & & \multicolumn{12}{|c|}{ L2 Effect } & & & & \\
\hline & & 0 & 0.2 & 0.5 & 0.8 & 0 & 0.2 & 0.5 & 0.8 & 0 & 0.2 & 0.5 & 0.8 & 0 & 0.2 & 0.5 & 0.8 \\
\hline \multirow{4}{*}{0} & 0 & 1.05 & 1.05 & 1.05 & 1.06 & 0.96 & 0.96 & 0.95 & 0.96 & 0.75 & 0.75 & 0.75 & 0.75 & 0.54 & 0.54 & 0.54 & 0.54 \\
\hline & 0.2 & 1.05 & 1.05 & 1.06 & 1.05 & 0.96 & 0.95 & 0.95 & 0.95 & 0.75 & 0.74 & 0.75 & 0.75 & 0.54 & 0.54 & 0.54 & 0.54 \\
\hline & 0.5 & 1.04 & 1.04 & 1.04 & 1.04 & 0.94 & 0.94 & 0.94 & 0.94 & 0.74 & 0.74 & 0.74 & 0.74 & 0.54 & 0.54 & 0.54 & 0.54 \\
\hline & 0.8 & 1.01 & 1.01 & 1.01 & 1.01 & 0.92 & 0.92 & 0.92 & 0.91 & 0.73 & 0.72 & 0.73 & 0.72 & 0.53 & 0.53 & 0.53 & 0.53 \\
\hline \multirow{4}{*}{0.2} & 0 & 1.06 & 1.06 & 1.06 & 1.05 & 0.95 & 0.95 & 0.96 & 0.96 & 0.75 & 0.75 & 0.75 & 0.75 & 0.54 & 0.54 & 0.54 & 0.54 \\
\hline & 0.2 & 1.05 & 1.05 & 1.05 & 1.05 & 0.95 & 0.95 & 0.95 & 0.95 & 0.75 & 0.74 & 0.74 & 0.75 & 0.54 & 0.54 & 0.54 & 0.54 \\
\hline & 0.5 & 1.04 & 1.04 & 1.04 & 1.04 & 0.94 & 0.94 & 0.94 & 0.94 & 0.74 & 0.74 & 0.74 & 0.74 & 0.54 & 0.53 & 0.54 & 0.53 \\
\hline & 0.8 & 1.01 & 1.01 & 1.00 & 1.01 & 0.92 & 0.91 & 0.92 & 0.91 & 0.73 & 0.72 & 0.72 & 0.73 & 0.53 & 0.52 & 0.53 & 0.53 \\
\hline \multirow{4}{*}{0.5} & 0 & 1.04 & 1.04 & 1.04 & 1.04 & 0.94 & 0.94 & 0.94 & 0.94 & 0.74 & 0.74 & 0.74 & 0.74 & 0.54 & 0.53 & 0.54 & 0.53 \\
\hline & 0.2 & 1.04 & 1.04 & 1.04 & 1.04 & 0.94 & 0.94 & 0.94 & 0.94 & 0.74 & 0.74 & 0.74 & 0.74 & 0.53 & 0.53 & 0.54 & 0.53 \\
\hline & 0.5 & 1.02 & 1.02 & 1.02 & 1.02 & 0.93 & 0.92 & 0.92 & 0.92 & 0.73 & 0.73 & 0.73 & 0.73 & 0.53 & 0.53 & 0.53 & 0.53 \\
\hline & 0.8 & 0.99 & 0.99 & 0.99 & 0.99 & 0.90 & 0.90 & 0.90 & 0.90 & 0.71 & 0.72 & 0.72 & 0.71 & 0.52 & 0.52 & 0.52 & 0.52 \\
\hline \multirow{4}{*}{0.8} & 0 & 1.01 & 1.01 & 1.01 & 1.01 & 0.91 & 0.91 & 0.91 & 0.91 & 0.72 & 0.72 & 0.72 & 0.72 & 0.53 & 0.52 & 0.53 & 0.53 \\
\hline & 0.2 & 1.00 & 1.01 & 1.01 & 1.01 & 0.91 & 0.91 & 0.91 & 0.91 & 0.72 & 0.72 & 0.72 & 0.72 & 0.52 & 0.52 & 0.53 & 0.53 \\
\hline & 0.5 & 0.99 & 0.99 & 0.99 & 0.99 & 0.90 & 0.90 & 0.90 & 0.90 & 0.71 & 0.72 & 0.71 & 0.71 & 0.52 & 0.52 & 0.52 & 0.52 \\
\hline & 0.8 & 0.96 & 0.96 & 0.96 & 0.97 & 0.88 & 0.88 & 0.88 & 0.88 & 0.70 & 0.70 & 0.70 & 0.70 & 0.52 & 0.52 & 0.51 & 0.51 \\
\hline
\end{tabular}

Note: ICC = Intraclass Correlation Coefficient. 
Table 6 cont.

Average Design Effect values for the cross-level interaction: Uniform Distribution conditions $(\mathrm{m}=20, \mathrm{k}=100)$

\begin{tabular}{|c|c|c|c|c|c|c|c|c|c|c|c|c|c|c|c|c|c|}
\hline \multirow{4}{*}{$\begin{array}{c}\text { L1 } \\
\text { Effect }\end{array}$} & \multirow{4}{*}{$\begin{array}{c}\text { Interaction } \\
\text { Effect }\end{array}$} & \multicolumn{16}{|c|}{ ICC Values } \\
\hline & & \multicolumn{4}{|c|}{0} & \multicolumn{4}{|c|}{0.1} & \multicolumn{4}{|c|}{0.3} & \multicolumn{4}{|c|}{0.5} \\
\hline & & \multicolumn{12}{|c|}{ L2 Effect } & & & & \\
\hline & & 0 & 0.2 & 0.5 & 0.8 & 0 & 0.2 & 0.5 & 0.8 & 0 & 0.2 & 0.5 & 0.8 & 0 & 0.2 & 0.5 & 0.8 \\
\hline \multirow{4}{*}{0} & 0 & 1.06 & 1.06 & 1.05 & 1.06 & 0.96 & 0.95 & 0.96 & 0.96 & 0.74 & 0.75 & 0.75 & 0.75 & 0.54 & 0.54 & 0.53 & 0.54 \\
\hline & 0.2 & 1.05 & 1.05 & 1.05 & 1.06 & 0.95 & 0.95 & 0.95 & 0.95 & 0.75 & 0.74 & 0.74 & 0.74 & 0.53 & 0.53 & 0.54 & 0.54 \\
\hline & 0.5 & 1.04 & 1.04 & 1.04 & 1.04 & 0.94 & 0.94 & 0.94 & 0.94 & 0.74 & 0.74 & 0.74 & 0.74 & 0.53 & 0.53 & 0.53 & 0.53 \\
\hline & 0.8 & 1.01 & 1.01 & 1.01 & 1.01 & 0.91 & 0.91 & 0.91 & 0.91 & 0.72 & 0.72 & 0.72 & 0.72 & 0.52 & 0.52 & 0.53 & 0.52 \\
\hline \multirow{4}{*}{0.2} & 0 & 1.05 & 1.05 & 1.05 & 1.05 & 0.95 & 0.96 & 0.95 & 0.95 & 0.74 & 0.75 & 0.74 & 0.75 & 0.53 & 0.53 & 0.54 & 0.54 \\
\hline & 0.2 & 1.05 & 1.05 & 1.05 & 1.05 & 0.95 & 0.95 & 0.95 & 0.94 & 0.74 & 0.74 & 0.74 & 0.74 & 0.54 & 0.54 & 0.53 & 0.53 \\
\hline & 0.5 & 1.03 & 1.03 & 1.04 & 1.04 & 0.94 & 0.94 & 0.94 & 0.93 & 0.73 & 0.73 & 0.74 & 0.73 & 0.53 & 0.53 & 0.53 & 0.53 \\
\hline & 0.8 & 1.01 & 1.00 & 1.00 & 1.00 & 0.91 & 0.91 & 0.91 & 0.91 & 0.72 & 0.72 & 0.72 & 0.72 & 0.52 & 0.52 & 0.52 & 0.52 \\
\hline \multirow{4}{*}{0.5} & 0 & 1.04 & 1.04 & 1.04 & 1.04 & 0.94 & 0.94 & 0.94 & 0.94 & 0.74 & 0.74 & 0.74 & 0.73 & 0.53 & 0.53 & 0.53 & 0.53 \\
\hline & 0.2 & 1.03 & 1.03 & 1.03 & 1.04 & 0.94 & 0.93 & 0.93 & 0.93 & 0.74 & 0.74 & 0.73 & 0.74 & 0.53 & 0.53 & 0.53 & 0.53 \\
\hline & 0.5 & 1.02 & 1.02 & 1.02 & 1.02 & 0.92 & 0.92 & 0.92 & 0.92 & 0.73 & 0.73 & 0.73 & 0.73 & 0.53 & 0.53 & 0.53 & 0.52 \\
\hline & 0.8 & 0.99 & 0.99 & 0.99 & 0.99 & 0.90 & 0.90 & 0.90 & 0.90 & 0.71 & 0.71 & 0.71 & 0.71 & 0.52 & 0.52 & 0.51 & 0.52 \\
\hline \multirow{4}{*}{0.8} & 0 & 1.01 & 1.01 & 1.01 & 1.00 & 0.91 & 0.91 & 0.91 & 0.91 & 0.72 & 0.72 & 0.72 & 0.72 & 0.52 & 0.52 & 0.52 & 0.52 \\
\hline & 0.2 & 1.00 & 1.01 & 1.01 & 1.01 & 0.91 & 0.91 & 0.91 & 0.91 & 0.72 & 0.72 & 0.72 & 0.72 & 0.52 & 0.52 & 0.52 & 0.52 \\
\hline & 0.5 & 0.99 & 0.99 & 0.99 & 0.99 & 0.90 & 0.90 & 0.90 & 0.90 & 0.71 & 0.71 & 0.71 & 0.71 & 0.52 & 0.52 & 0.52 & 0.52 \\
\hline & 0.8 & 0.96 & 0.96 & 0.96 & 0.96 & 0.87 & 0.87 & 0.87 & 0.87 & 0.70 & 0.69 & 0.70 & 0.70 & 0.51 & 0.51 & 0.51 & 0.51 \\
\hline
\end{tabular}

Note: ICC = Intraclass Correlation Coefficient. 
Table 6 cont.

Average Design Effect values for the cross-level interaction: Poisson Distribution conditions $(\mathrm{m}=5, \mathrm{k}=25)$

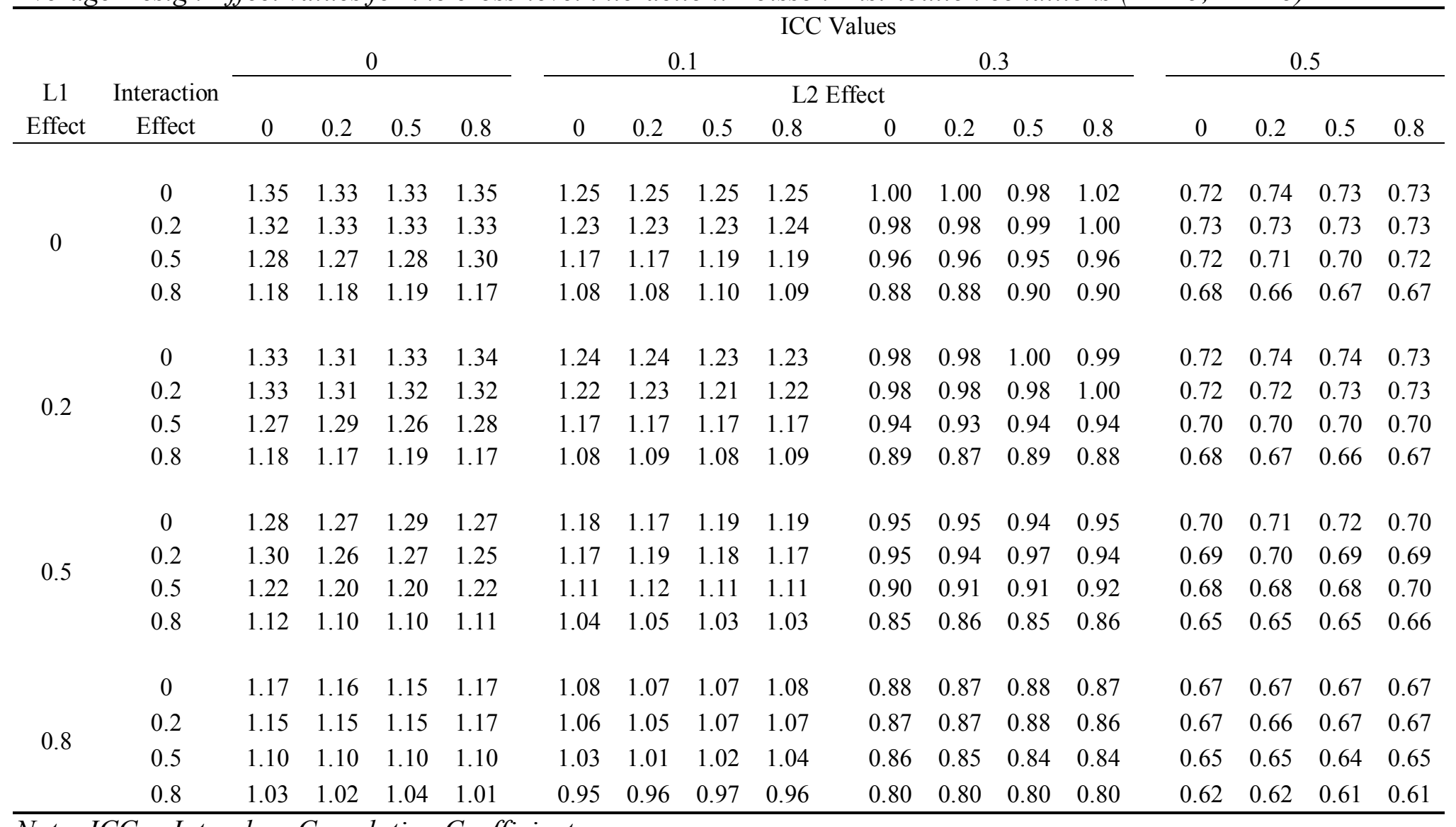

Note: ICC = Intraclass Correlation Coefficient. 
Table 6 cont.

Average Design Effect values for the cross-level interaction: Poisson Distribution conditions $(\mathrm{m}=5, \mathrm{k}=50)$

\begin{tabular}{|c|c|c|c|c|c|c|c|c|c|c|c|c|c|c|c|c|c|}
\hline \multirow{4}{*}{$\begin{array}{c}\text { L1 } \\
\text { Effect }\end{array}$} & \multirow{4}{*}{$\begin{array}{c}\text { Interaction } \\
\text { Effect }\end{array}$} & \multicolumn{16}{|c|}{ ICC Values } \\
\hline & & \multicolumn{4}{|c|}{0} & \multicolumn{4}{|c|}{0.1} & \multicolumn{4}{|c|}{0.3} & \multicolumn{4}{|c|}{0.5} \\
\hline & & \multicolumn{12}{|c|}{ L2 Effect } & & & & \\
\hline & & 0 & 0.2 & 0.5 & 0.8 & 0 & 0.2 & 0.5 & 0.8 & 0 & 0.2 & 0.5 & 0.8 & 0 & 0.2 & 0.5 & 0.8 \\
\hline \multirow{4}{*}{0} & 0 & 1.29 & 1.30 & 1.28 & 1.29 & 1.18 & 1.18 & 1.19 & 1.18 & 0.94 & 0.94 & 0.93 & 0.93 & 0.68 & 0.67 & 0.68 & 0.68 \\
\hline & 0.2 & 1.28 & 1.29 & 1.28 & 1.28 & 1.18 & 1.17 & 1.16 & 1.18 & 0.93 & 0.92 & 0.92 & 0.94 & 0.67 & 0.68 & 0.68 & 0.66 \\
\hline & 0.5 & 1.23 & 1.22 & 1.23 & 1.22 & 1.12 & 1.11 & 1.12 & 1.12 & 0.88 & 0.89 & 0.88 & 0.89 & 0.66 & 0.65 & 0.64 & 0.65 \\
\hline & 0.8 & 1.12 & 1.13 & 1.12 & 1.11 & 1.02 & 1.04 & 1.02 & 1.02 & 0.82 & 0.83 & 0.83 & 0.83 & 0.61 & 0.61 & 0.62 & 0.62 \\
\hline \multirow{4}{*}{0.2} & 0 & 1.29 & 1.28 & 1.28 & 1.28 & 1.18 & 1.19 & 1.17 & 1.18 & 0.92 & 0.93 & 0.92 & 0.93 & 0.68 & 0.67 & 0.68 & 0.66 \\
\hline & 0.2 & 1.28 & 1.27 & 1.26 & 1.27 & 1.16 & 1.16 & 1.16 & 1.16 & 0.92 & 0.91 & 0.92 & 0.92 & 0.66 & 0.67 & 0.66 & 0.68 \\
\hline & 0.5 & 1.21 & 1.23 & 1.21 & 1.21 & 1.10 & 1.10 & 1.10 & 1.11 & 0.88 & 0.89 & 0.88 & 0.89 & 0.64 & 0.65 & 0.64 & 0.65 \\
\hline & 0.8 & 1.10 & 1.11 & 1.11 & 1.11 & 1.01 & 1.01 & 1.01 & 1.02 & 0.82 & 0.81 & 0.82 & 0.82 & 0.62 & 0.61 & 0.61 & 0.61 \\
\hline \multirow{4}{*}{0.5} & 0 & 1.22 & 1.23 & 1.23 & 1.22 & 1.11 & 1.11 & 1.11 & 1.12 & 0.89 & 0.89 & 0.89 & 0.88 & 0.65 & 0.65 & 0.66 & 0.65 \\
\hline & 0.2 & 1.22 & 1.21 & 1.21 & 1.22 & 1.11 & 1.10 & 1.10 & 1.10 & 0.88 & 0.88 & 0.87 & 0.88 & 0.64 & 0.65 & 0.66 & 0.64 \\
\hline & 0.5 & 1.16 & 1.15 & 1.15 & 1.14 & 1.05 & 1.05 & 1.06 & 1.06 & 0.84 & 0.85 & 0.84 & 0.85 & 0.64 & 0.62 & 0.63 & 0.63 \\
\hline & 0.8 & 1.06 & 1.04 & 1.05 & 1.06 & 0.97 & 0.96 & 0.96 & 0.96 & 0.79 & 0.80 & 0.79 & 0.78 & 0.60 & 0.59 & 0.59 & 0.60 \\
\hline \multirow{4}{*}{0.8} & 0 & 1.11 & 1.12 & 1.10 & 1.11 & 1.00 & 1.01 & 1.02 & 1.02 & 0.82 & 0.82 & 0.82 & 0.83 & 0.62 & 0.60 & 0.63 & 0.62 \\
\hline & 0.2 & 1.10 & 1.09 & 1.09 & 1.11 & 1.01 & 1.01 & 1.00 & 1.01 & 0.82 & 0.83 & 0.83 & 0.82 & 0.61 & 0.61 & 0.62 & 0.62 \\
\hline & 0.5 & 1.04 & 1.05 & 1.04 & 1.05 & 0.95 & 0.97 & 0.96 & 0.95 & 0.79 & 0.79 & 0.78 & 0.79 & 0.59 & 0.59 & 0.60 & 0.60 \\
\hline & 0.8 & 0.97 & 0.96 & 0.96 & 0.97 & 0.89 & 0.90 & 0.88 & 0.89 & 0.74 & 0.74 & 0.74 & 0.74 & 0.57 & 0.58 & 0.56 & 0.56 \\
\hline
\end{tabular}

Note: ICC = Intraclass Correlation Coefficient. 
Table 6 cont.

Average Design Effect values for the cross-level interaction: Poisson Distribution conditions $(\mathrm{m}=5, \mathrm{k}=75)$

\begin{tabular}{|c|c|c|c|c|c|c|c|c|c|c|c|c|c|c|c|c|c|}
\hline \multirow{4}{*}{$\begin{array}{c}\text { L1 } \\
\text { Effect }\end{array}$} & \multirow{4}{*}{$\begin{array}{c}\text { Interaction } \\
\text { Effect }\end{array}$} & \multicolumn{16}{|c|}{ ICC Values } \\
\hline & & \multicolumn{4}{|c|}{0} & \multicolumn{4}{|c|}{0.1} & \multicolumn{4}{|c|}{0.3} & \multicolumn{4}{|c|}{0.5} \\
\hline & & \multicolumn{12}{|c|}{ L2 Effect } & & & & \\
\hline & & 0 & 0.2 & 0.5 & 0.8 & 0 & 0.2 & 0.5 & 0.8 & 0 & 0.2 & 0.5 & 0.8 & 0 & 0.2 & 0.5 & 0.8 \\
\hline \multirow{4}{*}{0} & 0 & 1.26 & 1.27 & 1.26 & 1.28 & 1.16 & 1.16 & 1.17 & 1.17 & 0.92 & 0.92 & 0.91 & 0.92 & 0.66 & 0.66 & 0.66 & 0.65 \\
\hline & 0.2 & 1.27 & 1.27 & 1.27 & 1.26 & 1.16 & 1.15 & 1.16 & 1.17 & 0.91 & 0.91 & 0.90 & 0.90 & 0.66 & 0.65 & 0.65 & 0.66 \\
\hline & 0.5 & 1.20 & 1.21 & 1.20 & 1.22 & 1.09 & 1.09 & 1.10 & 1.09 & 0.86 & 0.87 & 0.87 & 0.87 & 0.62 & 0.63 & 0.64 & 0.65 \\
\hline & 0.8 & 1.09 & 1.09 & 1.09 & 1.10 & 1.00 & 1.00 & 1.00 & 1.00 & 0.80 & 0.81 & 0.80 & 0.81 & 0.60 & 0.60 & 0.60 & 0.60 \\
\hline \multirow{4}{*}{0.2} & 0 & 1.26 & 1.27 & 1.27 & 1.28 & 1.16 & 1.16 & 1.15 & 1.15 & 0.91 & 0.91 & 0.90 & 0.91 & 0.66 & 0.65 & 0.65 & 0.65 \\
\hline & 0.2 & 1.26 & 1.26 & 1.25 & 1.25 & 1.13 & 1.14 & 1.14 & 1.14 & 0.90 & 0.90 & 0.89 & 0.90 & 0.65 & 0.65 & 0.65 & 0.65 \\
\hline & 0.5 & 1.20 & 1.20 & 1.20 & 1.20 & 1.09 & 1.09 & 1.09 & 1.08 & 0.85 & 0.86 & 0.87 & 0.86 & 0.63 & 0.63 & 0.63 & 0.63 \\
\hline & 0.8 & 1.08 & 1.09 & 1.08 & 1.08 & 0.99 & 0.99 & 1.00 & 1.00 & 0.80 & 0.79 & 0.79 & 0.80 & 0.60 & 0.59 & 0.60 & 0.61 \\
\hline \multirow{4}{*}{0.5} & 0 & 1.21 & 1.21 & 1.22 & 1.20 & 1.10 & 1.10 & 1.09 & 1.10 & 0.86 & 0.87 & 0.87 & 0.87 & 0.63 & 0.63 & 0.63 & 0.63 \\
\hline & 0.2 & 1.20 & 1.18 & 1.19 & 1.20 & 1.08 & 1.09 & 1.09 & 1.08 & 0.86 & 0.86 & 0.86 & 0.86 & 0.63 & 0.63 & 0.63 & 0.63 \\
\hline & 0.5 & 1.13 & 1.13 & 1.12 & 1.13 & 1.02 & 1.03 & 1.02 & 1.04 & 0.83 & 0.83 & 0.83 & 0.82 & 0.61 & 0.62 & 0.61 & 0.61 \\
\hline & 0.8 & 1.03 & 1.04 & 1.03 & 1.02 & 0.95 & 0.94 & 0.94 & 0.95 & 0.77 & 0.77 & 0.77 & 0.77 & 0.58 & 0.58 & 0.58 & 0.59 \\
\hline \multirow{4}{*}{0.8} & 0 & 1.09 & 1.08 & 1.09 & 1.08 & 0.99 & 0.99 & 0.99 & 1.00 & 0.80 & 0.79 & 0.80 & 0.80 & 0.60 & 0.60 & 0.60 & 0.61 \\
\hline & 0.2 & 1.07 & 1.08 & 1.08 & 1.08 & 0.98 & 0.99 & 0.97 & 0.99 & 0.79 & 0.80 & 0.80 & 0.80 & 0.59 & 0.59 & 0.59 & 0.60 \\
\hline & 0.5 & 1.02 & 1.03 & 1.02 & 1.02 & 0.94 & 0.94 & 0.94 & 0.94 & 0.77 & 0.77 & 0.77 & 0.77 & 0.58 & 0.58 & 0.58 & 0.57 \\
\hline & 0.8 & 0.93 & 0.94 & 0.94 & 0.94 & 0.87 & 0.87 & 0.87 & 0.86 & 0.72 & 0.72 & 0.72 & 0.72 & 0.55 & 0.54 & 0.56 & 0.55 \\
\hline
\end{tabular}

Note: ICC = Intraclass Correlation Coefficient. 
Table 6 cont.

Average Design Effect values for the cross-level interaction: Poisson Distribution conditions $(\mathrm{m}=5, \mathrm{k}=100)$

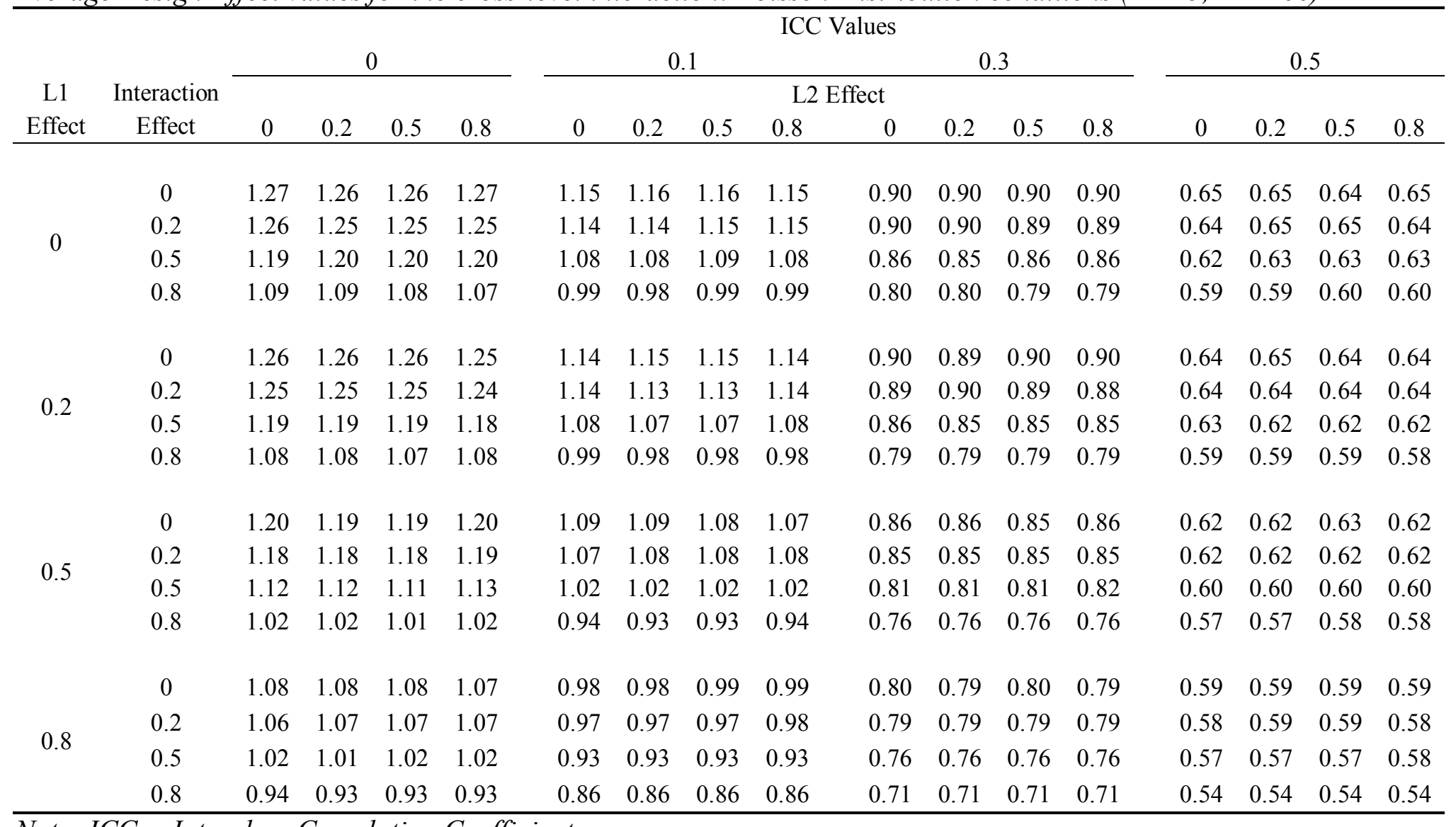

Note: ICC = Intraclass Correlation Coefficient. 
Table 6 cont.

Average Design Effect values for the cross-level interaction: Poisson Distribution conditions $(\mathrm{m}=10, \mathrm{k}=25)$

\begin{tabular}{|c|c|c|c|c|c|c|c|c|c|c|c|c|c|c|c|c|c|}
\hline \multirow{4}{*}{$\begin{array}{c}\text { L1 } \\
\text { Effect }\end{array}$} & \multirow{4}{*}{$\begin{array}{c}\text { Interaction } \\
\text { Effect }\end{array}$} & \multicolumn{16}{|c|}{ ICC Values } \\
\hline & & \multicolumn{4}{|c|}{0} & \multicolumn{4}{|c|}{0.1} & \multicolumn{4}{|c|}{0.3} & \multicolumn{4}{|c|}{0.5} \\
\hline & & & & & & \multicolumn{8}{|c|}{ L2 Effect } & & & & \\
\hline & & 0 & 0.2 & 0.5 & 0.8 & 0 & 0.2 & 0.5 & 0.8 & 0 & 0.2 & 0.5 & 0.8 & 0 & 0.2 & 0.5 & 0.8 \\
\hline \multirow{4}{*}{0} & 0 & 1.15 & 1.14 & 1.13 & 1.14 & 1.05 & 1.05 & 1.05 & 1.06 & 0.84 & 0.83 & 0.84 & 0.83 & 0.61 & 0.62 & 0.62 & 0.62 \\
\hline & 0.2 & 1.14 & 1.14 & 1.14 & 1.14 & 1.03 & 1.05 & 1.06 & 1.05 & 0.85 & 0.83 & 0.83 & 0.84 & 0.62 & 0.61 & 0.61 & 0.61 \\
\hline & 0.5 & 1.13 & 1.12 & 1.12 & 1.12 & 1.02 & 1.02 & 1.02 & 1.03 & 0.81 & 0.82 & 0.81 & 0.83 & 0.61 & 0.61 & 0.61 & 0.60 \\
\hline & 0.8 & 1.07 & 1.07 & 1.07 & 1.07 & 0.98 & 0.98 & 0.97 & 0.98 & 0.78 & 0.79 & 0.79 & 0.80 & 0.60 & 0.59 & 0.60 & 0.59 \\
\hline \multirow{4}{*}{0.2} & 0 & 1.14 & 1.14 & 1.14 & 1.14 & 1.04 & 1.04 & 1.05 & 1.05 & 0.84 & 0.82 & 0.83 & 0.84 & 0.62 & 0.61 & 0.62 & 0.62 \\
\hline & 0.2 & 1.14 & 1.13 & 1.14 & 1.14 & 1.06 & 1.05 & 1.04 & 1.04 & 0.84 & 0.84 & 0.83 & 0.83 & 0.61 & 0.60 & 0.62 & 0.62 \\
\hline & 0.5 & 1.11 & 1.11 & 1.11 & 1.11 & 1.01 & 1.02 & 1.02 & 1.02 & 0.81 & 0.82 & 0.81 & 0.81 & 0.60 & 0.60 & 0.60 & 0.61 \\
\hline & 0.8 & 1.06 & 1.06 & 1.07 & 1.06 & 0.98 & 0.97 & 0.98 & 0.97 & 0.78 & 0.79 & 0.77 & 0.78 & 0.60 & 0.59 & 0.59 & 0.59 \\
\hline \multirow{4}{*}{0.5} & 0 & 1.10 & 1.11 & 1.12 & 1.11 & 1.02 & 1.01 & 1.02 & 1.02 & 0.82 & 0.82 & 0.81 & 0.82 & 0.61 & 0.60 & 0.61 & 0.60 \\
\hline & 0.2 & 1.11 & 1.10 & 1.11 & 1.10 & 1.01 & 1.02 & 1.02 & 1.02 & 0.81 & 0.81 & 0.81 & 0.81 & 0.61 & 0.61 & 0.60 & 0.60 \\
\hline & 0.5 & 1.09 & 1.08 & 1.07 & 1.08 & 0.99 & 0.99 & 0.99 & 0.99 & 0.79 & 0.80 & 0.79 & 0.80 & 0.59 & 0.60 & 0.59 & 0.59 \\
\hline & 0.8 & 1.04 & 1.03 & 1.03 & 1.05 & 0.94 & 0.95 & 0.95 & 0.95 & 0.77 & 0.77 & 0.77 & 0.76 & 0.58 & 0.58 & 0.58 & 0.57 \\
\hline \multirow{4}{*}{0.8} & 0 & 1.06 & 1.06 & 1.06 & 1.06 & 0.97 & 0.97 & 0.98 & 0.97 & 0.79 & 0.78 & 0.79 & 0.79 & 0.58 & 0.59 & 0.59 & 0.58 \\
\hline & 0.2 & 1.06 & 1.06 & 1.05 & 1.06 & 0.97 & 0.96 & 0.96 & 0.96 & 0.78 & 0.78 & 0.78 & 0.78 & 0.58 & 0.59 & 0.59 & 0.59 \\
\hline & 0.5 & 1.04 & 1.02 & 1.04 & 1.04 & 0.95 & 0.95 & 0.95 & 0.95 & 0.77 & 0.76 & 0.77 & 0.76 & 0.58 & 0.57 & 0.58 & 0.57 \\
\hline & 0.8 & 0.98 & 0.98 & 0.98 & 0.98 & 0.90 & 0.91 & 0.91 & 0.90 & 0.74 & 0.75 & 0.74 & 0.74 & 0.56 & 0.57 & 0.57 & 0.56 \\
\hline
\end{tabular}

Note: ICC = Intraclass Correlation Coefficient. 
Table 6 cont.

Average Design Effect values for the cross-level interaction: Poisson Distribution conditions $(\mathrm{m}=10, \mathrm{k}=50)$

\begin{tabular}{|c|c|c|c|c|c|c|c|c|c|c|c|c|c|c|c|c|c|}
\hline \multirow{4}{*}{$\begin{array}{c}\text { L1 } \\
\text { Effect }\end{array}$} & \multirow{4}{*}{$\begin{array}{c}\text { Interaction } \\
\text { Effect }\end{array}$} & \multicolumn{16}{|c|}{ ICC Values } \\
\hline & & \multicolumn{4}{|c|}{0} & \multicolumn{4}{|c|}{0.1} & \multicolumn{4}{|c|}{0.3} & \multicolumn{4}{|c|}{0.5} \\
\hline & & \multicolumn{12}{|c|}{ L2 Effect } & & & & \\
\hline & & 0 & 0.2 & 0.5 & 0.8 & 0 & 0.2 & 0.5 & 0.8 & 0 & 0.2 & 0.5 & 0.8 & 0 & 0.2 & 0.5 & 0.8 \\
\hline \multirow{4}{*}{0} & 0 & 1.12 & 1.12 & 1.12 & 1.12 & 1.03 & 1.02 & 1.03 & 1.03 & 0.80 & 0.80 & 0.81 & 0.81 & 0.59 & 0.58 & 0.58 & 0.58 \\
\hline & 0.2 & 1.12 & 1.12 & 1.12 & 1.12 & 1.02 & 1.02 & 1.02 & 1.03 & 0.80 & 0.81 & 0.80 & 0.80 & 0.58 & 0.59 & 0.59 & 0.59 \\
\hline & 0.5 & 1.10 & 1.09 & 1.10 & 1.10 & 1.00 & 1.00 & 0.99 & 0.99 & 0.79 & 0.79 & 0.79 & 0.79 & 0.57 & 0.58 & 0.58 & 0.58 \\
\hline & 0.8 & 1.04 & 1.04 & 1.04 & 1.03 & 0.95 & 0.94 & 0.94 & 0.95 & 0.76 & 0.75 & 0.76 & 0.76 & 0.56 & 0.56 & 0.56 & 0.56 \\
\hline \multirow{4}{*}{0.2} & 0 & 1.12 & 1.12 & 1.11 & 1.12 & 1.02 & 1.02 & 1.02 & 1.02 & 0.80 & 0.80 & 0.80 & 0.81 & 0.58 & 0.58 & 0.59 & 0.58 \\
\hline & 0.2 & 1.12 & 1.12 & 1.12 & 1.11 & 1.02 & 1.01 & 1.01 & 1.02 & 0.80 & 0.80 & 0.80 & 0.80 & 0.58 & 0.58 & 0.58 & 0.58 \\
\hline & 0.5 & 1.08 & 1.09 & 1.08 & 1.09 & 0.99 & 0.99 & 1.00 & 0.98 & 0.78 & 0.78 & 0.78 & 0.79 & 0.57 & 0.57 & 0.57 & 0.57 \\
\hline & 0.8 & 1.04 & 1.03 & 1.03 & 1.05 & 0.94 & 0.94 & 0.94 & 0.94 & 0.75 & 0.75 & 0.76 & 0.75 & 0.56 & 0.56 & 0.56 & 0.56 \\
\hline \multirow{4}{*}{0.5} & 0 & 1.10 & 1.09 & 1.10 & 1.09 & 0.99 & 0.99 & 0.99 & 0.99 & 0.79 & 0.79 & 0.79 & 0.79 & 0.57 & 0.57 & 0.58 & 0.58 \\
\hline & 0.2 & 1.09 & 1.09 & 1.09 & 1.09 & 0.99 & 0.99 & 0.98 & 0.99 & 0.78 & 0.79 & 0.78 & 0.78 & 0.57 & 0.57 & 0.57 & 0.57 \\
\hline & 0.5 & 1.06 & 1.06 & 1.05 & 1.06 & 0.96 & 0.96 & 0.96 & 0.96 & 0.76 & 0.76 & 0.77 & 0.77 & 0.57 & 0.56 & 0.55 & 0.56 \\
\hline & 0.8 & 1.00 & 1.00 & 1.01 & 1.00 & 0.91 & 0.92 & 0.92 & 0.92 & 0.74 & 0.73 & 0.74 & 0.74 & 0.54 & 0.55 & 0.55 & 0.55 \\
\hline \multirow{4}{*}{0.8} & 0 & 1.04 & 1.02 & 1.03 & 1.04 & 0.94 & 0.94 & 0.94 & 0.94 & 0.76 & 0.76 & 0.76 & 0.75 & 0.56 & 0.55 & 0.55 & 0.56 \\
\hline & 0.2 & 1.03 & 1.03 & 1.03 & 1.03 & 0.94 & 0.93 & 0.94 & 0.94 & 0.75 & 0.75 & 0.75 & 0.76 & 0.55 & 0.56 & 0.55 & 0.55 \\
\hline & 0.5 & 1.00 & 1.00 & 1.00 & 0.99 & 0.91 & 0.91 & 0.91 & 0.92 & 0.74 & 0.74 & 0.73 & 0.74 & 0.54 & 0.54 & 0.55 & 0.54 \\
\hline & 0.8 & 0.95 & 0.96 & 0.95 & 0.96 & 0.87 & 0.87 & 0.88 & 0.87 & 0.71 & 0.71 & 0.72 & 0.71 & 0.53 & 0.53 & 0.54 & 0.53 \\
\hline
\end{tabular}

Note: ICC = Intraclass Correlation Coefficient. 
Table 6 cont.

Average Design Effect values for the cross-level interaction: Poisson Distribution conditions $(\mathrm{m}=10, \mathrm{k}=75)$

\begin{tabular}{|c|c|c|c|c|c|c|c|c|c|c|c|c|c|c|c|c|c|}
\hline \multirow{4}{*}{$\begin{array}{c}\text { L1 } \\
\text { Effect }\end{array}$} & \multirow{4}{*}{$\begin{array}{c}\text { Interaction } \\
\text { Effect }\end{array}$} & \multicolumn{16}{|c|}{ ICC Values } \\
\hline & & \multicolumn{4}{|c|}{0} & \multicolumn{4}{|c|}{0.1} & \multicolumn{4}{|c|}{0.3} & \multicolumn{4}{|c|}{0.5} \\
\hline & & & & & & \multicolumn{8}{|c|}{ L2 Effect } & & & & \\
\hline & & 0 & 0.2 & 0.5 & 0.8 & 0 & 0.2 & 0.5 & 0.8 & 0 & 0.2 & 0.5 & 0.8 & 0 & 0.2 & 0.5 & 0.8 \\
\hline \multirow{4}{*}{0} & 0 & 1.12 & 1.12 & 1.11 & 1.12 & 1.01 & 1.02 & 1.02 & 1.02 & 0.79 & 0.80 & 0.80 & 0.80 & 0.58 & 0.58 & 0.58 & 0.58 \\
\hline & 0.2 & 1.12 & 1.12 & 1.12 & 1.12 & 1.01 & 1.01 & 1.01 & 1.01 & 0.80 & 0.80 & 0.79 & 0.79 & 0.57 & 0.57 & 0.57 & 0.58 \\
\hline & 0.5 & 1.08 & 1.09 & 1.09 & 1.09 & 0.98 & 0.98 & 0.98 & 0.98 & 0.78 & 0.78 & 0.77 & 0.78 & 0.57 & 0.57 & 0.56 & 0.56 \\
\hline & 0.8 & 1.04 & 1.03 & 1.03 & 1.03 & 0.94 & 0.93 & 0.94 & 0.94 & 0.75 & 0.75 & 0.75 & 0.74 & 0.54 & 0.55 & 0.55 & 0.55 \\
\hline \multirow{4}{*}{0.2} & 0 & 1.11 & 1.12 & 1.11 & 1.11 & 1.01 & 1.01 & 1.01 & 1.01 & 0.79 & 0.79 & 0.79 & 0.79 & 0.57 & 0.57 & 0.58 & 0.58 \\
\hline & 0.2 & 1.11 & 1.10 & 1.11 & 1.11 & 1.00 & 1.01 & 1.00 & 1.01 & 0.79 & 0.79 & 0.79 & 0.79 & 0.57 & 0.57 & 0.57 & 0.57 \\
\hline & 0.5 & 1.09 & 1.08 & 1.08 & 1.08 & 0.97 & 0.98 & 0.98 & 0.98 & 0.78 & 0.77 & 0.77 & 0.78 & 0.57 & 0.56 & 0.56 & 0.56 \\
\hline & 0.8 & 1.02 & 1.02 & 1.02 & 1.03 & 0.93 & 0.93 & 0.93 & 0.93 & 0.74 & 0.74 & 0.74 & 0.74 & 0.54 & 0.54 & 0.55 & 0.54 \\
\hline \multirow{4}{*}{0.5} & 0 & 1.08 & 1.09 & 1.09 & 1.09 & 0.98 & 0.97 & 0.98 & 0.98 & 0.77 & 0.78 & 0.78 & 0.77 & 0.57 & 0.56 & 0.56 & 0.56 \\
\hline & 0.2 & 1.08 & 1.08 & 1.07 & 1.08 & 0.98 & 0.97 & 0.98 & 0.98 & 0.77 & 0.77 & 0.77 & 0.78 & 0.56 & 0.56 & 0.56 & 0.56 \\
\hline & 0.5 & 1.04 & 1.05 & 1.05 & 1.06 & 0.95 & 0.95 & 0.95 & 0.95 & 0.75 & 0.76 & 0.76 & 0.75 & 0.55 & 0.55 & 0.56 & 0.55 \\
\hline & 0.8 & 0.99 & 1.00 & 0.99 & 0.99 & 0.91 & 0.90 & 0.91 & 0.91 & 0.73 & 0.73 & 0.72 & 0.73 & 0.54 & 0.54 & 0.54 & 0.54 \\
\hline \multirow{4}{*}{0.8} & 0 & 1.02 & 1.03 & 1.03 & 1.03 & 0.93 & 0.94 & 0.94 & 0.93 & 0.75 & 0.74 & 0.75 & 0.74 & 0.54 & 0.55 & 0.55 & 0.55 \\
\hline & 0.2 & 1.02 & 1.02 & 1.02 & 1.02 & 0.93 & 0.93 & 0.92 & 0.93 & 0.74 & 0.74 & 0.74 & 0.74 & 0.54 & 0.55 & 0.54 & 0.55 \\
\hline & 0.5 & 1.00 & 0.99 & 0.99 & 0.99 & 0.90 & 0.91 & 0.90 & 0.90 & 0.72 & 0.73 & 0.73 & 0.73 & 0.53 & 0.54 & 0.54 & 0.54 \\
\hline & 0.8 & 0.94 & 0.94 & 0.95 & 0.94 & 0.86 & 0.86 & 0.86 & 0.87 & 0.70 & 0.70 & 0.70 & 0.70 & 0.52 & 0.52 & 0.52 & 0.52 \\
\hline
\end{tabular}

Note: ICC = Intraclass Correlation Coefficient. 
Table 6 cont.

Average Design Effect values for the cross-level interaction: Poisson Distribution conditions $(\mathrm{m}=10, \mathrm{k}=100)$

\begin{tabular}{|c|c|c|c|c|c|c|c|c|c|c|c|c|c|c|c|c|c|}
\hline \multirow{4}{*}{$\begin{array}{c}\text { L1 } \\
\text { Effect }\end{array}$} & \multirow{4}{*}{$\begin{array}{l}\text { Interaction } \\
\text { Effect }\end{array}$} & \multicolumn{16}{|c|}{ ICC Values } \\
\hline & & \multicolumn{4}{|c|}{0} & \multirow{2}{*}{\multicolumn{3}{|c|}{0.1}} & & \multicolumn{4}{|c|}{0.3} & \multicolumn{4}{|c|}{0.5} \\
\hline & & & & & & & & & \multicolumn{2}{|c|}{ L2 Effect } & & & & & & & \\
\hline & & 0 & 0.2 & 0.5 & 0.8 & 0 & 0.2 & 0.5 & 0.8 & 0 & 0.2 & 0.5 & 0.8 & 0 & 0.2 & 0.5 & 0.8 \\
\hline \multirow{4}{*}{0} & 0 & 1.12 & 1.11 & 1.12 & 1.12 & 1.01 & 1.02 & 1.01 & 1.02 & 0.79 & 0.79 & 0.80 & 0.79 & 0.57 & 0.57 & 0.57 & 0.57 \\
\hline & 0.2 & 1.12 & 1.12 & 1.11 & 1.11 & 1.01 & 1.01 & 1.00 & 1.01 & 0.79 & 0.79 & 0.79 & 0.79 & 0.57 & 0.57 & 0.57 & 0.57 \\
\hline & 0.5 & 1.08 & 1.08 & 1.08 & 1.08 & 0.98 & 0.98 & 0.98 & 0.98 & 0.77 & 0.77 & 0.77 & 0.77 & 0.56 & 0.56 & 0.56 & 0.56 \\
\hline & 0.8 & 1.03 & 1.03 & 1.02 & 1.03 & 0.93 & 0.93 & 0.94 & 0.93 & 0.74 & 0.74 & 0.74 & 0.74 & 0.54 & 0.55 & 0.55 & 0.54 \\
\hline \multirow{4}{*}{0.2} & 0 & 1.11 & 1.11 & 1.11 & 1.12 & 1.01 & 1.01 & 1.01 & 1.01 & 0.79 & 0.79 & 0.79 & 0.79 & 0.57 & 0.57 & 0.57 & 0.57 \\
\hline & 0.2 & 1.11 & 1.10 & 1.11 & 1.11 & 1.00 & 1.00 & 1.00 & 1.00 & 0.78 & 0.78 & 0.78 & 0.79 & 0.57 & 0.57 & 0.57 & 0.57 \\
\hline & 0.5 & 1.07 & 1.07 & 1.08 & 1.08 & 0.98 & 0.97 & 0.97 & 0.98 & 0.77 & 0.77 & 0.77 & 0.77 & 0.56 & 0.55 & 0.56 & 0.55 \\
\hline & 0.8 & 1.01 & 1.02 & 1.01 & 1.02 & 0.93 & 0.93 & 0.93 & 0.92 & 0.74 & 0.74 & 0.73 & 0.74 & 0.54 & 0.54 & 0.54 & 0.55 \\
\hline \multirow{4}{*}{0.5} & 0 & 1.09 & 1.08 & 1.09 & 1.09 & 0.98 & 0.98 & 0.98 & 0.98 & 0.77 & 0.77 & 0.77 & 0.78 & 0.56 & 0.56 & 0.56 & 0.56 \\
\hline & 0.2 & 1.07 & 1.08 & 1.07 & 1.08 & 0.97 & 0.97 & 0.98 & 0.97 & 0.77 & 0.77 & 0.77 & 0.77 & 0.55 & 0.56 & 0.56 & 0.55 \\
\hline & 0.5 & 1.04 & 1.04 & 1.04 & 1.05 & 0.95 & 0.95 & 0.95 & 0.95 & 0.75 & 0.75 & 0.76 & 0.75 & 0.55 & 0.55 & 0.54 & 0.55 \\
\hline & 0.8 & 0.98 & 0.98 & 0.99 & 0.98 & 0.90 & 0.90 & 0.90 & 0.90 & 0.72 & 0.72 & 0.72 & 0.72 & 0.53 & 0.53 & 0.53 & 0.53 \\
\hline \multirow{4}{*}{0.8} & 0 & 1.02 & 1.02 & 1.02 & 1.02 & 0.93 & 0.93 & 0.93 & 0.92 & 0.74 & 0.74 & 0.74 & 0.74 & 0.54 & 0.54 & 0.54 & 0.55 \\
\hline & 0.2 & 1.01 & 1.01 & 1.02 & 1.02 & 0.92 & 0.92 & 0.92 & 0.93 & 0.74 & 0.73 & 0.74 & 0.74 & 0.54 & 0.54 & 0.54 & 0.54 \\
\hline & 0.5 & 0.99 & 0.98 & 0.98 & 0.99 & 0.90 & 0.90 & 0.90 & 0.90 & 0.73 & 0.73 & 0.72 & 0.72 & 0.53 & 0.53 & 0.53 & 0.53 \\
\hline & 0.8 & 0.94 & 0.93 & 0.94 & 0.94 & 0.86 & 0.86 & 0.86 & 0.86 & 0.69 & 0.69 & 0.69 & 0.69 & 0.52 & 0.52 & 0.52 & 0.52 \\
\hline
\end{tabular}

Note: ICC = Intraclass Correlation Coefficient. 
Table 6 cont.

Average Design Effect values for the cross-level interaction: Poisson Distribution conditions $(\mathrm{m}=20, \mathrm{k}=25)$

\begin{tabular}{|c|c|c|c|c|c|c|c|c|c|c|c|c|c|c|c|c|c|}
\hline \multirow{4}{*}{$\begin{array}{c}\text { L1 } \\
\text { Effect }\end{array}$} & \multirow{4}{*}{$\begin{array}{c}\text { Interaction } \\
\text { Effect }\end{array}$} & \multicolumn{16}{|c|}{ ICC Values } \\
\hline & & \multicolumn{4}{|c|}{0} & \multicolumn{4}{|c|}{0.1} & \multicolumn{4}{|c|}{0.3} & \multicolumn{4}{|c|}{0.5} \\
\hline & & \multicolumn{12}{|c|}{ L2 Effect } & & & & \\
\hline & & 0 & 0.2 & 0.5 & 0.8 & 0 & 0.2 & 0.5 & 0.8 & 0 & 0.2 & 0.5 & 0.8 & 0 & 0.2 & 0.5 & 0.8 \\
\hline \multirow{4}{*}{0} & 0 & 1.06 & 1.06 & 1.07 & 1.07 & 0.97 & 0.97 & 0.98 & 0.97 & 0.77 & 0.78 & 0.78 & 0.78 & 0.57 & 0.57 & 0.57 & 0.57 \\
\hline & 0.2 & 1.07 & 1.07 & 1.06 & 1.06 & 0.97 & 0.97 & 0.98 & 0.97 & 0.77 & 0.78 & 0.77 & 0.78 & 0.57 & 0.57 & 0.57 & 0.57 \\
\hline & 0.5 & 1.05 & 1.06 & 1.04 & 1.05 & 0.96 & 0.96 & 0.96 & 0.96 & 0.77 & 0.76 & 0.77 & 0.77 & 0.57 & 0.56 & 0.56 & 0.56 \\
\hline & 0.8 & 1.03 & 1.03 & 1.03 & 1.03 & 0.94 & 0.94 & 0.93 & 0.93 & 0.75 & 0.75 & 0.76 & 0.75 & 0.56 & 0.56 & 0.56 & 0.56 \\
\hline \multirow{4}{*}{0.2} & 0 & 1.07 & 1.07 & 1.07 & 1.06 & 0.98 & 0.97 & 0.97 & 0.97 & 0.78 & 0.77 & 0.78 & 0.77 & 0.57 & 0.57 & 0.57 & 0.57 \\
\hline & 0.2 & 1.06 & 1.06 & 1.06 & 1.07 & 0.97 & 0.96 & 0.97 & 0.97 & 0.77 & 0.77 & 0.77 & 0.77 & 0.57 & 0.57 & 0.57 & 0.57 \\
\hline & 0.5 & 1.05 & 1.05 & 1.05 & 1.05 & 0.96 & 0.96 & 0.96 & 0.96 & 0.76 & 0.77 & 0.76 & 0.76 & 0.57 & 0.56 & 0.56 & 0.56 \\
\hline & 0.8 & 1.01 & 1.03 & 1.02 & 1.02 & 0.93 & 0.93 & 0.93 & 0.94 & 0.75 & 0.75 & 0.74 & 0.75 & 0.56 & 0.56 & 0.55 & 0.55 \\
\hline \multirow{4}{*}{0.5} & 0 & 1.05 & 1.05 & 1.05 & 1.05 & 0.96 & 0.96 & 0.97 & 0.96 & 0.76 & 0.77 & 0.76 & 0.76 & 0.56 & 0.56 & 0.57 & 0.56 \\
\hline & 0.2 & 1.05 & 1.05 & 1.05 & 1.05 & 0.96 & 0.96 & 0.95 & 0.96 & 0.77 & 0.76 & 0.77 & 0.76 & 0.57 & 0.57 & 0.56 & 0.56 \\
\hline & 0.5 & 1.03 & 1.03 & 1.04 & 1.04 & 0.94 & 0.95 & 0.95 & 0.95 & 0.76 & 0.76 & 0.76 & 0.76 & 0.56 & 0.56 & 0.57 & 0.56 \\
\hline & 0.8 & 1.02 & 1.02 & 1.01 & 1.01 & 0.93 & 0.92 & 0.93 & 0.92 & 0.75 & 0.75 & 0.75 & 0.74 & 0.55 & 0.55 & 0.55 & 0.55 \\
\hline \multirow{4}{*}{0.8} & 0 & 1.02 & 1.02 & 1.02 & 1.02 & 0.94 & 0.93 & 0.94 & 0.93 & 0.75 & 0.75 & 0.75 & 0.76 & 0.56 & 0.56 & 0.56 & 0.55 \\
\hline & 0.2 & 1.02 & 1.03 & 1.02 & 1.01 & 0.93 & 0.93 & 0.94 & 0.94 & 0.75 & 0.75 & 0.75 & 0.75 & 0.56 & 0.55 & 0.55 & 0.55 \\
\hline & 0.5 & 1.01 & 1.01 & 1.01 & 1.01 & 0.92 & 0.93 & 0.92 & 0.93 & 0.75 & 0.75 & 0.74 & 0.74 & 0.54 & 0.55 & 0.55 & 0.55 \\
\hline & 0.8 & 0.99 & 0.98 & 0.98 & 0.98 & 0.90 & 0.90 & 0.90 & 0.90 & 0.73 & 0.73 & 0.73 & 0.73 & 0.55 & 0.55 & 0.55 & 0.55 \\
\hline
\end{tabular}

Note: ICC = Intraclass Correlation Coefficient. 
Table 6 cont.

Average Design Effect values for the cross-level interaction: Poisson Distribution conditions $(\mathrm{m}=20, \mathrm{k}=50)$

\begin{tabular}{|c|c|c|c|c|c|c|c|c|c|c|c|c|c|c|c|c|c|}
\hline \multirow{4}{*}{$\begin{array}{c}\text { L1 } \\
\text { Effect }\end{array}$} & \multirow{4}{*}{$\begin{array}{c}\text { Interaction } \\
\text { Effect }\end{array}$} & \multicolumn{16}{|c|}{ ICC Values } \\
\hline & & \multicolumn{4}{|c|}{0} & \multicolumn{4}{|c|}{0.1} & \multicolumn{4}{|c|}{0.3} & \multicolumn{4}{|c|}{0.5} \\
\hline & & \multicolumn{12}{|c|}{ L2 Effect } & & & & \\
\hline & & 0 & 0.2 & 0.5 & 0.8 & 0 & 0.2 & 0.5 & 0.8 & 0 & 0.2 & 0.5 & 0.8 & 0 & 0.2 & 0.5 & 0.8 \\
\hline \multirow{4}{*}{0} & 0 & 1.06 & 1.06 & 1.06 & 1.05 & 0.96 & 0.96 & 0.96 & 0.96 & 0.75 & 0.76 & 0.76 & 0.75 & 0.55 & 0.55 & 0.55 & 0.54 \\
\hline & 0.2 & 1.05 & 1.05 & 1.06 & 1.05 & 0.96 & 0.96 & 0.96 & 0.95 & 0.75 & 0.75 & 0.76 & 0.76 & 0.55 & 0.55 & 0.54 & 0.54 \\
\hline & 0.5 & 1.04 & 1.04 & 1.04 & 1.04 & 0.95 & 0.95 & 0.94 & 0.95 & 0.74 & 0.75 & 0.75 & 0.75 & 0.54 & 0.54 & 0.55 & 0.55 \\
\hline & 0.8 & 1.01 & 1.02 & 1.01 & 1.02 & 0.92 & 0.92 & 0.92 & 0.92 & 0.73 & 0.73 & 0.73 & 0.73 & 0.53 & 0.54 & 0.53 & 0.54 \\
\hline \multirow{4}{*}{0.2} & 0 & 1.06 & 1.06 & 1.05 & 1.05 & 0.96 & 0.96 & 0.96 & 0.96 & 0.75 & 0.75 & 0.76 & 0.76 & 0.55 & 0.55 & 0.54 & 0.55 \\
\hline & 0.2 & 1.05 & 1.05 & 1.06 & 1.06 & 0.96 & 0.95 & 0.96 & 0.95 & 0.75 & 0.75 & 0.75 & 0.75 & 0.54 & 0.55 & 0.55 & 0.54 \\
\hline & 0.5 & 1.04 & 1.04 & 1.04 & 1.04 & 0.94 & 0.94 & 0.94 & 0.94 & 0.74 & 0.74 & 0.74 & 0.74 & 0.54 & 0.54 & 0.54 & 0.54 \\
\hline & 0.8 & 1.01 & 1.02 & 1.01 & 1.01 & 0.92 & 0.92 & 0.92 & 0.92 & 0.73 & 0.73 & 0.73 & 0.73 & 0.53 & 0.53 & 0.53 & 0.53 \\
\hline \multirow{4}{*}{0.5} & 0 & 1.04 & 1.04 & 1.04 & 1.04 & 0.95 & 0.94 & 0.94 & 0.95 & 0.74 & 0.75 & 0.74 & 0.74 & 0.54 & 0.54 & 0.55 & 0.55 \\
\hline & 0.2 & 1.04 & 1.04 & 1.04 & 1.04 & 0.94 & 0.94 & 0.94 & 0.94 & 0.74 & 0.75 & 0.74 & 0.75 & 0.54 & 0.54 & 0.54 & 0.54 \\
\hline & 0.5 & 1.02 & 1.02 & 1.02 & 1.03 & 0.93 & 0.93 & 0.93 & 0.93 & 0.74 & 0.73 & 0.74 & 0.73 & 0.54 & 0.53 & 0.53 & 0.54 \\
\hline & 0.8 & 1.00 & 1.00 & 1.00 & 1.00 & 0.91 & 0.91 & 0.90 & 0.91 & 0.72 & 0.73 & 0.72 & 0.72 & 0.53 & 0.53 & 0.53 & 0.53 \\
\hline \multirow{4}{*}{0.8} & 0 & 1.01 & 1.01 & 1.01 & 1.01 & 0.92 & 0.92 & 0.92 & 0.92 & 0.73 & 0.73 & 0.73 & 0.73 & 0.53 & 0.53 & 0.54 & 0.53 \\
\hline & 0.2 & 1.01 & 1.01 & 1.01 & 1.01 & 0.91 & 0.92 & 0.92 & 0.92 & 0.74 & 0.73 & 0.73 & 0.73 & 0.54 & 0.53 & 0.53 & 0.53 \\
\hline & 0.5 & 1.00 & 0.99 & 1.00 & 0.99 & 0.90 & 0.90 & 0.90 & 0.90 & 0.72 & 0.72 & 0.72 & 0.72 & 0.53 & 0.53 & 0.53 & 0.53 \\
\hline & 0.8 & 0.97 & 0.97 & 0.97 & 0.97 & 0.88 & 0.88 & 0.89 & 0.88 & 0.71 & 0.71 & 0.71 & 0.70 & 0.52 & 0.52 & 0.52 & 0.52 \\
\hline
\end{tabular}

Note: ICC = Intraclass Correlation Coefficient. 
Table 6 cont.

Average Design Effect values for the cross-level interaction: Poisson Distribution conditions $(\mathrm{m}=20, \mathrm{k}=75)$

\begin{tabular}{|c|c|c|c|c|c|c|c|c|c|c|c|c|c|c|c|c|c|}
\hline \multirow{4}{*}{$\begin{array}{c}\text { L1 } \\
\text { Effect }\end{array}$} & \multirow{4}{*}{$\begin{array}{c}\text { Interaction } \\
\text { Effect }\end{array}$} & \multicolumn{16}{|c|}{ ICC Values } \\
\hline & & \multicolumn{4}{|c|}{0} & \multicolumn{4}{|c|}{0.1} & \multicolumn{4}{|c|}{0.3} & \multicolumn{4}{|c|}{0.5} \\
\hline & & \multicolumn{12}{|c|}{ L2 Effect } & & & & \\
\hline & & 0 & 0.2 & 0.5 & 0.8 & 0 & 0.2 & 0.5 & 0.8 & 0 & 0.2 & 0.5 & 0.8 & 0 & 0.2 & 0.5 & 0.8 \\
\hline \multirow{4}{*}{0} & 0 & 1.05 & 1.06 & 1.06 & 1.06 & 0.96 & 0.96 & 0.96 & 0.96 & 0.75 & 0.75 & 0.75 & 0.75 & 0.54 & 0.54 & 0.54 & 0.54 \\
\hline & 0.2 & 1.06 & 1.05 & 1.05 & 1.06 & 0.95 & 0.95 & 0.96 & 0.96 & 0.75 & 0.75 & 0.75 & 0.75 & 0.54 & 0.54 & 0.54 & 0.54 \\
\hline & 0.5 & 1.04 & 1.04 & 1.04 & 1.04 & 0.94 & 0.94 & 0.94 & 0.94 & 0.74 & 0.74 & 0.74 & 0.74 & 0.53 & 0.54 & 0.54 & 0.53 \\
\hline & 0.8 & 1.01 & 1.01 & 1.01 & 1.02 & 0.92 & 0.92 & 0.91 & 0.92 & 0.73 & 0.73 & 0.72 & 0.72 & 0.53 & 0.53 & 0.53 & 0.53 \\
\hline \multirow{4}{*}{0.2} & 0 & 1.05 & 1.06 & 1.05 & 1.05 & 0.96 & 0.95 & 0.96 & 0.95 & 0.75 & 0.75 & 0.75 & 0.75 & 0.54 & 0.54 & 0.54 & 0.54 \\
\hline & 0.2 & 1.05 & 1.05 & 1.05 & 1.05 & 0.95 & 0.95 & 0.95 & 0.95 & 0.75 & 0.75 & 0.75 & 0.74 & 0.54 & 0.54 & 0.54 & 0.54 \\
\hline & 0.5 & 1.04 & 1.04 & 1.04 & 1.03 & 0.93 & 0.94 & 0.94 & 0.94 & 0.74 & 0.74 & 0.74 & 0.74 & 0.54 & 0.53 & 0.53 & 0.54 \\
\hline & 0.8 & 1.01 & 1.01 & 1.01 & 1.01 & 0.91 & 0.91 & 0.91 & 0.91 & 0.72 & 0.72 & 0.72 & 0.72 & 0.53 & 0.53 & 0.53 & 0.53 \\
\hline \multirow{4}{*}{0.5} & 0 & 1.04 & 1.04 & 1.04 & 1.04 & 0.94 & 0.94 & 0.94 & 0.94 & 0.74 & 0.74 & 0.74 & 0.74 & 0.53 & 0.53 & 0.53 & 0.54 \\
\hline & 0.2 & 1.03 & 1.04 & 1.03 & 1.03 & 0.93 & 0.94 & 0.94 & 0.94 & 0.74 & 0.73 & 0.74 & 0.74 & 0.53 & 0.53 & 0.54 & 0.53 \\
\hline & 0.5 & 1.02 & 1.02 & 1.02 & 1.02 & 0.93 & 0.92 & 0.92 & 0.93 & 0.73 & 0.73 & 0.73 & 0.73 & 0.53 & 0.53 & 0.53 & 0.53 \\
\hline & 0.8 & 0.99 & 0.99 & 0.99 & 0.99 & 0.90 & 0.90 & 0.90 & 0.90 & 0.72 & 0.71 & 0.71 & 0.71 & 0.52 & 0.52 & 0.52 & 0.52 \\
\hline \multirow{4}{*}{0.8} & 0 & 1.01 & 1.01 & 1.01 & 1.01 & 0.92 & 0.92 & 0.91 & 0.92 & 0.73 & 0.72 & 0.72 & 0.72 & 0.53 & 0.53 & 0.52 & 0.53 \\
\hline & 0.2 & 1.01 & 1.01 & 1.01 & 1.01 & 0.91 & 0.91 & 0.91 & 0.92 & 0.72 & 0.72 & 0.72 & 0.72 & 0.53 & 0.53 & 0.52 & 0.53 \\
\hline & 0.5 & 0.99 & 0.99 & 0.99 & 0.99 & 0.90 & 0.90 & 0.90 & 0.90 & 0.72 & 0.71 & 0.71 & 0.71 & 0.52 & 0.52 & 0.52 & 0.52 \\
\hline & 0.8 & 0.96 & 0.97 & 0.96 & 0.96 & 0.88 & 0.88 & 0.87 & 0.88 & 0.70 & 0.70 & 0.70 & 0.70 & 0.51 & 0.51 & 0.52 & 0.52 \\
\hline
\end{tabular}

Note: ICC = Intraclass Correlation Coefficient. 
Table 6 cont.

Average Design Effect values for the cross-level interaction: Poisson Distribution conditions $(\mathrm{m}=10, \mathrm{k}=100)$

\begin{tabular}{|c|c|c|c|c|c|c|c|c|c|c|c|c|c|c|c|c|c|}
\hline \multirow{4}{*}{$\begin{array}{c}\text { L1 } \\
\text { Effect }\end{array}$} & \multirow{4}{*}{$\begin{array}{c}\text { Interaction } \\
\text { Effect }\end{array}$} & \multicolumn{16}{|c|}{ ICC Values } \\
\hline & & \multicolumn{4}{|c|}{0} & \multicolumn{4}{|c|}{0.1} & \multicolumn{4}{|c|}{0.3} & \multicolumn{4}{|c|}{0.5} \\
\hline & & & & & & \multicolumn{8}{|c|}{ L2 Effect } & & & & \\
\hline & & 0 & 0.2 & 0.5 & 0.8 & 0 & 0.2 & 0.5 & 0.8 & 0 & 0.2 & 0.5 & 0.8 & 0 & 0.2 & 0.5 & 0.8 \\
\hline \multirow{4}{*}{0} & 0 & 1.05 & 1.06 & 1.05 & 1.05 & 0.96 & 0.96 & 0.95 & 0.96 & 0.74 & 0.75 & 0.75 & 0.74 & 0.54 & 0.54 & 0.54 & 0.54 \\
\hline & 0.2 & 1.05 & 1.05 & 1.05 & 1.05 & 0.95 & 0.95 & 0.95 & 0.95 & 0.74 & 0.75 & 0.75 & 0.75 & 0.54 & 0.54 & 0.53 & 0.54 \\
\hline & 0.5 & 1.04 & 1.04 & 1.04 & 1.04 & 0.94 & 0.94 & 0.94 & 0.94 & 0.74 & 0.74 & 0.74 & 0.74 & 0.53 & 0.53 & 0.53 & 0.53 \\
\hline & 0.8 & 1.01 & 1.01 & 1.01 & 1.01 & 0.91 & 0.91 & 0.91 & 0.91 & 0.72 & 0.72 & 0.72 & 0.72 & 0.52 & 0.52 & 0.52 & 0.52 \\
\hline \multirow{4}{*}{0.2} & 0 & 1.05 & 1.05 & 1.05 & 1.05 & 0.95 & 0.95 & 0.95 & 0.95 & 0.74 & 0.75 & 0.75 & 0.75 & 0.54 & 0.54 & 0.53 & 0.54 \\
\hline & 0.2 & 1.05 & 1.05 & 1.05 & 1.05 & 0.95 & 0.95 & 0.95 & 0.95 & 0.75 & 0.74 & 0.74 & 0.74 & 0.53 & 0.53 & 0.53 & 0.54 \\
\hline & 0.5 & 1.04 & 1.04 & 1.04 & 1.03 & 0.94 & 0.93 & 0.94 & 0.94 & 0.73 & 0.73 & 0.74 & 0.73 & 0.53 & 0.53 & 0.53 & 0.53 \\
\hline & 0.8 & 1.01 & 1.00 & 1.01 & 1.01 & 0.91 & 0.91 & 0.91 & 0.91 & 0.72 & 0.72 & 0.72 & 0.72 & 0.52 & 0.52 & 0.52 & 0.52 \\
\hline \multirow{4}{*}{0.5} & 0 & 1.04 & 1.04 & 1.04 & 1.04 & 0.94 & 0.94 & 0.94 & 0.94 & 0.73 & 0.74 & 0.74 & 0.74 & 0.53 & 0.53 & 0.53 & 0.53 \\
\hline & 0.2 & 1.04 & 1.04 & 1.04 & 1.03 & 0.94 & 0.93 & 0.94 & 0.93 & 0.73 & 0.73 & 0.73 & 0.74 & 0.53 & 0.53 & 0.53 & 0.53 \\
\hline & 0.5 & 1.02 & 1.02 & 1.02 & 1.02 & 0.92 & 0.92 & 0.92 & 0.92 & 0.73 & 0.73 & 0.73 & 0.73 & 0.53 & 0.53 & 0.53 & 0.53 \\
\hline & 0.8 & 0.99 & 0.99 & 0.99 & 0.99 & 0.90 & 0.90 & 0.90 & 0.89 & 0.71 & 0.71 & 0.71 & 0.71 & 0.52 & 0.52 & 0.52 & 0.52 \\
\hline \multirow{4}{*}{0.8} & 0 & 1.01 & 1.01 & 1.01 & 1.01 & 0.91 & 0.91 & 0.91 & 0.91 & 0.72 & 0.72 & 0.72 & 0.72 & 0.52 & 0.53 & 0.52 & 0.52 \\
\hline & 0.2 & 1.00 & 1.00 & 1.00 & 1.00 & 0.91 & 0.91 & 0.91 & 0.91 & 0.72 & 0.72 & 0.72 & 0.72 & 0.52 & 0.52 & 0.53 & 0.52 \\
\hline & 0.5 & 0.99 & 0.99 & 0.99 & 0.99 & 0.90 & 0.90 & 0.90 & 0.90 & 0.71 & 0.71 & 0.71 & 0.71 & 0.52 & 0.52 & 0.52 & 0.52 \\
\hline & 0.8 & 0.96 & 0.96 & 0.96 & 0.97 & 0.88 & 0.88 & 0.88 & 0.88 & 0.70 & 0.69 & 0.70 & 0.70 & 0.51 & 0.51 & 0.51 & 0.51 \\
\hline
\end{tabular}

Note: ICC = Intraclass Correlation Coefficient. 
Table 7.

Selected Articles from The Journal of Applied Psychology, Academy of Management Journal, and Personnel Psychology (2012- Spring 2015) Featuring Multilevel Models.

\begin{tabular}{|c|c|c|c|c|c|c|c|c|}
\hline Article & PY & Clusters & $\mathrm{N}$ & $\begin{array}{l}\text { Min. } \\
\text { Cluster } \\
\text { Size }\end{array}$ & $\begin{array}{c}\text { Max. } \\
\text { Cluster } \\
\text { Size }\end{array}$ & $\begin{array}{c}\text { Avg. } \\
m\end{array}$ & $\mathrm{SD}_{m}$ & ICC* \\
\hline Aime et al. & 2014 & 45 & 516 & 2 & 3 & - & - & - \\
\hline $\begin{array}{c}\text { Aryee et } \\
\text { al. }\end{array}$ & 2012 & 37 & 258 & - & - & - & - & 0.24 \\
\hline $\begin{array}{c}\text { Chang et } \\
\text { al. }\end{array}$ & 2014 & 238 & 1059 & 2 & 22 & 8.24 & - & 0.11 \\
\hline Chen et al. & 2015 & 31 & 238 & 4 & 10 & 7.9 & - & $.18, .28$ \\
\hline Chen et al. & 2013 & 95 & 428 & 3 & 14 & 4.51 & - & $.57, .09, .12$ \\
\hline Chen et al. & 2012 & 26 & 305 & - & 14 & - & - & $.30, .28$ \\
\hline $\begin{array}{c}\text { Chun \& } \\
\text { Choi }\end{array}$ & 2014 & 145 & 930 & 3 & 20 & 7.99 & 3.47 & 0.15 \\
\hline $\begin{array}{c}\text { Debus et } \\
\text { al. }\end{array}$ & 2012 & 24 & 15200 & - & - & - & - & 0.36 \\
\hline $\begin{array}{l}\text { deJong et } \\
\text { al. }\end{array}$ & 2014 & 73 & 334 & 3 & 13 & 4.6 & 2.2 & 0.28 \\
\hline $\begin{array}{l}\text { Dierdorff } \\
\& \\
\text { Morgeson }\end{array}$ & 2013 & 230 & 805 & - & - & - & - & $.04-.36$ \\
\hline $\begin{array}{l}\text { Dierdorff } \\
\& \\
\text { Ellington }\end{array}$ & 2012 & 64 & 338 & 4 & 6 & 5.23 & & - \\
\hline $\begin{array}{l}\text { * Studies wi } \\
\text { Morgeson } \\
\text { reported her } \\
\text { Table } 7 \text { con }\end{array}$ & $\begin{array}{l}\text { nult } \\
\text { 3) } n\end{array}$ & $\begin{array}{l}\text { depeno } \\
\text { led } 18\end{array}$ & $\begin{array}{l}\text { var } \\
\text { end }\end{array}$ & $\begin{array}{l}\text { es fea } \\
\text { variab }\end{array}$ & seve & $C C$ & es. & $\begin{array}{l}\text { erdorff \& } \\
\text { d ICCs is }\end{array}$ \\
\hline
\end{tabular}


Selected Articles from The Journal of Applied Psychology, Academy of Management Journal, and Personnel Psychology (2012- Spring 2015) Featuring Multilevel Models.

\begin{tabular}{|c|c|c|c|c|c|c|c|c|}
\hline Article & PY & Clusters & $\mathrm{N}$ & $\begin{array}{c}\text { Min. } \\
\text { Cluster } \\
\text { Size }\end{array}$ & $\begin{array}{l}\text { Max. } \\
\text { Cluster } \\
\text { Size }\end{array}$ & $\begin{array}{c}\text { Avg. } \\
m\end{array}$ & $\mathrm{SD}_{m}$ & ICC* \\
\hline Dong et al. & 2015 & 118 & 380 & - & - & - & - & 0.03 \\
\hline Eddy et al. & 2013 & 35 & 174 & 4 & 6 & 5 & - & $.36, .30, .29, .34$ \\
\hline $\begin{array}{l}\text { Farh \& } \\
\text { Chen }\end{array}$ & 2014 & 50 & 280 & 4 & 7 & 5.6 & - & $\begin{array}{c}.15, .26, .17, .17 \\
0.08\end{array}$ \\
\hline Fisher & 2014 & 337 & 6264 & - & - & 18.59 & - & 0.12 \\
\hline Gong et al. & 2013 & 100 & 485 & - & - & - & - & $\begin{array}{l}.24, .26, .19, .15 \\
.23, .18, .23, .16\end{array}$ \\
\hline $\begin{array}{l}\text { Gonzalez- } \\
\text { Mule et al. }\end{array}$ & 2014 & 102 & 1061 & - & - & 10.6 & 8.4 & 0.13 \\
\hline $\begin{array}{c}\text { Grandey et } \\
\text { al. }\end{array}$ & 2013 & 11 & 151 & - & - & 14 & - & - \\
\hline $\begin{array}{l}\text { Guillaume } \\
\text { et al. }\end{array}$ & 2014 & 69 & 316 & 4 & 5 & - & - & 0.15 \\
\hline $\begin{array}{c}\text { Hahn \& } \\
\text { Dormann }\end{array}$ & 2013 & 114 & 228 & 2 & 2 & 2 & - & 0.23 \\
\hline $\begin{array}{l}\text { Harman et } \\
\text { al. }\end{array}$ & 2014 & 25 & 160 & 1 & 17 & - & - & 0.28 \\
\hline
\end{tabular}

* Studies with multiple dependent variables feature several ICC values. $P Y=$ Publication Year. 
Table 7 cont.

Selected Articles from The Journal of Applied Psychology, Academy of Management Journal, and Personnel Psychology (2012-Spring 2015) Featuring Multilevel Models.

\begin{tabular}{|c|c|c|c|c|c|c|c|c|}
\hline Article & PY & Clusters & $\mathrm{N}$ & $\begin{array}{c}\text { Min. } \\
\text { Cluster } \\
\text { Size } \\
\end{array}$ & $\begin{array}{c}\text { Max. } \\
\text { Cluster } \\
\text { Size } \\
\end{array}$ & $\underset{m}{\text { Avg. }}$ & $\mathrm{SD}_{m}$ & ICC* \\
\hline Hirst et al. & 2015 & 11 & 223 & 14 & 68 & 25 & - & 0.18 \\
\hline $\begin{array}{l}\text { Hu \& } \\
\text { Liden }\end{array}$ & 2013 & 35 & 275 & 4 & 14 & 7.86 & - & 0.13 \\
\hline $\begin{array}{l}\text { Huang et } \\
\text { al. (Study } \\
\text { 1) }\end{array}$ & 2014 & 40 & 200 & 4 & 15 & 5 & 1.5 & 0.06 \\
\hline $\begin{array}{l}\text { Huang et } \\
\text { al. (Study } \\
\text { 2) }\end{array}$ & 2014 & 25 & 82 & 3 & 8 & 4.42 & 1.56 & 0.11 \\
\hline $\begin{array}{l}\text { Joshi \& } \\
\text { Knight }\end{array}$ & 2015 & 55 & 619 & - & - & - & - & - \\
\hline Liu et al. & 2012 & 175 & 5270 & - & - & 31 & 5.8 & - \\
\hline $\begin{array}{l}\text { Netemeyer } \\
\text { et al. }\end{array}$ & 2012 & 212 & 1484 & - & - & - & - & $.24, .33$ \\
\hline Probst & 2015 & 33 & 1238 & - & - & 37.52 & 78.17 & 0.22 \\
\hline $\begin{array}{l}\text { Raub \& } \\
\text { Liao }\end{array}$ & 2012 & 74 & 900 & - & - & - & - & - \\
\hline $\begin{array}{l}\text { Reiche et } \\
\text { al. }\end{array}$ & 2014 & 741 & 2111 & - & - & 3 & - & $.53, .49, .37, .36$ \\
\hline
\end{tabular}

* Studies with multiple dependent variables feature several ICC values. $P Y=$ Publication Year. 
Table 7 cont.

Selected Articles from The Journal of Applied Psychology, Academy of Management Journal, and Personnel Psychology (2012- Spring 2015) Featuring Multilevel Models.

\begin{tabular}{|c|c|c|c|c|c|c|c|c|}
\hline Article & PY & Clusters & $\mathrm{N}$ & $\begin{array}{l}\text { Min. } \\
\text { Cluster } \\
\text { Size }\end{array}$ & $\begin{array}{l}\text { Max. } \\
\text { Cluster } \\
\text { Size }\end{array}$ & $\underset{m}{\text { Avg. }}$ & $\mathrm{SD}_{m}$ & ICC* \\
\hline $\begin{array}{l}\text { Schaubroeck et } \\
\text { al. }\end{array}$ & 2012 & 78 & 172 & - & - & - & - & - \\
\hline Seo et al. & 2012 & 217 & 906 & 2 & 10 & 4.2 & - & 0.18 \\
\hline Stewart et al. & 2012 & 45 & 587 & - & - & 14 & - & $.40, .24$ \\
\hline Zhang et al. & 2012 & 74 & 361 & 4 & 7 & 4.88 & 1.24 & 0.35 \\
\hline Zhang et al. & 2014 & 88 & 339 & - & - & - & - & $.35, .20$ \\
\hline Zhou et al. & 2012 & 104 & 577 & 3 & 14 & 5 & - & 0.3 \\
\hline
\end{tabular}

* Studies with multiple dependent variables feature several ICC values. $P Y=$ Publication Year. 


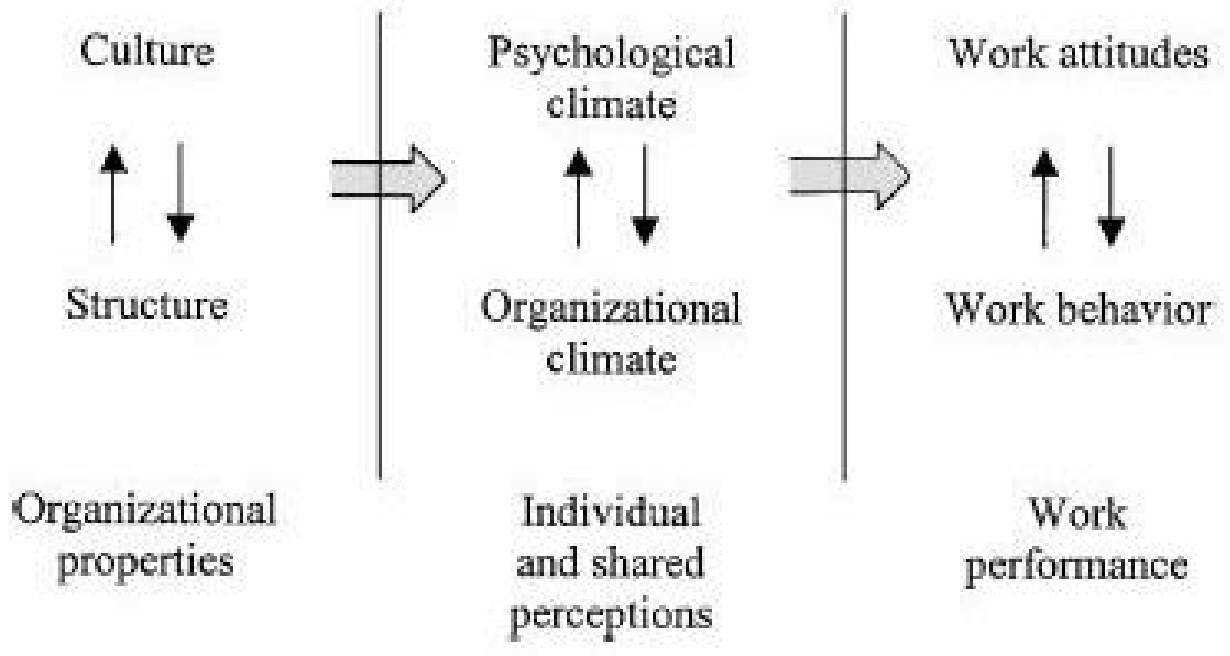

Figure 1. Model of Organizational Social Context proposed by Glisson (2002). 


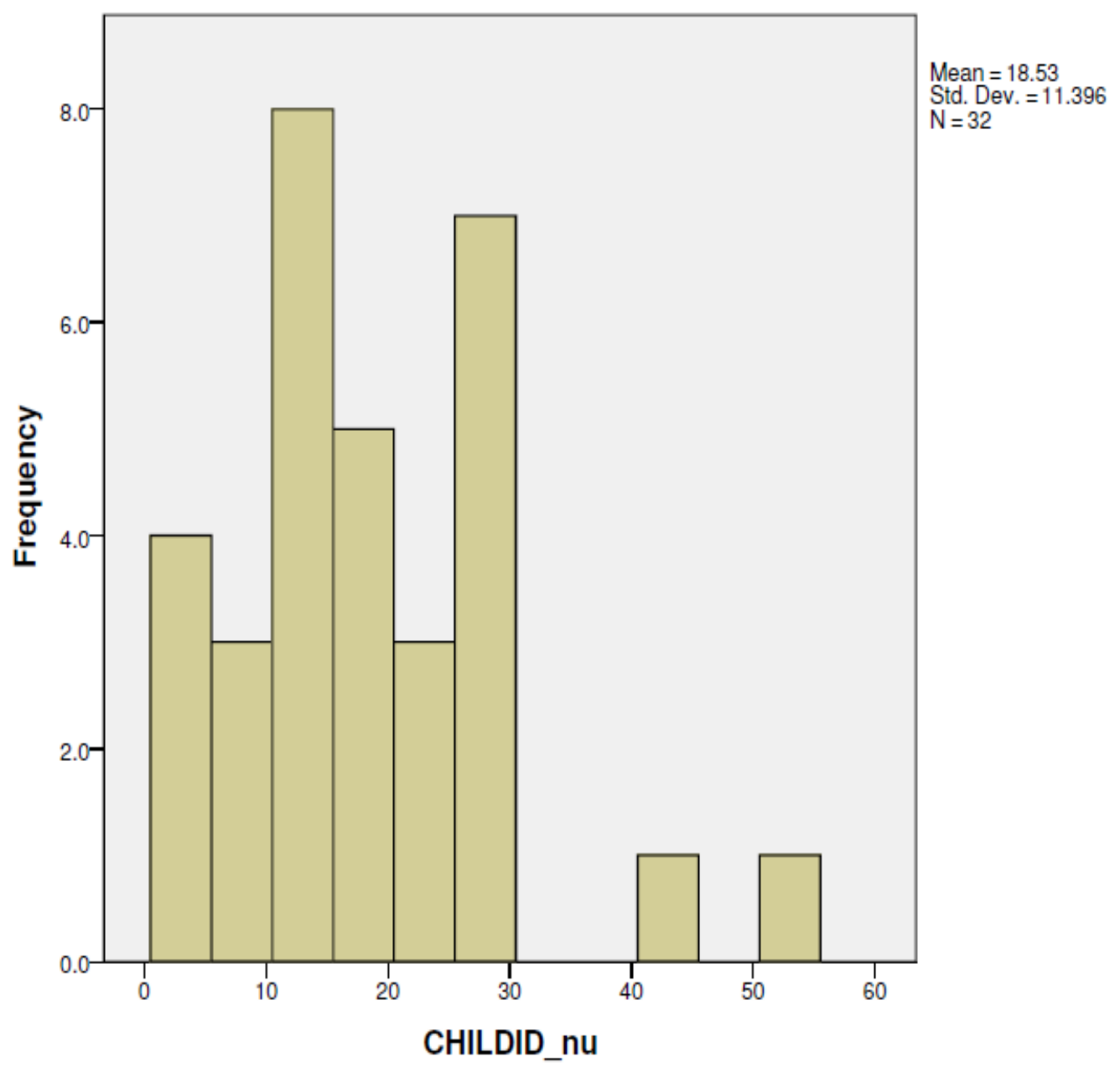

Figure 2. Distribution of matched parent/child respondents in the NAFASI dataset. Note: CHILDID_nu $=$ number of children per cluster. 


\section{REFERENCES}

Aguinis, H. \& Culpepper, S. A. (2015). An expanded decision-making procedure for examining cross-level interaction effects with multilevel modeling. Organizational Research Methods, 18, 155-176.

Aime, F., Humphrey, S., DeRue, D. S., \& Paul, J. B. (2014). The riddle of heterarchy: Power transformations in cross-functional teams. Academy of Management Journal, 57, 327-352.

Aryee, S., Walumbwa, F. O., Seidu, E. Y. M., \& Otaye, L. E. (2012). Impact of highperformance work systems on individual- and branch-level performance: Test of a multilevel model of intermediate linkages. Journal of Applied Psychology, 97, 287-300.

Chang, S., Jia, L., Takeuchi, R., \& Cai, Y. (2014). Do high-commitment work systems affect creativity? A multilevel combinatorial approach to employee creativity. Journal of Applied Psychology, 99, 665-680.

Chen, G., Farh, J. L., Campbell-Bush, E. M., Wu, Z., \& Wu, X. (2013). Teams as innovative systems: Multilevel motivational antecedents of innovation in R\&D teams. Journal of Applied Psychology, 98, 1018-1027.

Chen, X. P., Liu, D., \& Portnoy, R. (2012). A multilevel investigation of motivational cultural intelligence, organizational diversity climate, and cultural sales: Evidence from U.S. real estate firms. Journal of Applied Psychology, 97, 93-106.

Chen, Z., Zhu, J., \& Zhou, M. (2015). How does a servant leader fuel the service fire? A multilevel model of servant leadership, individual self identity, group competition climate, and customer service performance. Journal of Applied Psychology, 100, 511-521.

Chun, J. S. \& Choi, J. N. Members' needs, intragroup conflict, and group performance. Journal of Applied Psychology, 99, 437-450.

Clarke, P. (2008). When can group level clustering be ignored? Multilevel models versus single-level models with sparse data. Journal of Epidemiology and Community Health, 62, 752-758.

Debus, M. E., Probst, T. M., Konig, C. J., \& Kleinmann, M. (2012). Catch me if I fall! Enacted uncertainty avoidance and the social safety net as country-level moderators in the job-insecurity - job attitudes link. Journal of Applied Psychology, 97, 690-698. 
de Jong, J. P. \& Curseu, P. L., \& Leenders, R. T. A. J. (2014). When do bad apples not spoil the barrel? Negative relationships in teams, team performance, and buffering mechanisms. Journal of Applied Psychology, 99, 514-522.

Denison, D. R. (1990). Corporate culture and organizational effectiveness. New York, NY: John Wiley \& Sons, Inc.

Dierdorff, E. C. \& Ellingtion, J. K. (2012). Members matter in team training: Multilevel and longitudinal relationships between goal orientation, self-regulation, and team outcomes. Personnel Psychology, 65, 661-703.

Dierdorff, E. C. \& Morgeson, F. P. (2013). Getting what the occupation gives: Exploring multilevel links between work design and occupational values. Personnel Psychology, 66, 687-721.

Dong, Y., Liao, H., Chuang, A., Zhou, J., \& Campbell, E. M. (2015, March 16). Fostering employee service creativity: Joint effects of customer empowering behaviors and supervisory empowering leadership. Journal of Applied Psychology, Advance online publication.

Donner, A. \& Wells, G. (1986). A comparison of confidence interval methods for the intraclass correlation coefficient. Biometrics, 42, 401-412.

Eddy, E. R., Tannenbaum, S. I., \& Mathieu, J. E. (2013). Helping teams to help themselves: Comparing two team-led debriefing methods. Personnel Psychology, 66, 975-1008.

Eldridge, S. M., Ashby, D., \& Kerry, S. (2006). Sample size for cluster randomized trials: Effect of coefficient of variation of cluster size and analysis method. International Journal of Epidemiology, 35, 1292-1300.

Enders, C. K. \& Tofighi, D. (2007). Centering predictor variables in cross-sectional multilevel models: A new look at an old issue. Psychological Methods, 12, 121138.

Farh, C. I. C. \& Chen, Z. (2014). Beyond the individual victim: Multilevel consequences of abusive supervision in teams. Journal of Applied Psychology, 99, 1074-1095.

Farmer, S. M., Van Dyne, L., \& Kamdar, D. (2015). The contextualized self: How teammember exchange leads to coworker identification and helping OCB. Journal of Applied Psychology, 100, 583-595.

Fisher, D. M. (2014). A multilevel cross-cultural examination of role overload and organizational commitment: Investigating the interactive effects of context. Journal of Applied Psychology, 99, 723-736. 
Gabler, S., Haeder, S., \& Lahiri, P. (1999). A model based justification of Kish's formula for design effects for weighting and clustering. Survey Methodology, 25, 105-106.

Glisson, C. (2002). The organizational context of children's mental health services. Clinical Child and Family Psychology Review, 5, 233-253.

Glisson, C. \& James, L. R. (2002). The cross-level effects of culture and climate in human service teams. Journal of Organizational Behavior, 23, 767-794.

Glisson, C., \& Schoenwald, S.K. (2005). The ARC organizational and community intervention strategy for implementing evidence-based children's mental health treatments. Mental Health Services Research, 7, 243-259.

Gong, Y., Kim, T. Y., Lee, D.R., \& Zhu, J. (2013). A multilevel model of team goal orientation, information exchange, and creativity. Academy of Management Journal, 56, 827-851.

Gonzalez-Mule, E., DeGeest, D. S., McCormick, B. W., Seong, J. Y., \& Brown, K. G. (2014). Can we get some cooperation around here? The mediating role of group norms on the relationship between team personality and individual helping behaviors. Journal of Applied Psychology, 99, 988-999.

Goodman, R. (2001). Psychometric properties of the strengths and difficulties questionnaire. Journal of the American Academy of Child and Adolescent Psychiatry, 40, 1337-1345.

Gottfredson, D. C., Gerstenblith, S. A., Soule, D. A., Womer, S. C., \& Lu, S. (2004). Do after school programs reduce delinquency? Prevention Science, 5, 253-266.

Grandey, A. A., Chi, N. -W., \& Diamond, J. A. (2013). Show me the money! Do financial rewards for performance enhance or undermine the satisfaction from emotional labor? Personnel Psychology, 66, 569-612.

Guillaume, Y. R. F., Van Knippenberg, D., \& Brodbeck, F. C. (2014). Nothing succeeds like moderation: A social self-regulation perspective on cultural dissimilarity and performance. Academy of Management Journal, 57, 1284-1308.

Hahn, V. C. \& Dormann, C. (2013). The role of partners and children for employees' psychological detachment from work and well-being. Journal of Applied Psychology, 98, 26-36.

Harman, R. P., Ellington, J. K., Surface, E. A., \& Thompson, L. F. (2014, December 15). Exploring qualitative training reactions: Individual and contextual influences on trainee commenting. Journal of Applied Psychology, Advance online publication. 
Hartnell, C. A., Ou, A. Y., \& Kinicki, A. (2011). Organizational culture and organizational effectiveness: A meta-analytic investigation of the competing values framework's theoretical suppositions. Journal of Applied Psychology, 96, 677-694.

Hemmelgarn, A. L., Glisson, C., \& James, L. R. (2006). Organizational culture and climate: Implications for services and interventions research. Clinical Psychology: Science and Practice, 13, 73-89.

Hofstede, G. (1980). The cultural relativity of the quality of life concept. The Academy of Management Review, 9, 389-398.

Henry, G. T. (1990). Sample size. In Henry, G. T. (Ed.) Practical Sampling. p. 117-129. Thousand Oaks: Sage.

Hirst, G., Van Knippenberg, D., Zhou, J., Quintane, E., \& Zhu, C. (2015). Heard it through the grapevine: Indirect networks and employee creativity. Journal of Applied Psychology, 100, 567-574.

Hox, J. J. (2010). Multilevel Analysis: Techniques and applications. New York, NY: Routledge.

Hu, J. \& Liden, R. C. (2013). Relative leader-membership exchange within team contexts: How and when social comparison impacts individual effectiveness. Personnel Psychology, 66, 127-172.

Huang, X., Hsieh, J. J. P. A., He, W. (2014). Expertise dissimilarity and creativity: The contingent roles of tacit explicit knowledge sharing. Journal of Applied Psychology, 99, 816-830.

Ip, E. H., Wasserman, R., \& Barkin, S. (2011). Comparison of intraclass correlation coefficient estimates and standard errors between using cross-sectional and repeated measurement data: The safety check cluster randomized trial. Contemporary Clinical Trials, 32, 225-232.

Joshi, A. \& Knight, A. P. (2015). Who defers to whom and why? Dual pathways linking demographic differences and dyadic deference to team effectiveness. Academy of Management Journal, 58, 59-84.

Kish, L. (1965). Survey Sampling. New York: John Wiley \& Sons, Inc.

Kreft, I. G. G., de Leeuw, J., \& Aiken, L. S. (1995). The effect of different forms of centering in hierarchical linear models. Multivariate Behavioral Research, 30, 121. 
Lai, M. H. C. \& Kwok, O. (2015). Examining the rule of thumb of not using multilevel modeling: The "Design effect smaller than two" rule. Journal of Experimental Education, 83, 423-438.

Lauer, S. A., Kleinman, K. P., \& Reich, N. G. (2015). The effect of cluster size variability on statistical power in cluster-randomized trials. PLOS ONE, 10(4), 113.

Lazarsfeld, P. F. \& Menzel, H. (1971). On the relation between individual and collective properties. In A. Etzioni (ed.), A sociological reader on complex organizations, $\mathrm{p}$. 499-516.

LeBreton, J. M. \& Senter, J. L. (2008). Answers to 20 questions about interrater reliability and interrater agreement. Organizational Research Methods, 11, 815842 .

Littell, R. C., Milliken, G. A., Stroup, W. W., \& Wolfinger, R. D. (1999). SAS system for Mixed Models. Cary, NC: SAS Institute.

Liu, D., Mitchell, T. R., Lee, T. W., Holtom, B. C., \& Hinkin, T. R. (2012). When employees are out of step with coworkers: How job satisfaction trajectory and dispersion influence individual- and unit-level voluntary turnover. Academy of Management Journal, 55, 1360-1380.

Liu, Y., Wang, M., Chang, C. H., Shi, J. Zhou, L., \& Shao, R. (2015). Work-family conflict, emotional exhaustion, and displaced aggression toward others: The moderating roles of workplace interpersonal conflict and perceived managerial family support. Journal of Applied Psychology, 100, 793-808.

Luciano, M. M., Mathieu, J. E., \& Ruddy, T. M. (2014). Leading multiple teams: Average and relative external leadership influences on team empowerment and effectiveness. Journal of Applied Psychology, 99, 322-331.

McNeish, D. M. (2014). Modeling sparsely clustered data: Design-based, model-based, and single-level methods. Psychological Methods, 19, 552-563.

McNeish, D. M., Stapleton, L. M., \& Silverman, R. D. (2016). On the unnecessary ubiquity of hierarchical linear modeling. Psychological Methods. Advance online publication. DOI: $10.1037 /$ met0000078.

Metropolis, N. \& Ulam, S. (1949). The Monte Carlo method. Journal of the American Statistical Association, 44, 335-341. 
Muthen, B. O. \& Satorra, A. (1995). Complex sample data in structural equation modeling. Sociological Methodology, 25, 267-316.

Netemeyer, R. G., Heilman, C. M., \& Maxham, III, J. G. (2012). Identification with the retail organization and customer-perceived employee similarity: Effects on customer spending. Journal of Applied Psychology, 97, 1049-1058.

O’Connor, S. (1991). ASQ: Assessing school-age child care quality. Wellesley, MA: Wellesley College, Center for Research on Women.

Park, I. \& Lee, H. (2001, August). The design effect: What do we know about it? Paper presented at the annual meeting of the American Statistical Association

Paxton, P., Curran, P. J., Bollen, K. A., Kirby, J., Chen, F. (2001). Monte Carlo experiments: Design and implementation. Structural Equation Modeling, 8, 287312.

Peugh, J. L. \& Enders, C. K. (2005). Using the SPSS mixed procedure to fit crosssectional and longitudinal multilevel models. Educational and Psychological Measurement, 65, 717-741.

Pierce, K. M., Hamm, J. V., \& Vandell, D. L. (1999). Experiences in after-school programs and children's adjustment in first-grade classrooms. Child Development, 70, 756-767.

Probst, T. M. (2015, April 27). Organizational safety climate and supervisor safety enforcement: Multilevel explorations of the causes of accident underreporting. Journal of Applied Psychology. Advance Online Publication.

Raub, S. \& Liao, H. (2012). Doing the right thing without being told: Joint effects of initiative climate and general self-efficacy on employee proactive customer service performance. Journal of Applied Psychology, 97, 651-667.

Reiche, B. S., Cardona, P., Lee, Y. -T., \& Canela, M. A. (2014). Why do managers engage in trustworthy behavior? A multilevel cross-cultural study in 18 countries. Personnel Psychology, 67, 61-98.

Raudenbush, S. W. \& Bryk, A. S. (2002). Hierarchical Linear Models: Applications and data analysis methods. Thousand Oaks, CA: Sage.

Rosenthal, R., \& Vandell, D. L. (1996). Quality of Care at School-Aged Child-Care Programs: Regulatable Features, Observed Experiences, Child Perspectives, and Parent Perspectives. Child Development, 67, 2434-2445. doi:10.2307/1131632 
Sarndal, C. E., Swensson, B., \& Wretman, J. (1992). Model-Assisted Survey Sampling. New York: Springer-Verlag.

Schaubroek, J. M., Hannah, S. T., Avolio, B. J., Kozlowski, S. W. J., Lord, R. G., Trevino, L. K., Dimotakis, N., \& Peng, A. C. (2012). Embedding ethical leadership within and across organization levels. Academy of Management Journal, 55, 1053-1078.

Schein, E. H. (2010). Organizational culture and leadership. San Fransisco, CA: JosseyBass.

Seo, M. -G., Taylor, M. S., Hill, N. S., Zhang, X., Tesluk, P. E., \& Lorinkova, N. M. (2012). The role of affect and leadership during organizational change. Personnel Psychology, 65, 121-165.

Smith, C. A. B. (1956). On the estimation of intraclass correlation. Annals of Human Genetics, 21, 363-373.

Snijders, T. A. B. (2005). Power and sample size in multilevel modeling. In B. S. Everitt \& D.C. Howell (eds.) Encyclopedia of Statistics in Behavioral Science Volume 3 (pp. 1570-1573) Chicester (etc.): Wiley.

Snijders, T. A. B. \& Bosker, R. J. (2012). Multilevel Analysis: An introduction to basic and advanced Multilevel Modeling. Thousand Oaks, CA: Sage.

Stewart, G. L., Courtright, S. H., Barrick, M. R. (2012). Peer-based control in selfmanaging teams: Linking rational and normative influence with individual and group performance. Journal of Applied Psychology, 97, 435-447.

Susser, M. (1994). The logic in ecological: I. The logic of analysis. American Journal of Public Health, 84, 825-829.

van Mierlo, H., Vermunt, J. K, \& Rutte, C. G. (2009). Composing group-level constructs from individual-level survey data. Organizational Research Methods, 12, 368-392.

Vandell, D., Pierce, K., Brown, B., Lee, D., Bolt, D., Dadisman, K., Pechman, E., \& Reisner, E. (2006, March) Developmental outcomes associated with the afterschool contexts of low-income children and adolescents. Paper presented at the Society for Research on Adolescence Annual Meeting, San Fransisco.

Vandell, D. L., Shernoff, D. J., Pierce, K. M., Bolt, D. M., Dadisman, K., \& Brown, B. B. (2005). Activities, engagement, and emotion in after-school programs (and elsewhere). New Directions for Youth Development, 105, 121-129. doi:10.1002/yd.111 
Wang, L., \& Maxwell, S. E. (2015). On disaggregating between-person and withinperson effects with longitudinal data using multilevel models. Psychological Methods, 20, 63-83.

You, Z., Williams, D. O., Aban, I., Kabagambe, E. K., Tiwari, H. K., \& Cutter, G. (2011). Relative efficiency and sample size for cluster randomized trials with variable cluster sizes. Clinical Trials, 8, 27-36.

Zhang, Y., LePine, J. A., Buckman. B. R., \& Wei, F. (2014). It's not fair...or is it? The role of justice and leadership in explaining work stressor-job performance relationships. Academy of Management Journal, 57, 675-697.

Zhang, Z., Waldman, D. A., \& Wang, Z. (2012). A multilevel investigation of leadermember exchange, informal leader emergence, and individual and team performance. Personnel Psychology, 65, 49-78.

Zhou, L., Wang, M., Chen, G., \& Shi, J. (2012). Supervisors' upward exchange relationships and subordinate outcomes: Testing the multilevel mediation role of empowerment. Journal of Applied Psychology, 97, 668-680. 


\section{APPENDIX 1: SAS SIMULATION CODE}

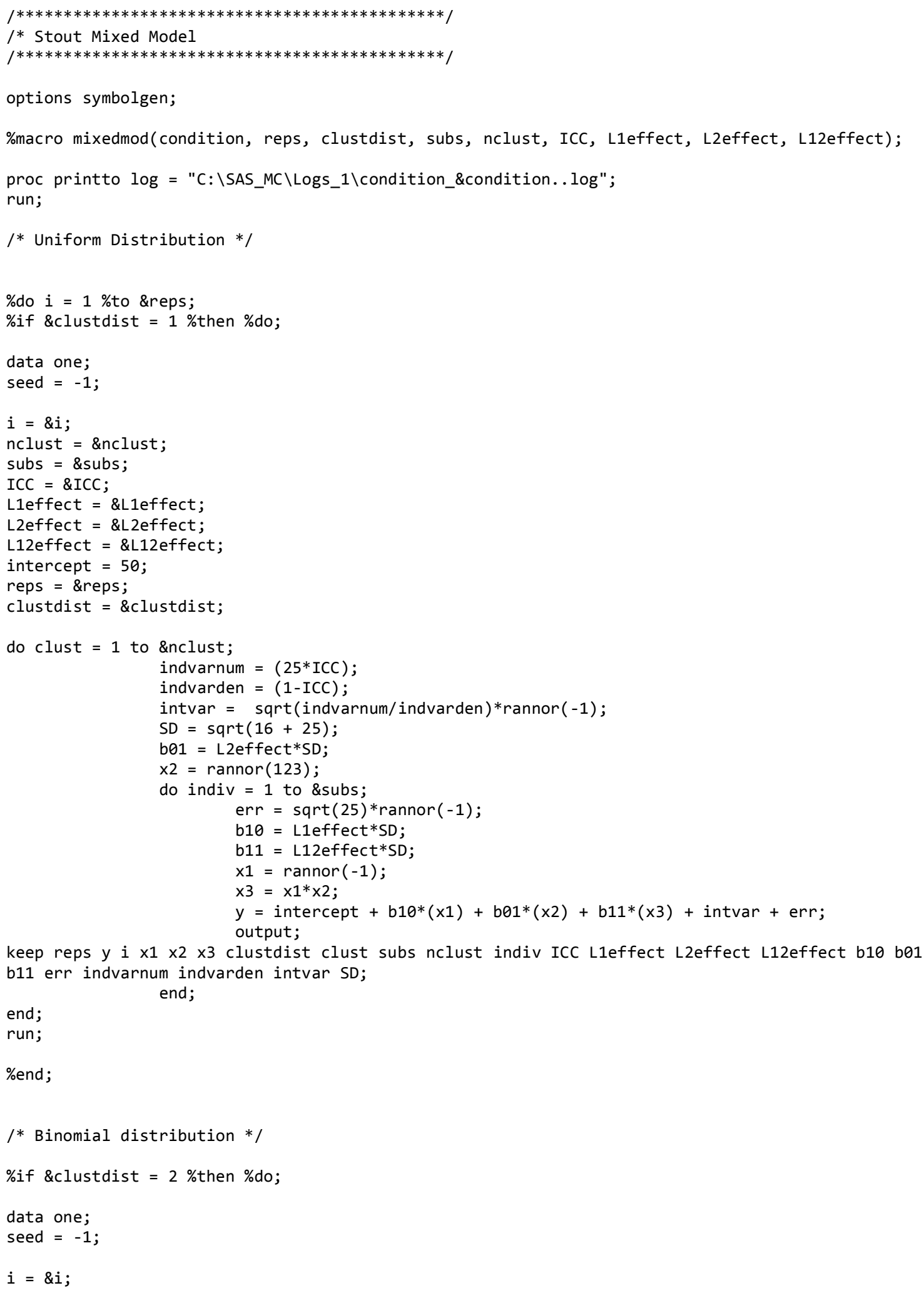




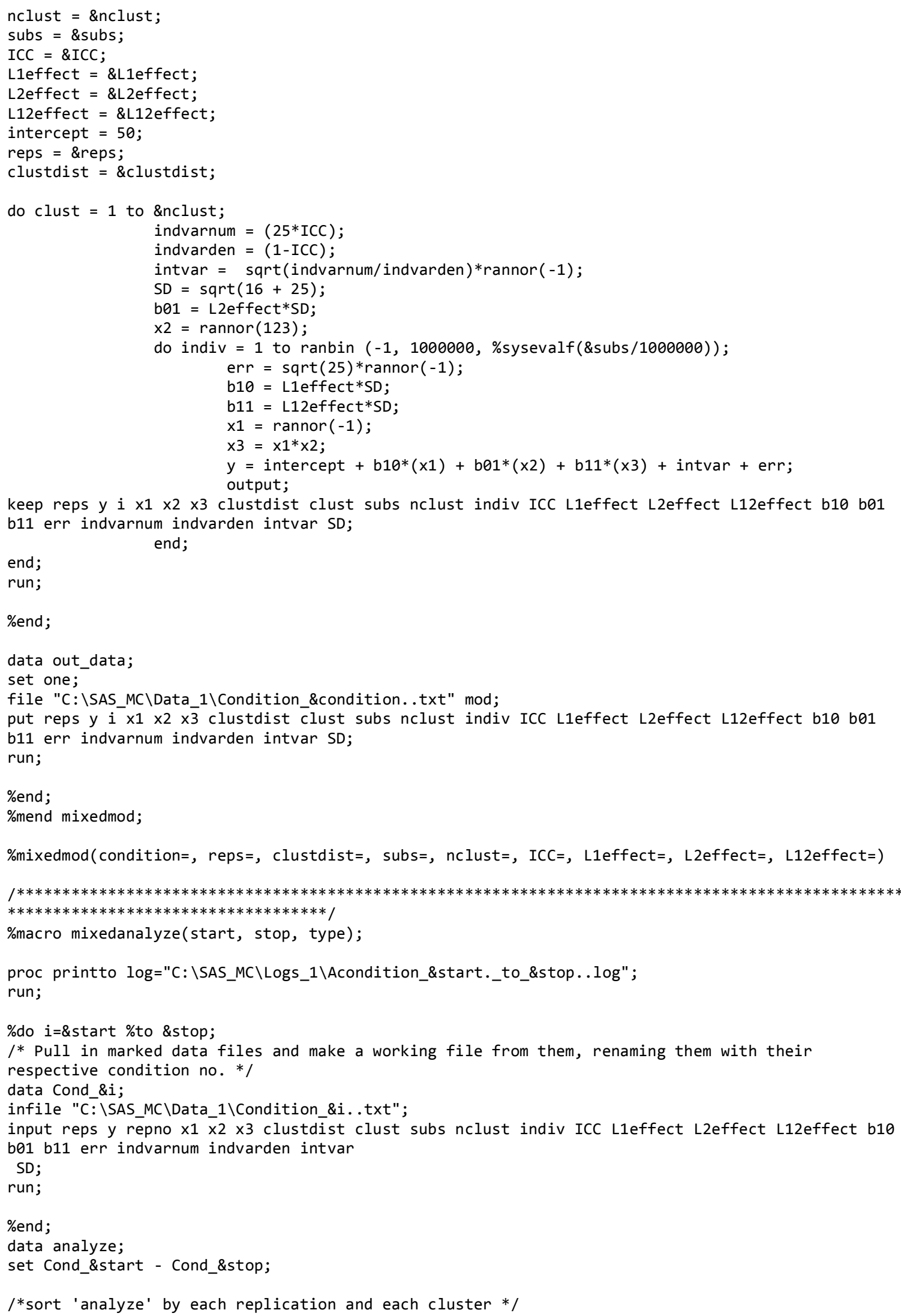




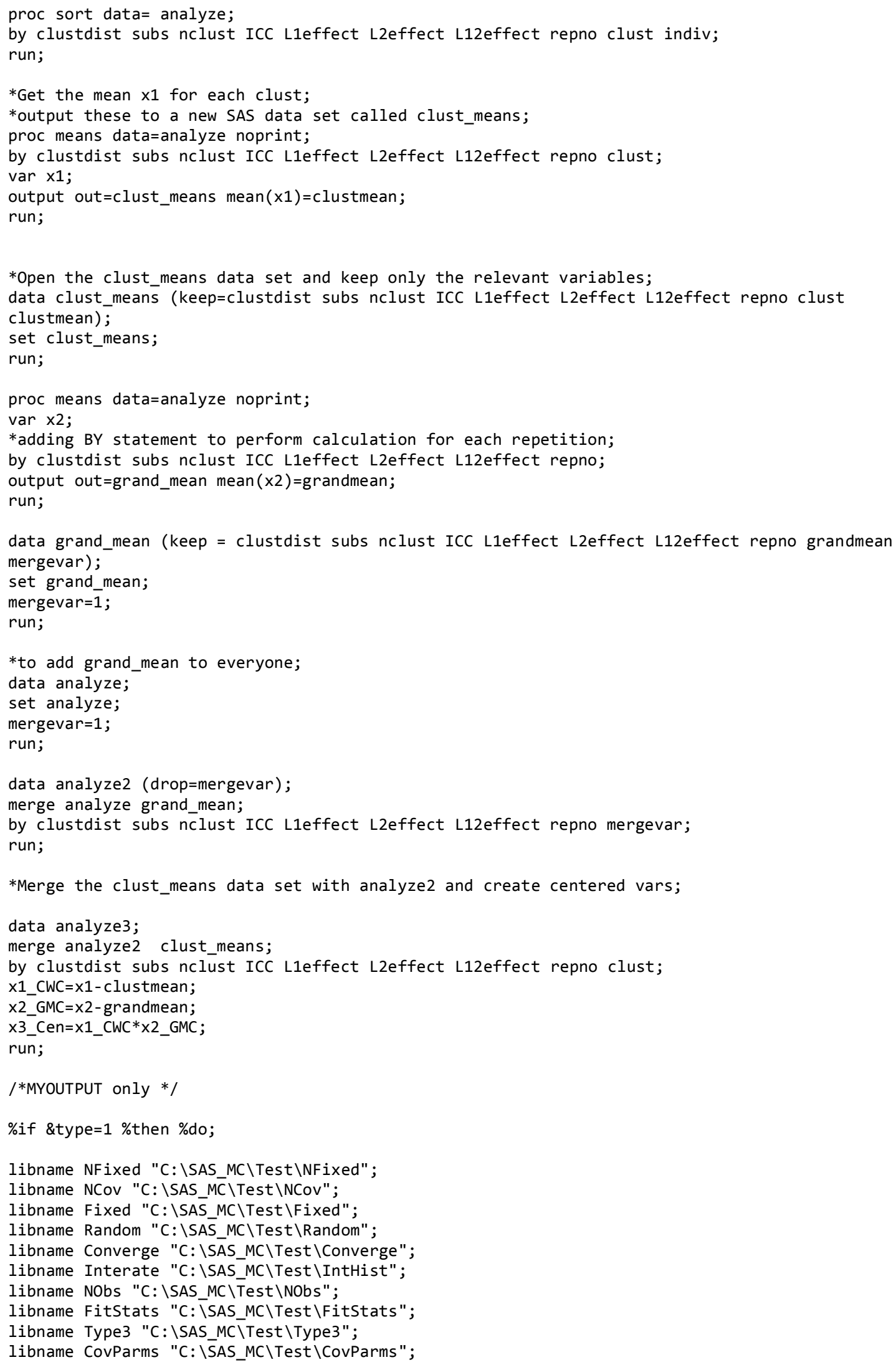




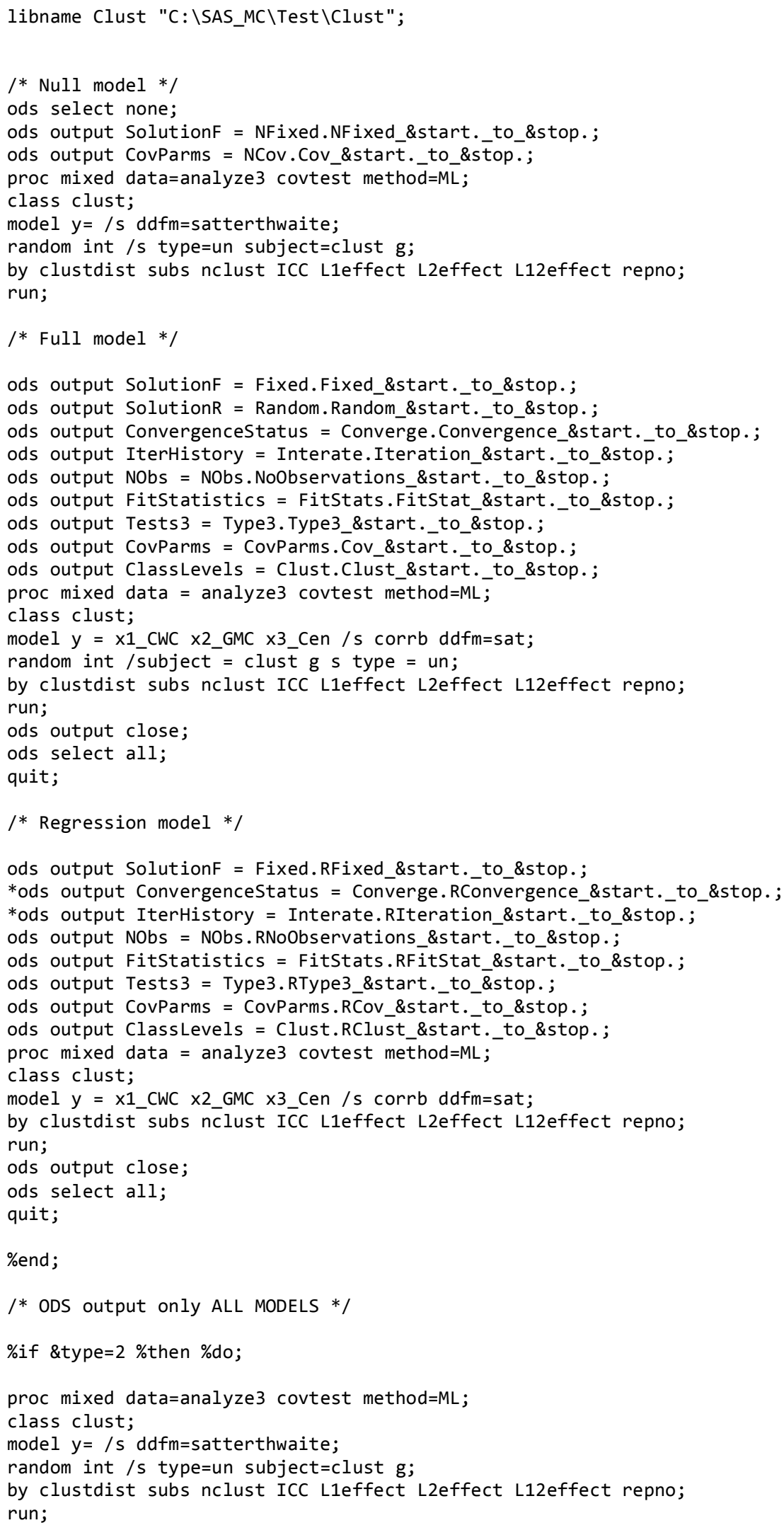




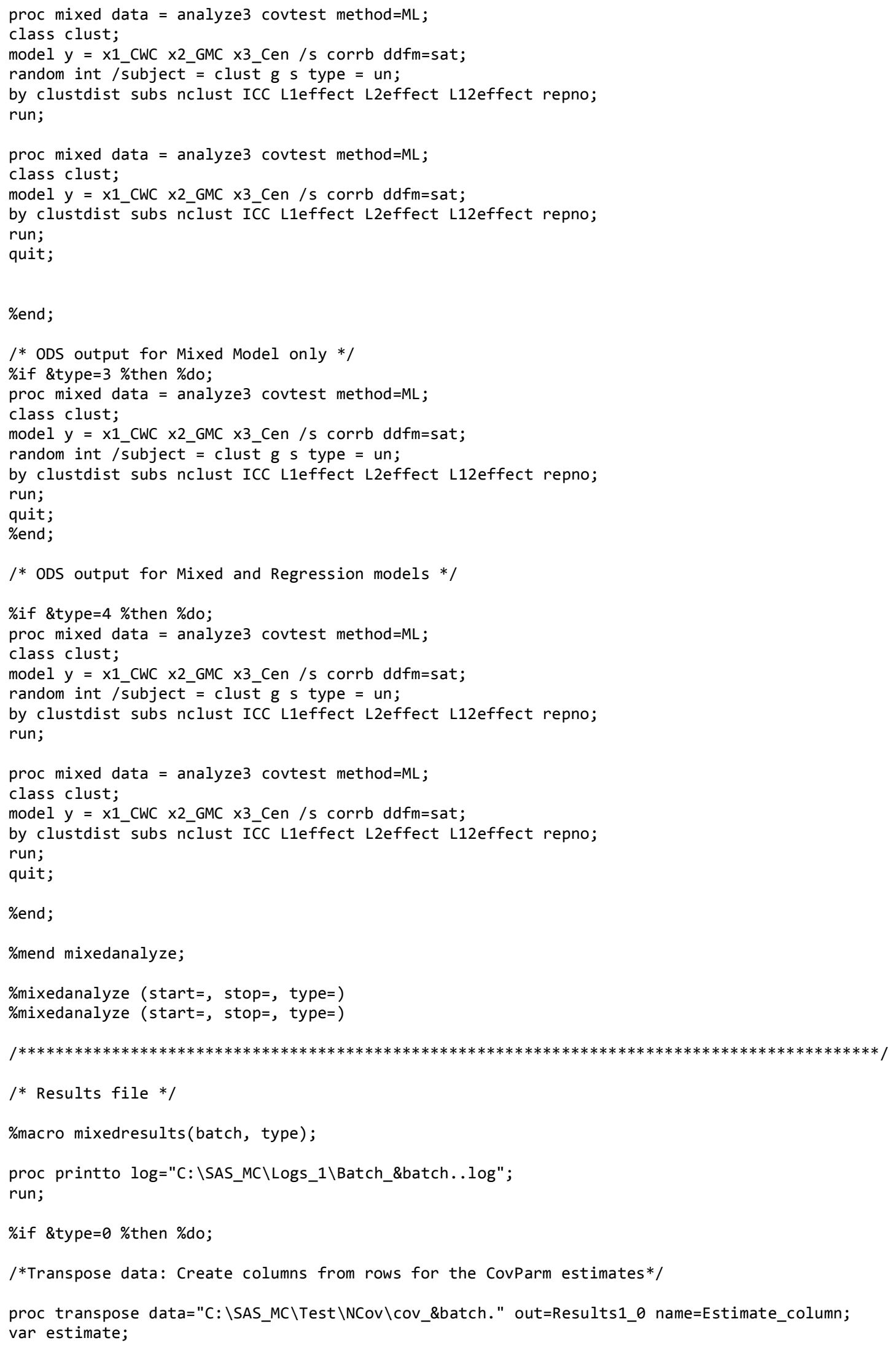




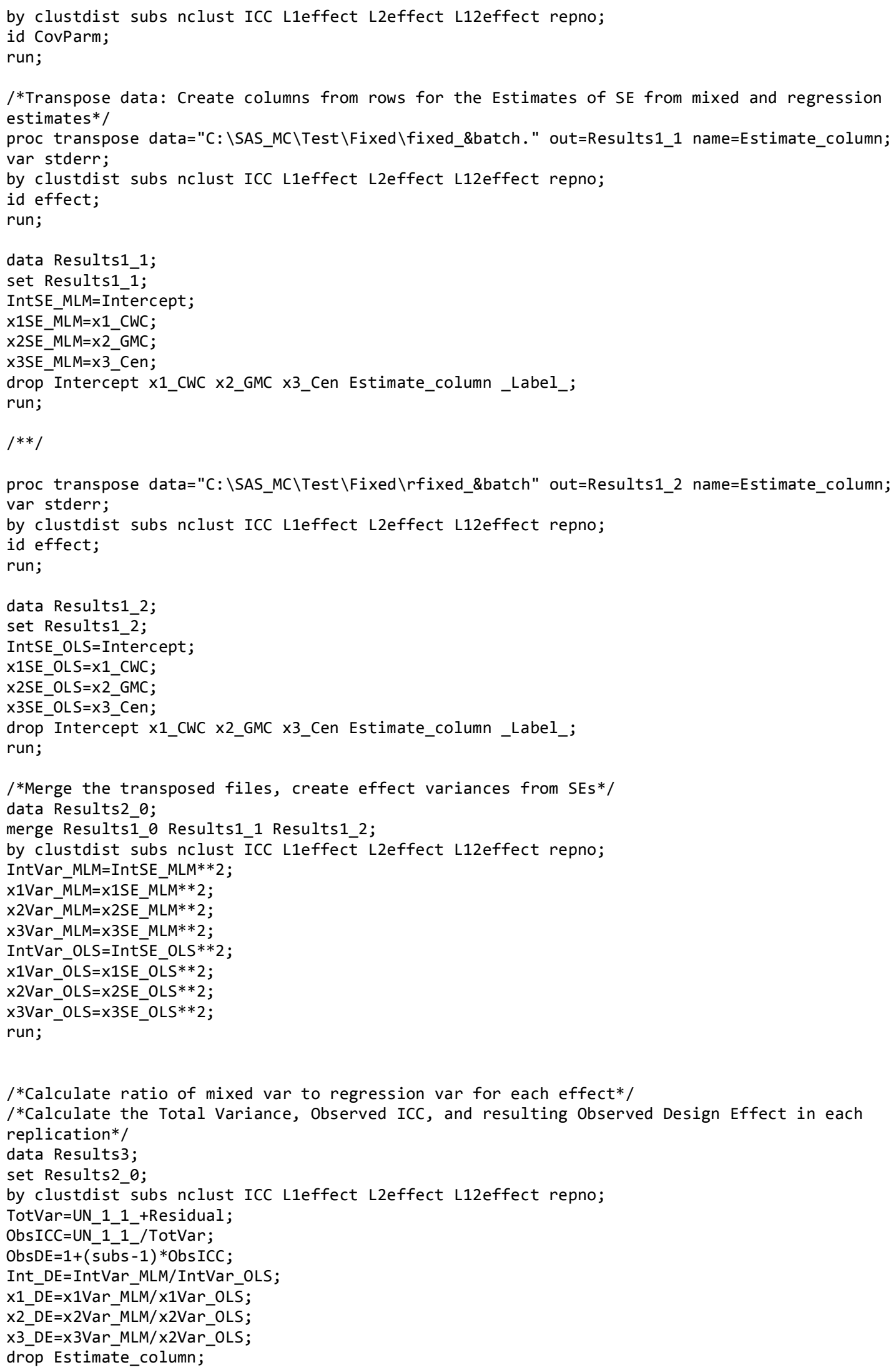




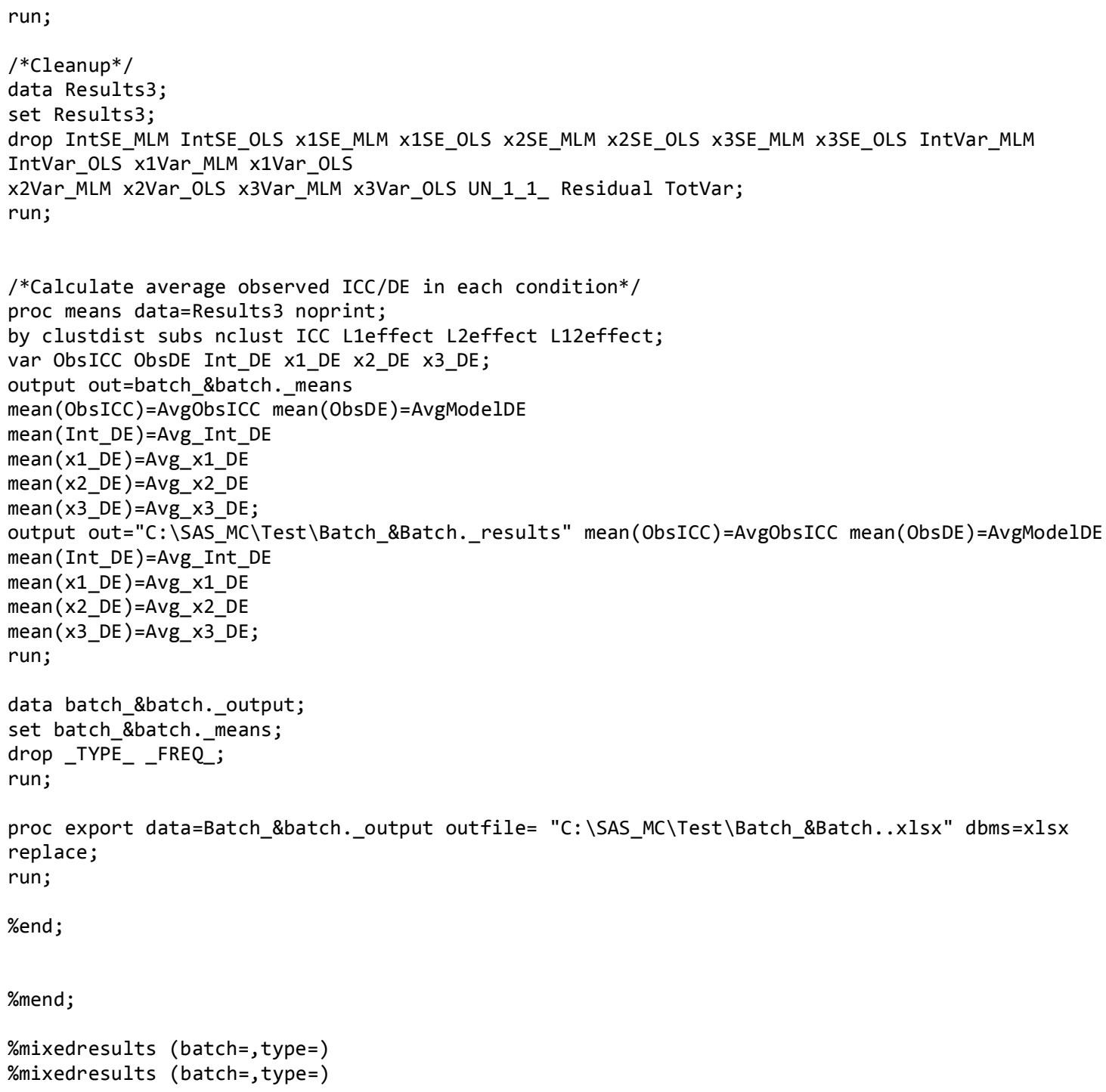


VITA

TYLER STOUT

\title{
EDUCATION AND INTERESTS
}

\author{
B.A. Psychology \\ Kansas State University \\ Manhattan, KS \\ M.S. Psychology \\ Florida International University \\ Miami, FL \\ Ph.D. Psychology \\ Florida International University \\ Miami, FL
}

- Substantive research interests include work-family conflict, engagement, flow, organizational culture and climate, stress, occupational health psychology, social support, talent poaching, motivation, and cross-cultural differences

- Quantitative research interests include modeling issues in mixed models, applied machine learning and data science, differences in measurement theory (i.e., CTT vs CFA vs IRT), estimation methods, differential item functioning, and ideal point vs dominance approaches to item response theory

\section{PUBLICATIONS AND PRESENTATIONS}

- Coxe, S. \& Stout, T. J. (2016, February) Missing data strategies for multilevel models. Poster presented at the Conference for Statistical Practice, San Diego, CA. February 18-20, 2016.

- Coxe, S. \& Stout, T. J. (2016, February) Missing data strategies for multilevel models. Poster presented at the Conference for Statistical Practice, San Diego, CA. February 18-20, 2016.

- Rudolph, C. W., Michel, J. S., Harari, M. B., \& Stout, T. J. (2014). Perceived social support and work-family conflict: A comparison of Hispanic immigrants and nonimmigrants. Cross Cultural Management: An International Journal, 21, 306-325.

- Frazier, S.L., Rusch, D., Coxe, S., Stout, T., Helseth, S.A., Glisson, C., \& Atkins, M.S. (In-progress) After-school programs and children's mental health: Organizational social context, program quality, and child outcomes. 
- Beyond CFA: Item Response Theory for Applied Research (2015, September)

- Workshop discussing basic Item Response Theory concepts, with a focus on estimating Graded Response Models in MPlus. Also discussion of Item Response Theory applications (CAT, DIF, etc.) and current research on the topic.

- Presented as inaugural workshop in the FIU Quantitative Psychology Workshop series. 